Studien aus dem Max-Planck-Institut

für ausländisches und internationales Sozialrecht

von Maydell/Pitschas/Pörtner/Schulte (Hrsg.)

\title{
Politik und Recht für Menschen mit Behinderungen in Europa und Asien
}

Unter den Bedingungen des demographischen Wandels kulturelle Voraussetzungen und Erklärungshypothesen 
Studien aus dem Max-Planck-Institut

für ausländisches und internationales Sozialrecht

Band 44 
Bernd von Maydell/Rainer Pitschas/Peter Pörtner/

Bernd Schulte (Hrsg.)

\section{Politik und Recht für}

Menschen mit Behinderungen in Europa und Asien

Unter den Bedingungen des demographischen Wandels kulturelle Voraussetzungen und Erklärungshypothesen 
Gefördert von der Robert-Bosch-Stiftung

Die Deutsche Nationalbibliothek verzeichnet diese Publikation in der Deutschen Nationalbibliografie; detaillierte bibliografische Daten sind im Internet über http://www.d-nb.de abrufbar.

Die Deutsche Nationalbibliothek lists this publication in the Deutsche Nationalbibliografie; detailed bibliographic data is available in the Internet at http://www.d-nb.de .

ISBN 978-3-8329-4798-9

\title{
Redaktion: Sarah Scholl
}

\begin{abstract}
1. Auflage 2009
(c) Nomos Verlagsgesellschaft, Baden-Baden 2009. Printed in Germany. Alle Rechte, auch die des Nachdrucks von Auszügen, der fotomechanischen Wiedergabe und der Übersetzung, vorbehalten. Gedruckt auf alterungsbeständigem Papier.
\end{abstract}

This work is subject to copyright. All rights are reserved, whether the whole or part of the material is concerned, specifically those of translation, reprinting, re-use of illustrations, broadcasting, reproduction by photocopying machine or similar means, and storage in data banks. Under $\S 54$ of the German Copyright Law where copies are made for other than private use a fee is payable to "Verwertungsgesellschaft Wort«, Munich. 


\section{Vorwort}

Das Recht der Rehabilitation und Teilhabe behinderter Menschen ist nicht erst mit der Verabschiedung des SGB IX vom Rand her in den Mittelpunkt des deutschen Sozialrechts gerückt. Es entfaltet als ein modernes Querschnittsgesetz ebenso Wirkungen auf das gesamte Sozialrecht, wie es vor allem das Schicksal zahlreicher Bürger in Deutschland bestimmt. Denn nahezu zehn Prozent der Bevölkerung sind schwerbehindert, davon etwa fünf Prozent seit ihrer Geburt. Wenn man davon ausgeht, dass jeder von ihnen zumindest über eine Betreuungsperson verfügt, gehört das Thema für jeden fünften Deutschen zur Lebenswirklichkeit. Diese ist allerdings durch eine tiefe Kluft zwischen der Normenwelt und der sozialen Wirklichkeit geprägt. Selbstbestimmung und gleichberechtigte Teilhabe behinderter Menschen sowie erfolgreiche Prävention vor einer Behinderung lassen erheblich zu wünschen übrig.

Aus dieser Situation resultiert zunächst ein ausgreifender Bedarf nach mehr Wissen über die grundlegenden Probleme der Umsetzung und des Vollzugs im Recht der Rehabilitation und Teilhabe behinderter Menschen. In Bezug auf die Problemlösungen geht es dabei um die Zusammenführung von sozialrechtlicher Dogmatik, Rechtssoziologie und Verwaltungswissenschaft, um bestandssichere Aussagen über den Rechtsvollzug durch die Sozialverwaltung in Verwaltungsverfahren und über die Wirkungsbedingungen des SGB IX zu treffen. Darüber hinaus und zweitens bleibt der Haushalt an einschlägigen Problemlösungen unter Berücksichtigung namentlich ausländischer Erfahrungen auszuschöpfen. Darin liegt die Aufforderung zum Rechtsvergleich, der bislang in Bezug auf die sozialrechtlichen Garantien für die Selbstbestimmung und gleichberechtigte Teilhabe behinderter Menschen allenfalls in der Europäischen Union (EU), kaum aber darüber hinausgehend mit Blick auf die übrige Staatenwelt begonnen hat. Schließlich und drittens muss sich dieser Vergleich auch auf die kulturellen Wurzeln des Rechts der Rehabilitation und Teilhabe behinderter Menschen beziehen. Dies wird deutlich, wenn man weiß, dass z.B. in Tansania noch heute Behinderungen als Strafe Gottes gelten und in der Volksrepublik China behinderte Kinder möglichst vor den Augen der Öffentlichkeit versteckt werden: Sozialpolitik und Sozialrecht für behinderte Menschen sind sowohl in der EU als auch in anderen Regionen dieser Welt kulturabhängig.

Zur Beantwortung der aufgeworfenen Fragen haben sich die ursprünglich drei heute vier - Herausgeber seit dem Jahr 2001 in einem gemeinsamen Projekt zusammengefunden, das gleichermaßen vom Max-Planck-Institut für ausländisches und internationales Sozialrecht in München (MPI) wie von der Deutschen Hochschule für Verwaltungswissenschaften (DHV) Speyer getragen wird. Aus der bisherigen gemeinsamen Arbeit an diesem Forschungsprojekt ist ein erster Tagungsband über die „Teilhabe behinderter Menschen an der Bürgergesellschaft in Asien und Europa (2002)“ und diesem folgend eine weitere umfangreiche Bestandsaufnahme zur „Be- 
hinderung in Asien und Europa im Politik- und Rechtsvergleich. Mit einem Beitrag zu den USA (2003)“ entstanden. Dabei hat sich deutlich gezeigt, in welchem Ausmaß die intra- und interdisziplinäre Arbeit zum Erkenntnisgewinn beitragen konnte. Die vielfältigen Informationen aufzuarbeiten und zu publizieren, hat allerdings einen längeren Zeitraum als gedacht in Anspruch genommen. So kann erst jetzt als Zwischenbilanz zu dem hiesigen Forschungsprojekt der Ertrag einer dritten Forschungskonferenz im Jahr 2007 vorgelegt werden, die - unter Erweiterung des Herausgeberkreises um einen Japanologen - gleichermaßen den „kulturwissenschaftlichen Hintergrund" staatendivergenter Politik und Rechtssetzung für Menschen mit Behinderung in Europa und Asien unter den Bedingungen des demografischen Wandels ausgeleuchtet als auch die Rechtsetzung als Lackmustest der Sozialstaatlichkeit in den genannten Weltregionen i. S. eines globalen Sozialstaates begriffen hat. Das Schwergewicht der vergleichenden Arbeit ist dabei auf die Europäische Union einerseits sowie auf Süd-, Südost- und Ostasien andererseits vor dem Hintergrund der sich immer stärker verdichtenden ökonomischen und sozialpolitischen Nähe beider Großregionen gelegt worden. Hiervon ausgehend dürfte es gelungen sein, die kulturellen und rechtlich-institutionellen Strukturen näher darzustellen, auf deren Hintergrund in anderen Staaten und Regionen funktionale Lösungen für die Probleme der Selbstbestimmung und gleichberechtigten Teilhabe behinderter Menschen in der jeweiligen Zivilgesellschaft zu ermitteln waren. Dem eiligen Leser, der sich über den Forschungsertrag informieren möchte, wird insoweit die Lektüre des Generalberichts empfohlen.

Der personelle und finanzielle Aufwand bei der Fortsetzung des Forschungsprojekts und für die Veranstaltung des Workshops im Herbst 2007 war (erneut) immens. Um so mehr ist deshalb der Robert Bosch Stiftung dafür zu danken, dass sie nicht nur die Forschungswerkstatt 2007 in München großzügig gefördert hat, sondern sich auch bereiterklärte, die Drucklegung dieses Bandes in jeder Hinsicht zu unterstützen. Zu dem Vorhaben konnten darüber hinaus und in bescheidenem Umfang das Max-Planck-Institut sowie die DHV Speyer mit ihren Ressourcen beitragen. Die Herausgeber, denen die inhaltliche Vorbereitung und Durchführung der Forschungswerkstatt oblag, danken darüber hinaus den Referentinnen und Referenten für die Zulieferung druckfertiger Manuskripte in überschaubarer Zeit; für die Unterstützung bei der Gestaltung der Tagung gilt unser besonderer Dank der studentischen Mitarbeiterin am MPI, Frau Sarah Scholl geb. Lempp, sowie den beteiligten Sekretärinnen Frau Roswitha Ellwanger in München sowie Frau Michaela Busche in Speyer.

München/Speyer, im Dezember 2008

Bernd von Maydell
Rainer Pitschas
Peter Pörtner
Bernd Schulte




\section{Inhaltsverzeichnis}

1. Behindertenrecht und Behindertenpolitik als Gegenstand vergleichender Betrachtung - Einführung in die Projektdiskussion Bernd von Maydell

2. Erscheinungsformen von Behinderung in einer alternden Gesellschaft:

Zur Kompetenz von älteren Menschen mit geistiger Behinderung Andreas Kruse

3. Kulturwissenschaftliche Überlegungen zur Behindertenpolitik in Asien?

- Einige sehr prinzipielle und unvorgreifliche Gedanken

Peter Pörtner

4. Entwicklung der Rechte behinderter Menschen in der Bundesrepublik Deutschland

4.1. Sozialpolitik im deutschen Sozialstaat seit der Vereinigung in ausgewählten Politikbereichen unter Einschluss des Behindertenrechts Werner Tegtmeier

4.2. Die Entwicklung der Rechte behinderter Menschen in der Bundesrepublik Deutschland - Eine Betrachtung aus Sicht der Bundesvereinigung Lebenshilfe für Menschen mit geistiger Behinderung Klaus Lachwitz

4.3. Behinderte Menschen in der kommunalen Sozialpolitik - Rehabilitation und Teilhabe junger Volljähriger sowie von Senioren im Zuständigkeits- und Leistungsgeflecht institutionalisierter Eingliederungshilfe nach dem Sozialhilfegesetzbuch -

Rainer Pitschas

4.4. Deutsche und Bayerische Politik für behinderte Menschen Burkard Rappl

4.5. Behindertenpolitik in Deutschland aus der Sicht behinderter Menschen

- Eine Streitschrift für eine bessere Behindertenpolitik aus Sicht eines Betroffenen und „Anwalts in eigener Sache“

Alexander Drewes 
5. Behindertenrecht und Behindertenpolitik im übrigen Europa

5.1. Vertretung der Interessen behinderter Menschen in der Sozialpolitik Belgiens

Jef van Langendonck

5.2. Die Maßnahmen für behinderte Menschen in den Niederlanden

Frans Pennings

5.3. Behindertenpolitik und Rechte behinderter Menschen in Schweden: „Riv Hindren“- „Beseitigt die Hindernisse“

Peter A. Köhler

5.4. Die Rolle der behinderten Menschen in der Zivilgesellschaft der Tschechischen Republik

Petr Tröster

5.5. Behinderte Menschen im türkischen Recht Yasemin Körtek

5.6. Die Rechte der behinderten Menschen in Russland: Zustandsanalyse unter Berücksichtigung der aktuellen Entwicklungen

Maksat Kachkeev

5.7. Teilhaberechte behinderter Menschen in den Mitgliedstaaten der Europäischen Union - Bericht über Leitbilder und Modelle

Peter Trenk-Hinterberger

5.8. Behindertenpolitik und Behindertenrecht in Europa: Behindertenpolitische und behindertenrechtliche Grundlegung: die Europäische Union Bernd Schulte

6. Behindertenrecht und Behindertenpolitik in Asien

6.1. Behinderte Menschen in der Volksrepublik China - Sicht einer Betroffenen

Ding Na

6.2. Politik und Recht der Eingliederung von behinderten Menschen in Indien William Gnanasekaran 
6.3. Behindertenrecht und Behindertenpolitik in Japan

6.3.1. Japan's Welfare System for People with Disabilities

Makoto Arai

6.3.2. Behinderung und Familie in Japan

Miyoko Motozawa

6.3.3. Die Rechtstellung behinderter Menschen in Japan - Unterschiede zwischen Deutschland und Japan

Hitohiro Takizawa

6.4. Behindertenrecht und Behindertenpolitik in Korea

6.4.1. Leistungen für behinderte Menschen in der Republik Korea

Kwang Seok Cheon

6.4.2. Bildungspolitik für junge behinderte Menschen in Korea und die Entwicklung von Gesetzgebung und Rechtsprechung

Soh-Yeon Won

6.5. The Foundation of Disabled Welfare Policy in Asia: Focusing on China Ming-Cheng Kuo

7. Generalbericht

Alexander Graser

Referenten- und Teilnehmerliste 


\title{
1. Behindertenrecht und Behindertenpolitik als Gegenstand vergleichender Betrachtung
}

Einführung in die Projektdiskussion

\author{
Von Bernd v. Maydell
}

1. Ursprünge 13

2. Zentrale Forschungsfragen 13

2.1. Besonderheiten des Risikos „Behinderung“ 13

2.2. Elemente eines Behindertenrechts $\quad 14$

2.2.1. Begriff des Behinderten 14

2.2.2. Ziele und Motive 15

2.2.2.1. Gewährleistung der öffentlichen Ordnung 16

2.2.2.2. Soziale Hilfe 16

2.2.2.3. Integration in das Erwerbs- und gesellschaftliche Leben 16

2.2.3. Gesellschaftliche Reaktionen 17

2.2.3.1. Verschiedene Wege der Reaktion 17

2.2.3.2. Inhalte von Behindertenhilfe 18

2.2.4. Akteure in der Behindertenpolitik 19

2.2.5. Rahmenbedingungen für Behindertenpolitik 19

2.2.6. Veränderungen der Rahmenbedingungen im Zeitablauf 20

2.2.6.1. Demographische Entwicklung 20

2.2.6.2. Globalisierung und Internationalisierung 21

3. Methodische Fragen 22

4. Bisherige Projektschritte 23

4.1. Forschungskonferenz in Speyer (2001) 23

4.2. Deutsch-japanischer Kongress in Berlin (2002) 23

4.3. Auswertung der Landesberichte 24

5. Zum weiteren Projektfortgang 25

5.1. Funktion des Münchner Workshops 25

5.2. Die (noch) zu behandelnden Forschungsfragen 25

5.2.1. Kulturwissenschaftlicher Hintergrund 25

5.2.2. Differenzierung nach Lebensabschnitten 25

5.2.3. Behinderung und Alterung der Bevölkerung 26

5.2.4. Koordination der verschiedenen Instrumente und Institutionen zur Förderung der Menschen mit Behinderung 27

5.2.5. Um- und Durchsetzung des gesetzten Rechts 27 
5.3. Vorbereitung des nächsten Kongresses

5.3.1. Auswahl der in den Mittelpunkt zu stellenden Forschungsfragen 28

5.3.2. Auswertung des Münchner Workshops

5.3.3. Asiatischer Länderschwerpunkt 


\section{Ursprünge}

Das Max-Planck-Institut für ausländisches und internationales Sozialrecht hat sich schon in den ersten Jahren seines Bestehens mit Behindertenrecht, vor allem im Zusammenhang mit der Pflegebedürftigkeit befasst ${ }^{1}$. Zacher hat insbesondere die Aufgaben, vor die die Behindertenproblematik die Rechtsordnung stellt, in einem umfassenden Ansatz untersucht ${ }^{2}$. In späteren Jahren ist diese Thematik zunächst im Institut nicht weiterverfolgt worden. Vor allem ist der ursprünglich auf Westeuropa bezogene $e^{3}$ rechtsvergleichende Ansatz nicht räumlich erweitert worden. Allerdings ergab sich im Laufe der Jahre eine steigende Nachfrage nach den europäischen und insbesondere den deutschen Erfahrungen und Lösungskonzepten aus anderen Teilen der Welt, vor allem aus Asien. Die Diskussion über die unterschiedlich ausgestalteten Behindertenrechte mit ausländischen Gästen führte immer wieder zu der Frage nach den Gründen für diese Unterschiede, die sich nicht nur auf die Instrumente sondern auch auf die sozialpolitische Rechtfertigung und die Intensität des Schutzes bezogen.

Abgesehen von diesem durch die Unterschiede in verschiedenen Teilen der Welt intensivierten Interesse an einem Rechtsvergleich führte auch die Binnensicht des deutschen Rechtszustandes ${ }^{4}$ mit seinen vielfältigen Verwerfungen und Unzulänglichkeiten zu der Frage nach besseren Lösungen, was wiederum den vergleichenden Ansatz nahe legte.

\section{Zentrale Forschungsfragen}

\subsection{Besonderheiten des Risikos „Behinderung“}

Die Behinderung ist im deutschen Sozialrecht nicht in einem selbstständigen eigenen Teilgebiet geregelt, vielmehr kann Behinderung als eine Beeinträchtigung, die sozialrechtliche Intervention notwendig macht, in verschiedenen Teilsystemen des Sozialrechts auftreten, in der Kriegsopferversorgung ebenso wie in der Unfallversicherung, der Krankenversicherung oder der Fürsorge. Behinderung lässt sich nicht auf eine einheitliche Ursache (wie z. B. Arbeitsunfall, Kriegsopferbeschädigung) zurückführen, sie kann bereits von Geburt an bestehen oder im Laufe des Lebens sich ergeben. Allerdings findet sich neuerdings eine Legaldefinition in SGB IX und

1 Siehe insb. Igl, Pflegebedürftigkeit und Behinderung im Recht der sozialen Sicherheit. Eine rechtsvergleichende Untersuchung für die Bundesrepublik Deutschland und Frankreich. Bd. V der Studien aus dem Max-Planck-Institut für ausländisches und internationales Sozialrecht, 1987.

2 Zacher, Die Lage der Behinderten: Eine Aufgabe des Sozialrechts, in: ZfS 1981, S. 257 ff.

3 Vgl. insb. die umfassende Studie von Igl (siehe Fn. 1).

4 Vgl. dazu nur die Dokumentation der Bundestagung des Deutschen Sozialrechtsverbandes e.V. am 27. und 28. September in Schwerin: „Die Behinderten in der sozialen Sicherung“, Schriftenreihe des Deutschen Sozialrechtsverbandes Bd. 49, 2002. 
- inhaltlich übereinstimmend - im Behindertengleichstellungsgesetz. Danach sind Menschen dann ,behindert, wenn ihre körperliche Funktion, geistige Fähigkeit oder seelische Gesundheit mit hoher Wahrscheinlichkeit länger als sechs Monate von dem für das Lebensalter typischen Zustand abweicht und daher ihre Teilnahme am Leben in der Gemeinschaft beeinträchtigt ist ${ }^{*{ }^{* 5}}$. Behinderung erfordert vielfältige sozialrechtliche Interventionen, wie Pflege, Rehabilitation oder finanzielle Leistungen zum Einkommensersatz; die Intervention erschöpfen sich aber nicht damit, vielmehr machen die Behinderungen Infrastrukturmaßnahmen, etwa im Verkehr oder bei der Einrichtung von Gebäuden notwendig, ebenso wie besondere Anpassungsstrategien bei der Ausbildung. Die für behinderte Menschen notwendige Regelungen und Maßnahmen sind damit über die gesamte Rechtsordnung verstreut ${ }^{6}$, sie umfassen aber auch den gesellschaftlichen Sektor. Insbesondere wird das mitmenschliche Miteinander gefordert, das sich nicht vollständig in rechtliche Ansprüche verfestigen lässt ${ }^{7}$. So vielfältig wie die Bedarfe und Bedürfnisse von Behinderten sind, so mannigfaltig sind auch die Institute und Regelungen, die zu ihrem Schutz und ihrer Unterstützung geschaffen werden oder geschaffen werden können. Eine bloß sektorspezifische Herangehensweise kann dieser Ausgangssituation nicht gerecht werden, vielmehr ist eine gesamtheitliche Betrachtung notwendig. Diese bereitet schon bei der Analyse eines einzigen nationalen Sicherungssystems Schwierigkeiten, die sich bei der vergleichenden Analyse potenzieren.

\subsection{Elemente eines Behindertenrechts}

Will man diese Gesamtheit erfassen und strukturieren, so muss man von den Fragestellungen ausgehen, die eine Rechtsordnung in Bezug auf Menschen mit Behinderung beantworten sollte.

\subsubsection{Begriff des Behinderten}

Jede vergleichende Untersuchung setzt eine Verständigung über den Lebenssachverhalt voraus, der der Untersuchung zugrunde gelegt werden soll. Eine erste Annäherung könnte darin bestehen, dass man von Menschen mit Behinderung dann spricht, wenn diese Menschen aufgrund körperlicher, geistiger oder psychischer

5 Vgl. Trenk-Hinterberger, Die Rechte behinderter Menschen und ihrer Angehörigen, 35. Aufl. 2007, S. 33, 34. Im 4. Bericht zur Lage der älteren Generation in der Bundesrepublik Deutschland wird allerdings eine Überprüfung dieses Behindertenbegriffs angeregt (S. 43).

6 Das gilt auch nach Schaffung des SGB noch, auch wenn die Rechtsgrundlage für Prävention, Beseitigung und Kompensation von Behinderung seit Inkrafttreten des SGB IX am 1.7.2001 übersichtlicher und systematischer geworden sind, worauf Welti (Schwerbehindertenrecht, in: SRH, 4. Aufl. 2008, § 27 Rz. 2) zutreffend hinweist.

7 Zu Recht fragt daher Zacher (siehe Fn. 2, S. 257): „Kann das Recht alle die Mittel mobilisieren, derer es bedarf, um den Behinderten alle Hilfen zu geben, die sie brauchen?“ 
Einschränkungen nicht oder nur vermindert in der Lage sind, am Arbeitsprozess und am gesellschaftlichen Leben teilzunehmen ${ }^{8}$. Diese Qualifizierung kann primär auf der Grundlage medizinischer Befunde, sie kann aber auch unter besonderer Berücksichtigung der aufgrund dieser Befunde bedingten beruflichen und gesellschaftlichen Einschränkungen erfolgen. Ausgehend von einem solchen allgemeinen Behindertenbegriff sind vielfältige Differenzierungen und Einschränkungen möglich. Es kann z. B. zwischen Behinderungen, die von Geburt an bestehen und später eintretende Behinderungen unterschieden werden, wobei diese Differenzierung in der gesellschaftlichen Wahrnehmung aber auch in den gesetzlichen Regelungen ihren Niederschlag finden kann. Bei den nachgeburtlichen Behinderungen kann man nach der Verursachung (beruflich, durch Unfall außerhalb der beruflichen Sphäre, durch Kriegs- oder Kriegsfolgen bedingt) unterscheiden, es kann aber auch allgemein final auf den vorliegenden Befund abgestellt werden. Eine weitere Unterscheidung knüpft an die Schwere der Behinderung an, wobei unterschiedliche Abstufungen möglich sind. Typischerweise spielt bei den Rechtsfolgen diese Differenzierung eine wichtige Rolle. So gelten häufig gewisse Rechtsfolgen nur für die Kategorie der Schwerbehinderten ${ }^{9}$, die ganz unterschiedlich definiert werden können.

Die Abgrenzung des Kreises der Behinderten, die für Teilbereiche des Behindertenrechts und in den einzelnen Staaten unterschiedlich erfolgen kann, erschwert die Vergleichbarkeit der Behindertenstatistik aber auch die qualitative Bewertung des Behindertenschutzes in den einzelnen Staaten. Innerhalb der Gruppe der behinderten Menschen in einer Gesellschaft bestehen Unterschiede auch hinsichtlich der Altersgruppe. Für Kinder und Heranwachsende, Erwerbstätige und aus dem Erwerbsleben ausgeschiedene Behinderte, insbesondere alte und sehr alte, können unterschiedliche, auf die jeweilige Lebenssituation abgestellte Regelungen bestehen, denen nicht ein einheitlicher Behindertenbegriff zugrunde liegen muss. Besonderer Schwerpunkt des Münchner Workshops liegt bei den älteren Behinderten ${ }^{10}$.

\subsubsection{Ziele und Motive}

Politik, die Behinderte zum Gegenstand hat, kann verschiedene Ziele verfolgen wobei diese Ziele ${ }^{11}$ jeweils von besonderen Motiven getragen werden.

$8 \quad$ Siehe bereits die Legaldefinition unter 2.1.

9 Vgl. für Deutschland die Regelungen im Teil 2 des SGB IX (Schwerbehindertenrecht).

10 Siehe dazu vor allem den Beitrag von Kruse, Erscheinungsformen von Behinderung in einer alternden Gesellschaft: Zur Kompetenz von älteren Menschen mit geistiger Behinderung, nachfolgend S. $29 \mathrm{ff}$.

11 Vgl. dazu Welti (siehe Fn. 6) Rz. 1 ff. 


\subsubsection{Gewährleistung der öffentlichen Ordnung}

Eine Wurzel des Fürsorgerechts ${ }^{12}$ ist das Bestreben, die von Armut ausgehende Störung der öffentlichen Ordnung zu beseitigen. Behinderung ist ein besonders sichtbarer Fall von Fürsorgebedürftigkeit, die staatliche Hilfsmaßnahmen erforderlich macht. Inwieweit diese Zielsetzung nach der Überlagerung durch andere Ziele der modernen Sozialpolitik heute noch eine Rolle spielt, mag dahinstehen.

\subsubsection{Soziale Hilfe}

Ziel der modernen staatlichen Sozialpolitik ist die Gewährung sozialer Hilfen dann, wenn der Einzelne sich nicht selbst helfen $\mathrm{kann}^{13}$. Das bedeutet, dass der Behinderte ohne eigenes Erwerbseinkommen eine Geldleistung erhält, die sicherstellt, dass ihm der Lebensbedarf zur Verfügung steht. Letztlich gilt dies auch für andere Leistungen an Behinderte.

\subsubsection{Integration in das Erwerbs- und gesellschaftliche Leben}

Allein durch soziale Hilfen können die Nachteile einer Behinderung nicht ausgeglichen werden. Vielmehr sollte der Behinderte in das Erwerbs- und das gesellschaftliche Leben eingegliedert und die Behinderung abgemildert, zumindest jedoch eine Verschlechterung verhindert werden. Dieses umfassende Ziel der Integration ${ }^{14}$ erfordert vielfältige Vorkehrungen in unterschiedlichen Lebensbereichen, etwa der Mobilität, der Ausbildung, des Arbeitslebens. Ein Tätigwerden des Gesetzgebers reicht nicht aus, vielmehr sind weitere Akteure gefordert, wie insbesondere die Arbeitgeber. Das Konzept einer umfassenden Integration der Menschen mit Behinderung kann abgeleitet werden aus einem weiterentwickelten Konzept von Sozialpolitik, das sich nicht auf die Gewährung von Sozialleistungen beschränkt.

Daneben ist jedoch auch ein anderer Ansatz möglich, der von dem Individuum und seiner Entfaltung ausgeht und Hindernisse für eine solche Entfaltung unter dem Aspekt der Diskriminierung bewertet. Schließlich kann eine Integrationspolitik auch ökonomisch motiviert werden. Wird der Behinderte in das Arbeitsleben eingegliedert $^{15}$, so wird er dadurch in die Lage versetzt, seinen Lebensbedarf ganz oder doch Hänlein/Tennstedt, Geschichte des Sozialrechts, in: SRH 4. Aufl. 2008, § 2 Rz. 2.

13 Diese Funktion der Sozialpolitik lässt sich bereits aus dem grundgesetzlich normierten sozialen Staatsziel ablenken, vgl. dazu Papier, Der Einfluss des Verfassungsrechts auf das Sozialrecht, in: SRH 4. Aufl. 2008, § 3 Rz. 9.

14 Zu der gesetzgeberischen Ausprägung dieses Ziels der Integration vgl. Welti (siehe Fn. 6) Rz. 6 ff.

15 Damit können auch arbeitsmarktpolitische Lenkungsinstrumente Bestandteil von Behindertenpolitik sein, vgl. dazu Welti (siehe Fn. 6) Rz. 4. 
zumindest teilweise durch eigene Arbeit sicher zu stellen und dadurch die öffentlichen Kassen zu entlasten, ganz abgesehen von dem gesteigerten Selbstwertgefühl, das eine solche Teilnahme am Arbeitsprozess vermittelt ${ }^{16}$.

Die bisherigen Projekterfahrungen deuten darauf hin, dass die gekennzeichneten unterschiedlichen Motivationen in den Vergleichsstaaten das Behindertenrecht in differenzierter Intensität beeinflusst haben, dass aber in vielen Staaten eine Kumulation festzustellen ist, die - jedenfalls auf den ersten Blick - eine Zuordnung des nationalen Systems zu einer der verschiedenen Motivationen erschwert.

\subsubsection{Gesellschaftliche Reaktionen}

Im speziellen Interesse des Rechtsvergleichs steht das „Wie“, die Frage also, wie eine Gesellschaft auf den Sachverhalt der Behinderung reagiert, wobei natürlich auch eine Reaktion in Form von Nichttätigwerden denkbar ist, weil die Behinderung als eine private, nur den Einzelnen bzw. die Familie betreffende Angelegenheit angesehen wird ${ }^{17}$.

Die Reaktionen kann man nach den eingeschlagenen Wegen differenzieren, aber auch nach den Inhalten.

\subsubsection{Verschiedene Wege der Reaktion ${ }^{18}$}

1. Zumeist wird das Regelwerk, das den Rahmen der Behindertenpolitik absteckt, durch den Gesetzgeber geschaffen: partielle Bereiche können auch durch die Tarifvertragsparteien geregelt werden. Insbesondere staatliche Regeln befassen sich mit der staatlichen Verpflichtung gegenüber den Behinderten. Adressaten des Regelwerks können auch private Organisationen oder Arbeitgeber, aber auch die Behinderten selbst sein, man denke nur an das Betreuungsrecht.

2. Die Lebensbedingungen von Behinderten werden maßgeblich durch die vorhandene Infrastruktur bestimmt ${ }^{19}$. Diese Infrastruktur (Verkehrswege, behindertengerechte Wohnungen etc.) werden durch den Staat, die Gebietskörperschaf-

16 Deshalb ist die Institution der Werkstatt für Behinderte auch außerhalb ihrer arbeitsmarktpolitischen Bedeutung so wichtig, vgl. dazu grundlegend: Institut für Sozialrecht (Hrsg.), Die Werkstatt für Behinderte. Ein interdisziplinärer Beitrag zur Rehabilitation der Behinderten, 1972.

17 Besonders extrem kann sich das auswirken, wenn in der gesellschaftlichen Anschauung die Behinderung eines Kindes ein schlechtes Licht auf die Familie wirft, wie es z. B. aus Indien berichtet wird, vgl. den Bericht von Schneider, Verloren und verdammt, in: Süddeutsche Zeitung Magazin vom 19.9.2008, S. 18 ff.. Vgl. im Übrigen die Länderberichte, die in den drei Kongressen des Projekts erstattet worden sind (siehe nachfolgend Fn. 36).

18 Dazu grundlegend Igl (siehe Fn. 1).

19 Die Bedeutung der Lebensumwelt für den Behinderten wird deutlich in Erfahrungsberichten der Betroffenen, vgl. etwa Dorner, Mein Dämon ist ein Stubenhocker. Aus dem Tagebuch eines Behinderten, 2008. 
ten aber auch durch private, etwa Arbeitgeber ${ }^{20}$ oder Vermieter geschaffen, zumeist in Realisierung des staatlichen Regelwerks. Fehlt die Infrastruktur, muss sie vom Behinderten selbst, seiner Familie oder in Nachbarschaftshilfe ersetzt werden.

3. Schließlich wird Behindertenpolitik durch finanzielle und persönliche Hilfen realisiert, die den Behinderten in die Lage versetzen sollen, seine fehlende oder eingeschränkte Erwerbsfähigkeit auszugleichen oder sich die notwendigen Hilfen zu beschaffen.

Die verschiedenen Wege einer Behindertenpolitik schließen sich nicht aus, sie treten vielmehr zumeist kumulativ auf, wobei die Gewichtung allerdings eine unterschiedliche ist, wie der Rechtsvergleich zeigt. Ob es eine ideale Gewichtung gibt, bleibt eine offene Frage.

\subsubsection{Inhalte von Behindertenhilfe}

Bereits die erwähnten Wege lassen erkennen, dass Behindertenhilfe unterschiedliche materielle Inhalte hat. Die Gewährleistung von Infrastruktur steht neben Geldleistungen sowie sozialen und persönlichen Diensten.

Eine andere Unterscheidung der Leistungen an und für behinderte Menschen setzt an der Lebenssituation an. Danach kann man differenzieren nach Leistungen in der Phase der Ausbildung ${ }^{21}$, der Berufsausübung oder der Arbeitslosigkeit und der Phase des Alters. Die Lebensphasen können von Bedeutung sein bei der Entstehung der Behinderung. So können die gesellschaftlichen Reaktionen unterschiedlich danach sein, ob es sich um eine Behinderung von Geburt an, um eine im Arbeitsleben zugezogene Behinderung oder um eine Altersbehinderung handelt, um nur drei typische Situationen zu nennen. Wachsende Bedeutung kommt in der alternden Gesellschaft den Behinderungen im Alter $z u$, insbesondere der Altersdemenz $z^{22}$, an der schon heute über eine Million alte Menschen in Deutschland leiden; für das Jahr 2050 rechnen Experten mit einer Verdoppelung ${ }^{23}$.

Das Leistungsspektrum ist für die verschiedenen Altersstufen typischerweise unterschiedlich ausgerichtet. Während bei der Behinderung von Geburt an ein Schwer-

20 Der Behindertenschutz wird im Rahmen des Arbeitsverhältnisses auch durch Mitbestimmung mitgestaltet, vgl. dazu Welti (siehe Fn. 6) Rz. 104-106.

21 So gibt es z. B. in verschiedenen Bundesländern Schulhilfen für behinderte Kinder, vgl. den Bericht von Köhler, in: Berliner Morgenpost vom 23.9.2008, S. 12.

22 Siehe den nachfolgenden Beitrag von Kruse, S. $29 \mathrm{ff}$.

23 Siehe den Bericht von Schmidt, Mediziner wollen Demenz-Abgabe, in: General-Anzeiger vom 22./23.5.2008, S. 4. Mit dieser Problematik beschäftigt sich die Wissenschaft und die Öffentlichkeit in Deutschland bislang eher zögerlich; vgl. allerdings den 4. Altenbericht der Bundesregierung und den großen Demenzkongress in Heidelberg am 19.5.2008, veranstaltet vom Institut für Gerontologie an der Universität Heidelberg. 
punkt bei der Ermöglichung einer optimalen Ausbildung ${ }^{24}$ liegt, liegt das Schwergewicht möglicher Maßnahmen im Falle von Behinderungen während des Berufslebens bei der Wiedereingliederung in den bisherigen oder einen zumindest adäquaten Beruf. Bei Altersbehinderung tritt die Rehabilitation gegenüber der Pflege häufig zurück. Es ist jedoch eine berechtigte Forderung, auch betagten Menschen mit Behinderungen Rehabilitationsmaßnahmen zu gewähren ${ }^{25}$.

\subsubsection{Akteure in der Behindertenpolitik}

Gerade der Rechtsvergleich zeigt, dass die Staaten hinsichtlich der Behindertenpolitik sich auch danach unterscheiden, wer die Akteure dieser Politik sind und wem die Durchführung obliegt. Neben dem Staat in seinen verschiedenen Organisationsgliederungen und den Religionsgemeinschaften sowie den gesellschaftlichen Organisationen $^{26}$ sind es vor allem die Familien, denen die Sorge für behinderte Menschen obliegt. Akteure sind schließlich auch die Behinderten selbst und ihre Organisatio$n^{27}{ }^{27}$. Das typische Bild, das sich bei der Analyse zeigt, ist ein Zusammenwirken dieser verschiedenen Akteure, wobei die jeweilige Gewichtung eine sehr unterschiedliche ist. Insbesondere das Maß, in dem der Staat sich in der Behindertenpolitik engagiert, ist in asiatischen Staaten deutlich geringer als in Europa. Ob die Gründe dafür in einem anderen Behindertenbild liegen, ist eine der Erkenntnisfragen, die durch das vorliegende Projekt beantwortet werden soll.

\subsubsection{Rahmenbedingungen für Behindertenpolitik}

Die Behindertenpolitik und das Behindertenrecht sind eingebunden ist das Rechts-, Wirtschafts- und Gesellschaftssystem des jeweiligen Staates. Es bestehen - zumeist wechselseitige - Abhängigkeiten und Einflüsse. Nur einige dieser Rahmenbedingungen sollen hier genannt werden.

1. Die Verfassung

Sie wirkt insbesondere mittels der Grundrechte in alle Rechtsbereiche und damit auch in das Behindertenrecht ein. Die nationale Verfassung kann durch supranationales und internationales Recht überlagert werden.

24 Aus der Fülle möglicher Maßnahmen seien nur die sozialpädagogische Begleitung von Kindern in der Grundschule genannt, wie sie z. B. in Bayern möglich ist, oder ein Mentoring für behinderte Studentinnen, gefördert von der Contergan-Stiftung für behinderte Menschen, durch das geholfen werden soll, die Hürden des Alltagslebens und der Karriereplanung zu meistern (vgl. den Bericht in: Gesundheit und Gesellschaft, Ausgabe 5/2008, S. 8). Zu den speziellen Konzepten für eine Schulbildung behinderter Kinder in Korea siehe den Beitrag von Won, in diesem Band S. 457 ff.

25 Siehe dazu nachfolgend Kruse, S. $29 \mathrm{ff}$.

26 So kann das Regelwerk für sportliche Wettbewerbe Behinderte diskriminieren, vgl. den Bericht von Reinsch, Eine Hand ist nicht genug, in: FAZ v. 19.4.2008, S. 32.

27 Vgl. dazu, soweit es Deutschland betrifft, die nachfolgenden Beiträge von Drewes und Lachwitz. 
2. Das allgemeine Rechts- und Verwaltungssystem

Dazu gehören, abgesehen von der Verfassung, nicht nur die gesetzten Normen sondern auch die Rechts- und Verwaltungskultur, von der die Durchführung des gesetzten Rechts entscheidend abhängt $t^{28}$.

3. Die wirtschaftlichen Verhältnisse, die den ökonomischen Rahmen für die Schaffung einer Infrastruktur zugunsten der Behinderten und für die Gewährung von Sozialleistungen abstecken.

4. Das Bestehen einer funktionierenden Zivilgesellschaft, die sich formiert und sich um das gesellschaftliche Miteinander und die Einbindung der Schwächeren kümmert.

5. Kulturelle und religiöse Traditionen, die das Gefühl der Verantwortlichkeit für die Mitmenschen begründen und prägen können.

Wie kaum ein anderer Bereich der Sozialordnung sind Behindertenrecht und Behindertenpolitik mit den Rahmenbedingungen, von denen vorstehend nur die wichtigsten genannt worden sind, verwoben. Das bedeutet aber, dass einerseits die Schaffung von Behindertenrecht von den Rahmenbedingungen abhängig ist, dass andererseits die Wirkung und das Funktionieren der geschaffenen Rechtsordnung für Behinderte ebenfalls nur in diesem Rahmen verstanden und bewertet werden kann.

\subsubsection{Veränderungen der Rahmenbedingungen im Zeitablauf}

Die Abhängigkeit des Behindertenrechts von den Rahmenbedingungen bedingt bereits bei statischer Betrachtung eine hohe Komplexität. Die Rahmenbedingungen sind jedoch, wenn auch in unterschiedlichem Maße, nicht statisch sondern verändern sich in der Zeit. Das soll an zwei Entwicklungen verdeutlicht werden, die Demographie und die Globalisierung.

\subsubsection{Demographische Entwicklung}

Die demographische Entwicklung, die Wirtschaft und Gesellschaft vor große Herausforderungen stellt, wird von zwei selbstständigen Faktoren bestimmt, die Geburtenhäufigkeit und die Lebenserwartung ${ }^{29}$. Die niedrigen Geburtenzahlen führen dazu, dass die Erwerbsbevölkerung im Verhältnis zu den alten und sehr alten Menschen schrumpft. Dadurch werden auch die sozialen Dienste beeinträchtigt, weil das dafür zur Verfügung stehende Potenzial kleiner wird. Das betrifft auch die Betreuung von Behinderten.

29 Hinzu kommt die Migration, die aber im Zusammenhang mit der Behindertenproblematik außer Betracht bleiben kann. 
Die Behindertenproblematik wird zusätzlich und in noch bedeutsamerer Weise durch die Alterung der Bevölkerung tangiert, und zwar in vielfacher Weise. Im Lebensverlauf des einzelnen Menschen mit Behinderung verändern sich die Beeinträchtigungen, die durch die Behinderung verursacht sind. Diese Entwicklung ist in den letzten Jahren für die Contergan-Geschädigten besonders registriert worden und hat zu einer Erhöhung der Zahlungen aus dem Stiftungsfonds an sie geführt. Dieser Trend gilt natürlich auch für andere Behinderte ${ }^{30}$.

Darüber hinaus hat die Steigerung der Lebenserwartung zur Folge, dass die Zahl der älteren und sehr alten Menschen zunimmt. Gerade für sehr alte Menschen (über 80jährige) gilt jedoch, dass typische Alterskrankheiten wie Alzheimer und andere Formen der Demenz überproportional zunehmen ${ }^{31}$. Dies bedeutet, dass es altersspezifische Behinderungen gibt, die zu den anderen Formen der Behinderung hinzutreten und zahlenmäßig immer gewichtiger werden. So wird die Zahl der Demenzkranken heute auf über 1 Mio. geschätzt; diese Zahl soll, so wird angenommen, bis zum Jahre 2050 auf über 2 Mio. steigen ${ }^{32}$. Die Versorgung dieser Altersbehinderten wird für die Gesellschaft eine zusätzliche gewaltige Aufgabe darstellen ${ }^{33}$.

\subsubsection{Globalisierung und Internationalisierung}

Die Sozialsysteme sind ursprünglich eine Einrichtung des Nationalstaates; das gilt auch in besonderem Maße für die Behindertenpolitik. Durch die Globalisierung der Finanz- und Warenmärkte und die Mobilität der Unternehmen und der Arbeitnehmer wird diese nationale Ausrichtung der Sozialsysteme zunehmend in Frage gestellt ${ }^{34}$. Tatsächlich gibt es auch für die Sozialpolitik Tendenzen einer Internationalisierung. In besonderem Maße gilt das für die Europäische Union, die sich auf dem Wege zu einer Sozialunion befindet ${ }^{35}$. Aber auch auf der universellen Ebene der UN und speziell der Internationalen Arbeitsorganisation gibt es solche Tendenzen, wenn auch in stark abgeschwächter Form. Ausprägungen der Internationalisierung sind vor allem die Schaffung supranationaler und internationaler Standards mit allerdings unterschiedlichem Verbindlichkeitsgrad. Daneben entwickelt sich ein internationaler Wettbewerb der Systeme, wie er im Rahmen der Europäischen Union vor allem durch die offene Methode der Koordinierung vorangetrieben werden soll. Darüber

30 Siehe dazu Havemann/Stöppler, Altern mit geistiger Behinderung, 2004.

31 Dazu das Statistische Bundesamt, Pressemitteilung vom 19.3.2008.

32 Vgl. den 4. Bericht zur Lage der älteren Generation in der Bundesrepublik Deutschland, der sich schwerpunktmäßig mit Risiken, Lebensqualität und Versorgung Hochaltriger - unter besonderer Berücksichtigung demenzieller Erkrankungen befasst.

33 Darauf wurde nachdrücklich auf dem Kongress „Lebensqualität bei Demenzerkrankung“ hingewiesen, der am 19. Mai 2008 in der Universität Heidelberg stattfand und vom Institut für Gerontologie, das von Prof. Andreas Kruse geleitet wird, organisiert wurde.

34 Vgl. etwa v. Maydell, in: Becker/v.Maydell/Nußberger (Hrsg.), Die Implementierung internationaler Sozialstandards, 2006, S. $11 \mathrm{ff}$.

35 Siehe dazu nachfolgend den Beitrag von Schulte, S. $307 \mathrm{ff}$. 
hinaus kommt dem Sozialrechtsvergleich auch im internationalen Rahmen bei der Transformation, der Modernisierung und der Reformierung der nationalen Sozialsysteme eine wachsende Bedeutung $\mathrm{zu}^{36}$.

\section{Methodische Fragen}

Die Komplexität der Behindertenproblematik macht deutlich, dass nur ein interdisziplinärer Einstieg es ermöglicht, den verschiedenen Dimensionen gerecht zu werden. Eine besondere Bedeutung kommt dabei, wie bereits die ersten Projektschritte nahegelegt haben, der kulturwissenschaftlichen Dimension ${ }^{37} \mathrm{zu}$. Dem wird in diesem Workshop Rechnung zu tragen sein.

Die Methodik wird im Übrigen dadurch bestimmt, dass das vorliegende Projekt vergleichend angelegt ist. Es sollen die Lösungsansätze in europäischen und asiatischen Staaten miteinander verglichen werden, um unterschiedliche und parallele Lösungsansätze und Regelungsmuster zu identifizieren. Für einen solchen vergleichenden Ansatz hat die Rechtswissenschaft die rechtsvergleichende Methodik entwickelt. Eine Erläuterung dieser Methodik kann hier nicht erfolgen. Es muss vielmehr genügen, auf die grundlegenden Arbeiten hinzuweisen, wie sie vor allem im Max-Planck-Institut entwickelt worden sind ${ }^{38}$.

Für den Rechtsvergleich ist die Auswahl der in den Vergleich einzubeziehenden Staaten eine entscheidende Frage. Die Auswahl wird sicherlich auch bestimmt durch die Verfügbarkeit von Experten aus den jeweiligen Staaten, wobei der Sprachenfrage eine wichtige Bedeutung zukommt. Letztlich sollten jedoch Kriterien, die sich aus der zu untersuchenden Sachproblematik ergeben, den Ausschlag geben. Die Kriterien, die Hinweise darauf ergeben haben, wo sich interessante Regelungen finden, haben sich bereits aus den Anfangsuntersuchungen ${ }^{39}$ ergeben. Im Übrigen sollen Staaten mit unterschiedlichem wirtschaftlichem Entwicklungsstand und unterschiedlicher Geschichte berücksichtigt werden.

Ein weiterer Sachgesichtspunkt ist der Umstand, dass in einem Staat Reformüberlegungen angestellt werden, die durch die Projekterkenntnisse unterstützt werden

Vgl. dazu v. Maydell/Nußberger (Hrsg.), Transformation von Systemen sozialer Sicherheit in Mittel- und Osteuropa, 2000.

37 Dazu nachfolgend der Beitrag von Pörtner, aber auch die Beiträge von Gnanasekaran und Ding Na.

38 Vgl. Zacher, Methodische Probleme des Sozialrechtsvergleichs. Colloquium der Projektgruppe für internationales und vergleichendes Sozialrecht der Max-Planck-Gesellschaft. Schritenreihe für internationales und vergleichendes Sozialrecht, Bd. 1, 1977; Ders. (Hrsg.), Sozialrechtsvergleich im Bezugsrahmen internationalen und supranationalen Rechts, Schriftenreihe für internationales und vergleichendes Sozialrecht, Bd. 2, 1978.

39 Siehe Pitschas/v. Maydell/Schulte (Hrsg.), Teilhabe behinderter Menschen an der Bürgergesellschaft in Asien und Europa - Eingliederung im Sozial- und Rechtsvergleich -, Speyrer Arbeitsheft Nr. 141, 2002; v. Maydell/Pitschas/Schulte (Hrsg.), Behinderung in Asien und Europa im Politikund Rechtsvergleich. Mit einem Beitrag zu den USA, Studien aus dem Max-Planck-Institut für ausländisches und internationales Sozialrecht, Bd. 26, 2003. 
können. Im Übrigen sind solche Reformpläne ein Indiz dafür, dass in dem jeweiligen Staat eine verstärkte Offenheit gegenüber den Problemen der Behinderten besteht. Schließlich sollten die für eine Region besonders wichtigen und bevölkerungsreichen Staaten berücksichtigt werden.

Der Workshop soll dabei helfen, diese Auswahlkriterien auf ihre Relevanz zu überprüfen und die Grundlagen für die nachfolgende asiatische Konferenz zu schaffen.

\section{Bisherige Projektschritte}

\subsection{Forschungskonferenz in Speyer (2001)}

Im Mittelpunkt der bisherigen Forschungsarbeit stand das Bemühen, durch Landesberichte Informationen aus europäischen und asiatischen Staaten über die Lage von Menschen mit Behinderung zu erlangen. Diesem Zweck diente eine erste Konferenz im Herbst 2001 in der Verwaltungshochschule Speyer. Die Berichte sind als Speyerer Arbeitsheft publiziert worden ${ }^{40}$. Sie offenbaren bei unterschiedlichen Ausgangsbedingungen und gesellschaftlichen und staatlichen Strukturen eine Palette von Strategien und umgesetzten Lösungen bei der Berücksichtigung von Bedarfen behinderter Menschen in den nationalen Gesellschaften Europas und Asiens. Zugleich lassen sich die strukturellen und institutionellen Ansätze erkennen, mit denen diese Gesellschaften und die jeweiligen Staaten auf nationale und internationale Forderungen nach Bedarfsdeckung reagiert haben. In Europa hat sich durch das Wachsen einer supranationalen Ordnung darüber hinaus ein Bestand an gemeinsamen Forderungen und - im Ansatz erkennbaren - Strukturen einer europäischen Behindertenpolitik entwickelt ${ }^{41}$. Politik und Recht für Menschen mit Behinderungen haben sich auf dieser Grundlage in Europa und Asien als eine Quelle von höchst unterschiedlichen Erfahrungen und Ansatzpunkten für die Formulierung von Forschungsfragen erwiesen.

\subsection{Deutsch-japanischer Kongress in Berlin (2002)}

Die Landesberichte waren damit Grundlage für eine zweite Forschungskonferenz im April 2002 in Zusammenarbeit mit dem Japanisch-Deutschen Zentrum Berlin $(\mathrm{JDZB})^{42}$. Was die Länderauswahl bei dieser zweiten Forschungskonferenz anbelangt, so wurden die europäischen und asiatischen Staaten ergänzt durch die USA,

41 Vgl. dazu Schulte, Behindertenpolitik und Behindertenrecht in der Europäischen Union, in: ZfSHSGB 2008, Heft 3, S. 131 ff. und Heft 4, S. 200 ff. 
weil in Anbetracht des stark durch den Diskriminierungsansatz geprägte amerikanische System zum Schutz behinderter Menschen sich interessante Aspekte für den Vergleich ergaben ${ }^{43}$.

Aus den nationalen Berichten waren Querschnittsthemen destilliert worden, die zum Gegenstand der Beratung des Berliner Kongresses gemacht wurden. In den Querschnittsthemen führten jeweils Referenten aus den verschiedenen europäischen und asiatischen Staaten ein. Die darauf fußenden weiterführenden Berichte und Querschnittsreferate der Berliner Konferenz sind in einer zweiten Veröffentlichung publiziert worden, die in der Schriftenreihe des Max-Planck-Instituts für ausländisches und internationales Sozialrecht erschienen ist ${ }^{44}$.

\subsection{Auswertung der Landesberichte}

Auf der Grundlage der beiden erwähnten Konferenzen und den umfangreichen Publikationen lassen sich bei allen Unterschieden in den Strategien und institutionellen Herangehensweisen an Problemlösungen doch letztlich ähnliche Entwicklungen in den relevanten Feldern der „Ausbildung von behinderten Kindern und Jugendlichen“, der „Teilhabe am Arbeitsleben“, dem „Umgang mit Behinderungen in alternden Gesellschaften“ sowie hinsichtlich der „Repräsentanz behinderter Menschen im gesellschaftlichen Leben“" erkennen. Die daraus resultierenden gleichartigen Fragestellungen sind weithin noch nicht bearbeitet. Hierfür dürfte vor allem verantwortlich zeichnen, dass die Forschungsziele bei der Erklärung der vorhandenen Divergenzen bislang diffus geblieben sind.

Die Forschungskonferenzen wurden vorbereitet und ausgewertet von den Projektverantwortlichen:

- Professor Pitschas von der Verwaltungshochschule Speyer,

- Professor v. Maydell und Dr. Schulte vom Max-Planck-Institut für ausländisches und internationales Sozialrecht und - später hinzutretend -

- Professor Pörtner, Japanologe an der Ludwig-Maximilians-Universität in München, der vor allem den kulturwissenschaftlichen Ansatz verstärken sollte.

Die Projektverantwortlichen trafen sich immer wieder zu ganz- oder halbtägigen Besprechungen. Insbesondere ist auch dieser Workshop auf diese Art und Weise vorbereitet worden. 


\section{Zum weiteren Projektfortgang}

\subsection{Funktion des Münchner Workshops}

Nachdem eine relativ lange Zeit seit dem letzten Projektschritt vergangen ist, soll der Münchner Workshop eine Zwischenbilanz ziehen und den das Projekt abschlieBenden Kongress in Asien vorbereiten. Insbesondere geht es darum,

- Die bisherigen Erkenntnisse zusammenfassen und zu analysieren,

- Die zentralen noch (oder weiter) zu behandelnden Forschungsfragen zu identifizieren und möglichst weitgehend zu präzisieren,

- Die Art und Weise, wie an diese Forschungsfragen herangegangen werden soll, zu klären.

\subsection{Die (noch) zu behandelnden Forschungsfragen}

\subsubsection{Kulturwissenschaftlicher Hintergrund}

Wie der Vergleich verschiedener Länder zeigt, ist die Wertung von Behinderung in den verschiedenen Gesellschaften eine sehr unterschiedliche. Behinderung kann als ein privates, nur die Familie betreffendes Schicksal, aber auch als Bestrafung der Familie $^{45}$ angesehen werden. Andererseits ist es auch möglich, dass die Hilfe und Förderung von behinderten Menschen als eine gesellschaftliche Aufgabe gesehen und anerkannt wird. $\mathrm{Ob}$ solche unterschiedliche Behindertenbilder auf religiösen oder kulturellen Rahmenbedingungen beruhen ${ }^{46}$, ist eine offene Frage, die weiterer Untersuchung bedarf. Ebenso ist zu beachten, dass das Individuum in verschiedenen Gesellschaften eine unterschiedliche Anerkennung erfährt ${ }^{47}$.

\subsubsection{Differenzierung nach Lebensabschnitten}

Die verschiedenen Lebensabschnitte (Jugend, Ausbildung, Erwerbsleben, Alter) können unterschiedliche Behindertenbilder bestehen, die mehr oder weniger mit den unterschiedlichen Regelungen korrespondieren können, die für die Behinderten in den verschiedenen Lebensabschnitten bestehen. Daraus folgt, dass der Vergleich sich auf einzelne Lebenssituationen beziehen kann, wie etwa die Behinderung im

47 Vgl. etwa den Bericht von Badde, „Ist der Behinderte denn nutzlos?“, in: Die Welt vom 5.2.2008, S. 10, in dem das Schicksal von autistischen Kindern in China geschildert wird. Aber auch in europäischen Staaten gibt es ,ein starkes gesellschaftliches Stigma gegen Menschen mit geistigen Behinderungen und ihren Familien“, so eine Studie der EU zur Lage in den Mitgliedstaaten unter Hinweis auf Bulgarien (siehe Meldung in SZ vom 30.1.2008, S. 8). 
Alter, für ein solches Vorgehen hat das Referat von $\mathrm{Kruse}^{48}$ eine wesentliche Fundierung geleistet.

Die bisherigen Projektarbeiten haben gezeigt, dass die nationalen Systeme den verschiedenen Lebensabschnitten ein unterschiedliches Gewicht beimessen. Dieser Befund, der noch verbreitert und vertieft werden sollte, ist als solcher bereits interessant; darüber hinaus stellt sich die Frage, inwieweit die Behinderung als solche, losgelöst vom Lebensalter des behinderten Menschen, im jeweiligen nationalen System als Hilfs- und Förderungstatbestand berücksichtigt wird.

\subsubsection{Behinderung und Alterung der Bevölkerung}

Die demographische Entwicklung, d. h. der Anstieg der Lebenserwartung und der Geburtenrückgang, wirkt sich auch auf Behindertenrecht und Behindertenpolitik aus, wie insbesondere in dem Beitrag von $\mathrm{Kruse}^{49}$ deutlich wird. Die Wirkung bezieht sich auf den Tatbestand der Behinderung, aber auch auf die gesellschaftlichen Reaktionen.

Der Tatbestand wird tangiert, weil die Alterung die Häufigkeit von spezifischen Formen der Altersbehinderung steigert. So wird vorhergesagt, dass sich die gegenwärtige Zahl von ca. 1 Mio. Demenzkranken bis zum Jahre 2050 mehr als verdoppeln dürfte ${ }^{50}$. Die Altersdemenz ist eine spezifische Form der Behinderung, die in besonderem Maße Pflegekapazitäten erfordert. Die dadurch notwendig werdenden Vorkehrungen sind bislang nur in Umrissen erkennbar und werden regelmäßig nicht eingeplant.

Die Alterung wirkt sich ebenfalls auf die bereits in früheren Jahren eingetretene Behinderung aus. Behinderte Menschen altern anders und zumeist früher, wie an den Contergan-Opfern zu beobachten ist. Die sich daraus ergebenden sozialpolitischen Folgerungen bedürfen noch einer Analyse und erfordern Änderungen der Gesetzgebung.

Gleichzeitig hat die demographische Entwicklung Auswirkungen auf das Angebot an Pflegediensten. Einerseits steigt durch die Zunahme von Altersbehinderungen die Nachfrage nach Pflege, andererseits führt die Schrumpfung der Erwerbsbevölkerung in Anbetracht des Geburtenrückgangs zu einer Verknappung von Pflegekräften. $\mathrm{Ob}$ in Anbetracht dieser Situation die Ersetzung von Pflegediensten durch technische Hilfsmittel oder sogar Automaten eine Entspannung herbeiführen kann, wird zu prüfen $\operatorname{sein}^{51}$. In Japan sind insoweit die technischen Entwicklungsarbeiten weiter

Siehe oben unter 2.2.3.2.

51 Zu der Bedeutung von technischen Hilfsmitteln für Behinderte vgl. Siebenbiedel, Die neue Freiheit hat vier Räder, in: Frankfurter Allgemeine Sonntagszeitung vom 11.5.2008, S. 46; Speziell zu Hilfsmitteln bei der Pflege siehe Strassmann, Die Zukunft liegt im Bett, in: Die Zeit vom 23.10.2008, S. 20. 
gediehen, als dies in Deutschland der Fall ist ${ }^{52}$. Bei einem Einsatz von Technik bei der Pflege muss es nicht darum gehen, dass Pflegekräfte dadurch überflüssig werden. Es könnte auch eine Umschichtung der Tätigkeiten dieser Pflegekräfte erfolgen weg von den manuellen Hilfsdiensten hin zu betreuender Zuwendung, wobei die manuellen Hilfsdienste von Maschinen erbracht werden könnten.

\subsubsection{Koordination der verschiedenen Instrumente und Institutionen zur Förderung der Menschen mit Behinderung}

Das Recht zum Schutz von Menschen mit Behinderungen wird dadurch gekennzeichnet, dass es sich nicht um ein geschlossenes einheitliches Gebiet handelt, sondern um eine Vielzahl verschiedener Institute und Institutionen in verschiedenen Lebensbereichen. Diese Vielfältigkeit muss den verschiedenen Lebenssituationen Rechnung tragen. Gleichzeitig bauen die Schutzregelungen für Behinderte auf dem allgemeinen System der Einrichtungen und Leistungen der Daseinsvorsorge auf. Daraus folgt für die Bewertung und den Vergleich verschiedener nationaler Systeme, dass es nicht genügen kann, einzelne Institutionen herauszugreifen und zu vergleichen. Vielmehr ist eine Gesamtschau notwendig, die die Integration der speziellen Schutzinstitution für Behinderte in das allgemeine Sicherungssystem ebenso berücksichtigt wie das Zusammenspiel der verschiedenen Institutionen zur Förderung von Menschen mit Behinderung ${ }^{53}$. Die Frage wie eine solche Gesamtschau gewonnen werden kann, ist auch für die weitere Projektarbeit eine Herausforderung.

\subsubsection{Um- und Durchsetzung des gesetzten Rechts}

Ein Vergleich, der nur das geschriebene Recht einbezieht, wäre unvollständig. Das gesetzte Recht bedarf zu seinem Wirksamwerden der Umsetzung durch eine funktionsfähige Verwaltung ${ }^{54}$ sowie der Durchsetzung durch die Betroffenen. Damit stellen sich eine Reihe weiterer Fragen für die Untersuchung.

Wie ist die Verwaltung, die mit der Umsetzung des Behindertenrechts betraut ist, organisiert? Ist der Verwaltungsvollzug effektiv? Inwieweit sind nichtstaatliche Organisationen in die Umsetzung der Rechtsnorm eingeschaltet? Haben insbesondere Organisationen der Behinderten Mitwirkungsrechte und wie ist diese Repräsen-

53 Dass ein solches koordiniertes Zusammenwirken verschiedener Institute und Hilfen zu Erfolgen bei der Integration führt, belegen viele individuelle Beispiele, von denen ein besonders eindrucksvolles der Sänger Thomas Quasthoff ist, siehe seine Autobiographie „Die Stimme“, 2006. Dieses Beispiel belegt aber auch gleichzeitig, dass Fehlschläge in der Ausbildung durch eine konsequente Unterstützung durch die Familie ausgeglichen werden können.

Siehe dazu insbesondere den Beitrag von Pitschas, nachfolgend S. $103 \mathrm{ff}$. 
tanz der Behinderten organisiert ${ }^{55}$ ? Welche Möglichkeiten hat schließlich der einzelne Behinderte, die ihm eingeräumten Rechte durchzusetzen? Und welche Unterstützung erhält er dabei?

\subsection{Vorbereitung des nächsten Kongresses}

\subsubsection{Auswahl der in den Mittelpunkt zu stellenden Forschungsfragen}

Die wenigen Hinweise haben deutlich gemacht, dass es eine Vielzahl von Forschungsfragen gibt, die im Behindertenrecht und der Behindertenpolitik noch einer vertiefenden Behandlung bedürfen, insbesondere unter dem vergleichenden Blickwinkel. Es wird nicht möglich sein, alle diese Fragen in der nächsten Konferenz umfassend zu behandeln. Vielmehr wird es notwendig sein, eine Auswahl zu treffen. Dabei kommt dem Münchner Workshop eine wichtige Funktion $\mathrm{zu}^{56}$. Neben den speziellen Problemen sollte die Analyse der Entwicklung des Behindertenrechts in den in die bisherige Untersuchung einbezogenen Staaten mitberücksichtigt werden, auch wenn die allgemeinen Landesberichte nicht mehr im Mittelpunkt stehen können.

\subsubsection{Auswertung des Münchner Workshops}

In Erweiterung der bisherigen Fragestellung befasst sich der Münchner Workshop schwerpunktmäßig, wenn auch nicht ausschließlich, mit den kulturwissenschaftlichen Rahmenbedingungen und den Auswirkungen der Alterung der Bevölkerung für die Behindertenpolitik. Die Auswertung der Referate und Diskussionen in diesem Band sind Grundlage für die Ausrichtung des geplanten Kongresses in Asien. Auch wird auf dieser Basis zu entscheiden sein, welche Forschungsfragen besonders dringend und zielführend sind.

\subsubsection{Asiatischer Länderschwerpunkt}

Bislang sind in den Ländervergleich verschiedene asiatische Staaten einbezogen worden, nämlich neben China, Japan, Indien, Korea auch Vietnam und Taiwan. Für die vertiefte Untersuchung spezieller Forschungsfragen, wie sie zuvor aufgeführt worden sind, bedarf es nun einer Konzentration auf ein oder maximal zwei asiatische Staaten. Nur dann wird es möglich sein, den kulturwissenschaftlichen Hinter-

56 Ein erster Schritt dabei ist der Generalbericht von Graser, siehe nachfolgend S. $489 \mathrm{ff}$. 
grund mit der notwendigen Intensität darzustellen und in seinem Bezug auf die Behindertenproblematik auszuwerten. Die Untersuchung hätte insoweit exemplarische Bedeutung. Inwieweit ein einzelner asiatischer Staat auch als Beispiel für Asien allgemein gelten könnte, ist allerdings eine andere Frage, die einer sorgfältigen Analyse bedürfte.

Bei der Entscheidung der Auswahl eines Schwerpunktlandes sind unterschiedliche Aspekte abzuwägen. Ein beachtlicher Gesichtspunkt ist die Aufgeschlossenheit für eine Weiterentwicklung und Neugestaltung des bestehenden Behindertenrechts. Eine solche sozialpolitische Aktualität könnte das Interesse an einer intensiven Mitwirkung an dem Projekt in dem jeweiligen Land anregen und dazu führen, dass konkrete Reformvorschläge für dieses Land entwickelt werden könnten. Diese Situation ist z. B. in Korea oder Vietnam gegeben.

Gleichzeitig ist jedoch der sozialwissenschaftliche und sozialrechtliche Diskussionsstand in dem jeweiligen Land zu berücksichtigen. Es müssen personelle, institutionelle und finanzielle Ressourcen gegeben sein, damit eine intensive Aufarbeitung der Problematik unter Einbeziehung der kulturwissenschaftlichen Fragestellung erfolgversprechend und eine reibungslose Organisation und Abwicklung eines größeren Kongresses im Land möglich ist. Diese Voraussetzung sind am ehesten in Japan gegeben, zumal mit japanischen Wissenschaftlern sehr weit verzweigte und intensive wissenschaftliche Beziehungen bestehen und der Workshop sich besonders intensiv mit Japan beschäftigt hat ${ }^{57}$. Dies würde dafür sprechen, den nächsten Folgekongress in Japan zu organisieren. 


\title{
2. Erscheinungsformen von Behinderung in einer alternden Gesellschaft: \\ Zur Kompetenz von älteren Menschen mit geistiger Behinderung
}

\author{
Von Andreas Kruse
}

Einführung

1. Zur Definition von Kompetenz 33

2. Kompetenz bei älteren Menschen mit geistiger Behinderung 36

3. Behinderungen als Folge spezifischer Person-Umwelt-Interaktionen 39

4. Die infrastrukturelle Umwelt: Inwieweit spiegelt sich die Kompetenzorientierung in den spezifischen Angeboten wider?

5. In welcher Hinsicht lassen sich die Kompetenz und Lebensqualität durch die Entwicklung innovativer Konzepte fördern?

5.1. Zentrale Merkmale der Kompetenz und Lebensqualität bei Behinderung im Alter aus einer Interventionsperspektive

5.2. Zentrale Kategorien innovativer Konzepte der Behindertenhilfe

5.3. Entwicklung innovativer Konzepte aus der Perspektive der Institutionen

6. Entwicklungsperspektiven 


\section{Einführung}

Die Lebenserwartung von Menschen mit geistiger Behinderung ist um fünf Jahre geringer als die der Normalbevölkerung, Menschen mit Down-Syndrom haben eine um zehn Jahre geringere Lebenserwartung als Menschen mit geistiger Behinderung und eine um 15 Jahre geringere Lebenserwartung als nicht behinderte Menschen (Haveman \& Stöppler, 2004'; Yang, Rasmussen \& Friedman, 2002²). In den letzten Jahrzehnten hat eine Angleichung der durchschnittlichen Lebenserwartung von Menschen mit geistiger Behinderung und ohne Behinderung stattgefunden (SchulzNieswandt, $2006^{3}$ ). Auch wenn Menschen mit Down-Syndrom eine um etwa 15 Jahre geringere Lebenserwartung zeigen, so trifft doch der Anstieg der Lebenserwartung gerade auf diese Personengruppe zu: Im Zeitraum von 1950 bis heute hat sich deren durchschnittliches Lebensalter fast vervierfacht, nämlich von 15 Jahren auf fast 60 Jahre. Der medizinische Fortschritt, hier vor allem die Antibiotika-Therapie, die Behandlung von Infektionskrankheiten, die korrigierende Herzchirurgie, sowie Bildungs- und Rehabilitationskonzepte haben zu dieser positiven Entwicklung beigetragen (Driller \& Pfaff, 2006 ${ }^{4}$ ). Die Definition von Grenzen der Lebenserwartung für Menschen mit geistiger Behinderung erscheint vor dem Hintergrund der Angleichung der Lebenserwartung nicht mehr als angemessen (Haveman \& Stöppler, $2004^{5}$ ).

Ergebnisse wissenschaftlicher Untersuchungen zur Kompetenz und Kompetenzförderung bei Menschen mit geistiger Behinderung deuten auf Plastizität und Veränderungskapazität auch im mittleren und hohen Erwachsenenalter (Ding-Greiner \& Kruse, 2004 ${ }^{6}$. Aus diesem Grunde ist es notwendig, in den Konzepten der Begleitung und Betreuung älterer Menschen mit geistiger Behinderung zum einen den Bildungsaspekt zu akzentuieren (Kraft, 2006 $)$, zum anderen den Umweltaspekt, wobei hier der anregende, ressourcenförderliche Charakter der Umwelt im Zentrum steht (Wacker \& Wahl, 2007 $)$.

1 Haveman/Stöppler (2004), Altern mit geistiger Behinderung. Grundlagen und Perspektiven für Begleitung, Bildung und Rehabilitation, Stuttgart: Kohlhammer.

2 Yang. et al. (2002), Mortality associated with Down's Syndrome in the USA, Lancet, 359, 10191025 .

3 Schulz-Nieswandt (2006), Alternsformen, Lebenserwartung und Altersstruktur behinderter Menschen - unter besonderer Berücksichtigung angeborener Formen geistiger Behinderung, in: Krueger./Degen (Hrsg.), Das Alter behinderter Menschen (S. 142-201), Freiburg: Lambertus. Driller/Pfaff (2006), Soziodemografische Struktur von Menschen mit Behinderung in Deutschland, in: Krueger/Degen (Hrsg.), Das Alter behinderter Menschen (S. 26-117), Freiburg: Lambertus.

$5 \quad$ Haveman/Stöppler (2004), Altern mit geistiger Behinderung. Grundlagen und Perspektiven für Begleitung, Bildung und Rehabilitation, Stuttgart: Kohlhammer.

6 Ding-Greiner/Kruse (2004), Alternsprozesse bei Menschen mit geistiger Behinderung, in: Kruse/Martin (Hrsg.), Enzyklopädie der Gerontologie (S. 519-532), Bern: Huber.

7 Kraft (2006), Pflege älterer Menschen mit Behinderungen: Tendenzen, Entwicklungen, Perspektiven, in: Krueger/Degen (Hrsg.), Das Alter behinderter Menschen (S. 192-206), Freiburg: Lambertus.

Wacker/Wahl (2007), Altersfreundliche und ressourcenförderliche Umwelten, in: Bertelsmann Stiftung (Hrsg.), Alter neu denken (S. 217-247), Gütersloh: Verlag Bertelsmann Stiftung. 
Aus diesem Grunde ist ausdrücklich nachfolgender Aussage zuzustimmen:

„Die Eingliederungshilfe mit ihrem ganzheitlichen Anspruch geht also weit über die Hilfe zur Pflege hinaus, weil sie auch Leistungen der Rehabilitation und Förderung umfasst. Es ist hier zunächst einmal von zentraler Bedeutung sowohl für die fachliche Weiterentwicklung wie auch für die Refinanzierung der Pflege älterer behinderter Menschen, dass diese weiterhin im Wirkungskreis der Eingliederungshilfe verbleibt" (Kraft, 2006, S.196 f. ${ }^{9}$ ).

\section{Zur Definition von Kompetenz}

Den Ausgangspunkt unserer Überlegungen bildet ein Verständnis von Kompetenz, das die Bedeutung der Umwelt für die Erhaltung der Leistungsfähigkeit des Menschen hervorhebt. Kompetenz umfasst dabei

„Fähigkeiten und Fertigkeiten des Menschen zur Erhaltung oder Wiederherstellung eines selbstständigen, selbstverantwortlichen und sinnerfüllten Lebens in einer anregenden, unterstützenden, zur selbstverantwortlichen Auseinandersetzung mit Anforderungen motivierenden sozialen, räumlichen und infrastrukturellen Umwelt" (Kruse, 2007 ${ }^{10}$; Kruse \& Schmitt, $\left.2006^{11}\right)$.

Für die Behindertenhilfe kann sich eine derartige Definition von Kompetenz in dreifacher Hinsicht als hilfreich erweisen:

Erstens: In dieser Definition wird zwischen Selbstständigkeit, Selbstverantwortung und sinnerfülltem Leben differenziert. Die in der Literatur zum Alter vielfach anzutreffende Beschränkung auf die Selbstständigkeit (zum Beispiel im Sinne der selbstständigen Ausführung von Aktivitäten des täglichen Lebens) wird hier vermieden. Selbstständigkeit bildet zwar ein bedeutsames Merkmal der Kompetenz, doch keinesfalls das einzige Merkmal. Hinzu kommt die Selbstverantwortung des Menschen, die als Fähigkeit und Bereitschaft verstanden wird, das Leben in einer den eigenen Leitbildern eines guten Lebens („Wer bin ich? Was will ich?“) folgenden Weise zu gestalten. In der Selbstverantwortung kommt nicht nur eine Fähigkeit zum Ausdruck (Reflektion eigenen Handelns), sondern auch eine Motivlage (Bereitschaft, Ziele zu definieren und diese zu verwirklichen). Dabei ist zu berücksichtigen, dass in der Behindertenhilfe nicht nur die Fähigkeiten, sondern eben auch die Motivlage des Menschen besondere Anforderungen an die Mitarbeiterinnen und Mitarbeiter stellt. Wie kann es - vor allem nach Ausscheiden des Menschen mit Behinderung aus der Werkstätte - gelingen, diesen dazu zu motivieren, neue Ziele zu definieren und sich verantwortlich mit den Entwicklungsaufgaben des höheren Lebensalters ven, in: Krueger/Degen (Hrsg.), Das Alter behinderter Menschen (S. 192-206), Freiburg: Lambertus.

10 Kruse (2007), Veränderbarkeit geistiger und körperlicher Fähigkeiten im Alter, in: Brandtstädter/Lindenberger (Hrsg.), Entwicklungspsychologie (S. 622-649), Stuttgart: Kohlhammer

11 Kruse/Schmitt (2006), Adult education, in: Birren (Ed.), Encyclopedia of Gerontology (pp.312332), Oxford: Elsevier. 
auseinander zu setzen (vgl. Wacker, Metzler \& Trost, $1996^{12}$ )? Diese Frage kann auch in anderer Weise ausgedrückt werden: Wie kann es gelingen, den Menschen dazu zu motivieren, seine Fähigkeiten (im kognitiven, im alltagspraktischen, im physischen Bereich) einzusetzen und aufrechtzuerhalten (vgl. Haveman, $2001{ }^{13}$ )? In der angeführten Definition wird neben der Selbstständigkeit und der Selbstverantwortung die sinnerfüllte Lebensgestaltung als bedeutsames Merkmal der Kompetenz gewertet. Auch hier haben wir es nicht nur mit einer Fähigkeit, sondern zudem mit einer Motivlage zu tun: Sinnerfahrung ist nur möglich, wenn Menschen offen für den Aufforderungscharakter, den Anregungsgehalt einer Situation sind. Bei der sinnerfüllten Lebensgestaltung wird deswegen von einer Fähigkeit gesprochen, da Menschen zum einen in der Lage sein müssen, zu beurteilen, in welchen Lebenssituationen sich die Erfahrung der Stimmigkeit einstellen kann, da sie zum anderen über Techniken verfügen müssen, solche Situationen aktiv aufzusuchen oder herzustellen. Die Angebote zur Tagesstruktur - die ein zentrales Element der Behindertenhilfe darstellen - lassen sich im Kern von dem Ziel leiten, ein „sinnstiftendes Angebot" zu unterbreiten, welches zum Beispiel nach Ausscheiden aus der Werkstätte für Behinderte die frühere Berufstätigkeit zu ersetzen vermag (Hawkins, $1999^{14}$; Hessisches Sozialministerium \& Landeswohlfahrtsverband Hessen $2000^{15}$; Walker et al., $1999^{16}$ ).

Zweitens: In dieser Definition wird die große Bedeutung der Umwelt für die Fähigkeiten des Menschen zu einem selbstständigen, selbstverantwortlichen und sinnerfüllten Leben betont. Damit wird zum Ausdruck gebracht, dass die personzentrierte Definition von Behinderung - die ausschließlich die bei einem Menschen bestehenden Einschränkungen im Blick hat, hingegen nicht die möglichen Umweltbarrieren eines selbstständigen, selbstverantwortlichen und sinnerfüllten Lebens aufgegeben werden muss zugunsten einer Definition von Behinderung, welche die Wechselwirkungen zwischen Person und Umwelt akzentuiert. In der vorgeschlagenen Kompetenzdefinition wird hervorgehoben, dass die Umwelt - und zwar die räumliche, die soziale, die infrastrukturelle Umwelt - durch Anregungen, Unterstützung und Motivation (nämlich zur selbstverantwortlichen Auseinandersetzung mit Anforderungen und Aufgaben) einen substanziellen Beitrag zur Erhaltung und Weiin: Berghau/Knapic/Sievert (Hrsg.), Abschied vom Fürsorgedenken (S. 56-78), Köln: Kuratorium Deutsche Altershilfe.

13 Haveman (2001), Perspektiven der Integration älterer Menschen mit geistiger Behinderung, in: Hessisches Sozialministerium, Landeswohlfahrtsverband Hessen \& Bundesvereinigung Lebenshilfe für Menschen mit geistiger Behinderung e.V. (Hrsg.), Lebensräume älterer Menschen mit Behinderung (S. 157-180), Marburg: Lebenshilfe-Verlag.

14 Hawkins (1999), Rights, place of residence, and retirement: Lessons from case studies on aging, in: Herr/Weber (Eds.), Aging, rights, and quality of life (pp. 93-108), Baltimore: Brookes.

15 Hessisches Sozialministerium, Landeswohlfahrtsverband Hessen (2000), Lebensräume älterer Menschen mit Behinderung. Fachliche Leitlinien und Empfehlungen, Wiesbaden: Hessisches Sozialministerium.

16 Walker et al. (1999), Quality of life as a matter of human rights, in: Herr/Weber (Eds.), Aging, rights, and quality of life (pp. 109-132), Baltimore: Brookes. 
terentwicklung von Fähigkeiten leistet. Die in Beiträgen zur Pflegeforschung nachgewiesenen Zusammenhänge zwischen einer selbstständigkeitsfördernden Umwelt, die zur selbstständigen Ausführung von Aktivitäten des täglichen Lebens motiviert und die die stellvertretende Ausübung von Aktivitäten durch die soziale Umwelt vermeidet, sowie dem Gewinn an Selbstständigkeit im Pflegeprozess (Baltes, 1996) weist auf die große Bedeutung der Umwelt für die Erhaltung und Weiterentwicklung von Fähigkeiten sowie - umgekehrt - für die Vermeidung, Linderung oder Überwindung von Einschränkungen hin. Speziell im Hinblick auf die Erhaltung von Selbstständigkeit bei chronischen oder chronisch-progredienten Erkrankungen ist dem „Frailty-Konzept“ hoher theoretischer und praktischer Wert zuzuordnen (DingGreiner \& Lang, 2004 ${ }^{17}$ ). Dieses Konzept geht davon aus (und diese Annahme konnte auch empirisch gestützt werden), dass der Übergang von chronischen Erkrankungen zu Hilfebedarf oder Pflegebedarf auch durch die psychische Befindlichkeit sowie durch die räumliche und soziale Umwelt vermittelt ist (Rockwood et al., $2000^{18}$ ).

Drittens: In dieser Definition sind Merkmale der Person und der Umwelt aufgeführt, die eng mit den in der Behindertenhilfe entwickelten Bildungs- und Förderkonzepten verwandt sind. Die Eingliederungshilfe und die auf dieser gründenden heilpädagogischen Konzepte bleiben ausdrücklich nicht bei der Selbstständigkeit stehen, sondern sehen auch die Schaffung von Rahmenbedingungen für ein selbstverantwortliches und sinnerfülltes Leben als bedeutsame Aufgabe an. Dabei wird hervorgehoben, dass sich die Funktion der Umwelt nicht allein auf die Unterstützung des Menschen mit Behinderung beschränkt, sondern auch anregende und motivierende Aspekte einschließt. Verbindet man diese drei Funktionen der Umwelt mit den Fähigkeiten, die - der vorgeschlagenen Definition von Kompetenz zufolge kompetentes Handeln konstituieren (Selbstständigkeit, Selbstverantwortung, Sinnerfahrung), so ergeben sich hier zentrale Komponenten eines Bildungsbegriffs. Die Behindertenhilfe versteht den Einsatz ihrer Fördermaßnahmen auch als Beitrag zur lebenslangen Bildung des Menschen. Sie stellt auch in der Arbeit mit älteren behinderten Menschen die Frage nach Potenzialen (Theunissen, 2002 ${ }^{19}$ ), das heißt nach Fähigkeiten und Interessen, wie auch nach möglichen Ressourcen für die Gemeinschaft.

17 Ding-Greiner/Lang (2004), Alternsprozesse und Krankheitsprozesse - Grundlagen, in: Kruse/Martin (Hrsg.), Enzyklopädie der Gerontologie (S. 182-206), Bern: Huber.

18 Rockwood/Hogan/MacKnight et al. (2000), Conceptualization and measurement of frailty in elderly people, Drugs Aging, 17, 295-302.

19 Theunissen (2002), Die Stärken-Perspektive. Impulse für die pädagogische und therapeutische Arbeit mit Menschen, die als geistig behindert gelten, Geistige Behinderung, 3, 2002. 


\section{Kompetenz bei älteren Menschen mit geistiger Behinderung}

Zunächst seien einige Aussagen zur körperlichen Gesundheit und physischen Kompetenz bei älteren Menschen mit geistiger Behinderung getroffen. In Bezug auf die generelle Morbidität und Mortalität unterscheiden sich ältere Menschen mit geistiger Behinderung nicht von jenen ohne geistige Behinderung (Ding-Greiner \& Kruse, $2004^{20}$ ). Allerdings muss damit gerechnet werden, dass gesundheitliche Probleme bei älteren Menschen mit geistiger Behinderung und der daraus resultierende Versorgungsbedarf unterschätzt werden, da (a) Menschen mit Behinderung gesundheitliche Probleme möglicherweise seltener ausdrücken, (b) gesundheitliche Probleme bei ihnen mit einer noch höheren Wahrscheinlichkeit als Folge natürlicher Alternsprozesse oder von Behinderung interpretiert werden (eine falsche Interpretation, die vielfach Ursache von Unterversorgung ist) (Janicki et al., 2002 ${ }^{21}$ ). Zu den Barrieren des Zugangs älterer Menschen mit geistiger Behinderung zum medizinischen Versorgungssystem gehören vor allem folgende (Evenhuis et al., 2001 ${ }^{22}$ ):
a) Kommunikationsprobleme
b) Motorische Einschränkungen
c) Mangelnde Ausbildung und mangelndes Training von Ärzten und Pflegefach- kräften
d) Zu hohe Kosten, zu hoher Zeitaufwand im Erleben der Angehörigen
e) Geringer sozialer Status von älteren Menschen mit Behinderung

Bei alten Menschen ohne Behinderung und alten Menschen mit geistiger Behinderung sind vergleichbare körperliche Erkrankungen erkennbar, wie HerzKreislauferkrankungen, Arthrosen, Frakturen mit anschließenden Funktionseinschränkungen, Diabetes mellitus Typ II und Lungenerkrankungen. Darüber hinaus ist bei Menschen mit geistiger Behinderung ähnlich wie bei Menschen ohne Behinderung ab dem 75. Lebensjahr das Risiko motorischer Einschränkungen, speziell aufgrund von Gon- und Coxarthrose, Parkinsonscher Erkrankung, Spondylitis und Osteoporose, erhöht. Seheinbußen und Hörbeeinträchtigungen sind bei Menschen mit geistiger Behinderung häufiger zu beobachten (Evenhuis, $1995 \mathrm{a}^{23}, 1995 \mathrm{~b}^{24}$ ). Es besteht ein signifikanter Zusammenhang zwischen Alter einerseits sowie Hilfebedarf und Pflegebedarf andererseits, dieser Zusammenhang ist dabei unabhängig sowohl

20 Ding-Greiner/Kruse (2004), Alternsprozesse bei Menschen mit geistiger Behinderung, in: Kruse/Martin (Hrsg.), Enzyklopädie der Gerontologie (S. 519-532), Bern: Huber.

21 Janicki et al. (2002), Health characteristics and health services utilization in older adults with intellectual disability living in community residences, Journal of Intellectual Disability Research, 46, 287-298.

22 Evenhuis et al. (2001), Healthy ageing - adults with physical health issues, Journal of Applied Research in Intellectual Disabilities, 14, 175-194.

23 Evenhuis (1995a), Medical aspects of ageing in a population with intellectual disability: Visual impairment, Journal of Intellectual Disability Research, 39, 19-25.

24 Evenhuis (1995b), Medical aspects of ageing in a population with intellectual disability: Hearing impairment, Journal of Intellectual Disability Research, 39, 27-33. 
vom Vorliegen einer geistigen Behinderung als auch vom Grad der Behinderung (Maaskant et al., 1996 ${ }^{25}$ ). Während geistige Behinderungen allgemein keinen Einfluss auf Hilfe- und Pflegebedarf haben, lässt sich dieser für das Vorliegen von Down-Syndromen nachweisen: Bei Menschen mit Down-Syndrom sind die Funktionseinbußen signifikant stärker ausgeprägt als bei Menschen mit einer geistigen Behinderung, aber ohne Down-Syndrom.

Menschen mit geistiger Behinderung verfügen über geringere psychische und kognitive Ressourcen, die als Schutz gegen Belastungen im Alltag dienen können. Dadurch ist das Risiko psychischer Erkrankungen im Alter erhöht. Diese Menschen sind anfälliger für umgebungsbedingte und soziale Belastungen.

Veränderungen im gesundheitlichen und funktionellen Bereich scheinen bei älteren Menschen mit geistiger Behinderung deutlich früher aufzutreten als bei Personen, die nicht geistig behindert sind. Insbesondere bei Menschen mit DownSyndrom scheint der Alterungsprozess nicht nur früher einzusetzen, sondern sie weisen offensichtlich auch ein höheres Risiko auf, an einer Demenz zu erkranken, und dies bereits in jungen Jahren. Dadurch werden vermutlich viele der alten, vielfach gesundheitlich und funktionell beeinträchtigten Eltern bei der Betreuung von älter werdenden Menschen mit geistiger Behinderung zusätzlich belastet, was möglicherweise einen Umzug der älter werdenden Person mit geistiger Behinderung in eine Einrichtung der Behindertenhilfe notwendig machen kann.

Nimmt man vor dem Hintergrund eines kompetenztheoretischen Konzepts eine Skizzierung der Fähigkeiten von älteren Menschen mit geistiger Behinderung vor, so lassen sich folgende Aussagen treffen: 1. Die Kompetenz im Alter (und zwar sowohl im physischen als auch im seelisch-geistigen Bereich) ist in hohem Maße vom Schweregrad der Behinderung beeinflusst; schon alleine aus diesem Grunde sind Verallgemeinerungen zu vermeiden. Für den Grad der physischen Kompetenz ist weniger die Frage, ob Menschen eine Behinderung aufweisen oder nicht, entscheidend. Vielmehr ist der Grad der Behinderung ein bedeutender Einflussfaktor $\left(\right.$ Cooper, $\left.1998^{26}\right)$. 2. Die Kompetenz im Alter ist in hohem Maße vom Grad der Förderung beeinflusst, die Menschen im Lebenslauf erfahren haben. 3. Die Kompetenz im Alter ist in hohem Maße vom Grad der sensorischen, kognitiven und sozialen Anregungen beeinflusst, die Menschen in früheren Lebensjahren erfahren haben und aktuell erfahren. Dabei ist speziell bei älteren Menschen mit geistiger Behinderung zu berücksichtigen, dass bei diesen Seh- und Höreinbußen häufiger auftreten als bei Menschen ohne Behinderung bzw. mit körperlicher oder seelischer Behinderung (Warburg, 2001 $1^{27}$ ). 4. Der Alternsprozess verläuft bei Menschen mit geistiger Behinderung nicht grundsätzlich anders als bei Menschen ohne geistige Behindeof a longitudinal study, Journal of Intellectual Disability Research, 40, 535-543.

26 Cooper (1998), Clinical study of the effects of age on the physical health of adults with mental retardation, American Journal on Mental Retardation, 102, 582-589.

27 Warburg (2001), Visual impairment in adult people with intellectual disability. Literature review, Journal of Intellectual Disability Research, 45, 424-438. 
rung (Evenhuis, $2001^{28}$ ). 5. Die Variabilität im Alter ist bei Menschen mit geistiger Behinderung noch stärker ausgeprägt als bei Menschen ohne geistige Behinderung. 6. Der Kreativität geistig behinderter Menschen ist im Alter genauso wenig eine Grenze gesetzt wie in früheren Lebensaltern: $\mathrm{Zu}$ nennen sind kreative Leistungen im künstlerischen Bereich. 7. Gefühle der Selbstverantwortung und Mitverantwortung sind bei Menschen mit geistiger Behinderung in gleicher Weise vorhanden wie bei Menschen ohne diese Behinderung. Aus diesem Grunde wird der Möglichkeit, auch etwas für andere - zum Beispiel für jüngere Menschen - zu tun, große Bedeutung beigemessen (Hensel et al., 2002 29 ; Meininger, 2000 $0^{30}$ ). 8. Fehlen systematische Anregung oder systematisches Training, so besteht bei Menschen mit geistiger Behinderung die besondere Gefahr, dass die im Lebenslauf entwickelten Fähigkeiten und Fertigkeiten rasch verloren gehen. Aus diesem Grunde ist für Menschen mit geistiger Behinderung das Training wichtig, weil sonst alle früheren Erfolge in Frage gestellt werden (Weltgesundheitsorganisation, 2001 31 ; Zigman et al., 2002 $2^{32}$ ). 9. Aufgrund verringerter affektiver und emotionaler Kontrolle sind die Belastungs- und Trauerreaktionen bei Menschen mit geistiger Behinderung intensiver. Aus diesem Grunde muss nach dem Auftreten von Verlusten eher mit tiefgreifenden psychischen Reaktionen gerechnet werden. 10. Die körperliche Ermüdung und seelische Erschöpfung nehmen bei Menschen mit geistiger Behinderung im Alter besonders stark zu, der Antrieb ist verringert. 11. Bei einzelnen Formen geistiger Behinderung - hier ist vor allem das Down-Syndrom zu nennen - ist die Gefahr des Auftretens einer Demenz im Alter erkennbar erhöht. Aus diesem Grunde ist hier dem alltagspraktischen und kognitiven Training besondere Bedeutung beizumessen, damit auch im Verlauf dieser Erkrankung die bestehenden Fähigkeiten und Fertigkeiten möglichst lange erhalten bleiben. 12. Bei der Entwicklung von Förder- und Rehabilitationskonzepten ist darauf zu achten, dass a. an den früheren Förderansätzen angeknüpft und das Training spezifischer Funktionen und Fertigkeiten im Alter fortgesetzt wird, dass b. auch nach Ausscheiden aus der Werkstätte ein ausreichendes Maß an Tagesstrukturierung und sinnerfüllter Aktivität gegeben ist, dass c. Möglichkeiten zur Kreativität bestehen, dass d. Möglichkeiten des körperlichen Trainings (vor allem: Ausdauer, Kraft, Geschicklichkeit) sowie des geistigen und alltagspraktischen Trainings gegeben sind, dass e. bei der Umweltgestaltung die Forderung nach Barrierefreiheit, nach optimalem Anregungsgehalt und nach Hilfsmittels berücksichtigt

Evenhuis (2001), Healthy aging - adults with intellectual disabilities: Physical health issues, Journal of Applied Research in Intellectual Disabilities, 14, 175-194.

29 Hensel et al. (2002), Subjective judgements of quality of life: a comparison study between people with intellectual disability and those without disability, Journal of Intellectual Disabi-lity Research, 46, 95-107.

30 Meininger (2000), Autonomy and professional responsibility in care for persons with intellectual disabilities, Nursing Philosophy, 2, 240-250.

31 World Health Organization (2001), Healthy Ageing - Adults with intellectual disabilities: Summative Report, Journal of Applied Research in Intellectual Disabilities, 14, 256-275.

32 Zigman et al. (2002), Incidence and temporal patterns of adaptive behavior change in adults with mental retardation, American Journal of Mental Retardation, 107, 161-174. 
werden (Jones et al., 1999 33 ; Kruse et al., 2002 $2^{34}$ ). 13. Es sollte darauf geachtet werden, dass sich Menschen auf das Ausscheiden aus der Werkstätte vorbereiten können und der Übergang in die nachberufliche Zeit gleitend erfolgt. Kontakte zur Werkstätte sollten auch nach Ausscheiden aus dem Beruf prinzipiell bestehen bleiben, wenn der Wunsch danach besteht. Ältere Menschen mit geistiger Behinderung sind gerade in der Phase des Übergangs in die nachberufliche Zeit in hohem Maße verunsichert, inwieweit sie auch in Zukunft Kontakte finden und persönlich bedeutsamen Tätigkeiten nachgehen können. Weiterhin gewinnt die Fragen der sozialen Anerkennung nach Ausscheiden aus dem Beruf Bedeutung (Gusset-Bährer, 2003 ${ }^{35}$ ). 14. Aufgrund abnehmender körperlicher Belastungsfähigkeit oder des Todes der Eltern ist die familiäre Betreuung der Menschen mit geistiger Behinderung nicht gesichert oder sogar unmöglich. Alternative Formen des gemeinschaftlichen Wohnens müssen geschaffen werden. Dabei ist in besonderem Maße auf die Erhaltung der sozialen Integration und Partizipation der Menschen mit geistiger Behinderung zu achten. Diese müssen die Möglichkeit haben, in einem ihnen vertrauten Umfeld mit ihnen vertrauten Menschen zu leben. Aus diesem Grunde ist im Falle von Pflegebedarf auch das Verbleiben in der Behinderteneinrichtung dem Umzug in ein Altenpflegeheim vorzuziehen. Der Umzug wäre vielfach mit einer Aufgabe des vertrauten sozialen Umfelds verbunden, die gerade von Menschen mit geistiger Behinderung nur schwer verarbeitet werden kann. Abgesehen davon ist in den meisten Altenpflegeheimen die für die Betreuung behinderter Menschen notwendige heilpädagogische Expertise nicht vorhanden. Einrichtungen der Behindertenhilfe stehen vor der Aufgabe, Expertise im Bereich der Altenpflege zu erwerben (sei es durch die Einstellung von Altenpflegern, sei es durch die Weiterbildung der Heilerziehungspfleger im Bereich der Altenpflege). Gegebenenfalls können in den Einrichtungen der Behindertenhilfe eigene Pflegeabteilungen geschaffen werden. Entscheidend ist die Integration von Elementen der Behinderten- und Altenhilfe - ein Aspekt, auf den später noch ausführlicher einzugehen sein wird.

\section{Behinderungen als Folge spezifischer Person-Umwelt-Interaktionen}

Die hier vorgeschlagene Definition von Kompetenz rückt sowohl die Person als auch die Umwelt in das Zentrum. Sie konzentriert sich zum einen auf die Fähigkeiten der Person, zum anderen betrachtet sie diese im Kontext der jeweils gegebenen räumlichen, sozialen und infrastrukturellen Umwelt: Inwieweit fördert, inwieweit

33 Jones et al (1999), Opportunity and promotion of activity among adults with severe intellectual disability living in community residences: the impact of training staff in active support, Journal of Intellectual Disability Research, 43, 164-178.

34 Kruse et al. (2002), Den Jahren Leben geben. Lebensqualität im Alter bei Menschen mit Behinderungen, Projektbericht, Stuttgart: Diakonisches Werk Württemberg.

35 Gusset-Bährer (2003), Menschen mit geistiger Behinderung beim Übergang vom Beruf in die nachberufliche Phase, Phil. Diss. Universität Heidelberg: Fakultät für Empirische Kultur- und Sozialwissenschaften. 
erschwert diese die Erhaltung oder Wiederherstellung eines selbstständigen, selbstverantwortlichen und sinnerfüllten Lebens? Mit der Akzentuierung der Wechselwirkungen zwischen Person und Umwelt wird verdeutlicht, dass Behinderungen nicht allein als Merkmal der Person zu verstehen sind, dass also die Umwelt Mitverantwortung für die Kompetenz des Menschen trägt.

Hingegen werden Behinderungen in der öffentlichen Diskussion primär oder ausschließlich aus einer personzentrierten, medizinisch-kurativen Perspektive betrachtet. Behinderung bildet danach allein ein Merkmal der Person; der Medizin, der Rehabilitation, der Heilpädagogik wird die Aufgabe gestellt, zur Linderung oder Kompensation bestehender Funktions- und Fähigkeitseinbußen beizutragen. Diese Perspektive verdeckt jedoch die Bedeutung der räumlichen, sozialen und infrastrukturellen Umwelt für die Behinderung. Behinderung ist auch Folge von behindernden Umweltbedingungen. Die nachfolgend angeführte Definition von Behinderung macht deutlich, dass eingeschränkte Funktionen und Fertigkeiten vor allem im umweltbezogenen Handeln des Menschen offenbar werden:

„Behinderung ist nicht in erster Linie ein Synonym für eine medizinische Diagnose, sondern ein umfassendes personales und soziales Geschehen. Behinderung stellt sich dar als ein auf mehreren Wirkungsebenen laufender Prozess. Diese Ebenen bezeichnen den unmittelbar aus dem klinischen Krankheitsgeschehen resultierenden Schaden (Impairment), die individuellen und funktionellen Einschränkungen mit der Folge von unterschiedlichen Fähigkeitsstörungen (Disability) und die soziale Beeinträchtigung (Handicap) und die sich daraus ergebenden vielfältigen persönlichen, familiären und gesellschaftlichen Folgen“ (Paeslack, 1998 ${ }^{36}$ ).

Für Heilpädagogik und Rehabilitation ergeben sich aus dieser Definition von Behinderung die folgenden fünf Aufgaben: 1. Umweltbedingungen sind so zu verändern, dass Menschen trotz bestehender Einbußen spezifischer Fähigkeiten in der Lage sind, in ihrer vertrauten (räumlichen und sozialen) Umwelt selbstständig und selbstverantwortlich zu leben sowie sozial zu partizipieren. 2. Barrieren in der räumlichen Umwelt, die sich negativ auf Mobilität und Selbstständigkeit auswirken, sind zu beseitigen. 3. (Technische) Hilfsmittel, die Mobilität, Selbstständigkeit und Kommunikationsfähigkeit fördern, sind in die räumliche Umwelt zu integrieren. 4. Beim Erwerb von Kenntnissen und Fertigkeiten zum effektiven Umgang mit diesen Hilfsmitteln ist Unterstützung anzubieten. 5. In unserer Gesellschaft ist auf ein verändertes Verständnis von Behinderung hinzuwirken, das a. Behinderung nicht mit Krankheit gleichsetzt, b. die Verschiedenartigkeit der Lebensbedingungen, Bedürfnisse und Fähigkeiten von Menschen mit Behinderung wahrnimmt und damit die Notwendigkeit eines differenzierten Spektrums an Angeboten (Tagesgestaltung, Wohnen, Bildung, Aktivierung) anerkennt, c. die große Bedeutung der räumlichen, sozialen und infrastrukturellen Umwelt für die Folgen von Behinderung akzentuiert. Die genannten Aufgaben der Rehabilitation lassen sich auch in der Weise charakterisieren, dass Abhängigkeit und Fremddefinition in den Lebensbereichen Wohnen, Arbeit und Freizeit zugunsten einer selbständigen und selbstverantwortlichen Le- 
bensgestaltung aufgehoben werden. Diese umfassende Zielsetzung liegt auch den Leitbegriffen Normalisierung und Integration zugrunde.

Vorbilder für dieses veränderte Verständnis von Behinderung finden sich unter anderem in Großbritannien. Dort nimmt die Umweltgestaltung (zu der auch die Ausstattung mit mobilitäts-, selbstständigkeits- und kommunikationsfördernden Hilfsmitteln gehört) bei der Entwicklung von Förderkonzepten sowie im Prozess der Betreuung und Rehabilitation eine hervorgehobene Stellung ein. Als Beispiel sei eine Erklärung der British Psychological Society aus dem Jahre 1989 angeführt, in der als Aufgabe des Sozial- und Gesundheitswesens vor allem die Linderung sozialer Handicaps genannt wird:

„Die Konzentration allein auf den behinderten Menschen geht an der Tatsache vorbei, dass die Gesellschaft in hohem Maße körperlich unversehrte Menschen bevorzugt, hingegen Menschen mit Fähigkeitsstörungen ausschließt; dies ist zum Beispiel in der Arbeitwelt der Fall, dies zeigt sich aber auch in der baulichen Gestaltung öffentlicher Gebäude. Es ist notwendig, soziale Handicaps zu verändern, anstatt Menschen mit Behinderung zu zwingen, sich willkürlich aufgestellten Normen anzupassen. Diese Normen werden in aller Regel von Menschen entwickelt, die körperlich unversehrt sind“".

Diese Aussage ist repräsentativ für jene Einrichtungen in Großbritannien, die an der Entwicklung von Förder-, Betreuungs- und Rehabilitationskonzepten für Menschen mit Behinderung beteiligt sind. Die dort entwickelten Modelle von Behinderung rücken die sozialen Barrieren in den Vordergrund. Aus diesen Modellen ergeben sich folgende Veränderungen in den bereitgestellten Hilfen: 1. Diese sollen sich nicht allein auf Gesundheit und Versorgung beschränken, sondern in gleichem Umfang Veränderungen in der Umwelt einschließen (erhöhter Anregungsgehalt, Zugänglichkeit von öffentlichen Räumen und sozialen Dienstleistungen, Stärkung der Kontrolle über die Umwelt, Stärkung der sozialen und kulturellen Teilhabe). 2. Hilfen für behinderte Menschen sollen nicht länger im Sinne verordneter und erbrachter Dienste verstanden werden, sondern im Sinne umweltbezogener Ressourcen, auf die behinderte Menschen einen gesetzlich geregelten Anspruch haben. 3. Hilfen für behinderte Menschen sollen in Richtung auf ein integriertes Unterstützungssystem gestaltet werden. Die von behinderten Menschen genutzten Hilfen - wie medizinische Dienste, Bildungsangebote, Gestaltung des Wohnraums, Transporthilfen müssen stärker aufeinander abgestimmt sein, sie sind als modulares System und nicht als unabhängig voneinander bestehende Komponenten zu verstehen. 4. Ausbildung und Training von Dienstleistenden im Bereich der (technischen) Hilfen sollen so beschaffen sein, dass deren Fertigkeiten noch stärker auf das Erkennen und die Beseitigung von Barrieren gerichtet sind. 5. Die gesetzlichen Bedingungen müssen in der Hinsicht weiterentwickelt werden, dass Menschen mit Behinderungen in ihrer eigenen Wohnung leben können und gleiche Möglichkeiten zur Nutzung von medizinischen Angeboten sowie von Bildungs- und Freizeitangeboten haben; darüber hinaus ist ihren Bedürfnissen bei der Gestaltung der Wohnung und des Wohnumfeldes Rechnung zu tragen. 


\section{Die infrastrukturelle Umwelt: Inwieweit spiegelt sich die Kompetenzorientierung in den spezifischen Angeboten wider?}

In einer Studie von Moss $\left(1992^{37}\right)$ wurden neun Länder in Bezug auf die spezifischen Formen der Behindertenhilfe miteinander verglichen. Vor allem in den Niederlanden und in Deutschland wurde die institutionelle Betreuung und Pflege als zentrale Komponente innerhalb des Spektrums von Dienstleistungen interpretiert. In Nordamerika, Australien und Großbritannien hingegen wurde deutlich größeres Gewicht auf den Abbau der Institutionalisierung und die Integration in der Kommune gelegt. - Allerdings, so hebt Moss (1992) hervor, ist die Verfügbarkeit von Plätzen außerhalb der Einrichtungen in den 80er Jahren des vergangenen Jahrhunderts nur in geringem Umfang gestiegen. Wenn Familien wünschen, dass Angehörige außerhalb von Einrichtungen leben, so müssen sie damit rechnen, auf eine lange Warteliste zu kommen. Moss (1992) deutete das Bedürfnis nach einem Platz außerhalb einer Einrichtung der Behindertenhilfe in den neun Ländern als das wichtigste der nicht verwirklichten Bedürfnisse. Für die Bundesrepublik Deutschland gilt auch heute noch die Aussage, dass Angebote der Behindertenhilfe primär institutioneller Art sind und eine „De-Institutionalisierung“ noch nicht wirklich eingeleitet wurde. Diese Aussage ist nicht als ein Vorwurf an Institutionen zu verstehen. Es wird hier auch nicht die Annahme vertreten, dass außerhalb von Institutionen grundsätzlich die besseren Angebote erfolgten und zudem der Individualisierungsgrundsatz eher $\mathrm{zu}$ verwirklichen sei. Vielmehr ist der positive Effekt einer zunehmenden DeInstitutonalisierung darin $\mathrm{zu}$ sehen, dass sich mehr Wohnalternativen ausbilden können. Diese sind angesichts der Tatsache, dass Menschen mit Behinderung eine heterogene Gruppe bilden, sinnvoll und können - im Sinne der anregenden und unterstützenden Umwelt - dazu beitragen, dass Selbstständigkeit, Selbstverantwortung und Sinnerfahrung erhalten bleiben oder gefördert werden (Moss, $1994^{38}$ ).

Die Forderung, vermehrt Angebote außerhalb von Institutionen zu schaffen, kann nur dann erhoben werden, wenn auch in der Kommune Möglichkeiten bestehen, ältere Menschen mit Behinderung bei der Verwirklichung des Bedürfnisses nach sozialer Integration und Teilhabe zu unterstützen - ohne ihnen allerdings die Selbstverantwortung bei der Gestaltung des Alltags und der sozialen Beziehungen zu nehmen. In den Vereinigten Staaten sind mehrere Modellprojekte durchgeführt und evaluiert wurden, die dazu dienten, Möglichkeiten der Förderung von sozialer Integration und Teilhabe außerhalb von Institutionen zu prüfen.

In einem US-amerikanischen Modell mit dem Titel 'Senior Center - Senior Companion' (Heller, 1999 ${ }^{39}$ ) wurde das Ziel verfolgt, die Integration älterer Men-

38 Moss (1994), Quality of life and aging, in: Goode (Ed.), Quality of life for persons with disabilities (pp. 218-234), Cambridge: Brookline Books.

39 Heller (1999), Emerging models, in: Herr/Weber (Eds.), Aging, right, and quality of life (pp.149166), Baltimore: Brookes. 
schen mit Behinderung in der Gemeinde durch Unterstützung von Mitarbeitern, ehrenamtlich Tätigen und Freunden zu erreichen. Die unterstützenden Personen wurden dabei als Mentoren angesehen, die ältere Menschen mit Behinderung in soziokulturelle Einrichtungen für ältere Menschen ohne Behinderung einführen sowie in andere kommunale Aktivitäten einbinden sollten. Dadurch sollten soziale Bezugssysteme geschaffen werden, die zur sozialen Integration und sozialen Teilhabe beitragen. Die Evaluation dieses Modell ergab Erfolge im Hinblick auf eine Steigerung der Lebensqualität durch 1. die vermehrte (objektive und subjektiv erlebte) soziale Integration, 2. das erweiterte Spektrum an Aktivitäten im Alltag, 3. das verbesserte Selbstkonzept.

Factor und Anderson $\left(1992^{40}\right)$ haben in einer für die Vereinigten Staaten repräsentativen Studie untersucht, welche innovativen Programme und Maßnahmen zur Individualisierung und sozialen Teilhabe älterer Menschen mit geistiger Behinderung entwickelt wurden. Die von ihnen als innovativ eingeschätzten Programme und Maßnahmen zeichneten sich vor allem durch folgende sechs Merkmale aus:

- Individuelle Trainingsangebote zur Förderung des Entscheidungsverhaltens

- Vermittlung von Informationen über Dienstleistungen in der Kommune

- Unterstützung bei der sozialen und kulturellen Teilhabe in der Kommune

- Bereitstellung von technischen Hilfen und Unterstützung bei deren Nutzung

- Vermehrte Individualisierung in der Arbeitsplatz- und Arbeitszeitgestaltung

- Einbeziehung von ehrenamtlich tätigen Menschen in die Arbeit mit behinderten Menschen

Im Kontext der Bereitstellung und Unterstützung bei der Nutzung von Technik ist folgender Befund von Bedeutung: Trotz der Fortschritte bei der Entwicklung von nutzerfreundlicher Technik wird diese speziell von älteren Menschen mit geistiger Behinderung nicht genutzt. Bereits zu Beginn der 90er Jahre wurde eine Untersuchung veröffentlicht, aus der hervorgeht, dass speziell jene älteren Menschen mit geistiger Behinderung, die außerhalb von Einrichtungen leben, nicht über technische Hilfsmittel verfügen, durch die deren Selbstständigkeit, Selbstverantwortung und soziale Integration gefördert werden könnte. In dieser Gruppe wurden durchschnittlich zwei nicht erfüllte Bedürfnisse nach technischen Hilfen pro Person ermittelt (Mann et al., $1993^{41}$ ).

Frage nachgegangen, durch welche Maßnahmen dazu beigetragen werden kann, dass Menschen mit Behinderung vermehrt am Leben in der Gemeinde teilhaben. Es wurden von den befragten Experten die folgenden sieben Maßnahmen als zentral erachtet:

1. Die Familie und die Gemeinde müssen als Ressourcen verstanden werden, die stärker genutzt werden sollten

40 Factor/Anderson (1992), Person-centered planning innovative approaches in case management and habilitation planning, New Orleans: American Association on Mental Retardation.

41 Mann et al. (1993), Needs of home-based older persons for assistive devices, Technology and Disability, 2, 1-11. 
2. Die zwischenmenschlichen Beziehungen, vor allem die Freundschaften, müssen eine deutlich größere Beachtung finden

3. Bei der Konzeptentwicklung sind die individuellen Lebenspläne sehr viel stärker zu berücksichtigen

4. Individuelle Entscheidungsprozesse müssen gefördert werden - es sollen eben nicht Entscheidungen abgenommen werden, sondern vielmehr soll zur selbstverantwortlichen Entscheidung motiviert werden. Wenn Unterstützung im Prozess der Entscheidung notwendig ist, so darf diese keinesfalls die individuellen Entscheidungsspielräume einschränken

5. Menschen müssen dabei unterstützt werden, Kontrolle über ihre Umwelt auszuüben

6. Die Assistenz in der vertrauten Umgebung sowie die unterstützende Technologie müssen sehr viel stärker beachtet werden

7. Es ist größeres Gewicht auf die Lebensqualität sowie auf die individuellen Kriterien für Lebensqualität zu legen

Zahlreiche Projekte zentrieren sich um die Förderung individueller Entscheidungskompetenz durch spezifische Bildungsansätze. Dabei kann immer wieder beobachtet werden, dass schon durch geringfügige Interventionen mit dem Ziel, die Entscheidungskompetenz des Individuums zu erhöhen (zum Beispiel in Bezug auf die Ausführung bestimmter Aktivitäten des täglichen Lebens oder die Gestaltung des Alltags), auch bei Menschen mit Behinderung eine Zunahme der Motivation, eigene Entscheidungen zu treffen, erreicht werden kann. Die Fähigkeit, Entscheidungen zu treffen, wird dabei als zentral für die Lebensqualität des Menschen betrachtet. Denn die Fähigkeit, Entscheidungen zu treffen, ist nicht nur Ausdruck von Autonomie und Kompetenz, sondern sie stellt zudem eine bedeutsame Maßnahme zur Erfüllung persönlicher Ziele dar.

Die Förderung der Entscheidungskompetenz dient auch der Stärkung persönlicher Kontrolle des Individuums über Ereignisse in seiner sozialen Umwelt (Beispiele: Einfluss auf das Verhalten anderer Menschen nehmen, das Verhalten anderer Menschen besser vorhersagen können, Gewissheit, dass eigene Werte und Bedürfnisse von anderen Menschen respektiert werden). Der Verlust persönlicher Kontrolle ist vielfach für die Entstehung von Depressionen verantwortlich. Zudem wird „schwieriges“ oder „herausforderndes“ Verhalten von Menschen, die nur über geringe Kontrolle verfügen, als Möglichkeit wahrgenommen, trotzdem Kontrolle über ihre Umwelt auszuüben.

Sowohl im Forschungsteam von Heller als auch im Forschungsteam von Haveman wurde Lernmaterial zur Förderung von Entscheidungskompetenz entwickelt und evaluiert. Dieses Material wird als Teil eines umfassenden Bildungskonzepts verstanden. Im Zentrum der von Heller und Mitarbeitern entwickelten Lerneinheit steht die Vermittlung von Wissen und Fertigkeiten in folgenden Bereichen: 1. Der Prozess der Entscheidungsfindung: Wie können individuell und sozial verantwortliche Entscheidungen getroffen werden? (Hier findet sich eine Übereinstimmung mit den beiden zentralen Kategorien der Selbstverantwortung und der Mitverantwor- 
tung.) 2. Die Rechte und Pflichten des Individuums. 3. Selbstbestimmung bei der Nutzung der freien Zeit sowie bei der Gestaltung des Alters. 4. Gesundheitsbewusste Lebensführung. - Die von Haveman ausgearbeitete Lerneinheit zum Thema „Selbstbestimmt älter werden" setzt sich aus folgenden sieben Lernschritten zusammen: 1. Selbstbestimmt Entscheidungen treffen. 2. Den Übergang in den Ruhestand vorbereiten (hier wird auch die Einbeziehung flexibler Arbeitszeitmodelle empfohlen). 3. Wohnformen kennen lernen. 4. Gründung und Pflege sozialer Beziehungen. 5. Erwerb von Kompetenzen für eine selbstverantwortliche Freizeitgestaltung. 6. Ausbildung der Bereitschaft und der Fertigkeiten zur gesundheitsbewussten Lebensführung. 7. Fähigkeit zur psychischen Auseinandersetzung mit Erkrankungen.

Ein spezielles Programm von Heller zielte auf die Vorbereitung von älteren Menschen mit geistiger Behinderung auf den Austritt aus dem Berufsleben (Heller et al., $1996^{42}$ ). Dieses Programm, welches unter der Überschrift „Person zentrierte Lebensplanung für das höhere Lebensalter " stand, umfasste folgende Bildungseinheiten:

- Training in Entscheidungsverhalten

- Training in Zieldefinition

- Informationen über Möglichkeiten, Gesundheit und Wohlbefinden zu erhalten

- Informationen über Freizeitaktivitäten und freiwillige Tätigkeiten im Ruhestand

- Informationen über Wohnformen und Freundschaften

- Training für Mitarbeiter von Einrichtungen und Familienangehörige mit dem Ziel, diese in die Lage zu versetzen, ältere Menschen mit geistiger Behinderung bei der Lebensplanung zu unterstützen

Die genannten Projekte sind Beispiele für die seit Beginn der 90er Jahre im internationalen und nationalen Raum zu beobachtende Akzentsetzung im Hinblick auf die Förderung von Selbstverantwortung. Die Hauptanliegen dieser Projekte lassen sich dabei in zwei Punkten zusammenfassen: Zum einen soll die Fähigkeit zum selbstständigen und selbstverantwortlichen Leben erhalten oder gefördert werden, zum anderen soll der Umzug in eine Pflegeeinrichtung aufgrund eingetretenen Hilfeoder Pflegebedarfs vermieden werden. Die Ergebnisse entsprechender Projekte (siehe auch Kruse et al., 2002 ${ }^{43}$ ) stützen die Annahme der auch bei älteren Menschen mit geistiger Behinderung gegebenen Lernfähigkeit. Schon ein drei bis vier Monate dauerndes Training in Selbstsicherheit und Entscheidungsverhalten führt zu statistisch signifikanten Verbesserungen in diesen beiden Merkmalen. Aus diesem Grunde ist es gerechtfertigt, die Förderprogramme für ältere Menschen mit geistiger Behinderung auch aus einer Bildungsperspektive zu betrachten (zum Beispiel Kruse,

42 Heller et al. (1996), Impact of person-centered later life planning training program for older adults with mental retardation, Journal of Rehabilitation, 62, 77-83.

43 Kruse et al. (2002), Den Jahren Leben geben. Lebensqualität im Alter bei Menschen mit Behinderungen, Projektbericht, Stuttgart: Diakonisches Werk Württemberg. 
$1997^{44}$; Kruse et al., 2002 ${ }^{45}$ ), die ihrerseits ein zentrales Merkmal der Eingliederungshilfe darstellt.

Die Ergebnisse deuten zudem auf die auch bei älteren Menschen mit geistiger Behinderung gegebene kognitive Plastizität und Verhaltensplastizität. Im allgemeinen Sinne beschreibt Plastizität die Reservekapazität der Nervenzelle, das heißt, das Potenzial zu erhöhter Reaktions- und Anpassungsfähigkeit, welches unter Aktivierung ausgeschöpft wird. Bei fehlender Aktivierung wird hingegen dieses Potenzial nicht genutzt; die Zelle zeigt nicht jene Reaktions- und Anpassungsfähigkeit, die sie potenziell erbringen könnte. Die Verhaltens- und kognitive Plastizität im hohen Lebensalter konnte zum Beispiel in Studien nachgewiesen werden, in denen potenzielle Wirkungen einer selbstständigkeitsfördernden Umwelt auf die alltagspraktische Kompetenz untersucht wurden (siehe dazu M. Baltes, 1995\%6 ; Zank \& Baltes, $1998^{47}$ ). In diesen Studien wurde zunächst aufgezeigt, dass das Verhalten von Pflegefachkräften als „selbstständigkeitsfördernd“ vs. „,abhängigkeitsfördernd“ klassifiziert werden kann: Im ersten Falle (selbstständigkeitsfördernd) unterstützen Pflegefachkräfte die Bewohner in deren Bemühen, Aktivitäten des täglichen Lebens möglichst selbstständig auszuführen - sie geben diesen mehr Zeit für die Ausführung dieser Aktivitäten, sie beraten diese, sie greifen möglicht selten in die Ausführung einer Handlung ein, sie verstärken selbstständigkeitsorientiertes Verhalten und ignorieren abhängigkeitsorientiertes Verhalten der Bewohner. Im letzteren Falle (abhängigkeitsfördernd) behindern Pflegefachkräfte die Bewohner in deren Bemühen, Aktivitäten des täglichen Lebens selbstständig auszuführen - indem sie in die Ausführung von Handlungen direkt eingreifen, stellvertretend für den Bewohner Handlungen übernehmen, selbstständigkeitsorientiertes Verhalten ignorieren und abhängigkeitsorientiertes Verhalten verstärken. Je nach Orientierung der Pflegefachkräfte zeigen die Bewohner unterschiedliche Grade von Selbstständigkeit: Im ersten Falle ist eine deutliche Zunahme der Selbstständigkeit, im letzteren Falle eine deutliche Zunahme der Abhängigkeit erkennbar. Selbstständigkeitsfördernde Umwelten können auch im Sinne der Aktivierung und Nutzung von Reservekapazitäten verstanden werden, abhängigkeitsfördernde Umwelten hingegen im Sinne von Kontexten, welche die Reservekapazität (Plastizität, Veränderungspotenzial) des Zentralnervensystems ungenutzt lassen. Das im Arbeitskreis von M. Baltes entwickelte Forschungsparadigma wurde von Kruse et al. $\left(2002^{48}\right)$ auf die Analyse der Konzepte in der Enzyklopädie der Psychologie - Pädagogische Psychologie: Psychologie in Erwachsenenbildung (S. 120-166), Göttingen: Hogrefe.

45 Kruse et al. (2002), Medizinpsychologische und -soziologische Aspekte des Alterns, in: Brähler/Strauß (Hrsg.), Handlungsfelder in der Psychosozialen Medizin (S. 163-184), Göttingen: Hogrefe.

Baltes (1995), Verlust der Selbstständigkeit im Alter: Theoretische Überlegungen und empirische Befunde, Psychologische Rundschau, 46, 159-170.

47 Zank/Baltes (1998), Förderung von Selbständigkeit und Lebenszufriedenheit alter Menschen in stationären Einrichtungen, in: Kruse (Hrsg.), Psychosoziale Gerontologie, Band 2: Intervention (S. 60-72), Göttingen: Hogrefe.

Kruse et al. (2002), Den Jahren Leben geben, Stuttgart: Verlag Diakonisches Werk. 
Behindertenhilfe übertragen. Auch hier wurde eine Differenzierung nach „selbstständigkeits- vs. abhängigkeitsfördernder“ Betreuung und Pflege vorgenommen; es wurden - ganz ähnlich wie im Arbeitskreis von M. Baltes - Interventionen bei Mitarbeitern durchgeführt, die dazu dienen sollten, deren Selbstständigkeitsorientierung im Betreuungs- und Pflegeverhalten zu fördern. Es ließ sich zeigen, dass selbstständigkeitsorientiertes Verhalten der Mitarbeiterinnen und Mitarbeiter mit einer signifikanten Zunahme der Selbstständigkeit, Selbstverantwortung und subjektiv erlebten Kompetenz bei den Bewohnern einhergeht. Diese Untersuchungsbefunde sprechen nicht nur für die positiven Effekte der selbstständigkeitsorientierten Betreuung und Pflege. Sie deuten auch auf die bei älteren Menschen mit geistiger Behinderung bestehende Verhaltens- und kognitive Plastizität. - In dieser Studie wurde an die Mitarbeiterinnen und Mitarbeiter abschließend folgende Frage gerichtet: „Was können Sie in Ihrer täglichen Arbeit tun, um die Kompetenz der älter werdenden und alten Menschen mit geistiger Behinderung möglichst lange zu erhalten und zu fördern"'? Nachfolgend sind die zentralen Kategorien aufgeführt, um die sich die Antworten zentrierten und die somit den Kern der Betreuungs- und Pflegekonzepte bilden:

1. Sicherheit bieten

- durch akzeptierende Grundhaltung

- durch feste Bezugspersonen

- durch gleiche Abläufe

2. Erhaltung des Aufgabenspektrums in der Wohngruppe

3. Aktivierung

- durch körperliche Mobilisierung

- durch anregende Umweltgestaltung

4. Unterstützung selbstständigkeitsorientierten Verhaltens

5. Förderung sozialer Kontakte

6. Individuelle Betreuungsplanung, Betreuung und Förderung

7. Anpassung an die Ressourcen der Person

- mehr Zeit geben

- die Anforderungen den Fähigkeiten anpassen

8. Ermöglichen von Erfolgserlebnissen

9. Entgegenbringen von Respekt

Vor dem Hintergrund der hier kurz skizzierten Modelle und Untersuchungsergebnisse lassen sich die zentralen Anforderungen an kompetenzfördernde Konzepte in der Behindertenhilfe wie folgt zusammenfassen: 1. Es ist an früheren Förderansätzen anzuknüpfen. 2. Die Nutzung und das Training spezifischer Funktionen und Fertigkeiten sind fortzusetzen. 3. Nach Ausscheiden aus der Werkstätte für Behinderte ist ein ausreichendes Maß an Tagesstrukturierung sicherstellen. 4. Es sind Möglichkeiten zur Kreativität, zum körperlichen Training (Ausdauer, Kraft, Geschicklichkeit) und zum alltagspraktischen Training zu schaffen. 5. Nach Ausscheiden aus der Werkstätte für Behinderte sollten die Kontakte zu dieser bestehen bleiben. 6. Bei der Entwicklung kompetenzfördernder Konzepte ist von lebenslanger 
Lern- und Entwicklungsfähigkeit auszugehen. Im Rahmen eines Gesamtplans (§ 46 BSHG) ist das angemessene individuelle Hilfeangebot fortzuschreiben. 7. Dem Wunsch- und Wahlrecht von behinderten Menschen kommt im Alter ganz besondere Bedeutung zu. Hier ist die Flexibilisierung der Angebote wichtig, auch erhält die Beratung großes Gewicht. 8. Menschen mit Behinderung sollen in der Einrichtung bzw. in dem ihn bekannten Wohnumfeld verbleiben können, um somit die Kontinuität des Lebensumfeldes wahren zu können. In Wohneinrichtungen der Eingliederungshilfe ist das Wohnen in altersheterogenen und altershomogenen Gruppen vorzusehen. 9. Für Menschen mit Behinderung ist es normal, mit spezifischen Hilfen, nämlich mit jenen der Eingliederungshilfe, ein möglichst selbstständiges Leben zu führen. An diesem Hilfebedarf ändert sich prinzipiell nichts bei Erreichen einer definierten Altersgrenze. Insoweit spricht das Normalitätsprinzip für die Beibehaltung der angestammten Lebenszusammenhänge auch im Alter, insbesondere aus sozialen Gründen. 10. Menschen mit Behinderung, die bereits in einer Einrichtung der Eingliederungshilfe gelebt haben, können für sich nach dem Normalitätsprinzip in Anspruch nehmen, dass die Kontinuität ihrer Lebenszusammenhänge gewahrt bleibt, wenn sie einen altersspezifischen Hilfebedarf entwickeln.

Das Pflegeheim ist für Menschen mit Behinderung in der Regel nicht die geeignete Wohn- und Betreuungsform. Ein Umzug nur aufgrund des Ausscheidens aus der Werkstätte für Behinderte oder des Erreichens des 65. Lebensjahres in Pflegeheim darf nicht erfolgen. Um einen Umzug in eine Pflegeeinrichtung zu vermeiden, sind in Einrichtungen der Behindertenhilfe Pflegeangebote zu schaffen. Es bedarf hier der entsprechenden räumlichen Gestaltung und sächlichen Ausstattung in den Wohneinrichtungen. Die Empfehlung, dass ältere Menschen mit Behinderung in ihrem gewohnten Lebensumfeld verbleiben sollten und aus diesem Grunde der Umzug in eine Einrichtung der Altenhilfe möglichst zu vermeiden sei, ist nur dann zu verwirklichen, wenn Mitarbeiter in Einrichtungen der Behindertenhilfe über ausreichende Kompetenzen in der Altenhilfe verfügen - zu nennen sind hier Grundlagenkenntnisse in Fragen der Gerontologie, der Geriatrie, der Gerontopsychiatrie, der aktivierenden Pflege. Umgekehrt müssen Mitarbeiter jener stationären Einrichtungen der Altenhilfe, die Menschen mit Behinderung aufnehmen, über ausreichende Kompetenzen in der Heilpädagogik (und zwar in beiden Bereichen: Bildung und Rehabilitation) verfügen.

\section{In welcher Hinsicht lassen sich die Kompetenz und Lebensqualität durch die Entwicklung innovativer Konzepte fördern?}

Zunächst werden Aussagen zu zentralen Merkmalen der Kompetenz und Lebensqualität bei Behinderung im höheren Lebensalter getroffen; auch hier steht die Lebenssituation von Menschen mit geistiger Behinderung im Vordergrund. Dabei werden Kompetenz und Lebensqualität noch stärker als bisher aus der Sicht der Intervention betrachtet. - In einem zweiten Schritt werden zentrale Kategorien innovativer Konzepte der Arbeit mit älteren Menschen, bei denen eine Behinderung besteht, disku- 
tiert. Die Diskussion orientiert sich allem an den ,Fachlichen Leitlinien und Empfehlungen', die vom Hessisches Sozialministerium und dem Landeswohlfahrtsverband Hessen in Kooperation mit Wissenschaft und Praxis entwickelt wurden (Hessisches Sozialministerium \& Landeswohlfahrtsverband, 2000 ${ }^{49}$ ). - In einem dritten Schritt wird die Entwicklung innovativer Konzepte aus der Perspektive der Einrichtungen der Behindertenhilfe sowie der Mitarbeiterinnen und Mitarbeiter dieser Einrichtungen untersucht: In welchen Bereichen der Arbeit mit älteren Menschen, bei denen eine geistige, eine körperliche oder eine seelische Behinderung vorliegt, sehen Mitarbeiterinnen und Mitarbeitern der Behindertenhilfe in Deutschland besonderen Handlungsbedarf? Bei der Beantwortung dieser Frage stützen wir uns auf eine in 45 Einrichtungen der Behindertenhilfe durchgeführte Expertenbefragung.

\subsection{Zentrale Merkmale der Kompetenz und Lebensqualität bei Behinderung im Alter aus einer Interventionsperspektive}

Den Ausgangspunkt jener Arbeiten, in denen der Versuch unternommen wird, Merkmale der Kompetenz und der Lebensqualität zu differenzieren, orientieren sich vielfach an Arbeiten zum ,erfolgreichen Altern“. In einer von P. Baltes \& M. Baltes $\left(1990^{50}\right)$ herausgegebenen Monographie zum „erfolgreichen Altern“ werden sieben Merkmale genannt, die bei einer Definition des Konstrukts zu berücksichtigen sind.

1. Lebensdauer

2. Positiver psychischer Status

3. Gute allgemeine Gesundheit

4. Erhaltene kognitive Kompetenz

5. Kontrolle über das eigene Leben und über die zu verfolgenden Ziele

6. Erhaltene soziale Kompetenz und fortdauerndes produktives Engagement

7. Hohe subjektive Zufriedenheit mit dem eigenen Leben

Es liegen Arbeiten vor, welche die hier genannten Merkmale erfolgreichen Alterns mit Merkmalen der Kompetenz und Lebensqualität von Menschen mit Behinderung verbinden (Hawkins, 1999 $9^{51}$; Schalock, 1996 ${ }^{52}$, 1997 ; Schalock et al., $1999^{54}$; Taylor \& Bogdan, $1996^{55}$ ). In diesen Arbeiten werden vor allem folgende

49 Hessisches Sozialministerium \& Landeswohlfahrtsverband Hessen (2000), Lebensräume älterer Menschen mit Behinderung. Fachliche Leitlinien und Empfehlungen, Wiesbaden: Hessisches Sozialministerium.

50 Baltes/Baltes (Ed.) (1990), Successful aging: Perspectives from the behavioral sciences, New York: Cambridge University Press.

51 Hawkins (1999), Rights, Place of Residence, and Retirement: Lessons from Case Studies on Aging, in: Herr/Weber (Eds.), Aging, rights, and quality of life (pp. 93-108), Baltimore: Brookes.

52 Schalock (Ed.) (1996), Quality of Life, Vol. I: Conceptualization and measurement, Washington, DC: American Association of Mental Retardation.

53 Schalock (Ed.) (1997), Quality of Life, Vol. II: Application to persons with disabilities, Washington, DC: American Association of Mental Retardation.

54 Schalock et al. (1999), Enhancing quality of life, in: Herr/Weber (Eds.), Aging, rights, and quality of life (pp. 81-92), Baltimore: Brookes. 
Merkmale genannt, die bei Aussagen zur Kompetenz und Lebensqualität von älteren Menschen mit Behinderung zu berücksichtigen sind und die aus diesem Grunde auch als konstitutiv für erfolgreiches Altern gewertet werden:

1. Gesundheitsstatus

2. Wahrgenommene Lebenssituation

3. Die Möglichkeit mitverantwortlichen Lebens, das heißt, die Möglichkeit, sowohl zur Gemeinschaft als auch zur Gesellschaft aktiv beizutragen.

Im Arbeitskreis von Schalock wurde ein Fragebogen zur Erfassung der Lebensqualität bei älteren Menschen mit geistiger Behinderung entwickelt (Schalock, 1997). Dieser Fragebogen umfasst 40 Items, die sich auf die von der Person selbst eingeschätzten Grade der
a) Selbstständigkeit
b) Produktivität
c) Integration in die Gemeinschaft
d) Zufriedenheit

beziehen. Dieser Fragebogen wurde in der Klientel des ,Mid-Nebraska Mental Retardation Service Program' eingesetzt und diente als Grundlage für die jährlich erfolgende Evaluation des Programms. Eine Analyse von 21 60jährigen und älteren Personen ergab, dass im Laufe eines fünfjährigen Beobachtungszeitraums die selbst bewertete Lebensqualität um 3 Prozent zurückging - und mit leichten Rückgängen in den Graden der Selbstständigkeit, der Produktivität und der Integration in die Gemeinschaft. Zugleich fand sich in diesem Zeitraum eine leichte Zunahme in der selbst eingeschätzten Zufriedenheit (Schalock, 1997).

In Tabelle 1 ist eine von Schablock et al. (1999) erarbeitete Aufstellung von Dimensionen der Lebensqualität sowie von Indikatoren der einzelnen Dimensionen wiedergegeben, die - wie die Autoren auf der Grundlage einer größeren Anzahl von empirischen Studien bei älteren Menschen belegen können - zentral für die Erfassung von Lebensqualität bei Behinderung sind. Vor allem sind diese Dimensionen und Indikatoren für eine Evaluation von Programmen geeignet, die für Menschen mit Behinderungen entwickelt wurden. 


\begin{tabular}{|c|c|}
\hline Dimension & Indicators \\
\hline Emotional well-being & $\begin{array}{l}\text { Freedom from fear } \\
\text { Spirituality } \\
\text { General mood } \\
\text { Self-concept } \\
\text { Freedom from anxiety }\end{array}$ \\
\hline Interpersonal relationships & $\begin{array}{l}\text { Family } \\
\text { Friends } \\
\text { Neighbours } \\
\text { Activity personnel } \\
\text { Caregivers }\end{array}$ \\
\hline Material well-being & $\begin{array}{l}\text { Home / Residence } \\
\text { Money } \\
\text { Personal possessions } \\
\text { Clothing } \\
\text { Insurance }\end{array}$ \\
\hline Personal development & $\begin{array}{l}\text { Educational } \\
\text { Activities } \\
\text { Intellectual } \\
\text { Reading materials } \\
\text { Helping others }\end{array}$ \\
\hline Physical well-being & $\begin{array}{l}\text { Physical health } \\
\text { Food } \\
\text { Health care } \\
\text { Medication level } \\
\text { Energy level }\end{array}$ \\
\hline Self-determination & $\begin{array}{l}\text { Decisions about daily activities } \\
\text { Decisions about what I eat and drink } \\
\text { Decisions about how I spend time } \\
\text { Personal opinions } \\
\text { Personal goals }\end{array}$ \\
\hline Social inclusion & $\begin{array}{l}\text { Accepted by cohabitants } \\
\text { Accepted by staff } \\
\text { Receiving help from cohabitants } \\
\text { Receiving help from staff } \\
\text { Participation }\end{array}$ \\
\hline
\end{tabular}


Rights
Privacy
Input into decisions
Protection of possessions
Compliance with legal and human rights Safety

Tabelle 1: Dimensionen und Indikatoren der Lebensqualität (aus: Schalock et al., 1999)

\subsection{Zentrale Kategorien innovativer Konzepte der Behindertenhilfe}

Mit den „Fachlichen Leitlinien und Empfehlungen“ des Hessischen Sozialministeriums und des Landeswohlfahrtsverbandes Hessen zur Zukunft der Behindertenhilfe wird das Ziel verfolgt, Anregungen zur Entwicklung innovativer Konzepte in der Behindertenhilfe zu geben. Unternimmt man den Versuch, die zentralen Kategorien dieser Leitbilder und Empfehlungen herauszuarbeiten, so sind zunächst drei Bereiche zu nennen, um die sich diese Leitbilder und Empfehlungen gruppieren:

1. Wohnen

2. Tagesgestaltung

3. Übergang von der Arbeit in den Ruhestand (auch im Sinne der individuellen Planung)

Darüber hinaus werden in den Leitbildern und Empfehlungen grundlegende Orientierungen sichtbar, die sich in folgenden fünf Kategorien zusammenfassen lassen:

1. Planung von Angeboten - und zwar regional wie überregional.

2. Flexibilität - und zwar sowohl bei der Planung als auch bei der Umsetzung von Angeboten sowie bei der Ansprache von Menschen mit Behinderung.

3. Übergänge zwischen verschiedenen Angeboten - vor allem zwischen jenen der Behindertenhilfe und der Altenhilfe.

4. Entscheidungskompetenz und Wahlrecht - dies vor allem mit Blick auf den Wohn- und Lebensort, auf die Gestaltung des Alltags sowie auf die Art der Begleitung, der Förderung, der Betreuung, der Unterstützung und der Pflege.

5. Beratung - mit dem Ziel, die Entscheidungskompetenz sowie das Wahlrecht nachhaltig zu stützen.

Die in den Leitbildern und Empfehlungen entwickelten Handlungsempfehlungen lassen sich wie folgt zusammenfassen:

1. Vorbereitung und Begleitung des Übergangs von der Arbeit in den Ruhestand

2. Erhaltung der individuellen Selbstorganisation durch ambulante Dienste

3. Sicherstellung ambulanter Pflegeleistungen

4. Ausbau des Betreuten Wohnens

5. Weiterentwicklung der Tagesstrukturierung und von Freizeitangeboten 
6. Förderung des Wohnens in altersheterogenen und altershomogenen Gruppen

7. Einrichtung von Wohngruppen

8. Einrichtung von Wohngruppen mit pflegerischem Betreuungsschwerpunkt

\subsection{Entwicklung innovativer Konzepte aus der Perspektive der Institutionen}

Es wurden Mitarbeiterinnen und Mitarbeiter aus 45 Einrichtungen der Behindertenhilfe dazu befragt, in welchen Bereichen der Behindertenhilfe sie Handlungsbedarf sehen, um damit einen Beitrag zur Förderung der Kompetenz und Lebensqualität bei älteren Menschen mit Behinderung zu leisten.

Die Antworten der Experten zentrierten sich um die folgenden sieben Bereiche:

1. Verstärkte Kompetenzorientierung (anstelle einer einseitigen Defizitorientierung) in der Arbeit mit älteren Menschen mit geistiger Behinderung

2. Vorbereitung auf den Berufsaustritt sowie Unterstützung beim Berufsaustritt, Ermöglichung eines allmählichen (nicht abrupten) Übergangs in die nachberufliche Zeit, Ausbau tagesstrukturierender Angebote

3. Vermehrte Berücksichtigung individueller Bedürfnisse, Kompetenzen und Interessen

4. Entwicklung spezifischer Angebote für Menschen mit seelischer Behinderung

5. Verstärkter Ausbau des betreuten Wohnens sowie von Wohngruppen in der Gemeinde, wobei diese Angebote als Ergänzung, nicht als Alternative zu stationären Einrichtungen zu verstehen sind

6. Vorbereitung der Familienangehörigen auf einen möglichen Umzug der Angehörigen mit Behinderung in eine stationäre Einrichtung, in bereutes Wohnen oder in eine Wohngruppe

7. Entwicklung einer engeren Kooperation zwischen Behindertenhilfe und Altenhilfe

Die zu den einzelnen Bereichen getroffenen Aussagen, in denen sich der wahrgenommene Handlungsbedarf widerspiegelt, seien nachfolgend zusammenfassend dargestellt.

\section{Themenbereich 1:}

\section{Vermehrte Kompetenzorientierung in der Arbeit mit älteren Menschen}

Die Einrichtungen der Behindertenhilfe sollen sich auch in der Arbeit mit älteren Menschen sehr viel stärker als heute an Kompetenzmodellen orientieren und die einseitige Orientierung an Defizitmodellen aufgeben. Diese Aussage gilt zum einen für Bildungsangebote, die auch ältere Menschen ansprechen müssen, zum anderen für die Förderung der Kompetenz im Alltag, zum dritten für Maßnahmen, die auf eine Stärkung der sozialen Teilhabe zielen, zum vierten für Maßnahmen der Aktivierung, die sich am Leitbild der Rehabilitation orientieren sollten. 
Es wird kritisch angemerkt, dass sich noch zu viele Einrichtungen an den Defizitmodellen des Alters orientieren und ältere Menschen mit Behinderung im Alter nicht ausreichend fordern und fördern. Durch die Unterschätzung der Lern- und Veränderungsfähigkeit im Alter werden Entwicklungspotenziale dieser Lebensphase nicht ausreichend ausgeschöpft.

\section{Themenbereich 2:}

\section{Übergang in den Ruhestand, tagesstrukturierende Angebote im Alter}

1. Der Übergang in den Ruhestand muss begleitet werden - zu nennen sind als Beispiel die gemeinsamen Nachmittage in einer Tagesstätte für ältere Menschen, in Wohnheimen wird eine Tagesstruktur angeboten, Finanzierung des Werkstattplatzes über das 65. Lebensjahr hinaus wird auf der Grundlage von Vergütungsvereinbarungen übernommen; anzustreben ist die Entwicklung eines kontinuierlichen Freizeit- und Bildungsangebots

2. Nach Ausscheiden aus der Werkstatt ist Verbleib in der vertrauten Umgebung wichtig

3. Es sollten tagesstrukturierende Angebote in der Nähe von Wohneinrichtungen geschaffen werden - notwendig ist der flächendeckende Ausbau solcher Angebote

4. Tagesstätten und den alltagsgestaltenden Angeboten kommt wachsende Bedeutung zu

5. Die Tagesstruktur in kleinen Wohneinheiten ist weiter zu entwickeln; Altentagesstätten und gemeindenahe Angebote müssen geschaffen werden

6. Freizeitangebote unter Einbeziehung ehrenamtlicher Helfer sind bedeutsam und müssen ausgebaut werden

7. Für die Tagesstruktur sind noch keine praktikablen Lösungsversuche entwickelt worden - eine enge Kooperation der Werkstattträger mit den Wohnheimträgern ist notwendig

\section{Themenbereich 3:}

\section{Stärkere Beachtung der individuellen Bedürfnisse und Kompetenzen bei der Entwicklung von Angeboten}

1. ,Präventive‘ Beratung mit dem Ziel, Berührungsängste gegenüber Wohnheimen möglichst weit abzubauen - Freizeit- und Urlaubsangebote in Wohnheimen, Kurzzeitpflege

2. Über die Bedürfnisse von älteren Menschen mit Behinderungen ist zu wenig bekannt

3. Unterstützende Hilfen können Fertigkeiten erweitern

4. Vergleiche behinderte Menschen nicht mit pflegebedürftigen Menschen, sondern vergleiche sie mit nicht behinderten Menschen

5. Die Bedürfnisse von Menschen mit körperlicher Behinderung müssen bei der Entwicklung von Angeboten stärkere Beachtung finden 
6. Besondere Berücksichtigung der Lebenssituation behinderter Frauen. Bei der Pflege ist behinderten Frauen weibliche Assistenz zur Verfügung zu stellen

7. Ausbau des Case Management, um Menschen mit Behinderung und ihre Angehörigen besser begleiten und betreuen zu können

\section{Themenbereich 4: \\ Kooperation zwischen Behindertenhilfe und Altenhilfe (Vernetzung, Integration)}

1. Vernetzung zwischen Behindertenhilfe, Pflegediensten, (ambulanter, teilstationärer und stationärer) Altenhilfe und Infrastruktur in den Gemeinden

2. Kooperation zwischen Altenhilfe und Behindertenhilfe kann nur auf regionaler Ebene erfolgen - Überlegungen zur Einrichtung einer entsprechenden Regionalkonferenz werden angestellt; für die Strukturentwicklung - mit einer engeren Kooperation zwischen der Behinderten- und der Altenhilfe - sind solche Regionalkonferenzen wichtig

3. Die Eingliederungshilfe bietet mehr als die Pflegeversicherung

4. Integration von Sozialhilfeleistungen und Pflegeversicherungsleistungen

5. Aufrechterhaltung der Versorgungsverträge für Pflegeabteilungen in Einrichtungen der Behindertenhilfe

6. Angebote der Altenhilfe sollten geöffnet werden für Menschen mit Behinderung

7. Pflegerische Leistungen gehören bei Menschen mit Behinderung zur Eingliederungshilfe

8. Die fachlichen Anforderungen bei entstehendem Pflegebedarf sind ungeklärt

\section{Themenbereich 5: \\ Angebote für Menschen mit seelischer Behinderung}

1. Es sind auch mit Blick auf psychisch erkrankte Menschen Übergänge vom ambulanten in den stationären Bereich zu schaffen

2. Es sind Tagesstättenangebote für psychisch erkrankte Menschen zu schaffen

3. Vernetzungen im Sinne eines gemeindepsychiatrischen Verbundes sind wichtig - die Hilfeleistungen der älter werdenden seelisch behinderten Menschen sollen an bereits existierende oder neu aufzubauende gemeindepsychiatrische Angebote angebunden werden, um auf diese Weise eine gemeindenahe Versorgung zu ermöglichen (Betreuung am bisherigen Lebensort)

4. Belegungskonferenzen sind notwendig, auf deren Grundlage für Menschen mit seelischer Behinderung ein Versorgungsnetz geschaffen werden kann, welches sich auf individuelle Bedarfe und Bedürfnisse einstellen kann

5. Entwicklung von Konzepten für Wohnheime für Menschen mit seelischer Behinderung, die auch bei körperlichen Einbußen den Verbleib am Wohnort ermöglichen 
6. Pflegekassen betrachten Wohneinrichtungen für Menschen mit seelischer Behinderung nicht als Wohnort, sondern als Einrichtung; aus diesem Grunde wird nur ein monatlicher Festbetrag gezahlt

\section{Themenbereich 6: Wohnen}

1. Das Betreute Wohnen (Wohnen im Verbund) ist auszubauen; die Flexibilisierung bestehender Wohnangebote ist notwendig; neue Einrichtungen müssen nicht geschaffen werden; Formen betreuten Wohnens sind für alle Gruppen behinderter Menschen zu schaffen

2. Bildung von Wohngruppen mit Schwerpunkt ,Pflege“ wird vorgenommen

3. In Heimen sollten verschiedene Wohngruppen eingerichtet werden - doch sind zwei Fragen ungeklärt: (a) Wer übernimmt die Betreuung? Inwieweit sind die Berufsgruppen für die spezifischen Betreuungsaufgaben qualifiziert? (b) In welcher Trägerschaft stehen die Einrichtungen, wer ist für deren Finanzierung verantwortlich?

4. Persönliche Assistenz und unterstütztes Wohnen

\section{Themenbereich 7: Kommunale Perspektive}

1. Die kommunale Perspektive ist zu stärken - sie wird bislang zu wenig beachtet

2. Die Übernahme von Verantwortung der Kommunen für die Weiterentwicklung bestehender Angebote sowie für die Planung neuer Angebote ist vielfach zu gering

3. Der Austausch zwischen Kommunen ist zu stärken, ebenfalls die Vernetzung zwischen den Regionen - Regionalkonferenzen sind auf kommunaler Ebene auszurichten; nur wird diese Forderung praktisch nicht umgesetzt, da keiner die Initiative ergreift

4. Eine einheitliche Haltung der kommunalen Spitzenverbände ist nicht erkennbar

5. Zeitdruck und Regelungsbedarf werden unterschätzt - in Kommunen wird vielfach die Notwendigkeit zusätzlicher Angebote nicht gesehen oder es wird streng nach bisherigen Vorgaben gearbeitet, eine Flexibilisierung der Angebote findet nicht statt

6. Bedarfsanalysen und Planungskonferenzen sind bedeutsam: Eine regionalspezifische Bedarfskalkulation ist zu leisten; in diesem Kontext kommt Prognosen des Pflegebedarfs großes Gewicht zu

7. Streit um Kostenzuständigkeit bildet eine Barriere der Umsetzung

8. Klare Kompetenzen und Zuständigkeiten der Träger - dies ist auch unter dem Aspekt verstärkter und effektiver Kooperation in der Kommune wichtig.

Bedeutsam erscheint hier vor allem die Tatsache, dass zahlreiche Anforderungen an die Kommune gestellt werden. In der Diskussion über die Zukunft der Behinder- 
tenhilfe wird die kommunale Perspektive vielfach nicht ausreichend beachtet. Folgt man den Ergebnissen der Befragung, so ist die Mitverantwortung der Kommunen für die Behindertenhilfe zu stärken, darüber hinaus wird die Bedeutung einer engeren Kooperation von Institutionen für die Versorgungsqualität hervorgehoben. Die Vernetzung von Angeboten wird als große Herausforderung für die Zukunft gewertet; eine Aufgabe der Kommunen wird darin gesehen, Anstöße zu dieser Vernetzung zu geben.

Auch in einem weiteren thematischen Bereich setzen die Ergebnisse der Befragung neue Akzente. Angesprochen ist hier der Bereich der Begleitung, Betreuung, Förderung, Unterstützung und Pflege älterer Menschen mit seelischer Behinderung. Keine der drei Gruppen - Menschen mit körperlicher, mit geistiger, mit seelischer Behinderung - darf benachteiligt werden; bei der Entwicklung von Konzepten ist in gleicher Weise auf alle drei Gruppen zu achten. Die Tatsache, dass in den Befragungen ausführlich auf die Situation der älteren Menschen mit seelischer Behinderung eingegangen und großer Handlungsbedarf gerade in Bezug auf die Versorgung dieser Gruppe konstatiert wurde, zeigt, dass in Zukunft noch mehr Gewicht auf diese Personengruppe gelegt werden muss. Diese scheint - folgt man den Aussagen in den Stellungnahmen - in der Diskussion von Konzepten und Angeboten nicht selten vernachlässigt zu werden.

Die in den Leitbildern und Empfehlungen wie auch in den Befragungen sichtbar werdenden Kategorien, mit denen die Interventionskonzepte für Menschen mit Behinderung beschrieben werden, vermitteln ein modernes Bild der Behindertenhilfe.

Dieses moderne Bild der Behindertenhilfe lässt sich am besten mit den drei folgenden Kategorien charakterisieren:

- Selbstständigkeit (im Sinne möglichst weiter Unabhängigkeit bei der Ausführung von Aktivitäten des täglichen Lebens)

- Selbstverantwortung (im Sinne möglichst weiter Selbstbestimmung bei der Gestaltung des Alltags, im Sinne von Entscheidungskompetenz und Wahlfreiheit, im Sinne der bewussten Auseinandersetzung mit der eigenen Person, ihren Werten, ihren Interessen, ihren Bedürfnissen: Wer bin ich? Was möchte ich tun?)

- Mitverantwortung (im Sinne möglichst weiter sozialer Partizipation an der Gesellschaft und an der Kultur, im Sinne des Engagements für andere Menschen)

\section{Entwicklungsperspektiven}

In der Behindertenhilfe - dies gilt sowohl für die Diskussion in Deutschland als auch für die Diskussion im internationalen Raum - wird vermehrt Gewicht auf Möglichkeiten zur sozialen Teilhabe (Partizipation) gelegt. Neben der Fähigkeit zur Vorbereitung und zum Treffen von Entscheidungen (Entscheidungskompetenz) sowie der Wahlfreiheit nehmen Möglichkeiten zur sozialen Teilhabe den breitesten Raum in der Diskussion neuer Konzepte ein. Dabei wird zum einen die Bedeutung des Wohnens für die soziale Teilhabe unterstrichen: Das Betreute Wohnen und das 
Wohnen im Verbund sind zentrale Elemente des Wohnens in der Gemeinde, durch die zur vermehrten sozialen Teilhabe beigetragen wird (wie auch zur Förderung von Selbstständigkeit und Selbstverantwortung). Zudem akzentuieren stationäre Einrichtungen der Behindertenhilfe die engen Beziehungen zwischen einer Einrichtung und der Gemeinde, um auf diese Weise soziale Teilhabe zu fördern und soziale Segregation abzubauen. Neben dem Wohnen wird der Gestaltung der sozialen Beziehungen große Bedeutung für die soziale Teilhabe beigemessen. Dabei ist es wichtig, dass sich die Beziehungen nicht allein auf Menschen mit Behinderung beschränken, sondern auch Beziehungen zu Menschen ohne Behinderung umfassen. In den USA gibt es zahlreiche ehrenamtliche Initiativen, die darauf zielen, ältere Menschen aus Einrichtungen der Behindertenhilfe und ältere Menschen ohne Behinderung, die Bildungseinrichtungen besuchen oder am Vereinsleben aktiv teilnehmen, zusammenzubringen, sodass (für beide Gruppen!) ein erkennbarer Zuwachs an sozialer Teilhabe möglich wird.

In vielen Einrichtungen der Behindertenhilfe ist ein hohes Maß an Kreativität in Bezug auf die Entwicklung von Konzepten für ältere Menschen mit Behinderung erkennbar. Die Grundlage dieser Konzepte bilden dabei die Kategorien: Selbstständigkeit (im Sinne der Fähigkeit, die Aktivitäten des täglichen Lebens selbständig ausführen zu können), Selbstverantwortung (im Sinne der Autonomie und Entscheidungskompetenz) und Mitverantwortung (im Sinne der sozialen Teilhabe). Es handelt sich hier vor allem um jene Einrichtungen, die bereits über Erfahrungen in der Arbeit mit älteren Menschen, bei denen Behinderung besteht, verfügen, und vor dem Hintergrund dieser Erfahrungen Aussagen über die Lern- und Veränderungspotenziale im Alter treffen können. - Das Verständnis der Situation von Menschen mit Behinderung ist somit nicht mehr (allein) von dem Gedanken der Fürsorge geprägt, sondern (auch) von dem Gedanken der Autonomie, der Kompetenz sowie der aktiven Teilhabe an Gemeinschaft. Der Austritt aus dem Beruf ist als eine Phase des Übergangs zu verstehen, in der sich wieder vermehrt die Frage nach Möglichkeiten der selbstständigen, selbstverantwortlichen und mitverantwortlichen Lebensführung stellt. Wie müssen die Förderkonzepte beschaffen sein, um auch nach Austritt aus dem Beruf die Fähigkeit und Bereitschaft des Individuums zur Selbstständigkeit, Selbstverantwortung und Mitverantwortung zu erhalten? Dabei ist zu bedenken, dass von der Berufstätigkeit vielfach kompetenzerhaltende und kompetenzfördernde Anregungen ausgehen, die nach Austritt aus dem Beruf fortfallen. Die Einrichtungen der Behindertenhilfe sind nun in besonderer Weise gefordert, Konzepte zur Erhaltung von Kompetenz anzubieten. Zentrale Bedeutung gewinnen hier Angebote zur Tagesstrukturierung, zur sozialen Teilhabe sowie zum Wohnen (betreutes Wohnen, Wohnen in Wohngruppen). In der Weiterentwicklung bestehender Angebote sehen die Einrichtungen der Behindertenhilfe eine zentrale Zukunftsaufgabe. Der demographische Wandel führt in Einrichtungen der Behindertenhilfe zu neuen Anforderungen.

Die Zunahme des durchschnittlichen Lebensalters der Bewohnerschaft erfordert neue Akzentsetzungen im Hinblick auf heilpädagogische Angebote. Diese lassen sich charakterisieren im Sinne: 
a) der Erhaltung von Fähigkeiten und Interessen, die Menschen in früheren Lebensjahren ausgebildet haben

b) der Erschließung von neuen Möglichkeiten sozialer und kultureller Teilhabe

c) der Erhaltung von Selbstständigkeit und Selbstverantwortung auch im Falle eintretender funktioneller Einbußen.

Diese Vielfalt von Anforderungen, die Einrichtungen der Behindertenhilfe mit Blick auf ein „gutes Leben“ im Alter zu erfüllen haben, legt die Forderung nach Aufrechterhaltung der Prinzipien der Eingliederungshilfe - und damit eines heilpädagogischen, bildungsbezogenen Konzepts - auch für ältere Menschen mit Behinderung nahe.

Die potenzielle Lern- und Veränderungsfähigkeit von älteren Menschen mit Behinderung wird erkannt - der Konzeptentwicklung liegt nicht selten ein Bildungsverständnis zugrunde, welches von der Bildbarkeit, dem Interesse an Bildung und der Notwendigkeit der Bildung über den gesamten Lebenslauf ausgeht.

In der internationalen Literatur werden vier Konzepte genannt, die für die Entwicklung der Behindertenhilfe als zentral erachtet werden:

1. Förderung von sozialer Integration, sozialer Partizipation und sozialem Engagement

2. Akzentuierung von Förderkonzepten, die von den Werten, Neigungen, Fertigkeiten und Bedürfnissen der einzelnen Person ausgehen

3. Akzentuierung der Förderung und Assistenz in der vertrauten Umwelt der Person

4. Orientierung am Leitbild des 'Ageing in place' (mit diesem Begriff wird ausgedrückt, dass Menschen auch im Alter in ihrer vertrauten Umgebung bleiben)

Dabei wird die Entwicklung der Behindertenhilfe vielfach in drei Phasen untergliedert, die unterschiedliche Akzentsetzungen der Art Dienstleistungen beschreiben:

Erste Phase: Institutionalisierung, vielfach auch Segregation. Die in der dieser Phase entwickelten Dienstleistungen orientierten sich vor allem an einem ,medizinischen Modell“. Behinderung wurde als eine Störung verstanden, die vor allem medizinische Diagnostik, Therapie und Rehabilitation erfordere. Als geeigneter Wohnund Lebensort für Menschen mit Behinderung wurde die Institution angesehen, da nur diese die Bereitstellung der medizinischen Diagnostik, Therapie und Rehabilitation gewährleisten könne.

Zweite Phase: De-Iinstitutionalisierung und verstärkte Entwicklung des Gemeindegedankens. Die in dieser Phase entwickelten Dienstleistungen bildeten vor allem das Resultat der Normalisierungsdebatte, wie diese speziell im nordeuropäischen Raum, zum Teil auch in den Vereinigten Staaten, Kanada und Australien geführt wurde. Menschen mit Behinderung sollte die Möglichkeit gegeben werden, in gleicher Weise am Leben in der Gemeinde teilzunehmen wie Menschen ohne Behinderung. Diese Zieldefinition ging von der grundlegenden Annahme aus, dass Menschen mit Behinderung dann umfassend am gemeinschaftlichen Leben teilhaben 
können, wenn gesellschaftliche Barrieren (und hier vor allem Stereotype) abgebaut werden. Behinderung wurde nicht länger als zentrales Merkmal des Menschen gesehen. Aus diesem Grunde wurde auch nicht mehr von behinderten Menschen, sondern vielmehr von Menschen mit einer Behinderung gesprochen. Darüber hinaus wurde in dieser Phase mehr und mehr die Lern- und Veränderungsfähigkeit des Menschen mit Behinderung in den Vordergrund gestellt und die Forderung nach Entwicklung von heilpädagogischen Förderkonzepten erhoben. Man kann hier auch von einer vermehrten Kompetenz-Orientierung in der Behindertenhilfe sprechen.

Dritte Phase: Leben in der Gemeinde, aktives Mitglied der Gemeinde, individualisierende Formen der Förderung, der Unterstützung, der Assistenz. Diese Phase charakterisiert die gegenwärtige Konzeptentwicklung in der Behindertenhilfe. Die Möglichkeit des Lebens in der Gemeinde und der Aktivität in der Gemeinde ist eng verbunden mit den Wohnformen. Betreutes Wohnen oder Wohnen im Verbund bilden eine Grundlage für soziale Partizipation und soziales Engagement. Im Falle des Wohnens in einer stationären Einrichtung der Behindertenhilfe ist es notwendig, dass diese Einrichtung ausreichend in die Gemeinde integriert ist. Denn nur dann sehen sich die Bewohner ausreichend motiviert, auch wirklich am Leben in der Gemeinde teilzunehmen. - Die individualisierenden Förderkonzepte gehen von der Annahme aus, dass Menschen im Lebenslauf spezifische Fertigkeiten und Interessen entwickelt haben, die in einem biographisch orientierten Bildungs- und Förderansatz aufgegriffen werden müssen. Darüber hinaus wird die Annahme vertreten, dass die Notwendigkeit der Bildung und Förderung über den gesamten Lebenslauf hinweg besteht, somit auch ältere Menschen mit Behinderung ausdrücklich in derartige Bildungs- und Förderkonzepte einbezogen werden müssen. Die Hilfen zur Wiedereingliederung enden also nicht mit einem bestimmten Lebensalter. 


\title{
3. Kulturwissenschaftliche Überlegungen zur Behindertenpolitik in Asien?
}

Einige sehr prinzipielle und unvorgreifliche Gedanken.

\author{
Von Peter Pörtner
}

Die in diesem Band versammelten Berichte und Statements bezeugen, wie verschieden die Interpretation und Umsetzung der Grundprinzipien der Behindertenpolitik selbst innerhalb der Grenzen der heutigen Europäischen Union sind. Dabei handelt es sich um Prinzipien, die sich zum größten Teil (zumindest) in und aus der gemeinsamen christlich-abendländischen Tradition entwickelt haben. Innerhalb dessen, was der Jean-Luc Nancy das „religiöse Schema des Okzidents“ nennt, das sich auf den Monotheismus gründet, den Alltag beherrscht, und selbst dem okzidentalen Atheismus sein besonderes Gepräge verleiht.

Wenn sich schon innerhalb desselben Kulturkreises, in dem die (geteilten) Grundprinzipien ihren Ursprung haben, solch auffällig unterschiedliche gesellschaftlich-kulturellen „Dialektformen“, wie man sie nennen könnte, herausbilden, muss die Frage virulent werden, was die Übernahme dieser - ihrem Wesen nach - abendländisch-alteuropäisch geprägten Grundprinzipien und Grundideen (zumal in so avancierten Formen wie Rechts- und Politiksystemen) für die Gesellschaften und Kulturen des Fernen Ostens - zum Beispiel - bedeuten. Und zwar nicht in einem (nur) „funktionalen“ Sinne: Ob importierte „Systeme“ in Gesellschaften, die diese Systeme und die damit verbundenen Verfahrensweisen nicht ,aus sich selbst“ hervorgebracht haben, „funktionieren“, das sagt im besten Falle etwas über die Adaptionsbereitschaft und Adaptionsfähigkeit dieser Gesellschaften aus; aber letztlich nichts über deren kulturelles „Format“. Es scheint daher klug und geraten, anzunehmen, dass etwa der westliche Gesetzesbegriff, wie Japan ihn im 19. Jahrhundert zusammen mit dem westlichen Rechtssystem übernommen hat, den Japanerinnen und Japanern, die bis zum Jahr 1868 keine eigene, der christlich-abendländischen entsprechende „universalistische“ Denkweise hervorgebracht haben (schon diese Tatsache ist bezeichnend), noch heute fremd ist. unabhängig davon, wie gut die alltägliche japanische Rechtsprechung „,funktioniert“; eine Rechtsprechung, die sich - wieder bezeichnenderweise - in der Praxis am britischen Modell, also an „Präzedenzfällen“ orientiert: Die Anwendung von Gesetzen setzt eben - re vera - ein Wissen vom Ursprung und „Geist“ der Gesetze nicht voraus. Das Verfahren bedarf, wie man in Anlehnung an Niklas Luhmann zu sagen legitimiert ist, als Legitimation nur seiner selbst. Das gilt auch für andere Grundbegriffe und Vorstellungen, wie „Person“, „Gesundheit" und „Normalität“. Oder die Klassifikation von „Behinderung“ durch die WTO (1980) in: impairment (also Funktionsdefizit in einem medizinischen Sinne), disability (in Bezug auf das persönliche (Er-)Leben) und handicap (in 
Bezug auf das gesellschaftliche Leben - die „Sozialität“). Dieser Klassifikationsversuch setzt ein Menschenbild voraus, ein Bild von „Gesundheit“, „Persönlichkeit“ und von „sozialem“ Leben voraus, das - über eine sehr lange Zeit - im „Westen“ gezeichnet wurde. Es kann hier natürlich nicht darum gehen, die gewollte oder (direkt/indirekt) erzwungene (zumindest formale) Übernahme von Vorstellungen, Ideen, Verfahrensweisen etc., rückgängig machen zu wollen (das wäre absurd), sondern sich zu fragen, was Geber- und Nehmer-Länder heute voneinander lernen können. In diesem Kontext bieten sich zahlreiche Fragestellungen an; etwa: Hat sich der europäische Persönlichkeitsbegriff seit dem Ende des 19. Jahrhunderts dem mehr „kontextualistischen“ Begriff vom Individuum in Japan angeähnelt? (Viele Phänomene der europäischen Jugendkultur, die sich noch niemals so wie zur Zeit, im ersten Jahrzehnt des 21. Jahrhunderts, an der japanischen Pop-Kultur orientierte, sprechen dafür.)? Auch: Woher kommt die hohe Akzeptanz humanoider Roboter im Pflegebereich in Japan (und der unglaubliche Nachdruck, mit dem in Japan an der Entwicklung und Vervollkommnung solcher Roboter in Japan gearbeitet wird)? Es gibt keinen Grund, die Unterschiede, die sich hier zeigen, nicht als kulturell motiviert zu beschreiben, - solange, bis sie sich genauer qualifizieren lassen - , und dabei unter „Kultur“ nichts anderes als einen optionalen „Themenvorrat“ zu sehen, den sich einzelne Gesellschaften im Laufe ihrer Geschichte erarbeitet haben. Auch „Behinderung“ ist nicht nur ein sozial konstruierter Begriff (wenigstens das wird wohl niemand mehr bezweifeln), sondern es ist in seiner weltweit bekannten, gleichsam praktizierten, Form ein im Sinne der abendländischen Denk- und Wertetradition konstruierter Begriff. Könnte es - in diesem Sinne - nicht sein, dass der vergleichsweise hohe Grand an Diskrimination Behinderter, wie man ihn heute noch in Japan beobachten kann, sich gerade einer Art Überadaption an ein ursprünglich fremdes System verdankt? So ist z.B. die fast 1000jährige (!) Tradition eines geradezu ,antipsychiatrischen" Umgangs mit geistig Behinderten, wie sie in Iwakura (bei Kyôto) exitsierte, unter dem Einfluss westlicher Psychiatrie im ersten Drittel des 20. Jahrhunderts verschwunden, bzw. abgeschafft worden. Ein „kulturellen“ oder „kulturwissenschaftlichen“ Argumenten gegenüber nicht verschlossenes Denken wird zumindest vor allzu formalistischen und funktionalistischen Schlüssen bewahrt bleiben.

Es ist nicht leicht, über „kulturwissenschaftliche Grundlagen“ von Behindertenpolitik zu sprechen. Überdies ist das Thema meines Statements einerseits zu hoch gegriffen; andererseits schon dadurch problematisch, dass der „Kultur“-Begriff selbst problematisch und umstritten ist - und von manchem für obsolet erachtet wird. Aber auch der Begriff der Kultur ist letztlich nicht mehr als eine Unterscheidungskategorie. Und in diesem Sinne, als Unterscheidungen markierende und ermöglichende „Themenvorräte“ verschiedener Gesellschaften möchte ich hier den Begriff „Kultur(en)“ verstehen und verstanden wissen; als nicht mehr - aber auch nicht als weniger. Soviel zum Problembegriff „Kultur“. Aber da haben wir auch noch die „Grundlagen“, die Fundamente, von denen etwa Descartes sich - philosophisch - wünschte, dass sie eines, ein „fundamentum“ und obendrein ,inoncussum“, also „unerschütterlich“ sein sollten. So haben wir mit dem Begriff der Grundlagen 
schon unausweichlich in das Labyrinth des abendländischen Denkens begeben. Auch das ein Schlag ins Kontor ,kultur-vergleichender“ Absichten. Der Teufel des Eurozentrismus steckt schon in den ersten Begriffen; ja gerade in den „ersten“ Begriffen. Immerhin hat sich das europäische Denken unter der Leitung der Frage nach dem „Warum“ entwickelt; lautet eine ihrer fundamentalen Fragen doch, wie ich es selbst ganz am Anfang meines ersten Philosophiekurses an der Oberschule gehört und gelernt habe; „Warum ist überhaupt etwas und nicht vielmehr nichts?“ - von Parmenides über Leibniz bis Heidegger. Eine Frage, die die „asiatischen Denker“, wie ich sie hier pauschal nennen möchte, erst von den Abendländern lernen mussten. Zwar wird auch im östlichen Denken immer wieder nach dem „Ursprung“ gefragt; aber bei genauerer Betrachtung ergibt sich, dass damit nicht die Frage nach dem „Warum das alles überhaupt und eigentlich sein muss“, sondern die Frage nach dem „Wie“, nach dem Wie? des Funktionierens der Welt, des Kosmos oder, im Jargon der chinesischen und japanischen Philosophie gesagt, nach dem Funktionieren der „Zehntausend Dinge“ gemeint ist. Am ausgeprägtesten im Buddhismus, den es nicht interessiert, warum die „Schaumwelt“ überhaupt da ist, sondern wie man ihr Funktionieren durchschaut, um sie abschaffen zu können; auf dem Weg der Erkenntnis und der Erleuchtung.

Ein allzu ungehemmtes Vertrauen in einen ungehemmten Erkenntnisoptimismus ist problematisch; wenn nicht gar gefährlich. Gerade im Zuge der so genannten Globalisierung sehen wir uns - sozusagen schon aus Zeit- und Termingründen gezwungen, Evidenzen, oder das, was wir dafür halten, als bare Münze zu nehmen. Wir folgen der Maxime „Schau hin und sag, was du siehst!“ - was an sich nicht schlecht und auch nicht falsch ist; bedenklich wird es aber, wenn wir es nicht für möglich halten, dass Evidenzen auch die Funktion haben (können), etwas zu verdecken. Vielleicht sogar meistens. Gerade im interkulturellen Verständnis ist die Gefahr, einen Anschein von Ähnlichkeit zu leicht und zu schnell als ein Beruhigungsmittel für den kritischen Zweifel zu akzeptieren, sehr groß.

Grundentscheidungen, die Gesellschaften - oder Kulturen, im oben beschriebenen Sinne - im Laufe ihrer Entwicklung treffen - oder im Laufe ihrer Evolution „selbst“" treffen, „färben“ die ganze betroffene Kultur gleichsam ein; geben ihr einen eigenen „Ton“. Das heißt, dass die Folgen und Spuren sehr elementarer Entscheidungen bis in die kleinsten Verzweigungen des Alltags sichtbar bleiben. Jede Kultur entspricht einem bestimmten geistigen „Milieu“; in dem die einzelnen aufwachsen, in dem sie „sozialisiert“, mit einem Wort: geformt werden. Dabei müssen etwa die religiösen und weltanschaulichen Grundvorstellungen nicht bewusst akzeptiert oder gar „geglaubt“ werde. Sie sind einfach nur das allgemeine Element, in dem die Einzelnen „zu sich selbst kommen“. So wie die Sprache, etwas ursprünglich Fremdes, das die Einzelnen sich aneignen müssen, nicht nur um mit anderen zu kommunizieren, sondern auch um mit sich selbst reden zu können. Die Sprache ist das unverzichtbar Fremde in uns. Aber die Sprache ist nur ein - mediales - Beispiel. Ähnlich verhält es sich auch mit den mehr inhaltlichen „Imprägnierungen“ der Einzelnen, wie man es nennen könnte: Goethes Mephisto nennt - im Gespräch mit dem Herrn höchstpersönlich - den Menschen ,den kleinen Gott der Welt“; und bringt damit die 
ganze Dialektik des abendländischen Menschen auf einen unüberbietbaren Begriff. Sehr verkürzt gesagt: Der abendländische Mensch formiert sich selbst, bringt sich in Positur, indem er sich als das Ebenbild eines Gottes denkt, den er sich wiederum als vor aller Zeit existierend, allmächtig und mit einer Persönlichkeit begabt denkt. Nicht nur das; er denkt ihn sich zudem als Schöpfer; und zwar als einen, der die Welt nicht - wie noch Platons Demiurg - nur gezimmert hat, aus vorhandenen Materialen. Nein der Gott der Abendländer kreiert die Welt aus dem Nichts - und zwar dem Nichts seiner Gedanken. Er stülpt ein gedankliches Konzept sozusagen in materiale Wirklichkeit um. Die abendländische Welt ist also insofern aus dem Nichts entstanden, als sie eine Kopfgeburt ist, eine Kopfgeburt Gottes. Man wundert sich nicht, dass der österreichische Stararchitekt Hollein die Arbeit seiner Zunft in genau dieser Metaphorik beschreibt. Er vergisst dabei aber, dass er die Materialen (Beton. Glas, Stahl) jener Ur-Szene verdankt, an der er metaphorisch schmarotzt. Wichtiger für das abendländische Denken überhaupt ist aber die Tatsache, dass die unterstellte Allmacht des als ,personenhaft“ gedachten Schöpfer-, Vater- und Rächergottes auch dessen Universalität impliziert. Daran konnten sich dann Gesetzes- und Rechtsvorstellungen orientieren, genauer ,okzidentieren“, die mittlerweile - zumindest formal und verfahrenstechnisch - die ganze Welt beherrschen. Dass diese abendländischen Grundentscheidungen auch das „Repräsentationsdenken“ ermöglicht, vielleicht sogar generiert haben, das die Politik, die Kunst, ja den Umgang mit „Zeichen“ überhaupt durchgehend prägt, braucht hier nur erwähnen zu werden.

Wie anders sieht das zum Beispiel im sino-japanischen Bereich aus: Bei allen Feinunterschieden, die man hier ausmachen kann: Man findet nirgends einen - als Person - gedachten Schöpfer-, Vater- und Rächergott. Überhaupt ist die Welt nicht geschaffen; sie ,ging einfach hervor“. Plötzlich war sie da oder hat sich aus einem unbestimmten Chaos herausgearbeitet; oder begann damit, wie es in der japanischen Mythologie heißt, dass „Himmel und Erde sich zu trennen begannen“ und so einen Raum eröffneten, in dem sich das Weltgeschehen - bis heute - abspielt. In diesem Raum sind dann zwar auch Schilfgräser gewachsen, dia „,kami to nareri“, zu „Gottheiten wurden“. Aber diese Gottheiten sind Folgen der Evolution, nicht die Impulsgeber der Evolution. Und als Modelle für das „Universale“ sind sie völlig ungeeignet. Sie sind Teil der Welt, eben der „Zehntausend Dinge“, die Götter sind Teil der Welt und deren Gesetzen unterworfen. Insofern kann in diesem Modell nur das Universum selbst und unmittelbar das Universale sein. Hinter, über oder neben diesem einen kosmischen Kontinuum gibt es auch keine Hinterwelt; wo zum Beispiel die eigentliche Wirklichkeit etwa in der Form der adamantinen Ideen Platons gelagert sein konnte. Und erst recht keinen Gott, einen Hüter der eigentlichen Wahrheit; auf den die Zeichen dieser Welt nur repräsentierend hinweisen könnten. Einen Satz wie den, den Hegel am Anfang seiner „Logik“ geschrieben hat, und der so repräsentativ ist für das abendländische Denken, ist in dieser Welt nie formuliert worden; weil er hier nicht formuliert werden konnte. Hegel schreibt in seiner „Wissenschaft der Logik":

„Die Logik ist sonach als das System der reinen Vernunft, als das Reich des reinen Gedankens zu fassen. Dieses Reich ist die Wahrheit, wie sie ohne Hülle an und für sich selbst ist. Man 
kann sich deswegen ausdrücken, daß dieser Inhalt die Darstellung Gottes ist, wie er in seinem ewigen Wesen vor der Erschaffung der Natur und des endlichen Geistes ist."

Wenn schon die Grundentscheidungen, Grundkonzepte und -vorstellungen verschiedener Kulturen geradezu unvereinbar erscheinen, muss dies auch für die jeweiligen Menschenbilder gelten. Und ist es nicht gerade der Unterschied der Menschenbilder, der verantwortlich ist für den „clash“ der Kulturen? Solange man diese Zusammenhänge nicht ausreichend bedenkt, kann etwa der Dialog zwischen dem „Westen“ und China über das, was im Westen „Menschenrecht(e)“ genannt wird, nicht sinnvoll geführt werden. Aber vor allem über Art und Qualität der Behindertenpolitik entscheidet, auf welchen Grundideen sie aufbaut: welches Menschenbild vorausgesetzt wird, wie Normalität und Gesundheit, wie Menschenwürde definiert werden, ja, was unter „Leben“ verstanden wird. Solange in einem „Gespräch“ der Kulturen die oft sehr verschiedenen Grundentscheidungen, die das geistige Milieu der jeweiligen Gesellschaften prägen, mitbedacht werden und zu ihrem „Recht“ kommen können, werden keine dauerhaften, d.h. nachhaltig „global“ akzeptablen Lösungen gefunden werden können.

Bei einem Symposium im Frühjahr 2001 mit dem Titel „Reisen als Urbild des Lebens und Sterbens" der stark körperlich Behinderte Japaner Ôtsuru Tadashi einen Vortrag mit dem Titel „Behindert-Sein als eine Reise zu sich selbst: Ost-WestBegegnung in der Überwindung des gewöhnlichen Rückerstattungsgedankens" beigetragen, worin er durchaus kritisch - auch den ,eigenen“, asiatischen, Traditionen gegenüber - zeigt, wie gerade kulturgeschichtliche (und auch religionsgeschichtliche) Reflexionen eine Revision des Status quo befördern können. Für Ôtsuru selbst scheint der Begriff der Kultur dabei sogar eine zentrale Rolle zu spielen: Wenn man unter chronischen Behinderung nicht das Gegenteil von Gesundheit, sondern das Andere der Normalität versteht, bedeutet das auch, dass der Behinderte seine Defizite hinnimmt und ein - in einem eigenen Sinne - gesundes Verhältnis zu ihnen aufbaut. „In diesem Sinne ist ein Behinderter nicht „,normal“ und kann vielleicht nicht mehr „normal“ werden, aber er kann wohl „gesund“ werden.“ Behinderung erscheint nun nicht mehr als reine Negativität, sondern als eine Herausforderung mit eigenen Maßstäben, als eine Aufgabe, der der Behinderte sich stellt, ohne dass die Möglichkeiten z.B. medizinischer Hilfe vernachlässigt werden.

„Dadurch wird es für die Behinderten selbst vielleicht möglich, ihre eigenen mannigfaltigen „Kulturen“ aufzubauen, ohne der Kultur des Normalen nachzulaufen. Ein Beispiel dafür ist die Kultur der Fingersprache bei den Hörbehinderten. Es gehört allerdings zum Begriff der Behinderung, dass die Aufgabe, die Behinderung zu erleichtern und zu korrigieren, nie einfach beseitigt werden sollte. Es muss aber möglich sein und ist auch nötig, diese Aufgabe im Ausgleich mit dem eigenständigen Bestreben und der eigenen Kultur des Behinderten selbst zu begreifen und sie in ihren eigenen Grenzen zu platzieren." 
4. Entwicklung der Rechte behinderter Menschen in der Bundesrepublik Deutschland 


\title{
4.1. Sozialpolitik im deutschen Sozialstaat seit der Vereinigung in ausgewählten Politikbereichen unter Einschluss des Behindertenrechts
}

\author{
Von Werner Tegtmeier
}

1. Einleitung $\quad 70$

2. Herausforderungen und Gestaltungserfordernisse der sozialstaatlichen $\begin{array}{ll}\text { Entwicklung } & 72\end{array}$

2.1. Demographische Entwicklung 73

2.2. Segmentierte und divergierende Zeit- und Sachhorizonte der Entscheidungsträger verschiedener Ebenen

3. Ausgewählte Politikbereiche 77

3.1. Arbeitsmarkt / Arbeitsförderung 77

3.2. Alterssicherung $\quad 82$

3.3. Rehabilitation und Teilhabe Behinderter 85

4. Schlussbetrachtung $\quad 88$ 


\section{Einleitung}

Sozialpolitik seit der deutschen Vereinigung erschließt sich dem Verständnis zutreffend eigentlich nur dann, wenn hierbei auch einige vor und im Prozess der Herstellung der staatlichen Einheit relevante Sachverhalte und Abläufe mit beleuchtet und bedacht werden.

Die einleitenden Bemerkungen sollen hieran in aller Kürze erinnern.

Erstens: Die Monate November 1989 und Oktober 1990 markieren in der jüngeren deutschen Geschichte, insbesondere auch der Sozialpolitik, diese bedeutsamen Zeitpunkte und Zäsuren:

Am 9. November 1989 fand im Deutschen Bundestag gerade die abschließende 2. u. 3. Lesung und Beschlussfassung einer weitreichenden Reform der Alterssicherung statt. ${ }^{1}$ Mitten in diese Beratungen fiel die Nachricht von der Öffnung der DDRGrenze. Der Prozess der deutschen Vereinigung nahm seinen Lauf - schneller und dynamischer als dies seinerzeit überhaupt für möglich gehalten werden konnte.

Am 18. Mai 1990 wurde der Staatsvertrag über die Herstellung einer Währungs-, Wirtschafts- und Sozialunion unterzeichnet. Die seinerzeitige Erwartung war, dass bis zur Wiederherstellung der staatlichen Einheit wohl noch mindestens $12-18$ Monate vergehen würden. Die Realität: Bereits knapp 5 Monate später, nämlich zum 3. Oktober 1990, war dies mit dem Staatsvertrag zwischen der Bundesrepublik Deutschland und der Deutschen Demokratischen Republik über die Herstellung der Deutschen Einheit im Wege des Beitritts bereits vollzogen. ${ }^{2}$

Zweitens: Es gab keine Blaupause für die inhaltliche, organisatorische und finanzielle Bewältigung eines derartig hoch komplexen Vorgangs. Es fehlte an fundierten Kenntnissen der DDR-Gegebenheiten. Entsprechende Arbeiten - wie man sie vielleicht beim früheren Bundesministerium für gesamtdeutsche Fragen (bis 1969), später Bundesministerium für innerdeutsche Beziehungen (aufgelöst zum 18.1.1991) hätte vermuten können - waren längst eingestellt oder nicht verfügbar. Vermutlich wären sie ohnehin auch nicht hinreichend gewesen, denn bei den Staatsvertragsverhandlungen (und danach) zeigte sich wiederholt, dass zahlreiche Sachverhalte (z.B. hinsichtlich von Sonderversorgungsystemen) sowohl inhaltlich als auch in den Regelsetzungen selbst hochrangigen Verhandlungspartnern nicht bekannt waren.

Fazit: Zeit- und Handlungsdruck eröffneten realiter und administrativ beherrschbar letztlich nur die Übertragung des in der Bundesrepublik geltenden Rechts- und Regelungssystems - angereichert um anwendungsorientierte Flexibilitätsklauseln.

Die Konsequenzen dieser Vorgehensweise waren vielfältig. Die - meistens auf Zeit - eingebauten Flexibilitätsklauseln ${ }^{3}$ erwiesen sich für das praktische Handeln

1 Zur Bedeutung des Rentenreformgesetzes vgl. Flecken, Sozialgesetzbuch -6 . Buch - Rentenversicherung, in: Übersicht über das Sozialrecht, Bundesministerium für Arbeit und Soziales (Hrsg.), 4. Auflage, Bonn 2007, S. 258.

2 Vgl. hierzu die ausführliche, mit einer Vielzahl von Quellenhinweisen belegte Darstellung von Ritter, Der Preis der deutschen Einheit, München 2006, S. 11 ff.

3 Vgl. z.B. NN: Übersicht über das Sozialrecht, Bundesministerium für Arbeit und Sozialordnung (Hrsg), 4. überarbeitete und erweiterte Auflage, Bonn, Juni 1997, S. 63, S. 67, S. 109 ff.. 
als sehr hilfreich. Andererseits: Die Übertragung beinhaltete - mangels anderer getroffener Regelungen - allerdings auch die Transformation längst als reformbedürftig erkannter Sachverhalte.

In letzter Konsequenz führte die praktizierte Vorgehensweise auch dazu, dass ein großer Teil der Kosten der deutschen Vereinigung über die Sozialversicherungssysteme abgewickelt wurde und noch abgewickelt werden. ${ }^{4}$ Versuche, diese - sowohl verteilungspolitisch als auch beschäftigungspolitisch - unbefriedigenden Finanzierungsregelungen zu einem späteren Zeitpunkt nachhaltig zu ändern, waren nicht erfolgreich. Hierin liegt einer der Gründe für den enormen finanziellen Konsolidierungsdruck insbes. in den Sozialversicherungssystemen, der im weiteren Verlauf der 90er Jahre zu bewältigen war.

Drittens: Als ebenso gravierend erwiesen sich die Fehleinschätzungen zum Zeitbedarf und zu den auftretenden Problemen bei der Transformation eines zentralverwaltungswirtschaftlich organisierten in ein marktwirtschaftlich verfasstes System. Die ursprüngliche Erwartungshaltung, dass mit der Bereitstellung eines funktionierenden Rechtsrahmens und eines leistungsfähigen Finanzierungssystems eine zügige Transformation und alsbaldige Stabilisierung auch der Beschäftigung bewirkt werden könne, waren falsch - es wurde kein Sturm der Marktkräfte entfacht.

Binnen kurzem waren die bisherigen (östlichen) Absatzmärkte - u.a. auch wegen der Bezahlung in harten Devisen - weitgehend weggebrochen. Die Produktivität großer Teile der Wirtschaft war schlecht und eröffnete ohne entschiedene Modernisierung des Produktionsapparates und der Produktionsabläufe keine wirkliche Überlebensperspektive in einem konkurrenzwirtschaftlich verfassten Markt. Und dies verlangte Zeit, erforderte viel Geld und „kostete“ Beschäftigung. Binnen vier Jahren reduzierte sich die Zahl der Erwerbstätigen um rd. ein Drittel - 1989: 9,7 Mio. Erwerbstätige, 1993: 6,38 Mio. Erwerbstätige. ${ }^{5}$

Die Konsequenz: Die sozialen Sicherungssysteme wurden mit Auffangaufgaben, finanziellen Belastungen und personellen Bindungen zur administrativen Bewältigung dieser Transformation extensiv in Anspruch genommen - mit Auswirkungen, die noch weit in die Zukunft ausstrahlen würden.

Die arbeitsmarktlichen Instrumente, konzeptionell eher im Hinblick eines Brückenschlags bei zeitweiliger Arbeitslosigkeit ausgelegt, wurden mehr und mehr mangels anderer Alternativen - auch zur langfristigen Flankierung und Bewältigung von Beschäftigungsanpassungen und Reduktionen eingesetzt. Auch Frühverrentungen gehörten dazu - etwa in Gestalt des sog. Altersübergangsgeldes, das instrumentell nichts anderes war als die Kombination von Arbeitslosengeld und Arbeitslosenhilfe in einer einheitlichen (administrativ leicht anwendbaren) Leistung, dem ALÜG, das für einen begrenzten Zeitraum die Phase der Arbeitslosigkeit bis zum schaftlichen Entwicklung, S. 371; Tegtmeier, Arbeitsmarktpolitik - Anspruch und Wirklichkeit von Reformen, ZeS-Arbeitspapier Nr. 5/2007 (Universität Bremen), S. 9

5 Quelle: Bundesministerium für Arbeit und Sozialordnung und Bundesanstalt für Arbeit. IIa1 - 214 9, S. 6, vgl. auch die Angaben im Jahresgutachten 1994/1995 des Sachverständigenrates zur Begutachtung der gesamtwirtschaftlichen Entwicklung, S. 339 f. 
Renteneintritt überbrücken half. Passive Leistungen mit erheblicher zeitlicher Bindungswirkung hierfür eingesetzter Instrumente und erheblichen Mittelaufwand (insbes. Arbeitslosenversicherung, Rentenversicherung und auch Bundeshaushalt), aber mit einem hohem Befriedungseffekt, ohne den der ökonomische Transformationsprozess (Treuhand) wegen seiner gravierenden sozialen Folgen vermutlich überhaupt nicht zu bewältigen gewesen wäre. Die Vorgänge um die seinerzeitige Stilllegung eines Stahlstandortes (Rheinhausen) im Ruhrgebiet indizieren jedenfalls den potentiellen sozialen Sprengstoff, den eine nicht hinreichend flankierte Entwicklung hätte haben können.

\section{Herausforderungen und Gestaltungserfordernisse der sozialstaatlichen Entwicklung}

Prinzipiell gilt, dass sozialstaatliche Leistungen einer Periode immer auch nur aus der Wertschöpfung dieser Periode finanziert werden können. Soziales Handeln hat in aller Regel diese ökonomische und finanzielle Dimension und deshalb auch die Mitverantwortung dafür, dass finanzielle Leistungsfähigkeit und volkswirtschaftliches Leistungsvermögen in Balance gehalten werden. Dieser Anpassungs- und Konsolidierungsdruck, insbes. als Folge schwächelnder Konjunktur und andauernder Unterbeschäftigung, bestand bereits im Jahrzehnt vor der Vereinigung und hatte zu zahlreichen Konsolidierungsmaßnahmen geführt.

Während in der Vereinigung und in der unmittelbaren Phase danach diese konsolidierenden Maßnahmen zeitweilig nachrangig und im Gegenteil bestimmte ausgabewirksame Instrumente aus den oben beschriebenen Gründen deutlich ausgeweitet wurden. Das Ungleichgewicht zwischen finanzieller Leistungsfähigkeit der Institutionen und volkswirtschaftlichem Leistungsvermögen nahm wieder zu. Zur Illustration: Die Sozialleistungsquote (Sozialleistungen gemessen am Bruttosozialprodukt) betrug zeitweilig (in 1992) für die neuen Bundesländer 70,5 \% und für die alten Bundesländer 29,8 \% (Gesamtdeutschland: 33,1 \%). ${ }^{6}$ Es war also nur eine Frage der Zeit, dass - bei gegebenem Finanzreglement - die Konsolidierungserfordernisse wieder handlungsbestimmend werden würden. Die 90er Jahre werden insoweit Ausdruck beständigen Ringens zwischen Konsolidierungszwängen einerseits und überfälligen Gestaltungserfordernissen andererseits.

Im Übrigen kann Beschreibung und Beurteilung politischen Handelns mit dem Hintergrund der beschriebenen „Sachzwänge“ auch nur dann zutreffend eingeordnet werden, wenn gleichzeitig die großen sozialstaatlich relevanten Herausforderungen endogener und exogener Art in die Betrachtung einbezogen werden.

Auch dies soll - nachstehend - in Kurzform geschehen. 


\subsection{Demographische Entwicklung}

Generatives Verhalten, zunehmende Lebenserwartung, veränderte Lebensstile (Singularisierung) führen dazu, dass sich der Bevölkerungsaufbau, das Verhältnis zwischen aktiven und nicht mehr aktiven Bevölkerungsteilen nachhaltig verschieben und damit die bisherige Architektur und Statik zwischen Leistungserbringern und Leistungsnehmern nachhaltig ins Ungleichgewicht geraten. Hieran werden auch Zuwanderungen nichts mehr spürbar verändern. Unsere Gesellschaft wird in den nächsten Jahrzehnten schrumpfen und sich altersstrukturell anders, nämlich älter, zusammensetzen Es besteht immenser Handlungsbedarf.

Ausdruck hierfür ist, dass die sog. Nettoreproduktionsrate ${ }^{7}$ in Deutschland seit mehr als 4 Jahrzehnten mit 0,59-0,69 deutlich unter dem für die Bestandserhaltung notwendigen statistischen Wert 1 liegt. Es werden weniger Kinder pro Frau während ihrer Lebenszeit geboren, zahlreiche Frauen bleiben ein Leben lang kinderlos (z.B. im Geburtsjahrgang 1960: rd. 23\%). Exogene Ereignisse wirken zusätzlich ein: So hat z.B. der ökonomische und soziale Umbruch, die hohe Arbeitslosigkeit, in den neuen Bundesländern dazu geführt, dass sich die Geburtenzahl dort zeitweilig halbierte - Nettoreproduktionsrate 1993: 0,37!! ${ }^{8}$

In einigen Großstädten, z.B. Berlin, liegt der Anteil der sog. Ein-PersonenHaushalte bei über 50\%; eine nachhaltig veränderte Sozialstruktur greift Platz.

Zugleich hat die Lebenserwartung der Menschen in den letzten Jahrzehnten - und noch andauernd - zugenommen. So weisen z.B. allein die aus den sog. abgekürzten Sterbetafeln 1991/93 bis 1997/98 abgeleiteten Annahmen über die fernere Lebenserwartung folgende Entwicklung auf

$\begin{array}{lll}\text { bei Neugeborenen: } & \text { Männer } & \text { Frauen } \\ \text { 1991/93 } & \text { 72,5 Jahre } & \text { 79 Jahre } \\ \text { 1997/98 } & \text { 74,4 Jahre } & \text { 80,6 Jahre }\end{array}$

bei Personen im Alter 60

$\begin{array}{lll}1991 / 93 & 17,8 \text { Jahre } & 22,1 \text { Jahre } \\ 1997 / 98 & 19,0 \text { Jahre } & 23,3 \text { Jahre }\end{array}$

Und mit einer weiteren Zunahme der Lebenserwartung um rd. 4 Jahre im Zeitraum bis 2050 wird gerechnet. ${ }^{9}$

Bereits im Jahr 2040 wird die Hälfte der Bevölkerung älter als 50 Jahre sein. D.h., auch die Zusammensetzung des Erwerbspersonenpotentials wird sich nachhaltig verändern. Der technologische Wandel muss mit deutlich älteren Erwerbspersonen bewältigt werden.

7 Zur Entwicklung der Nettoreproduktionsrate im Zeitraum 1960 - 2005 vgl. Flecken, a.a.O, S. 258 f.

8 Vgl. Sozialbericht 2001 der Bundesregierung, a.a.O.,S. 217

9 Zur Entwicklung der durchschnittlichen Lebenserwartung in Deutschland (West) seit 1970 vgl. Flecken, a.a.O, S. 259. 
Der Deutsche Bundestag hatte 1992 hierzu eine Enquête-Kommission „Demographischer Wandel“ eingesetzt, die die „Herausforderungen unserer älter werdenden Gesellschaft an den Einzelnen und die Politik" in 2 Zwischenberichten und 2002 in einem 688 Seiten starken Schlussbericht vorgelegt und der Öffentlichkeit zugänglich gemacht hat. ${ }^{10}$

(Inwieweit diese Erkenntnisse die jeweiligen Handlungsträger auch tatsächlich oder nur virtuell erreicht haben, kann - bis in die jüngste Zeit hinein - bei den streitigen Fragen zur Gestaltung einiger Rahmenbedingungen der Sozialversicherung: Bezugsdauer des Arbeitslosengeldes I und Renteneintrittsalter besichtigt werden. $)^{11}$

\subsection{Segmentierte und divergierende Zeit- und Sachhorizonte der Entscheidungs- träger verschiedener Ebenen}

Allein aus der skizzierten fundamentalen Bedeutung der Bevölkerungsentwicklung für Politik, Wirtschaft und Gesellschaft ergibt sich, dass zur Bewältigung dieser Herausforderung eine die Politikbereiche, Institutionen und Handlungsebenen übergreifende Lösungsstrategie entwickelt und durchgesetzt werden müsste und auch die Fähigkeit gestärkt sein sollte, bereits präventiv gestaltend zu handeln. Ein solches Gesamtkonzept, aus dem auch in sich konsistente Regelsetzungen für verschiedene Bereiche und Handlungsebenen abgeleitet werden könnten, gibt es nicht.

Für einzelne Politikbereiche, etwa der gesetzlichen Rentenversicherung, sind - gesetzlich - Verfahrensweisen etabliert, die die Betrachtung eines längerfristigen Zeitraumes (15-Jahres-Vorausberechnung von Einnahmen und Ausgaben bei alternativen Annahmen zur Wirtschaftsentwicklung mit Ausweis der Folgewirkungen für die Beitragsentwicklung) verlangen, so dass rechtzeitig gestaltend reagiert werden konnte, um Einnahmen und Ausgaben ins Gleichgewicht zu bringen. Die Lösungskonzepte waren - mangels einer übergreifenden Gesamtstrategie - fast durchgängig segmentspezifisch, nämlich im bestehenden System, angelegt, ohne dass denkbare und langfristig belastbarere Alternativen in Betracht gezogen wurden bzw. gezogen werden mussten. ${ }^{12}$

10 NN: Enquête-Kommission Demographischer Wandel. Herausforderungen unserer älter werdenden Gesellschaft an den Einzelnen und die Politik., Deutscher Bundestag (Hrsg), Berlin 2002

11 Zur Verlängerung der Bezugsdauer des Arbeitslosengeldes auf 24 Monate für langjährig Versicherte und ältere Arbeitnehmer vgl. SZ Nr. 297(2007), S. 27 und Generalanzeiger v. 29.12.2007, S. 25; die Umsetzung ist -rückwirkend zum Jahresbeginn 2008- mit dem 7. Gesetz zur Änderung des SGB III und anderer Gesetze erfolgt. Vgl. hierzu NN: Soziale Sicherung im Überblick, Bundesministerium für Arbeit und Soziales (Hrsg), Bonn 2008, S. 25

12 Ein typisches Beispiel: Die Ablösung der früher beitragslosen Zeiten zur Rentenversicherung im Falle von Arbeitslosigkeit durch Beitragszeiten, finanziert aus der Arbeitslosenversicherung, zunächst orientiert an $90 \%$ des letzten Nettoeinkommens - mit erheblichen Folgewirkungen für das Übergangsverhalten in andere Beschäftigungsformen, z.B. Teilzeit, oder aber des Einmündens in den Vorruhestand. Diese veränderte Regelsetzung ist mit einer der Gründe für das seinerzeit zunehmende Auseinanderdriften der beiden Fürsorgesysteme Arbeitslosenhilfe und Sozialhilfe. 
Einen herben Widerspruch zwischen einzel- und gesamtwirtschaftlicher Rationalität kann gerade in diesem Zusammenhang auch im Verhalten einzelwirtschaftlicher Entscheidungsträger beobachtet werden. Während einerseits - oft sogar unter Hinweis auf die demographische Entwicklung - eine längere Lebensarbeitszeit eingefordert wird, die Höhe der Lohnnebenkosten als beschäftigungsfeindlich qualifiziert wird, erfolgen Beschäftigungsanpassungen - bis heute - in erheblichen Umfang über Frühverrentungen, d.h. über die Externalisierung einzelwirtschaftlicher Kosten in die Sozialversicherung, und damit lohnnebenkosten-erhöhend! Diese gemeinhin als „sozialverträgliche Beschäftigunganpassung“ bezeichnete Verfahrensweise ist im Ergebnis also eine - milliardenschwere - Einigung zu Lasten Dritter. Eine derart missweisende Rationalität kann nur durch Änderung der dies begünstigenden Regelungen und öffentliche Transparenz erreicht werden. Und hilfreich wäre dabei natürlich insbesondere, wenn auch die öffentliche Hand in ihrem eigenen Verantwortungsbereich entsprechend handeln würde und nicht immer wieder neue Ausnahmen für bestimmte Personenkreise beschließen würde.

Von besonderer Bedeutung erscheint es auch, dass gerade bei langfristig wirksam werdenden, heute in der Entwicklungstendenz bereits bekannten Sachverhalten, z.B. der Entwicklung des Arbeitskräftepotentials, die verantwortlichen Entscheidungsträger gleichwohl in ihrem Handlungsrahmen einen sehr kurzen Zeithorizont aufweisen: Quartalszahlen, Marktkapitalisierung, Bilanzkennziffern scheinen das Beschäftigungsverhalten oft mehr zu beeinflussen als dies einer gedeihlichen Beschäftigungsentwicklung förderlich ist. Fehlende Ausbildungsplätze, frühverrentete Ingenieure und die Klage eines Defizits an Fachkräften - wie derzeit vielfach bekundet und mit dem Verlangen verbunden, die Zuwanderung qualifizierter Fachkräfte zu ermöglichen - sollte allemal Anlass sein, zunächst die eigenen Regeln darauf hin zu überprüfen, ob sie den längerfristigen Herausforderungen, den gewollten Zielsetzungen und den eigenen Verhaltensweisen noch gerecht werden oder in Widerspruch dazu stehen.

Von mindestens ebenso großer Relevanz sind die aus der Globalisierung und Internationalisierung resultierenden Ausstrahlungen. Krisen auf den Finanz- und Gütermärkten (1.Ölkrise 1973, Währungsturbulenzen um das britische Pfund 1992, Asienkrise 1997, Immobilienkrise in den USA 2007) strahlen durch die weltwirtschaftlichen Verflechtungen ganz unmittelbar auf die nationalen Volkswirtschaften aus, berühren Wachstum und Beschäftigung. Nicht selten fallen standortrelevante Entscheidungen multinationaler Unternehmen weit entfernt von jenen Regionen, in denen sie sich auswirken. Und oft ohne die regionalen Verantwortungsträger jemals auch nur annähernd in solche Entscheidungen einbezogen zu haben, werden diese gleichwohl mit den Folgen konfrontiert. Und ebenso die Systeme sozialer Sicherheit.

Am 20. Oktober 2007 haben sich die Staats- und Regierungschefs der EU in Lissabon auf den Grundlagenvertrag der EU verständigt, der dann mit dem für 2008 angestrebten Abschluss der Ratifizierung durch die Mitgliedstaaten im Jahr 2009 
wirksam werden kann. ${ }^{13}$ Damit erhält die EU eine neue Qualität. Das bisherige Einstimmigkeitsprinzip des Ministerrats wird - unbeschadet fortbestehender Ausnahmeregelungen - im Grundsatz durch Mehrheitsentscheidungen abgelöst (doppelte Mehrheit: 55\% der Mitglieder mit 65\% der Bevölkerung).

Zwar gehört Sozialpolitik auch künftig in die Kompetenz der Mitgliedstaaten, aber die Auswirkungen über die ökonomischen und finanziellen Rahmenbedingungen werden die weitere Gestaltung der Sozialpolitik spürbar berühren, wie heute ohnehin über die Freizügigkeit der Arbeitnehmer, die Niederlassungsfreiheit von Selbständigen, Entscheidungen des EuGH, aber auch die Methode der offenen Koordinierung zahlreiche Ausstrahlungen bestehen. ${ }^{14}$

Und prinzipiell gilt natürlich, dass in einem gemeinsamen Wirtschaftsraum mit einer gemeinsamen Währung auch die unterschiedlichen Steuer- und Sozialsysteme im Wettbewerb miteinander stehen. So macht es strukturell und im Ergebnis einen erheblichen Unterschied, ob die Finanzierung sozialer Sicherung budgetfinanziert (wie in Dänemark), also unter Heranziehung der gesamten Wertschöpfung einer Volkswirtschaft erfolgt oder ob, wie in Deutschland, lediglich der auf Einkommen entfallende Teil der Wertschöpfung der Volkswirtschaft - und noch zusätzlich limitiert durch eine Beitragsbemessungsgrenze - zur Finanzierung herangezogen wird. Die Beitragsfinanzierung der Sozialversicherung hat ja nicht nur verteilungspolitische Implikationen, sondern wirkt sich - bei vergleichbaren Sicherungsniveaus der EU-Länder - nachteilig auf die Kosten des Faktors Arbeit aus: Arbeit wird hierdurch teurer.

In Deutschland ist in 2007 eine Anhebung der Mehrwertsteuer von 16\% auf 19\% wirksam geworden. Eine vertiefte Diskussion um eine auch unter beschäftigungspolitischen Aspekten vorteilhaftere Gestaltung der Finanzierungsstruktur sozialer Sicherung (oder von Teilen sozialer Sicherung) hat es gleichwohl nicht gegeben. Stattdessen ist ein Teil des zusätzlichen Mehrwertsteueraufkommens in ein Teilsystem sozialer Sicherung ${ }^{15}$ gelenkt worden und im Gegenzug - und von der Öffent-

13 Der Deutsche Bundestag hat dem Vertragswerk am 24.4.2008 mit breiter Mehrheit zugestimmt, die abschließende Zustimmung durch den Bundesrat wird für Mai 2008 erwartet.

14 Vgl. Becker, Das Gemeinschaftsrecht, die deutschen Sozialleistungssysteme und die Debatten um deren Reformen, in: Reformen des deutschen Sozial- und Arbeitsrechts im Lichte supra- und internationaler Vorgaben, Studien aus dem Max-Planck-Institut für ausländisches und internationales Sozialrecht Bd. 35, 1. Aufl. 2005, S. 21 ff; Schulte, Sozialpolitik und europäische Integration. Nach dem Euro und vor der Erweiterung, in: ZFSH/SGB 2 (6), S. 328 ff und ZFHS/SGB 2 (7), S. 387 ff.; $\mathrm{zu}$ den operativen Ausstrahlen politischen Handelns vgl. NN: Nationales Reformprogramm Deutschland 2005 - 2008, Umsetzungs- und Fortschrittsbericht 2006, Bundesministerium für Wirtschaft und Technologie (Hrsg), Dokumentation 558, insbes. S. 46 ff.

15 Aus der Mehrwertsteueranhebung waren für die Arbeitslosenversicherung in 2007 hierfür 6,468 Mrd. $€$ vorgesehen und für 2008 7,583 Mrd. € geplant. Im Gegenzug wurden die Beiträge zur Arbeitslosenversicherung für 2007 auf 4,2\% zurück genommen und für 2008 mit 3,9\% geplant. (Zahlenangaben zum MWST-Aufkommen aus: Entwurf Haushalt 2008 der Bundesagentur für Arbeit VR 144/2007, Anlage 3. 
lichkeit weitgehend unbemerkt - sind gesamtgesellschaftliche Aufgaben aus der Budget- in die Beitragsfinanzierung verlagert worden. ${ }^{16}$

\section{Ausgewählte Politikbereiche}

\subsection{Arbeitsmarkt / Arbeitsförderung}

Der Übergang vom zentralverwaltungswirtschaftlichen in ein marktwirtschaftlich verfasstes System in Verbindung mit der Währungsunion führte - wie eingangs bereits erwähnt - binnen kürzester Frist in den neuen Bundesländern zum Verlust von rd. 3,5 Mio. Arbeitsplätzen. ${ }^{17}$ Allein im Bereich „Land und Forstwirtschaft, Fischerei“" sank die Beschäftigung auf ein Viertel (1989: 976 Tsd., 1995: 224 Tsd.) und im produzierenden Gewerbe halbierte sich die Zahl der Erwerbstätigen (1989: 4,386 Mio., 1996: 2,143 Mio.). Entsprechend schnell stieg die Zahl der registrierten Arbeitslosen an, und entsprechend schnell mussten über die Arbeitsförderung Auffanglösungen bereitgestellt werden. Einige der AFG-Instrumente: Kurzarbeit und Frühverrentung (ALÜG, Vorruhestand §105c AFG) wurden in der Spitze von fast 1 Mio. bzw. 0,8 Mio. Personen in Anspruch genommen.

Die nachstehende Zahlenreihe vermittelt einen Überblick über den arbeitsmarktlichen Umbruch in den neuen Bundesländern:

\begin{tabular}{|c|c|c|c|}
\hline Jahr & $\begin{array}{l}\text { Zahl der Arbeits- } \\
\text { losen: }\end{array}$ & $\begin{array}{l}\text { Arbeitslosen- } \\
\text { quote: }\end{array}$ & $\begin{array}{l}\text { Personen in } \\
\text { AFG-Maßnahm.: }\end{array}$ \\
\hline 1990 & 240 Tsd. & $2,7 \%$ & 543 Tsd. \\
\hline 1991 & 913 Tsd. & $11,1 \%$ & 1881 Tsd. \\
\hline 1992 & 1170 Tsd. & $15,6 \%$ & 1984 Tsd. \\
\hline 1993 & 1149 Tsd. & $15,6 \%$ & 1672 Tsd. \\
\hline 1994 & 1142 Tsd. & $15,3 \%$ & 1335 Tsd. \\
\hline 1995 & 1047 Tsd. & $14,0 \%$ & 1105 Tsd. \\
\hline
\end{tabular}

16 „Nach dem Entwurf eines 6. Gesetzes zur Änderung des SGB III und anderer Gesetze ist vorgesehen, den Aussteuerungsbetrag entfallen zu lassen und einen Eingliederungsbeitrag der BA vorzusehen. Danach hat die BA sich zur Hälfte an den Ausgaben für Verwaltung und Leistungen zur Eingliederung in Arbeit nach dem SGB II zu beteiligen. Hierfür sind nach dem Haushaltsplan des Bundes insgesamt $10 \mathrm{Mrd}$. EUR vorgesehen, so dass die BA $5 \mathrm{Mrd}$. EUR zu übernehmen hätte, die an den bisherigen 4 Überweisungsterminen für den Aussteuerungsbetrag an den Bund überwiesen werden sollen."

Ebenda, S. 6. Man darf gespannt sein, wann der Bund auch den restlichen Ausgabenblock in das Sozialversicherungssystem verladen haben wird.

17 Diese und die folgenden Zahlenangaben jeweils den entsprechenden Statistiken des Bundesministeriums für Arbeit und Sozialordnung entnommen worden. 
Die finanziellen Dimensionen waren gewaltig. Allein für das Altersübergangsgeld (ALÜG) wurden 1992: 9,3 Mrd. DM (515 Tsd. Empfänger), 1993: 14,1 Mrd. DM (612 Tsd. Empfänger) verausgabt; die Vorruhestandsregelung erforderte 1992: 5,1 Mrd. DM (292 Tsd. Empfänger), 1993: 4,5 Mrd. DM (209,9 Tsd. Empfänger).

Insgesamt dominierten in den ersten Jahren nach der Vereinigung die - unter dem Aspekt ausfallender Wertschöpfung - eher passiven Instrumente, während die aktiven Instrumente (Fortbildung und Umschulung, Arbeitsbeschaffungsmaßnahmen, $\S 249$ h AFG -Maßnahmen) vergleichsweise zeitversetzt, dann aber mit erheblichen Gewicht aufwuchsen:

FuU: 1991: 170 Tsd. Personen; 1992: 432 Tsd. Personen

ABM 1991: 257 Tsd. Personen; 1992: 543 Tsd. Personen

Selbst neue Ausformungen der Instrumente (z.B. § 249 h AFG) mit Einsatzmöglichkeiten in den Bereichen „Umweltsanierung, soziale Dienste“ wurden zügig angenommen und umfassten Mitte 1994 bereits knapp 88 Tsd. Personen. Dieses neue Instrument $\S 249$ h AFG stieß dann vornehmlich aus 2 Gründen sehr bald an Grenzen: Einerseits wurde es zunehmend als Konkurrenz zu den langsam entstehenden privatwirtschaftlichen Aktivitäten wahrgenommen (und diskreditiert) und die konzeptionell als Ko-Finanziers eingeplanten Kommunen begannen - angesichts ihrer Finanzschwäche - damit, gemeindliche Pflichtaufgaben durch Auslagerung mit diesem Instrumentarium zu finanzieren. Was als zusätzlicher Beschäftigungsimpuls gedacht war, wurde sehr bald zu einem Umfinanzierungsinstrument denaturiert.

Einsatz und Wirkung der Instrumente kann im übrigen nur zutreffend eingeordnet werden, wenn bedacht wird, dass das AFG-Instrumentarium zuvor in der DDR unbekannt war, die neuen institutionellen, infrastrukturellen und personellen Voraussetzungen überhaupt erst und zwar in kürzester Frist geschaffen werden mussten (Pesonalbestand der Ämter für Arbeit der DDR: 3600 Mitarbeiter, Personalbestand der Arbeitsämter nBL Ende 1991: rd. 25000 Personen).

Als administrativ sehr hilfreich erwies sich die - auf Zeit - vorgenommene Flexibilisierung bestimmter Instrumente. So konnte Kurzarbeitergeld (KUG: 68\% bzw. $63 \%$ des Nettolohnes für die ausgefallene Arbeitszeit) auch dann gezahlt werden, wenn die Arbeitsplätze voraussichtlich nicht erhalten werden konnten und zeitweilig die Arbeitszeit den Wert „Null“ annahm. Die hiervon betroffenen Arbeitnehmer wurden in einer betriebsorganisatorisch eigenständigen Einheit zusammengefasst, ohne dass unmittelbar Kündigungen ausgesprochen werden mussten. D.h., die Unternehmen konnten umstrukturiert werden und gewannen Zeit für die Personaldispositionen. Zugleich übernahmen sie - auf Zeit - die administrative Bewältigung dieser Form der Beschäftigungslosigkeit: Die Arbeitsämter (noch im Aufbau) wurden entlastet, lange Warteschlangen von Antragstellern vermieden. Nach Inkrafttreten der Währungsunion (1.7.1990) schnellten die Empfängerzahlen von KUG zeitweilig auf 2 Mio. (!!) Personen hoch. ${ }^{18}$ 
Der West-Ost-Finanztransfer war gewaltig. Er umfasste im Zeitraum 1991 - 1994 allein im Segment Bundesanstalt für Arbeit 134 Mrd. DM, davon im Wege des Bundeszuschusses 57,5 Mrd. DM. Der Bundeszuschuss zur Bundesanstalt für Arbeit, der qua Defizithaftung des Bundes die Differenz zwischen Einnahmen und Ausgaben auszugleichen hatte, schnellte von 0,7 Mrd. DM (1990) auf 24,4 Mrd. DM (1993) empor.

Die Jahre 1993/94 waren auf diesem Hintergrund vor allem von der Neugestaltung der Lastentragung zwischen Bund und Ländern - Föderales Konsolidierungsprogramm (FKP - wirksam ab 26.6.1993) - und weiteren Konsolidierungsanstrengungen in verschiedenen Politikbereichen, auch dem der sozialen Sicherung, geprägt. Letztere fanden mit Kabinettbeschluss v. 13.7.1993 ihren Niederschlag im Spar-, Konsolidierungs- und Wachstums-Programm (SKWP). Die während dieses Zeitfensters erfolgten Bemühungen, die Finanzierung von großen Teilen der Einigungskosten über die - beitragsfinanzierten - Sozialversicherungssysteme zugunsten einer gesamtgesellschaftlichen Lastentragung zu korrigieren, sind allesamt gescheitert.

Die Folge: Die Spar- und Konsolidierungsmaßnahmen führten zu spürbaren Veränderungen auf der Einnahmen- und Ausgabenseite. Auch im arbeitsmarktlichen Bereich: Zum 1.4.1991 werden die Beiträge zur Arbeitslosenversicherung um 2,5\% auf 6,8\% angehoben, für das Jahr 1992 werden 6,3\% und für die Folgejahre auf $6,5 \%$ festgesetzt.

Die Prozentsätze der Lohnersatzleistungen werden um einen Prozentpunkt (bei Arbeitslosen ohne Kinder um 3 Prozentpunkte) gesenkt. Im Einzelnen :

Arbeitslosengeld:

(KUG/SWG)

Arbeitslosenhilfe:

(Eingliederungshilfe)

Unterhaltsgeld:

Übergangsgeld:

(Behinderte)

$$
\text { mit Kind: }
$$

ohne Kind:

$\begin{array}{ll}\text { von } 68 \% \text { auf } 67 \% & \text { von } 63 \% \text { auf } 60 \% \\ \text { von } 58 \% \text { auf } 57 \% & \text { von } 56 \% \text { auf } 53 \% \\ \text { von } 73 \% \text { auf } 67 \% & \text { von } 65 \% \text { auf } 60 \% \\ \text { von } 80 \% \text { auf } 75 \% & \text { von } 70 \% \text { auf } 68 \%\end{array}$

Die ursprüngliche Streichung der originären Arbeitslosenhilfe und die Befristung der Arbeitslosenhilfe scheitern im Vermittlungsverfahren. Bei der originären Arbeitslosenhilfe erfolgt - zunächst - eine Reduktion auf 1 Jahr. In einem weiteren Gesetzgebungsverfahren wird die originäre Arbeitslosenhilfe später (1.1.2000) ganz gestrichen.

Diese und andere Regelungen des SKWP traten zum 1.1.1994 in Kraft. Zu den anderen in Kraft getretenen Regelungen gehörte auch die Verpflichtung der ALÜGEmpfänger, „zum frühestmöglichen Zeitpunkt in Rente zu gehen“. Dies bedeutete, dass sich der Bund aus seiner bisherigen Mitfinanzierung des Instruments verabschiedete. 
Bei näherer Betrachtung der Maßnahmenstruktur, die in diesem Fall einmal bewusst ausführlich gehalten worden ist, fällt auf, dass vielfach weniger die Effektivität und Sinnhaftigkeit der Maßnahmen und ihre Einbettung in einem größeren strukturellen Zusammenhang dominant erschien, sondern schlicht die finanziellen Auswirkungen auf die Gebietskörperschaften (Länder/ Kommunen), Bund und auch auf die Sozialversicherungszweige. Wenn dabei in Erinnerung gerufen wird, dass in etwa zeitgleich um die Frage der Einführung einer Pflegeversicherung gerungen wurde, erklärt dies zwar Einiges, aber exkulpiert natürlich nicht von der insgesamt doch segmentspezifischen und auf die kurzfristige Finanzentwicklung fokusierte Handlungsweise.

Auch in den Folgejahren wird die Arbeitsmarktpolitik durch zahlreiche Konsolidierungsanstrengungen gekennzeichnet. Ein überproportionaler Mitteleinsatz ist nach wie vor für die neuen Bundesländer erforderlich - angesichts der fortdauernden Diskrepanz zwischen der Nachfrage nach Arbeit und dem Angebot an Arbeitsplätzen; etliche Orte weisen Arbeitslosenquoten von 20\% und mehr auf. Zahlreiche Arbeitnehmer pendeln in die westlichen Bundesländer, junge Menschen beabsichtigen zunehmend, ihre Heimatregion dauerhaft zu verlassen.

Aber es werden auch zahlreiche neue Modellansätze ${ }^{19}$ (verbesserte Zusammenarbeit von Arbeits- und Sozialämtern, Sonderprogramme zur Bekämpfung der Jugendarbeitslosigkeit - JumP (Jugend mit Perspektive) - und zur Förderung von Geringqualifizierten und Langzeitarbeitslosen - CAST (Chancen und Anreize zur Aufnahme sozialversicherungspflichtiger Tätigkeiten) erprobt und durchgeführt. Mit dem Job-AQTIV-Gesetz (Aktivieren, Qualifizieren, Trainieren, Investieren, Vermitteln) wird der Aspekt des „Fordern und Fördern“ instrumentell unterstützt und zugleich werden „beschäftigungspolitische Leitlinien“ der EU umgesetzt. Insgesamt löbliche Maßnahmen, denen angesichts der Strukturprobleme und insbesondere der flauen Konjunktur zunächst jedoch ein sichtbarer arbeitsmarkt- und beschäftigungsrelevanter Erfolg versagt blieb. Erst mit deutlich anziehender Konjunktur in 2007 bilden sich die Zahlen registrierter Arbeitslosigkeit spürbar zurück. (Oktober 2007: 3,434 Mio. Arbeitslose)

Die wiederholten Versuche, die Rahmenbedingungen zur Inanspruchnahme von Frühverrentungen zu verschärfen, waren - vorübergehend - insoweit erfolgreich, als gesetzlich die Anrechnung gewährter Abfindung auf das Arbeitslosengeld verschärft, sowie die steuerliche Behandlung von Abfindungen restriktiver gefasst wurden. Doch noch vor dem Inkrafttreten dieser Regelung wurde sie durch die neu gewählte Koalition (rot/grün) ,,auf Wunsch“ des Bündnisses für Arbeit wieder weitgehend entschärft (Aufhebung des $§ 140$ SGB III mit dem EntlassungsentschädigungsÄnderungsgesetzes v. 24.3.1999). D.h. Frühverrentungen haben bis in die jüngste Gegenwart statt gefunden und die Öffentliche Hand ist daran durchaus selbst beteiligt auf Bundes- und Landesebene! 
Eine spürbare Zäsur in der Arbeitsmarktpolitik kündigte dann die Regierungserklärung von Bundeskanzler Schröder am 14.3.2003 an. Diese gemeinhin als „Agenda 2010“ in Bezug genommene Erklärung ${ }^{20}$ enthält u.a. folgende Aussagen:

„Die Lohnnebenkosten haben eine Höhe erreicht, die für die Arbeitnehmer zu einer kaum mehr tragbaren Belastung geworden ist und die auf der Arbeitgeberseite als Hindernis wirkt, mehr Beschäftigung zu schaffen. ${ }^{، 21}$

„Die Struktur unserer Sozialsysteme ist seit 50 Jahren praktisch unverändert geblieben. An manchen Stellen, etwa bei der Belastung der Arbeitskosten, führen Instrumente der sozialen Sicherheit heute sogar zu Ungerechtigkeiten“. ${ }^{22}$

„Wir werden die Kommunen ab dem 1 Januar 2004 von der Zahlung für die arbeitsfähigen Sozialhilfeempfänger entlasten. Das heißt, für bis zu1 Million Sozialhilfeempfänger wird künftig die Bundesanstalt für Arbeit materiell zuständig sein.“23

Basierend auf den Vorschlägen der „Kommission Moderne Dienstleistungen am Arbeitsmarkt" (Vorsitz: Peter Hartz, zum damaligen Zeitpunkt Arbeitsdirektor der VW AG, Berichtspräsentation am 16.8.2002 im Französischen Dom (!) in Berlin), werden dann in 4 Gesetzen für moderne Dienstleistungen am Arbeitsmarkt und einem Gesetz zu Reformen am Arbeitsmarkt die tief greifenden Reformen ins Werk gesetzt $^{24}$ :

Kernstück: Zusammenführung der Fürsorgesysteme Arbeitslosenhilfe (bis dato finanziert aus dem Bundeshaushalt) und Sozialhilfe für Erwerbsfähige (bis dato finanziert aus Länder- und Kommunalhaushalten) in eine neue Leistung: Grundsicherung für Arbeitsuchende (SGB II), hierbei insbes. das sog. Arbeitslosengeld II. Soweit erwerbsfähige Hilfebedürftige nicht bereits über eine Familienversicherung abgesichert sind, besteht Pflichtversicherung zur Kranken- und Pflegeversicherung, sowie - auf der Basis von Mindestbeiträgen - zur gesetzlichen Rentenversicherung.

Organisationsreform der BA, u.a. Ersetzung der Landesarbeitsämter durch sog. Regionaldirektionen mit Steuerungsfunktion für die örtlichen Agenturen für Arbeit (früher: Arbeitsämter). Diese Agenturen haben neben den Leistungen nach SGB III auch die SGB II relevanten Leistungen (u.a. Arbeitslosengeld II) zu erbringen - in aller Regel in Arbeitsgemeinschaften, sog. ARGEN, mit den kommunalen Trägern, die ihrerseits zuständig sind für das frühere Sozialhilfe-Spektrum (Unterkunft, soziale Dienste, Schuldner-, Suchtberatung etc.). Für 6 Jahre ist es insges. 69 Kommunen gestattet, alle relevanten Funktionen, auch die der Arbeitsämter, also Auszahlung des Arbeitslosengeldes II, zu übernehmen. Die vorgesehene wissenschaftliche Evaluation wird zeigen, ob dies sinnvolle Ergebnisse zu erbringen vermag.

Zahlreiche Instrumente werden gestrafft oder neu gestaltet. so z.B. die Möglichkeit, statt des Übergangsgeldes die auf 3 Jahre ausgelegte Existenzförderung, die

Regierungserklärung von Bundeskanzler Schröder am 14. März 2003 vor dem Deutschen Bundestag in: REGIERUNG online

21 Ebenda, S. 1

22 Ebenda, S. 3

23 Ebenda, S. 4

24 Vgl. hierzu die ausführliche Darstellung im Sozialbericht 2005, S. 11 ff. 
sog. "Ich AG“ in Anspruch zu nehmen, die Schaffung von „vermittlungsorientierter Leiharbeit in sog. Personal-Service-Agenturen (PSA), die Förderung im Bereich „geringfügiger Beschäftigung“, z.B. Mini-Jobs (bis $400 €$ p.m.) bzw. Midi-Jobs (401 $-800 €$ p.m.) bei pauschalierter Beitragszahlung des Arbeitgebers zur Steuer (2\%) und zur Sozial-Versicherung ( $12 \%$ zur RV, $11 \%$ zur KV, bei privaten Haushalten jeweils $5 \%$ ), etc.

Zahlreiche zwischenzeitlich erfolgte Gesetzesänderungen überlagern diese Neugestaltung. Gleichwohl - alle Instrumente sind zur wissenschaftlichen Evaluation vorgesehen, so dass zur geg. Zeit empirische Fakten die Bewertung dieser Neugestaltung erleichtern werden.

Begrenzung der Bezugsdauer des Arbeitslosengeldes I auf 12 Monate (für über 55-jährige auf 18 Monate) mit Wirksamkeit ab 1.2.2006. Noch vor dem Wirksamwerden werden die Übergangsfristen für den längeren Bezug von Arbeitslosengeld auf den 31. Jan. 2008 verlängert. Und im Oktober 2007 ist die Bezugsdauer des ALG I selbst neuerlich in die politisch kontroverse Diskussion geraten - mit dem eingangs bereits erwähnten Ergebnis einer neuerlichen Verlängerung der Bezugsdauer von ALG I für ältere und langjährig Versicherte auf bis zu 24 Monate. ${ }^{25}$

Die eingangs geschilderten Herausforderungen (demographische Entwicklung, längerfristige Orientierung des Sach- und Entscheidungshorizonts) spielen keine oder eher eine nachrangige Rolle.

Strukturell und finanziell bedeutsame Grundsatzfragen werden von der Öffentlichkeit gar nicht wahrgenommen, geschweige denn erörtert. So etwa:

Ist es Aufgabe einer Sozialversicherung über die Erfüllung seiner Kernfunktion hinaus gesamtgesellschaftliche Aufgaben zu erfüllen und zu finanzieren?

Sind die Regelsetzungen innerhalb des Systems und zu korrespondierenden Politikbereichen konsistent oder kontraproduktiv? Beispiel: Nach dem neu geschaffenen $\S 46$ SGB II hatte die - beitragsfinanzierte - Arbeitslosenversicherung bei jedem Übergang von ALG I zu ALG II einen kompletten Jahresbetrag ALG II an den Bundeshaushalt (!) abzuführen. ${ }^{26}$ Das Finanzvolumen umfasst mehrere Milliarden Euro. Abgesehen von der höchst fragwürdigen Steuerungswirkung und der faktischen Sozialisierung von Sozialversicherungsbeiträgen ist das natürlich das genaue Gegenteil einer Entlastung der Lohnnebenkosten.

\subsection{Alterssicherung}

Die am 9. November 1989 verabschiedete, 1992 wirksam gewordene Rentenreform war eine erste Antwort auf sich nachhaltig verändernde Rahmenbedingungen von Wirtschaft und Gesellschaft, insbes. der Demographie.

Vgl. S. 6, Fußnote 11.

Dieser sog. Aussteuerungsbetrag ist inzwischen - durch Verlagerung der Hälfte der Eingliederungsbetrages aus dem Bundeshaushalt in die Arbeitslosenversicherung - ersetzt worden. Vgl. im einzelnen S. 8, Fußnote 10 
„Um eine angemessene, automatische Verteilung der sich hieraus ergebenden Belastungen auf Rentner, Beitragszahler und den Bund zu erreichen, wurde mit dem RRG 1992 Rentenanpassung, Beitragssatzfestlegung, Festsetzung des Bundeszuschusses in einem selbst regulierenden Mechanismus eingebunden, so dass auch bei sich ändernden ökonomischen, sozialen und demographischen Rahmenbedingungen ohne weitere gesetzgeberische Eingriffe ein angemessener Ausgleich von Einnahmen und Ausgaben sichergestellt ist“.(BMA) ${ }^{27}$

Schon sehr bald stellte sich heraus, dass die andauernde hohe Arbeitslosigkeit, die zunehmende Praxis der Frühverrentungen, der hohe Finanztransfer West/Ost (allein 1992-94: 28 Mrd. DM) eine stärkere Beitragssatzanhebung von 17,5\% in Richtung auf 19,2\% (1996), 20,3 (1997) notwendig machen würde.

Eine neuerliche, intensive Rentendiskussion setzte ein, die mit dem RRG 1999 u.a. zum Abflachen der Ausgaben-Entwicklung und der Rentenanpassungen und zur Einfügung eines ,demographischen Faktors in die Rentenformel führte. Die neue (rot/grüne) Bundesregierung setzte diese Regelung dann 1998 aus, um Zeit für einen modifizierten Reformansatz zu finden. Die Rentenanpassung 2000 folgte lediglich der Preissteigerungsrate des Vorjahres. ${ }^{28}$

Zugleich wurden ab Juni 1999 direkte Beitragszahlungen des Bundes für Zeiten der Kindererziehung eingeführt (und aus Mitteln der 1. Stufe der „ökologischen Steuerreform“ finanziert) und der Rentenversicherung wurden einigungsbedingte Lasten (ohne Anrechnung auf den zusätzlichen Bundeszuschuss) erstattet. Dies gestattete es, den Beitragssatz ab 1.4.1999 von 20,3\% auf 19,5\% zu senken. ${ }^{29}$

Neu in der rentenpolitischen Diskussion waren:

- die explizite Zielsetzung, den Beitragssatz bis zum Jahr 2020 unter 20\% zu halten und ihn bis 2030 nicht über $22 \%$ steigen zu lassen ${ }^{30}$

- die Forderung (und Förderung) des individuellen Aufbaus von zusätzlicher Altersvorsorge, ,da sonst ein angemessener Lebensstandard im Alter nicht erreicht werden könne".

Im Kern bedeutete dies die Verabschiedung von der Philosophie der lebensstandardsichernden Funktion der gesetzlichen Rentenversicherung.

Die Aufwendungen für die zusätzlichen Aufwendungen werden anstiegsabflachend in die Rentenformel eingestellt. Bis zu 4\% der Beitragsbemessungsgrenze (der Rentenversicherung) werden bei Aufwendungen für Alters-Vorsorge in betrieblichen Pensionsfonds steuer- und sozialversicherungsfrei gestellt. Als bevorzugte Umsetzungsform haben die Tarifvertragsparteien vor allem das Instrument der Entgeltumwandlung genutzt. ${ }^{31}$

27 Übersicht über das Sozialrecht (Stand 1997), a.a.O, S. 117 ff,, insbes. Ziff. 11

28 Vgl. Sozialbericht 2001. a.a.O., S. 49,

29 Ebenda, S. 50. Ziff. 100.

30 Ebenda, S. 49 Ziff. 96 und Ziff. 101

31 Durch den Beitragsausfall in der Rentenversicherung haben die Geförderten faktisch ihre Förderung in Teilen selbst bezahlt. Zugleich ist innerhalb der Versichertengemeinschaft ein Umverteilungsprozess insoweit bewirkt worden, als die Auswirkungen auch die Personen berühren, die von der Förderung keinen Gebrauch gemacht haben. 
Seit dem Jahresbeginn 2003 gibt es das Instrument „Grundsicherung im Alter“, das als steuerfinanzierte Leistung der Sozialhilfe ausgeformt ist. Bedürftige ab dem 65. Lebensjahr, sowie dauerhaft erwerbsgeminderte Personen haben hierdurch einen (von Unterhaltsansprüchen gegen Eltern oder Kinder) unabhängigen Sozialleistungsanspruch erhalten. ${ }^{32}$

In den Folgejahren werden weitere einschneidende Konsolidierungsmaßnahmen ${ }^{33}$ auf den Weg gebracht:

- 2004: Aussetzung der Rentenanpassung,

- Absenkung der Schwankungsreserve auf $20 \%$ einer Monatsausgabe

- (wird bei verbesserter Kassenlage später wieder angehoben werden).

- Verlegung des Auszahlungstermins für Neurentner auf das Monatsende

- Volle Beitragsleistung der Rentner an die Pflegeversicherung

- Mit dem RV-Nachhaltigkeitsgesetz wird ein Nachhaltigkeitsfaktor in die Rentenformel eingefügt. (Berücksichtigung des Verhältnisses von Leistungsbeziehern und versicherungspflichtig Beschäftigten).Und die Lohnentwicklung wird stärker auf die beitragspflichtige Lohn- und Gehaltssumme abgestellt.

- $\quad$ Eine Niveau-Sicherungsklausel wird eingeführt

bis 2020: $46 \%$ vor Steuern

bis 2030: $43 \%$ vor Steuern

Die vom Bundesverfassungsgericht seit langem wegen der unterschiedlichen steuerlichen Behandlung von Pensionen und Renten (bislang Ertragsanteilbesteuerung) angemahnte Neuordnung wird - mit langen Übergansfristen (2005 - 2040) in dem Sinne umgesetzt, dass die Renten in Schritten in die volle Besteuerung einbezogen und im Gegenzug die Vorsorgeaufwendungen steuerfrei gestellt werden. ${ }^{34}$

Die Verfahrensweise: Renten aus der gesetzlichen Rentenversicherung werden in $2005 \mathrm{zu}$ 50\% für die Besteuerung herangezogen. Der verbleibende steuerfreie Teil der Jahresbruttorente wird individuell auf Dauer festgeschrieben. Für jeden neu hinzukommenden Rentnerjahrgang wird der steuerlich zu erfassende Rentenanteil in Schritten von 2 Prozentpunkten auf $80 \%$, anschließend mit 1 Prozentpunkt (bis 2040) auf $100 \%$ angehoben.

Nach heftiger - und derzeit erneut aufflammender - innenpolitischer Diskussion ist mit dem am 30.03.2007 vom Bundesrat verabschiedeten „Gesetz zur Anpassung der Regelaltersgrenze“ (Inkrafttretenstermin: 1.1.2008) mittlerweile auch die gesetzgeberische Voraussetzung für ein späteres Renteneintrittsalter geschaffen worden. Betroffen sind die Geburtsjahrgänge ab 1947: Ab 2012 werden sie in 1-MonatsSchritten pro Geburtsjahrgang, (in 2-Monats-Schritten ab Jahrgang 1958) erhöht, bis mit Jahrgang 1964 die neue Regelaltersgrenze von 67 Jahren erreicht sein wird.

Vgl- hierzu Schmähl, Entgeltumwandlung und die Finanzen der Sozialversicherung - ein Problemaufriss, (Universität Bremen, Zentrum für Sozialpolitik) ZeS-Arbeitspapier 7/2007, S. 16 f. 
Neben zahlreichen Sonderregelungen in der Übergangsphase (z.B. für Altersteilzeit, Erwerbsminderung) gilt im Grundsatz, dass für vorzeitiges „In-Rente-gehen“ $0,3 \%$ Abschlag p.m. für die gesamte Rentenlaufzeit getragen werden muss. ${ }^{35}$

Für Schwerbehinderte wird es weiterhin ein vorzeitiges, abschlagsfreies Altersruhegeld geben. Die Altersgrenze hierfür wird (ab Jahrgang 1952) schrittweise vom 63. auf das 65. Lebensjahr angehoben. Die Altersgrenze für die frühestmögliche Inanspruchnahme (dann mit Abschläge von 0,3\% p.m.) wird ebenfalls in Schritten vom 60. auf das 62. Lebensjahr angehoben.

Die prinzipielle Neuregelung der Regelaltersgrenze soll auch in den Pensionsregelungen für den Beamtenbereich nachvollzogen werden.

Fazit: Die Überleitung des Rentenrechts im Gefolge der Vereinigung (also im Kern die Neuberechnung der Renten auf der Grundlage von Arbeitsjahren und individuellen Durchschnittseinkommen) war reibungslos bis Ende Dezember 1991 abgeschlossen worden. Infolge des besonderen Anpassungsmodus konnten die Renten Ost sehr schnell aufholen. Die Durchschnittsrente (Ost) stieg von 475 Mark (Juni 1990) auf 1214 DM im Juli $1994 .{ }^{36}$ Die Rentenpopulation in der früheren DDR hat von der Vereinigung materiell am Stärksten profitiert.

In Gegenwart und Zukunft werden die Mitglieder dieser erweiterten Solidargemeinschaft allerdings - wie geschildert - ebenfalls in vollem Umfang von den großen Herausforderungen, insbes. der demographischen Entwicklung, aber auch der strukturellen Arbeitslosigkeit gerade in Teilen Ostdeutschlands, auch auf dem Feld der Alterssicherung nachhaltig berührt sein.

\subsection{Rehabilitation und Teilhabe Behinderter}

In Deutschland leben rund 8,5 Mio. Menschen mit einer - amtlich - anerkannten Behinderung. Rund 6,7 Mio. Personen sind als schwerbehindert eingestuft, z.T. von Geburt an, die Mehrzahl in Folge von Krankheit, Unfall, Alter. ${ }^{37}$ Die quantitative Bedeutung wird allein schon aufgrund der eingangs skizzierten Bevölkerungsentwicklung noch zunehmen.

Im Jahr 1999 waren rd. 858 Tsd. Schwerbehinderte in Beschäftigung, als arbeitslos waren rd. 191 Tsd. Schwerbehinderte registriert (Schwerbehinderten-Arbeitslosenquote: 17.9\%). Als Folge der allgemeinen Arbeitsentwicklung waren binnen 5 Jahren (seit 1995) rd. 90.000 Schwerbehinderte weniger in Beschäftigung. Unterbeschäftigung, Dysfunktionalitäten der Märkte, regionale Disparitäten führten unmittelbar und mittelbar zur Beeinträchtigung der Teilhabe am realen Wertschöpfungsprozess. Erst gezielte Sonderprogramme vermochten diesen Trend zu brechen. Aber

Vgl. Soziale Sicherung im Überblick, a.a.O., S. 103 ff., bes. S.105., NN. Übersicht über das Sozialrecht. a.a.O., S. $283 \mathrm{ff}$.

36 Statistik des Bundesministeriums für Arbeit und Sozialordnung, ferner NN: Übersicht über das Sozialrecht, a.a.O, S. $252 \mathrm{f}$.

37 Vgl. Sozialbericht 2005, a.a.O, S. 95. 
auch im Jahr 2000 waren noch 183.563 Schwerbehinderte arbeitslos, davon allein 37.690 (Quote: $24.7 \%$ ) im östlichen Bundesgebiet. ${ }^{38}$

Insgesamt ist dieser Bereich von Rehabilitation und Teilhabe Behinderter seit der Vereinigung durch drei einander folgende, z.T. überlagernde Phasen geprägt: Transformation und Neuaufbau Ost, Sparen und Konsolidieren, gestaltende Weiterentwicklung.

Allein die mit der Übertragung des Schwerbehindertenrechts notwendig gewordene Ausstellung von „neuen“ Schwerbehindertenausweisen stellte die damit nicht hinreichend vertraute und personell unterbesetzte dortige Versorgungsverwaltung vor administrativ kaum zu bewältigende Probleme. Ende Dezember 1992 waren von rd. 980 Tsd. Anträgen lediglich $67 \%$ beschieden und 373 Tsd. neue Ausweise ausgestellt. Und es vergingen noch weitere 15 Monate bis der „Rückstau“ überwiegend abgearbeitet und die bis dahin ausgefertigten Ausweise eine Gesamtzahl von rd. 900 Tsd. erreicht hatte.

Als ähnlich schwierig und zeitaufwendig erwies sich die Neuerrichtung bzw. Modernisierung von - z.T. dramatisch unzureichenden - Einrichtungen der medizinischen Rehabilitation und der beruflichen Eingliederung. Mit ursächlich hierfür waren die Erfordernisse der Ko-Finanzierung. Neben Bund und Reha-Trägern im Bereich der beruflichen Rehabilitation sollten sich auch die neuen finanzschwachen Bundesländer an den Investitionskosten bei den Berufsförderungswerken (7) und bei den Berufsbildungswerken (8) mit rd. einem Drittel (bis zu 35\% bei Werkstätten, $40 \%$ bei Wohnungen) beteiligen. Allein im Zeitraum 1991 - 1996 sind vom Bund für den Bereich Werkstätten für Behinderte und Wohnungen rd. 835 Mio. DM. eingesetzt worden.

Während sich die infrastrukturelle Ausstattung in den neuen Bundesländern im Bereich der medizinischen und beruflichen Rehabilitation schrittweise verbesserte, verschlechterte sich der Arbeitsmarkt und damit die tatsächlichen Eingliederungschancen nachhaltig.

Trotz der Konsolidierungsmaßnahmen, u.a. Kürzungen des Unterhaltsgeldes mit dem SKWP, werden Rehabilitation und Teilhabe der Behinderten konzeptionell vorangebracht: Das Grundgesetz wird im Zuge der Verfassungsreform 1994 um eine Maxime ergänzt, „wonach niemand wegen seiner Behinderung benachteiligt werden darf“. ${ }^{39}$ Zum 1. Juli 2001 tritt das SGB IX in Kraft, ein Meilenstein für „ein bürgernahes Rehabilitations- und Teilhaberecht“. Es fasst eine Vielzahl von für die verschiedenen Leistungsbereiche und Träger der Rehabilitation gemeinsam geltenden Vorschriften in einem Gesetzbuch zusammen, fördert damit die Zusammenarbeit der Träger (z.B. Schaffung gemeinsamer örtlicher Servicestellen) und eröffnet einen einfachen Weg für behinderte Menschen zu Beratung und Unterstützung. RehaTräger können Leistungen zur Teilhabe auch in Form eines Persönlichen Budgets bereitstellen, so dass behinderte Menschen ihre Leistungen selbst „organisieren“ können. Zunächst sind derartige Leistungserbringungen im Rahmen eines Persönli- 
chen Budgets als Ermessensleistung ausgeformt, ab 2008 wird daraus ein Rechtsanspruch.

Politik für behinderte Menschen findet seinen Niederschlag u.a. in dem steten Bemühen zur Barrierefreiheit. Mit dem Behindertengleichstellungsgesetz (in Kraft seit 1.5.2002) wird die öffentliche Verwaltung zu einem Barriere freien Verwaltungsverfahren verpflichtet, z.B. in der Kommunikation, beim Zugang zu Dokumenten etc. Im öffentlichen Personennahverkehr haben die Verkehrspläne Maßnahmen zur Barriere-Freiheit zu beinhalten bzw. es sind entsprechende Programme aufzustellen.

Teilhabe muss nicht zuletzt heißen, auch realen Zugang zu Beschäftigung und Arbeitsmarkt zu erhalten. Mit dem Gesetz zur Bekämpfung der Arbeitslosigkeit Schwerbehinderter (29.9.2000) und dem Gesetz zur Förderung der Ausbildung und Beschäftigung schwerbehinderter Menschen (1.5.2004) ${ }^{40}$ sind - auch in Umsetzung der auf europäischer Ebene eingegangenen Verpflichtungen - entsprechende Anstrengungen auf den Weg gebracht worden.

Obwohl im Rahmen dieser Darlegungen und des Workshop-Kontexts andere wichtige, sozialstaatsbedeutsame Politikbereiche - wegen des Umfangs - nicht dargelegt werden können, soll doch - weil in besonderer Weise themenrelevant - kurz auf die seit 1. Jan. 1995 wirksame Pflegeversicherung hingewiesen werden. 2005 waren 1,952 Mio. Leistungsempfänger registriert, den Einnahmen von 17,49 Mrd. $€$ standen 17,86 Mrd. $€$ Ausgaben gegenüber. D.h. die Pflegeversicherung deckt ein gesellschaftsweit bedeutsames und aufgrund der Bevölkerungsentwicklung noch zunehmendes Risiko ab. Und das gilt auch für die Finanzentwicklung. ${ }^{41}$ Derzeitige Beschlusslage ist, dass neben einer Leistungsausweitung in der ambulanten und stationären Pflege und der Einbeziehung demenziell Erkrankter, ab 1.7.2008 die Beitragssätze zur Pflegeversicherung von 1,7\% auf 1,95\% steigen werden, Kinderlose zahlen $0,25 \%$ mehr. $^{42}$ Letztere Differenzierung beruht auf einer Entscheidung des Bundesverfassungsgerichts zum Familienlastenausgleich (3.April 2001 BVR 1629/94), mit der dem Gesetzgeber aufgegeben wird, bis Ende 2004 eine Regelung zu treffen, die Versicherte mit Kindern (beitragsmäßig) besser stellt, als kinderlose Versicherte.

In der Konzeptionsphase der Pflegeversicherung ist heftig um das „Ob“ und das „Wie“ der Finanzierung gerungen worden, nämlich Budgetfinanzierung oder Beitragsfinanzierung qua Sozialversicherung. Die Entscheidung fiel zugunsten der Beitragsfinanzierung. Eine segmentspezifische Entscheidung - mit weit reichenden Konsequenzen ..... 


\section{Schlussbetrachtung}

Der Historiker Gerd A. Ritter wird am 9. November 2007 in München für ein exzellent recherchiertes und geschriebenes Buch mit dem Preis des Historischen Kollegs ausgezeichnet.

Der Buch-Titel lautet: „Der Preis der deutschen Einheit - Die Wiedervereinigung und die Krise des Sozialstaats"

In seinem Resumé schreibt er:

„Das Überleben des deutschen wie des Sozialstaats überhaupt wird schließlich davon abhängen, inwieweit er fähig sein wird, dem ständigen Wandel von Staat, Wirtschaft und Gesellschaft sowie der internationalen Rahmenbedingungen Rechnung zu tragen, die Balance zwischen Eigenvorsorge, Solidarität der Gesamtgesellschaft oder kleinerer Gemeinschaften und staatlicher Hilfe immer neu auszutarieren und die Bedingungen für wirtschaftliches Wachstum zu schaffen, ohne den Zusammenhalt der Gesellschaft und die politische Freiheit zu gefährden. ${ }^{\text {“43 }}$

Recht hat er. 


\title{
4.2. Die Entwicklung der Rechte behinderter Menschen in der Bundesrepublik Deutschland
}

Eine Betrachtung aus der Sicht der Bundesvereinigung Lebenshilfe für Menschen mit geistiger Behinderung

\author{
Von Klaus Lachwitz
}

\section{Vorbemerkung}

1. Der Mensch mit geistiger Behinderung als gleichberechtigter Staatsbürger

2. Die Stellung behinderter Menschen im Deutschen Sozialrecht 


\section{Vorbemerkung}

Das Behindertenrecht in Deutschland ist ein komplexes Gebilde. Die nachfolgenden Ausführungen versuchen, die Entwicklung dieses Rechtsgebiets seit der Gründung der Bundesrepublik Deutschland im Jahr 1949 aus dem Blickwinkel von Menschen mit geistiger und mehrfacher Behinderung darzustellen.

Das deutsche Behindertenrecht ist nicht am „Grünen Tisch“ entstanden, sondern das Ergebnis eines engen Zusammenspiels zwischen den jeweiligen Bundesregierungen bzw. Regierungsparteien und einer großen Zahl von Behindertenverbänden und Selbsthilfegruppen, die selbstständig agieren, bei Bedarf jedoch ein Aktionsbündnis bilden, das unter dem Namen „Deutscher Behindertenrat (DBR)“ bekannt geworden ist.

Eine der wichtigsten Akteure ist die Bundesvereinigung Lebenshilfe für Menschen mit geistiger Behinderung, die im Jahr 1958 von Eltern geistig behinderter Menschen und Fachleuten gegründet wurde, heute über 130.000 Mitglieder in 540 Orts- und Kreisvereinigungen verfügt und etwa 3.000 ambulante Dienste und Einrichtungen - darunter zahlreiche Wohnheime und Werkstätten für behinderte Menschen und ein flächendeckendes Netz von Frühförderstellen und Familienunterstützenden Diensten - unterhält.

Die Bundesvereinigung Lebenshilfe verfolgt das Ziel, Menschen mit geistiger Behinderung in ihrem Bestreben, gleichberechtigt am Leben in der Gesellschaft teilzunehmen, zu begleiten und zu unterstützen.

Der Kampf um gleichberechtigte Bürgerrechte dieser Personengruppe und ihre leistungsrechtliche Absicherung im Sozialrecht steht deshalb seit Jahrzehnten im Vordergrund der politischen Lobbyarbeit der Lebenshilfe.

Im ersten Teil dieses Beitrags soll im Rück- und Ausblick untersucht werden, wie sich der Rechtsstatus von Menschen mit geistiger Behinderung in unserer Gesellschaft ausgebildet hat. Im zweiten Teil soll die leistungsrechtliche Absicherung von Menschen mit geistiger Behinderung im gegliederten System der sozialen Sicherung in Deutschland nachgezeichnet werden.

\section{Der Mensch mit geistiger Behinderung als gleichberechtigter Staatsbürger}

Für Menschen mit geistiger und mehrfacher Behinderung war die Gründung der Bundesrepublik Deutschland ein Akt der Befreiung und ein Neuanfang: Während der Schreckensherrschaft der Nationalsozialisten wurden mehr als 100.000 behinderte Menschen durch SS-Ärzte und SS-Pflegekräfte ermordet. Diese unter der euphemistischen Überschrift „Euthanasie“ oder „Aktion Gnadentot“ bekannt gewordenen Massentötungen hatten ihre Ursache in rassenhygienischen Vorstellungen und kriegswirtschaftlichen Erwägungen. 
Aus rechtlicher Sicht lassen sich die Euthanasiemorde insbesondere auf das Gesetz zur Verhütung erbkranken Nachwuchses vom 14. Juli $1933^{1}$ zurückführen, das die Zwangssterilisation „erbkranker“ Menschen anordnete und insbesondere die Menschen erfasste, die an ,angeborenem Schwachsinn, Schizophrenie, manisch depressivem Irresein, erblichem Veitstanz, erblicher Blindheit, erblicher Taubheit, schwerer erblicher körperlicher Missbildung“" usw. (vgl. § 1 Abs. 2 des genannten Gesetzes) litten. Mehr als 400.000 Menschen wurden auf der Grundlage dieses Gesetzes zwangssterilisiert. Im Jahr 1939 begann die planmäßige Vernichtung der als erbkrank bezeichneten Menschen durch Vergasung. Sie beruhte auf einer Anweisung Adolf Hitlers und wurde als Aktion „T 4“ von der in der Tiergartenstraße 4, Berlin, residierenden Zentrale für die Leitung der Ermordung behinderter Menschen im Deutschen Reich organisiert.

Nur wenige Menschen mit geistiger Behinderung überlebten die Schreckensherrschaft der Nationalsozialisten. Sie wurden nach dem Ende des Zweiten Weltkriegs entweder in den von den Kirchen unterhaltenen Anstalten bzw. in psychiatrischen Einrichtungen untergebracht oder von verängstigten Eltern und Angehörigen versteckt.

Mit der Gründung der Bundesrepublik Deutschland trat Art. 3 Abs. 1 GG in Kraft: „Alle Menschen sind vor dem Gesetz gleich.“

Doch für viele behinderte Menschen stand das Menschenrecht auf Gleichbehandlung in der Nachkriegszeit zunächst nur auf dem Papier. Erst Anfang der 70er Jahre des vergangenen Jahrhunderts war der Deutsche Bundestag bereit, eine Sachverständigenkommission zu berufen, die beauftragt wurde, einen Bericht über die Lage der Psychiatrie in der Bundesrepublik Deutschland zu erarbeiten.

Im Oktober 1973 legte die Kommission einen Zwischenbericht vor, in dem festgestellt wurde, ,dass eine sehr große Anzahl psychisch kranker und behinderter Menschen in den stationären Einrichtungen unter elenden, zum Teil als menschenunwürdig zu bezeichnenden Umständen leben muss.“

Um diese Zustände möglichst schnell zu verändern, sprach sich die Sachverständigenkommission in ihrem im September 1975 fertiggestellten Bericht ${ }^{2}$ dafür aus, das mit dem Bürgerlichen Gesetzbuch von 1896 in das Zivilrecht eingeführte Rechtsinstitut der Entmündigung abzuschaffen und durch die Feststellung von „Betreuungsbedürftigkeit“ bei gleichzeitiger Bestellung eines „Betreuers“ mit unterschiedlichen, auf den jeweiligen Einzelfall abstellenden Aufgabenkreisen zu ersetzen $^{3}$.

Doch es vergingen erneut mehrere Jahre, bis mit dem Gesetz zur Reform des Rechts der Vormundschaft und Pflegschaft für Volljährige (Betreuungsgesetz) vom 12.09.1990 endlich die Voraussetzungen dafür geschaffen werden konnten, auf die Entmündigung und die damit verknüpfte Fiktion der Geschäftsunfähigkeit des entmündigten Menschen zu verzichten und mit dem Institut der rechtlichen Betreuung 
eine Form der rechtlichen Vertretung einzuführen, die nicht automatisch zu einem Verlust der Geschäftsfähigkeit führt und die rechtliche Handlungsfähigkeit eines Menschen im zivilrechtlichen Bereich grundsätzlich unberührt lässt.

Damit waren entscheidende Weichen gestellt, um Menschen mit geistiger Behinderung oder psychischer Erkrankung aus ihrem Schattendasein am Rande der Gesellschaft zu befreien und schrittweise in die Gesellschaft einzugliedern.

Wie sehr jedoch auch die Sachverständigenkommission der Psychiatrie-Enquête fast 30 Jahre nach dem Zusammenbruch des Dritten Reiches noch einem Menschenbild verhaftet war, das wie selbstverständlich davon ausging, in die körperliche Unversehrtheit eines einwilligungsunfähigen Menschen eingreifen zu dürfen, ergibt sich aus folgender Empfehlung:

„Eine gesetzliche Regelung, die auch die Sterilisation solcher geistig behinderten Personen zulässt, die einwilligungsunfähig sind und bei denen der Eingriff in ihrem eigenen wohlverstandenen Interesse angezeigt ist, wäre anzustreben“"4.

Es verwundert angesichts dieses Vorschlags nicht, dass noch mehr als ein Jahrzehnt verging, bevor die Praxis der Sterilisation geistig behinderter Menschen ,in der Grauzone des Rechts" kritisch hinterfragt und vom Bundesministerium der Justiz darauf hingewiesen wurde, dass pro Jahr etwa eintausend Sterilisationen an überwiegend jungen geistig behinderten Frauen durchgeführt werden ${ }^{5}$.

Erst ab 1988 bewirkten die rechtspolitischen Diskussionen zur Einführung des Betreuungsrechts, dass bei vielen Abgeordneten des Deutschen Bundestages die Bereitschaft wuchs, sich vertieft mit den Problemen behinderter Menschen zu befassen. Die von zahlreichen Behindertenverbänden und Selbsthilfegruppen behinderter Menschen erhobene Forderung, endlich rechtliche Vorkehrungen $\mathrm{zu}$ treffen, um insbesondere Menschen mit geistiger Behinderung und/oder psychischer Erkrankung zu vollwertigen Bürgerinnen und Bürgern zu erklären, fiel auf fruchtbaren Boden: Im Jahr 1994 verankerte der Bundesgesetzgeber das Verbot der Benachteiligung behinderter Menschen in Art. 3 Abs. 3 des Grundgesetzes und erkannte damit an, dass viele Menschen in unterschiedlichen Bereichen des gesellschaftlichen Lebens noch immer benachteiligt werden.

Alle Beteiligte waren sich darin einig, dass mit Art. 3 Abs. 3 GG das Signal dafür gegeben werden sollte, Benachteiligungen behinderter Menschen in Recht und Gesetzgebung abzubauen.

Wie zäh der beschriebene Veränderungsprozess abgelaufen ist und noch immer abläuft, zeigt ein Blick in das Bürgerliche Gesetzbuch: Nach wie vor sind die mehr als 100 Jahre alten Vorschriften der $\S \S 104$ Nr. 2, 105 BGB in Kraft, wonach die Willenserklärung eines Menschen, der sich ,in einem die freie Willensbildung ausschließenden Zustand krankhafter Störung der Geistestätigkeit befindet“, nichtig ist. Zwar hat der Gesetzgeber die Reichweite dieser Vorschrift eingeschränkt, indem er im Jahr 2002 beschlossen hat, auf die Fiktion der Nichtigkeit eine Willenserklärung

Psychiatrie-Enquête - BT-Drs. 7/4200, S. 34 Ziff. 14.

vgl. Diskussions-Teilentwurf des Bundesministeriums der Justiz zum Betreuungsgesetz, November 1987,71 
zu verzichten, wenn die erwachsene Person, die als geschäftsunfähig gilt, eine Willenserklärung abgibt, die sich nur auf ein mit geringfügigen Mitteln bewirktes Rechtsgeschäft bezieht ${ }^{6}$. Er hat außerdem angeordnet, dass Heimverträge und Verträge, die den arbeitnehmerähnlichen Rechtsstatus von behinderten Personen in Werkstätten für behinderte Menschen regeln, auch dann rechtsgültig sind, wenn der behinderte Mensch bei Abschluss des Vertrages geschäftsunfähig war, die Vertragsparteien jedoch ihre gegenseitig vereinbarten Leistungen bewirkt haben ${ }^{7}$.

Auf eine grundlegende Überarbeitung des Rechts der Geschäftsfähigkeit wurde jedoch verzichtet, obwohl Canaris, ein namhafter Rechtswissenschaftler, der das deutsche Wirtschaftsrecht mit zahlreichen Fachbeiträgen geprägt hat, schon im Jahr 1987 die Auffassung vertreten hatte, die Regelung des § 105 BGB, mit der die Totalnichtigkeit jeder Rechtshandlung eines Geschäftsunfähigen angeordnet wird, verstoße ,in einer geradezu dramatischen Weise“ gegen die im Grundgesetz geschützte allgemeine Handlungsfreiheit und das Persönlichkeitsrecht des Art. $2 \mathrm{GG}^{8}$.

Der Weg zur vollen Anerkennung als Bürger dieses Landes war also für viele Menschen mit Behinderung mit Steinen übersät.

Dass auch das viel zitierte Verbot der Benachteiligung behinderter Menschen (Art. 3 Abs. 3 GG) an seine Grenzen stößt, und zumindest die Einflussmöglichkeit dieser Vorschrift auf die zivilrechtliche Rechtsprechung überschätzt worden ist, zeigt ein Urteil des Oberlandesgerichts Köln vom 08.01.19989. Das Oberlandesgericht Köln hat in einem Nachbarschaftsstreit entschieden, der Kläger, ein Musiklehrer, müsse es nicht hinnehmen, dass er während seiner Freizeit, an Wochenenden und an den Feiertagen Geräuschen ausgesetzt sei, die von den auf dem Nachbargrundstück wohnenden geistig behinderten Menschen erzeugt würden. Die auf sein Grundstück dringenden Lärmeinwirkungen seien das Ergebnis „misslungener Sprechversuche“. Daraus resultierten „stimmliche Laute nicht verbaler Art“, die von einem „unvoreingenommenen Zuhörer als unharmonisch, fehlmoduliert und damit als unangenehm empfunden würden.“ Der Landschaftsverband Rheinland als Träger der benachbarten Wohneinrichtungen wurde deshalb verurteilt, dafür zu sorgen, dass das „Schreien, Stöhnen und Kreischen“ der geistig behinderten Menschen an Sonntagen und gesetzlichen Feiertagen ab 12:30 Uhr, mittwochs und samstags ab 15:30 Uhr und an den übrigen Werktagen ab 18:30 Uhr unterbleibt.

Dieses Urteil hatte deshalb negative Auswirkungen auf den Integrationsprozess von Menschen mit geistiger Behinderung in das gesellschaftliche Leben, weil es sich ausführlich mit dem Benachteiligungsverbot des Art. 3 Abs. 3 Satz 2 GG auseinandergesetzt hat und trotzdem zu dem Ergebnis gekommen ist, dass das Ruhebedürfnis der Nachbarn Vorrang vor der mit der Vorschrift des Art. 3 Abs. 3 Satz 2 GG ange- 
strebten „erhöhten Toleranzbereitschaft“ gegenüber Menschen mit Behinderung hat $^{10}$.

Die politischen Auseinandersetzungen zur Anerkennung behinderter Menschen als Bürger der Bundesrepublik Deutschland sind durch dieses Urteil allerdings eher positiv beeinflusst worden.

Zur Stärkung der Stellung und Anerkennung behinderter Menschen im gesellschaftlichen Leben haben in den Folgejahren insbesondere das Behindertengleichstellungsgesetz (BGG) vom 27.04.2002 $2^{11}$ und das Allgemeine Gleichbehandlungsgesetz (AGG) vom 14.08.2006 ${ }^{12}$ beigetragen. Beide Gesetze machen jedoch auch deutlich, dass der Bundesgesetzgeber teilweise rechtliche Regelungen trifft, die sich in der Praxis nicht immer umsetzen lassen. So will z. B. das Behindertengleichstellungsgesetz erreichen, dass die Barrierefreiheit im zivilrechtlichen Bereich insbesondere durch Zielvereinbarungen zwischen Unternehmen und Behindertenverbänden hergestellt wird. Inzwischen hat sich jedoch gezeigt, dass die behinderten Menschen, die sich an der Verhandlung derartiger Zielvereinbarungen beteiligen, geradezu hoffnungslos benachteiligt sind, wenn sie mit der ,geballten Fachkompetenz" und Finanzkraft von Rechtsabteilungen und Verhandlungsführern große Unternehmen wie der Deutschen Bundesbahn konfrontiert werden. So verwundert es nicht, dass im Bericht der Bundesregierung über die Lage behinderter Menschen 2004 ausgeführt wird, es sei noch kein Abschluss einer Zielvereinbarung i. S. d. BGG mitgeteilt worden, sondern nur Verhandlungen in vier Fällen bekannt gewor$\operatorname{den}^{13}$. Wenn weiterhin ein Ungleichgewicht auf der Verhandlungsebene der Zielvereinbarungen besteht, wird das Instrument der Zielvereinbarung wirkungslos bleiben.

$\mathrm{Ob}$ dies auch für das Allgemeine Gleichbehandlungsgesetz gilt, wird abzuwarten sein. Ein Ziel dieses Gesetzes ist es, die Benachteiligung geistig behinderter, psychisch kranker oder blinder Menschen beim Abschluss von Haftpflicht- und Lebensversicherungsverträgen zu beseitigen. Sie zählen zu den sogenannten zivilrechtlichen Massengeschäften ( $§ 19$ AGG), die dem Verbot der Diskriminierung unterliegen. Doch der Gesetzgeber hat diese Verbotsregelung eingeschränkt und eine unterschiedliche Behandlung von Versicherungsnehmern für zulässig erklärt, wenn einer der in Art. 20 AGG genannten Gründe vorliegt, wie z. B. ein versicherungsmathematisch begründeter Risikozuschlag bei einer Versicherung.

Welche Perspektiven eröffnen sich für Menschen mit geistiger Behinderung und/oder psychischer Erkrankung, wenn man ihren Rechtsstatus als Bürger festigen will?

Große Bedeutung wird dem Übereinkommen der Vereinten Nationen über die Rechte behinderter Menschen zukommen, das die Bundesrepublik Deutschland gemeinsam mit inzwischen 125 Vertragsstaaten der Vereinten Nationen ${ }^{14}$ am 
30. März 2007 unterzeichnet hat und so schnell wie möglich ratifizieren, d. h. in nationales deutsches Recht umsetzen will.

Mehr als vier Jahre hat sich das Ad-hoc-Komitee der Vereinten Nationen, das den Text des Übereinkommens erarbeitet hat, mit der Rechtstellung behinderter Menschen befasst. Keine Menschenrechtsfrage war so umstritten und wurde so ausführlich diskutiert wie Art. 12 des Konventionstextes. Dieser erkennt an, dass alle behinderten Menschen unabhängig vom Schweregrad ihrer Behinderung die volle Rechts- und Handlungsfähigkeit besitzen.

Die Endfassung des Art. 12 hat nach der inzwischen vorliegenden - mit Österreich, der Schweiz und Liechtenstein abgestimmten - amtlichen deutschen Übersetzung folgenden Wortlaut:

Art. 12 Gleiche Anerkennung vor dem Recht

1) Die Vertragsstaaten bekräftigen, dass Menschen mit Behinderungen das Recht haben, überall als Rechtssubjekte anerkannt zu werden.

2) Die Vertragsstaaten anerkennen, dass Menschen mit Behinderungen in allen Lebensbereichen gleichberechtigt mit anderen Rechts- und Handlungsfähigkeit genießen.

3) Die Vertragsstaaten treffen geeignete Maßnahmen, um Menschen mit Behinderungen Zugang zu der Unterstützung zu verschaffen, die sie bei der Ausübung ihrer Rechts- und Handlungsfähigkeit ggf. benötigen.

4) $\ldots .$.

Bemerkenswert ist zweierlei:

Art. 12 enthält keine Sonderregelung für Menschen mit geistiger Behinderung oder psychischer Erkrankung. Dieser Artikel hat zwar für den genannten Personenkreis besondere Bedeutung; die Generalversammlung der Vereinten Nationen hat jedoch bewusst darauf verzichtet, im Text des Art. 12 einzelne Behinderungsarten besonders hervorzuheben, weil insbesondere aus Staaten Südamerikas, Afrikas und Asiens berichtet wurde, dass nicht nur Menschen mit geistiger Behinderung oder psycho-sozialen Problemen entmündigt, einer umfassenden gesetzlichen Vertretung unterstellt oder für geschäftsunfähig erklärt werden, sondern auch taubstumme, taubblinde oder in ihrer Sprachfähigkeit beeinträchtigte Menschen.

Bis zuletzt war umstritten, ob der im englischen Originaltext der UN-Konvention zur Anwendung kommende Begriff „Legal capacity“ nur die Rechtsfähigkeit oder auch die Handlungsfähigkeit eines behinderten Menschen umfasst. Die Entwicklung der Textvorlagen zu Art. 12 zeigt ganz eindeutig auf, dass sich letztlich in der Generalversammlung der Vereinten Nationen die Erkenntnis durchgesetzt hat, Art. 12 sei nur dann mit den Zielen der in diesem Übereinkommen geregelten Menschenrechte in Einklang zu bringen, wenn allen behinderten Menschen die volle Rechts- und Handlungsfähigkeit zugesprochen wird.

Sind behinderte Menschen in dieser Fähigkeit beeinträchtigt, weil die jeweilige Gesellschaft, in der sie leben, außerstande ist, z. B. den Schriftverkehr von Behörden mit geistig behinderten Menschen in „leichter Sprache“ zu organisieren, so haben 
die betroffenen behinderten Menschen einen Anspruch auf Unterstützung bzw. Assistenz.

Die Person, die diese Unterstützungsfunktion ausübt, agiert nach Sinn und Zweck des Art. 12 jedoch nicht als gesetzlicher Vertreter, sondern als rechtlicher Begleiter des behinderten Menschen.

Viele international organisierte Behindertenverbände und Selbsthilfegruppen, die an den Beratungen der UN-Konvention über die Rechte behinderter Menschen beteiligt waren, diskutieren deshalb bereits jetzt die Fragestellung, wie das weltweit verbreitete System der Entmündigung bzw. Anordnung einer gesetzlichen Vertretung durch ein rechtliches Assistenzmodell ersetzt werden kann, das die Handlungsfähigkeit und damit auch die Geschäftsfähigkeit als Teil der rechtlichen Handlungsfähigkeit $^{15}$ des behinderten Menschen unberührt lässt.

Auch an das im internationalen Vergleich als fortschrittlich geltende deutsche Betreuungsrecht wird nach einer Ratifikation der UN-Konvention die Frage heranzutragen sein, ob z. B. die Anordnung eines Einwilligungsvorbehaltes, mit dem in die Geschäftsfähigkeit eines Menschen eingegriffen wird (vgl. § 1903 BGB), mit Sinn und Zweck des Art. 12 des Übereinkommens zu vereinbaren ist. Desgleichen wird sich die Frage stellen, ob die von Canaris bereits im Jahr 1987 beanstandete Vorschrift des $\S 105$ BGB nicht endgültig aus dem Bürgerlichen Gesetzbuch zu entfernen ist.

\section{Die Stellung behinderter Menschen im Deutschen Sozialrecht}

Menschen mit geistiger Behinderung oder psychosozialen Problemen sind in besonderer Weise darauf angewiesen, dass das in Art. 20 GG verankerte Sozialstaatsgebot umgesetzt und soziale Leistungen zur Verfügung gestellt werden, die ihnen ein menschenwürdiges Leben innerhalb der Gesellschaft ermöglichen.

Während die Ausformung des rechtlichen Status eines Menschen mit geistiger Behinderung als Staatsbürger im Nachkriegsdeutschland lange auf sich warten ließ, markierte ein schon fast in Vergessenheit geratenes Urteil des Bundesverwaltungsgerichts bereits im Jahr 1954 eine bahnbrechende Wende: Unter Hinweis auf den Schutz der Menschenwürde (Art. 1 GG) und das Sozialstaatsgebot (Art. 20 GG) erhielt der Gedanke der öffentlichen Daseinsfürsorge für bedürftige Menschen seinen entscheidenden Impuls: „Der Pflicht der öffentlichen Hand zur Fürsorge für Bedürftige steht ein entsprechender einklagbarer Rechtsanspruch der Betroffenen auf Hilfe gegenüber“'16.

Darauf aufbauend entwickelte sich in den 50er Jahren ein differenzierter Fürsorgebegriff, der dazu führte, dass die Hilfe in besonderen Lebenslagen einschließlich der Eingliederungshilfe für behinderte Menschen neben der Hilfe zum Lebensunter- 
halt im Jahr 1961 in enger Anlehnung an das Körperbehindertengesetz vom 27.02.1957 ${ }^{17}$ und das Gesetz über die Tuberkulosenhilfe vom 23.07.1959 ${ }^{18}$ als eigenständige Hilfeart im Bundessozialhilfegesetz (BSHG) verankert wurde.

Um die Eigenständigkeit der Eingliederungshilfe wird seit dem Inkrafttreten des Bundessozialhilfegesetzes vom 30.06.1961 ${ }^{19}$ immer wieder gerungen. Je nach wirtschaftlicher Lage in der Bundesrepublik Deutschland wurden der Leistungskatalog der Eingliederungshilfe und die Vorschriften über die Anrechnung von Einkommen und Vermögen der leistungsberechtigten Menschen und ihrer unterhaltspflichtigen Angehörigen entweder erweitert oder eingeengt.

Die Blütezeit der Eingliederungshilfe waren zweifellos die Jahre der Hochkonjunktur zwischen 1974 und 1981, als ein nahezu flächendeckendes Netz von Frühförderstellen, Wohnheimen und Werkstätten für behinderte Menschen (WfbM) geschaffen werden konnte.

Besonders erwähnenswert sind die Rechtsgrundlagen für die inzwischen in nahezu fast jeder kreisfreien Stadt bzw. in fast jedem Landkreis anzutreffenden WfbM, denn kaum ein Bereich der Behindertenhilfe ist vom Gesetzgeber so detailliert geregelt worden wie die WfbM, die den behinderten Menschen eine Arbeitsmöglichkeit verschaffen soll, die nicht, noch nicht oder noch nicht wieder auf dem allgemeinen Arbeitsmarkt beschäftigt werden können, jedoch in der Lage sind, ,ein Mindestmaß an wirtschaftlich verwendbarer Arbeitsleistung“ zu erbringen ( $\$ 52$ SchwbG a. F. jetzt $\S 136$ SGB IX).

Die Weichen für den Aufbau von Werkstätten wurden mit dem Gesetz zur Sicherung der Eingliederung Schwerbehinderter in Arbeit Beruf und Gesellschaft SchwbG - vom 29.04.1974 ${ }^{20}$ gestellt. Die maßgeblichen Vorschriften sehen in $\S 55$ SchwbG (jetzt $\S 142$ SGB IX) ein Anerkennungsverfahren vor und setzen einerseits eine Mindestgröße von 120 Arbeitsplätzen für behinderte Menschen und andererseits eine Aufnahmeverpflichtung für den Träger der Werkstatt voraus. Insgesamt gibt es zurzeit etwa 690 anerkannte Werkstätten mit über 260.000 Plätzen. Zu den Trägern zählen insbesondere die Lebenshilfe für Menschen mit geistiger Behinderung, das Diakonische Werk der EKD und der Deutsche Caritasverband.

Neben den $\S \S 39$ bis 43 SGB IX, 136 bis 144 SGB IX zählen die Werkstättenverordnung (WVO) vom 13.08.1980 ${ }^{21}$ und die Werkstättenmitwirkungsverordnung (WMVO) vom 28.06.2001 ${ }^{22} \mathrm{zu}$ den wichtigsten gesetzlichen Grundlagen für die Werkstatt.

Während die in den 70er und 80er Jahren des vergangenen Jahrhunderts eingeführten Rechtsvorschriften für die WfbM bis heute nahezu unverändert in Kraft sind, war es im Jahr 1982 mit dem Höhenflug der Sozialhilfe zunächst vorbei. Die wirt- 
schaftliche Rezession bewirkte, dass am Rechtsanspruch auf Sozialhilfe gerüttelt wurde.

So hieß es in einem damals weit verbreiteten Leitartikel der Frankfurter Allgemeinen Zeitung vom 20.11.1982, dass es notwendig sei, den Rechtsanspruch auf Hilfe zum Lebensunterhalt zu einer Ermessensleistung herabzustufen und als „Geschenk der Gesellschaft an ihre Ärmsten“ darzustellen ${ }^{23}$. Vor allem die Eltern von Menschen mit geistiger Behinderung bekamen diese „Zeitenwende“ zu spüren, denn sie wurden, soweit ihre Töchter und Söhne vollstationäre Eingliederungshilfe erhielten, teilweise zu hohen Unterhaltszahlungen herangezogen ${ }^{24}$.

Auch die Folgejahre waren immer wieder geprägt von einem „Auf und $A b^{\text {“ der }}$ Gesetzgebung im Bereich der Sozialhilfe. So ist die Eingliederungshilfe für behinderte Menschen immer wieder neuen gesellschaftlichen, fachlichen und politischen Erkenntnissen und Entwicklungen angepasst worden. Dies gilt nicht nur für die in den 90er Jahren des vergangenen Jahrhunderts vollzogene Abkehr vom Selbstkostendeckungsprinzip im Bereich der stationären Eingliederungshilfe und für die sich daran anschließende Hinwendung zu konkreten Leistungs-, Vergütungs- und Prüfungsvereinbarungen ( $\S 93 \mathrm{ff}$. BSHG; jetzt $\S \S 75$ ff. SGB XII), sondern auch für die Änderung zahlreicher BSHG-Vorschriften im Zuge des mit dem Sozialgesetzbuch Neuntes Buch (Rehabilitation und Teilhabe - SGB IX) vom 19. Juni $2001^{25}$ eingeleiteten Paradigmenwechsels in der Behindertenhilfe.

So schwierig es auch ist, die Entwicklung der Eingliederungshilfe rückblickend zu bewerten, lässt sich doch folgendes feststellen:

- Der offene Leistungskatalog der Eingliederungshilfe (§ 54 SGB XII) hat sich bewährt. Er hat sich als flexibel und anpassungsfähig erwiesen. Dies gilt z. B. für den Anspruch von behinderten Kindern auf heilpädagogische Leistungen, die seit Jahrzehnten den Grundstock der flächendeckend im ganzen Bundesgebiet anzutreffenden Frühförderstellen bilden.

- Nicht bewährt haben sich die finanziellen Strukturen, die der Eingliederungshilfe für behinderte Menschen zugrunde liegen. Solange die Verantwortung zur Finanzierung der Eingliederungshilfe ausschließlich den Kommunen obliegt und der Bund sich weigert, sich an der Finanzierung der Kosten der Eingliederungshilfe zu beteiligen, ist der Bestand an Hilfen für behinderte Menschen, die auf die Leistungen der Eingliederungshilfe angewiesen sind, gefährdet. Je mehr die Städte und Gemeinden finanziell unter Druck geraten, desto lautstärker wird die Forderung erhoben werden, Leistungen der Eingliederungshilfe zu kürzen oder die Einkommens-, Vermögens- und Unterhaltsheranziehungsregelungen des SGB XII zu verschärfen. Dies hat insbesondere der Gesetzentwurf des Bundesrates vom 05.11.2005 zur Entlastung der Kommunen im sozialen Bereich 
(KEG) gezeigt ${ }^{26}$, mit dem der Versuch gemacht wurde, die Leistungen der Eingliederungshilfe für behinderte Menschen und Leistungen der Jugendhilfe von der Finanzkraft der öffentlichen Hand abhängig zu machen.

Inzwischen hat sich die Lage wieder beruhigt. Die Wirtschaft wächst und die Gemeinden profitieren von steigenden Einnahmen aus der Gewerbesteuer. Doch der Wind kann sich sehr schnell drehen. Deshalb ist es wichtig, auch einen Blick in die Zukunft zu wagen:

Seit Jahren wird darüber diskutiert, ob es nicht an der Zeit ist, die Eingliederungshilfe für behinderte Menschen aus der im SGB XII geregelten Sozialhilfe herauszunehmen und in ein eigenes Leistungsgesetz für Menschen mit Behinderungen zu überführen. Doch keiner der bisher bekannt gewordenen Vorschläge hat bisher „Gesetzesreife" erlangt. Auch die Koalitionsvereinbarung vom 11. November 2005 enthält keine entsprechenden Hinweise, sondern kündigt stattdessen an, dass die Koalitionsparteien die Eingliederungshilfe weiterentwickeln wollen. Doch Vorkehrungen zur Novellierung des SGB XII im Bereich der Eingliederungshilfe hat die Bundesregierung bisher nicht getroffen. Im Gegenteil: Am 13. Dezember 2007 hat der Parlamentarische Staatssekretär beim Bundesminister für Arbeit und Soziales auf zwei mündliche Fragen des Abgeordneten Markus Kurth, Bündnis 90/DIE GRÜNEN, zur Reform der Eingliederungshilfe geantwortet:

„Die Bundesregierung hält es nicht für sachgerecht, einen Gesetzentwurf zur Weiterentwicklung der Eingliederungshilfe vorzulegen. Bevor gesetzgeberische Maßnahmen ergriffen werden, sollten zunächst die Strukturen und die Organisation der Eingliederungshilfe durch die Träger und die Leistungsanbieter so reformiert werden, dass bestehende Hemmnisse für die Teilhabe und Selbstbestimmung von Menschen mit Behinderungen abgebaut werden. Die Bundesregierung ist weiterhin unverändert bereit, zusammen mit den Ländern, den Kommunen und den Verbänden behinderter Menschen nach Lösungen zu suchen, die Eingliederungshilfe weit mehr als bisher auf den Paradigmenwechsel auszurichten“, der insbesondere mit dem Sozialgesetzbuch Neuntes Buch (SGB IX) eingeleitet worden ist ${ }^{27}$.

Der Verweis auf den Paradigmenwechsel in der Behindertenhilfe lädt dazu ein, erneut das von der Generalversammlung der Vereinten Nationen am 13.12.2006 verabschiedete - von der Bundesrepublik Deutschland am 30.03.2007 unterzeichnete - Übereinkommen über die Rechte behinderter Menschen in den Blick zu nehmen.

Das UN-Übereinkommen umfasst nicht nur die klassischen Freiheitsrechte, sondern auch das Recht auf „Unabhängige Lebensführung und Teilhabe an der Gemeinschaft" (Art. 19), das Recht auf Habilitation und Rehabilitation" (Art. 26) und das Recht auf angemessenen Lebensstandard und sozialen Schutz" (Art. 28).

Die Verfassung der Bundesrepublik Deutschland kennt bisher keine sozialen Grundrechte wie sie in den genannten Artikeln 19, 26 und 28 der Konvention geregelt sind. Deutschland kann jedoch darauf verweisen, das Verbot der Benachteili-

27 vgl. Plenarprotokoll der 132. Sitzung des Deutschen Bundestages vom 12.12.2007,S. $13838 \mathrm{f}$. 
gung behinderter Menschen im Jahr 1994 in Art. 3 Abs. 3 GG verankert und eine Sozialgesetzgebung entwickelt zu haben, die in weiten Teilen darauf zielt, behinderte Menschen so zu unterstützen, dass sie als Bürger am gesellschaftlichen Leben teilhaben können.

Nicht in Einklang mit den Zielvorgaben der UN-Konvention steht allerdings die Verankerung der Eingliederungshilfe für wesentlich behinderte Menschen im System einer nachrangigen - von Bedürftigkeitsprüfungen abhängigen - Sozialhilfe. Dies ergibt sich aus Zielstellung der Konvention, die der Vorsitzende des von den Vereinten Nationen eingesetzten Ad-hoc-Komitees zur Einarbeitung des Konventionstextes bei der Vorstellung der Konvention in der Generalversammlung der Vereinten Nationen wie folgt umschrieben hat: „The support of disabled persons is no longer a question of charity, but a human right ${ }^{628}$.

Wer sich an der UN-Konvention orientiert, muss ein Teilhabegesetz für behinderte Menschen fordern, das seinen Namen verdient und nicht länger auf die Grundsätze des Nachrangs der Sozialhilfe und des Nachweises von Bedürftigkeit gestützt wird. Strukturell ist ein Teilhabegesetz für behinderte Menschen auf dem Grundgedanken des Nachteilsausgleichs aufzubauen.

Auch hierfür enthält die UN-Konvention wichtige Anhaltspunkte: Der Begriff der Behinderung wird in der Präambel des Konventionstextes so umschrieben, „dass Behinderung entsteht, wenn Menschen mit Beeinträchtigungen auf einstellungs- und umweltbedingte Barrieren stoßen, die sie an der vollen, wirksamen und gleichberechtigten Teilnahme am gesellschaftlichen Leben hindern." Dem entsprechend wird der Begriff „behinderte Menschen“ in Art. 1 Satz 2 der Konvention dahin definiert, dass er „Menschen mit langfristigen körperlichen, seelischen, geistigen oder Sinnesschädigungen umfasst, die sie im Zusammenwirken mit verschiedenen Barrieren daran hindern können, gleichberechtigt mit anderen uneingeschränkt und wirksam an der Gesellschaft teilzunehmen.“

Die „Barrieren“ sind also die entscheidenden Hindernisse, die überwunden werden müssen, wenn eine Behinderung beseitigt oder gemildert werden soll. Es ist deshalb kein Zufall, dass die konkrete Aufzählung von Menschenrechten in der UNKonvention mit dem Recht auf Zugänglichkeit (Barrierefreiheit) beginnt (Art. 9). Je mehr Barrierefreiheit in der Gesellschaft hergestellt wird, desto größer sind die Chancen behinderter Menschen im gleichen Umfang wie nicht behinderte Menschen, als vollwertige Bürger anerkannt und in das allgemeine gesellschaftliche Leben einbezogen zu werden. Umgekehrt gilt: Je größer die Barrieren sind, die sich vor behinderten Menschen auftürmen, desto umfassender sind die gesellschaftlichen Bereiche, in denen behinderte Menschen in ihren Teilhabemöglichkeiten begrenzt oder vom gesellschaftlichen Leben ausgeschlossen sind.

Selbst in hoch entwickelten Staaten, zu denen die Bundesrepublik Deutschland zählt, ist es bisher nur ansatzweise gelungen, im Bereich des Bauwesens, des Verkehrs, der Kommunikation usw. Barrierefreiheit herzustellen. Solange dies so ist, 
müssen die Vertragsstaaten der UN-Konvention zum Schutz der Rechte behinderter Menschen dafür sorgen, dass die Nachteile, die durch einstellungs- und umweltbedingte Barrieren entstehen, ausgeglichen werden.

Geschieht dies auf dem Wege des Leistungsrechts, so muss der Nachteilsausgleich gesetzlich festgeschrieben und entweder durch Sach- oder Geldleistungen verwirklicht werden. Einige Länder (Niederlande, Dänemark u. a.) gewähren behinderten Menschen deshalb eine Behindertenrente, d. h. sie zahlen entweder ab Eintritt der Behinderung, ab Volljährigkeit oder zu einem späteren Zeitpunkt eine pauschalierte Geldleistung zum Ausgleich der behinderungsbedingten Nachteile.

Das vom Deutschen Verein für öffentliche und private Fürsorge vorgeschlagene Bundesteilhabegeld beruht ebenfalls auf dem Grundgedanken des Nachteilsausgleichs in der Form einer Geldleistung ${ }^{29}$.

Als Alternative bietet sich das insbesondere in Schweden praktizierte Assistenzmodell an: Behinderte Menschen erhalten zweckgebundene Geldbeträge, um sich die Assistenzdienste „einkaufen“ zu können, die sie benötigen, um arbeiten (Arbeitsassistenz), selbstständig wohnen (Betreuungsassistenz, Pflege u. a.) und ihre Mobilität sicherzustellen zu können (abrufbare Fahrdienste zu verträglichen Bedingungen und Preisen).

Schon diese Hinweise zeigen, dass ein Leistungsgesetz, das behinderungsbedingte Nachteile ausgleichen soll, auf dem individuellen Bedarf eines behinderten Menschen aufbauen muss. Es empfiehlt sich deshalb, ein flexibles System des Nachteilsausgleichs zu entwickeln. Dieses könnte aus einem pauschaliertem Sockelbetrag bestehen, der bei entsprechendem Bedarf und unter Berücksichtigung des Wunschund Wahlrechts behinderter Menschen durch individuell bemessene Geldleistungen oder durch Sachleistungen ergänzt wird.

Unverzichtbar ist, dass der Nachteilsausgleich einkommens- und vermögensunabhängig gewährt wird. Dafür ist die Überlegung maßgebend, dass ein leistungsrechtlich ausgestalteter Nachteilsausgleich nicht an der Bedürftigkeit, sondern an der Behinderung eines Menschen ansetzt. Würde man den Nachteilsausgleich von Bedürftigkeitsprüfungen abhängig machen, so würde man neue Barrieren schaffen und die gleichberechtigte „Inklusion“ behinderter Menschen in die Gesellschaft beeinträchtigen. 
4.3. Behinderte Menschen in der kommunalen Sozialpolitik

- Rehabilitation und Teilhabe junger Volljähriger sowie von Senioren im Zuständigkeits- und Leistungsgeflecht institutionalisierter Eingliederungshilfe nach dem Sozialhilfegesetzbuch -

\author{
Von Rainer Pitschas
}

1. Kommunale Sozialpolitik in Deutschland 105

1.1. Gegliederte Sozialverantwortung im „sozialen Bundesstaat“

1.2. „Sozialer Bundesstaat“ und kommunale Sozialpolitik 106

$\begin{array}{ll}\text { 1.3. Innovationsgehalte von Sozialpolitik } & 107\end{array}$

1.4. Kommunale Sozialpolitik im europäischen Sozialstaat 107

1.5. Kommunale Politikverantwortung im Zeichen des demographischen Wandels und der „Ökonomisierung“: Zur Ausgrenzung von jungen und alten behinderten Menschen aus der Bürgerkommune

2. Verrechtlichung kommunaler Politik für behinderte Menschen:

Gestaltungsfreiheit und - grenzen für Rehabilitation und Teilhabe in ausgewählten Handlungsfeldern

2.1. Kommunale Entscheidungen über Rehabilitation und Teilhabe im „rechtlichen Gehäuse“ gesetzlicher Intervention

2.1.1. Gesetzliche Ausgangslage nach dem Neunten Buch

Sozialgesetzbuch - Rehabilitation und Teilhabe behinderter

Menschen (SGB IX): Ein Überblick

2.1.2. Gestaltungsfreiheit und Gestaltungsgrenzen allgemeiner oder kommunaler Vollzugszuständigkeit

2.2. Ausgewählte Handlungsfelder kommunaler Sozialpolitik für behinderte Menschen: Soziale Existenzsicherung nach dem SGB XII

2.2.1. Soziale Existenzsicherung behinderter Menschen als kommunales Handlungsfeld

2.2.2. Schnittstellen zum Rehabilitationsrecht (SGB IX) 114

2.2.2.1. Das "neue" Sozialhilferecht als Rehabilitationsrecht 114

2.2.2.2. Nachrang der Rehabilitationsleistungen und Persönliches Budget

2.2.2.3. Umfang der Leistungen 115

2.3. Kinder- und Jugendhilfe als kommunales Handlungsfeld 116

2.3.1. Kinder- und Jugendhilfe als „offene“ Form kommunaler

Sozialpolitik

2.3.2. Rehabilitation als Aufgabe der Kinder- und Jugendhilfe $\quad 117$

2.3.3. Insbesondere: Eingliederungshilfe 
2.4. Behinderte junge Volljährige im Spannungsfeld von Kinder- und Jugendhilfe zur Sozialhilfe

2.4.1. Zuordnung zur Kinder- und Jugendhilfe

2.4.2. Abgrenzung der Zuständigkeiten zwischen Jugendamt,

Sozialamt und der Gesundheitsfachverwaltung - Ein Grundproblem des bürokratisierten Sozialstaats

2.4.3. Der ,institutionelle“ Politikansatz vor dem Versagen? 


\section{Kommunale Sozialpolitik in Deutschland}

\subsection{Gegliederte Sozialverantwortung im ,sozialen Bundesstaat“}

Kommunale Sozialpolitik als Gesamtheit der Reaktion von Gemeinden, Kreisen und Städten der Bundesrepublik Deutschland auf soziale Notlagen und Bedürfnisse des Kommunalbürgers wurzelt in der Sozialverantwortung des dezentralisierten modernen Staates. ${ }^{1}$ Dessen sozialpolitisches Handeln ist als ein komplexer staatlicher Steuerungsprozess angelegt, der aus der Idee eines ,sozialen“ Gemeinwesens seinen spezifischen und grundgesetzlich vorgezeichneten Auftrag zur Herstellung sozialer Gerechtigkeit und Herbeiführung sozialen Ausgleichs innerhalb der Zivilgesellschaft empfängt. Dem verpflichtete gesamtstaatliche Sozialpolitik unterliegt als ein Teilbereich politischen Handelns im kooperativen Staat der Gegenwart sowie unter den Bedingungen sozialstaatlicher Governance in Deutschland einer gegliederten Verantwortung des „sozialen Bundesstaates“, die auch die kommunale Sozialpolitik einbezieht. $^{2}$

Verwiesen ist damit auf die Ausdifferenzierung der deutschen Sozialpolitik in einem bundesstaatlichen Gefüge. Sie trifft hierbei einerseits auf konkurrierende TeilPolitiken in anderen Politikfeldern wie z. B. auf die Bildungs- oder Familienpolitik. ${ }^{3}$ Auf der anderen Seite vertraut die grundgesetzliche Sozialverfassung das „Soziale“ keinem einheitsstaatlichen Gemeinwesen und damit einer Sozialpolitik ,aus einem Guss" an. Deren Eigenheit und Vielfalt hat sich statt dessen und auf dem Fundament regionaler Einflüsse, der Sonderheiten europäischer Regionalpolitik und in Verfolg vielfacher „Public Private Partnership“ in einem „sozialen Bundesstaat“ zu entfalten bzw. zu bewähren. Dies geschieht vor allem unter den Bedingungen des demographischen Wandels im ergrauenden Europa einerseits, angesichts der „Ökonomisierung" aller Sozialpolitik andererseits. ${ }^{4}$

Das genuin „Soziale“ der staatlichen Politik, also qualitative Sozialpolitik versteht sich insoweit und von vorneherein als Element eines (bundes-)staatlich gegliederten und innerhalb eines jeden Landes dann ausdifferenzierten Organisationsrahmens sowie Wertegefüges. Der Bundesstaat als ganzes, der Zentralstaat, seine Gliedstaaten bzw. Regionen, aber auch deren ,interner“ kompetenzieller und institutioneller Aufbau - sollen „sozial“ sein. In diesem Sinne fügt das Grundgesetz seiner Sozialverfassung eine bundes- bzw. gliedstaatlich und regional differenzierte Di-

1 Pitschas, Kommunale Sozialpolitik, in: von Maydell/Ruland (Hrsg.),Sozialrechtshandbuch (SRH), 3. Aufl. 2003, C. 24 Rz. 1; zur „Dezentralisierung“ als weltweiter Prozess am Beispiel Süd- und Ostasiens s. die Beiträge in ders. (Hrsg.), Dezentralisierung im Vergleich, 2008.

2 Zacher, FS W. Schmitt Glaeser, 2003, S. 199 ff.; Pitschas, Sozialer Bundesstaat und Föderalismusreform, in: Blanke (Hrsg.), Die Reform des Sozialstaats zwischen Freiheitlichkeit und Solidarität, 2007, S. 23 (25 ff., 32 ff.).

3 Vgl. nur Ricken, Familiale Gemeinschaften im Sozialrecht, FS F. E. Schnapp, 2008, S. 509 ff.

4 Streeck, Politik in einer alternden Gesellschaft: Vom Generationenvertrag zum Generationenkonflikt?, in: Gruss (Hrsg.), Die Zukunft des Alterns, 2007, S. 279 ff.; auf ökonomische Zwänge weist Stolleis hin, ebd., S. 258 (273 f.). 
mension ein: Ist nämlich die Bundesrepublik Deutschland ein „sozialer Bundesstaat“ (Art. 20 Abs. $1 \mathrm{GG}$ ) und hat jedes Bundesland in diesem Sinne ein Sozialstaat zu sein (Art. 28 Abs. 1 S. 1 GG), dann bewahrt dieses Land sich zugleich eine eigene Zuständigkeit im Hinblick auf die Landes- und kommunale Sozialpolitik sowie auf den Zuschnitt der Aufgaben und Governance länderstaatlicher wie kommunaler Sozialverwaltung. ${ }^{5}$

Der dadurch aufgeworfenen Frage nach der kompetenziellen Reichweite und effektiven Durchschlagskraft staatlicher Sozialpolitik, die sich in die virulente Problematik zunehmender, auch finanzieller Spaltung zwischen sozialer Gleichheit und bundesstaatlicher Vielfalt einfügt, kann hier nicht näher nachgegangen werden. Durch die Föderalismusreform I sind insoweit auch für die kommunale Sozialpolitik entscheidende Weichen neu gestellt worden. Kraft des „Wettbewerbsföderalismus“ nimmt nunmehr das Grundgesetz durchaus gewollt länderbezogene und regionale Differenzierungen, die zu kommunalen bzw. regionalen Disparitäten führen können auch im Sozialsektor in Kauf. ${ }^{6}$

\section{2. „Sozialer Bundesstaat“ und kommunale Sozialpolitik}

Stärker als je zuvor konkurrieren auf diese Weise und in Verfolg wie Ausdeutung des sozialen Staatsziels nicht nur der Bund und die Länder untereinander. Und auch gliedstaatliche Vielfalt steht nicht allein für das Problem der Entwicklung zu einem dezentralen bzw. regionalisierten Sozialstaat in der Europäischen Gemeinschaft. Die bundesstaatliche Offenheit des „Sozialen“, die parallel dazu verlaufenden Ansätze zur Ausdifferenzierung sozialer Politik und ihre Niveaudivergenzen setzen sich vielmehr länderinkorporiert nach unten auf der kommunalen Ebene fort (Art. 28 Abs. 1 S. 2, Abs. 2 GG). Sozialpolitik im sozialen Bundesstaat stellt in diesem Sinne eine in sich „gebrochene“ Gesamtheit der Handlungsvollzüge von Zentralstaat, Gliedstaaten, Regionen, Städten und Kommunen sowie Landkreisen dar. Dadurch entsteht zwar noch keine dritte bzw. vierte staatliche Ebene. Immerhin aber gewährleistet die grundgesetzliche Garantie der kommunalen Selbstverwaltung den Regionen, Landkreisen, Städten und Gemeinden im Gestaltungsrahmen des sozialen Bundesstaates gem. Art. 28 Abs. 2 GG das Recht, alle Angelegenheiten der örtlichen Gemeinschaft auf der Grundlage der allgemeinen Gesetze in eigener Verantwortung zu regeln. ${ }^{7}$

Diese Funktionszuweisung umschließt einerseits den Spielraum der Kommunen und - in Einzelfällen durchaus vom Bund eingeschränkt (vgl. Hartz IV-Gesetz-

5 Merten/Pitschas, Sozialverwaltung im Reformprozess, 1997, S. 23 ff.; Pitschas (Fn. 2), S. 32 ff.

6 Pitschas, Zukunftsfähige Kinder- und Jugendhilfe nach der Föderalismusreform?, in: Arbeitsgemeinschaft für Kinder- und Jugendhilfe-AGJ (Hrsg.), Auswirkungen der Föderalismusreform auf die Kinder- und Jugendhilfe, 2006, S. 67 (73 ff.).

7 Losch, Zum gegenwärtigen Stand des Kommunalrechts in Deutschland, in: Pitschas (Hrsg.), Dezentralisierung im Vergleich (Fn. 1), S. 17 (20 f., 24 f.). 
gebung) ${ }^{8}$ - die Befugnis, soziale Aufgaben selbst zu entdecken sowie eigenverantwortlich wahrzunehmen. Darin liegt der eine normative Ansatzpunkt einer spezifisch kommunalen Sozialpolitik. Sie ist dazu aufgerufen, im Wirkbereich der kommunalen Zuständigkeiten das soziale Staatsziel selbstständig, d. h. eigenverantwortlich und innovativ zu erfüllen. Auf der anderen Seite unterliegen die Kommunen allerdings ebenso und gleichzeitig der Zuweisung von Aufgaben durch die Bundesländer, dies zur Erfüllung als ,übertragene Aufgaben“ oder in eigener Zuständigkeit, aber fremdverantwortlich. ${ }^{9}$

\subsection{Innovationsgehalte von Sozialpolitik}

Die dahinter aufscheinenden Bewirkungsgehalte von Sozialpolitik empfangen über die sog. Homogenitätsklausel des Art. 28 Abs. 1 S. 1 GG i. V. mit Art. 23 Abs. 1 GG bindende Direktiven einer wert- und substanzgeprägten Sozialstaatlichkeit, die sich - europarechtlich durch den Gemeinschaftsrechtsvorrang überlagert - im Zentralstaat entwickelt und die sodann über die Länder - gliedstaatlich kompetenziell wie institutionell zur gemeinwohlorientierten Entfaltung und zu Vollzug in den Gemeinden und Gemeindeverbänden aufgegeben ist. ${ }^{10}$ Darüber hinaus verbleibt den Kommunen noch immer ein ausgreifendes eigenes Mandat zur sozialen Daseinsvorund -fürsorge entsprechend den Bedarfen und Bedürfnissen in der örtlichen Gemeinschaft bzw. im lokalen Gemeinwesen. Auch wenn nicht zu bestreiten ist, dass dabei das Entdeckungsprinzip und -verfahren „Sozialität“ zunehmend stärkerer und vor allem ausgabenwirksamer Steuerung durch Gemeinschafts- und Landesgesetze, zentrale Fach- und Entwicklungsplanungen sowie präventive Aufsichtsmaßnahmen bei immer schwierigeren kommunalen Investitionen bzw. Unterhaltungsaufwendungen für die sozial Infrastruktur unterliegt, können doch auch in Zeiten höchster finanzieller Bedrängnis gerade die sozialen Aufgaben ein stets von neuem erfolgreich zu bestellendes Feld sozialer Innovationen darstellen. ${ }^{11}$

\subsection{Kommunale Sozialpolitik im europäischen Sozialstaat}

In diesem Gefüge offenbart sich zugleich eine gewisse inhaltliche Veränderungsprogrammatik der kommunalen Sozialpolitik durch die gemeinschaftsrechtlichen Direktiven und Aktionsformen (Programme, Projekte etc.) des Europäischen Sozialstaats. Dieser umschließt als Rechtsbegriff diejenigen gemeinschaftlichen Rechtsgrundlagen, strukturellen Rahmenbedingungen und Politikaufträge, die in der sozia-

8 Dazu statt aller BVerfGE 119, 331 (332 ff.).

9 Vgl. zuletzt BVerfGE 119, 331 (362 ff.).

10 BVerfGE 119, 331 (363).

11 Näher ausgeführt bei Pitschas, Kommunale Sozialpolitik (Fn. 1), Rz. 24, 149. 
len Dimension der Europäischen Gemeinschaft geborgen sind. ${ }^{12}$ Einwirkungen dieser auf die kommunale Sozialpolitik in den Mitgliedstaaten und auch in Deutschland umfassen sowohl formelle und/oder informelle unmittelbare soziale Absprachen mit (Groß-)Kommunen, andererseits die Erarbeitung und Umsetzung finanzieller Förderprogramme im Rahmen der Strukturfonds, vergleichbare Initiativen im Rahmen der Aktionsprogramme im Sozialsektor, die Förderung des „Sozialen Dialogs" sowie Elemente der Umsetzung der Gemeinschaftscharta der Sozialen Grundrechte der Arbeitnehmer. In alledem liegt der Kern einer künftigen Gemeinschaftsverantwortung für die kommunale Sozialpolitik. Diese bildet das Gegenstück zum inzwischen deutlicher gewordenen Begehren der Städte, Gemeinden und Kreise sowie ihrer Verbände, durch Lobbyarbeit und durch die Vertretung im „Ausschuss der Regionen“ zu einem „Europa der Kommunen“ auf einer eigenen europäischen Organisations- und Politikebene zu finden. Freilich bedarf diese „Europäisierung von unten" noch weiterer Ausprägung.

\subsection{Kommunale Politikverantwortung im Zeichen des demographischen Wandels} und der „Ökonomisierung“: Zur Ausgrenzung von jungen und alten behinderten Menschen aus der Bürgerkommune

Allerdings stößt diese Entwicklung auch und gleichzeitig auf die im Gemeinschaftsrecht prinzipiell verankerte wettbewerbliche Dimension der kommunalen Daseinsvorsorge. ${ }^{13}$ Immerhin empfängt kommunale Sozialpolitik nicht nur hieraus neue Gestaltungsdirektiven. Die Verpflichtung der Kommunen zu einer Anwendung z. B. des Vergaberechts auch in diesem Sektor bei gleichzeitigem Übergang zur wettbewerblichen Organisation kommunaler Sozialpolitik hat in dieser europäischen Anbindung einen tieferen Grund. ${ }^{14}$

Parallel hierzu sehen sich die Kommunen auch in der Entwicklung ihrer Sozialpolitik von den nationalen Initiativen des „New Public Management“ betroffen, Kommunen als „Konzerne“ zu restrukturieren und das Neue Steuerungsmodell in den kommunalen Verwaltungen vorbehaltlos umzusetzen. Näherhin folgt daraus die Verpflichtung der Kommunen zu einer verstärkt wettbewerbsgeöffneten Organisation ihrer sozialen Leistungserbringung zwischen den Polen einer kommunalen Sozialverwaltung als „Unternehmen“ einerseits und der Berücksichtigung von Solidarität und ,sozialem Zusammenhalt“ (Art. 158 a EGV) durch „Sozialwirtschaft" andererseits. Sowohl das Gemeinschaftsrecht wie auch die deutsche Verfassung gehen da-

12 Weniger weitgehend Eichenhofer, Geschichte des Sozialstaats in Europa. Von der ",sozialen Frage“ bis zur Globalisierung, 2007, S. 97 ff. („Auf dem Weg zum Sozialstaat in Europa“).

13 Pielow, „Soziale“ Daseinsvorsorge und „Dienste von allgemeinem Interesse“, FS F. E. Schnapp, 2008, S. $491 \mathrm{ff}$.

14 Rixen, Sozialrecht als öffentliches Wirtschaftsrecht, 2005, S. 40 ff., 334 ff.; Schlüter/Scholz, Rollenwandel der Wohlfahrtspflege in der Europäischen Union: Organisatorische und rechtliche Aspekte, in: Linzbach/Lübking/Scholz/Schulte (Hrsg.), Globalisierung und Europäisches Sozialmodell, 2007, S. 189 (201 ff.). 
von aus, dass die kommunale Sozialpolitik weder von Seiten der Gemeinschaft unangemessen „ökonomisiert“ werden darf (vgl. Art. 16, 86 Abs. 2 S. 2 EGV; Art. 20, 28 GG), noch in ihrer Eigendynamik den sozialen Schutzcharakter der Politik außer Kurs setzen dürfte. ${ }^{15}$

Vor diesem Hintergrund ist kommunaler Sozialpolitik im europäischen Sozialstaat und besonders in der Bundesrepublik Deutschland im Einzelfall immer wieder aufgegeben zu prüfen, ob eine Aufgabe bzw. Leistung wie etwa die Eingliederung und Teilhabe behinderter Menschen ${ }^{16}$ aus dem öffentlichen Bereich zu entlassen und im Wege der Aufgabenprivatisierung einer privatwirtschaftlichen Wahrnehmung stärker als bisher zu überantworten wären. Wie schwierig dies ist, zeigt sich zumal an der Rehabilitation und Teilhabe junger Volljähriger sowie von Senioren im Zuständigkeits- und Leistungsgeflecht institutionalisierter Eingliederungshilfen nach dem deutschen Sozialgesetzbuch. Die für diesen Zweck intern gegliederte behördliche Zuständigkeit auf Seiten der kommunalen Sozialleistungsträger entwickelt unter dem Zwang budgetierter Finanzzuweisungen im jeweiligen öffentlichen Haushalt ausgeprägte Abschottungsstrategien gegenüber ganzheitlichen Politikvorstellungen. Davon sind nicht nur behinderte junge Volljährige, sondern auch alte behinderte Menschen in besonderem Maße betroffen. ${ }^{17}$ Während es nämlich gesetzliche Regelungen dem alten und behinderten Mitbürger ermöglichen wollen, bis in das hohe Alter hinein in seiner eigenen Wohnung zu bleiben, fehlt es an ausreichenden Kapazitäten der Sozialstationen und anderer institutioneller Akteure, in dekonzentrierter Form einzelne Hilfeangebote für „zu Hause“ miteinander zu verbinden. Daran dürften die nunmehr im Rahmen der Weiterentwicklung sozialer Pflegeversicherung einzurichtenden „Stützpunkte“ zur Beratung von alten und behinderten Bürgern sowie deren Familien nichts ändern. ${ }^{18}$ Gleiches gilt für die wachsenden Aktivitäten privatwirtschaftlicher Pflegedienste, von Seniorenstiften und anderen privaten Einrichtungen, für die in der Regel der behinderte alte Mensch tatsächlich nur zählt, wenn er vermögend ist.

Dieser rechtstatsächlichen Einschätzung und einer insoweit ähnlichen Situation von jungen volljährigen behinderten Menschen und behinderten Senioren im Rahmen der deren Lebensverlauf verbindenden Betrachtung von Lebensphasen ${ }^{19}$ entspricht die Rechtsprechung des BSG in diesem Punkt: So verneint dessen Großer Senat die Leistungspflicht einer Krankenkasse für einen Krankenhausaufenthalt, „wenn der Versicherte aus anderen, nicht mit der Behandlung zusammenhängenden

15 Pitschas, Kommunale Sozialpolitik (Fn. 1), Rz. 21 f., 29 f.

16 Dazu der Bericht aus dem Dt. Bundestag über die Lage behinderter Menschen und die Entwicklung ihrer Teilhabe, BT-Drs. 15/4575.

17 Völlig irreführend, was die Realität angeht, demgegenüber Keupp, Von der Fürsorge zur Selbstsorge - Eine Empowerment-Perspektive für eine solidarische Sozialpolitik, in: Linzbach u. a. (Fn. 14), S. 441 (448 f., 455 f.).

18 Ähnlich Welti, Systematische Stellung des SGB IX im Sozialgesetzbuch - Zusammenarbeit der Leistungsträger und Koordinierung der Leistungen, SGb 2008, 321 (327).

19 Hierzu Pitschas, Zur rechtlichen Verfassung der Lebenslage „Alter“ - Ein persönlichkeitsrechtliches Strukturkonzept der seniorenpolitischen Aktivbürgerschaft in einer Gesellschaft des langen Lebens, FS O. E. Krasney, 1997, S. 355 (366 ff.). 
Gründen, eine spezielle Unterbringung oder Betreuung benötigt und wegen des Fehlens einer geeigneten Einrichtung vorübergehend im Krankenhaus verbleiben muss." Betroffen hiervon sind vor allem Pflegebedürftige, Demenzkranke und psychisch Kranke, die auf die Weiterbehandlung in einer besonderen Einrichtung angewiesen sind.

\section{Verrechtlichung kommunaler Politik für behinderte Menschen: Gestaltungsfreiheit und-grenzen für Rehabilitation und Teilhabe in ausgewählten Handlungsfeldern}

2.1. Kommunale Entscheidungen über Rehabilitation und Teilhabe im ,,rechtlichen Gehäuse" gesetzlicher Intervention

An diesem Beispiel wird einerseits deutlich, dass die Grundlinien der kommunalen Reaktion auf soziale Notlagen und Bedürfnisse behinderter Menschen, die Gemeindebürger sind, vom deutschen Bundes- und Landesrecht weitgehend vorgezeichnet werden. In zahlreichen Gesetzen und Rechtsverordnungen haben Legislative und Exekutive der Länder und des Bundes vollzugsbedürftiges Sozialrecht geschaffen, das auch für behinderte Menschen einen allgemeinen Handlungsrahmen absteckt. Dem können sich weder kommunale Verwaltungsinstitutionen noch die Sozialrechtsprechung entziehen.

Zudem und andererseits wird erkennbar, dass eine durchgreifende eigenständige Initiative zur Überwindung abschottender Sozialpolitik und Rechtsprechung den Kommunen schwerfallen muss. Denn das geltende Sozialrecht einschließlich der Eingliederungshilfe für Menschen mit Behinderung stellt nach den Büchern VIII, IX und XII des Sozialgesetzbuchs (SGB) für administratives Sozialhandeln sowohl ein „rechtliches Gehäuse“ des Vollzugs als auch ein Instrument der gesetzlich in festen Bahnen vorgezeichneten Einzelsteuerung sozialrechtlicher Entscheidungen dar. Den Kommunen ist vielfach eine direktive Handlungsorientierung vorgegeben, die mit einer institutionellen Subjektivierung der Rechtsanwendung verbunden ist. ${ }^{20}$

2.1.1. Gesetzliche Ausgangslage nach dem Neunten Buch Sozialgesetzbuch - Rehabilitation und Teilhabe behinderter Menschen (SGB IX): Ein Überblick

Die rechtliche Ausgangslage für kommunale Entscheidungen über Rehabilitation und Teilhabe behinderter Bürger am Berufs- und Alltagsleben ist darüber hinaus

20 Pitschas, Organisationsrecht als Steuerungsressource in der Sozialverwaltung, in: SchmidtAßmann/Hoffmann-Riem (Hrsg.), Verwaltungsorganisationsrecht als Steuerungsressource, 1997, S. 151 (182 ff., 190). 
überaus kompliziert. Denn das Rehabilitationsrecht ist nach wie vor kompetenziell und materiell zersplittert, wie der knappe Blick auf seine Fundamente zeigt. ${ }^{21}$

Zwar ist nach mehrjährigen Vorarbeiten zum 1. Juli 2001 das von Bundestag und Bundesrat mit breiter Mehrheit beschlossene SGB IX in Kraft getreten. ${ }^{22}$ Das neue Gesetzeswerk ist nunmehr Teil des deutschen Sozialrechts; es enthält leistungs-, verwaltungs- und arbeitsrechtliche Regelungen. Diese umschließen in ihrer Gesamtheit eine Reihe sozialrechtlicher Gebiete, die weiterhin über ein eigenes abgegrenztes Leistungsrecht verfügen. Denn die Rehabilitation behinderter Menschen bleibt nach wie vor in den Besonderen Teilen des Sozialgesetzbuches verankert, z. B. in der gesetzlichen Krankenversicherung, der Arbeitsförderung, der Kriegsopferversorgung und selbstverständlich auch in dem Kinder- und Jugendhilferecht sowie in der Sozialhilfe. ${ }^{23}$

Im Mittelpunkt des SGB IX steht daher das Bestreben, behinderten und von Behinderung bedrohten Menschen vom Grundsatz her ein selbstbestimmtes Leben zu ermöglichen und dafür zentrale Direktiven an alle anderen Regelungen zu formulieren. Deren Ziel ist in Anlehnung an das sog. "Partizipationsmodell" der Weltgesundheitsorganisation die Förderung der Teilhabe behinderter Menschen am gesellschaftlichen und insbesondere am Arbeitsleben. Dieses Ziel soll mit medizinischen, erzieherischen und sozialen sowie mit beruflichen Leistungen schnell, wirkungsvoll, wirtschaftlich und nachhaltig zugleich erreicht werden. Zu diesem Zweck werden die Sozialleistungen als "Leistungen zur Teilhabe" zusammengefasst. ${ }^{24}$

Der Gesetzgeber hat näherhin das Recht der Rehabilitation und Teilhabe behinderter Menschen in zwei Teile gegliedert. In seinem ersten Teil schafft das SGB IX für das sozialrechtliche Rehabilitationsrecht über die Einzelbücher des Sozialgesetzbuchs hinweg für diese einen allgemeinen Rahmen, eine Art „Allgemeiner Teil“. Er gilt etwa in der gesetzlichen Renten- wie für die Alterssicherung der Landwirte, aber eben auch für die Sozial-, Kinder- und Jugendhilfe. Dieser Rahmen löst das bisherige Rehabilitations-Angleichungsgesetz ab. Gleichwohl sind die in den jeweiligen einzelnen Leistungs-Gesetzen bestehenden Anspruchsvoraussetzungen weitgehend erhalten geblieben. Im zweiten und Besonderen Teil des neuen Gesetzbuchs wird sodann derjenige Bereich geregelt, der bislang im Schwerbehindertengesetz als Recht der Schwerbehinderten und dieser gleichgestellten Personen normiert worden war. Demgemäss ist das Schwerbehindertengesetz ebenfalls aufgehoben worden.

$21 I g l$, Das SGB IX und seine Wirkungen auf das System des Sozialrechts, ZSR 2004, $385 \mathrm{ff}$.

22 BGB1. 2001 I S. 1046; zu ersten Erfahrungen bei der Umsetzung in der Praxis vgl. BT-Drs. $14 / 8972$.

23 Dazu der Überblick bei Auktor, Landesbericht Deutschland, in: von Maydell/Pitschas/Schulte (Hrsg.), Behinderung in Asien und Europa im Politik- und Rechtsvergleich, 2003, S. 27 (39 ff.).

Vgl. auch $\S \S 14,33,55$ SGB IX. 
2.1.2. Gestaltungsfreiheit und Gestaltungsgrenzen allgemeiner oder kommunaler Vollzugszuständigkeit

Im Mittelpunkt fachspezifischer und kommunaler Vollzugszuständigkeit für das Behindertenrecht stehen die nunmehr durch das SGB IX hervorgehobene Pflichten der Rehabilitationsträger, wie sie in § 6 SGB IX einzeln aufgeführt werden, zu individueller Leistungserbringung und zum Qualitätsmanagement. Der Gesetzgeber stärkt das Prinzip der individuellen Konkretisierung von Leistungen dadurch noch, das nunmehr den berechtigten Wünschen der Leistungsbezieher mehr als früher entsprochen werden muss; diese selbstverantworteten Vorstellungen sollen die zentrale Leitlinie im Rehabilitationsprozess verkörpern. ${ }^{25}$ Wird den Wünschen nicht entsprochen, muss der Träger dies durch Bescheid begründen, der anfechtbar ist. Sachleistungen zur Teilhabe können dabei, soweit sie nicht in Einrichtungen zu erfüllen sind, auf Antrag der Berechtigten auch als Geldleistungen erbracht werden.

In diesem Zusammenhang bleibt das Persönliche Budget gem. § 18 Abs. $1 \mathrm{~S} .1$ Nr. 4 SGB IX hervorzuheben. Es soll in Zukunft eine gleichberechtigte Form der Leistungserbringung darstellen und die Selbstbestimmung behinderter Menschen sichern. Persönliche Budgets müssen nach dem Willen des Gesetzgebers so bemessen sein, dass die Deckung des individuellen Bedarfs unter Beachtung der Grundsätze von Wirtschaftlichkeit und Sparsamkeit möglich ist. Allerdings erinnern diese limitierenden Voraussetzungen sehr stark an den Versuch in der gesetzlichen Krankenversicherung, bedarfsdeckende Leistungen in den Rahmen einer vorrangigen Wirtschaftlichkeitsverpflichtung zu "pressen", wodurch dann „budgetierte Qualitätsprobleme" auftreten. ${ }^{26}$ Möglicherweise verbirgt sich hinter der vom Gesetzgeber gewählten Formulierung doch bloß ein verdeckter Sparzwang. Ihm soll aber entgegenwirken, dass die Leistungsträger nach $\S 20$ SGB IX die Qualität der Hilfeanstrengungen sichern. Hierfür ist ein entsprechendes Qualitätsmanagement bereitzuhalten.

Über die Defizite der rechtlichen Regelung einer Rehabilitation behinderter Menschen ist hier nicht im einzelnen zu handeln. An einer Reihe von weiteren Gesichtspunkten lässt sich jedenfalls aufzeigen, dass die Neuordnung des deutschen Rehabilitationsrechts nach wie vor wenig zur Integration behinderter Menschen in die Gesellschaft beiträgt. Verantwortlich hierfür sind u. a. die Schnittstellenprobleme in der Leistungsrechtsetzung und der Leistungserbringung. vention - Chancen für Effizienzsteigerung in den Sozialleistungsbereichen, SDSRV Bd. 52, 2004, S. $85 \mathrm{ff}$.

26 Fahlbusch, Rechtsfragen des Persönlichen Budgets, NJV 2006, 227 ff.; wie hier krit. auch und wiederum Welti, Das Persönliche Budget - Herausforderung an soziale Dienste, Einrichtungen und Berufe, Betreuungsmanagement 2007, $117 \mathrm{ff}$. 
2.2. Ausgewählte Handlungsfelder kommunaler Sozialpolitik für behinderte Menschen: Soziale Existenzsicherung nach dem SGB XII

Die Umsetzung gesetzlicher Regelungen in der Sozialpolitik speziell als gegliedertes Handlungskonzept durch Kommunen bezieht sich insbesondere auf die Handlungsfelder des „neuen“ Sozialhilferechts (SGB XII), der Kinder- und Jugendhilfe (SGB VIII) sowie des Rechts der Rehabilitation und Teilhabe behinderter Menschen (SGB IX).

\subsubsection{Soziale Existenzsicherung behinderter Menschen als kommunales Handlungsfeld}

Einen Schwerpunkt der kommunalen Sozialpolitik bildet insoweit die Anwendung des Sozialhilferechts (SGB XII). Es steckt den hierin enthaltenen und die frühere traditionsreiche „Armenpflege“ ablösenden spezifischen Sicherungsauftrag der Kommunen vor Armut durch die Hilfe zum Lebensunterhalt, die Grundsicherung im Alter und bei Erwerbsminderung sowie durch besondere Leistungen (ehedem „Hilfe in besonderen Lebenslagen“) rahmenhaft ab. Zu den „besonderen Leistungen“ zählen insbesondere die Eingliederungshilfe für behinderte Menschen, die Hilfe zur Überwindung besonderer sozialer Schwierigkeiten sowie die Pflege- und Altenhilfe. Jede dieser Hilfen wird - wie auch die Hilfe zum Lebensunterhalt - im Einzelfall als Teil eines umfassenden sozialhilferechtlichen Betreuungsverhältnisses erbracht. ${ }^{27}$

Institutionelles Kernstück des sozialhilferechtlichen Sicherungsauftrags ist das gemeindliche/städtische bzw. bei dem Kreis eingerichtete Sozialamt. Es nimmt der kreisfreien Stadt oder dem Landkreis als örtlichem Träger der Sozialhilfe zugewiesenen Aufgaben wahr. Regelmäßig sind die örtlichen Aufgaben der Sozialhilfe dann auch Selbstverwaltungsangelegenheiten der Kommunen. Für einzelne besondere Hilfearten werden dagegen überörtliche Träger von den Ländern bestimmt.

Vom Umfang der finanziellen Aufwendungen her stehen die besonderen Leistungen im Vordergrund. Der Idee des Gesetzgebers nach erhält die darin geborgene Hilfe derjenige, der sich in einer Lebenslage befindet, in der er sich nicht durch den zumutbaren Einsatz eigener Ressourcen zu behaupten vermag. Besonders kostenintensiv ist in diesen Fällen die Hilfe in Einrichtungen. ${ }^{28}$

So hat sich in der Hilfe für behinderte junge Menschen ein dichtes Netz von stationären (und ambulanten) Hilfs- und Betreuungsangeboten herausgebildet: Sonderschulkindergärten, „Hausfrühbetreuung“ mit Anleitung der Erziehungsberechtigten, Behindertenwerkstätten, Tagesbildungseinrichtungen und behindertengerechte Wohnformen legen neben anderen Zeugnis über die einschlägigen Bemühungen der 
kommunalen - und z. 'T. auch überörtlichen - Leistungsträger ab. Ohne Rücksicht auf das Lebensalter gilt jeweils das Prinzip, dem Sozialhilfeempfänger möglichst viele von denjenigen Leistungen zuzuwenden, auf die behinderte Menschen aus anderen Anspruchsverhältnissen im „sozialen Netz“ einen Rechtsanspruch haben. In der Folge dessen werden denn auch Sozialhilfeleistungen der Behindertenhilfe neben solchen erbracht, die sich auf andere Rechtsgrundlagen stützen können. Leistungsträger können ferner kommunal geförderte Selbsthilfegruppen und andere Einrichtungen des „Dritten Sektors“ sein.

Gravierende Defizite der Hilfe für behinderte Menschen nach dem SGB VII sind freilich nicht zu übersehen. Die bereits erwähnte Teil-Reform des Pflegeversicherungsrechts ${ }^{29}$ hatte auch darin einen ihrer Auslöser. Vor allem die Kommunen sind mit ihren bescheidenen finanziellen und personellen Kräften nicht in der Lage, die auch für behinderte Menschen überdeutliche Verschärfung sozialer Lebensverhältnisse im Zeichen der „,neuen Armut“ aufzufangen. Ähnlich begrenzt sind die Instrumente bzw. Möglichkeiten, die den Trägern der Sozialhilfe zur Verfügung stehen, um behinderten Menschen in der sozialen Wirklichkeit effektiv die Teilnahme am Leben in der Gemeinschaft zu ermöglichen oder zu erleichtern. Dies gilt auch und vor allem für alte Bürger, die im Falle einer Behinderung - gleichsam durch „Doppelbeeinträchtigung“ - nicht in der Lage sind, eigenverantwortlich für sich zu sorgen.

\subsubsection{Schnittstellen zum Rehabilitationsrecht (SGB IX)}

\subsubsection{Das "neue" Sozialhilferecht als Rehabilitationsrecht}

Die Rehabilitation und Förderung behinderter Menschen zur Teilhabe am Leben in der Gemeinschaft ist eine, wie bereits betont, gewichtige und Schnittstellen herbeiführende Aufgabe unterschiedlicher Rechtsbereiche. Zu ihnen gehört das Sozialhilferecht in Gestalt seiner Einordnung in das Sozialgesetzbuch. Die darauf beruhende Sozialhilfe springt aber - wie schon zuvor nach dem Bundessozialhilfegesetz (BSHG) - nur nachrangig als Rehabilitationsträger ein. Wie im gesamten Fürsorgerecht ist daher die Bedürftigkeit eine, wenn auch eingeschränkte, Leistungsvoraussetzung. Nebenbei bemerkt, handelt es sich bei der sog. Eingliederungshilfe kostenmäßig um den größten Leistungsbereich der Sozialhilfe.

Die Träger der Sozialhilfe sind nach § 6 SGB IX in den Kreis der Rehabilitationsträger einbezogen. Im Rahmen ihrer Zuständigkeit erbringen sie nach § 5 SGB IX Leistungen zur medizinischen Rehabilitation, Leistungen zur Teilhabe am Arbeitsleben und solche zur Teilhabe am Leben in der Gemeinschaft. Nach § 7 SGB IX gelten für die Leistungen zur Teilhabe die Vorschriften des SGB IX, soweit sich aus 
dem SGB XII und den dazu erlassenden Rechtsverordnungen nichts abweichendes ergibt. Die Zuständigkeit und die Voraussetzungen für die Leistungen zur Teilhabe richten sich insoweit nach dem Sozialgesetzbuch XII (§ 53 Abs. 4 SGB XII). ${ }^{30}$

\subsubsection{Nachrang der Rehabilitationsleistungen und Persönliches Budget}

Die Träger der Sozialhilfe sind also gleichberechtigte Rehabilitationsträger, allerdings dem Nachrangprinzip unterworfen. Gleichwohl bleibt die Eingliederungshilfe des SGB XII eine eigenständige Leistung der Sozialhilfe. Ihre Voraussetzungen und die Zuständigkeit zur Leistungserbringung bestimmen sich ausschließlich nach dem SGB XII; im übrigen gelten die Regelungen des SGB IX, soweit die Sozialhilfe nichts abweichendes bestimmt.

Zum 1. Juli 2004 wurde mit einer Erprobungsphase bis zum 31. Dezember 2007 ergänzend die Leistungserbringung in Form des Persönlichen Budgets durch das Gesetz zur Einordnung des Sozialhilferechts in das Sozialgesetzbuch geregelt (§§ 57, 61 Abs. 2 Sätze 3 und 4 SGB XII). Materiell gesehen, enthält das neue Recht der Eingliederungshilfe im Verhältnis zum vorgängigen BSHG wenig Veränderungen.

\subsubsection{Umfang der Leistungen}

Die Leistungen zur Eingliederungshilfe nach dem SGB XII sind denn auch, wie schon bisher diejenigen nach dem BSHG, vielgestaltig. Sie erstrecken sich gem. $\S 54$ SGB XII von den Hilfen zu einer angemessenen Schulbildung bis hin zur nachgehenden Hilfe zur Sicherung der Wirksamkeit von ärztlichen und ärztlich verordneten Leistungen sowie zur Sicherung der Teilhabe behinderter Menschen am Arbeitsleben. Um diese Leistungsversprechen so wirksam wie möglich zu erfüllen, stellt der Träger der Sozialhilfe nach $\S 58$ SGB XII so frühzeitig wie möglich einen Gesamtplan zur Durchführung der einzelnen Leistungen auf. Dabei soll der Träger der Sozialhilfe nicht nur mit den behinderten Menschen und den sonst im Einzelfall Beteiligten zusammenwirken, sondern auch - wie in einem Netzwerk - mit anderen öffentlichen Institutionen. Darunter fallen insbesondere das Jugendamt (vgl. § 59 SGB XII) und das Gesundheitsamt. ${ }^{31}$ Es gehört zu den Merkwürdigkeiten der Rechtsetzung durch das SGB XII, das die mit dem Vorgänger, dem BSHG, einhergehende Verkümmerung des Instruments "Gesamtplan" vom Gesetzgeber nicht zur Kenntnis genommen worden ist. Auch heute stellt sich wiederum die Frage, ob und in wel-

30 Großmann/Melzer, Sozialhilfe. BSHG - SGB XII, SGB I, II, IX, X, 2004, S. 30; im Übrigen gelten die Regelungen des SGB IX, soweit das Sozialhilferecht nicht abweichendes bestimmt (§ 7 SGB IX); vgl. auch nachfolgend im Text.

31 Zum „Gesamtplan“ vgl. Kroneberger, ArchSozArb 2006 (H. 2), 34 ff.; Trenk-Hinterberger (Fn. 27), Rz. 143. 
chem Ausmaß der "Gesamtplan" und seine Abstimmung mit den genannten Behörden/Ämtern tatsächlich und erfolgreich herbeigeführt wird. Jedenfalls für junge behinderte Menschen ist dies selten der Fall.

\subsection{Kinder- und Jugendhilfe als kommunales Handlungsfeld}

\subsubsection{Kinder- und Jugendhilfe als „offene“ Form kommunaler Sozialpolitik}

In ihrer Notlage - freilich in den „Lebensphasen“ gegeneinander verschoben - treffen sich behinderte alte Menschen mit jungen behinderten Menschen, also Kindern, Jugendlichen und jungen Volljährigen. Nicht nur deshalb liegt ein zweiter Schwerpunkt kommunaler Sozialpolitik im Handlungsfeld der Kinder- und Jugendhilfe. ${ }^{32}$ Vielfach verschlungen mit den Sozialhilfeaktivitäten, gestaltet sich hier das soziale Engagement der Städte, Gemeinden und Kreise als kommunale Jugendpolitik, die - ohne besondere Berücksichtigung des eigenständigen Status von Kindern - im wesentlich als Jugendhilfepolitik für Personen mit abweichenden Verhalten und eben nicht als kommunale Integration behinderter junger Menschen einschließlich Kinder durch entsprechende Förderungsmaßnahmen gehandhabt wird. Während nämlich aus fachlicher Perspektive (kommunale) Kinder- und Jugendpolitik in der heutigen Zivilgesellschaft die Gesamtheit der Einwirkungen auf die Lebenssituation von Kinder- und Jugendlichen bzw. Heranwachsenden und jungen Volljährigen weniger durch Festsetzung von Rechten und Pflichten meint, sondern vor allem als ideelle und finanzielle Förderung wünschenswerter Aktivitäten sowie die Gewährung von Schutz für die körperliche, geistig-seelische und soziale Entwicklung umfasst $^{33}$, bezieht sich die durch das SGB VIII vorrechtliche Jugendhilfe(-politik) lediglich auf einen (engeren) Ausschnitt hieraus, der - angeleitet durch sozialpädagogische Theorien - die Familienerziehung unterstützen, ergänzen oder ersetzen will.

Institutioneller Kern der Kinder- und Jugendhilfe ist das Jugendamt. ${ }^{34}$ Diesem ist die Durchführung kommunaler Jugendpolitik zur sozialen Integration aller der örtlichen Gemeinschaft zuzurechnen Kinder/Jugendlichen/jungen Erwachsenen anvertraut. Einbezogen darin sind auch spezialisierte Dienste und Einrichtungen wie z. B. die der Eingliederungshilfe und Teilhabesicherung für behinderte Kinder, Jugendliche, Heranwachsende und volljährige junge Bürger. Darauf wird zurückzukommen sein.

Gefährdungen für die Qualität der Kinder- und Jugendhilfe vornehmlich im Bereich der Eingliederung und Teilhabe sind allerdings unübersehbar. Abgesehen von den möglichen Problemen, die durch die Föderalismusreform I verursacht wor-

Vgl. Pitschas (Fn. 1), Rz. 58 ff.

Heilmann, Hilfe oder Eingriff?, in: Zentralblatt für Jugendrecht (ZfJ) 2000 (H. 2), S. 41 ff.

Pitschas, Strukturen und Verfahrensweisen des Jugendamts im kooperativen Rechts- und Sozialstaat, in: Sachverständigenkommission 11. Kinder- und Jugendbericht (Hrsg.), Bd. 1: Strukturen der Kinder- und Jugendhilfe. Eine Bestandsaufnahme, 2002, S. 163 (203 ff.). 
den sein könnten, droht das KJHG (SGB VIII) als „Strukturgesetz“ durchgängiger Wirtschaftlichkeitsanforderungen an die Kinder- und Jugendhilfe seiner ursprünglichen Entstehungsintention entfremdet zu werden. Das produkt- und kostenorientierte Handeln der behördlichen Kinder- und Jugendhilfe, das in der Praxis gegenwärtig weg von einer „sozialen Betreuung“ und hin zu einer verhaltensabgewandten und scheinbaren Mess- und Rechenbarkeit betreuungsintensiver Angebote führen will, entlarvt teilweise die Effizienzorientierung von Kinder- und Jugendhilfemaßnahmen aller Art als einen ökonomischen Mythos. ${ }^{35}$

Dies zeigt sich insbesondere, wenn man behinderte junge Volljährige und ihre Familien im Zuständigkeits- und Leistungsgeflecht der Eingliederungshilfe nach dem Sozialgesetzbuch näher ins Auge fasst und untersucht, wie sich deren Hilfe im Angebot kommunaler Institutionen als Spannungsfeld erweist. ${ }^{36}$ Die These ist, dass im Schnittpunkt der rechtlichen Gewährleistungen von SGB VIII, IX und XII durch gesetzgeberische Effizienzüberlegungen intendierte und bürokratisch vollzogene Grenzziehungen der Eingliederungshilfe und Teilhabesicherung bei der Anwendung im Alltag unter den Bedingungen des Schnittstellenmanagements die Gesetzgebungspolitik in ihrer Wirkung als symbolische Politik ausweisen. Dies gilt pars pro toto auch für behinderte Senioren und Seniorinnen. Beide Feststellungen sehen sich durch die in der Beratungsarbeit gewonnenen empirischen Erfahrungen des Verfassers belegt.

\subsubsection{Rehabilitation als Aufgabe der Kinder- und Jugendhilfe}

Völlig aus dem Blick der Öffentlichkeit ist daneben die familiäre und jugendhilfepolitische Komponente der Rehabilitation behinderter Kinder, Jugendlicher und junger Volljähriger geraten. Einerseits stärkt nämlich der durch das SGB IX eingeschlagene Weg gerade nicht die familiären Kräfte. Dies gilt andernfalls auch und z. B. für das Kinder- und Jugendhilferecht: So lässt etwa $\S 35$ a SGB VIII den Unterstützungsauftrag des Jugendamts gegenüber Eltern und anderen Erziehungsberechtigten merkwürdig "blass" erscheinen. Nicht von ungefähr ist auch darauf hingewiesen worden, dass "insbesondere die Neuregelungen (des SGB IX) zur Zuständigkeitsklärung, zur Leistungsplanung und -koordination sowie zur Komplexleistungsfrühförderung ... dringend ein übergreifendes und einheitliches Grundverständnis der Rehabilitationsträger (benötigen), dessen regionsspezifische Konkretisierung und Umsetzung in der Praxis ... in den 26 Jahren Gültigkeit des Rehabilitationsangleichungsgesetzes ... nicht immer optimal gelungen ist." ${ }^{37}$ Dieser Vorbehalt gilt auch heute noch und z. B. im Land Berlin. Vom Gesetzgeber bzw. den zuständigen Länderbehörden hätte man hiernach erwartet, den bekannten Implementationsdefiziten zukünftig durch die 
Aufnahme einer gesetzlichen Verpflichtung zur vertraglichen Regelung der Zusammenarbeit vorsorglich entgegenzutreten. Statt dessen herrscht heute in den allermeisten Bundesländern ein Zustand des geregelten Nebeneinanders von Behörden der Rentenversicherung, Krankenversicherung, Unfallversicherung, aber auch der Sozialhilfe neben der Kinder- und Jugendhilfe. ${ }^{38}$

Eine ähnliche Problematik der Strukturineffizienz stellt sich auf kommunaler Ebene. Denn das neue Recht enthält (lediglich) ein Gebot an die Rehabilitationsträger, fachgerechte und ortsnahe, insbesondere ambulante sozialräumliche Angebotsstrukturen zu schaffen. Im übrigen aber ist der Sicherstellungsauftrag an die Rehabilitationsträger, in jeder kreisfreien Stadt bzw. jedem Landkreis bzw. Bezirk im Stadtstadt Servicestellen mit einem integrativen Aufgabenprofil zu schaffen, finanziell kaum erfüllbar. Bundesstaatliche Kompetenzprobleme haben zudem verhindert, den Auftrag zur Qualitätssicherung gem. § 20 Abs. 1 SGB IX auf die Träger der Sozial- und Jugendhilfe auszudehnen. Die Rehabilitationskette wird dadurch unterbrochen; der Tatbestand dürfte unbestreitbar sein.

\subsubsection{Insbesondere: Eingliederungshilfe}

Im Verhältnis zur Sozialhilfe besteht allerdings für seelisch behinderte junge Volljährige und von einer solchen Behinderung bedrohte Kinder und Jugendliche ein vorrangiger Anspruch auf Eingliederungshilfe im Rahmen der Kinder- und Jugendhilfe ( $\$ 35$ a SGB VIII i. V. m. $§ 10$ Abs. 2 SGB VIII). Entsprechendes gilt für junge Volljährige (§ 41 Abs. 2 SGB VIII). Dieser Vorrang der Kinder- und Jugendhilfe für seelisch behinderte oder von einer seelischen Behinderung bedrohte Kinder gilt zwar nicht bei der Frühförderung, soweit diese das Landesrecht regelt. Aber die Regelungen bieten die Möglichkeit, an der Schnittstelle von Kinder- und Jugendhilfe zur Sozialhilfe einen Konflikt zu lösen, der seit dem Inkrafttreten des BSHG im Jahre 1961 diskutiert wird. ${ }^{39}$

Dieser Konflikt resultiert aus dem unterschiedlichen Stellenwert der (seelischen) Behinderung in beiden Leistungssystemen. Denn während die spezifische Hilfe für seelisch behinderte junge Menschen (und junge Volljährige) aus der Sicht der Sozialhilfe einen Ausschnitt aus einem umfassenden Hilfesystem darstellt, das alle Arten von Behinderung und alle Altersgruppen erfassen will, wird doch aus der Sicht der Kinder- und Jugendhilfe die Ausgrenzung junger Menschen bzw. Volljähriger (und auch Jugendlicher) als willkürliche Abtrennung einer umfassend zu verstehenden

Vgl. auch Welti (Fn. 18), 329 und dort insbes. zum Fehlen regionaler Arbeitsgemeinschaft en ( $\$ 12$ Abs. 2 SGB IX) und zu den Defiziten gemeinsamer Servicestellen gem. § 22 SGB IX; ähnlich Wiesner (Fn. 35), RdNr. 24. Damit erfüllen sich leider meine Prognosen, vgl. Pitschas, Integration behinderter Menschen in verschiedenen Lebensphasen als Politikauftrag - „Potentiale wecken statt Menschen entmündigen": ein kategorialer Rahmen, in: von Maydell/Pitschas/Schulte (Hrsg.), Behinderung (Fn. 23), S. 389 (401 ff., 403 f., 409 ff.). 
Förderung von Erziehung und Entwicklung angesehen. Dementsprechend ist es im Verhältnis zwischen Kinder- bzw. Jugend- und Sozialhilfe bis heute nicht gelungen, eine durchweg praktikable Abgrenzung zwischen den Zuständigkeitsbereichen zu finden - oder gar die Problematik durch Kostenvereinbarungen zu entschärfen.

\subsection{Behinderte junge Volljährige im Spannungsfeld von Kinder- und Jugendhilfe zur Sozialhilfe}

\subsubsection{Zuordnung zur Kinder- und Jugendhilfe}

Freilich kann schon von einem echten Spezialitätsverhältnis zwischen Eingliederungshilfe nach dem SGB XII und der Kinder- bzw. Jugendhilfe nach dem SGB VIII deshalb nicht gesprochen werden, weil die beiden Normenkomplexe sich nicht an einen grundsätzlich gleichen Adressatenkreis richten und nicht das gleiche Ziel verfolgen. Die Entwicklung junger Menschen zu einer eigenverantwortlichen Persönlichkeit wird eben nicht nur von Art und Schwere einer Behinderung, sondern ebenso von ihrer Erziehung, dem familiären Umfeld und der Art der bisherigen persönlichen Förderung beeinflusst. Es besteht ein untrennbarer Zusammenhang zwischen Entwicklung der Behinderung und Entwicklung der Persönlichkeit. Die Entscheidung, ob eine Entwicklungsverzögerung ausschließlich durch die Behinderung verursacht wird oder nicht, darf deshalb nicht allein zur Grundlage für die $\mathrm{Zu}$ ständigkeitswahl gemacht werden. ${ }^{40}$

Eine generelle Vorrangigkeit der Leistungen der Eingliederungshilfe nach dem SGB XII vor den Leistungen der Kinder- und Jugendhilfe kann m. a. W. nicht festgestellt werden. Die Eingliederungshilfe des Sozialhilfeträgers kann nur mit einem unmittelbaren Bezug zur Behinderung greifen, nicht aber die in schwierigen Lebensphasen $-\mathrm{z}$. B. bei Schulbesuch unter Mehrfachbehinderungen - die von den Familien benötigte intensive pädagogische oder auch therapeutische Begleitung leisten. Die dazu notwendige fachliche Kompetenz liegt nun einmal bei den Jugendämtern, aber nicht bei den Gesundheits- bzw. Sozialämtern.

Auch die Vollendung des 18. Lebensjahres darf die Behinderung nicht als lediglich gesundheitliches oder leistungsrechtliches Problem ansehen lassen. Zwar mag in Grenzfällen strittig sein, ob die erforderliche Hilfe einen vorrangig behinderungsspezifischen oder erzieherischen Bezug hat; doch spricht in aller Regel nach der Lebenserfahrung die Tatsache der (seelischen) Behinderung dafür, jedenfalls die Fortsetzung pädagogischer Hilfen in früheren Jahren oder bei der Verselbständigung durch das Jugendamt zu prüfen. Aus fachlicher Sicht erschiene mir deshalb die $\mathrm{Zu}$ ordnung der Leistungen für alle behinderten jungen Menschen zur Kinder- bzw. Jugendhilfe als beste Lösung, weil sie Abgrenzungsschwierigkeiten vermeidet und 
dem Gedanken der Integration durch die Gleichbehandlung behinderter mit nichtbehinderten Kindern und Jugendlichen umfassend Rechnung tragen kann. ${ }^{41}$

Diese Änderung der gegenwärtigen rechtlichen Zuordnung wäre zwar mit erheblichen personellen und finanziellen Konsequenzen verbunden. Die derzeitige Finanzausstattung der örtlichen Jugendämter lässt eine solche Vorgehensweise schon überhaupt nicht zu. Doch müssen sie zur Vermeidung gefährlicher Entwicklungen unbedingt personell deutlich verstärkt und in ihrer Haushaltssituation erheblich aufgebessert werden. Eingedenk dessen hatte sich der Gesetzgeber des KJHG für die sog. "kleine Lösung" entschieden, aber nach § 35a SGB XIII immerhin Maßnahmen der Eingliederungshilfe für solche jungen Menschen in das SGB VIII integriert, die seelisch behindert oder von einer solchen Behinderung bedroht sind.

\subsubsection{Abgrenzung der Zuständigkeiten zwischen Jugendamt, Sozialamt und der}

Gesundheitsfachverwaltung - Ein Grundproblem des bürokratisierten Sozialstaats

Damit allerdings tritt wiederum das Zuständigkeitsproblem verschärft hervor; dies gilt insbesondere bei jungen Volljährigen mit seelischer Behinderung. Verschiedentlich wird versucht, durch „Bordmittel“ der Fachaufsicht zu einer Lösung zu finden. $\mathrm{Zu}$ Recht stellten manche Bundesländer auf den individuellen Bedarf ab und gehen davon aus, dass die Ausdehnung der Hilfe für junge Volljährige ein wesentliches Reformanliegen des KJHG ist. Die Voraussetzungen für die Hilfegewährung sind deshalb auch bewusst vom Gesetzgeber weitgezogen worden. Es ist in der Tat sinnvoll, dass die Jugendämter ggf. erforderliche Eingliederungshilfe leisten, wenn nach $\S 41$ SGB VIII das Jugendamt zugleich pädagogische Hilfe übernehmen muss. Allerdings sind bislang zahlreiche "Grenzfälle" praktischer Abgrenzung aufgetreten, die "an der Front" schnelle und nachvollziehbare Entscheidungen zugunsten der betroffenen und behinderten jungen Menschen bzw. der Familien insgesamt nicht ermöglichen konnten.

Das Land Berlin bietet hierfür ein treffliches Beispiel. Ob die dort erlassenen „Ausführungsvorschriften für den Prozess der Planung und Durchführung von Hilfe zur Erziehung und Eingliederungshilfe für seelisch behinderte Kinder und Jugendliche und Hilfe für junge Volljährige (AV-Hilfeplanung)“ vom 31.01.2005 der Senatsverwaltung für Bildung, Jugend und Sport insoweit Abhilfe schaffen, erscheint mir fraglich. Gleiches gilt gegenüber den Hinweisen der Senatsverwaltung für Gesundheit, Soziales und Verbraucherschutz des Landes zur Abgrenzung der Hilfen für junge Volljährige nach $\S 41$ des SGB VIII und $\S \S 67,68$ des SGB XII. Schon das gewillkürte Nebeneinander beider Hilfearten, mit dem eine Nähe der sozialhilfe-

41 Zu Unrecht rückt angesichts fragwürdiger Mittelverteilung Wiesner (Fn. 36), RdNr. 6, die personellen und finanziellen Konsequenzen in den Vordergrund - von Integrationsaspekten in Bezug auf ausländische behinderte Kinder ganz zu schweigen. Ganz und gar irreführend der Hinweis a. E. seiner Kommentierung auf die Vorteile der ,örtliche(n) Ebene“. 
rechtlichen Leistungen zur Überwindung besonderer sozialer Schwierigkeiten zur erzieherischen Pädagogik suggeriert wird, scheint mir bedenklich. Viel eher müsste es doch um eine substanzielle Abgrenzung der Leistungspflichten gehen.

Anzuerkennen sind deshalb die Bemühungen der Hinweise zur „Abgrenzung der Hilfe für junge Volljährige nach $\S 41$ SGB VIII und $\S \S 67,68$ SGB XII“ im Rundschreiben Jug 2/2005 vom 28. Januar 2005. Hierin wird zu Recht der Grundsatz betont, dass eine Kollision der Normen nicht gegeben ist, "wenn sozialpädagogische Hilfen im Vordergrund stehen, die direkt oder indirekt einer eigenverantwortlichen Lebensführung der/des jungen Volljährigen dienen".

\subsubsection{Der ,institutionelle“ Politikansatz vor dem Versagen?}

Wo aber liegen die Auswege? Notwendig ist allemal der Fachaustausch über rechtliche und umsetzungsorientierte Fragen sowie über die zu bewerkstelligende Kooperation an den Schnittstellen der Zuständigkeiten. Das Ziel kann nur sein, Zuständigkeitskonflikte zu Lasten der Betroffenen zu vermeiden. Freilich bedarf es dazu auch der Analyse von Defiziten einerseits, der Entwicklung kooperativer Lösungen andererseits. Analytisch i. S. der Abstandnahme wäre etwa die Entspezialisierung der jugendamtlichen Behindertenhilfe zu diskutieren oder auch die vorherrschende Budgetierung von Hilfen im Rahmen der Verwaltungsmodernisierung. Lösungen ließen sich andererseits im Rahmen einer sozialräumlichen Behindertenhilfe für junge Menschen andenken, die nicht pädagogisch spezialisierte Hilfeangebote kompensieren kann.

\section{Zusammenfassung}

Nach alledem lässt sich ein klarer Befund konstatieren: Im Zeichen des demografischen Wandels und der „Ökonomisierung“ aller Sozialleistungen sowie sozialen Dienste kommt es in der kommunalen Sozialpolitik zu der partiellen Ausgrenzung von jungen und alten behinderten Menschen. In dieser von den politischen und institutionellen Akteuren sowie von der Personalpolitik in Einrichtungen zu verantwortenden Entwicklung offenbart sich die Logik der gegliederten Sozialverantwortung im Bundesstaat. Dieser trifft ebenso auf konkurrierende Teil-Politiken wie die erstere Implementationsdefizite im Mehrebenen-System des Europäischen Sozialraumes bzw. im Rahmen der Sozialgesetzgebung von Bund und Ländern hinnehmen muss. Namentlich qualitative kommunale Sozialpolitik ist dadurch sowie mit Blick auf knappe finanzielle Ressourcen der Kommunen in der alltäglichen Praxis zum Scheitern verurteilt.

Von dieser Entwicklung sind vor allem sog. Randgruppen und darunter wiederum in besonderem Maße behinderte junge Volljährige und Senioren/innen betroffen. Einerseits fehlt es an einem vollzugsteuernden Sozialrecht. Die einschlägigen Rechtnormen der Bücher VIII, IX und XII des Sozialgesetzbuches räumen den 
Kommunen vielfach dirigiertes Ermessen auf der Grundlage „offen“ formulierter Normen ein, dessen Anwendung mit einer institutionenbezogenen Subjektivierung der Rechtskonkretisierung verbunden ist. Diese aber unterwirft bislang die Lebensschicksale einzelner Betroffener absurden Zuständigkeits- und Wirtschaftlichkeitserwägungen - eine verwaltungsrechtlich weithin fehlerhafte Vorgehensweise. Auf der anderen Seite führt die Fragmentierung des Rechts für behinderte Menschen zu institutionellen Abschottungen der das Recht anwendenden Behörden untereinander. Hinzu treten Koordinationskonflikte an den Schnittstellen der Institutionen und Rechtskonkretisierung im Einzelfall. Der empirisch informierte Beitrag arbeitet diese Defizite heraus. 


\title{
4.4. Deutsche und Bayerische Politik für behinderte Menschen
}

\author{
Von Burkard Rappl
}

1. Situation und Fakten

2. Grundsätzliche Aussagen zur Weiterentwicklung der Behindertenpolitik

3. Bayerische Schwerpunkte

4. Fazit

5. Anhang: Auszug aus dem Informationsangebot des Bayerischen

Staatsministeriums für Arbeit und Sozialordnung, Familie und Frauen $\quad 128$

5.1. Grundsätze bayerischer Behindertenpolitik 128

$\begin{array}{lr}\text { 5.2. Schwerbehindertenrecht } & 129\end{array}$

5.3. Wohnformen für körperlich und/oder geistig behinderte Menschen $\quad 130$

5.4. Bayerisches Gesetz zur Gleichstellung, Integration und Teilhabe von Menschen mit Behinderung

5.5. Der Bayerische Landesbehindertenrat

5.6. Berufliche Eingliederung von Menschen mit Behinderung 135

5.7. Bayerisches Blindengeldgesetz

5.8. Dienste der offenen Behindertenarbeit $\quad 140$

5.9. Hilfen für Kinder und Jugendliche 141

5.10. Psychisch kranke und behinderte Menschen - Psychiatrie-Grundsätze Bayern 
Zum Einstieg ein Zitat: „Sag mir, was du vom behinderten Menschen denkst, wie du zu ihm stehst, wie du mit ihm lebst, und ich sage dir, was dir der Mensch überhaupt bedeutet, was dein Reden über Menschenrechte wert ist.“ (Professor Alfred Läpple)

\section{Situation und Fakten}

In Bayern gibt es ca. 1,1 Mio. schwerbehinderte Menschen, das sind rd. 8,95\% der Gesamtbevölkerung (Stand: 30.09.2008). Diese Quote entspricht in etwa dem Durchschnitt in den übrigen Ländern Deutschlands. Es handelt sich hier um schwerbehinderte Menschen, wenn bei ihnen ein Grad der Behinderung von wenigstens $50 \mathrm{v}$. Hundert festgestellt ist bzw. sie schwerbehinderten Menschen gleichgestellt sind. Dazu gehören u. a. 16.000 blinde Menschen, rd. 30.000 schwerstbehinderte Menschen, die in Heimen oder besonderen Einrichtungen leben. Die Beschäftigungsquoten schwerbehinderter Menschen im Bund und in Bayern (Pflichtquote: Derzeit müssen auf mindestens 5\% der Arbeitsplätze schwerbehinderte Menschen beschäftigt werden, wenn mindestens 20 Arbeitsplätze bestehen) sind derzeit (2006; letzte insgesamt verfügbare Zahl): Bayern (jeweils alle Arbeitgeber) 3,9\%, Bund 4,3\%. Die bayerische Staatsregierung hat als Arbeitgeber erstmals 2006 die gesetzliche Schwerbehindertenquote erreicht. Staatsregierung (2007) insgesamt 5,21\% Staatskanzlei 12,54\%, Sozialministerium 12,86\%, und Wirtschaftsministerium $8,02 \%$.

Insgesamt ist ein Anstieg von Menschen mit schweren Behinderungen in den letzten Jahren und einhergehend damit auch ein deutlicher Anstieg der Ausgaben der Eingliederungshilfe (Finanzierung der laufenden Kosten schwerbehinderter Menschen durch die Sozialhilfeträger) zu verzeichnen. So sind die Empfänger bundesweit von 1991 mit rd. 240.000 auf rd. 640.000 im Jahr 2006 angestiegen. Für Bayern wirkt sich dies z.B. in einem erheblichen Anstieg der Ausgaben der Eingliederungshilfe für die überörtlichen Sozialhilfeträger von $1991 \mathrm{rd}$. 600 Mio. $€$ auf rd. 1,4 Mrd. $€$ (Nettoausgaben) im Jahr 2006 aus.

Zur Verbesserung der beruflichen Eingliederung schwerbehinderter Menschen werden vom Integrationsamt beim Zentrum Bayern Familie und Soziales nach dem Neunten Buch Sozialgesetzbuch umfassende Leistungen aus Mitteln der Ausgleichsabgabe bewilligt (2007: ca. 52,2 Mio. Euro). Neben Leistungen an die schwerbehinderten Menschen zur begleitenden Hilfe im Arbeitsleben (2007: rd. 2,3 Mio. Euro) sind davon insbesondere die Leistungen an Arbeitgeber (2007: rd. 30 Mio. Euro) sowie an Einrichtungen der beruflichen Rehabilitation (2007: 13,6 Mio. Euro) zu erwähnen. 


\section{Grundsätzliche Aussagen zur Weiterentwicklung der Behinderten- politik}

Folgende Leitlinien prägen den Paradigmenwechsel in Deutschland von der reinen Fürsorge hin zur Teilhabe: Unterstützung von Selbständigkeit, Selbsthilfe und Selbstbestimmung als gesellschaftliche Aufgabe. Fortsetzung des eingeleiteten Prozesses zur Verwirklichung einer umfassenden Teilhabe behinderter Menschen in der Gesellschaft. Weiterentwicklung der Leistungsstrukturen der Eingliederungshilfe mit Ländern, Kommunen und Verbänden behinderter Menschen nach den Grundsätzen „Ambulant vor Stationär“, Verzahnung ambulanter und stationärer Dienste, Umsetzung der Einführung des persönlichen Budgets. Förderung der Beschäftigungsmöglichkeiten für behinderte Menschen, auch außerhalb von Werkstätten im allgemeinen Arbeitsmarkt.

\section{Bayerische Schwerpunkte}

Berufliche Integration verbessern durch gezielte Unterstützung des betrieblichen Eingliederungsmanagements; gezielte Beratung und begleitende Hilfe im Arbeitsleben durch Integrationsämter und Integrationsfachdienste; Umsetzen des Modellprojekts „Vernetzung Arbeitsmarkt für schwerbehinderte Menschen“ mit dem Ziel, alle Verantwortlichen für Beschäftigung schwerbehinderter Menschen auf kommunaler und Landkreisebene zu vernetzen und so eine dauerhafte träger- und zuständigkeitsübergreifende Zusammenarbeit herzustellen; Umsetzung des Modellprojekts „Übergang Förderschule in den Beruf" mit dem Ziel, durch die Integrationsfachdienste adäquate Praktikums- und Arbeitsplätze für geeignete Förderschulabgänger mit Förderbedarf im Förderschwerpunkt geistige Entwicklung zu akquirieren; Beteiligung an der Bund-/Länderinitiative „Job 4000“; Festhalten und Unterstützung an den Werkstätten für behinderte Menschen als wichtiger Pfeiler der Rehabilitation von schwer- und schwerstbehinderten Menschen in Bayern.

Neben der beruflichen Integration ist die gesellschaftliche Integration und die Teilhabe am Leben wichtiges Ziel der Maßnahmen für Menschen mit Behinderung. Teilhabe, Integration und Inklusion sollen künftig den Weg bestimmen. Beispiele dafür:

- Unterstützung durch Gesetzgebung: Bayern hat als eines der ersten Länder ein Gleichstellungsgesetz für Menschen mit Behinderung erlassen. Es trat zum 01.08.2003 in Kraft. Zielrichtung: Abbau von Barrieren in allen Formen (sowohl baulicher Art als auch im kommunikativen Bereich und der Barrieren in den Köpfen); dazu auch Erlass von Verordnungen u. a. Bayerische barrierefreie Informationstechnikverordnung, Verordnung zur Zugänglichmachung von Dokumenten für Blinde, erblindete und sehbehinderte Menschen im Verwaltungsverfahren, Gebärdensprachkursleiterprüfungsverordnung, Verordnung zum Landesbehindertenrat, gesetzliche Verankerung eines/r Behindertenbeauftragten der Staatsregierung. 
- Gezielte Unterstützung vor allem auch von Kindern und Jugendlichen mit Behinderung: Interdisziplinäre Frühförderung und qualifizierte pädagogische Förderung und Betreuung behinderter Kinder. Förderung und Betreuung von ca. 20.000 Kindern in den Frühförderstellen und von rd. 27.000 Kindern und Jugendlichen in heilpädagogischen Tagesstätten, schulvorbereitenden Einrichtungen, integrativen Kindergärten und Heimen.

- Ambulante Betreuung von behinderten Menschen sowie Unterstützung von Familien mit behinderten Angehörigen durch derzeit 200 Dienste der offenen Behindertenarbeit.

- Unterstützung der Selbsthilfe für Menschen mit Behinderung als hochkompetente Beteiligung Betroffener zur Verbesserung ihrer eigenen Lebens- und Gesundheitssituation und vor allem auch als wertvolle Unterstützung für die betroffenen Menschen.

- Derzeit wichtiges Gesetzgebungsvorhaben Neuordnung der Zuständigkeiten in der Sozialhilfe. Die bisherige Zuständigkeitsaufteilung in der Eingliederungshilfe (Bezirke als überörtliche Träger zuständig für teilstationäre und stationäre Leistungen, Landkreise und kreisfreien Gemeinden als örtliche Träger für ambulante Leistungen) hat sich nicht bewährt. Sie hindert den Ausbau des ambulanten Sektors und steht damit dem Wunsch vieler Menschen, ihr Leben möglichst lange in privater Umgebung verbringen zu können, entgegen. Die Staatsregierung hat deshalb zum 01.01.2008 eine Neuordnung der Zuständigkeiten in der Sozialhilfe dahingehend vorgenommen, dass ambulante, teilstationäre und stationäre Leistungen der Eingliederungshilfe in der Hand eines Kostenträgers, der Bezirke, vereinigt werden. Die Zusammenführung der Zuständigkeiten bei der Hilfe zur Pflege steht in einem zweiten Schritt an.

- Auf der Bundesebene ein wichtiges Vorhaben ist die Ratifzierung des UNÜbereinkommens über die Rechte von Menschen mit Behinderungen. Das enstprechende Gesetz soll zum 1.1.2009 in Kraft treten. Bayern unterstützt das Vorhaben, das die Behindertenpolitik auf der ganzen Welt beeinflussen und verbessern soll.

\section{Fazit}

In diesem Rahmen konnten nur einige Aspekte der modernen, dem Teilhabegedanken zugewandten Behindertenpolitik in Deutschland und Bayern dargestellt werden. In Details und Einzelheiten zu gehen würde den Rahmen sprengen. Bereits die dargestellten Beispiele zeigen, dass in Bayern und Deutschland große Anstrengungen unternommen werden, das Recht der Menschen mit Behinderung auf gleichberechtigte Teilhabe und ein selbstbestimmtes Leben umzusetzen. Weitere Anstrengungen sind nötig, etwa im Hinblick auf die Zunahme älterer Menschen mit Behinderung. Nötig ist auch, dieses Gebiet, das nicht im Rampenlicht steht, immer wieder an die Öffentlichkeit zu holen, für Aufgeschlossenheit zu sorgen und für den richtigen Umgang zu werben. Als zuständiger Abteilungsleiter für Fragen für Menschen mit 
Behinderung bin ich dem Max-Planck-Institut für ausländisches und internationales Sozialrecht in München dankbar, die Frage der Rechte der Menschen mit Behinderung in Europa und Asien in vielfältigen Facetten beleuchtet zu haben. Hier wird ein weiterer wichtiger Grundstein im Umgang von Menschen mit und ohne Behinderung gelegt. Es lohnt sich sicher, dieses Projekt unter neuen Blickwinkeln fortzuführen. 


\section{Anhang: Auszug aus dem Informationsangebot des Bayerischen Staatsministeriums für Arbeit und Sozialordnung, Familie und Frauen}

\subsection{Grundsätze bayerischer Behindertenpolitik ${ }^{1}$}

Die Behindertenpolitik ist seit langem ein Schwerpunkt bayerischer Sozialpolitik. Dabei sind zwei Grundsätze richtungsweisend:

- Schutz des Lebens und der Würde von Menschen mit Behinderung

- Stärkung der Fähigkeit und der Möglichkeit von Menschen mit Behinderung, über ihr Leben selbst zu bestimmen bzw. es selbst zu gestalten.

Im Zentrum bayerischer Behindertenpolitik steht der Paradigmenwechsel von der Fürsorge und Versorgung hin zur gleichberechtigten Teilhabe für Menschen mit Behinderung.

Vorrangiges Ziel der bayerischen Behindertenpolitik ist es deshalb, die volle und gleichberechtigte Einbeziehung der Menschen mit Behinderung in das gesellschaftliche und berufliche Leben zu erreichen. Eine effektive und nachhaltige gesellschaftliche Teilhabe setzt zu aller erst voraus, dass behinderte Menschen nicht wegen ihrer Behinderung benachteiligt werden. Mit Aufnahme des Benachteiligungsverbots für behinderte Menschen in die Bayerische Verfassung 1998 wurde daher ein wichtiges Ziel bayerischer Behindertenpolitik erreicht. Gleichzeitig wurde durch die Aufnahme eines staatlichen Schutz- und Förderauftrags eine wichtige Weichenstellung für die zukünftige Behindertenpolitik vorgenommen. Das am 1. Mai 2002 in Kraft getretene Behindertengleichstellungsgesetz des Bundes und das zum 1. August 2003 in Kraft getretene Bayerische Behindertengleichstellungsgesetz konkretisieren den Verfassungsauftrag der Gleichstellung für den Bereich des öffentlichen Rechts.

Zur Teilhabe von Menschen mit Behinderung am Leben in der Gemeinschaft gehört auch eine sinnvolle Gestaltung der Freizeit, die Möglichkeit, Kontakte zu knüpfen und Neues kennen zu lernen. Hierzu bedarf es ggf. spezieller Angebote und besonderer Unterstützung, damit Menschen mit Behinderung, möglichst zusammen mit Nichtbehinderten, Gemeinschaft erleben. Ca. 200 Dienste der offenen Behindertenarbeit bieten insbesondere Beratung, Begleitung und betreute Freizeitmaßnahmen an. Auch der Behindertensport leistet wesentliche Integrationsarbeit. Allein an den vom Freistaat geförderten Sportmaßnahmen für Menschen mit Behinderung nehmen jährlich rund 830.000 Menschen mit Behinderung teil. Hervorzuheben ist dabei auch der oftmals unentbehrliche Einsatz ehrenamtlicher Helferinnen und Helfer.

Zur Erreichung gleichwertiger Lebensbedingungen von Menschen mit und ohne Behinderung sind auch in Zukunft weitere Anstrengungen erforderlich. Dies gilt insbesondere hinsichtlich der Mobilität, beruflichen Integration und gesellschaftlichen Teilhabe behinderter Menschen. 


\subsection{Schwerbehindertenrecht $t^{2}$}

Das Schwerbehindertengesetz wurde zum 1. Juli 2001 als Teil 2 in das Sozialgesetzbuch - Neuntes Buch - SGB IX eingegliedert). Das SGB IX verfolgt das Ziel, die Teilhabe behinderter Menschen im Arbeitsleben und am Leben in der Gesellschaft zu verbessern. Ferner wurde mit dem SGB IX ein einheitliches Rehabilitationsrecht geschaffen. Wesentliche Inhalte sind:

- Regelungen über Zuständigkeiten und einheitliche Rehabilitation;

- Errichtung gemeinsamer Beratungs- und Servicestellen;

- Verbesserung der Zusammenarbeit der Rehabilitationsträger durch trägerübergreifendes Reha-Management;

- Eingliederung des Schwerbehindertengesetzes in das SGB IX;

- Verzicht auf die Bedürftigkeitsprüfung bei Leistungen zur medizinischen und beruflichen Rehabilitation im Rahmen der Eingliederungshilfe nach dem SGB XII.

Am 1. Mai 2004 trat das Gesetz zur Förderung der Ausbildung und Beschäftigung schwerbehinderter Menschen in Kraft, mit dem das SGB IX bereits nach kurzer Zeit novelliert wurde. Die Regelungen dieses Gesetzes sollen die Ausbildungsund Beschäftigungssituation behinderter und schwerbehinderter Menschen und die betriebliche Prävention optimieren.

Ein wichtiger Schwerpunkt des Gesetzes ist die Verbesserung der Ausbildungsmöglichkeiten. Das Gesetz sieht vor, betriebliche und überbetriebliche Ausbildung besser miteinander zu verzahnen: Möglichst viele Jugendliche, die sich in überbetrieblicher Ausbildung befinden - zum Beispiel in einem Berufsbildungswerk sollen in Zukunft Teile ihrer Ausbildung im Betrieb absolvieren. Ziel ist ferner, die Beschäftigung behinderter Menschen in kleinen und mittleren Betrieben zu verbessern.

Vielen Arbeitgebern sind die Förderinstrumente nicht hinreichend bekannt. Viele wissen nicht, welcher Träger für welche Leistung zuständig ist. Die Integrationsfachdienste sollen als Hauptansprechpartner für die Arbeitgeber zur Verfügung stehen, ihnen helfen, sie beraten und informieren. Dabei sollen sie stärker als bisher mit den Handwerks- sowie Industrie- und Handelskammern zusammenarbeiten.

Das Gesetz beinhaltet einen ganzen Katalog konkreter Verbesserungen:

- Verbesserung der Möglichkeiten für eine betriebliche Ausbildung

- Verbesserte Beratung, Information und Unterstützung der Arbeitgeber zur Beseitigung von Einstellungshindernissen und zur Sicherung der Beschäftigung

- Ausbau betrieblicher Prävention im Sinne von "Rehabilitation statt Entlassung"

- Fortentwicklung der bisherigen Verfahrensregelungen im SGB IX zu einem wirksamen betrieblichen Eingliederungsmanagement

- Ausbau des Instruments der Integrationsvereinbarungen 
- Verbesserung der Arbeitsmöglichkeiten der Schwerbehindertenvertretungen

- Zusätzliche Klarstellungen im Hinblick auf die Rechte der Schwerbehindertenvertretungen

- Erweiterte Einbeziehung stellvertretender Mitglieder der Vertretungen in größeren Betrieben

- Beibehaltung der mit dem Gesetz zur Bekämpfung der Arbeitslosigkeit Schwerbehinderter im Jahre 2000 verbesserten Rahmenbedingungen für die Arbeit-geber durch Festhalten an der auf 5 Prozent abgesenkten Beschäftigungspflichtquote

- Ausbau der Integrationsfachdienste

- Übertragung der organisatorischen Verantwortung für die Integrationsfachdienste von der Bundesagentur für Arbeit auf die Integrationsämter

- Aufgabe der Integrationsfachdienste als zentrale Ansprechpartner für die Arbeitgeber

- Zusätzliche Klarstellungen zur Dauer der Förderung der beruflichen Bildung in Werkstätten für behinderte Menschen

- Weitere Verbesserung der Instrumente zur Förderung des Übergangs aus Werkstätten für behinderte Menschen auf den allgemeinen Arbeitsmarkt

- Gewährung besonderer Leistungen an Arbeitgeber zum Ausgleich von Minderleistungen und Mehraufwand

- Mehrfachanrechnung von Werkstattbeschäftigten auf Zahl der Pflichtarbeitsplätze des Arbeitgebers.

\subsection{Wohnformen für körperlich und/oder geistig behinderte Menschen ${ }^{3}$}

Es lassen sich grundsätzlich drei verschiedene Wohnformen für behinderte Menschen unterscheiden:

- Die Einzelwohnung, in der ein Mensch mit Behinderung alleine, mit seinem Partner (und ggf. seinen Kindern) oder seiner Herkunftsfamilie wohnt. Betreutes Wohnen in der Einzelwohnung findet statt, wenn ambulante Hilfen notwendig sind (z. B. Dienste), damit diese Form des Wohnens auf Dauer realisiert werden kann.

- Die Wohngemeinschaft, in der der behinderte Mensch zusammen mit anderen Menschen (mit und/oder ohne Behinderung) auf freiwilliger Basis wohnt. Betreutes Wohnen in der Wohngemeinschaft findet statt, wenn ambulante Hilfen notwendig sind, damit die BewohnerInnen diese Wohnform dauerhaft verwirklichen können.

- Das Heim, in dem ein Mensch mit Behinderung in einer nicht frei bestimmten Gruppe zusammen mit anderen Menschen mit Behinderung wohnt. Wohnen im Heim bedeutet, dass ein Mensch mit Behinderung einer Gruppe (Wohngruppe 
als Organisationseinheit eines Heims und nicht als freiwillig gewählte Wohngemeinschaft) zugeteilt wird und in dieser lebt, seine Wohnkosten meist nicht selbst trägt (Pflegesatz), festes Personal zur Betreuung bereit steht und ein Träger den Betrieb dieses Heims sicherstellt.

Die meisten Menschen mit Behinderung wollen in der eigenen Wohnung leben.

Bei den Befragungen nach den Wohnwünschen von Menschen mit Behinderung lässt sich ein eindeutiger Trend feststellen: Menschen mit Behinderung geben nichtinstitutionellen Wohnformen den Vorzug, obwohl viele Befragte sich mehrere Wohnformen für sich vorstellen können. Maßgeblichen Einfluss auf die Wohnwünsche haben Art und Schwere der Behinderung, das Alter, die soziale Eingliederung der Betroffenen sowie Umfang, Qualität und Kosten von Betreuungsangeboten.

Gesetzgeber räumt Wohnen in der eigenen Wohnung Vorrang ein.

Unter dem Aspekt „ambulant vor stationär“sollen Menschen mit Behinderung möglichst lange selbständig und selbstbestimmt in ihrer Wohnung leben können. Nach dem Sozialgesetzbuch XII besteht deshalb ein grundsätzlicher Vorrang der ambulanten Hilfen (Einzelwohnung, Wohngemeinschaft) vor den stationären Hilfen (Wohnen im Heim). Hierbei ist zu beachten, dass der Vorrang der ambulanten Leistungen nicht gilt, wenn eine Leistung für eine geeignete stationäre Einrichtung zumutbar und im Vergleich dazu eine ambulante Leistung mit unverhältnismäßigen Mehrkosten verbunden ist. Im ambulanten Bereich bestehende, sehr unterschiedliche Wohnformen (wie zum Beispiel Integriertes Wohnen, Betreutes Wohnen, Gruppenwohnen) können im Rahmen des Bayerischen Wohnungsbauprogramms über die allgemeine Miet- und Eigenwohnraumförderung gefördert werden (www.wohnen. bayern.de).

\section{Einheitliche Zuständigkeit für alle Wohnformen}

Dem verstärkten Ausbau der betreuten Wohngemeinschaften und des intensiv betreuten Einzelwohnens stellte sich ein strukturelles Problem entgegen: für die stationäre Unterbringung (somit für die laufenden Kosten) waren die Bezirke als überörtliche Sozialhilfeträger zuständig und kostentragungspflichtig. Für die ambulanten Hilfen waren dies die Landkreise und kreisfreien Gemeinden als örtliche Sozialhilfeträger. Das bedeutete: bei einem im Einzelfall möglichen Wechsel von einer stationären Einrichtung zum Wohnen in einer Wohngemeinschaft oder im betreuten Einzelwohnen erfolgte auch ein Wechsel des Kostenträgers. Örtliche Sozialhilfeträger hatten aber kein Interesse, durch den verstärkten Ausbau ambulanter Wohnformen die Voraussetzungen dafür zu schaffen, dass eine Reihe bisher stationär vom Bezirk 
versorgter Menschen in die eigene örtliche Zuständigkeit überführt wird. Es wurde daher ab 1.1.2008 eine einheitliche Zuständigkeit herbeigeführt. Seit 1.1.2008 sind die Bezirke für ambulante wie auch stationäre Wohnformen und Hilfen für Menschen mit Behinderung zuständig.

Auch die Zahl der behinderten Menschen in Heimen steigt weiter

Unbeschadet des erforderlichen Ausbaus ambulanter Wohnformen steigt nach einschlägigen Untersuchungen (u.a. des Deutschen Vereins für öffentliche und private Fürsorge) aufgrund der unstrittigen Zunahme von Menschen mit schweren Behinderungen (bessere medizinische Versorgung) und der Zunahme älterer Menschen insbesondere mit geistiger und geistig-mehrfacher Behinderung auch die Zahl derer an, die stationärer Betreuung bedürfen. Im Jahr 2006 gab es nach der Erhebung des Statistischen Landesamtes (vgl. unten) in Bayern 660 Einrichtungen mit nahezu 31.000 Plätzen für erwachsene Menschen mit Behinderung.

Statistischer Bericht zu Wohnformen für Menschen mit Behinderung

Das Bayerische Landesamt für Statistik und Datenverarbeitung veröffentlicht alle zwei Jahre den statistischen Bericht und das Verzeichnis der "Heime und betreute Wohnformen für volljährige Menschen mit Behinderung in Bayern".

5.4. Bayerisches Gesetz zur Gleichstellung, Integration und Teilhabe von Menschen mit Behinderung ${ }^{4}$

Der Bayerische Landtag hat am 25. Juni 2003 das Gesetz zur Gleichstellung, Integration und Teilhabe von Menschen mit Behinderung (Behindertengleichstellungsgesetz) einstimmig beschlossen. Es ist am 1. August 2003 in Kraft getreten.

"Das Bayerische Behindertengleichstellungsgesetz ist ein Meilenstein bayerischer Behindertenpolitik und ein weiterer Schritt von der Fürsorge und Versorgung hin zur gleichberechtigten Teilhabe von Menschen mit Behinderung am gesellschaftlichen Leben. Oberste Leitlinien des Gesetzes, das zahlreiche Verbesserungen für Menschen mit Behinderung mit sich bringt, sind die Würde von Menschen mit Behinderung und die Stärkung ihrer Fähigkeit, ihr Leben selbst $\mathrm{zu}$ gestalten und es selbst zu bestimmen", erklärte die damalige Bayerische Sozialministerin Stewens zum Inkrafttreten.

Das Gesetz lehnt sich eng an die Bestimmungen des Bundesgleichstellungsgesetzes an, das am 1. Mai 2002 in Kraft getreten ist und ergänzt es in vielen wichtigen Lebensbereichen. Schwerpunkte des Gesetzes sind insbesondere die Verbesserung 
der Barrierefreiheit und Mobilität behinderter Menschen, die Erleichterung der Kommunikation unter anderem durch Anerkennung der deutschen Gebärdensprache und die Einrichtung von Beauftragten für die Belange von Menschen mit Behinderung auf kommunaler Ebene. Außerdem erhalten anerkannte Verbände unter bestimmten Voraussetzungen ein Verbandsklagerecht etwa bei einem Verstoß gegen das Benachteiligungsverbot oder gegen Vorschriften zur Herstellung der Barrierefreiheit.

Oberste Leitlinien des Bayerischen Gleichstellungsgesetzes sind die Würde von Menschen mit Behinderung sowie die Stärkung ihrer Fähigkeit, über ihr Leben selbst zu bestimmen und es selbst zu gestalten. Die Behindertenpolitik hat sich in den letzten Jahren stark gewandelt. Mit Fug und Recht kann von einem Paradigmenwechsel gesprochen werden: Von der Fürsorge und Versorgung hin zur gleichberechtigten Teilhabe von Menschen mit Behinderung am Leben der Gesellschaft. Dieser Paradigmenwechsel spiegelt sich auch im Landesgleichstellungsgesetz wider.

Entscheidend für die uneingeschränkte Teilhabe behinderter Menschen am täglichen Leben ist die Verbesserung der Mobilität. Die Staatsregierung wird deshalb die Förderung eines barrierefreien öffentlichen Personennahverkehrs verstärkt fortführen. Außerdem soll die barrierefreie Erreichbarkeit und Nutzbarkeit von öffentlichen Gebäuden verbessert und auch im privaten Wohnungsbau verstärkt auf barrierefreies Bauen geachtet werden. Eine barrierefreie Umwelt ermöglicht nicht nur Menschen mit Behinderung ein Leben in Teilhabe, sondern allen Bürgerinnen und Bürgern. Insbesondere bringt Barrierefreiheit älteren Menschen und jungen Müttern oder Vätern mit kleinen Kindern ebenso wie den zeitweise durch Unfall oder Krankheit in ihrer Bewegungsfreiheit eingeschränkten Menschen sehr oft eine deutliche Erleichterung im Alltag.

Zur Verbesserung der Kommunikation von Menschen mit Sinnesbehinderungen ist die deutsche Gebärdensprache im Umgang mit den bayerischen Behörden anerkannt worden. Außerdem haben behinderte Menschen einen Anspruch auf Kostenerstattung bei Hinzuziehung eines Gebärdensprachdolmetschers im Verwaltungsverfahren. Internetauftritte der öffentlichen Hand werden künftig barrierefrei gestaltet. Bei Landtagswahlen wird blinden Menschen die Möglichkeit eröffnet, mittels einer Stimmzettelschablone abzustimmen.

Alle Bezirke, Landkreise und kreisfreien Gemeinden wurden verpflichtet, Beauftragte für die Belange von Menschen mit Behinderung zu bestellen. Mittlerweile geschah dies in fast allen. Dadurch soll nicht nur eine verstärkte Einbindung der Betroffenen erreicht, sondern auch eine Instanz zur Wahrnehmung behindertenspezifischer Interessen geschaffen werden. Ergänzt wird diese Regelung durch ein Benachteiligungsverbot für Menschen mit Behinderung durch die Träger öffentlicher Gewalt in Bayern.

Sozialministerin Stewens: "Mit dem Inkrafttreten des Bayerischen Landesgleichstellungsgesetzes sind die gesetzlichen Rahmenbedingungen abgesteckt. Darüber hinaus brauchen wir jedoch die Unterstützung jedes einzelnen, um die Gleichstellung behinderter Menschen im Alltag mit Leben zu erfüllen und so allen Bürgern - mit und ohne Behinderung - eine echte gemeinsame Teilhabe am gesellschaftlichen Leben zu ermöglichen." 
In $\S 9$ wurde die Geltung des BayBGG bis zum 31.07.2008 befristet. Das BayBBG hat sich bewährt und gilt nun aufgrund des Novellierungsgesetzes vom 22.07.2008 ab 31.07.2008 unbefristet, ebenso die dazugehörigen Verordnungen. Zu den wesentlichen Neuerungen zählen:

- Bessere Berücksichtigung der speziellen Belange von Menschen mit seelischer Behinderung

- Änderungen in der BayBO, wonach barrierefreie zugängliche Wohnungen in bestimmten Teilen auch barrierefrei nutzbar sein müssen

- Erhöhung des Anteils barrierefreien Wohnraums

Folgende Verordnungen wurden auf Grundlage des BayBGG erlassen:

- Bayerische Verordnung zur Zugänglichmachung von Dokumenten für blinde, erblindete und sehbehinderte Menschen im Verwaltungsverfahren (BayDok ZugV) vom 24. Juli 2006

- Bayerische Verordnung zur Verwendung der Deutschen Gebärdensprache und anderer Kommunikationshilfen im Verwaltungsverfahren und in der Kommunikation mit der Schule (Bayerische Kommunikationshilfenverordnung - Bay KHV) vom 24. Juli 2006

- Verordnung über die Anerkennung der Prüfung für Gebärdensprachkursleiterinnen und Gebärdensprachkursleiter (Gebärdensprachkursleiter-Prüfungsordnung - GKPO) vom 17. Oktober 2006

- Landesbehindertenratsverordnung vom 14. Januar 2005

- Bayerische Verordnung zur Schaffung barrierefreier Informationstechnik (Bayerische Barrierefreie Informationstechnik-Verordnung - BayBITV) vom 24. Oktober 2006

\subsection{Der Bayerische Landesbehindertenrat ${ }^{5}$}

Der Bayerische Landesbehindertenrat unterstützt und berät die Staatsregierung in allen Fragen der Behindertenpolitik.

Dem Gremium gehören 17 Mitglieder an; neben der/dem Sozialminister/in, der/die den Vorsitz führt, der beauftragten Person für die Belange der Menschen mit Behinderung,der Bayerischen Staatsregierung,, Vertreter der Behindertenverbände, der Selbsthilfeorganisationen und der kommunalen Beauftragten für die Belange von Menschen mit Behinderung sowie die Vertreter der Freien und Öffentlichen Wohlfahrtspflege.

Der Landesbehindertenrat kommt nach Bedarf zu seinen Sitzungen zusammen und kann so jeweils auf aktuelle, für die Behindertenpolitik relevante Ereignisse reagieren. 


\subsection{Berufliche Eingliederung von Menschen mit Behinderung ${ }^{6}$}

Gesellschaftliche Anerkennung wird in besonderem Maße davon bestimmt, auf welche Weise und in welchem Umfang der einzelne seinen Lebensunterhalt selbst sicherstellen kann. Der Teilhabe am Arbeitsleben kommt dabei eine Schlüsselrolle zu. Für behinderte Menschen ist die Integration in das Berufs- und Erwerbsleben besonders wichtig. Aus diesem Grunde erhalten behinderte Menschen gezielte und umfassende Hilfestellung.

Integrationsamt Bayern $^{7}$

Die bisher bei den Bezirksregierungen arbeitenden Integrationsämter wurden zum 1. August 2005 in das Zentrum Bayern Familie und Soziales (www.zbfs.bayern.de) als Integrationsamt Bayern eingegliedert.

Das Integrationsamt Bayern ist in allen Regionalstellen des Zentrums Bayern Familie und Soziales operativ tätig und steht dort Menschen mit schweren Behinderungen und Arbeitgebern als sachkundiger Ansprechpartner in allen Fragen, die mit der Integration schwerbehinderter Menschen zusammenhängen, zur Verfügung. Der gewohnte Service der bisherigen Integrationsämter wird vom Integrationsamt Bayern weiterhin erbracht.

Aufgabe des Integrationsamts ist die begleitende Hilfe, die Teilhabe schwerbehinderter und gleichgestellter Menschen am Arbeitsleben zu stärken und zu fördern, Arbeitsverhältnisse zu sichern und Arbeitgeber bei der Beschäftigung von schwerbehinderten Menschen zu beraten und zu unterstützen (www.integrationsaemter.de).

Begleitende Hilfen im Arbeitsleben können sein: Beratung zur Gestaltung behindertengerechter Arbeitsbedingungen, Geldleistungen für technische Arbeitshilfen, zur Erreichung des Arbeitplatzes, zur Gründung und Erhaltung einer selbständigen beruflichen Existenz, zur Beschaffung, Ausstattung und Erhaltung einer behinderungsgerechten Wohnung, zur Teilnahme an Fortbildungsmaßnahmen, Leistungen in besonderen Lebenslagen sowie Übernahme von Kosten einer notwendigen Arbeitsassistenz. Arbeitgeber können Leistungen zur behinderungsgerechten Einrichtung von Arbeitsplätzen für schwerbehinderte Menschen sowie zum Ausgleich außergewöhnlicher und nicht zumutbarer Belastungen (Minderleistung/Betreuungsaufwand) erhalten.

6 http://www.stmas.bayern.de/behinderte/integration/index.htm.

7 http://www.stmas.bayern.de/behinderte/integration/index.htm. 
Integrationsfachdienste ${ }^{8}$

Integrationsfachdienste sind im Auftrag der Arbeitsagenturen, der Arbeitsgemeinschaft nach dem SGB II, der Optionskommunen, der Integrationsämter oder Rehabilitationsträger tätig. Sie sollen die Teilhabe schwerbehinderter Arbeitnehmerinnen und Arbeitnehmer am Arbeitsleben sichern helfen. Sie vermitteln behinderungsgerechte Arbeitsplätze und bieten psychosoziale Beratung für schwerbehinderte Arbeitnehmer an. Integrationsfachdienste arbeiten eng mit Arbeitgebern, Betriebsräten und Schwerbehindertenvertretungen zusammen.

\section{Berufsbildungswerke ${ }^{9}$}

Berufsbildungswerke sind überregionale Einrichtungen zur beruflichen Erstausbildung von behinderten jungen Menschen, die wegen Art oder Schwere ihrer Behinderung auf besondere ausbildungsbegleitende Hilfen angewiesen sind. Die Berufsbildungswerke umfassen in der Regel Ausbildungswerkstätten, Berufschule, differenzierte Wohnmöglichkeiten sowie Freizeiteinrichtungen und verschiedene Fachdienste.

Die Lerninhalte und Ausbildungsbedingungen sind auf die Belange der behinderten Auszubildenden abgestimmt. Neben Berufen, die für Behinderte wie Nichtbehinderte gleichermaßen geeignet sind, ist in Berufsbildungswerken auch eine Ausbildung in speziellen Berufen für behinderte Menschen möglich. Zur Unterstützung und Begleitung stehen den behinderten jungen Menschen in Berufsbildungswerken besondere pädagogische, medizinische und psychologische Fachdienste zur Verfügung. Ziel ist die möglichst dauerhafte Eingliederung der behinderten jungen Menschen in Beruf, Arbeit und Gesellschaft.

In Bayern stehen rund 2.600 Plätze für behinderte junge Menschen in Berufsbildungswerken zur Verfügung. Über die Notwendigkeit einer Ausbildung in einem Berufsbildungswerk entscheidet die für den Wohnort zuständige Agentur für Arbeit - Berufsberatung für Behinderte.

\section{Berufsförderungswerke ${ }^{10}$}

Berufsförderungswerke sind außerbetriebliche Bildungseinrichtungen zur Fortbildung und Umschulung von behinderten Menschen, die in der Regel bereits berufstätig waren und sich wegen ihrer Behinderung beruflich neu orientieren müssen. Die 
Berufsförderungswerke verfügen über Ausbildungsstätten, Internate mit Wohngruppen, verschiedene Fachdienste sowie Freizeiteinrichtungen.

Berufliche Rehabilitation in Berufsförderungswerken soll den behinderten Menschen befähigen, seinen künftigen Beruf weitgehend ohne Beeinträchtigung auszuüben und damit eine dauerhafte Teilhabe am Arbeitsleben und gesellschaftlichem Leben ermöglichen. Inhalte und Methoden der Maßnahmen in Berufsförderungswerken sind behinderungs- und erwachsenengerecht ausgerichtet. Die Qualifizierung erfolgt in anerkannten Ausbildungsberufen oder speziellen Ausbildungsberufen für Behinderte. Den behinderten Menschen stehen in den Berufsförderungswerken spezielle berufsbegleitende Fachdienste zur Verfügung.

Der Zugang zu den Berufsförderungswerken erfolgt über den zuständigen Rehabilitationsträger. Das sind in der Regel die Rentenversicherungsträger oder die Bundesagentur für Arbeit, die Unfallversicherungsträger im Falle eines Arbeitsunfalls.

\section{Werkstätten für behinderte Menschen ${ }^{11}$}

Die Werkstätten für behinderte Menschen und Blindenwerkstätten im Sinne des Sozialgesetzbuches Neuntes Buch (SGB IX) sind Einrichtungen zur Teilhabe behinderter Menschen am Arbeitsleben. Aufgabe der Werkstätten ist es, denjenigen Menschen, die wegen Art und Schwere ihrer Behinderung nicht, noch nicht oder noch nicht wieder auf dem allgemeinen Arbeitsmarkt tätig sein können,

- eine angemessene berufliche Bildung und Beschäftigung zu einem ihrer Leistung angemessen Entgelt aus dem Arbeitsergebnis der Werkstatt sowie

- die Entwicklung, Erhöhung, Erhaltung oder Wiedergewinnung ihrer Leistungsund Erwerbsfähigkeit und die Weiterentwicklung ihrer Persönlichkeit zu ermöglichen.

Die Werkstatt steht allen behinderten Menschen unabhängig von Art, Schwere und Ursache ihrer Behinderung offen, sofern erwartet werden kann, dass sie spätestens nach Teilnahme an Maßnahmen im Berufsbildungsbereich ein Mindestmaß an wirtschaftlich verwertbarer Arbeitsleistung erbringen können. Neben Fachkräften, die geeignet sind, behinderte Menschen unter Berücksichtigung ihrer individuellen Bedürfnisse, Fähigkeiten und Leistungsvermögen anzuleiten und zu fördern, beschäftigt eine Werkstatt Personal zur Betreuung der behinderten Menschen (Sozialdienst, medizinische und psychologische Dienste).

Insgesamt gibt es in Bayern rd. 180 Werkstätten für behinderte Menschen mit etwa 30.000 Plätzen. Die Anerkennung als Werkstatt für behinderte Menschen im Sinne des SGB IX erfolgt über die Bundesagentur für Arbeit, die auch ein Verzeichnis der anerkannten Werkstätten für behinderte Menschen führt. Das Verzeichnis kann über www.rehadat.de abgerufen werden 
Integrationsprojekte $^{12}$

Die Integrationsprojekte in Bayern sind überwiegend Klein- oder Mittelbetriebe aus verschiedenen Wirtschaftsbranchen, die entsprechend den gesetzlichen Vorschriften der $\S § 132$ ff. Neuntes Buch Sozialgesetzbuch schwerbehinderte Menschen beschäftigen, deren Teilhabe an einer sonstigen Beschäftigung auf dem allgemeinen Arbeitsmarkt aufgrund von Art und Schwere der Behinderung oder aufgrund sonstiger Umstände auf besondere Schwierigkeiten stößt. Ursprünglich wurden die Integrationsprojekte für psychisch kranke und behinderte Menschen geschaffen, um diesen eine Alternative zur Beschäftigung in Werkstätten für behinderte Menschen zu bieten. Die Integrationsprojekte unterscheiden sich von Werkstätten für behinderte Menschen darin, dass mit den Betroffenen Ausbildungs- und Arbeitsverträge mit allen sich daraus ergebenden arbeitsrechtlichen, tarifrechtlichen und sozialrechtlichen Rechten und Pflichten geschlossen werden. Zwischenzeitlich existieren auch Integrationsprojekte für körperbehinderte, lern-, geistig behinderte Menschen sowie für Menschen mit anderen Behinderungen.

Ziel ist die Schaffung von sozialversicherungspflichtigen Dauerarbeitsplätzen für schwerbehinderte Menschen sowie die Zusammenarbeit und gemeinsame Beschäftigung von Menschen mit und ohne Behinderung. Die Integrationsprojekte verstehen sich nicht als "Sonderbetriebe", sondern arbeiten wie andere Unternehmen marktund wettbewerbsorientiert.

Eine aktuelle Übersicht über die Integrationsprojekte in Bayern (Stand: März 2008) entnehmen Sie der nachfolgenden Tabelle:

\begin{tabular}{|c|c|c|c|c|c|c|c|c|c|c|}
\hline \multirow{3}{*}{$\begin{array}{l}\text { Regierungs- } \\
\text { bezirk }\end{array}$} & \multirow{3}{*}{$\begin{array}{c}\text { Firmen } \\
\text { bzw. } \\
\text { Projekte }\end{array}$} & \multirow{3}{*}{$\begin{array}{l}\text { Beschäf- } \\
\text { tigte } \\
\text { insgesamt }\end{array}$} & \multirow{3}{*}{$\begin{array}{l}\text { schwer- } \\
\text { behinderte } \\
\text { Beschäf- } \\
\text { tigte }\end{array}$} & \multicolumn{7}{|c|}{ davon } \\
\hline & & & & psB & $\mathrm{KB}$ & GB & LB & SB & HSB & SHV \\
\hline & & & & 1 & 2 & 3 & 4 & 5 & 6 & 7 \\
\hline Oberbayern & 35 & 1.599 & 734 & 366 & 163 & 137 & 22 & 19 & 19 & 2 \\
\hline Niederbayern & 4 & 119 & 62 & 37 & 10 & 3 & 2 & 7 & 2 & 1 \\
\hline Oberpfalz & 8 & 311 & 147 & 80 & 45 & 5 & 7 & 2 & 4 & 4 \\
\hline Oberfranken & 6 & 222 & 173 & 77 & 64 & 14 & 3 & 3 & 6 & 6 \\
\hline Mittelfranken & 17 & 602 & 341 & 240 & 86 & 11 & 3 & 1 & 0 & 0 \\
\hline Unterfranken & 9 & 353 & 208 & 38 & 72 & 37 & 33 & 8 & 17 & 3 \\
\hline Schwaben & 6 & 225 & 124 & 90 & 8 & 2 & 11 & 4 & 8 & 1 \\
\hline Summe & 85 & 3.431 & 1.789 & 982 & 448 & 209 & 81 & 44 & 56 & 22 \\
\hline
\end{tabular}


$\mathrm{GB}=$ geistige Behinderung

$\mathrm{LB}=$ Lernbehinderung

$\mathrm{SB}=$ Sehbehinderung

HSB $=$ Hör-/Sprachbehinderung

SHV $=$ Schädel-/Hirnverletzte

\subsection{Bayerisches Blindengeldgesetz ${ }^{13}$}

"Ein Tor ist zugetan, doch tausend stehn noch offen" (Friedrich Rückert)

Das Zentrum Bayern Familie und Soziales (bzw. seine Regionalstellen) ist für die Zahlung von Blindengeld zuständig. Mit dem Blindengeld als reiner Landesleistung trägt der Freistaat Bayern der besonderen Situation seiner blinden Mitbürgerinnen und Mitbürger Rechnung. Erblindete Menschen haben einen hohen Aufwand für Hilfs- und Pflegeleistungen durch dritte Personen oder die Anschaffung von blindengerechten Hilfsmitteln. Zum Ausgleich dieser blindheitsbedingten Mehraufwendungen dient das Blindengeld, das keine Pflegebedürftigkeit voraussetzt und einkommensunabhängig gezahlt wird.

Blindengeld erhält nach den Bestimmungen des Bayerischen Blindengeldgesetzes, wer seinen Wohnsitz oder gewöhnlichen Aufenthalt in Bayern hat. Ausländische Mitbürger mit Wohnsitz oder gewöhnlichem Aufenthalt in Bayern haben Anspruch auf Blindengeld, wenn sie sich rechtmäßig im Geltungsbereich des Gesetzes aufhalten. Es muss Blindheit vorliegen. Das Blindengeld muss schriftlich beantragt werden. Nach einer beabsichtigten Änderung des europäischen Rechts werden wohl auch im Ausland lebende EU-Bürger unter bestimmten Voraussetzungen Leistungen erhalten können.

Der Anspruch auf Blindengeld entsteht mit dem ersten Tag des Monats, in dem die gesetzlichen Voraussetzungen vorliegen, frühestens mit dem ersten Tag des Antragsmonats. Das Blindengeld wird monatlich im Voraus gezahlt. Es kann bereits ab Geburt zustehen, sofern im ersten Lebensmonat des Kindes Antrag gestellt und Blindheit nachgewiesen wird.

Kein Blindengeld erhalten Personen, die wegen der Blindheit Leistungen nach dem Bundesversorgungsgesetz oder den Gesetzen, die seine entsprechende Anwendung vorsehen, z.B.: Opferentschädigungsgesetz, Soldatenversorgungsgesetz, erhalten. Dasselbe gilt für Blinde, die wegen der Erblindung Leistungen aus der gesetzlichen Unfallversicherung oder aus öffentlichen Kassen aufgrund gesetzlich geregelter Unfallversorgung oder Unfallfürsorge erhalten.

Das volle Blindengeld beträgt ab 01.07.2008 monatlich $505 €$. Das Blindengeld wird nur zur Hälfte gezahlt, wenn sich die blinde Person in einem Heim oder in einer gleichartigen Einrichtung aufhält und die Kosten des Aufenthaltes ganz oder teilweise aus Mitteln öffentlich-rechtlicher Leistungsträger, z.B.: Sozialhilfeträger, 
gesetzliche Krankenkassen, Pflegekassen oder Beihilfeträger bestritten werden oder Leistungen der privaten Pflegeversicherung in Anspruch genommen werden.

Das Blindengeld wird gekürzt

- auf $376 €$, wenn der Blinde Leistungen (Pflegegeld, Sachleistungen oder Kombinationsleistungen) der Pflegestufe I aus der gesetzlichen oder privaten Pflegeversicherung erhält,

- $\quad$ auf $337 €$, wenn der Blinde Leistungen der Pflegestufe II oder III erhält,

- auf höchstens $376 €$, wenn der Blinde Leistungen zum Ausgleich blindheitsbedingter Mehraufwendungen nach anderen Rechtsvorschriften erhält.

Der Anspruch auf Blindengeld entfällt mit Ablauf des Monats, in dem die Voraussetzungen weggefallen sind. Ein Wegzug aus Bayern ist daher unverzüglich mitzuteilen.

Blindheit bedingt nach dem SGB IX einen Grad der Behinderung (GdB) von 100. Außerdem sind die Voraussetzungen für die Zuerkennung folgender Merkzeichen gegeben: Bl (blind), $\mathrm{G}$ (erhebliche Beeinträchtigung der Bewegungsfähigkeit im Straßenverkehr), B (Notwendigkeit ständiger Begleitung), H (Hilflosigkeit), RF (Befreiung von der Rundfunkgebührenpflicht). Einen entsprechenden Schwerbehindertenausweis stellt dieselbe Regionalstelle des Zentrums Bayern, Familie und Soziales auf Antrag aus, die in der Blindengeldangelegenheit zuständig ist.

Hinweis: Eine ergänzende Blindenhilfe nach dem Sozialgesetzbuch Zwölftes Buch -Sozialhilfe- (SGB XII) können volljährige Blinde erhalten, die die Einkommens- und Vermögensvoraussetzungen des SGB XII erfüllen. Entsprechende Anträge sind bei dem für den jeweiligen Wohnort zuständigen Bezirk zu stellen.

\subsection{Dienste der offenen Behindertenarbeit ${ }^{14}$}

Die meisten Menschen mit Behinderung oder chronischer Krankheit leben nicht in einem Heim, sondern in ihren Familien oder einer eigenen Wohnung und möchten diese Lebensform auch beibehalten. Die bayerische Behindertenpolitik hat es daher zu einem ihrer Grundprinzipien gemacht, die Fähigkeit und die Möglichkeiten behinderter Menschen zu stärken, über ihr Leben selbst zu bestimmen und es selbst zu gestalten.

Die Dienste der Offenen Behindertenarbeit tragen diesem Prinzip in beispielhafter Form Rechnung. Seit 1988 fördert der Freistaat Bayern im Rahmen einer Richtlinie diese regionalen und überregionalen Beratungs- und Betreuungsdienste für Menschen mit Behinderung, die

- die ambulante Betreuung und hauswirtschaftliche Versorgung von Menschen mit Behinderung oder chronischer Krankheit sicherstellen, 
- Menschen mit einer Behinderung bei der Führung eines selbstständigen und selbstbestimmten Lebens helfen und

- die Familien mit behinderten Angehörigen unterstützen und entlasten.

Träger dieser Dienste sind die Spitzenverbände der freien Wohlfahrtspflege in Bayern, deren Mitgliedsorganisationen oder die Landesbehindertenverbände. In einigen Regionen haben mehrere Anbieter in Kooperation miteinander die Offenen Hilfen aufgeteilt.

Zielgruppe der regionalen Dienste, die meist auf der Ebene eines Landkreises oder einer kreisfreien Stadt tätig sind, sind körperlich und geistig behinderte und chronisch kranke Menschen aller Behinderungsarten und alle Altersstufen.

Das Angebotsspektrum der Dienste umfasst Informationen und Beratung zu allen Fragen des (Schwerpunkte) täglichen Lebens und Vermittlung von Hilfen, vor allem

- $\quad$ psychosoziale Beratung und Betreuung der behinderten bzw. chronisch kranke Menschen und deren Familien

- Pflege und Betreuung - nur außerhalb des Zuständigkeitsbereiches der Pflegeversicherung

- familienentlastende Dienste

- Organisation und Durchführung von Freizeit- und Begegnungsmaßnahmen

- Schulungsmaßnahmen für behinderte Menschen und ihre Familien

- Öffentlichkeitsarbeit, Gemeinwesenarbeit

Für spezielle Behindertengruppen - wie z.B. Blinde, Gehörlose, MS-Kranke usw.gibt es Spezialdienste auf der Ebene der Regierungsbezirke, sogenannte überregionale Dienste der Offenen Behindertenarbeit.

Derzeit gibt es überregionale Dienste für:

- Blinde

- Gehörlose

- Aphasiker

- Autisten

- MS-Kranke

- Krebskranke

- Muskelkranke

- Rheumakranke

- Schädel-Hirn-Verletzte

- Epilepsiekranke

- Mukoviszidosekranke

\subsection{Hilfen für Kinder und Jugendliche}

"Ihr Kind ist behindert" - die Mitteilung dieser Diagnose ist für die meisten Eltern zunächst schockierend und stellt sie vor eine schwere Lebensaufgabe. Ein behindertes Kind zu haben und zu erziehen, bedeutet für die betroffenen Familien große 
zusätzliche Anforderungen psychischer, physischer und finanzieller Art. Familien mit behinderten Kindern und Jugendlichen brauchen fachkundige Beratung sowie Rat und Hilfe in allen Situationen von der Geburt bis zum Arbeitsleben aber auch Informationen über Unterstützungs- und Fördermöglichkeiten.

\section{Frühförderung von behinderten oder von Behinderung bedrohten Kindern ${ }^{15}$}

Die frühe und rechtzeitige Förderung von Kindern, die behindert, von Behinderung bedroht, teilleistungsgestört, entwicklungsverzögert oder verhaltensgestört sind, ist seit über 30 Jahren ein wichtiges Anliegen bayerischer Behindertenpolitik. Von Anfang an stand dabei die Überzeugung im Vordergrund, dass sich Hilfen, die Kindern im frühen Alter gewährt werden, besonders günstig auf den weiteren Verlauf einer Behinderung, Entwicklungsverzögerung oder Auffälligkeit auswirken. Rechtzeitig und fachlich qualifiziert geförderte Kinder sind in späteren Jahren oftmals völlig unauffällig in ihrem Verhalten und Erleben. Dies ist ein Erfolg, der zu einem großen Teil auf die verschiedenen Frühförderangebote, insbesondere der Frühförderstellen zurückzuführen ist.

Unter Frühförderung ist ein fachliches Hilfe- und Förderangebot für Kinder im Alter von der Geburt bis zum Schuleintritt zu verstehen. Die spezielle Institution "Frühförderstelle", die ambulant und/oder mobil arbeitet, ist dabei der wichtigste Anbieter von Frühförderung. Frühförderung geschieht dort in einem interdisziplinären Team, d.h. medizinisch orientierte und pädagogisch-psychologisch ausgerichtete Frühförderung und die entsprechenden Berufsgruppen ergänzen sich.

Für behinderte oder von Behinderung bedrohte Säuglinge, Kleinkinder und Kinder im Vorschulalter steht heute ein bedarfsgerechtes, flächendeckendes Netz von 144 Frühförderstellen in Bayern zur Verfügung.

Aufgaben der Frühförderstellen:

- Behinderungen, Schädigungen, Defizite, Verzögerungen, Störungen und Auffälligkeiten bei Kindern möglichst früh zu erkennen,

- diese Kinder entsprechend zu fördern und zu therapieren,

- die Eltern der betroffenen Kinder zu beraten, zu unterstützen und zu begleiten,

- notwendige Kontakte und weitere Hilfen zu vermitteln.

Daneben leisten 12 Sozialpädiatrische Zentren in Altötting, Coburg, Erlangen, Garmisch-Partenkirchen, Hof, Landshut, Memmingen, München, Passau, Regensburg, Traunstein und Würzburg einen weiteren wichtigen Beitrag u.a. bei der Diagnose, bei der Beurteilung und Therapie körperlicher, geistiger und seelischer Gesundheitsstörungen vom Säuglings- bis ins Jugendalter.

Über diese Themen informiert auch das 306-seitige Buch "Die Sozialpädiatrischen Zentren in Bayern", das im Dezember 2003 von den bayerischen Sozialpädiatrischen 
Zentren herausgegeben wurde. Das Sozialministerium war an der Finanzierung maßgeblich beteiligt.

Tagesstätten für Kinder und Jugendliche mit Behinderung ${ }^{16}$

Für Kinder und Jugendliche mit Behinderung gibt es die folgenden Formen der Tagesstättenbetreuung:

- Integrative Kindertagesstätten

- Heilpädagogische Tagesstätten

- Schulvorbereitende Einrichtungen

Heilpädagogische Tagesstätten sind teilstationäre Fördereinrichtungen für Kinder mit Behinderung im Vorschulalter oder im Schulalter. Sie orientieren sich bei ihrem jeweiligen Förder- und Betreuungsangebot an der individuellen Behinderung des einzelnen Kindes. In Heilpädagogischen Tagesstätten bestehen die Gruppen aus 6-12 Kindern und sind mit speziell heilpädagogisch ausgebildeten Fachkräften besetzt. Darüber hinaus stehen den Kindern zahlreiche heilpädagogische und medizintherapeutische Fachdienste wie Logopädie, Physiotherapie-, Ergotherapie, Musiktherapie oder Spieltherapie zur Verfügung. Die Aufnahme betroffener Kinder erfolgt nach Aufnahme eines entsprechenden ärztlichen Gutachtens. Die Kosten der Betreuung übernimmt bis auf einen geringen Beitrag zur häuslichen Ersparnis der zuständige Sozialhilfeträger. Die Zahl der Heilpädagogischen Tagesstätten wurde in den letzten zehn Jahren mit erheblicher staatlicher Unterstützung auf den heutigen Stand von rund 15.000 Plätzen ausgebaut.

Die Schulvorbereitenden Einrichtungen betreuen Kinder mit sonderpädagogischem Förderbedarf meist ab dem vierten Lebensjahr. Auch von der Förderschule zurückgestellte Kinder werden dort aufgenommen. Die Schulvorbereitenden Einrichtungen befinden sich bei den Förderschulen für Kinder mit Behinderung. Nähere Informationen finden Sie auf den Seiten des Bayerischen Staatsministeriums für Unterricht und Kultus (www.stmuk.bayern.de).

Heime und Internate für behinderte Kinder und Jugendliche ${ }^{17}$

In Bayern gibt es derzeit 95 Heime und Internate mit 4.940 Plätzen für Kinder und Jugendliche mit Behinderung.

Die bestehende Heimlandschaft ist in verschiedene Heimarten unterteilt und differenziert zwischen verschiedenen Angebotsformen einerseits und Behinderungsar-

16 http://www.stmas.bayern.de/behinderte/kinder/tagesstaetten.htm.

17 http://www.stmas.bayern.de/behinderte/kinder/heime.htm. 
ten andererseits. So umfasst das Spektrum der Heime sämtliche Behinderungsarten bis zu schweren Mehrfachbehinderungen. Es reicht in seiner Angebotsstruktur vom

- Kurzzeitpflegeheim

- über das 5-Tages-Internat an Förderschulen

- bis zum vollstationären Heim für schwerstbehinderte Kinder und Jugendliche.

Daneben gehören auch Kur-, Genesungs- und Erholungsheime für chronisch kranke oder behinderte Kinder zu diesem Spektrum. Diese Einrichtungen bieten zeitlich befristete, spezielle Behandlungen und Therapien für chronisch kranke und behinderte Kinder und Jugendliche sowie Familienerholungen an.

Personal, Betreuungsqualität und Raumangebot der Heime und Internate werden von der zuständigen Aufsicht bei den Regierungen nach den Bestimmungen des Kinder- und Jugendhilfegesetzes (SGB VIII) laufend überprüft.

\section{Geschwister behinderter Kinder ${ }^{18}$}

Was bedeutet es für ein Kind, mit einem behinderten Bruder oder einer behinderten Schwester aufzuwachsen?

Die Geschwister sind gleich nach den Eltern die wichtigsten Personen in unserer Kindheit. Mit ihnen verbringen Kinder und oft auch noch Jugendliche die meiste Zeit. Sie haben daher großen Einfluss auf die psychische und soziale Entwicklung. In Familien mit behinderten oder chronisch kranken Kindern wachsen die anderen Kinder jedoch unter besonderen Rahmenbedingungen auf, denn das Augenmerk wird meist auf die behinderten Kinder gerichtet. Die besondere Lebenssituation von nicht behinderten Geschwistern dagegen wurde bislang eher vernachlässigt. Dass auch sie Hilfe und Unterstützung brauchen, belegt eine Studie des Staatsinstituts für Familienforschung an der Universität Bamberg eindrucksvoll.

Im Auftrag des Bayerischen Staatsministeriums für Arbeit und Sozialordnung, Familie und Frauen hat sich das Staatsinstitut für Familienforschung intensiv mit der besonderen Lebenssituation der Geschwister von behinderten Kindern befasst. Tatsächlich werden an sie schon frühzeitig ungewöhnliche Erwartungen herangetragen und sie müssen sich mit Schwierigkeiten auseinandersetzen, von denen andere Kinder meist verschont bleiben. Aus diesen Erfahrungen und nach vielen Gesprächen mit Eltern und Geschwistern haben wir ein spezielles und ansprechendes Informationspaket für sie erstellt:

- DER ELTERNBRIEF wendet sich an Mütter und Väter von behinderten oder chronisch kranken Kindern. Ilse Achilles und Dr. Nicosia Nieß berichten aus eigener Betroffenheit über ihre Erfahrungen und sagen, weshalb ihnen das Thema "Geschwister" so besonders am Herzen liegt.

- DIE BILDERGESCHICHTE mit dem Titel "Teddy ist ein guter Zuhörer" von Charlotte Knees richtet sich an 3- bis 6-jährige Kinder - zum Vorlesen und zum 
Erzählen. Denn Teddy beobachtet sehr genau, warum sich die kleine Laura über Gregor, ihren behinderten Bruder, manchmal ärgert.

- DER BRIEFWECHSEL "Freunde" zwischen Saskia und Marko, entworfen von Marlies Winkelheide, zeigt 7- bis 12-Jährigen, wie wichtig Freunde sind, selbst wenn man sie nicht täglich sieht und mit ihnen reden kann. Brieffreundschaften eignen sich besonders gut, um sich Kummer von der Seele zu schreiben.

- DER CHECK-UP regt 13- bis 17-Jährige dazu an, sich Gedanken über ihre Situation zu machen, die in diesem Alter besonders schwierig ist. Mit seiner Hilfe können die Jugendlichen "checken", ob bei ihnen alles im "grünen Bereich" ist oder "Alarmstufe Rot" droht.

- DAS INFOBLATT enthält Anschriften und Hinweise auf Literatur und Filme zum Thema.

Finanzielle Unterstützung und weitere Leistungen ${ }^{19}$

Landesstiftung "Hilfe für Mutter und Kind"

- Angebot: schnelle und unbürokratische finanzielle Hilfe für kinderreiche Familien oder Alleinerziehende im Einzelfall, bspw. zur Beschaffung eines behindertengerechten Fahrzeugs oder zum behindertengerechten Umbau der Wohnung

- Weitere Infos: Landesstiftung Hilfe für Mutter und Kind, im Internet unter www.zbfs.bayern.de/stiftung/

Jugendamt, Sozialamt

- Angebot: verschiedene Leistungen der Eingliederungshilfe und Leistungen nach dem Kinder- und Jugendhilfegesetz, unter anderem Hilfen zur Erziehung, Leistungen zur vorschulischen und sozialen Eingliederung

- Weitere Infos: bei der jeweiligen Kommunalverwaltung

Besondere Leistungen der Krankenversicherung und bei Pflegebedürftigkeit

- Angebot: Besonderheiten für Menschen mit Behinderung bei Leistungen zur Früherkennung und Behandlung von Krankheiten sowie bei Pflegebedürftigkeit (z. B. Verordnung von nicht-verschreibungspflichtigen Medikamenten für behinderte Jugendliche bis 18 Jahre, Fahrkosten, Befreiungsregelungen von $\mathrm{Zu}$ zahlungen)

- Weitere Infos: Zuständige Kranken- und Pflegeversicherungen sowie Broschüre des Bundesverbands für Körper- und Mehrfachbehinderte e. V. "Mein Kind ist behindert - diese Hilfen gibt es", www.bvkm.de

Nachteilsausgleiche

- Angebot: Ausgleich behinderungsbedingter Nachteile oder Mehraufwendungen (überwiegende Voraussetzung ist die Eigenschaft als schwerbehinderter Mensch) (z. B. unentgeltliche Beförderung, Steuererleichterungen) 
- Weitere Infos: Broschüre des StMAS „Wegweiser für Menschen mit Behinderung"; Broschüre des StMF "Steuertips für Menschen mit Behinderung"; Broschüre des Bundesverbands für Körper- und Mehrfachbehinderte e. V. "Mein Kind ist behindert - diese Hilfen gibt es" sowie "Steuermerkblatt für Familien mit behinderten Kindern"

Kindergeld

- Angebot: Anspruch auf Kindergeld für ein behindertes Kind auch nach Eintritt der Volljährigkeit (unter bestimmten Voraussetzungen)

- Weitere Infos: Broschüre des StMF "Steuertips für Familien" und "Steuertips für Menschen mit Behinderung"

Fördereinrichtungen sowie Unterstützung bei der Ausbildung und auf dem Arbeits$\operatorname{markt}^{20}$

\section{Frühförderung}

- Angebot: Frühe Förderung für behinderte oder von Behinderung bedrohte Säuglinge, Kleinkinder und Kinder im Vorschulalter (allgemeine Frühförderstellen mit zahlreichen Außenstellen auf Landkreisebene und spezielle Einrichtungen mit einem größeren Einzugsbereich für sinnesgeschädigte Kinder sowie Sozialpädiatrische Zentren)

- Weitere Infos: Arbeitsstelle Frühförderung, Seidlstr. 4, 80335 München, www.fruehfoerderung-bayern.de; Broschüre des StMAS „Die sozialpädiatrischen Zentren in Bayern“"

Integrative Kinderkrippen, Kindergärten

- Angebot: Selbstverständlicher Umgang mit Gleichaltrigen, Persönlichkeit entfalten und Entwicklungsmöglichkeiten verwirklichen.

- Weitere Infos: www.stmas.bayern.de/behinderte/kinder/tagesstaetten/

Schulvorbereitende Einrichtungen, Heilpädagogische Tagesstätten

- Angebot: Für Kinder ab dem dritten Lebensjahr sowie zur schulbegleitenden Betreuung und Förderung gibt es ein differenziertes teilstationäres Betreuungsangebot bestehend aus Schulvorbereitenden Einrichtungen (SVE) und Heilpädagogischen Tagesstätten (HPT)

- Weitere Infos: Über SVE beim Bayerischen Staatsministerium für Unterricht und Kultus, über HPTs bei den Kommunen und den regional ansässigen Behindertenverbänden

Förderschulen

- Angebot: Gezielter und behindertengerechter Unterricht für Schülerinnen und Schüler mit Behinderung (z. B. in Schulen für Gehörlose, Sehbehinderte) 
- Weitere Infos:

www.stmuk.bayern.de $/ \mathrm{km} / \mathrm{schule} /$ schularten/allgemein/foerderschule/index. shtml

Fortbildungsmaßnahmen für Familien mit behinderten Kindern

- Angebot: Fachspezifische Bildungsmaßnahmen für Familien mit behinderten Kindern.

- Weitere Infos: Zentrum Bayern Familie und Soziales im Internet: www.zbfs. bayern.de

Unterstützung bei Berufsausbildung und Studium

- Angebot: speziell geschulte Berater in den Arbeitsagenturen, Berufsbildungswerke, Leistungen im Rahmen der Eingliederungshilfe (z. B. Kosten für Gebärdensprachdolmetscher an der Universität)

- Weitere Infos: Arbeitsagenturen, Berufsbildungswerke, Broschüre des Deutschen Studentenwerks "Studium und Behinderung"

Unterstützung bei Berufstätigkeit

- Angebot: Integrationsfachdienste, die bei der Eingliederung in das Arbeitsleben helfen, Vorschriften zum Schutz erwerbstätiger Menschen mit Behinderung, Werkstätten für Menschen mit Behinderung, Tagesförderstätten, Integrationsämter

- Weitere Infos: Broschüre des StMAS "Werkstätten für behinderte Menschen", Broschüre der Bundesarbeitsgemeinschaft der Integrationsämter und Hauptfürsorgestellen "ABC Behinderung und Beruf", www.integrationsaemter.de

\section{Beratungseinrichtungen $^{21}$}

Staatlich anerkannte Schwangerenberatungsstellen und kirchliche Beratungsstellen

- Angebot: Umfassende Beratung in allen die Schwangerschaft betreffenden sozialen, medizinischen und psychologischen Fragen; psychosoziale Beratung vor, während und nach einer PND sowie Beratung über Hilfemöglichkeiten bei einer zu erwartenden Schädigung des Kindes; Vermittlung von sozialen und finanziellen Hilfen

- Weitere Infos: www.stmas.bayern.de/familie/beratung/schwangere/; Broschüre Schwangerschaft und Beratung mit ausführlichem Anschriftenverzeichnis

Eltern-, Familien- und Lebensberatungsstellen sowie Erziehungsberatungsstellen

- Angebot: Unterstützung der Ratsuchenden in verschiedenen Lebenslagen (z. B. bei Problemen mit dem Partner, der Familie [z. B. behindertem Kind] und der Umwelt)

- Weitere Infos: www.stmas.bayern.de/familie/beratung/ 
Sozialberatungsstellen

- Angebot: Information und Beratung von Menschen mit Behinderung über gesetzliche Ansprüche und Hilfe bei der Durchsetzung der Ansprüche, Information über vorhandene soziale Dienste freier und öffentlicher Träger im Einzugsbereich

- Weitere Infos: Adressen der örtlichen Stellen zur Beratung zu behinderungsspezifischen Themen (z. B. Beratungsstelle für Blinde und hochgradig Sehbehinderte) unter www.intakt.info

Psychosoziale Beratung, Krisenintervention

- Angebot: diagnostisches, beratendes und therapeutisches Angebot für Familien mit behinderten Angehörigen/Kindern

- Weitere Infos: kommunale und kirchliche Träger (z. B. Krisendienst der Telefonseelsorge), detaillierte Auflistung der Beratungsstellen nach Regierungsbezirken unter www.intakt.info

Behindertenbeauftragte/r der Staatsregierung

- Angebot: Beratung, Vermittlung von Kontakten für die Belange behinderter Menschen (z. B. im Bereich Gesundheit, Mobilität)

- Weitere Infos: www.behindertenbeauftragte.bayern.de

Behindertenbeauftragte der Städte und Landkreise

- Angebot: Beratung, Vermittlung von Kontakten für die Belange behinderter Menschen

- Weitere Infos: jeweilige Kommunalverwaltung

Dienste der Offenen Behindertenarbeit

- Angebot: regionale und überregionale Beratungs- und Betreuungsdienste für Menschen mit Behinderung (Fachberatung bei speziellen Fragen aus dem Bereich der Behindertenhilfe, Familienentlastende Hilfen, Kurzzeitbetreuung, Fahrdienste, Assistenz und Integrationshilfen im Freizeit-, Wohn- und Arbeitsbereich u. a.)

- Weitere Infos: www.stmas.bayern.de/behinderte/offeneba/

Gemeinsame Servicestellen der Rehabilitationsträger

- Angebot: Gemeinsame Servicestellen der verschiedenen Rehaträger (u. a. ges. Krankenkassen, Bundesagentur für Arbeit, Sozialhilfeträger, Unfallversicherung, Rentenversicherung) bieten Beratung für Menschen mit Behinderung und Angehörige über Leistungsvoraussetzungen, Leistungen der Rehabilitationsträger sowie über Verwaltungsabläufe

- Weitere Infos: Übersicht über Adressen und Öffnungszeiten der örtlichen gemeinsamen Servicestellen unter www.vdr.de 
5.10. Psychisch kranke und behinderte Menschen - Psychiatrie-Grundsätze Bayern ${ }^{22}$

Die Bayerische Staatsregierung hat am 13. März 2007 die Grundsätze zur Versorgung von Menschen mit psychischen Erkrankungen in Bayern verabschiedet, die vom Bayerischen Staatsministerium für Arbeit und Sozialordnung, Familie und Frauen erarbeitet wurden. Damit wurde der Zweite Bayerische Landesplan zur Versorgung psychisch kranker und psychisch behinderter Menschen von 1990 fortgeschrieben.

Bei der Erarbeitung der Grundsätze zur Versorgung von Menschen mit psychischen Erkrankungen in Bayern (kurz: Psychiatrie-Grundsätze Bayern) wurden die psychiatrische Fachöffentlichkeit und die an der psychiatrischen, psychotherapeutischen und psychosomatischen Versorgung beteiligten Vereinigungen und Verbände in Bayern intensiv eingebunden. Im Rahmen des hierfür vom Bayerischen Sozialministerium eingerichteten Runden Tisches Psychiatrie hatten zehn Arbeitsgruppen spezifische Themen bearbeitet und ihre Berichte vorgelegt. Die zehn Arbeitsgruppenberichte bildeten die Grundlage für die Erarbeitung der Psychiatrie-Grundsätze Bayern. Diese Berichte der Arbeitsgruppen wurden gemeinsam mit den PsychiatrieGrundsätzen Bayern auf dieser Internetseite veröffentlicht.

Die psychiatrische, psychotherapeutische und psychosomatische Versorgungslandschaft hatte sich seit dem Zweiten Bayerischen Psychiatrieplan in allen Bereichen sowohl quantitativ als auch qualitativ in eindrucksvoller Weise weiter entwickelt. Mit den Grundsätzen zur Versorgung von Menschen mit psychischen Erkrankungen in Bayern wird diese Entwicklung weiter unterstützt und gefördert werden. Die Psychiatrie-Grundsätze Bayern sollen eine Orientierung für die Planung und Umsetzung von Maßnahmen zur Weiterentwicklung der Versorgung von Menschen mit psychischen Erkrankungen geben. 


\title{
4.5. Behindertenpolitik in Deutschland aus der Sicht behinderter Menschen - Eine Streitschrift für eine bessere Behindertenpolitik aus Sicht eines Betroffenen und „An- walts in eigener Sache“"
}

\author{
Von Alexander Drewes
}

1. Vorwort und Danksagung

2. Behindertenpolitiken aus der Sicht behinderter Menschen

2.1. Einstrahlung inter- und supranationalen Rechts in die deutschen Behindertenpolitiken

2.2. Behindertenpolitiken in Deutschland als Querschnittaufgaben versus Anhängsel der Sozialpolitik?

2.3. Organisationsrahmen, innerhalb dessen Behindertenpolitiken in Deutschland stattfinden

2.3.1. Der Deutsche Behindertenrat (DBR)

2.3.2. Behindertenbeiräte auf kommunaler und regionaler Ebene

2.3.3. Beauftragte für die Belange behinderter Menschen bei der Bundesregierung und bei den Landesregierungen

2.3.4. Grundsätzliches Demokratiedefizit

2.3.5. Lokale Ebene als eigentlicher Entscheidungsrahmen für Behindertenpolitik

2.3.6. Behindertenpolitiken als Anscheins-, Schimären- und Alibipolitiken

2.4. Einige Lebensbereiche, in denen fortschrittliche behindertenpolitische Ansätze greifen oder greifen sollten

2.4.1. Kindergarten/Hort

2.4.2. Schule

2.4.3. Arbeitsleben

2.5. Querschnittbereiche, in denen Behindertenpolitiken von Relevanz sind 178

2.5.1. Recht der umfassenden gleichberechtigten Partizipation behinderter Menschen als Ausfluss verfassungsrechtlicher Normierung und einfachrechtlicher Zielsetzung kontra Eingliederungshilfe als politisch gewolltem und zementiertem Fortbestand 
2.5.3. Zivilrechtliche Antidiskriminierung und öffentlich-rechtliches Benachteiligungsverbot

2.5.4. Barrierefreiheit

2.5.5. Kommunikation und Medien 


\section{Vorwort und Danksagung}

Während der diesem Werk zugrunde liegenden Tagung in München vom 20. bis 24 . Oktober 2007 reifte bei mir der Entschluss, entgegen der ursprünglichen Überlegung die Behindertenpolitiken in Deutschland insgesamt und unter Beteiligung so vieler Betroffenenverbände wie irgend möglich und sinnvoll darzustellen. Daneben, sozusagen als korrespondierendes Element, sollten die Beauftragten für die Belange behinderter Menschen bei der Bundesregierung wie den Landesregierungen und die behindertenpolitischen Sprecher der fünf Bundestagsfraktionen umfänglich Berücksichtigung finden, sodass sich auch sowohl die kontrapunktische Intention von Behindertenpolitiken als auch deren Auswirkungen hätte darstellen lassen.

Dem folgte eine - mehrfach wiederholte - Anfrage an die Verbände, die Behindertenbeauftragten ${ }^{1}$ und die behindertenpolitischen Sprecher. Die Anfrage bezog sich ausschließlich auf bei den Angefragten vorhandenes Material. Natürlich konnte man von den Angeschriebenen im Einzelnen nicht erwarten, dass sie eigenständig auf den umfangreichen Fragenkatalog in der Form eingehen würden, als das extra noch Thesenpapiere zu meinen Fragepunkten erarbeitet werden konnten.

Insgesamt ist zu konstatieren, dass sich in einem erschreckenden und auch deprimierenden Umfang sowohl die Verbände als auch die Behindertenbeauftragten und Fraktionssprecher unkooperativ gezeigt haben. Die Anfragen wurden vielfach überhaupt nicht beantwortet, von einer Antwort wurde teilweise unter den fadenscheinigsten Gründen abgesehen und in wenigstens einem Falle ging eine Antwort trotz Zusage bis heute nicht ein.

Bei den Verbänden bleiben einzig die beiden zu nennen, zu loben und positiv hervorzuheben, die sich auf Bundesebene mit den Belangen sehgeschädigter Menschen beschäftigen. Hier habe ich dem Deutschen Blinden- und Sehbehindertenverband (DBSV) sowie dem Deutschen Verein der Blinden und Sehbehinderten (DVBS) zu danken.

Als einziger Verband der Freien Wohlfahrtspflege hat sich DER PARITÄTISCHE um eine Antwort bemüht, wenngleich dieselbe auch auf das Persönliche Budget beschränkt blieb.

Ganz besonderen Dank sage ich Hubert Hüppe von der Fraktion der CDU/CSU im Deutschen Bundestag sowie seinem Parlamentskollegen Markus Kurth, der der Fraktion von Bündnis 90/DIE GRÜNEN angehört. Beide sind behindertenpolitische Sprecher ihrer jeweiligen Fraktionen, beide haben sich deutlich engagiert gezeigt und ausgesprochen umfänglich Material beigesteuert.

1 Der Begriff der Behindertenbeauftragten wird hier allenfalls zu Vereinfachungszwecken verwendet, da er - rein sprachlich - unsinnig ist. Korrekter wäre die längere und umständlichere Bezeichnung Beauftragter für die Belange behinderter Menschen, wie ihn z.B. die Beauftragte der Bundesregierung verwendet. Soweit in diesem Beitrag weiterhin Funktionsbezeichnungen auftauchen, sind dieselben ab und an lediglich in einer Geschlechtsform gewählt worden (dies dient ausschließlich der besseren Lesbarkeit des Beitrages und meint in keiner Weise die Nichtberücksichtigung der jeweils anderen Geschlechtsbezeichnung, die in jedem Falle mitgemeint ist). 
Als am hilfreichsten für die gesamte Arbeit hat sich Matthias Rösch erwiesen, der im Auftrag der damaligen - das Amt hat seit dem 14.01.2008 Ottmar Miles-Paul inne - Landesbeauftragten für die Belange behinderter Menschen in Rheinland-Pfalz Marita Boos-Waidosch Materialien zusandte.

Sehr weitergeholfen haben mir auch Klaus Lachwitz, stellvertretender Bundesgeschäftsführer und Justiziar der Bundesvereinigung Lebenshilfe als auch Prof. Dr. Volker Neumann, Ordinarius an der Humboldt-Universität Berlin, ersterer mit allgemeinen Tipps und Ratschlägen, letzterer mit sowohl sachdienlichen Hinweisen zum Thema Persönliches Budget als auch dem Zur-Verfügung-Stellen von Material.

Dank habe ich weiter zu sagen dem Behindertenbeauftragten des Landes BadenWürttemberg Dieter Hillebrand und dem Landesbeauftragten für die Belange von Menschen mit Behinderungen im Saarland Wolfgang Gütlein, die jeweils teilweise brauchbares Material zur Verfügung gestellt haben. Detlef Jähnert, persönlicher Referent des Behindertenbeauftragten des Landes Niedersachsen Karl Finke, hat mir wertvolle Hinweise auf die Beschäftigung von Menschen in Werkstätten für Behinderte gegeben.

So ist dies denn eine Arbeit geworden, die in Teilbereichen spekulativ ist, dies auch bleiben muss, weil sich das Fehlen der Beantwortung mancher Fragestellung nicht einmal durch die intensive Nutzung des Mediums Internet heilen ließ und die (nicht antwortenden) Angeschriebenen großenteils nicht einmal in der Lage sind, ihre eigenen behindertenpolitischen Ansätze in schriftlicher Form auf ihren Webseiten darzulegen.

Deshalb ließ sich auch der ursprüngliche Titel des Beitrages „Behindertenpolitik in Deutschland aus der Sicht der Interessenvertretungen behinderter Menschen“" nicht mehr halten. Wo die entsprechenden Verbände nicht willens oder in der Lage waren, zumindest in rudimentärer Form informativ tätig zu werden, kann über eine Gesamtschau der Behindertenpolitik in Deutschlang nicht mehr die Rede sein.

Deswegen war eine Änderung am ursprünglichen Titel des Beitrages vorzunehmen. Der zweite Grund neben dem fast völligen Desinteresse der Verbände liegt darin, dass die Politikansätze, die auf Bundes-, Länder- und kommunaler Ebene verfochten werden, teilweise dermaßen unterschiedlich ausgestaltet sind, dass man von Politik im Singular nicht mehr sprechen kann. Da zunehmend der internationale und insbesondere supranationale Ansatz in das deutsche Sozialrecht stetig zunimmt, ist auch hier eher von Politiken zu sprechen.

Der ursprüngliche wissenschaftliche Ansatz, über Behindertenpolitiken in Deutschland objektiv und umfassend berichten zu wollen, ließ sich nach all dem so nicht mehr durchhalten. Darunter leiden gezwungener Maßen in Teilen der Fußnotenapparat und wohl auch der Tiefgang der Arbeit. Allerdings habe ich mich nichtsdestotrotz bemüht, soweit dies irgend möglich war, ein Substrat dessen, was für behinderte Menschen auf der politischen Agenda steht und ihnen „unter den Nägeln brennt", herauszuarbeiten. 


\section{Behindertenpolitiken aus der Sicht behinderter Menschen}

\subsection{Einstrahlung inter- und supranationalen Rechts in die deutschen Behinderten- politiken}

Wie der Beitrag von Schulte in diesem Band eindrücklich zeigt, ist das deutsche Recht ohne internationale Einflüsse, insbesondere aber denjenigen der Europäischen Union überhaupt nicht mehr denkbar.

So verhält es sich - auch, gerade und schon seit längerer Zeit - im Bereich des Sozialrechts und zunehmend mehr auch im spezifischen Bereich der Benachteiligungsverbote für behinderte Menschen und des (zivilrechtlichen) Schutzes vor Diskriminierung.

$\mathrm{Zu}$ nennen sind hier vorrangig die Behindertenrechtskonvention der Vereinten Nationen (VN) vom 06.12.2006 ${ }^{2}$, die Grundrechte-Charta der Europäischen Union (EU) vom 07.12.2000 ${ }^{3}$ und die Gleichbehandlungsrichtlinie der EU vom 27.11.20004. Ausfluss der Grundrechte-Charta und der Gleichbehandlungsrichtlinie waren in der Folge das Behindertengleichstellungsgesetz (BGG) ${ }^{5}$ wie auch das Allgemeine Gleichbehandlungsgesetz (AGG) ${ }^{6}$ auf Bundesebene. Auf ein Teilhabesicherungsgesetz warten behinderte Menschen allerdings seit Jahren vergeblich ${ }^{7}$. Um ein solches Gesetz zu verhindern, entblödet sich die Bundesregierung nicht einmal mehr, die VN-Behindertenrechtskonvention falsch übersetzen zu lassen ${ }^{8}$. Ohne die

2 Convention on the Rights of Persons with Disabilities, United Nations General Assembly, Dec., 06., 2006, Sixty-first session, item 67 (b); in Deutschland noch nicht ratifiziert, international seit dem 03.05.2008 in Kraft; vgl. hierzu: http://www.kobinet-nachrichten.org/cipp/kobinet/ custom/pub/content,lang,1/oid,17947/ticket,g_a_s_t (Stand: 30.05 .2008$)$.

3 Amtsblatt (AB1.) der Europäischen Gemeinschaften (EG) vom 18.12.2000, C. 164/1.

4 Richtlinie 2000/78/EG des Rates vom 27.11.2000 zur Festlegung eines allgemeinen Rahmens für die Verwirklichung der Gleichbehandlung in Beschäftigung und Beruf, Amtsblatt der EG vom 21.12.2000, L 303/16.

5 Gesetz zur Gleichstellung behinderter Menschen (Behindertengleichstellungsgesetz [BGG]) vom 27.04.2002 (BGB1. I S. 1467, 1468), zuletzt geändert durch Art. 12 des Gesetzes zur Änderung des Vierten Buches Sozialgesetzbuch und anderer Gesetze vom 19.12.2007 (BGB1. I [Nr. 67], S. 3024, 3034). Zur Entstehungsgeschichte des BGG vgl.: Miles-Paul/Drewes, Bundesgleichstellungsgesetz setzt neue Standards, auf der Website des Netzwerks Artikel 3 e.V. vom 18.04.2002: http://www.netzwerk-artikel-3.de/wsite/nstandards.htm (Stand: 30.05.2008).

6 Allgemeines Gleichbehandlungsgesetz (AGG) vom 14.08.2006 (BGBl. I [Nr. 39], S. 1897), zuletzt geändert durch Art. 19 Ziffer 10 des Gesetzes zur Neuregelung des Rechtsberatungsrechts vom 12.12.2007 (BGB1. I [Nr. 63], S. 2840).

7 Die Forderung nach einem Teilhabesicherungsgesetz wird zwischenzeitlich nicht mehr nur von Betroffenen und Teilen des politischen Establishments, sondern neuerdings sogar von Seiten der Justiz, hier insonderheit von Masuch, dem seit dem 01.01.2008 amtierenden Präsidenten des BSG, forciert. S. hierzu: Frankfurter Allgemeine Zeitung (FAZ), Nr. 24/2008 vom 29.01.2008, S. 18 Sp. 1 bis 4 .

8 S. hierzu die u.a. auf einer durch die Behindertenaktivistin Arnade veranlasste (s. hierzu: http://www.kobinet-nachrichten.org/cipp/kobinet/custom/pub/content,lang,1/oid,17066/ticket,g_a s_t [Stand: 30.05.2008)) - teilweise mdl. - Anfragen des Abgeordneten Kurth (Fraktion Bündnis 90/DIE GRÜNEN) im Deutschen Bundestag an die Bundesregierung sowie die mdl. Antworten des Parlamentarischen Staatssekretärs im Bundesministerium für Arbeit uns Soziales (BMAS), Thön- 
supranationalen Vorgaben der EU wäre die deutsche Behindertenpolitik heute - trotz des teilweise immensen Engagements hauptsächlich von Einzelpersonen, aber auch von einzelnen Verbänden der Behindertenbewegung - noch nicht einmal so weit, wie sie es mittlerweile ist, bei allen Mängeln, von denen hier in der Folge noch einige aufzuzeigen sein werden.

\subsection{Behindertenpolitiken in Deutschland als Querschnittaufgaben versus Anhängsel der Sozialpolitik?}

Obschon weiter unten noch näher auf die Ansiedlung der Behindertenbeauftragten von Bund und Ländern im politischen Umfeld einzugehen ist, ist bereits hier festzustellen, dass der Ansatz, den die Politik - insbesondere auf Bundesebene - zeitweilig selbst verfochten hat, dass nämlich Behindertenpolitik eine umfassende und qualitativ hochwertige und eigenständige Querschnittaufgabe darstelle (und eben kein schieres Anhängsel der Sozialministerialbürokratie), spätestens mit Beginn der GroBen Koalition auf Bundesebene nach der Bundestagswahl im Jahre 2005 nicht weiter verfochten wird. Die Stellung des bzw. der Beauftragten der Bundesregierung für die Belange behinderter Menschen wurde in den letzten Legislaturperioden zwei Mal hin- und hergeschoben. Nach der Bundestagswahl $2002^{9}$ zunächst vom damaligen Bundesministerium für Arbeit und Sozialordnung (BMAS) hin zum neu entstandenen Bundesministerium für Gesundheit und Soziale Sicherung (BMGS), nur um - nach einem neuerlichen Revirement der Ministerialzuschnitte - 2005 zum sodann neuerlich geschaffenen BMAS, nunmehr allerdings mit der - richtigeren Bezeichnung Soziales statt Sozialordnung, zurück zu wechseln. Weder das häufige „Bäumchen-wechsel-Dich-Spiel“ des Verschiebebahnhofs Behindertenbeauftragte/r

nes, vom 23.01.2008 (Plenarprotokoll 16/138 vom 23.01.2008, Punkt 25, S. 14562 A und Punkt 26, S. 14563 A) sowie meinen Leserbrief in den kobinet-nachrichten hierzu vom 27.01.2008 unter dem Titel „Was interessiert uns der Deutsche Behindertenrat?““: http://www.kobinet-nachrichten.org/ cipp/kobinet/custom/pub/content,lang,1/oid,17192/ticket,g_a_s_t). Hinsichtlich des Behinderungsbegriffs hinkt die Bundesregierung der neuesten Diskussion ganz bewusst hinterher: vgl. hierzu die Antwort (BT-Drs. 16/9283) auf die Große Anfrage von Bündnis '90/DIE GRÜNEN (BT-Drs. 16/6825), S. 11 auf die Fragen 25 und 26, wo die neuere Terminologie der WHO einfach nicht zur Kenntnis genommen wird. Es lohnt sich, die gesamte Beantwortung dieser Großen Anfrage durchzulesen, weil in ihr auf bedrückende Weise das offenkundige Desinteresse, um nicht zu schreiben die Gleichgültigkeit offenbar wird, die diese Bundesregierung beim Thema behinderte Menschen umtreibt.

9 Nach dem Wechsel im Sozialministerium von Walter Riester (SPD) hin zu Ulla Schmidt (SPD), der dadurch „fällig“ wurde, dass für Wolfgang Clement (ebenfalls SPD) ein „Superministerium“ für Wirtschaft und Arbeit (BMWA) geschaffen wurde, wanderte die Funktion des damaligen Behindertenbeauftragten Karl Hermann Haack auch zum Gesundheits- und Sozialministerium (das sich damals BMGS kürzte). Nun hätte man Clement aufgrund seiner - damals schon - stark wirtschaftspolitischen Ausrichtung auch nicht wirklich zugetraut, sich für behindertenpolitische Fragestellungen in irgendeiner Form zu engagieren. Allerdings war auch von Ulla Schmidt in der Zeit, als die Funktion des Behindertenbeauftragten im BMGS angesiedelt war, erstaunlich wenig zu den vielfältigen Thematiken zu hören oder zu lesen. 
noch das nahezu vollständige Fehlen von Interesse der höchsten Ministerialebene an Topoi der Behindertenpolitik müssen jedoch zwangsläufig dafür sorgen, dass auch eine schlechte Politik gemacht wird.

Eine mehr denn löbliche Ausnahme stellte hier der Arbeits- und Sozialminister im ersten Kabinett Gerhard Schröder (SPD), Walter Riester (SPD), dar. Dieser ehe stille und für einen Politiker ungewöhnlich fachlich interessierte Minister hat wesentlich zu der Schaffung beispielsweise des BGG mit beigetragen (er hatte allerdings mit Karl Hermann Haack auch einen „Lautsprecher“, der das Anliegen derart penetrant in der Öffentlichkeit vertrat, dass zum Schluss selbst die - ursprünglich skeptische CDU/CSU-Bundestagsfraktion - zum Glück - „einknickte“ und dem Gesetz im Deutschen Bundestag zustimmte ${ }^{10}$ ).

So ist die Einordnung verschiedener sozialrechtlicher Materien in das Sozialgesetzbuch (SGB), wie die Jugendhilfe, die Renten- und Unfallversicherung und die Arbeitsförderung weitgehend geräuschlos gelungen (weil die Materien nicht im politischen Diskurs „zerrieben“ wurden, sondern fast ausschließlich von fachlich kompetenten Ministerialbeamten der oberen und mittleren Ebene erarbeitet und von Sozialpolitikern ihren Fraktionen ,verkauft“ wurden). Anders verhielt es sich lediglich bei den - seit jeher - politisch problematischen Bereichen der Gesundheitsfürsorge, der Pflege und den Grundsicherungssystemen (und seit einigen Jahren auch bei der Rente, seitdem klar ist, dass die Gesetzliche Rentenversicherung wohl spätestens Mitte des Jahrhunderts eine Grund sichernde Rente nicht mehr wird garantieren können).

Eine tatsächlich als solche zu bezeichnende Behindertenpolitik auf Bundesebene gibt es eigentlich erst seit dem ersten Kabinett Schröder, also seit 1998, und dies auch im Grunde genommen nur deshalb, weil sich mit Karl Hermann Haack ein ursprünglich von der Behindertenbewegung mehr als argwöhnisch beäugter „Sachwalter“ der Behindertenpolitik fand. Sein Vorgänger Otto Regenspurger (CSU) war in den Jahren zwischen 1982 und 1998 überwiegend durch schiere Untätigkeit aufgefallen und trieb vielen Vertretern behinderter Menschen schon die Zornesröte ins Gesicht, wenn sein Name nur fiel. Regenspurger fiel vor allem dadurch unangenehm auf, dass er meinte, sich selber jeglichen Ansatz von Fortentwicklung im Bereich selbst der Rehabilitation (und viel mehr war Behindertenpolitik unter den fünf Kabinetten Helmut Kohl nicht) zuschreiben zu müssen. Man kann die Politik für behinderte Menschen in den Jahren nach Schaffung des Schwerbehindertengesetzes (SchwbG) $1974^{11}$ bis zum Jahre 1998 vielleicht als schiere Rehabilitationspolitik bezeichnen; einziger tatsächlicher Lichtblick in dieser Zeit war die Einfügung

$10 \mathrm{Zu}$ der überwiegend skeptischen Grundhaltung der CDU/CSU-Bundestagsfraktion vgl. die Aussagen der Abgeordneten Nolte (Plenarprotokoll des Deutschen Bundestages 14/221 vom 28.02.2002, S. 21862 C)

11 Gesetz zur Sicherung der Eingliederung Schwerbehinderter in Arbeit, Beruf und Gesellschaft in der Fassung (i.d.F.) der Bekanntmachung vom 26.08.1986 (BGB1. I, S. 1421, 1550), zuletzt geändert durch Art. 20 des Gesetzes vom 20.12.2000 (BGBl. I [Nr. 57], S. 1827, 1844), außer Kraft gesetzt durch Art. 63 des Sozialgesetzbuch - Neuntes Buch (SGB IX) - Rehabilitation und Teilhabe behinderter Menschen vom 19.06.2001 (BGBl. I [Nr. 27], S. 1046, 1138). 
der Textmarke „Niemand darf wegen seiner Behinderung benachteiligt werden“ als Art. 3 Abs. 3 Satz 2 des Grundgesetzes ${ }^{12}$. Auch das eigentlich nur ein Wahlkampfschlager, weil der damalige Bundeskanzler Helmut Kohl meinte, hier mit dem Versprechen auf einer Verbandstagung noch ein paar Wählerstimmen behinderter Menschen ,einheimsen“ zu können.

Verblüffend ist weiterhin, dass Behindertenpolitik in der Deskription des Behindertenrechts innerhalb der Sozialrechtswissenschaft praktisch überhaupt nicht stattfindet. Liest man sich die kürzlich in Neuauflage erschienenen Lehrbücher zum Thema Sozialrecht von Bley, Kreikebohm und Marschner ${ }^{13}$, Eichenhofer ${ }^{14}$, Igl und Welt $^{15}$ i, Kokemoor $^{16}$, Muckel $^{17}$ sowie von Waltermann ${ }^{18}$ durch, stellt man fest, dass dort zwar regelmäßig von Behindertenrecht geschrieben wird, der verrechtlichte Anteil aber fast ausschließlich auf das materielle Recht des vormaligen SchwbG reduziert wird. Selbst ein so umfängliches Werk wie das kürzlich erst neu erschienene und neben dem „Sozialrechtshandbuch“ von v. Maydell/Ruland/Becker ${ }^{19}$ umfänglichste monographische Werk "Sozialrecht“ von Erlenkämper und Fichte ${ }^{20}$ handelt die Teilhabeaspekte allenfalls en passant und mit sichtlichem Desinteresse am Detail ab, wenngleich man es dem Werk schon beinahe anrechnen muss, sich mit der Materie überhaupt auseinanderzusetzen.

Dies, also die fast vollständig fehlende Berücksichtigung der über den reinen Rehabilitationsaspekt hinausgreifenden Fragestellungen, trägt allerdings der Tatsache Rechnung, dass Teilhabeaspekte wie auch der - zumindest im Arbeitsrecht formulierte - Antidiskriminierungsgrundsatz juristisch nach wie vor kaum eine Rolle spie-

12 Eingefügt durch Art. 1 Ziff. 1.b. des Gesetzes zur Änderung des Grundgesetzes vom 27.10.1994 (BGBl. I, S. 3146).

13 Bley/Kreikebohm/Marschner, „Sozialrecht“, 9. Aufl., 2007, Neuwied (Luchterhand), S. 293-295; bei denen behinderte Menschen allenfalls als Randbemerkung stattfinden, im Sachregister nach wie vor unter „Behinderte“ firmieren und die auch ansonsten, selbst in der gewählten, schier unglaublichen Kürze ausgesprochen „schlampig“ zitieren.

14 Eichernhofer, „Sozialrecht“; 6. Aufl., 2007, Tübingen (Mohr Siebeck), S. 285-291 (Rn. 518-531, § 25).

15 Igl/Welti, „Sozialrecht“, 8. Aufl., 2007, Neuwied (Werner), S. 341-356 (Kapitel 13), die dem Rehabilitations- und Teilhaberecht für behinderte Menschen immerhin noch ein eigenes Kapitel einräumen.

16 Kokemoor, „Sozialrecht“, 2. Aufl., 2006, Köln/Berlin/München (C. Heymanns), S. 242-244 (Rn. 433-437; Kap. 4.II.8), der es tatsächlich schafft, das gesamte Rehabilitations- und Teilhaberecht auf ganzen zweieinhalb Seiten abzuhandeln. Dementsprechend oberflächlich, nichtssagend und miserabel ist sodann auch die Qualität der Abhandlung.

17 Muckel, „Sozialrecht“, 2. Aufl., 2007, München (C. H. Beck), S. 251-264 (§ 14.VI, Rn. 50-71).

18 Waltermann, „Sozialrecht“, 6. Aufl., 2006, Heidelberg (C. F. Müller), S. 258-261 (Rn. 537.-542, § 16 III), der gleich das ganze Behindertenrecht innerhalb des Bereiches soziale Förderung verortet, obwohl er in seiner Absatzüberschrift noch - richtiger Weise - von der Teilhabe behinderter Menschen schreibt.

19 Das grundlegende und kürzlich in 4. Aufl., Baden-Baden (Nomos) neu erschienene, von v. Maydell, Ruland und Becker hrsg. „Sozialrechts-Handbuch“ konnte aufgrund des späten Erscheinungstermins leider nicht mehr berücksichtigt werden.

20 Erlenkamper/Fichte, „Sozialrecht. Allgemeine Rechtsgrundlagen. Sozialgesetze. Verfahrensrecht“, unter Mitarbeit von Fock und Fischer; 6. Aufl., Köln (Luchterhand), hier insbes. die Ziffer 19.1. auf den S. 616-665 des Werkes. 
len. Bereits hier ist weiterhin zu konstatieren, dass der antidiskriminatorische Sinngehalt des Allgemeinen Gleichbehandlungsgesetzes (AGG) so schwammig und selbst für Juristen kaum nachvollziehbar formuliert ist, dass es nicht wirklich wundert, dass sich bislang kaum Fallgestaltungen haben finden lassen, auf die das Gesetz passen würde. Nicht dass deshalb in Deutschland auch nur einen Deut weniger diskriminiert würde, die gesetzliche Grundlage gibt nur kaum etwas her, um gegen diese Missstände auch tatsächlich vorgehen zu können.

Weshalb das alles so ist wie es ist, weshalb es also elementar an nahezu jeglicher tatsächlichen Umsetzung der neueren gesetzlichen Möglichkeiten über die reine politische Rhetorik von Selbstbestimmung und gleichberechtigter Teilhabe hinausgehend mangelt, davon erzählt der Abschnitt 2.3., der einige der Protagonisten darstellt, die in Deutschland - entweder auf gesetzlicher Grundlage oder durch Eigeninitiative - Politik für behinderte Menschen als selbst Betroffene „machen“.

\subsection{Organisationsrahmen, innerhalb dessen Behindertenpolitiken in Deutschland stattfinden}

\subsubsection{Der Deutsche Behindertenrat (DBR)}

Seit dem 03. Dezember 1999 existiert ein Deutscher Behindertenrat, der die drei wesentlichen „Säulen“ der Behinderten(selbst)hilfe umfasst, also die traditionellen Sozialverbände (VdK und SoVD, nicht hingegen die ausschließlich in Ostdeutschland agierende Volkssolidarität), die Bundesarbeitsgemeinschaft (BAG) Selbsthilfe. mit einigen ihrer Mitgliedsverbände als die „traditionelle“ Behindertenselbsthilfe und die sog. ,autonome Behindertenbewegung“" (bestehend z.B. aus dem Club Behinderter und ihrer Freunde [CeBeeF] und der Interessenvertretung „Selbstbestimmt Leben" in Deutschland [ISL]). Der DBR koordiniert sich durch einen Arbeitsausschuss und wird durch einen Sprecherrat vertreten. Beide Gremien zeichnen sich durch eine jährliche Rotation innerhalb der Führung aus, die alle drei „Säulen“ gleichermaßen berücksichtigt.

Vergegenwärtigt man sich, welch einen Kraftakt es dargestellt hat, die drei „Säulen“ der Behindertenbewegung überhaupt „unter einen Hut" zu bekommen, macht es das einem vielleicht ein wenig verständlicher, dass der DBR sich seit seiner Gründung weitgehend darin erschöpft, sich alle paar Monate einmal zu einem mehr oder weniger wichtigen behindertenpolitischen Thema in der Öffentlichkeit zu Wort zu melden und mit wiederum mehr oder weniger stichhaltigen Argumenten (das hängt sehr stark davon ab, wer gerade die „Führung“ in den Gremien innehat) ein Thema problematisiert: und in die Sachdebatte eingreift. Zeichnen sich die traditionellen Sozialverbände überwiegend dadurch aus, dass sie mit populistischem „Wortgeklingel“" selbst dieses Gremium noch als allgemeinpolitische Plattform missbrauchen, steht bei der Selbsthilfe und den „Autonomen“ die Sacharbeit im Vordergrund.

Doch selbst, wenn das Gremium einmal inhaltlich Vorschläge macht, wird es meistenteils von der Politik torpediert oder überhaupt nicht wahrgenommen. Ein 
besonders anschauliches Beispiel hierfür war ein - durchaus vermeidbares - Missverständnis zwischen dem DBR und der ggw. Behindertenbeauftragten der Bundesregierung im Jahre 2007. Die Beauftragte hatte eingeladen, um über neue Ansätze innerhalb des Rechts der Eingliederungshilfe zu diskutieren und ... alle, alle kamen sie. Allerdings war die Veranstaltung lediglich vor kurzer Dauer, um genau zu sein, sie hielt gerade einmal vierzehn Tage vor ${ }^{21}$. Hernach verkündete die Beauftragte - es liest sich selbst die Presseerklärung noch so, als sei nicht in Wahrheit mit nichts anderem zu rechnen gewesen - die Verbände hätten doch tatsächlich ein Teilhabeleistungsgesetz gefordert, sie sei allerdings nur bereit und willens gewesen, über die „Reform“ der Eingliederungshilfe zu diskutieren.

Dies Beispiel zeigt eindrücklich, wie Politik zwischen den Betroffenen und der Exekutive nach wie vor funktioniert. Die Kommunikation besteht im günstigsten Fall häufig aus Missverständnissen, im ungünstigeren Fall - wie unter der gegenwärtigen Großen Koalition ständig - wird Behindertenpolitik schlicht und einfach nicht mehr betrieben. Probleme bleiben hier nicht deshalb liegen, weil man sich zwischen den verschiedenen Parteien nicht einigen könnte. Selten hat die SPD so eindrücklich bewiesen, wie gleichgültig ihr die Klientel behinderter Menschen ist, wie unter der von ihr mit zu verantwortenden Regierungsägide seit 2005. Unter dem vorherigen Bundeskanzler Gerhard Schröder liefen benachteiligte und diskriminierte Minderheiten wenigstens noch unter dem Terminus „Gedöns“, unter der Kanzlerin Angela Merkel findet eine wie auch immer geartete Behindertenpolitik überhaupt nicht mehr statt. Selbst wenn der DBR also das Aktionsbündnis wäre, für das er sich ausgibt (ein Bündnis mag er wohl sein, einzig, es ermangelt weitgehend an der nach außen hin sichtbaren Aktion): Bei nahezu vollständigem Desinteresse des politisch gestaltenden Apparates können Betroffene über weite Strecken allenfalls agitatorisch agieren. Eine wirkliche Einflussnahme auf politische Prozesse ist schier unmöglich, wie eindrücklich ein Überblick über die gesetzgeberischen Initiativen aus dem Bereich der Behindertenpolitik im Deutschen Bundestag zeigt. Im Verlauf der gegenwärtigen 16. Wahlperiode stammen nahezu sämtliche Gesetzgebungsinitiativen, Anfragen und Beschlussvorlagen zum Thema Behindertenpolitik aus der Feder von Abgeordneten der Oppositionsfraktionen von Bündnis 90/DIE GRÜNEN und DIE LINKE. Das kann nicht alleine mit dem bürgerschaftlichen Impetus der Grünen und dem Sozialfetisch der Linken und genauso wenig damit erklärt werden, dass die wesentlichen Gesetzgebungswerke im Bereich der Behindertenpolitik ,abgefrühstückt" seien. Arbeitsgebiete gibt es nach wie vor zuhauf, wie die regelmäßig erscheinenden Berichte der behindertenpolitischen Sprecher der CDU/CSU-Bundestagsfraktion und derjenigen von Bündnis '90/DIE GRÜNEN, Hubert Hüppe und Markus Kurth, zeigen. Einzig, in der Regierung gibt es offenkundig niemanden, den

21 Die Nachricht „Arbeitskreis Eingliederungshilfe stellt seine Arbeit ein“ von Miles-Paul vom 18.09.2007 in den kobinet-nachrichten: http://www.kobinet-nachrichten.org/cipp/kobinet/ custom/pub/content,lang,1/oid,16074/ticket,g_a_s_t; hierzu ein Kommentar von Drewes unter dem Titel „Wer ist Koch und wer ist Kellner?“ im selben Medium: http://www.kobinet-nachrichten.org/ cipp/kobinet/custom/pub/content,lang,1/oid,16310/ticket,g_a_s_t (Stand: jeweils 30.05.2008). 
das auch nur in Ansätzen zu interessieren scheint oder das Personal ist mit eher minderer Kompetenz auf dem hier in Rede stehenden Sachgebiet ,gesegnet“.

So bleibt dem DBR denn nur zu wünschen, dass er bei veränderten politischen Bedingungen ab dem kommenden Jahr auf politisches Personal treffen möge, das seine Initiativen ernster nimmt und den DBR mehr dazu veranlassen könnte, tatsächlich das „Sprachrohr“ behinderter Menschen auf Bundesebene zu sein.

\subsubsection{Behindertenbeiräte auf kommunaler und regionaler Ebene}

Zunächst einmal mag man sich beim Durchlesen der obigen Gliederung gewundert haben, dass hier zunächst die Beiräte und erst hernach die Beauftragten auftauchen. Das hat seinen guten Grund darin, dass in den Beiräten, so sie denn überhaupt existieren, regelmäßig selbst betroffene, also behinderte Menschen sitzen, die Problemschwerpunkte alltäglich ,am eigenen Leibe“ erfahren.

Dabei sind die einzelnen Bundesländer bei der Schaffung ihrer Gleichstellungsgesetze höchst unterschiedliche Wege gegangen. Teilweise findet sich zu den Beiräten kein einziges Wort in den Gleichstellungsgesetzen (ein auch hier unrühmliches Beispiel sind Baden-Württemberg und der Freistaat Sachsen). In anderen Bundesländern - wie in Hessen - gibt es zwar kommunale Beiräte, die allerdings lediglich aufgrund Satzung geschaffen sind. Der Schaffung eines landesgesetzlichen Rahmens zur Einführung von kommunalen Beiräten steht mittlerweile - spätestens seit der ersten Föderalismusreform - vermutlich auch das Konnexitätsprinzip entgegen, wonach diejenige Instanz, die durch eine Gesetzgebung Kosten an eine andere Instanz verlagert, die daraus entstehenden Kosten auch zu tragen hat.

Auf regionaler Ebene, also in den Freistaaten, Bundesländern und Stadtstaaten, sind Behindertenbeiräte fast ausnahmslos installiert worden, allerdings regelmäßig mit ausschließlich beratender und der Ministerialverwaltung zuarbeitender Funktion. Nun ist es eine Selbstverständlichkeit, dass ein - im Übrigen demokratisch in keiner Weise legitimierter - Funktionsträger wie ein Behindertenbeirat zur Gesetzesinitiative (oder zur Satzungsinitiative auf kommunaler Ebene) nicht berufen sein kann, da es hiefür schon an der parlamentarischen und mithin demokratischen Legitimationsgrundlage fehlt. Ein Initiativrecht wäre mithin auch nur aufgrund Wahl vorstellbar, ähnlich den Instrumenten wie des Volksbegehrens und der Volksabstimmung. Dem steht jedoch durchaus ein umgreifendes Interesse vonseiten der Betroffenenverbände entgegen. Nicht alleine, dass man sich über die Jahre und Jahrzehnte natürlich relativ gut eingerichtet hat. Eine Wahl birgt ja auch immer das Risiko, nicht gewählt zu werden, zudem unterliegt man in einem Wahlkampf auch dem Zwang, bestimmtes Wahl- und Stimmverhalten sowie politische Opportunitätsgrundsätze erläutern zu müssen und steht deshalb unter einem gewissen Erklärungs"zwang", warum man eigentlich wiedergewählt werden will. So scheint es nicht nur mein individueller Eindruck zu sein, dass man innerhalb des Verbandswesens letztlich ganz zufrieden damit ist, auf regionaler Ebene regelmäßig mehr oder minder „ernannt“ zu werden. Zugegeben, es hat ja auch etwas von Huld und Gottesgnadentum, vom demokratisch 
selbst nicht legitimierten Beauftragten bzw. - legalistischer - von immerhin entweder durch die oder den Ministerpräsidenten/in ernannten oder sogar vom Landtag gewählten Minister oder der Ministerin „ernannt“" worden zu sein. Nur, mit Demokratie hat das alles nichts zu tun, und das bei einer Vertretung von immerhin $10 \%$ der Gesamtbevölkerung.

\subsubsection{Beauftragte für die Belange behinderter Menschen bei der Bundesregierung und bei den Landesregierungen}

Im Gefolge der Schaffung des BGG haben sämtliche Länder, Stadt- und Freistaaten (zwischenzeitlich, mit reichlicher Verspätung, sogar die Hansestadt Bremen) in ihren Landesgleichstellungsgesetzen Beauftragte für die Belange behinderter Menschen bei der jeweiligen Landesregierung installiert ${ }^{22}$. In aller Regel ernennt der Regierungschef für die jeweils laufende Legislaturperiode. Das hat den Nachteil, dass die Beauftragten letztlich von der Exekutive abhängig sind (was sie hinsichtlich der Gutwilligkeit der Informationspreisgabe durch die Verwaltung jedoch grundsätzlich sind, steht ihnen doch, anders als z.B. den Bürgerbeauftragten, nicht einmal ein Akteneinsichtsrecht zu). Nur in wenigen Ländern - wie dem Freistaat Bayern - kann ohne schwerwiegenden Grund nicht vor Ablauf der Legislaturperiode abberufen werden. Welches Gewicht alleine dem Umstand beizumessen ist, dass ein Beauftragter gewählt ist (abgesehen davon, dass es dies der einzige Beauftragte ist, den das Grundgesetz explizit erwähnt), zeigt sich an der Funktion der Wehrbeauftragten, der vom Deutschen Bundestag gewählt wird und der weitreichende Befugnisse innerhalb des Verwaltungsapparates der Bundeswehr hat. Vergleichbares - allerdings mit einer wesentlich umfänglicheren Rechts vertretenden Möglichkeit - kennt nur das schwedische Ombudsmannwesen ${ }^{23}$, das ich für den deutschen Rechtsraum als für durchaus vorbildhaft halte. Der Ombudsmann ist gegenüber der Exekutive vollständig unabhängig, an Weisungen nicht gebunden und funktioniert gerade deshalb als außerprozessual ungewöhnlich effektive Rechtsinstanz.

Durchaus nicht nötig ist es deshalb, dass der oder die Beauftragte selbst Volljurist ist. Das würde allerdings bedeuten, dass man eine „Ombudsperson für die Belange behinderter Menschen“ mit einem sowohl personellen als auch sächlichen und finanziellen Apparat ausstatten müsste, der dem Gewicht der Aufgabe auch die nötige Geltung und Außenwirkung verleihen kann. Gegen welche politischen Widerstände

22 In Mecklenburg-Vorpommern wird das Amt des Behindertenbeauftragten vom Bürgerbeauftragten im wahrsten Sinne des Wortes „,mit erledigt“. Bürgerbeauftragte kennen ansonsten noch der Freistaat Thüringen und das Land Schleswig-Holstein, hier gibt es neben den Bürgerbeauftragten allerdings jeweils noch Behindertenbeauftragte mit eigenständiger Funktionsbezeichnung und einem eingeschränkten - Aufgabenkatalog.

23 Vgl. zum schwedischen Ombudsleutewesen den ausgesprochen informativen und sehr gute lesbaren „Landesbericht Schweden“ von Köhler, in: von Maydell/Pitschas/Schulte (Hrsg), Behinderung in Asien und Europa im Politik- und Rechtsvergleich (Studien aus dem Max-Planck-Institut für ausländisches und internationales Sozialrecht, Bd. 26), 2003, Baden-Baden (Nomos), S. 57, 72 ff. 
ein solcher neuer Funktionsparameter stößt, hat sich eindrücklich bei der Installation der Antidiskriminierungsstelle des Bundes ${ }^{24}$ in der Folge der Schaffung des AGG gezeigt. Die Antidiskriminierungsstelle darf nichts, sie soll nichts dürfen und sie kann nicht einmal - anders als der Löwe, der später zum Papiertiger mutiert - springen, um sodann allenfalls noch flatternd zu landen.

Vor der Installation eines solchen Ombudswesens im Sozialrecht schreckt die deutsche Gesetzgebung in ihrer - in dieser Hinsicht nach wie vor vorhandenen Obrigkeitshörigkeit nach wie vor zurück. Nicht alleine, dass die Funktionsparameter der Behindertenbeauftragten meistenteils überhaupt nicht präzise um- und beschrieben sind, sie hängen vielfach auch erheblich von dem- oder derjenigen ab, der oder die das Amt innehat (was letzten Endes dazu führt, dass das Amt als solches für exponierte und qualifizierte Vertreter der Behindertenbewegung eigentlich völlig unattraktiv ist). Bereits unter 2.2. habe ich aufzuzeigen versucht, wie abhängig das Amt des Beauftragten bislang von seinem Inhaber ist. Das ist auf Bundesebene nicht anders als auf Landesebene. Auch hier gibt es „starke“ Beauftragte (RheinlandPfalz, Freistaat Bayern), solche, die zwar eine gute Öffentlichkeitsarbeit betreiben, aber keine wirkliche politisch starke Funktion in der Landesverwaltung haben (z.B. Niedersachsen, Nordrhein-Westfalen) sowie solche, die in einer breiteren Öffentlichkeit überhaupt nicht wahrgenommen werden (z.B. Schleswig-Holstein, Hessen).

Unstreitig scheint mir, dass es Sinn macht, als Behindertenbeauftragte nur selbst behinderte Menschen einzusetzen (man käme ja wohl eher selten auf die Idee, als Gleichstellungsbeauftragten einen Mann einzusetzen, wenn das kürzlich wohl auch einmal versucht worden ist; allerdings erfolglos). Dies zeigt sich durchaus darin, dass die wenigen Beauftragten, die selbst behindert sind (z.B. Miles-Paul in Rheinland-Pfalz, Hase in Schleswig-Holstein, Steinbrück in Bremen, Marquard in Berlin), teilweise durchaus in der Lage sind, ihre behinderungsbedingte Herkunft auch in greifbare Politik umzusetzen (am besten schaffen dies wohl Miles-Paul allgemein und Marquard insonderheit bei der Umsetzung von Barrierefreiheit im öffentlich gestalteten Raum).

\subsubsection{Grundsätzliches Demokratiedefizit}

Bereits oben unter 2.3.2. und 2.3.3. wurde jeweils darauf hingewiesen, dass sowohl die Beiräte als auch die Beauftragten demokratisch nicht legitimiert sind. Man mag das für unbedenklich halten, schließlich ist kaum ein Beauftragter der Bundesregierung gewählt (vom Wehrbeauftragten einmal abgesehen, der allerdings auch nicht der Bundesregierung, sondern dem Bundestag verpflichtet ist). Das zeigt allerdings nach meiner Einschätzung lediglich zweierlei:

- Zum einen maßt sich die Exekutive mittlerweile für nahezu jeden politischen Bereich ein eigenständiges Beauftragtenwesen an, 
- das ihr demokratisch nicht zusteht,

○ für das es regelmäßig keinerlei gesetzliche Legitimation gibt (lässt man sinnvolle Beauftragte wie denjenigen für den Datenschutz einmal außen vor; dieser ist allerdings durch das Bundesdatenschutzgesetz auch vorgesehen)

○ und die - obwohl regelmäßig nur mit Aufwandsentschädigungen versehen - finanzielle Spielräume ausreizen, ohne dass auch nur ansatzweise geklärt wäre, mit welcher Zielsetzung über den politischen Pragmatismus hinausgehend, jedes Problem solange „outzusourcen“, bis es als Problem nicht mehr wahrgenommen wird, die Beauftragten überhaupt installiert werden. Der gleiche Unsinn findet sich seit Jahrzehnten in sämtlichen Ministerien mit der Bestallung sog. „Parlamentarischer Staatssekretäre“, die auch lediglich der Pfründensicherung von Abgeordneten dienen, die ansonsten ,nichts mehr werden durften oder noch nichts werden konnten“.

- Die Aufgabenstellung ist zudem regelmäßig allenfalls ansatzweise umrissen (,der Beauftragte soll ..." regelmäßig, es besteht also nicht einmal eine verbindliche Verpflichtung, den Beauftragten dann zu konsultieren, wenn es um originäre behinderungsbedingte Belange geht). Das hat jedoch mit strukturiertem Verwaltungshandeln und mit organisierter Gesetzgebung nichts mehr zu tun, das ist organisatorische Willkür und gesetzgeberischer Wildwuchs.

Wenn man denn auch im Weiteren auf die Funktion von Behindertenbeauftragten nicht verzichten will ${ }^{25}$, müssen dieselben allerdings (und das sind lediglich Mindeststandards):

- $\quad$ selbst behindert sein,

- gegenüber der Verwaltung ein umfassendes Auskunftsrecht erhalten,

- die Betroffenen juristisch vertreten können und

- in Musterverfahren auch ohne Beteiligung von unmittelbar Betroffenen Benachteiligungs- und Diskriminierungstatbestände problematisieren und hierdurch einen deutlich kontinuierlichen gesellschaftlichen Diskurs anstoßen können.

Problematischer noch als die Beauftragten sind die Beiräte. Diese werden - vollkommen bar jeder Wahl - auf Landesebene regelmäßig entweder durch den Beauftragten oder den zuständigen Minister ernannt. Immerhin finden sich hier nahezu ausschließlich lediglich Betroffene (der Freistaat Bayern bildet hier eine interessante Ausnahme). Das Prinzip der Selbstbetroffenheit wird auf kommunaler Ebene vielfach auch dadurch gebrochen, dass sich teilweise Fraktionsvertreter aus den Kommunalparlamenten (dann allerdings ohne Stimmrecht) in den Beiräten wiederfinden. 
Die Erfahrung der letzten Jahre lehrt, dass die Beiräte auf kommunaler Ebene wiederum stark abhängig von ihrer personellen Besetzung und also der Durchsetzungsfähigkeit ihrer Mitglieder - innerhalb der Kommunen eine durchaus fruchtbare Rolle spielen können, wenn ihnen auch jegliches Initiativrecht in die Kommunalparlamente hinein fehlt. Teilweise werden die Beiräte (so z.B. in Hessen) in ordentlichen Wahlverfahren auch gewählt, wobei allerdings bei der Wahlaufstellung eine Prärogative für die traditionellen Verbände festzustellen ist.

\subsubsection{Lokale Ebene als eigentlicher Entscheidungsrahmen für Behindertenpolitik}

Somit ist eine Lanze zu brechen für die Stärkung sowohl von Beiräten als auch von Beauftragten für die Belange behinderter Menschen auf kommunaler Ebene. Oder, durchaus favorisiert, auch hier eines Systems der für die betroffenen behinderten Menschen Rechts sichernd wirkenden, neben der Verwaltung und nicht in ihr integriert stehenden, demokratisch legitimierten Ombudsperson. Nur weil wir hier in Deutschland keinerlei Tradition eines Ombudswesens haben, heißt das ja nicht, dass wir nicht auch in der Lage sein können, neue Instrumente auszuprobieren. Es gibt auf der kommunalen Ebene weitgehend ein Sonnenkönigtum im Berech der Bestallung des oder der Beauftragten, das man mit einer Devise Ludwig XIV „l'état c'est moi“" umschreiben kann. Regelmäßig stellt die Verwaltung eine oder einen Mitarbeiter/-in als Behindertenbeauftragten - unter teilweiser Freistellung von dessen gewöhnlicher Tätigkeit - ab. Das hat für die Verwaltung ausschließlich Vorteile, für die betroffenen behinderten Menschen hängt alles ausschließlich davon ab, wie unabhängig sich der oder die Beauftragte von der Verwaltung zu machen in der Lage ist. Dabei soll überhaupt nicht verhehlt werden, dass es Beauftragte innerhalb der Verwaltung gibt, die sowohl segensreich für die Betroffenen wirken als auch in Kooperationsbereitschaft mit der Verwaltung durchaus anerkennenswerte Fortschritte erzielen ${ }^{26}$. Beschäftigt man sich langjährig mit Behindertenpolitik, fällt eine Binsenweisheit auf, dass sich Veränderungen im Sinne tatsächlicher Fortschritte nämlich weitgehend auf lokaler Ebene nicht nur am ehesten (nach-)vollziehen lassen, sondern dass - bei entsprechender Disposition der Beteiligten,

- also einem gerüttelt Maß guten Willens auch von Seiten der Verwaltung,

- einer mehr als nur in Ansätzen vorhandenen Sachkompetenz vonseiten der betroffenen behinderten Menschen

- und einer nach beiden Seiten hin kooperativ wirkenden Schnittstelle des Beauftragten

- diese Veränderungen auch viel eher „,nah am Menschen“ sind, um eine der neuesten Phrasen des SPD-Bundesvorsitzenden Kurt Beck hier einmal sinnvoll in Anwendung zu bringen. Vielfach wird auf dieser ,unteren“ Ebene viel eher als im ,pro-

26 Vgl. hierzu: Drewes, „Behinderung im Alter auch als Chance begreifen“, auf der Website des handicap-netzwerkes vom 10.01.2008: http:/www.handicap-netzwerk.de/content/detail.php? subaction $=$ showfull\&id $=1199945949 \&$ archive $=\& u c a t=6$ (Stand: 30.05 .2008$)$. 
fessionelleren“ Politbetrieb auf Bundes- oder Landesebene begriffen, dass Maßnahmen nicht nur einer „schmalen“" Minderheit von behinderten Menschen, sondern als tatsächliche Erleichterung im täglichen Leben praktisch jedermann zugute kommen.

\subsubsection{Behindertenpolitiken als Anscheins-, Schimären- und Alibipolitiken}

Die unter eben 2.3.5. erarbeiteten Aussagen zeigen jedoch eines überdeutlich: Dort, wo sich die Politik als eine spezielle Materie für behinderte Menschen geriert, gerät sie in aller Regel zu bloßer Anscheins-, Schimären- und Alibipolitik ${ }^{27}$.

Es war schon zu Zeiten der rot-grünen Koalition auf Bundesebene so, dass das Bündnis 90/DIE GRÜNEN die SPD bei der Gleichstellungsgesetzgebung eher „zum Jagen tragen“ musste, will heißen, die in ihren Grundsätzen am Ideal der Solidarität geschulten Sozialdemokraten waren eher zweifelnd, ob und was ein solches Gesetz denn bringen solle. Wie die Zeitläufte erwiesen haben: Sie hatten Recht!

Dass die Sozialdemokraten Recht hatten, ist aber gar nicht der springende Punkt. Das Problem hinsichtlich der Politik für Minderheiten, wie dies behinderte Menschen innerhalb des politischen Diskurses nach wie vor sind, ist: Wir sind schlicht und einfach nach wie vor nicht in der Gesellschaft angekommen, nach wie vor gilt der Begriff des „Menschen mit Behinderung“ als der vermeintlich fortschrittlichere gegenüber dem viel realistischeren und die Sachlage wesentlich präziser aufzeigenden „behinderten Menschen“. Im letzteren Falle werde ich durch meine Umgebung behindert, obwohl ich - abgesehen von der mich hindernden Einschränkung - ein völlig normaler Mensch bin. Im ersteren Fall wird fein säuberlich dahingehend ziseliert, dass ich für mein Schicksal in gewisser Weise selber verantwortlich zeichne (das tue ich grundsätzlich gerne, aber doch nicht für einen Umstand wie meine Behinderung, für die ich als Geburtsbehinderter ja nichts kann). Letzten Endes weicht man mit der vermeintlich „modernen“ Bezeichnung des „Menschen mit Behinderung“" in Wahrheit auch vom Finalitätsprinzip ab und konkludiert - zumindest

27 Natürlich erfüllt es mich als Teilnehmer des „Forums behinderter Juristinnen und Juristen“ immer noch mit Stolz, zu wissen, dass mit zweien unserer wesentlichen Protagonisten (nämlich Horst Frehe und Dr. Andreas Jürgens) Teilnehmer eines Forums in die Gesetzgebung beim BGG im BMAS mit eingebunden waren, bei der zweiten und dritten Lesung des Gesetzes neben der Regierungsbank in ihren Rollstühlen saßen und heute beide als Abgeordnete für das Bündnis 90/DIE GRÜNEN im Hessischen Landtag (Jürgens) bzw. der Bremischen Bürgerschaft (Frehe) sitzen. Beide machen, was man so mitverfolgen kann, eine gute Politik, allerdings machen sie kaum - das bringt der Parlamentsbetrieb insgesamt so mit sich - dem Grunde nach Behindertenpolitik. Dabei dürfen beide als behindertenpolitisch außerordentlich engagiert und fachlich hoch versiert gelten. Wenn jedoch das Thema „behinderte Menschen“ selbst in einer Fraktion wie der der bürgerschaftlich orientierten Ökopaxe von Bündnis 90/DIE GRÜNEN nur eine eher randständige Rolle spielt, wie steht es dann erst um diejenigen Parteien, die nicht derart engagierte Vertreter aufweisen können, die auch willens und in der Lage sind, sich politisch derart zu exponieren (beide, Frehe wie Jürgens, sind z.B. exzellente Rechtspolitiker und als solche auch in ihren Fraktionen durchaus anerkannt)? 
sprachlich - ein Kausalitätsprinzip, wo es spätestens seit der Schaffung des SchwbG im Jahre 1974 nichts mehr verloren hat.

Und so lässt sich - vom Sprachlichen zum Juristischen - die Politik des Talmi und des Lug und Trug unendlich fortsetzen. So schafft der Gesetzgeber zwar die Möglichkeit, dass die Verbände für die Betroffenen im Rahmen der Gleichstellungsund Antidiskriminierungsgesetzgebung klagen können, wenn die Betroffenen selbst benachteiligt oder diskriminiert worden sind. Allerdings gibt es in Deutschland bislang überhaupt keine Kultur eines solchen Verbandsklagerechts.

Genauso wenig, wie man vor dem Jahre 2002 gewusst hätte, was eine Zielvereinbarung $^{28}$ sein soll. Nun gut, die meisten können selbst mit den Begrifflichkeiten bis heute nicht wirklich etwas anfangen, aber immerhin haben sich die Verbände zwischenzeitlich daran gemacht, sich Personal für letzteres heranzuziehen .Für die Möglichkeit der Verbandsklage haben die Verbände bis heute - woher auch? - nach wie vor kein Geld.

Man hat also die Verbände zwar in die Lage versetzt, rein formell bestimmte Rechte wahrzunehmen, hat es allerdings - und das in voller Absicht - unterlassen, sie hierfür materiell in angemessener Weise auch finanziell auszustatten. Frei nach dem fiskalpolitisch umgedrehten St.-Florians-Prinzip „Oh heiliger Florian, lass mein Haus steh'n, zünd' and're an“. Wenn man, was der Gesetzgeber ja vorgeblich tut, die Teilhabe behinderter Menschen als eine gesamtgesellschaftliche Aufgabe sieht, müssten die Verbände auch als Teilhabeleistungsgaranten in die Lage versetzt werden, die Teilhabe - notfalls auch juristisch - durchzusetzen. ${ }^{29}$ Auch hier greift wieder das Motto seitens der Bundesregierung: Wortgeklingel ja, aber es darf absolut nichts kosten!

Die wissentliche und gewollte Unfähigkeit der Politik, in größeren Entwürfen auch nur zu denken, setzt sich fort, gleich welches behindertenpolitische Thema man nimmt. Das aktuellste Beispiel ist wohl die unsägliche Diskussion um die Erhöhung der Entschädigungsrenten für Menschen mit einer durch das Schlafmittel „Contergan“ verursachten Körperschädigung. Hier bedurfte es erst eines außerordentlich deutlichen Aufschreis seitens der Betroffenen, ehe sich die Regierung wenigstens dazu bereit fand, die Entschädigungssummen auf das doppelte Maß dessen zu bemessen, was sie in den letzten Jahren den Betroffenen an ,peanuts“ zugestanden hatte.

Doch bleiben wir bei den - vermeintlich - großen Themen. Ganz gleich, ob man die Teilhabeaspekte im Rahmen des SGB IX nimmt oder die Eingliederungshilfe im

28 Es gibt- nach Erkenntnissen der Bundesregierung (BT-Drs. 16/9283, S. 12 ff.) - bislang lediglich acht Zielvereinbarungen binnen sechs Jahren; eine geradezu lächerliche Zahl in Anbetracht dessen, welche Hoffnungen man ursprünglich in das Instrument gesetzt hatte.

29 Daran gedenkt die Bundesregierung wohl auch längerfristig nichts Wesentliches zu ändern, wie sich in der Antwort (BT-Drs. 16/9283) auf die Große Anfrage von Bündnis '90/DIE GRÜNEN (BT-Drs. 16/6825) „Fünf Jahre Behindertengleichstellungsgesetz“, S. 2 letzter Abs. zu Frage 1 sowie S. 16 f. zu den Fragen 35, 37 und 39 entnehmen lässt. Obwohl die Bundesregierung selbst sieht, dass das Instrument bislang nicht greift, verweigert sie sich ohne jede Argumentation einer Verschärfung; vgl. hierzu die Antwort auf S. 17 f. zu Frage 40. 
Rahmen des SGB XII: Grundsätzlich wird staatlicherseits die preisgünstigste Lösung favorisiert oder es werden zumindest langjährig eingefahrene und zementierte Strukturen mit weiterem Zementmörtel versehen.

Dass es zum Beispiel nach wie vor eine stete Förderung von Heimeinrichtungen gibt, obwohl mittlerweile umfänglich der Grundsatz ,ambulant vor stationär“ in der Rahmengesetzgebung des $\S 9$ Abs. 2 Satz 2 SGB XII gilt, ist ein Skandal, bei dem einem spätestens dann die Worte fehlen, wenn man sich mit dem Entstehen und dem leichtfertigen Vertuschen von Pflegeskandalen ein wenig intensiver auseinandergesetzt hat.

Dass es der Gesetzgeber weiterhin nicht geschafft hat, bei der Reform des Rehabilitations- und Teilhaberechts einen eigenständigen Rehabilitationsleistungsträger zu schaffen ist genauso ein Unsinn wie die Schaffung sogenannter Servicestellen, die zwar dafür gedacht waren, die Betroffenen durch das Verwaltungsverfahren zu lotsen,

- zum einen aber gerade bei den Betroffenen nach wie vor weitgehend unbekannt sind,

- teilweise nach wie vor nicht barrierefrei zugänglich und nutzbar sind,

- durch die Rehabilitationsträger auch nicht propagiert werden,

- soweit ersichtlich (es würde lohnen, dies einmal wissenschaftlich zu evaluieren) überwiegend drittklassige Arbeit leisten

- und dadurch ihre fehlende Notwendigkeit jeden Tag aufs Neue unter Beweis stellen.

Dass es bei Politik in aller Regel um zwei Dinge geht, nämlich um Macht und um Geld, zeigt sich augenfällig an der Eingliederungshilfe. Weiter unten (2.5.1.) wird noch erläutert, weshalb die gegenwärtige Heranziehung von Leistungsempfängern hinsichtlich ihrer Einkünfte und ihres Vermögens auch aus sozialpolitischer Sicht vollkommen ungerecht ist. Zudem stelle ich ausdrücklich in Frage, ob die durch die Sozialhilfeverwaltungen beigetriebenen Eigenbeteiligungsbeträge wenigstens die Kosten decken, die durch ihre Beitreibung seitens der Sozialhilfeträger auflaufen.

Alles in allem darf man - ohne larmoyant wirken zu wollen - konstatieren: Der große Wurf war in der Behindertenpolitik in den letzten Jahren, auch unter vermeintlich „fortschrittlichen“ Regierungen, nicht wirklich dabei. Seit mindestens drei Jahren, also seit den Zeiten der jetzigen Großen Koalition, findet Behindertenpolitik als Fortentwicklung überhaupt nicht mehr statt. Nun könnte man natürlich festhalten, dass die in den letzten Jahren geschaffenen neuen „Werkzeuge“ erst einmal Zeit bräuchten, ihren Nutzen zu erweisen (was nach über sieben Jahren für das Rehabilitations- und Teilhaberecht aber auch kein wirklich fundiertes Argument mehr darstellt). Jedoch, das Gegenteil ist der Fall. Bei der momentanen Konstruktion der Neuheiten darf man eher festhalten: Es liest sich schön, es klingt auch gut, einzig, einen tatsächlichen Nutzeffekt für die Betroffenen haben die meisten Instrumente nicht. Sie wirken beruhigend selbst auf die „Avantgarde“ der Behindertenbewegung, weil man meint, man habe in den letzten Jahren endlich einmal etwas bewegt. Das Gegenteil ist der Fall! Die Politik hat die „Avantgarde“ ein wenig im Hamsterrad 
laufen lassen. Dieselbe läuft immer noch und merkt nicht einmal, dass sich das politische Establishment längst anderen Themen zugewendet hat und sich andere Klientel (gegenwärtig sind es die Familien) gesucht hat, denen einige Glasperlchen als Edelsteine unter der Prämisse „verhökert“ werden: „Zum einen halten die dann für einige Zeit die Klappe und wir können wenigstens so tun, als hätten wir politisch endlich einmal etwas Sinnvolles getan“.

\subsection{Einige Lebensbereiche, in denen fortschrittliche behindertenpolitische Ansätze greifen oder greifen sollten}

Natürlich gibt es viele Bereiche, in denen man sich vorstellen kann, dass die Lebensbedingungen für behinderte Menschen verbessert werden müssen. Mit absoluter Sicherheit gehört der Sektor des Pflegens hierzu. Weshalb in diesem Aufsatz lediglich die Lebensabschnitte Kindergarten/Hort, Schule und Arbeitsleben auftauchen, hat einen einfachen Grund. Die Pflegeversicherung ist eine reine Komplementärversicherung, die darüber hinausreichenden Assistenzleistungen wie für die Haushaltsführung, die Mobilität, den rein pflegenden Bereich werden - ähnlich der Eingliederungshilfe - durch Leistungen der Sozialhilfe finanziert, sofern der oder die Betroffene nicht in der Lage ist, sie selber zu finanzieren. Die Eingliederungshilfe als Subsidiärleistung, die aber immerhin als letzte verbleibende Leistung neben der Pflege nicht pauschaliert, sondern Bedarf deckend zu gewähren ist, wird weiter unten in 2.5.1. abgehandelt.

Mit den Bereichen Vorschule, Schule und die Grundschule begleitende pädagogische Versorgung sowie den Bereich des Arbeitslebens habe ich drei Lebensabschnitte herausgegriffen, an denen sich das bisherige Versagen tatsächlicher Teilhabe im Sinne einer Inklusion überdeutlich aufzeigen lässt.

\subsubsection{Kindergarten/Hort ${ }^{30}$}

Bereits im Vorschulbereich entscheidet sich, ob ein behindertes Kind segregiert oder inkludiert aufwächst. Die pädagogischen Erfahrungen des letzten halben Jahrhunderts haben eindrücklich nachgewiesen, dass es sowohl für das Selbstbewusstsein des behinderten Kindes als auch für die Sozialkompetenz nichtbehinderter Kinder gegenüber behinderten von elementarer Bedeutung ist, ob und inwieweit ein normaler Umgang miteinander bereits im vorschulischen Bereich stattfindet. Hier lässt sich festmachen, ob und inwiefern nichtbehinderte Kinder bereits Vorurteile, Vor-

30 S. zu allen drei Bereichen, also sowohl dem Lebensabschnitt „Kindergarten/Hort“ als auch denjenigen von „Schule“ und „Arbeitsleben“ den hoch instruktiven Beitrag von Trenk-Hinterberger in diesem Band. 
behalte oder auch nur Scheu gegenüber behinderten Menschen antrainiert oder dieselben eben gerade erst gar nicht entwickelt werden.

Das Land Hessen hält sich seit einigen Jahren viel darauf zugute, dass es dort keine Sonderkindergärten mehr gibt. Hessen ist damit das einzige Bundesland, das auf den ersten Blick die Segregation bereits in der Vorschule abgeschafft zu haben scheint. Jedes Kind, gleich mit welcher Behinderung, erhält einen Platz in einem Kindergarten. Für jedes behinderte Kind wird eine gesonderte pädagogische Fachkraft abgestellt, die die sonderpädagogischen Förderbedarfe abdecken soll.

Und damit sind wir schon beim wesentlichen Dilemma dieses dem Grunde nach hochlobenswerten Ansatzes. Gefordert und auch tatsächlich erbracht wird nämlich in aller Regel tatsächlich lediglich eine pädagogische Fachkraft, das Hessische Sozialministerium geht allen Ernstes auch mehrere Jahre nach Initiierung des Modells davon aus, dass sich die pädagogischen Fachkräfte in ihrer Freizeit unentgeltlich fortbilden.

Der Modellansatz mag deshalb für Kinder mit einer Körperbehinderung noch ansatzweise hinreichen (obwohl man auch hier natürlich im Bereich Pflege und Mobilität nicht zwingend davon ausgehen kann, dass dies von einer pädagogischen Fachkraft ohne Weiteres geleistet werden kann). Schwieriger wird dies bereits beim Bereich Sinnesbehinderung (weder wird eine pädagogische Fachkraft in der Lage sein, ein blindes Kind sonderpädagogisch zu betreuen, noch wird sie in aller Regel bei einem gehörlosen Kind in der Lage sein, die Deutsche Gebärdensprache zu verwenden). Teilweise unsinnig wird das Konzept bei Kindern mit einem sehr hohen Pflegebedarf und bei solchen mit erheblichen Lerneinschränkungen. Natürlich soll hier nicht dem Konzept sonderpädagogischer Förderzentren das Wort geredet werden. Diese sind zum einen hinsichtlich des Inklusionsgedanken problematisch, zum anderen würden derartige Förderzentren lediglich in größeren Städten vorgehalten, behinderte Kinder auf dem Land fallen folglich hintenüber und könnten ein Kindergartenangebot bei dieser Konstruktion überhaupt nicht nutzen.

Auch im Bereich Kindergarten funktioniert folglich meine These, dass Behindertenpolitik alles Mögliche darf, nur kein Geld kosten. Das hessische Konzept, das sich teilweise schon auf den zweiten Blick nicht mehr sonderlich schlüssig liest, geht in seinem Ansatz (wenn es diesen denn überhaupt hat, was man trotz der Tatsache bezweifeln kann, dass es zu dem Modell sogar dicke wissenschaftliche „Wälzer“ $\mathrm{zu}$ lesen gibt ${ }^{31}$ ) davon aus, dass es einer sonderpädagogischen Förderung im Kindergarten oder Hort regelmäßig nicht bedürfe, die Bedarfslagen könnten mehr oder minder problemlos vom vorhandenen Personal aufgefangen werden.

Das ist natürlich Unsinn. Der sonderpädagogische Förderbedarf kann unmöglich ausschließlich durch die Frühförderung abgedeckt werden, allzumal die Kosten der Termine für das Kind hier regelmäßig budgetiert sind (immerhin ist die Frühförderung einer der wenigen Bereiche, in denen innerhalb der Sozialhilfe keine Einkommens- und Vermögensprüfung stattfindet). So verkommt manches behinderte Kind

31 Hessisches Sozialministerium/Institut für Kinder- und Jugendhilfe, ,Qualitätsentwicklung Integrationsplatz. Implementierung. Abschlussbericht“, 2007, Wiesbaden/Main. 
in hessischen Kindergärten als bloßem Verwahrort, weil es sowohl an der schieren Anleitung für das pädagogische Personal fehlt und meistenteils für graduell schwerwiegendere Schädigungen die fachliche Kompetenz schlichtweg nicht vorhanden ist.

Es mangelt hier folglich an der Fachlichkeit, dieser Mangel wiederum fußt darauf, dass das Land sich weigert, umfänglich in die verbesserte Ausbildung der Fachkräfte zu investieren. Damit werden fiskalpolitisch gleich zwei Fliegen mit einer Klappe geschlagen: Das Land muss nicht für eine Ausbildung aufkommen und muss auch nicht - auch in Hessen gilt mittlerweile das Konnexitätsprinzip - die verbesserte fachliche Ausstattung der Fachkräfte bezahlen. Dieselben sollen sich zwar selber schulen (und für die Schulmaterialien auch noch selber aufkommen), haben sie sodann jedoch die fachliche Qualifikation (die allerdings nicht mittels einer Prüfung nachzuweisen ist, dann griffe ja wieder der höhere Entgelttarif), haben sie nicht einmal etwas davon.

Hat die hessische Regelung wenigstens noch den Charme, dass sie Inklusion suggeriert, wo dieselbe teilweise wohl eher schwer gelebt werden kann, eben weil es einen Bedarf von behinderten Kindern an sonderpädagogischer Fachlichkeit gibt, der sich großenteils nicht wegleugnen lässt, gehen andere Länder wie der Freistaat Bayern oder Baden-Württemberg geradezu den umgekehrten Weg. Hier greifen Sondersysteme nach wie vor vollumfänglich und bevorzugt bereits im kindlichen Vorschulalter. Das ist - wie oben bereits gezeigt - vielfach, so in bevölkerungsschwachen Gebieten wie z.B. großen Teilen des Allgäus oder der Schwäbischen Alb, schon deshalb problematisch, weil hier Sondereinrichtungen für Vorschulkinder mit auch nur halbwegs finanziell vertretbaren Kosten gar nicht vorgehalten werden können (und demzufolge auch nicht werden). Folglich greift hier das Prinzip „Vogel friss oder stirb“, wobei es sowohl die Bayerische Staatsregierung wie auch die baden-württembergische Landesregierung dann eher beim Sterben in der Fläche belassen, will heißen, wo es keine Sondereinrichtung gibt und die gewöhnlichen Kindergärten nicht verpflichtet werden können, einen sonderpädagogischen Förderbedarf aufrecht zu erhalten, findet ein Kindergarten- oder Hortbesuch erst gar nicht statt, Garantie eines Kindergartenplatzes ab dem dritten Lebensjahr hin oder her.

Jedoch werden Eltern behinderter Kindern, völlig gleich in welchem Bundesland sie leben, schon sehr frühzeitig für das Leben gestählt, erfahren sie doch regelmäßig schon im Vorschulalter ihrer Kinder, dass man sich für ihre Probleme herzlich wenig bis gar nicht interessiert. Dass sich Eltern behinderter Kinder so etwas mittlerweile nicht mehr gefallen lassen und selbst initiativ werden, um ihren Kindern eine möglichst frühzeitige vollständige Teilhabe am gesellschaftlichen Leben zu ermöglichen, wo es keine oder nur unzureichende Lösungen gibt, ist wohl der wesentliche Fortschritt, den die Behindertenselbsthilfe neben den Gedanken von Barrierefreiheit, Benachteiligungsverbot und gleichberechtigter und diskriminierungsfreier Teilhabe in den letzten Jahren im politischen Diskurs erreicht hat.

Wenn denn nur der Gedanke zählte, wäre das ein evidenter Fortschritt, einzig: Die Gedanken sind frei und Papier ist, wie weiter unten in 2.5. aufgezeigt werden wird, gerade in der behindertenpolitischen Gesetzgebung ausgesprochen geduldig. 


\subsubsection{Schule}

Noch weitaus problematischer als im Vorschulalter stellt sich die Situation für behinderte Kinder im Bereich der Beschulung dar. Deutschland kennt ein staatliches Pflichtschulsystem, dem sich die Erziehungsberechtigten in aller Regel entweder nur durch Ausweichen ins Ausland oder die Beschulung in (staatlich zugelassenen) privaten Schuleinrichtungen entziehen können.

Kein einziges Bundesland kennt bislang eine ausschließlich inkludierende Beschulung behinderter Kinder, nahezu überall greift der Vorrang von segregierenden Sondereinrichtungen gegenüber dem Angebot der Beschulung an allgemeinen Schulen. Mit diesem Absonderungsmodell hat Deutschland europaweit dann „endlich“ einmal ein Alleinstellungsmerkmal, eine derart radikale Zuweisungspraxis in Sondereinrichtungen kennt kein einziger anderer Mitgliedsstaat der Europäischen Union.

Dass hier in Deutschland derart umfänglich abgesondert wird, hat ganz unterschiedliche - durchaus auch pädagogische - Ursachen. Zum einen steht im politischen Raum nach wie vor das Argument (und in Deutschland ist das selbst im Bereich der Pädagogik immer ein vorrangiges), dass eine Sonderbeschulung preisgünstiger sei. Tatsächlich mag das auf den ersten Blick auch so scheinen, allerdings besitzen die meisten Sondereinrichtungen bei der Berechnung ihrer Budgets durchaus die Chuzpe, ihre fixen Kosten im Immobiliarsachenbereich einfach nicht in die Kostenrechnung zu übernehmen, da dieselben nicht aus dem regelmäßig, durch den Träger der Sozialhilfe zu finanzierenden Kostensatz, sondern aus einem gesonderten „Topf" finanziert werden. So ist es durchaus auch unredlich, wenn Sozialhilfeträger den Grundsatz ,ambulant vor stationär“ mit dem Argument aushebeln, schließlich sehe selbst das Sozialhilferecht vor, eine ambulante Zuweisung sei nur dann statthaft, wenn sie nicht deutlich kostspieliger als die stationäre Heimunterbringung sei.

Nun will ich nicht per se jedem Leistungsträger Herzlosigkeit unterstellen, aber kein Mitarbeiter eines Sozialhilfeträger kann sich ausmalen, was er einem behinderten Kind regelmäßig damit antut, es aus seinem familiären Umfeld zu reißen, um es während der Woche durch eine Ersatzgroßfamilie erziehen zu lassen; dies mit dem - auch noch falschen - Argument, schließlich spare dies immerhin Kosten.

Auch greifen die Schulträger durch die Zuweisung von behinderten Kindern in weitgehend unzulässiger Weise in das vollumfänglich verfassungsmäßig garantierte elterliche Sorge- und Aufenthaltsbestimmungsrecht ein. Es liegt ja in Fällen der Zuweisung in eine Sondereinrichtung durchaus kein erzieherisches Versagen seitens der Erziehungsberechtigten vor. Ganz im Gegenteil wird bei der sonderpädagogischen Zuweisung ausschließlich mit der besseren individuellen Schulversorgung und - und das ist in Wahrheit das schlagende Argument - mit einer evidenten Unterversorgung mit sonderpädagogischem Förderbedarf an allgemeinbildenden Schulen argumentiert.

Allerdings versagt das System der Fördereinrichtungen nahezu vollständig, vergegenwärtigt man sich, dass vier Fünftel aller Schüler, die in Fördereinrichtungen 
unterrichtet werden, nicht einmal einen Berufs qualifizierenden Abschluss erlangen. ${ }^{32}$

Ein wesentliches Problem segregierender Einrichtungen wie der schulischen Förderzentren ist, dass die Betroffenen förmlich zur Unmündigkeit erzogen werden. Dagegen helfen selbst fortschrittliche pädagogische Konzepte nicht wirklich weiter, wie ich selbst am eigenen Leib erfahren musste. Ich habe - schulisch - eine vollständige segregative „Karriere“ hinter mich gebracht, von der Grundschule bis zum Abitur. Selbst wenn man positiv in Anrechnung bringt,

- dass ich immerhin mit einer Hochschulzugangsberechtigung die letzte Fördereinrichtung verlassen habe

- und man unterstellt, dass es mich hätte pädagogisch weit schlimmer hätte treffen können, als es mich in den mich „,betreuenden“ Einrichtungen getroffen hat, meine Probleme (und das ist meine Lebenserfahrung, die sich nicht nur wissenschaftlich belegen lässt, sondern auch aus vielen Gesprächen mit ebenfalls selbst Betroffenen herrührt) fingen im Grunde erst nach dem Schulbesuch so richtig an. Ich wurde eben, Erziehung zur Selbstständigkeit hin oder her, nicht wirklich „fit fürs Leben" gemacht, weil es auch in der Einrichtung, in der ich in meinen letzten drei Schuljahren bis zum Abitur ,gebüffelt“ habe, immer noch Rückzugsräume gab, die mir ein Elternhaus - zum Glück - nicht hätte bieten können. Angefangen von Schwierigkeiten im kommunikativen Bereich mit Kommilitonen bis zur fehlenden Durchsetzungsfähigkeit gegenüber unwilligem Lehrpersonal und hin zu Fragen der Organisation eines Studiums und dem selbstständigen wissenschaftlichen Arbeiten kann ich nicht behaupten, durch meine schulische „Karriere“ wirklich mit dem Prädikat „fitting for the best" versehen worden zu sein.

Nun bin ich ein Mensch, der durchaus in der Lage ist, auf verschiedenen Ebenen zu kommunizieren, ich habe ein gewisses Organisationstalent und bin - im persönlichen Bereich vielleicht weniger als beruflich - durchaus durchsetzungsstark. Aber selbst ich bin hier in Deutschland als multipel Behinderter im Studium (zumindest im rechts-wissenschaftlichen) gescheitert und habe meinen Abschluss letztlich im Ausland erlangt; nicht weil der Abschluss dort leichter zu bewältigen gewesen wäre, sondern einzig deshalb, weil man dort auf meine vielfältigen Behinderungen sachgerechter ein- und damit umzugehen in der Lage war als hier an einer deutschen Universität.

Weshalb führe ich hier mein persönliches Beispiel an? Es lässt sich in diesem Fall eindrücklich aufzeigen, dass, zieht man mein persönliches Schicksal eines sogenannten „hochqualifizierten“, von Geburt an behinderten Menschen zurate, wie es dann erst um weniger „qualifizierte“ behinderte Menschen stehen muss?

Dabei breche ich durchaus eine Lanze gegen das pädagogische Gutmenschentum der Sonderpädagogik. Man hat im Bereich des sonderpädagogischen Förderbedarfs die Fehler, die durch die erste PISA-Studie im deutschen Schulsystem aufgezeigt worden sind, noch dadurch erheblich verstärkt, dass Sonderpädagogik dazu neigt,

32 S. hierzu: mittendrin e.V., „Warum macht Integration schlau? Materialien zum Kongress „Eine Schule für Alle““‘ vom 16. - 18. November 2007 in Köln; Köln, 2008. 
„Kuschelpädagogik“ mit einem extremen Behütungselement gegenüber behinderten Kindern und Jugendlichen darzustellen. Das wirkt sich jedoch regelmäßig als vollkommen fatal aus. Ich rede hier - natürlich - keinem Darwinschen Ausleseprinzip im Schulsystem das Wort, aber man hat in Deutschland in den letzten Jahrzehnten vor lauter vermeintlich gut gemeinter Förderpädagogik völlig vergessen, dass im Schulbetrieb nach wie vor das Motto gelten muss: „Nicht für die Schule lernen wir, sondern fürs Leben“. Die pädagogischen Ansätze aus den letzten Jahrzehnten waren teilweise dermaßen selbst referentiell, dass sie überhaupt nicht mehr in Betracht gezogen haben, wozu Pädagogik überhaupt gut sein soll, nämlich dafür, jungen Menschen letztlich das Lernen beizubringen und - mit Glück - vielleicht auch noch den Spaß daran, sich unbekannte Lerninhalte selber zu erarbeiten.

Diese pädagogischen Negativelemente wurden (und werden nach wie vor) in der Sonderpädagogik mit einer Verve vertreten, dass man manchmal das Gefühl hat, bei behinderten Kindern müsse es sich um Marsmenschen und nicht um völlig normale Kinder mit einer Körper-, Sinnes-, mit einer psychischen oder intellektuellen Schädigung handeln. Das Schlagwort, das hier wieder und wieder in den Raum zu werfen ist, heißt Normalität. Behinderte Kinder haben genauso wie nichtbehinderte einen Anspruch darauf, unter Berücksichtigung ihrer besonderen „Anders“artigkeit als normal behandelt zu werden.

Sie merken schon, ich schreibe hier absichtlich nicht Schädigung oder Benachteiligung, weil das Leben mit einer Behinderung weder als schädigendes Ereignis noch als Benachteiligung an sich gewertet werden kann. Behindert bin ich deshalb, weil - in meinem konkreten Falle - ich gewisse körperliche Einschränkungen habe (ich bin blind mit einem minimalen Restvisus, ich bin hochgradig hörbehindert, ich habe ein Körpersyndrom, das sich Stickler nennt, was dazu führt, dass ich Asthma, Zucker und zu kurze Gliedmaßen habe). Diese behindern mich jedoch nicht in meinem Leben (sieht man vielleicht einmal von ab und an auftretenden asthmatischen Anfällen $a b)$. Behindert werde ich deshalb, weil meine Umwelt nicht so gestaltet ist, dass ich gleichberechtigt mit jedem nichtbehinderten Menschen daran teilhaben kann, also dass gestaltete Lebensbereiche im öffentlichen Raum mir als blindem oder schwerhörigem Menschen eben regelmäßig nicht in der gleichen Weise zugänglich sind oder ich sie nur eingeschränkt (oder manchmal auch gar nicht) nutzen kann.

Einmal abgesehen davon, dass es sich diese Gesellschaft - spätestens mittelfristig - gar nicht mehr wird leisten können, bei dem bereits jetzt gegebenen Fachkräftemangel und der bereits offenkundigen Unterversorgung der privatwirtschaftlichen Unternehmen und der öffentlichen Verwaltung mit Akademikern, auf vorhandenes Potenzial zu verzichten (und mit dem weiteren Vorhalten von Sondereinrichtungen konstruiert der Staat ja, dass er diesen Personenkreis nicht für wert befindet, normal und gleichberechtigt am gesellschaftlichen Leben teilzuhaben), ist es auch zutiefst un-, wenn nicht asozial, sowohl behinderten Kindern als auch nichtbehinderten das Zusammenleben miteinander vorzuenthalten. Dies suggeriert doch, dass Absonderung ein gesellschaftlich akzeptierter Zustand sei, und gerade wir in Deutschland sollten nach den erschreckenden Erfahrungen, die wir mit Ab- und Aussonderung in 
der ersten Hälfte des 20. Jahrhunderts gemacht haben, doch die notwendige Sensibilität und Empathie besitzen, „Anders“artigkeit als Normalität zu (er)leben.

\subsubsection{Arbeitsleben}

Ein weiteres Kuriosum vieler postmoderner Gesellschaften, also auch der meisten europäischen, ist der Umgang mit behinderten Menschen im Arbeitsleben.

Man mag es für verständlich und nachvollziehbar halten (ich tue das nicht), dass es für behinderte Menschen ab einem bestimmten Grad der Minderleistungsfähigkeit im Arbeitsleben einen gesonderten Arbeitsmarkt geben muss. Man mag es weiterhin für nachvollziehbar halten (auch das tue ich nicht), dass es für Arbeitgeber ein Anreiz- oder / und ein Sanktionssystem geben muss, behinderte Menschen einzustellen und zu beschäftigen. Auch mag man eine Sonderverwaltung für sinnvoll erachten, die dafür sorgt, dass behinderten Menschen im Arbeitsleben diejenigen technischen, sächlichen oder personellen Hilfen an die Seite gestellt werden, die sie benötigen, um ihrer Arbeit angemessen nachgehen zu können und die sich darum zu kümmern haben, im Kündigungsverfahren ein gesondertes Verwaltungsverfahren durchzuführen, in dem geklärt werden soll, ob eine arbeitgeberseitige Kündigung nach den Regelungen des SGB IX rechtmäßig erfolgt ist oder nicht.

Diese beiden Bereiche einer Sonderverwaltung zuzuweisen ist jedoch sowohl sachlich als auch juristisch unsinnig. Zum einen ist nicht ersichtlich, weshalb die Ausstattung mit besonderen Hilfen nicht durch die allgemeine Arbeitsverwaltung erfolgen können soll (sog. Technische Berater gab es bei den Arbeitsverwaltungen früher durchaus, ehe dieselben in Arbeitsagenturen „umgetauft“ wurden).

Völliger Unsinn ist jedoch das Verwaltungsverfahren im besonderen Kündigungsschutz für behinderte Menschen. Es ist in keiner Weise einsichtig, dass es ein zweites verwaltungsverfahrensrechtliches Kündigungsverfahren neben dem ordentlichen vor der Arbeitsgerichtsbarkeit gibt. Dies stellt ein schwerwiegendes Einstellungshemmnis für behinderte Menschen dar und verschafft allenfalls einigen Menschen in der Verwaltung der sog. Integrationsämter ein einträgliches Leben. Kein einziger behinderter Mensch wird seinen Arbeitsplatz tatsächlich deswegen behalten, weil ein Integrationsamt der Ansicht ist, einer Kündigung zunächst widersprechen zu müssen. Auch im normalen arbeitsgerichtlichen Verfahren sind sämtliche Sozialbelange im Kündigungsschutzverfahren umfassend zu gewichten, zudem sind diese Verfahren regelmäßig wesentlich zügiger abgeschlossen als der Verwaltungsrechtsweg im besonderen Kündigungsschutz nach dem SGB IX vor den Integrationsämtern (und nachfolgend gegebenenfalls vor den Verwaltungsgerichten). ${ }^{33}$

33 Zum besonderen Kündigungsschutz für behinderte Menschen s. Drewes, „Zweigleisigen Kündigungsschutz abschaffen", in den kobinet-nachrichten vom 09.01.2008: http://www.kobinetnachrichten.org/cipp/kobinet/custom/pub/content,lang,1/oid,17047/ticket,g_a_s_t

(Stand: 30.05.2008). 
Jedoch, Absonderung hat in Deutschland nicht erst im Berufsleben Methode und Tradition. Ab- und ausgesondert wird bereits im Stadium der Ausbildung in Berufsbildungs- und Berufsförderungswerken, deren einziger Zweck darin besteht, „dem ach so armen behinderten Menschen" eine berufliche Ausbildung zuteil werden zu lassen, die er ansonsten vorgeblich nicht erhalten haben würde.

Wenn denn dieses System - ,ambulant vor stationär“, wir erinnern uns immer wieder in diesem Beitrag dieses Grundsatzes sowohl des Rehabilitations- und Teilhaberechts als auch desjenigen des Rechts der Eingliederungshilfe - irgendeiner konsequenten Logik folgen würde, hätte ich im Grunde an einer Förderuniversität studieren müssen. ${ }^{34}$

Die Grundlegungen werden jedoch eh - völlig gleichgültig, welchen Lebensabschnitt man nimmt und betrachtet - immer dort durch Gesetz oder Verwaltungshandeln durchbrochen, wo es - aus finanziellen Gründen oder einfach deshalb, weil man sich das Denken ersparen will - opportun erscheint, besondere Systeme vorhalten zu wollen.

Sinnvoll wäre es bei Schaffung des SGB IX ja durchaus gewesen, einen eigenständigen Rehabilitationsleistungsträger zu schaffen, aber hier haben sich in aller Deutlichkeit die „großen“ Leistungsträger aus Renten- und Krankenversicherung durchgesetzt, die mindestens um einen Teil ihrer Pfründen fürchteten. Eines solchen „besonderen“ Leistungsträger würde es ja gar nicht bedürfen, wenn denn das Leistungssystem in sich schlüssig wäre und tatsächlich umfassend greifen würde. Das ist jedoch ersichtlich nicht der Fall, Wesentliches hieran hat auch die Schaffung des SGB IX im Jahre 2001 nicht geändert. Die Leistungsträger verweisen nach wie vor auf den Klageweg, wenn sie - was immer noch der Regelfall ist - nicht in der Lage sind, binnen angemessener Fristen zu entscheiden.

Besonders eklatant ist dieses Dilemma bei der Arbeitsverwaltung. Nicht alleine, dass:

- Antragsteller auf Rehabilitationsmaßnahmen im Arbeitsleben großenteils wochen- bis monatelang warten müssen,

- sich in vielen Arbeitsagenturen nicht einmal ein sachkundiger und fachlich versierter Ansprechpartner findet,

- $\quad$ eine besondere Beratung und Vermittlung (schwer)behinderter Arbeitsuchender (die im gegenwärtigen System zwingend erforderlich ist) nicht mehr vorgesehen ist,

- die finanziellen Mittel für rehabilitative Maßnahmen zur Vermittlung sog. schwer Vermittelbarer auf einen Bruchteil früherer Summen heruntergefahren worden sind und

- eine Arbeitsvermittlung von sog. schwer Vermittelbaren (worunter behinderte Menschen nach der Terminologie der Bundesagentur für Arbeit [BA] zweifellos zählen) mindestens im Bereich der Arbeitsvermittlung durch die sog. Job-

34 Auf einen solchen Schwachsinn ist allerdings bislang noch kein Bildungspolitiker gekommen und ich hoffe inständig, jetzt nicht irgendjemanden durch diesen Ansatz noch auf eine solche Idee zu bringen. 
Center, also für behinderte Menschen, die länger als ein Jahr arbeitslos gemeldet sind, praktisch nicht mehr stattfindet,

ist die BA „sinnvoller Weise“ im vergangenen Jahr auf die „glorreiche“ Idee verfallen, die Akademikerarbeitsvermittlung für schwerbehinderte Menschen bei der Zentralstelle für Arbeitsvermittlung (ZAV) in Bonn einfach ersatzlos aufzulösen. Unter anderem hieran wird klar ersichtlich, dass selbst hocheffiziente Arbeitsvermittlungsinstrumente (um bei dem Beispiel zu bleiben: die Akademikerarbeitsvermittlung der ZAV war ein solches) für die Sanierung des Etats der BA ohne Not und auch ohne jeden Sinn und Verstand anheimgegeben werden.

Der einzige Fortschritt, der sich in den letzten Jahren verzeichnen lässt, ist der seit 2001 gegebene Rechtsanspruch auf eine Arbeitsassistenz im Arbeitsleben. Diese erhalten behinderte Menschen im Arbeitsleben dann, wenn es behinderungsbedingt Tätigkeiten gibt, die sie nicht selbstständig ausführen können.

Jedoch, wie im deutschen Behindertenrecht üblich, auch hier greift „Absurdistan“. Gibt es nämlich über die Arbeitsassistenz hinausgehend noch einen pflegerischen Bedarf, so ist hierfür nicht mehr die BA, sondern die Pflegeversicherung (bzw. zur Abdeckung des vollständigen Bedarfs der örtliche Sozialhilfeträger) zuständig.

Dem Gesetzgeber hat allerdings bereits bei Schaffung des SGB IX „gedämmert“, dass es in der Verwaltungspraxis langfristig so nicht weitergehen konnte. Deshalb hat er das - jetzt zum 01. Januar 2008 vollumfänglich in Kraft getretenes ${ }^{35}-$ Persönliche Budget geschaffen, durch das der behinderte Mensch in die Lage versetzt werden soll, bei sog. Komplexleistungen (also dem Vorhandensein von mehr als einer Leistungsart, die durch einen oder mehrere Leistungsträger erbracht werden soll) die Leistung durch ein pauschaliertes Budget selbst zu finanzieren ${ }^{36}$. Das klingt zunächst sehr fortschrittlich und sehr im Sinne von Autonomie und Selbstständigkeit der behinderten Menschen gedacht, der Widerhaken findet sich - man hat es fast schon erwartet - im finanziellen Bereich. Nicht umsonst geht in der Behindertenszene seit dem 01. Januar 2008 ein wenig die Furcht um, die Leistungsträger würden auch dieses neue Instrument - wieder einmal, wie bei vielen vermeintlichen „Fortschritten" in der Vergangenheit - dazu benutzen, die Bedarfslagen behinderter Menschen als finanzielle „Abrissbirne“ zu missbrauchen.

Auf weitere neuere Instrumente wie Integrationsfachdienste oder Integrationsprojekte wird hier schon deswegen nicht eingegangen, weil sie in wesentlichem Umfang auch in den letzten sieben Jahren nicht in der Lage waren, behinderte Menschen in einem quantitativ bemerkenswerten Umfang dauerhaft im ersten Arbeitsmarkt ,unterzubringen“. worden ist.

36 Hinsichtlich von Leistungen durch einzelne Träger, die auch auf Dauer erbracht werden, galt bereits bisher die Möglichkeit der Finanzierung durch eine Geldleistung; vgl. hierzu: § 9 Abs. 2 SGB IX. 
Die Quote arbeitsloser (schwer)behinderter Menschen dümpelt seit Jahren auf einem erschreckend viel höheren Niveau als diejenige nichtbehinderter Menschen. Und selbst letztere Quote wird von der BA gezielt „,schön gerechnet“, wie sich aus dem Statistikmaterial des der BA zugehörigen Instituts für Arbeitsmarkt- und Berufsforschung (IAB) regelmäßig ablesen lässt. ${ }^{37}$

\subsection{Querschnittbereiche, in denen Behindertenpolitiken von Relevanz sind}

Behindertenpolitiken sind, wie in diesem Beitrag schon mehrfach angeklungen, fast ausnahmslos Querschnittspolitiken, werden in Deutschland allerdings bislang weder politisch noch juristisch so verstanden.

Man mag sich beim Durchlesen der obigen Gliederung ein wenig gewundert haben, dass ein momentan subsidiärer Bestandteil eines Leistungssystems hier an den Anfang gestellt wird. Das hat jedoch seinen guten Grund.

2.5.1. Recht der umfassenden gleichberechtigten Partizipation behinderter Menschen als Ausfluss verfassungsrechtlicher Normierung und einfachrechtlicher Zielsetzung kontra Eingliederungshilfe als politisch gewolltem und zementiertem Fortbestand

Die hier - und mit mir nahezu von der gesamten Behindertenbewegung und auch des sozialpolitisch ambitionierten Teils in Deutschland - vertretene Ansicht geht davon aus, dass es nicht länger persönliches Schicksal bis hinein in die Einkommen und Vermögen der betroffenen behinderten Menschen sein kann und darf, dass Teilhabe oder selbst Rehabilitation, die nicht durch die Sozialversicherungsträger oder die BA (und neuerdings die Job-Center) zu leisten ist, stattfindet oder nicht. Insofern hat sich an meiner - damals noch eher kurz und prägnant - formulierten Forderung nach einem vermögens- und einkommensunabhängigen Teilhabeleistungsgesetz aus dem Jahre $2003^{38}$, das sich weitgehend aus Steuermitteln des Bundes finanzieren muss, nichts geändert.

Warum einkommens- und vermögensunabhängig? Schließlich gibt es ansonsten auch kein System der Grundsicherung in Deutschland, das vollständig ohne die Heranziehung der Leistungsbezieher von Einkommen und Vermögen bis zum Abschmelzen auf gewisse Schonbeträge bei den Einkünften bzw. bestimmten Werthal(BT-Drs. 16/9283) auf die Große Anfrage von Bündnis '90/DIE GRÜNEN (16/6825) „Fünf Jahre Behindertengleichstellungsgesetz“, S. 5 zu Frage 9. von Maydell/Pitschas/Schulte (Hrsg), Behinderung in Asien und Europa im Politik- und Rechtsvergleich (Studien aus dem Max-Planck-Institut für ausländisches und internationales Sozialrecht, Bd. 26), 2003, Baden-Baden (Nomos), S. 463, 474. 
tigkeitsgrenzen bei den Vermögen auskommt (was man durchaus auch kritisch betrachten kann, was aber spätestens seit der Schaffung des SGB II wohl in weiten Kreisen des politischen Establishments Komment ist).

Während jedoch z.B. die Grundsicherung für Arbeitsuchende nach dem SGB II nicht als Dauerleistung angelegt ist ${ }^{39}$, geht das Recht der Eingliederungshilfe nach wie vor davon aus, dass es eine vollumfängliche Bedarfsdeckung geben muss, die allerdings nahezu ausschließlich nachrangig erfolgen soll. Nun ist dem Grunde nach gegen einen Nachrangigkeitsgrundsatz nichts einzuwenden, wenn man denn auch langfristig das gegliederte System der Sozialleistungsträger auch im Rehabilitationsund Teilhabebereich aufrechterhalten will ${ }^{40}$. Dass der Gesetzgeber jedoch bereits bei Schaffung des Bundessozialhilfegesetzes (BSHG) Anfang der 1960er Jahre von einer vollumfänglichen Bedarfsdeckung im Bereich des Rechts der Eingliederung behinderter Menschen ausgegangen ist, hat ja gute Gründe - und an dieser Werthaltigkeit hat sich in den letzten fünfundvierzig Jahren absolut nichts geändert. Dass ein behinderter Mensch jedoch mit Teilen seines Einkommens und Vermögens dafür herangezogen wird, dass Teilhabe am gesellschaftlichen Leben für ihn (oder sie) überhaupt stattfinden kann, ist schon in sich widersinnig ${ }^{41}$. Solange jedoch insbesondere auch die gestalteten Lebensbereiche im öffentlichen Raum in großem Umfang nicht barrierefrei zugänglich und nutzbar sind, ist eine Teilhabesicherung zwingend erforderlich (für bestimmte Bereiche und bei bestimmten Schädigungsarten wird sie das auch im privaten Raum immer bleiben).

Deshalb ist es ein Postulat sozialstaatlicher Gerechtigkeit, behinderten Menschen die Teilhabesicherung zu ermöglichen, ohne dass die Schaffung von Normalität die Betroffenen auch noch regelmäßig Geld bis knapp oberhalb der Grenze der Grundsicherung kostet ${ }^{42}$. Da der Gesetzgeber die Einkommens- und Vermögensgrenzen bei Schaffung des SGB XII auch noch radikal „eingedampft“, also im Wesentlichen

39 Schließlich soll der Leistungsempfänger trotz regelmäßig miserabler Beratung seitens der meisten Job-Center und dem Nicht-Vorhandensein selbst gesetzlicher Gewährleistungen wie des vom Gesetzgeber tatsächlich euphemistisch so genannten „Fall-“ oder „Case-Managers“ ja schnellstmöglich in die Lage versetzt werden, jede legale Tätigkeit zu jedem legalen Tarif unter gewissen Zumutbarkeitsgrenzen des Ortswechsels, der Fahrstrecken zum Arbeitsplatz und der - mindestens zeitweiligen - Trennung von der Familie auszuüben. Daneben sind die Leistungen, die nach dem SGB II gewährt werden, nicht mehr bedarfsdeckend, sondern umfänglich pauschaliert, zum „CaseManagement" hat Rixen am 21.11.2007 einen außerordentlich instruktiven Vortrag unter dem Titel „Abschied von der Solidarität? - Hartz IV und das Verhältnis von Sozialer Arbeit, Sozialrecht und Sozialpolitik" anlässlich seiner Antrittsvorlesung an der Universität Kassel gehalten

40 Auch hiergegen gibt es allerdings schwerwiegende Bedenken, die ich weiter oben bereits ausgeführt habe.

41 Genauso übrigens wie die Kostenbeteiligung im Bereich der Pflege, sofern die Kostenübernahme durch den Sozialhilfeträger erfolgt und die neuerdings in sämtlichen Bundesländern um sich greifenden Praxis der Landesgesetzgeber, die bereits bislang pauschalierte Leistungen des Landesblindengeldes zu bestimmten Teilen auch noch über die Einkünfte und Vermögen der Betroffenen refinanzieren zu lassen, also eine weitere - einkommens- und vermögensabhängige - Pauschale innerhalb einer Pauschale zu schaffen

42 Was letztlich entweder zu einer Verarmung der Betroffenen oder dazu führt, dass selbst zwingend notwendige Teilhabeleistungen erst gar nicht in Anspruch genommen werden. Auch dieses Problemfeld bedarf dringend einer wissenschaftlichen Bearbeitung. 
nivelliert hat, ist das Armutsrisiko im Falle der Notwendigkeit von Leistungen der Eingliederungshilfe seit dem Jahre 2005 (dem Inkrafttreten des SGB XII) evident angestiegen. Man muss diesbezüglich ja nur einmal die Einkommens- und Vermögensgrenzen des alten BSHG mit dem des SGB XII vergleichen.

Dass jedoch - insbesondere auf Seiten des Bundes - die Politik gegenwärtig überhaupt nicht daran interessiert ist, eine Änderung des behinderte Menschen eklatant und offensichtlich diskriminierenden Zustandes herbeizuführen, zeigte sich bereits am Koalitionsvertrag 2005. Ausdrücklich wird - das auch noch unter der Titelüberschrift „Gesellschaftliche Teilhabe von Menschen mit Behinderung ${ }^{\text {‘43 }}$ daran festgehalten, dass die Eingliederungshilfe lediglich fortentwickelt werden $\mathrm{soll}^{44}$. Von einer Teilhabesicherung ist in diesem Vertragswerk nirgendwo die Rede. Dies haben sich bislang in dieser Legislaturperiode lediglich die Fraktionen von Bündnis 90/DIE GRÜNEN ${ }^{45}$ und DIE LINKE ${ }^{46}$ mit Entschließungsanträgen auf die Fahne geschrieben.

Insofern kann man die Beauftragte der Bundesregierung für die Belange behinderter Menschen Fr. Evers-Mayer sogar ein klein wenig verstehen, dass sie von der - aus ihrer Sicht - Blockadehaltung der Behindertenbewegung im letzten Jahr ein wenig enttäuscht gewesen sein muss. Die Teilnehmer der Behinderten(selbst)hilfe hätten halt einfach den Koalitionsvertrag vorher mal ein wenig gründlicher lesen sollen.

\subsubsection{Persönliches Budget}

Es glitzert wie ein Juwel, es wirkt wie eine Wunderdroge, es liest sich, als sei eine Revolution im Behindertenrecht ausgebrochen, man ist beinahe versucht, glänzende Augen zu bekommen, ahnte man nicht, dass die Sozialleistungsträger auch dieses Modell wieder umgreifend torpedieren werden. Die Rede ist vom trägerübergreifen-

43 Koalitionsvertrag zwischen CDU, CSU und SPD vom 11.11.2005 unter dem Titel „Gemeinsam für Deutschland - mit Mut zur Menschlichkeit“, Teil B. Die Handlungsfelder, Abschnitt IV. Soziale Sicherheit verlässlich und gerecht gestalten, Unterabschnitt 5.

44 Koalitionsvertrag (ebd.), S. 84, Zeile 4123-4126.

45 Antrag des Abgeordneten (Abg.) Markus Kurth, weiterer Abg. sowie der Fraktion von Bündnis 90/DIE GRÜNEN: „Die Eingliederungshilfe für Menschen mit Behinderungen weiterentwickeln“, BT-Drs. 16/7748 vom 16.01.2008, wobei selbst die Fraktion von Bündnis 90/DIE GRÜNEN ein Teilhabeleistungsgesetz ggw. aufgrund der vielfältigen Widerstände der Rehabilitationsleistungsträger für „,kaum erfolgsversprechend“ (S. 2, 2. Abs.), allerdings - wenn auch nur langfristig - ,als Perspektive handlungsleitend“" (ebd.).

46 Deutlich weitergehend als der Antrag von Bündnis 90/DIE GRÜNEN deshalb der des Abg. Dr. Ilja Seifert, weiterer Abg. sowie der Fraktion DIE LINKE: „Gesetz zum Ausgleich behinderungsbedingter Nachteile vorlegen (Nachteilsausgleichsgesetz - NAG)“, BT-Drs. 16/3698 vom 30.11.2006, der sich auch insofern innovativ zeigt, als nach diesem Vorschlag erstmalig sämtliche Materien des Rehabilitations- und Leistungsrechts in einem einzigen Gesetzgebungswerk zusammengefasst würden (S. 4 letzter Abs. des Antrages). 
den Persönlichen Budget ${ }^{47}$, das - und das ist die Revolution im Behindertenrecht an die Leistungsempfänger regelmäßig als Geldleistung ${ }^{48}$ auszukehren ist. Eine weitere Besonderheit besteht darin, dass die Pflegekassen und die Integrationsämter, die ansonsten keine Rehabilitationsträger im Sinne des SGB IX $\operatorname{sind}^{49}$, bei der Leistungsausführung der Persönlichen Budgets zu beteiligen sind ${ }^{50}$.

Obwohl es bereits wissenschaftliche Literatur zur Begleitforschung ${ }^{51}$ des im zweiten Halbjahr 2007 modellhaft erprobten Persönlichen Budgets gibt, kann bislang überhaupt nicht näher verifiziert werden, ob und inwiefern das Modell, das bislang wohl allenfalls minimal genutzt wird, tatsächlich greifen wird ${ }^{52}$. Allerdings erfuhr das Modell bereits - teilweise von den Sozialverbänden - vor seiner Einführung erhebliche $\mathrm{Kritik}^{53}$.

Es bleibt folglich abzuwarten, ob das Persönliche Budget zu einem Erfolgsmodell werden kann. Allerdings ist dem - nach den gemachten Erfahrungen mit Neuerungen wie Selbstbeschaffung der Leistung, Servicestellen und Verkürzung der Fristen - wie dem gesamten reformerischen Ansatz im SGB IX mittlerweile mit viel Skepsis und einem gerüttelt Maß der Erwartung, dass sich auch hier (wie bislang eigentlich immer) die Rehabilitationsträger als fundamentalistische Quertreiber gerieren werden, zu begegnen.

$47 \S 17$ Abs. 2 Satz 1 des Sozialgesetzbuch - Neuntes Buch (SGB IX) - Rehabilitation und Teilhabe behinderter Menschen vom 19.06.2001 (BGBl. I [Nr. 27], S. 1046), zuletzt geändert durch Artikel 8 Abs. 2 des Gesetzes zur Modernisierung der landwirtschaftlichen Sozialversicherung (LSVMG) vom 18. Dezember 2007 (BGBl. I [Nr. 66] S. 2984).

$48 \S 17$ Abs. 3 Satz 1 SGB IX.

49 Vgl. § 6 SGB IX.

$50 \S 17$ Abs. 2 Satz 2 SGB IX; allerdings werden deren Leistungen bislang nur durch Gutscheine gewährt. Vgl. hierzu die „Erklärung zum integrierten trägerübergreifenden Budget“ von Klie et al. vom 29.02.2008, veröffentlicht auf der Website des Forums selbstbestimmter Assistenz e.V. (ForseA): http://www.forsea.de/projekte/pers_budget/Erklaerung_zum_integrierten_traeger uebergreifenden\%20Budget_29_02_2008.pdf (Stand: 30.05.2008).

51 So z.B. von Welti, „Gutachten im Rahmen der wissenschaftlichen Begleitung der modellhaften Erprobung Persönlicher Budgets nach $\S 17$ Abs. 6 SGB IX“", Juni 2007, veröffentlicht auf der Website des Bundesministeriums für Arbeit und Soziales: http://www.bmas.de/coremedia/generator /23074/property=pdf/f367_forschungsbericht_anlage_zu+f366.pdf (Stand: 30.05.2008).

52 Die Rede ist von bislang 1000 Nutzern, was in Anbetracht von 643.000 Leistungsempfängern der Eingliederungshilfe geradezu verschwindend gering ist

53 PARITÄTISCHER (Wohlfahrtsverband) e.V., „,2 Jahre Modelle zum Persönlichen Budgets für behinderte Menschen. Ein Zwischenfazit aus Sicht des PARITÄTISCHEN Kompetenzzentrums Persönliches Budget" vom 23.09.2006, veröffentlicht auf der Website des PARITÄTISCHEN: http://www.infothek.paritaet.org/pid/persbuget.nsf/902102cb4478366dc1256f6c0039d503/83d1d97 7ee7df59ac12571f900317d41/\$FILE/Fazit-23-09-2006.pdf (Stand: 30.05.2008). 


\subsubsection{Zivilrechtliche Antidiskriminierung und öffentlich-rechtliches Benach- teiligungsverbot}

Antidiskriminierung und Benachteiligungsverbot sind im Grunde lediglich zwei Seiten ein und derselben Medaille. In beiden Fällen geht es letztlich um Gleichbehandlung behinderter Menschen mit nichtbehinderten.

Während Träger der öffentlichen Gewalt gem. § 7 Abs. 2 Satz 1 BGG behinderte Menschen nicht benachteiligen dürfen, also sie im Bereich der gesellschaftlichen Teilhabe ohne zwingenden Grund gegenüber nichtbehinderten Menschen nicht anders behandeln dürfen ${ }^{54}$, ergibt sich auch im Zivilrecht ein Verbot der Benachteiligung (also eine weniger günstige Behandlung als für eine andere Person, die das Merkmal, dessentwegen benachteiligt wird, nicht aufweis ${ }^{55}$ oder neutral wirkende Vorschriften, Kriterien oder Verfahren, die bereits nach dem Anschein eine Benachteiligung von Merkmalsträgern gegenüber solchen Menschen, die dieses Merkmal nicht haben, benachteiligend wirken können ${ }^{56}$ ), die bei Massengeschäften ${ }^{57}$, privatrechtlichen Versicherungen ${ }^{58}$ und im Arbeitsrecht greift $^{59}$, wobei behinderte Menschen lediglich eine von sechs verschiedenen Gruppen darstellen, die im AGG als benachteiligungsfähig benannt werden ${ }^{60}$. Ein besonderes Kuriosum stellt es allerdings dar, dass Kündigungen im Arbeitsverhältnis vom Benachteiligungsverbot im AGG gerade ausgenommen sind ${ }^{61}$, was aber nicht heißt, dass nicht Schadensersatz wegen einer in der Behinderung liegenden Kündigung auch nach dem AGG geltend gemacht werden $\mathrm{kann}^{62}$. Im Arbeitsverhältnis kann darüber hinaus bei Nichtvermögensschäden auf Entschädigung geklagt werden ${ }^{63}$ (die Höchstsumme ist allerdings bei Nichteinstellung auf drei Monatsgehälter beschränkt ${ }^{64}$, zudem besteht kein Anspruch auf Einstellung wegen der Verletzung des Benachteiligungsverbotes; auch

Das haben sämtliche deutschen Landesgesetzgeber in ihren Gleichstellungsgesetzen für behinderte Menschen - teilweise identisch bis in den Wortlaut der Bestimmung hinein - mit ähnlichen Formulierungen für die jeweiligen Landesverwaltungen und den ihnen nach geordneten Dienststellen ähnlich formuliert.

$55 \S 3$ Abs. 1 AGG.

$56 \S 3$ Abs. 2 AGG.

$57 \S 19$ Abs. 1 Ziff. 1 AGG.

$58 § 19$ Abs. 1 Ziff. 2 AGG.

$59 \S 7$ Abs. 1 AGG.

$60 \S 14$. Var. AGG; unglaublicher Weise verwendet der Gesetzgeber hier selbst im Jahre 2006 für Ethnien auch noch den Begriff der Rasse; vgl. § 1 Var. 1. 1. Alt. AGG; dabei entblödet sich der Regierungsentwurf in seiner Begründung nicht einmal, den Terminus der Rasse mit dem Schlagwort Rassismus in unmittelbaren Zusammenhang zu bringen und merkt allenfalls rudimentär (schiebt dies aber letztlich im Verantwortungsbereich auf die EU-Vorgabe, dass er bereits durch schiere Wortwahl diskriminiert; vgl. zur Darstellung des Begriffs: Gesetzentwurf der Bundesregierung. Entwurf eines Gesetzes zur Umsetzung europäischer Richtlinien zur Verwirklichung des Grundsatzes der Gleichbehandlung, BT-Drs. 16/1780 vom 06.06.2006, S. 30 f.

$61 \S 2$ Abs. 4 AGG.

$62 \S 15$ Abs. 1 Satz 1 AGG.

$63 \S 15$ Abs. 2 Satz 1 AGG.

$64 \S 15$ Abs. 2 Satz 2 AGG. 
ein Ausbildungsverhältnis oder gar der berufliche Aufstieg kann deswegen nicht „durchgeklagt" werden ${ }^{65}$ ).

Entgegen den Befürchtungen der deutschen Wirtschaftsverbände - insbesondere des Bundesverbandes der Deutschen Industrie (BDI), der Bundesvereinigung der Deutschen Arbeitgeberverbände (BDA) und des Deutschen Industrie- und Handelskammertages (DIHK) - ist es nicht zu einer „Prozessflut“ auf Seiten betroffener benachteiligter Menschen gekommen, was aber weniger daran liegen wird, dass heutzutage weniger diskriminiert wird als früher. Das macht man heutzutage wesentlich geschickter als vor den Zeiten des AGG, indem man z.B. einem Bewerber für ein Arbeitsverhältnis keinerlei Begründung mehr dafür liefert, warum er nicht eingestellt wurde. Fehlt ein Begründungszusammenhang, nützt auch die schönste Beweislastumkehr ${ }^{66}$ nichts; es gibt einige Anwaltskanzleien, die sich mittlerweile darauf spezialisiert haben, Personalabteilungen von Unternehmen so zu schulen, dass das Gesetz völlig legal umgangen werden kann. Die gesetzlichen Voraussetzungen, um klagen zu können, sind denn auch so schwammig und für den Benachteiligten nachteilhaft formuliert, dass es bislang kaum Judikate zum AGG gibt. Auch hier kann man, wenn man die unsägliche Entstehung des Gesetzes verfolgt hat, getrost davon ausgehen, dass genau dies gewollt war $^{67}$.

\subsubsection{Barrierefreiheit}

Barrierefrei sind gestaltete Lebensbereiche wie z.B. bauliche Anlagen, Verkehrsmittel, der Bereich der gesamten technischen Kommunikation und die Medien dann, wenn sie für behinderte Menschen in der allgemein üblichen Weise, ohne besondere Erschwernis und regelmäßig eigenständig zugänglich und nutzbar sind ${ }^{68}$.

Dabei sind die Begrifflichkeiten Zugänglichkeit und Nutzbarkeit umfassend zu verstehen, gemeint sind folglich nicht nur die Möglichkeit, die gestalteten Lebensbereiche als körper- und sinnesbehinderter Mensch tatsächlich nutzen zu können; dies gilt in gleicher Weise für Menschen mit intellektuellen Defiziten. Einen elementaren Punkt stellt es für diese Gruppe von Menschen dar, dass Texte in möglichst einfacher Sprache geschrieben werden.

Es ergibt sich also nicht nur die Notwendigkeit (um hier mit einem Beispiel zu arbeiten), dass ein körperbehinderter Mensch ein Gebäude auch mit einem Rollstuhl problemlos erreichen und sämtliche für ihn relevanten Bereiche „befahren“ kann. D.h., es muss Aufzüge geben, die Korridore und die Türen benötigen eine gewisse

\$22 AGG geht hier von einer Beweiswirkung von Indizien aus, die die Beweislast umkehren

67 S. hierzu auch meinen Kommentar „Gedanken zum Allgemeinen Gleichbehandlungsgesetz“ vom 03.10.2007 in den kobinet-nachrichten: http://www.kobinet-nachrichten.org/cipp/kobinet/custom/ pub/content,lang, 1/oid,16196/ticket,g_a_s_t.

$68 \S 4$ BGG und im Gefolge sämtliche deutschen Landesgleichstellungsgesetze, die regelmäßig auch hier selbst den gleichen Wortlaut verwenden wie die Norm des BGG. 
Breite, die Zimmerfluchten müssen so gestaltet sein, dass sie auch für z.B. einen Elektrorollstuhlfahrer nutzbar sind oder sich ein sinnesbehinderter Mensch im Gebäude zurecht findet. Es muss hierbei eine Gestaltung erfolgen, die die Nutzbarkeit auch für seh- und hörbehinderte Menschen ermöglicht, z.B. durch bestimmte Farbund Kontrastschemata und durch eine plausible Erläuterung auch der deutschen Lautsprache vielleicht nicht vollständig mächtiger Menschen, sondern eben auch, dass ein Mensch mit Lernbeeinträchtigung sich in dem Gebäude zu Recht finden kann. Das kann eben, wie bereits geschrieben, durch leichte Sprache oder durch sinnvolle Piktogramme erfolgen. Problematisch können sich jedoch selbst die an und für sich sinnvollen Regelungen auswirken, wenn sie auf die ,anerkannten Regeln der Technik“"69 verweisen.

Zwar spricht überhaupt nichts dagegen, immer dann nach den Regelungen einer „best practice“ zu verfahren, wo dies sinnvoll und nützlich ist. So kann von einem Einzelhändler mit durchschnittlichem Umsatz sicherlich auch in einer Zielvereinbarung nicht verlangt werden, dass er binnen kürzester Zeit umfassende Barrierefreiheit in seinem Ladengeschäft herstellt. Hier wird man mit mittel- und langfristigen Regelungen, die dem Stand der Technik entsprechen, arbeiten müssen. Allerdings sind die Vertragsparteien ja nicht gehindert, Lösungen zu finden, die - günstiger den gleichen oder zumindest den weitgehend gleichen Effekt erzielen.

Dass man sich jedoch auch an den ,,anerkannten Regeln der Technik“ mächtig stoßen kann, hat kürzlich in unrühmlicher Weise die Bundesarchitektenkammer im Lenkungsausschuss des DIN bewiesen, als es darum ging, die DIN 18040 in zwei Teilen zu verabschieden. War die Barrierefreiheit hinsichtlich des Teils 1, der sich mit der Barrierefreiheit öffentlich zugänglicher Gebäude befasst, kein streitiges Thema (was auch ein wenig merkwürdig gewesen wäre in Anbetracht der diesbezüglich eindeutigen gesetzlichen Lage), stand die Verabschiedung des Teiles 2, der sich mit Wohnungen beschäftigt, längere Zeit „,auf der Kippe“70.

Des Themas ,leichte Sprache“ hat sich zunächst - bereits vor mehreren Jahren der Verein „Mensch zuerst - People First Deutschland.“ angenommen. Dieser darf bis heute als zentraler Vorreiter selbst Betroffener im Bereich der Lerneinschränkungen gelten. In Zusammenarbeit mit „Mensch zuerst“ hat sodann das „Netzwerk Artikel 3“ den Versuch unternommen, erstmalig in Deutschland ein Gesetz, nämlich das BGG, auch in leichter Sprache zu erläutern ${ }^{71}$.

Während sich der Bund und auch die Landesverwaltungen darauf verpflichtet haben, wenigstens Neubauten barrierefrei herzustellen (und bei wesentlichen Um- und

$69 \S 8$ Abs. 1 Satz 1 BGG und im Gefolge sämtliche Landesgleichstellungsgesetze.

70 S. hierzu die Beiträge auf der Website des Deutschen Behindertenrates „Konsens für Norm zum barrierefreien Bauen gefährdet. DBR kritisiert Blockadehaltung der Bundesarchitektenkammer im DIN-Ausschuss“ vom 15.02.2008: http://www.deutscher-behindertenrat.de/ID62457, „Mogelpackung zum barrierenfreien Bauen verhindern“ vom 04.03.2008: http://www.deutscherbehindertenrat.de/ID63235 und „Künftig muss barrierefrei gebaut werden“ vom 24.04.2008: http://www.deutscher-behindertenrat.de/ID64872 (Stand: jeweils 30.05.2008).

71 Netzwerk Artikel 3 e.V. (Hrsg.), Gleichstellungsregelungen leicht gemacht! (Red.: Hans-Günter Heiden; juristische Beratung: Alexander Drewes), 2003, Berlin. 
Erweiterungsbauten ebenfalls Barrierefreiheit zu garantieren), tun sich die Verkehrsträger, insonderheit die auch ansonsten in der Öffentlichkeit in den letzten Jahren (meistenteils zurecht) vielgescholtene Deutsche Bahn mit der Herstellung von Barrierefreiheit reichlich schwer. So hat man es hier - auch um nur wieder ein Beispiel zu nennen - selbst bei der Bestellung des ICE der dritten Generation verabsäumt, zumindest einen Triebwagen barrierefrei zugänglich zu machen, wie dies bei Zügen und Triebfahrzeugen im Nahverkehr mittlerweile weitgehend üblich ist. Barrierefrei nutzbar sind die Züge und Triebfahrzeuge der Deutschen Bahn im Nahverkehr allerdings mittlerweile großenteils, wenn man denn von einer auf körperbehinderte Menschen reduzierten Nutzbarkeit ausgeht. Vielfach werden jedoch - gerade bei Verkehrsträgern und auch in Anschauung der Tatsache, dass im Nahverkehr mittlerweile auch bei dessen Beplanung die Belange behinderter Menschen zu berücksichtigen sind - nach wie vor behinderte Menschen hinsichtlich der Ausgestaltung von Fahrplänen, Fahrausweisen und sonstigen Informationen schlichtweg „vergessen“.

\subsubsection{Kommunikation und Medien}

Eine besondere Ausprägung der Barrierefreiheit stellen die Sektoren Kommunikation und Medien dar.

Hier hat zunächst der Bundesgesetzgeber im Rahmen seiner Gesetzgebungskompetenz umfänglich Regelungen geschaffen. Er:

- hat die Deutsche Gebärdensprache (DGS) als eigenständige Sprache anerkannt $^{72}$;

- lässt im Verwaltungsverfahren die DGS als auch andere Kommunikationshilfen für in der Kommunikation behinderte Menschen $\mathrm{zu}^{73}$;

- hat dafür gesorgt, dass auch blinden und sehbehinderten Menschen sie selbst betreffende Bescheide sowie Allgemeinverfügungen, Vordrucke oder öffentlich-rechtliche Verträge in einer auch für sie wahrnehmbaren Form zugänglich gemacht werden müssen ${ }^{74}$ (z.B. mittels Blindenschrift, Audiocassette oder

$72 \S 6$ Abs. 1 BGG und im Gefolge sämtliche Landesgleichstellungsgesetze.

$73 \S 9$ Abs. 1, Abs. 2 BGG i.V.m. der Verordnung zur Verwendung von Gebärdensprache und anderen Kommunikationshilfen im Verwaltungsverfahren nach dem Behindertengleichstellungsgesetz (Kommunikationshilfenverordnung - KHV) vom 17.07.2002 (BGBl. I (Nr. 49] S. 2650), zuletzt geändert durch Art. 15 durch Art. 12 des Gesetzes zur Änderung des Vierten Buches Sozialgesetzbuch und anderer Gesetze vom 19.12.2007 (BGB1. I [Nr. 67], S. 3024, 3034). Die DGS und andere Kommunikationshilfen dürfen mittlerweile auch in Gerichtsverfahren und im Sozialverwaltungsverfahren verwendet werden. Sämtliche Landesgleichstellungsgesetze sehen die Deutsche Gebärdensprache und andere Kommunikationshilfen in ihren Landesgleichstellungsgesetzen und den zugehörigen Kommunikationshilfeverordnungen mittlerweile als Rechtsanspruch in ihrer Kommunikation akustisch behinderter Menschen vor.

$74 \S 10$ Abs. 1, Abs. 2 BGG i.V.m. der Verordnung zur Zugänglichmachung von Dokumenten für blinde und sehbehinderte Menschen im Verwaltungsverfahren nach dem Behindertengleichstellungsgesetz (Verordnung über barrierefreie Dokumente in der Bundesverwaltung - VBD) vom 17.07.2002 (BGB1. I (Nr. 49] S. 2652). Dieser Rechtsanspruch greift auch aufgrund der Landes- 
- ganz einfach, aber für manche Behörde nach wie vor ein schieres Unding, wie ich selbst schon regelmäßig erfahren habe - mittels e-Mail) und

- hat sich verpflichtet, seine öffentlich zugängliche Informationstechnik barrierefrei zu gestalten ${ }^{75}$.

Während sich der Bund wenigstens im Bereich der Kommunikation umfänglich auf die Umsetzung von Barrierefreiheit „eingelassen“ hat, haben sich die Länder dem Ansinnen der Verbände, ähnlich umfassende Regelungen auch für den Bereich der Medien zuzugestehen (die Medien unterfallen dem Kultusbereich, sind also Ländersache), weitgehend verweigert. Das Forum behinderter Juristinnen und Juristen (FbJ) hatte im August 2002 zwar vollumfänglich auch Vorschläge zum Rundfunkrecht unterbreitet ${ }^{76}$, die Landesgesetzgeber haben sich hiermit jedoch nirgendwo in den Ausschuss- oder Plenarsitzungen bei Schaffung der Landesgleichstellungsgesetze positiv auseinandergesetzt. So sind blinde und sehbehinderte Menschen hinsichtlich der Audiodeskription, gehörlose, ertaubte und schwerhörige Menschen hinsichtlich der Gebärdung und / oder Untertitelung jeweils von Fernsehsendungen nach wie vor ausschließlich auf den guten Willen der Rundfunkanstalten angewiesen.

\section{Nachspruch und Fazit}

Aus der Darstellung im Vorwort, dass eine Beteiligung von Verbänden, Behindertenbeauftragten und behindertenpolitischen Sprechern der Bundestagsfraktionen kaum erfolgt ist, können im Grunde nur die wiederholt im Beitrag behaupteten Thesen geschlussfolgert werden. Behindertenpolitiken in Deutschland erschöpfen sich vielfach lediglich darin, als Alibi, zur Beruhigung der Betroffenen oder zur Vernebelung zu fungieren. Mit Behindertenpolitik wird vielfach Talmi betrieben und es werden Potemkinsche Dörfer aufgebaut. Das am meisten verblüffende Ergebnis der Arbeit ist wohl dieses, dass die meisten Ansätze, die von den Verbänden selbst ge-

gleichstellungsgesetze und der dort jeweils erlassenen Verordnungen für die Träger öffentlicher Gewalt im Bereich der Bundesländer sowie durch das GVG auch in Gerichtsverfahren, kurioser Weise jedoch - bislang - noch nicht im Bereich des Sozialverwaltungsverfahrens.

$75 \S 11$ Abs. 1 BGG i.V.m. der Verordnung zur Schaffung barrierefreier Informationstechnik nach dem Behindertengleichstellungsgesetz (Barrierefreie Informationstechnik-Verordnung - BITV) vom 17.07.2002 (BGBl. I (Nr. 49] S. 2654). Auch hierzu haben die Länder weitgehend wortgleiche Normierungen sowohl hinsichtlich des gesetzlichen Rahmens als auch im Hinblick auf Durchführungsverordnungen auf die Vorlage des Bundes zurückgegriffen. $\mathrm{Zu}$ dem Gesamtkomplex s. Drewes, „Zum Begriff der Barrierefreiheit im Internet für behinderte Menschen - juristische Aspekte“, in: JurPC Web-Dok. 252/2004 vom 17.09.2004, Abs. 1 - 31, auf: http://www.jurpc.de/ aufsatz/20040252.htm (Stand: 30.05.2008).

76 S. hierzu den Art. 5 „Änderung rundfunkrechtlicher Vorschriften“ des Entwurfs eines Landesgesetzes zur Gleichstellung behinderter Menschen und zur Änderung anderer Gesetze (LBGG), vorgelegt vom Forum behinderter Juristinnen und Juristen im August 2002, S. 54 - 60, veröffentlicht auf der Website des Netzwerks Artikel 3 e.V.: http://www.netzwerk-artikel-3.de/dokum/lbgg.pdf 
fordert werden, in der tatsächlichen Durchsetzung durch die Verbände als Fata Morgana oder als Beschäftigungstherapie für einige wenige privilegierte selbst Behinderte funktioniert.

Erstaunlich ist, wie wenig sich behinderte Menschen im politischen Prozess engagieren, was sich daraus ablesen lässt, eine wie kleine Anzahl selbst Behinderter sich tatsächlich als Mandatsträger in den Parlamenten finden lassen.

Das hat zum einen - natürlich - etwas damit zu tun, dass eine Diskriminierung im politischen Prozess der Aufstellung von Wahllisten nach wie vor gegeben ist. So finden sich behinderte Menschen in aller Regel auf hinteren Plätzen bei der Aufstellung von Wahllisten in den Parlamenten. Nimmt man den Bundestagsabgeordneten Dr. Ilja Seifert von der Partei DIE LINKE sowie den Abgeordneten der Bremischen Bürgerschaft Horst Frehe und das Mitglied des Hessischen Landtages Dr. Andreas Jürgens einmal heraus, findet sich kein einziger prominenter selbst behinderter Abgeordneter im Bundestag oder einem Landesparlament. Ähnlich erschreckend sieht es auf der Ebene der Kommunalparlamente aus. Auch hier finden sich, bis auf wenige Ausnahmen, allenfalls „Alibi-Behinderte“ in den Sitzungssälen.

Zum anderen - und damit sind wir beim wesentlichen Punkt und Ergebnis dieser Arbeit - haben aber auch die Verbände vielfach augenscheinlich überhaupt kein Interesse daran, Ihre Positionen politisch fruchtbar zu machen und sie in einem wissenschaftlichen Vergleich darzulegen.

Als Fazit - liest man sich zum Beispiel die Beiträge von Trenk-Hinterberger und Köhler in diesem Band durch - bleibt eigentlich nur festzuhalten: Hinsichtlich der Politiken für behinderte Menschen macht man es - häufig - in anderen Staaten besser als in Deutschland. Rechtsvergleichende Ansätze sind und bleiben dringend notwendig, Implementation internationalen, supranationalen oder auch nur ausländischen Rechts (wie, wie dieser Beitrag aufs Eindrücklichste aufzuzeigen versucht hat, deren Rechtsdurchsetzung) stellen eine dringend erforderliche Alternative zur gegenwärtigen Verballhornung dessen dar, was man in Deutschland momentan Behindertenpolitik zu nennen beliebt.

Allerdings würde man den deutschen behindertenpolitischen Protagonisten ab und an wünschen, sie nähmen die Ergebnisse der Disziplin Sozialrechtsvergleichung, als einer deren Nestoren Bernd Baron v. Maydell gelten muss, wenigstens in Ansätzen Ernst oder auch nur zur Kenntnis und versuchten nicht bei jedem neuen Projekt - sei es das AGG oder das BGG, die nächste Pflege"reform" oder die sicherlich bald wieder ins Haus stehenden nächsten Kranken- und Rentenversicherungs"reformen" oder das unsägliche und hier massiv kritisierte Beauftragten- und Beiratsunwesen - „das Rad neu zu erfinden“. Der große politische Wurf gelingt seit Jahrzehnten nicht (mehr) und scheint wohl auch gar nicht gewollt zu sein. Vielleicht ist das auch zu viel verlangt in einer Verbändedemokratie ${ }^{77}$ wie der unseren.

77 Dazu, wie die „Verbändedemokratie“ das demokratische System in Deutschland zunehmend aushöhlt, vgl. z.B.: Darnstädt, Die Konsens-Falle. Wie das Grundgesetz Reformen blockiert; 2004, München (Deutsche Verlags-Anstalt). 
Behindertenpolitik kann sich nicht in schierer Interessen(be)wahrung für einzelne Behindertengruppen erschöpfen, sie kann sich auch nicht auf Klientelpolitik zurückziehen, ohne die Öffentlichkeit (mit) einbeziehen zu wollen. Behindertenpolitik hat als Querschnittpolitik immer einen sozialrevolutionären Impetus, dass auch der behinderte Mensch ein tatsächlich Gleicher unter Nichtbehinderten sein will. Deshalb steht Behindertenpolitik viel mehr als sonstige z.B. sozialpolitische Einzeldisziplinen unter einem öffentlichen Rechtfertigungsdruck, gerade weil behindertenpolitische Ansätze regelmäßig nicht kostenneutral und im Kostenrahmen nicht einmal wirklich „günstig“ zu haben sind. Was ich hingegen bei der Ausarbeitung dieser Schrift erlebt habe, darf man getrost von nahezu allen Angeschriebenen unter die Rubriken:

„Wir sind etabliert und bekommen unsere finanziellen Zuwendungen, weshalb sollen wir noch unsere Arbeit öffentlich rechtfertigen oder uns sogar um einen fortschrittlichen politischen Ansatz bemühen?" oder

unter der politischen Formel „Ich kann in der Politik ansonsten nichts (mehr) werden, also bin ich behindertenpolitische/r Sprecher/in oder Behindertenbeauftragte/r geworden" zusammenfassen.

Es wird allerhöchste Zeit, dass - wie in den 1980er Jahren - wieder einmal einige politisch denkende behinderte Menschen deutlich Lärm schlagen, um das - erstarrte, ermüdete und vollkommen lethargisch gewordene - behindertenpolitische Establishment aufzuwecken und die vor wenigen Jahren begonnenen Reformansätze nicht nur endlich fruchtbar zu machen, sondern in tatsächliche reformerische Ansätze auswachsen zu lassen. 
5. Behindertenrecht und Behindertenpolitik im übrigen Europa 


\title{
5.1. Vertretung der Interessen behinderter Menschen in der Sozialpolitik Belgiens
}

\author{
Von Jef van Langendonck
}

$\begin{array}{lr}\text { 1. Strukturell } & 192 \\ \text { 2. Föderale Ebene } & 192 \\ \text { 3. Flämische Gemeinschaft } & 193 \\ \text { 4. Andere } & 194 \\ \text { 5. Interessen und ihre Vertretung } & 194\end{array}$ 


\section{Strukturell}

Die Zuständigkeit für die Sozialpolitik bezüglich der Behinderten ist in Belgien einigermaßen unglücklich über die verschiedenen Verwaltungsebenen verteilt. Wie man weiß, hat Belgien neben der föderalen Regierung und Parlament sechs andere Regierungen und Parlamente: drei für die Gemeinschaften (Flämische, Französischsprachige und Deutschsprachige) und drei für die Regionen (Flandern, Wallonien und Brüssel).

Nach dem Institutionellen Sondergesetz vom 8. August 1980 gehört die soziale Sicherheit zu der Zuständigkeit der föderalen Autoritäten. Die Gemeinschaften dagegen sind zuständig für Hilfe an Personen, sowie für bestimmte Aspekte der sozialen Förderung. Die Regionen befassen sich dann wieder mit bestimmten Aspekten der Arbeitsvermittlung.

Die Flämische Gemeinschaft und die Flämische Region haben sich in eine Flämische Regierung mit einem Flämischen Rat vereinigt. Die Französischsprachige Gemeinschaft hat, wegen Finanzierungsschwierigkeiten, Teile ihrer Zuständigkeit, auch für die Behinderten, an die Wallonische und Brüsseler Region übergetragen. Und in Brüssel sind die Flämische und die Französischsprachige Gemeinschaft beide zuständig, nachdem die Bürger es bevorzugen sich an niederländischsprachige oder an französischsprachige Leistungserbringer zu wenden.

Kompliziert wird es, wenn Anstalten und Institutionen zweisprachig sind. Diese können nur von einer Gemeinschaft offiziell anerkannt sein, aber es besteht die Möglichkeit, mit der anderen (meistens die Flämische) einen Sondervertrag zu schliessen. Dann müssen die Empfänger der Leistungen wählen unter welcher Gemeinschaftsgesetzgebung sie ihre Leistungen erhalten wollen.

Das Leben eines Behinderten in Belgien ist nicht immer einfach

\section{Föderale Ebene}

Die föderale soziale Sicherheit umfasst einen Sonderbereich, der finanzielle Beihilfen für Behinderte bietet (Gesetz vom 27. Februar 1987). Anders als die übrigen Branchen der sozialen Sicherheit kennt diese keine Selbstverwaltung durch Vertreter der Betroffenen. Die Leistungen werden, nach anglo-amerikanischem Modell, direkt von einer Regierungsstelle, unter der Verantwortung des zuständigen Ministers, erbracht.

Zwar wird bei dieser Stelle eine Beratungskommission installiert, die aus Personen besteht, "die, wegen ihrer Beteiligung an Aktivitäten von Institutionen, die sich mit der Sorge für Personen mit einer Behinderung, oder wegen anderer sozialer Tätigkeiten besonders geeignet sind" (Art. 31, Kön. Erlass 22. Mai 2003), aber dies müssen nicht notwendigerweise Vertreter der Behinderten oder deren Organisationen sein. Diese Kommission hat ihren Nutzen darin, dass sie bei Rückforderungsklagen der Verwaltung wegen unrechtmäßig erhaltener Leistungen 
(aufgrund Irrtums der Behörde oder falscher Angaben des Versicherten), die oft für die Betroffenen peinlich sind, in jedem Fall eine Empfehlung abgeben muss.

Das Gesetz (Art. 24 des früheren Gesetzes vom 27. Juni 1969) hat auch eine mehr allgemeine Beratungsstelle gegründet, die Hoher Rat für Personen mit einer Behinderung heißt. Dieser Rat besteht zur Hälfte aus Vertretern der Behindertenorganisationen und zur anderen Hälfte aus Vertretern der zuständigen Regierungsbehörden. Sie hat eine sehr weite Kompetenz bezüglich aller Angelegenheiten, die mit der Behindertenpolitik zu tun haben. Sie ist aber in den letzten Jahren wenig aktiv gewesen und hat keine sehr große Bedeutung.

Von größerer Bedeutung ist, sei es auch auf einem etwas beschränkten Gebiet, das Gesetz über Patientenrechte vom 22. August 2002. Dieses Gesetz betrifft jede Art von Dienstleistung zur Förderung der Gesundheit und damit auch viele Dienstleistungen an Behinderte. Es garantiert den Patienten ein klares Recht auf Information und auf informierte Zustimmung zu jeder Behandlung, auf Einsicht in ihre Dossiers und ein Klagerecht bei einem Ombudsdienst, der in jeder Anstalt vorhanden sein muss. Die Artikel 12 bis 14 organisieren die Vertretung eines Patienten, wenn er unfähig ist seine Rechte selber auszuüben. In der Beratungskommission, welche nach diesem Gesetz gegründet wird, sind Vertreter der Patienten, aber nicht der Behinderten vorgesehen.

\section{Flämische Gemeinschaft}

Bei der Flämischen Gemeinschaft spielt die Behindertenpolitik eine sehr bedeutende Rolle. Sie wird getragen von einer Institution, die früher Flämischer Fonds für die soziale Eingliederung von Personen mit einer Behinderung, jetzt aber mehr in moderner Managementsprache Flämische Agentur für Personen mit einer Behinderung genannt wird. Zu dieser Behörde gehört eine Beratungskommission, in der drei Interessensgruppen zu gleichen Teilen vertreten sind: Vertreter der Leistungsberechtigten, der Verwalter und der Arbeitnehmer der Leistungserbringer. Die Vertreter der Leistungsberechtigten werden von den Behindertenorganisationen bestimmt. Die wichtigste dieser Organisationen ist die Katholieke Vereniging voor Gehandicapten (KVG), die für die Behinderten das ist, was der Boerenbond für die Landwirte ist: eine Vertretung, eine Dienstleistungsorganisation und eine politische Lobby. Hier liegt oft die wirkliche Interessenvertretung der Behinderten.

Die Flämische Regierung hat darüber hinaus noch einige Regelungen verabschiedet, die für unseren Bereich von Bedeutung sind. Die wichtigste davon ist das Dekret über Qualitätsüberwachung in den Institutionen für die soziale Eingliederung von Personen mit einer Behinderung vom 15. Dezember 2000. Dieses Dekret verpflichtet diese Institutionen dazu, einen Qualitätsplan und ein Qualitätshandbuch auszuarbeiten. Für die verschiedenen Bereiche der Behindertenpflege werden "sektorspezifische Qualitätsnormen" festgelegt. Angestellte der Agentur kontrollieren die Erfüllung dieser Normen. Dazu gehören Regelungen über die "Benutzerbezogenheit", u.a. über das Recht auf Auskunft und Beratung, das 
Mitspracherecht der Leistungsempfänger und die Antwortpflicht der Institutionen in allen gemeinsamen Angelegenheiten, die Einbeziehung der Erfahrung der Kunden und die Förderung deren Mündigkeit. Die Institutionen sollen die Rechte und Pflichten der Leistungsempfänger klar in einem Dokument beschreiben, die Privatsphäre respektieren, sich über deren Zufriedenheit informieren und eine interne Beschwerdestelle organisieren. Auch soll in Anstalten, wo sich die Kunden länger als zwei Jahre aufhalten ein "Benutzerrat" gegründet werden, wovon ein Vertreter an den Sitzungen des Vorstands der Institution teilnehmen kann.

Das alles sind wichtige Garantien für die Behinderten, die solche Dienstleistungen in Anspruch nehmen - insoweit als sie sich in der Wirklichkeit auch tatsächlich auswirken. Die einzige Sanktionierung dieser Vorschriften läuft jedoch über den Entzug der Anerkennung der Institution, was den Behinderten nicht besonders viel helfen kann.

Von größerer praktischer Bedeutung ist die Möglichkeit für Behinderte, sich an den Vlaamse Ombudsdienst zu wenden, der jede Beschwerde mit den entsprechenden Behörden diskutieren und Problemlösungen vorschlagen kann.

\section{Andere}

Neben der Flämischen Agentur bestehen noch in anderen Teilen Belgiens:

- die Wallonische Agentur für die Eingliederung von Personen mit einer Behinderung

- der Französichsprachige Brüsseler Dienst für Personen mit einer Behinderung

- die Dienststelle für Personen mit Behinderung der Deutschsprachigen Gemeinschaft.

Sie haben meistens ähnliche Strukturen und Vorschriften wie die Flämische Agentur.

\section{Interessen und ihre Vertretung}

Behinderte haben meist unterschiedliche Interessen, die auf den verschiedensten Ebenen vertreten werden müssen. Leichtbehinderte und Schwerbehinderte sind nicht in der gleichen Lage. Blinde, Gehörgeschädigte, Gelähmte, geistig Behinderte, psychiatrische Patienten usw. haben andere Probleme und brauchen andere Dienstleistungen. Behinderte haben oft schwierige Beziehungen, nicht nur zu öffentlichen Behörden und Institutionen, sondern auch zu privaten Organisationen, Firmen, und zu Personen im privaten Bereich, wie Nachbarn und Familienmitgliedern. Sie haben Interessen im Familienleben, im Berufsleben, in der Beteiligung an der Kultur und an der ganzen menschlichen Gesellschaft. Das alles kann nicht auf einmal durch eine einzige Stelle vertreten und organisiert werden. 
Man hört oft in Belgien - sowie in anderen Ländern - scharfe Kritik an der chaotischen Organisation von Dienstleistungen für Behinderte. Mein alter Doktorvater, Rector Roger Dillemans, ist dafür bekannt, dass er gesagt hat: "Behinderter sein in Belgien ist wie abgeworfen werden in einem juristischen Urwald." Aber darin liegt auch ein gewisser Vorteil verborgen. Aus der Vielfalt an Stellen und Institutionen können sich die Behinderten diejenigen wählen, von denen sie glauben, dass ihre Interessen am besten vertreten werden. Und wenn sie von einer Stelle enttäuscht sind, können sie einfach zu einer anderen wechseln. Wenn sie gut beraten sind, liegt ein weiterer Vorteil darin, die verschiedensten Leistungen so zu kombinieren, dass sie daraus den größt möglichen Nutzen ziehen - auch wenn diese Kombinationen nicht immer vom Gesetzgeber so gemeint waren.

Die Katholieke Vereniging voor Gehandicapten (KVG) spielt diese Rolle auf hervorragende Weise. Sie ist eine Vereinigung, die völlig auf der Seite der Behinderten steht, auch gegen die öffentlichen Behörden und Institutionen. Sie hat einfach als Ziel, den Behinderten die größt möglichen Vorteile zukommen zu lassen. Die Behinderten in Belgien haben großes Vertrauen in diese Vereinigung. Sie ist zweifellos der wichtigste Interessenvertreter der Behinderten aller Art.

Sie stellt aber keine demokratische Vertretung der Behinderten dar. In den offiziellen Beratungsgremien, wo die Interessen der Behinderten vertreten werden sollen, repräsentiert sie zwar ihre Mitglieder, aber die Mitglieder sind nicht alle Behinderte und die Repräsentanten sind nicht demokratisch gewählt. Außerdem besteht auch die Gefahr, dass bestimmte Behinderte mit dieser Vereinigung in Konflikt geraten, z.B. bezüglich einer der vielfältigen Dienstleistungen, welche die Vereinigung bietet. Dann kommt man in die klassische Situation, wo der Hilfeempfänger gegen den Hilfebieter geschützt werden muss. Und das ist die Frage, die im Sozialrecht am schwierigsten zu lösen ist. In Belgien hat sie allerdings bisher noch keine Lösung gefunden.

An einem kann man ja nie vorbei: eine Person mit Behinderung ist schwächer als eine ohne (oder mit weniger) Behinderung. Und auch im Sozialstaat sind immer noch die Schwachen schwächer und die Starken stärker. Auch im Sozialbereich besteht was unser verstorbener Kollege Herman Deleeck den Matthäuseffekt genannt hat: „Denn wer da hat, dem wird gegeben werden, daß er Fülle habe; wer aber nicht hat, von dem wird auch genommen, was er hat.“ (Matthäus 25,29). Es geht nicht nur darum, dass die Nicht-Behinderten meistens sozial und wirtschaftlich stärker sind. Sie sind auch besser fähig die Dienste und Leistungen zu nutzen, die der Sozialstaat zur Verfügung stellt.

Ferner muss man bedenken, dass gerade die Bereiche, wo Behinderte am meisten Probleme haben können, sich dort befinden, wo es schwierig ist, sich Beweise für erlittenes Unrecht zu besorgen. In Familien, in Heime, in Betriebe hat die Außenwelt keinen Einblick. Wer dort gedemütigt, beraubt oder verprügelt wird, kann sich kaum wehren. Wer Klage einlegen will gegen Familienmitglieder, Heimpersonal oder Arbeitgeber kann nur sein Wort gegen das Wort des anderen stellen. Es gibt keine Unterlagen und keine unparteiischen Zeugen, die das wirklich Geschehene bestätigen können. Die Heimlichkeit wirkt immer zum Vorteil der Starken. Bei 
Verwaltung und Justiz werden Klagen ohne Beweis nicht ernst genommen. Solange es in den Familienheimen, in den Anstalten und in den Betrieben keine CCTV gibt, werden die Schwachen immer die Schwachen bleiben, welche Interessenvertretung man auch zustande bringen kann.

Belgien ist nicht besser oder nicht weniger gut gestellt als andere Länder, was die Interessenvertretung der Behinderten betrifft. Belgien hat etwas weniger formale Vertretungsstrukturen und vertraut etwas mehr auf nicht amtliche Organisationen. In vielen Fällen, sogar in den meisten Fällen, scheint das Ergebnis sehr zufriedenstellend zu sein. Aber gerade dort, wo Behinderte in einer delikaten Lage sind und ihre Interessen am meisten bedroht sind, bietet diese Struktur nicht genügend Garantie. Und man sieht auch nicht, wie sie das besser tun könnte.

Vielleicht kann dieses Projekt hier Aussicht auf neue Lösungen bringen. 


\title{
5.2. Die Maßnahmen für behinderte Menschen in den Niederlanden
}

\author{
Von Frans Pennings
}

1. Allgemeines 198

2. Die gesetzliche Regelung für Rollstühle, Anpassung der Wohnung und Transport

3. Rollstühle und Anpassungen der Wohnungen 200

4. Die persönliche Versorgung 200

5. Hilfe im Haushalt $\quad 202$

$\begin{array}{ll}\text { 6. Transport } & 203\end{array}$

7. Die Eingliederung in den Arbeitsprozess 204

7.1. Die unterschiedliche Behandlung der Behinderten 204

7.2. Die Implementierung der Antidiskriminierungsrichtlinien 204

8. Einkommensleistungen 205

$\begin{array}{ll}\text { 9. Schlussfolgerungen } & 207\end{array}$ 


\section{Allgemeines}

Das Hauptziel der niederländischen Behindertenpolitik ist es, den Behinderten zu ermöglichen, ein 'normales' Leben zu führen und am Arbeitsleben teilzunehmen. Natürlich ist dieses Ziel manchmal nicht einfach zu realisieren. Wichtige Faktoren für den Erfolg dieser Politik sind u.a. die Erkenntnisse der Behinderten selber, die Umgebung und die Art der Behinderung oder der Krankheit. Für die Eingliederung in die Arbeit sind auch die Haltung und Meinungen der Arbeitgeber sehr wichtig. (Vor)urteile über die Integrationsmöglichkeiten können zwar teilweise von öffentlichen Informationskampagnen beeinflusst werden, aber damit kann nicht immer das Hauptziel erreicht werden.

In diesem Beitrag werde ich die Maßnahmen beschreiben, die für Behinderte bestehen, um am Leben (inkl. Arbeitsleben) teilzunehmen. Über den Erfolg kann ich nur sehr wenig sagen.

Die Bestimmungen, die Maßnahmen für Behinderte betreffen, finden sich in verschiedenen Gesetzen wieder. Im niederländischen Grundgesetz gibt es keine behinderten-spezifischen Bestimmungen. Trotzdem können einige Grundrechtsbestimmungen Bedeutung für Behinderte haben, weil sie eine Arbeitsförderungspflicht des Staates, die Förderung der Einkommenssicherheit und die Verteilung des Wohlstandes verlangen. Diese sogenannten sozialen Grundrechte fordern deshalb Maßnahmen der öffentlichen Organe, aber sie spezifizieren nicht die Formen und den Inhalt der geforderten Aktivitäten. Die Grundrechtsbestimmungen enthalten auch keine subjektiven Rechte, die man gerichtlich durchsetzen könnte.

Ich werde darum die gesetzlichen Regelungen besprechen, die konkretere Maßnahmen beinhalten. Erstmal werde ich grundsätzlich die gesetzlichen Regelungen für Rollstühle, für Anpassungen der Wohnung und für Transport besprechen (unten 2). Danach werde ich diese Regelungen im Besonderen erörtern. Zunächst diskutiere ich die Mittel, die für den Behinderten im täglichen Leben wichtig sind, das sind z.B. Rollstühle und Anpassungen der Wohnung (z.B. Aufzüge) (unten 3). Ein spezielles Gebiet betrifft die Unterstützung der persönlichen Versorgung (unten 4) und die Hilfe im Haushalt (unten 5). Für die Teilnahme am sozialen Leben sind die Transportmöglichkeiten von großer Bedeutung (unten 6). Dann werde ich die Unterstützung der Eingliederung in den Arbeitsprozess diskutieren (unten 7) und die darauf abgestimmten Einkommensleistungen (unten 8).

Ich werde stets spezielles Augenmerk legen auf die sogenannten persönlichen Budgets, die unter bestimmten Bedingungen für Behinderte verfügbar sind: Sie können mit einem solchen Budget zum Beispiel entscheiden, mit wem sie für ihre persönliche Versorgung kontrahieren möchten. Auf Grund eines persönlichen Budgets können sie auch einen Versorgungsvertrag mit einem Familienmitglied schließen. Diese Wahlfreiheit gibt den Behinderten Möglichkeiten, ihr Leben so normal wie möglich zu führen.

Ein persönliches Budget kann auch für die Eingliederung ins Arbeitsleben genutzt werden, zum Beispiel für Schulungen oder einen Jobcoach, die vom Behinderten selber ausgewählt werden. 


\section{Die gesetzliche Regelung für Rollstühle, Anpassung der Wohnung und Transport}

In den 90er Jahren des zwanzigsten Jahrhunderts wurden die Maßnahmen für Behinderte in einem Gesetz, De Wet voorzieningen gehandicapten (WVG - Gesetz über Maßnahmen für Behinderte) zusammengefasst. Dieses Gesetz ersetzte die bis dahin bestehenden Bestimmungen in dem Wet op de algemene arbeidsongeschiktheidsverzekering (AAW - Allgemeines Arbeitsunfähigkeitsversicherungsgesetz), welches 1987 aufgehoben wurde, und andere Gesetze mit Behindertenmaßnahmen. Dies brachte einige grundlegende Änderungen im Behindertenrecht mit sich: In dem neuen Gesetz wurde nicht mehr dem Staat, sondern nun den lokalen Gemeinden die Verpflichtung auferlegt, adäquate Versorgung zu gewähren in Form von Rollstühlen, im Bereich des Transportwesens und in Bezug auf behindertengerechte Anpassungen der Wohnung. Die Verpflichtung in diesem Bereich wurde also dezentralisiert. Die Gemeinden bekamen die alleinige (auch finanzielle) Verantwortlichkeit für diese Maßnahmen übertragen. Zwar werden ihnen zur Durchführung dieser Maßnahmen pauschale Summen vom Staat bezahlt (die Summe, die früher auf Grund des AAWs für solche Maßnahmen verfügbar war, wird jetzt den Gemeinden bezahlt), aber die Gemeinden haben grundsätzlich die Freiheit, das Geld auch für andere Sachen als die im Gesetz genannten Maßnahmen zu bezahlen.

Zudem ergab sich eine wichtige Änderung in Bezug auf den Berechtigtenkreis: Während nach dem AAW der Anspruch auf solche Maßnahmen auf arbeitsunfähige Personen beschränkt war, erstreckt sich die Verantwortlichkeit der Gemeinden nach dem WVG nun allgemein auf alle Personen, die dieser Unterstützung bedürfen, wodurch insbesondere ältere Personen über 65 Jahre als Leistungsempfänger neu einbezogen wurden. Diese können natürlich auch Transportunterstützung oder einen Umbau der Wohnung benötigen. Dies bedeutete eine wichtige Ausweitung des Berechtigtenkreises - allerdings mit gleichbleibenden Mitteln. Die neue gesetzliche Verpflichtung stellt die Gemeinden also vor die Herausforderung, einerseits adäquate Versorgung für Behinderte zu gewährleisten, andererseits aber auf eine ökonomische Art und Weise. Die Versorgung soll also so billig wie möglich sein, aber sie muss trotzdem adäquat sein. Zu billig darf sie also nicht werden. Die Gemeinden können zwar aus den eigenen Haushaltsmitteln bezahlen, wo der Staatszuschuss nicht ausreicht, aber man kann verstehen, dass darüber leicht politische Diskussionen in der Gemeindevertretung entstehen können.

Hierzu soll außerdem noch angemerkt werden, dass in den vergangenen Jahren eine Neugliederung der Gemeinden stattgefunden hat. Die frühere Anzahl wurde um gut die Hälfte reduziert. Die neuen Gemeinden sind ziemlich groß und haben also auch mehr Möglichkeiten ihre Verantwortlichkeiten professionell zu gestalten. Im Allgemeinen kann man sagen, dass eine solche Professionalisierung auch tatsächlich stattgefunden hat.

Das WVG verpflichtete hierfür jede Gemeinde, eine Regelung zu erlassen, in der ausgestaltet wird, wie die Gemeinde ihrer gesetzlichen Verpflichtung zur Versorgung der Behinderten nachkommen will. Um diese Verpflichtung zu erleichtern, hat 
die Vereinigung der Gemeinden eine Musterverordnung gemacht; die Gemeinden können diese nach eigener Einsicht übernehmen.

Die genauen Ansprüche der Unterstützungsbedürftigen werden individuell festgestellt; dazu wird der Antragsteller zunächst ärztlich untersucht, um seine Bedürfnisse feststellen zu können. Die Ansprüche können angepasst werden, wenn sich die Einschränkungen des Betroffenen verändert haben. Behinderte, die unzufrieden sind mit den für sie festgestellten Ansprüchen, können die Entscheidung vor Gericht anfechten. Die genauen Prozeduren werden hiernach noch besprochen. ${ }^{1}$

Seit 1. Januar 2007 ist das WVG wiederum ersetzt worden durch das Wet maatschappelijke ondersteuning (WMO - Gesetz der sozialen Unterstützung), das für die im folgenden Paragrafen genannten Maßnahmen allerdings wenig Änderungen gebracht hat.

\section{Rollstühle und Anpassungen der Wohnungen}

Auf Grund des WMOs ist die Gemeinde verpflichtet, Rollstühle und Anpassungen der Wohnungen zu finanzieren, wenn der Leistungsberechtigte dafür eine Indikation bekommen hat.

Da die Gemeinde bei der Leistungserbringung ökonomisch wirtschaften muss, ist sie berechtigt, bestimmte Rollstühle einzukaufen (z.B. mit Mengenrabatt) und den Berechtigten zuzuweisen, sofern eine adäquate Versorgung im Einzelfall gewährleistet bleibt.

Ähnliches gilt bei einer Indikation für einen Umbau der Wohung. Auch hier hat die Gemeinde das Recht, dem Behinderten zunächst andere Wohnungen zuzuweisen, die schon früher angepasst wurden. Es ist dabei aber wichtig, dass eine solche andere Wohnung adäquat ist für den jeweiligen Antragsteller. Diese individuelle Frage ist nicht immer einfach $\mathrm{zu}$ beantworten; die gesundheitlichen Faktoren sind dafür am wichtigsten.

Seit dem WMO müssen die Gemeinden allerdings auch in diesem Bereich ein persönliches Budget anbieten, das dem Leistungsempfänger unter bestimmten Voraussetzungen erlaubt, selbst Anpassungen in der eigenen Wohnung - allerdings unter Einbeziehung eigener finanzieller Mittel - vorzunehmen.

\section{Die persönliche Versorgung}

Versorgung kann auf verschiedene Weise stattfinden, z.B. im Krankenhaus, in einem Pflegeheim und auch zu Hause. Dafür gibt es viele Institutionen wie das Pflegeheim, und Organisationen, die Hilfe zu Hause anbieten. Weil dieser Beitrag

1 Siehe im Allgemeinen zum WVG: Vermaat, Voorzieningen in de Wet voorzieningen gehandicapten: verantwoorde voorziening of participatievoorziening?, Den Haag 2003 
hauptsächlich die (Un)möglichkeiten der Behinderten betrifft, in der Gesellschaft und im Arbeitsleben zu partizipieren, werde ich mich bei der Behandlung der Versorgung auf das persönliche Budget beschränken.

Auf dem Algemene wet bijzondere ziektekosten (AWBZ - Allgemeines Gesetz betreffend besondere gesundheitliche Ausgaben) basieren verschiedene Budgets für die persönliche Versorgung. Die persönlichen Budgets geben dem Individuum eine ziemlich große Wahlfreiheit, weil das Individuum mit dem 'Provider' ein Übereinkommen über die gewünschte Versorgung treffen kann. Der Budgetinhaber kann also die Versorgung auf seine individuellen Wünsche abstimmen lassen. Für diejenigen, die nicht in der Lage sind, die persönlichen Budgets zu beantragen und zu administrieren, besteht noch immer die Möglichkeit, die institutionelle Versorgung zu nutzen, wie z.B. in einem Pflegeheim oder von einer Organisation, die Hilfe zu Hause anbietet. Eine Kombination traditioneller und moderner Methoden ist auch möglich.

Im Bereich der persönlichen Versorgung sind Budgets verfügbar für die alltägliche Versorgung (z.B. Hilfe bei tägliche Handlungen wie aufstehen, duschen, ankleiden, essen, trinken, Toilettenbesuch), die Pflegeversorgung (z.B. Hilfe bei Medizingebrauch, Wundversorgung, Beatmung), die unterstützende Begleitung (Unterstützung bei Aktivitäten zu Hause oder anderswo), die Hilfe zur Aktivierung (z.B. um zu lernen mit der Behinderung zu leben oder selbständig leben zu lernen oder zu arbeiten), und für den zeitlichen Verbleib außer Haus (z.B. einige Wochen während des (Schul)Urlaubs, um die Eltern etwas zu entlasten, Maximum 104 Tagen pro Jahr).

Die Entscheidungen über die Budgets werden vom Centrum Indicatiestelling Zorg (Zentrum für Indikationsfeststellung für Versorgung) oder vom Bureau Jeugdzorg (für Personen jünger als 18) vorgenommen.

Das Zentrum stellt fest, welche Versorgung individuell benötigt wird. Dies wird in einer Entscheidung festgelegt, die angibt auf welche Versorgungsfunktionen der einzelne einen Anspruch hat. Bei jeder Funktion wird auch eine sogenannte Klasse angegeben, womit die Zahl der Stunden pro Woche gemeint ist, in denen ein Anspruch auf diese konkrete Versorgung besteht. Die Entscheidung kann zunächst beim CIZ und danach auch beim Gericht angefochten werden.

Das CIZ oder das Bureau Jeugdzorg senden die Entscheidung an die Leistungsadministration des AWBZ. Diese entscheidet über das Budget für die Versorgung. Die Beiträge werden festgestellt vom College voor Zorgverzekeringen. ${ }^{2}$

Die Budgets kommen der Wahlfreiheit der Behinderten sehr zu Gute. Wer ein Budget bekommen hat, kann sich die versorgende Person - zum Beispiel auch Bekannte oder Familienmitglieder - selber aussuchen und die Bedingungen der Versorgung selbstständig mit ihr regeln. Der Versorger wird dann aus dem Budget bezahlt. Auf den ersten Blick könnte man dabei Bedenken haben, dass auch Familienmitglieder bezahlt werden für Hilfe, die traditionell auf Grund des Familienbandes 
gegeben wurde. Die Versorgung verlangt aber manchmal viel mehr als man von Familienmitgliedern erwarten darf oder will. Zudem ist die Versorgung häufig nicht zu vereinbaren mit der Arbeitsstelle des versorgenden Familienmitglieds oder Bekannten, wodurch also ein Einkommensverlust eintritt und somit eine Bezahlung für die Versorgung durchaus gerechtfertigt ist.

Außerdem ermöglicht das Budget auch professionelle Vereinbarungen mit dem Versorgenden zu treffen. Dies gibt dem Versorgungsbedürftigen mehr Selbstständigkeit in Entscheidungen über seine Versorgungssituation und verkleinert seine Abhängigkeit von anderen Personen. Auch kann ein Budget für eine kurzfristige Unterbrechung der Versorgung (z.B. in einem Urlaubscamp für ein behindertes Kind) die Familie entlasten und ihr deswegen ermöglichen, die (unbezahlte) Versorgung dieser Person langfristig aufrecht zu erhalten.

Der Budgetinhaber kann den Lohn für die Versorgung selber mit dem Versorgenden verabreden, aber hier gelten natürlich die gesetzlichen Bestimmungen, wie der Mindestlohn und Urlaubsbestimmungen.

Die Vereinbarung wird schriftlich festgehalten; es gibt Musterkontrakte, die man hierfür benutzen kann.

Außerdem bieten die Vereinigung der Budgethalter ${ }^{3}$ und ein Servicezentrum, eingerichtet bei der Sociale Verzekeringsbank (Leistungsadministration für die Volksversicherungen $)^{4}$, Hilfe bei der Gestaltung des Budgetvertrags an. Das gilt auch für die anderen Budgets, wie z.B. im Rahmen des WMO.

Der Budgetinhaber muss seine Ausgaben nachweisen; er kann die Kosten für die Versorgenden, inklusive Vergütungen für Reisekosten, sowie die Vermittlungskosten bei der Suche nach einem Versorgenden deklarieren. Ausgenommen von der Nachweispflicht sind 1,5\% des Netto-Jahresbetrags (Minimum 250 Euro und maximal 1250 Euro); dieser Anteil kann flexibel für die Lösung von Problemen eingesetzt werden.

Seit 2007 gibt es auch persönliche Budgets für eine langfristige Versorgung. Wer eine Indikation für einen langfristigen Verbleib in einer Institution bekommen hat und die Versorgung trotzdem lieber zu Hause organisieren möchte, kann auch hierfür ein PGB erhalten. Das Budget darf nicht geringer sein als der Tagespreis für eine institutionelle Versorgung

\section{Hilfe im Haushalt}

Die Hilfe im Haushalt ist jetzt ebenfalls Bestandteil des WMOs. Für die Beschäftigung einer Hilfskraft kann daher, wie bei der persönlichen Versorgung, ein persönliches Budget gewährt werden. 
Für die Verwaltung und Kontrolle der Budgets kann die Gemeinde entweder selbst die Verantwortung übernehmen und diesbezügliche Regelungen treffen, oder aber das Servicezentrum PGB der SVB beauftragen.

Um die Indikation festzustellen wird eine Gemeinde das schon genannte Centrum Indicatiestelling Zorg einschalten.

\section{Transport}

Auch im Bereich des Transports wurden die Gemeinden nach dem WVG und später nach dem WMO dazu verpflichtet, die Maßnahmen für Behinderte so zu organisieren, dass adäquate und zugleich billige Versorgung gewährleistet ist. Die Gemeinden haben daher sogenannte Regiotaxis organisiert, die auf Abruf verfügbar sind. Diese müssen aber manchmal mit anderen behinderten Kunden geteilt werden und kennen (deshalb) auch Wartezeiten. Die Kunden müssen die örtlichen Preise für den öffentlichen Transport bezahlen. Wenn die beschränkte Mobilitat solches fordert, z.B. um in die Wohnung oder in andere Gebäude hinein zu kommen oder um einkaufen zu gehen, dann wird die Taxiregelung ergänzt z.B. durch ein Scootmobil oder einen Behindertenwagen. Nur für Personen, die unmöglich mit einem Taxi reisen können, gibt es teurere Maßnahmen, z.B. ein behindertengerechtes Fahrzeug.

Für Personen, die schon unter dem AAW diesbezügliche Ansprüche hatten, bedeutet die neue Regelung manchmal eine Verschlechterung, da unter der alten Regelung viel mehr Personen einen angepassten privaten Wagen bekamen. Jetzt sind sie auf das Regiotaxi angewiesen, was - wie nun einmal bei kollektivem Transport oft der Fall - weniger angenehm ist. Aber man kann dies noch adäquat nennen und für die Gemeinschaft ist es billiger; auf diese Weise kann für viel mehr Personen Transport organisiert und bezahlt werden.

Außerdem ist das Regiotaxi in der Regel auf das Territorium der Gemeinde beschränkt; in Zusammenarbeit mit Nachbargemeinden kann der Bereich der Taxis aber ausgeweitet werden.

Weil die Gemeinden im Rahmen des WMO eine diskretionäre Kompetenz haben, die sie in ihren örtlichen Regelungen ausgestaltet haben, darf das Gericht die Entscheidungen auf Grund dieses Gesetzes nur marginal prüfen. Das bedeutet, dass die Klagen der Behinderten in vielen Fällen nicht erfolgreich sind. Wenn es aber offensichtlich ist, dass eine Maßnahme nicht tatsächlich abgestimmt ist auf die gesundheitlichen Einschränkungen des individuellen Behinderten (also nicht adäquat ist), dann wird die Entscheidung nicht gültig bleiben. So hat das Gericht z.B. schon entschieden, dass die Beschränkung der Transportmaßnahmen auf Gemeindegrenzen nicht angemessen ist, wenn soziale Isolierung und Einsamkeit für das Individuum droht. Dies wird aber nicht schnell vom Gericht angenommen.

Seit 1. Januar 2007 ist das WVG ersetzt vom Wet maatschappelijke ondersteuning, wie wir schon gesehen haben. Für die Transportmaßnahmen bedeutet dies nur wenige Änderungen, außer dass Gemeinden nun auch die Möglichkeit haben, ein persönliches Budget für Transport zu gewähren. Dies kann bedeuten, dass eine be- 
hinderte Person statt einer spezifischen Maßnahme in Natura eine Summe Geld bekommen kann, um eine gesetzlich nicht gewährte, aber individuell gewünschte Maßnahme zu realisieren. So kann ein Behinderter mit dem persönlichen Budget und einer eigenen Zuzahlung ein behindertengerechtes Fahrzeug erwerben.

Für die Prozedur siehe oben unter 5. (Hilfe im Haushalt.)

\section{Die Eingliederung in den Arbeitsprozess}

\subsection{Die unterschiedliche Behandlung der Behinderten}

Für die Unterstützung der (Wieder)eingliederung in den Arbeitsprozess von Behinderten gibt es einige Subventionsregelungen, die meistens in den jeweilig relevanten Leistungsgesetzen zu finden sind. Die Art der Subvention hängt also von der Art der Leistung ab. Behinderte mit einem Sozialhilfeanspruch sind demnach auf andere Subventionstypen angewiesen als Behinderte, die eine Versicherungsleistung (WIAleistung) erhalten.

Formen der Unterstützung sind ein Job Coach, die Freistellung des Arbeitgebers von der gesetzlichen Verpflichtung den Mindestlohn zu bezahlen (in diesem Fall wird der Lohn durch Leistungen ergänzt, um das Mindestlohnniveau zu erreichen), Leistungsubventionen und Subventionen für die Einrichtung behindertengerechter Arbeitsplätze. Einige dieser Subventionen können auch in Form eines persönlichen Budgets zuerkannt werden. ${ }^{5}$

\subsection{Die Implementierung der Antidiskriminierungsrichtlinien}

Für den tatsächlichen Zugang zum Arbeitsprozess kann auch die Antidiskriminierungsgesetzgebung Bedeutung haben. Die EU Gleichbehandlungsrichtlinie 2000/78 wurde in den Niederlanden im Gesetz über gleiche Behandlung auf Grund eines Handicaps oder chronischer Krankheit umgesetzt. Es gibt also ein spezielles Gesetz für diese Antidiskriminierungsbestimmungen; sie sind kein Unterteil des Allgemeinen Gleichbehandlungsgesetzes. Die unterschiedliche Behandlung der Diskriminierungsmerkmale - der auch bei Unterscheidungen auf Grund Alters gefolgt wird - ist darauf gegründet, dass im Allgemeinen Gleichbehandlungsgesetz nur wenige, limitativ genannte Rechtfertigungsgründe für Ungleichbehandlungen möglich sind. Im Fall der Ungleichbehandlung auf Grund einer Behinderung gibt es solche Beschränkungen nicht. Der Gesetzgeber konnte also nicht von vornherein absehen, wenn ein Unterschied gerechtfertigt ist. Jetzt wird aber ein Entwurf für ein neues Allgemeines Gleichbehandlungsgesetz vorbereitet, in dem alle Gründe integriert werden. rechtsvergelijkende analyse, Deventer 2000. 
Das Gesetz verbietet Diskriminierung auf Grund eines Handicaps oder chronischer Krankheit in Berufsausbildung, Berufsvermittlung, bei Prüfungen und beim Zugang zu Arbeit. Auf Grund des Gesetzes sind Arbeitgeber verpflichtet, wirksame Anpassungen der Arbeitsplätze zu realisieren, es sei denn, dass diese eine unverhältnismäßige Last darstellen. Eine Anpassung ist wirksam, wenn sie geeignet und notwendig ist, um einen Behinderten in der Gesellschaft partizipieren zu lassen. Sie ist geeignet, wenn die Anpassung Hindernisse für eine unabhängige, volle Teilnahme oder Integration einer behinderten Person - welcher Art auch immer - beseitigen kann. Die Anpassungen können immaterieller Art sein, wie eine Anpassung der Arbeitszeiten und Pausen, aber auch materieller Art, wie behindertengerechte Stühle oder Computer mit Spracherkennung. Der Ausnahmegrund der unverhältnismäßigen Last bedeutet, dass die Interessen der Behinderten abgewogen werden müssen mit den Interessen des Arbeitgebers. Hierfür sind der Umfang der Organisation oder Unternehmung und die Kosten der Investition wichtig, wobei verfügbare Subventionen berücksichtigt werden können.

Das Gesetz lässt positive Maßnahmen zu, wenn diese dazu beitragen, dass bestehende Nachteile für Behinderte ausgeglichen werden.

Das Gesetz betrifft noch nicht alle gesellschaftlichen Bereiche, zum Beispiel noch nicht den öffentlichen Transport, das Wohnungswesen und die Produkt- und Dienstleistungen, aber im Laufe der Zeit wird der Wirkungsbereich des Gesetzes ausgedehnt werden.

\section{Einkommensleistungen}

Im Jahr 2006 ist ein ganz neues Gesetz für Arbeitsunfähigkeitsleistungen in Kraft gesetzt geworden. Nachdem dieses Gesetz auf ganz neue und radikale Weise die Arbeitseingliederung von Behinderten fördert, werde ich diese Elemente beschreiben.

Die Leistungsgesetze sind in einer ziemlich kurzen Zeit ganz radikal geändert worden. In den 60er Jahren, einem Jahrzehnt wirtschaftlichen Wachstums, war eine schnelle Entwicklung des Wohlfahrtsstaates möglich; die Verabschiedung des Gesetzes, genannt Wet op de arbeidsongeschiktheidsverzekering (WAO - Gesetz über Arbeitsunfähigkeitsversicherung), im Jahre 1966 war ein richtiger Meilenstein. In den ersten Jahren waren generöse Leistungen von 80\% des ehemaligen Lohns, möglich, doch gab es in dieser Periode nur sehr wenige Arbeitseingliederungsinstrumente.

10 Jahre später wurde eine Volksversicherung für Behinderte eingeführt, das $\mathrm{Al}$ gemene Arbeidsongeschiktheidswet (AAW - Allgemeines Behindertengesetz). Auf Grund dieses Gesetzes wurden alle Einwohner der Niederlanden für den Fall der Arbeitsunfähigkeit versichert. Die Höhe der Leistung war maximal 70\% des Mindestlohns, d.h. ebenso hoch wie die Sozialhilfe für einen Alleinstehenden. Für die Behindertenleistung wurden aber nicht das Vermögen und Einkommen von anderen (Ehegatte) in Anspruch genommen. 
In den 80er Jahren wurde das WAO in der Praxis manchmal benutzt als eine Einkommensregelung für Arbeitnehmer, die von einer Betriebsschließung betroffen waren. Die Arbeitsunfähigkeitsrente war in diesen Fällen viel attraktiver als Arbeitslosengeld, weil letztere Leistung nur für eine bestimmte Frist bezahlt wurde. Selbstverständlich mussten die von Betriebsstilllegungen betroffenen Antragsteller für die WAO-Leistung arbeitsunfähig sein, aber zu dieser Zeit war eine solche Beurteilung ziemlich leicht zu erhalten - sie fand manchmal nur auf Grund eines Dossiers statt. Für diese Entwicklung war auch eine Regelung des Gesetzes sehr wichtig, die auch teilweise Behinderten eine Leistung für völlig Behinderte gewährte, wenn sie auf Grund ihres Handicaps nicht im Stande waren Arbeit zu finden. Das war der Fall, wenn man sagen konnte, dass sie arbeitslos waren, weil kein Arbeitgeber sie wegen ihrer Behinderung annehmen mochte. Die Arbeitsunfähigkeit war also durch die behinderungsbedingte Arbeitslosigkeit indiziert.

Weil diese Anzahl der Arbeitsunfähigkeitsleistungempfänger sehr schnell zunahm, wurde das Gesetz einige Male geändert. Wichtige Elemente dieser Änderungen waren die Beendigung der durch die Arbeitslosigkeit indizierten Arbeitsunfähigkeit und die Änderung der Definition von Arbeit, die für die Schätzung der Einkommenskapazität benutzt wird; die theoretische Einkommenskapazität bestimmt den Grad der Arbeitsunfähigkeit.

Die Schätzung nimmt nun jede mögliche Arbeit in Betracht, also nicht nur passende Arbeit (Arbeit, die der Arbeitsunfähige bis zu seiner Krankheit ausübte). Mit den Worten des Gesetzgebers: der Focus soll nicht mehr auf der Vergangenheit liegen (was kann man nicht mehr?) sondern auf der Zukunft (was kann man noch?). Es darf aber nicht übersehen werden, dass als Folge dieser Änderung auch viele Leistungsempfänger ihren Anspruch verloren (für ältere Leistungsempfänger galten aber Übergangsbestimmungen, auf Grund deren sie ausgenommen waren von dieser neuen Schätzungsmethode).

Im Jahr 2006 wurde diese Tendenz verstärkt von einem ganz neuen Gesetz, dem Wet Werk and Inkomen naar Arbeidsvermogen (WIA - Arbeit und Einkommen nach Arbeitsleistungskapazitätgesetz), das für neue Antragsteller gilt (für diejenigen, die bereits eine WAO-Leistung bekommen bleibt das WAO bestehen).

Das Hauptziel des Gesetzes ist es, die Leistungen zu beschränken auf völlig und dauerhaft Arbeitsunfähige; sie sollten eine höhere Leistung bekommen als unter dem WAO. ${ }^{6}$ Die anderen, also auch die völlig Arbeitsunfähigen, bei denen dieser $\mathrm{Zu}-$ stand noch nicht als permanent eingeschätzt wird, sollten dagegen sehr starke Incentives bekommen, um wieder mit Arbeit anzufangen. In der Hauptsache bestimmt das Gesetz, dass man eine viel höhere Leistung bekommt, wenn man mindestens 50\% der Verdienkapazität aus Arbeitseinkommen realisiert. Die anderen bekommen eine sehr kleine Leistung. Für den Arbeitsunfähigen ist es also sehr wichtig, die Arbeit zu behalten oder neue Arbeit zu bekommen.

6 Die Personen bekommen nach einer Wartefrist von 104 Wochen eine Leistung von 75\% ihres früheren Lohn. Es gibt sehr strikte Bestimmungen um festzustellen, ob sie dauerhaft völlig arbeitsunfahig sind. 
Es gibt, wie wir oben gesehen haben, auch Instrumente um Arbeitsunfähigen bei der Eingliederung in Arbeit zu helfen. Man kann aber sagen, dass die Einkommenspolitik, wie in dem WIA Gesetz zum Ausdruck gebracht, das wichtigste Instrument war. Das Gesetz ist 2004 eingeführt worden und es gibt noch nicht sehr viele Informationen über die Effekte. Im Allgemeinen besteht aber der Eindruck, dass es für Arbeitsunfähige noch immer sehr schwierig ist, Arbeit zu bekommen und dass diese Regelung nach wie vor zu viel Einkommensverlust bringt für Menschen, die arbeitsunfähig geworden sind. Auf der anderen Seite sieht man auch, dass die Anzahl der Antragsteller sehr gesunken ist; man kann also auch vermuten, dass Personen, die krank geworden sind, alles tun, um in Arbeit zu bleiben und keine Arbeitsunfähigkeitsleistung zu beantragen. Das könnte ein positiver Effekt des Gesetzes sein, aber nur umfangreiche empirische Untersuchungen können mehr Klarheit schaffen.

\section{Schlussfolgerungen}

In der oben gegebenen Beschreibung des niederländischen Systems kann man einige interessante Entwicklungen sehen, die den Behinderten einige Wahlfreiheit geben. Man kann aber auch einschränkende Entwicklungen erkennen, weil das Kriterium der Kostenökonomie nötig wurde, u.a. weil mehr und mehr Leute Unterstützung bedürfen (Überalterung der Gesellschaft).

Man kann sich fragen, ob und wie diese Entwicklung kulturell beeinflusst ist. Ich meine, dass man dies nur sehr schwer feststellen kann. In jedem Fall geht „die Kultur" nicht sehr weit zurück: Vor den 70er Jahren des 20sten Jahrhunderts bestand sehr wenig Unterstützung für Behinderte.

Seitdem können wir eine schnelle Entwicklung sehen, von einer ziemlich generösen und wenig kontrollierten Leistungszuerkennung (am Anfang des Versorgungsstaates) zu einem mehr rationellen und restriktiven System, das graduell auch mehr Wahlfreiheit zulässt. Die persönlichen Budgets sind formell kein Experiment mehr, aber man sieht schnelle Änderungen, wenn es Probleme gibt. Wir können also noch kein Ende der Entwicklung feststellen.

An die größere Wahlfreiheit sind in vielen Fällen auch größere eigene Beiträge geknüpft. Man kann natürlich solche eigenen Beiträge bedauern, aber es ist sehr gut möglich, dass diese Beiträge einfacher zu akzeptieren sind (als überhaupt eine geringere Leistung), wenn man mehr Wahlfreiheit bekommt. Dies könnte ein interessantes Thema sein für weitere Untersuchungen. 


\title{
5.3. Behindertenpolitik und Rechte behinderter Menschen in Schweden: \\ „Riv Hindren“ - „Beseitigt die Hindernisse“
}

\author{
Von Peter A. Köhler
}

1. Behindertenpolitik und Recht der Behinderten nach dem Regierungswechsel 2006

2. Gesetzliche Sanktionen bei mangelnder Rechtsgewährung

2.1. Die „Sanktionsabgabe“ im LSS und SoL

2.2. Die Rechtsprechung zur „Sanktionsabgabe“

2.3. Die Gründe der Verzögerungen bei der Rechtsgewährung

3. Behindertenrecht und Praxis: Ombudsman, Koordination und Hilfsmitteloptimierung

3.1. Der Handikappsombudsman, HO

3.2. „Handisam“ - die Koordinationsbehörde der Behindertenpolitik

3.3. Das Hjälpmedelsinstitut (HI) / Institut für Hilfsmittel

4. Behindertenleistungen in der Praxis: Ergebnisse eines empirischen Forschungsprojekts

5. Ausblick: Aktuelle Reformdiskussion 


\section{Behindertenpolitik und Recht der Behinderten nach dem Regierungswechsel 2006}

Kulturgeschichtlich unterscheidet sich die gesellschaftliche Reaktion auf körperlich oder geistig Behinderte in Schweden nicht grundsätzlich von der in vielen anderen Ländern: Menschen mit Behinderung waren über die Benachteiligung durch ihre individuell zu tragende Last hinaus lange Zeit meist auch noch weitgehend von der Teilnahme am gesellschaftlichen Leben ausgeschlossen. Wegen des Fehlens institutioneller Garantien für Hilfe und Unterstützung waren Behinderte vom Wohlwollen ihrer Mitmenschen abhängig. Am Behindertenrecht wird ganz besonders deutlich, dass Recht ein kulturelles Produkt ist, das seinerseits wieder auf die Kultur zurückwirkt ${ }^{1}$ : Die Anfänge einer Sozialpolitik zur Verbesserung des Lebens von Behinderten beruhten auf dem höchst ambivalenten, den gesellschaftlichen Realitäten entsprechenden Ansatz, einerseits Behinderte vor Diskriminierung durch die Gesellschaft zu schützen, wie andererseits die Gesellschaft vor der Begegnung mit der Not der Behinderten zu bewahren (Segregation).

Die aktuelle Behindertenpolitik in Schweden hat ihre Wurzeln im kulturellen Wandel der 1960er Jahre, insbesondere in den auf vielfältige gesellschaftliche Bereiche ausgreifenden Gleichheitsforderungen. Bis dahin fasste man die Probleme behinderter Menschen als eine auf einem individuellen Mangel beruhende subjektive Belastung auf. Schwierigkeiten bei der persönlichen Lebensführung oder in der Arbeitswelt wurden allein auf die jeweilige individuelle Behinderung zurückgeführt, der man primär mit medizinischen Maßnahmen abzuhelfen trachtete.

Nur langsam setzte sich die Erkenntnis durch, dass es erst die von der Gesellschaft oft ungewollt errichteten Hindernisse sind, die Menschen mit eingeschränkten Funktionen von der Teilhabe am „normalen“ Leben ausschließen. Nun zielte die sozialpolitische Diskussion auf eine Veränderung der Gesellschaft im Interesse der Behinderten. An der Beschleunigung dieses Prozesses hatten die Betroffenen selbst einen bedeutenden Anteil, da ihnen durch ihren hohen Organisationsgrad, ihre Präsenz in zahlreichen Verbänden und in allen politischen Parteien erhebliches politisches Gewicht zugemessen wird. Auf diese Weise wurde ein neuer, auf die Kausalität „Behinderungen durch die Umwelt“ abstellender Behindertenbegriff gesellschaftlich durchgesetzt. Im Programm des Zentralverbands der Behinderten, Handikappförbundens Centralkomittè (HCK), „Ett samhälle för alla” (= „Eine Gesellschaft für alle”) aus dem Jahr 1972 hieß es folgerichtig:

„Behinderung ist zu einem großen Teil eine Folge gesellschaftlicher Mängel. Deshalb kann Behinderung beseitigt werden. Dies geschieht durch Veränderung der Gesellschaft““.

1 S. statt vieler Rehbinder, Rechtssoziologie, 5. Aufl., München 2003 und Hotz, Gedanken zur Rechtsvergleichung als einer Kulturwissenschaft und über Europa hinaus, in: Senn/Puskás, (Hrsg), Rechtswissenschaft als Kulturwissenschaft? Stuttgart 2007, S. 201.

2 „Handikapp är in stor utsträckning en följd av brister i samhället. Darför kan handikapp elimineras. Det gör man att förändra samhället." 
Seither beruht die Zielsetzung der Behindertenpolitik auf dem Herstellen voller Teilhaberechte der Behinderten, der Durchsetzung dieser Rechte und auf der Gleichstellung mit Menschen ohne Behinderung, also auf der Anerkennung des gleichen Werts aller Menschen.

Dieser grundsätzliche Konsens überdauerte seither diverse Regierungswechsel und auch das Wahlergebnis vom September 2006 wird hieran wohl nichts ändern. Nach zwölfjähriger Regierung verloren die Sozialdemokraten 2006 die Macht an die bürgerlich-konservative „Allians för Sverige“. Dieser Reichstagswahl wird aus vielfältigen Gründen historische Bedeutung zugemessen. Man erinnert sich: während der letzten 74 Jahre haben die Sozialdemokraten 65 Jahre die Regierung bestimmt. In dieser Zeitspanne konnte das bürgerliche Lager zwar zweimal die Wahlen gewinnen, doch fanden diese Machtwechsel jeweils im Zeichen tiefer ökonomischer Krisen statt. Im Gegensatz zu den Jahren 1976 und 1991 befand sich Schweden im Wahljahr 2006 aber in einer Phase der Hochkonjunktur ${ }^{3}$. Mit einem Wachstum des Bruttoinlandprodukts von über 4\% erreicht die Konjunktur erst Ende 2006 ihren Zenit; für die beiden Folgejahre wird nur eine leichte Verlangsamung auf rund 3\% erwartet. Die Staatsfinanzen sind geordnet und die Wirtschaft agiert erfolgreich auf dem Weltmarkt - an sich kein Szenario, das eine Wechselstimmung bei den Wählern erzeugt. Dennoch mussten die Sozialdemokraten mit 35,0\% ihr schlechtestes Wahlergebnis seit Einführung des allgemeinen Wahlrechts im Jahr $1911^{4}$ hinnehmen. Sie blieben aber, obwohl die Moderaten gleichzeitig mit 26,2 \% den größten Erfolg ihrer Geschichte erzielen konnten, immer noch die weitaus stärkste Fraktion im Reichstag. Zusammen mit den anderen bürgerlichen Parteien lagen die Moderaten aber um knapp 2\% der Stimmen vor der Linken und der „Miljöparti“ (= die Grünen) und konnten so die Regierung bilden. Vor diesem Hintergrund wird allgemein hervorgehoben, dass das eigentlich Neue am Wahlergebnis 2006 und das womöglich dafür entscheidende Moment die bisher nicht gekannte Einigkeit der vier bürgerlichen Parteien sei.

Für die meisten Politikbereiche und insbesondere für die Sozialpolitik wird freilich wenig Veränderung durch den Regierungswechsels erwartet, schon deshalb, weil es Fredrik Reinfeldt gelang, seine „,neue“ Moderate Partei während des Wahlkampfes als die eigentliche „Arbeiterpartei“ mit dem sozialpolitischen Hauptziel der Bekämpfung der (verdeckten) Arbeitslosigkeit darzustellen ${ }^{5}$. Es ist folglich kein

Die Notenbank versucht, mit ihrem schrittweisen Anziehen der geldpolitischen Zügel einer Überhitzung vorzubeugen. Die Inflation dürfte bis 2008 auf 2,6\% steigen. Dazu kommen ein Budgetüberschuss im Bereich von 2,8\% und ein Aussenhandelsplus von etwa 7\%. S. die Analyse des Wahlausgangs von Henrik Brors in: Dagens Nyheter (DN), 18. Sept. 2006, S. 7 und den materialreichen Artikel von Göran Persson, Ingen tillträdande regering har haft bättre förutsättningar, in: DN, 5. Oktober 2006, der dafür das Bild benutzt, dass sich die neue Regierung „an einen gedeckten Tisch setzen kann” (="den borgerlia regeringen får ta över ett dukat bord...") Damals wurden nur 28,5 \% erreicht; im Zeitraum 1911 bis 2006 kamen die Sozialdemokraten nur zweimal über 50 \%, das war während des Krieges im Jahr 1940 und dann im Jahr 1968 bei der dritten Regierung von Tage Erlander.

5 S. das Interview mit Palme: „In Schweden bleibt alles anders.“, in: Die Tageszeitung vom 27.September 2006, S. 12. 
Paradox, wenn die von den Sozialdemokraten verlorene Wahl deshalb sogar als ein „Triumph für die Sozialdemokratie“ interpretiert wurde, weil die Moderaten deren Politik übernommen hätten. Die konservative Allianz hat sich nicht mehr gegen eine Sozialpolitik stellen könnten, von deren Leistungsangebot ein Grossteil auch ihrer Wähler abhängig ist. Damit sei in Schweden der Neo-Liberalismus sowohl ideologisch wie politisch niedergerungen. Der schwedische Staatsrechtler Bo Rothsteins zieht als Fazit:

„Die wirklichen politischen Siege bestehen darin, dass es glückt, seine Gegner dahin zu bringen, dass sie denken und fühlen wie man selbst - wenn das eigene Weltbild und politische Ideal zu dem wird, was die Gesellschaftswissenschaftler das vorherrschende nennen." ${ }^{66}$

Trotz des Regierungswechsels gibt es somit wenig Anlass, an der institutionellen Stabilität des Behindertenrechts zu zweifeln. Ein Blick auf die aktuelle Praxis der Umsetzung dieser Rechte bestätigte diese Vermutung ebenso, wie die Reformdiskussionen, die alle nicht auf Leistungsabbau, sondern auf Effizienzsteigerung des Angebotsspektrums zielen.

\section{Gesetzliche Sanktionen bei mangelnder Rechtsgewährung}

Von der Regierungs-Proposition 1999/2000:79: „Från patient till medborgare - en nationell handlingsplan för handikappolitiken“ (= „Vom Patienten zum Mitbürger ein nationaler Handlungsplan für die Behindertenpolitik“) war im erwähnten Landesbericht Schweden ausführlich die Rede ${ }^{7}$. Darin wurden aufgrund eines häufig zu konstatierenden Sachverhalts Maßnahmen gegen den sog. „Domstolstrots“ (wörtlich "Rechtsprechungstrotz") angekündigt, also die von vielen Behindertenorganisationen kritisierte Zurückhaltung der Gemeinden als verantwortliche Leistungsträger, Urteile, die Behinderten Sachleistungen zusprachen, unverzüglich in die Praxis umzusetzen ${ }^{8}$.

6 Rothstein, Valet en triumf för socialdemokraterna, in: DN vom 20. Sept. 2006, S. 6, „De verkliga segrarna i politiken kommer när man lyckas få sine motståndare att tänka och känna som man själv - när ens verklighetsuppfattning och politiska ideal blir det som samhällsvetare benämner hegemonisk.” Ders. erinnert daran, dass während der 1990er Jahre „ein Bataillon von schwedischen Nationalökonomen ihre wissenschaftliche Ehre dafür verpfändet hätten“, dass die ökonomischen Probleme in Schweden nur durch Steuersenkungen und Einsparungen im öffentlichen Wohlfahrtssystem überwunden werden könnten, eine Ansicht, um die es nun „sehr still geworden“ sei.

$7 \quad$ S. Anm. 1, S. 58 ff.

8 Das Problem wurde schon lange diskutiert und war Gegenstand mehrerer öffentlicher Untersuchungen, i.e., Statens offentliga utredningar, SOU, s. z. B. SOU 1993:109 Förtroendevaldas ansvar vid domstolstrots och lagtrots; SOU 2004:118 Beviljats men inte fătt. 


\subsection{Die „Sanktionsabgabe“ im LSS und SoL}

Der Gesetzgeber hat inzwischen entsprechend gehandelt: In das Gesetz betr. Unterstützung und Service für bestimmte funktional Behinderte, LSS $^{9}$, wurde ein neuer Abschnitt: „Besondere Abgaben“ eingeführt ${ }^{10}$. Dabei handelt es sich um ein Zwangsgeld als Sanktionsmittel gegenüber solchen Gemeinden oder Regierungsbezirken (län), die „ohne rechtfertigenden Grund die Umsetzung von Maßnahmen verzögern, zu denen jemand aufgrund eines Gerichtsurteils berechtigt ist“"11. Die Sanktion wird von der jeweils höheren Verwaltungseinheit festgesetzt ${ }^{12}$. Sie beträgt mindestens 10.000 SEK und höchstens 1 Million SEK. Bei der Festsetzung ist zu beachten, wie lange und mit welchen Folgen für den leistungsberechtigten Behinderten das Gerichtsurteil nicht umgesetzt worden ist ${ }^{13}$. Die ,sanktionsavgift“ (= Sanktionsabgabe) soll über das Abschöpfen von Einsparungen, die sich der Verzögerung der Urteilsumsetzung verdanken, hinausgehen und auch „repressiv“, also als empfindliche Sanktion wirken ${ }^{14}$. Kommt eine Kommune trotz Entrichtung des Zwangsgeldes ihren Pflichten aus dem LSS immer noch nicht nach, wird ein weiteres Zwangsgeld fällig ${ }^{15}$.

Gleichzeitig wurde eine wortgleiche Vorschrift auch in das „Sozialdienstgesetz“16 eingefügt. Mit Wirkung zum 1. Juli 2006 wurde für diese eine praxisnahe Änderung durchgeführt ${ }^{17}$. Im Rahmen der Sozialhilfeleistungen und -dienste wird ein Zwangsgeld in gleicher Höhe und nach den gleichen Maßstäben wie im speziellen Behindertenschutz nunmehr dann fällig, wenn eine Kommune „nicht in angemessener Zeit die Hilfe nach Kap. 4 des Sozialdienstgesetzes anbietet, zu der jemand aufgrund eines Beschlusses des Sozialamtes berechtigt ist ${ }^{18}$. Hilfsbedürftige, und das sind oftmals auch behinderte Menschen, müssen also bei Leistungen der Sozialhilfe nicht erst den Rechtsweg beschreiten und ein Urteil erwirken, um die Sanktion für die säumige Gemeinde auszulösen.

Der Katalog von Leistungen für Behinderte, zu dem die Gemeinden nach dem LSS verpflichtet sind, hat durch die „Sanktionsabgabe“ erheblich an Gewicht für die

9 i. e. Lag (1993:387) om stöd och service till vissa funktionshindrade, LSS.

10 Mit Lag 2002:439, Särskild avgift, $\S \S 28$ lit a bis lit d.

11 LSS § 28 a: En kommun eller ett landsting som underlåter att utan oskäligt dröjsmål tillhandahålla en insats enligt $9 \S$ som någon är berättigad till enligt en domstols avgörande, skall åläggas att betala en särskild avgift.

12 LSS § 28 c. Sie kann nur binnen zwei Jahren nach dem Urteil ergehen, gegen den Zwangsgeldbeschluss ist für die Kommune der Verwaltungsrechtsweg möglich.

13 LSS $\S 28$ b.

14 In der Reg.-Prop. 2001/02:122 zur Gesetzesänderung des LSS heißt es, die Sanktion habe ,,ett vinsteliminerande och ett repressivt inslag“" (S. 30).

15 LSS $328 \mathrm{~d}$.

16 S. Socialtjänstlag (2001:453), Kap. 16, § 6 lit. A bis lit d.

17 Mit Lag 2006:495.

$18 \S 6 \mathrm{a}:$,En kommun som inte inom skälig tid tillhandahåller bistånd enligt 4 kap. $1 \S$ som någon är berättigad till enligt ett beslut av socialnämnden, skall åläggas att betala en särskild avgift.” 
Praxis bekommen. Ein Blick auf die dazu inzwischen bereits ergangene höchstrichterliche Rechtsprechung verdeutlicht die Problemstrukturen der Praxis:

\subsection{Die Rechtsprechung zur „Sanktionsabgabe“}

Den drei am 23.07.2007 ergangenen Urteilen des Obersten Gerichts (regeringsrätten) lagen jeweils Ansprüche von Behinderten auf behindertengerechten Ausbau der Wohnung bzw. auf Zuteilung einer solchen Wohnung mit Seviceleistungen (betreutes Wohnen ${ }^{19}$ ) zugrunde.

Der ersten Entscheidung lag folgender Sachverhalt zugrunde: Die Gemeinde Mölndal lehnte am 07.08.2002 den Antrag einer 1956 geborenen, aufgrund Entwicklungsstörungen funktionsbehinderten ${ }^{20}$ Frau, die mit ihren 82-jährigen Eltern zusammenlebte, auf Zuweisung einer Wohnung mit besonderem Service für Erwachsene nach $\S 9$ Ziff. 9 LSS ab. Die Antragstellerin klagte beim Länsrätt in Göteborg, das am 10.09.2002 in I. Instanz entschied, dass die Gemeinde eine entsprechende Wohnung zu stellen hat. Nach Rechtskraft des Urteils mahnte die Bezirksregierung die Kommune, das Urteil bis 01.02.2003 umzusetzen. Darauf kündigte diese an, bis Herbst 2003 neue Servicewohnungen zu errichten und eine davon für die Antragstellerin zu reservieren. Die Bezirksregierung verhängte daraufhin ein Zwangsgeld nach $\S 23$ a ff. LSS in Höhe von monatlich 61.000 SEK für den Zeitraum Dezember 2002 bis April 2002, weil für eine Verzögerung über drei Monate nach Rechtskraft des Urteils hinaus ein rechtfertigender Grund nicht vorliege ${ }^{21}$. Dagegen klagte Mölndal und begründete dies damit, dass es sehr wohl wichtige Gründe für die Verzögerung gegeben habe: So lägen der Gemeinde mehrere bereits bewilligte Anträge auf Servicewohnungen vor, wobei es sich z. T. um dringlichere Fälle als dem der Antragstellerin handele. Die begrenzten Ressourcen der Gemeinde machten es zudem unmöglich, bei aufwendigen Bauvorhaben in der geforderten Schnelligkeit ökonomisch umzudisponieren. Im Übrigen sei der steigende Bedarf an betreuten Wohnungen nicht vorhersehbar gewesen.

Das Länsrätt Göteborg stellte in seinem Urteil dazu nur knapp fest, dass Mölndal objektiv sein Pflichten gegenüber der Antragstellerin nicht erfüllt habe. Abzuwägen sei nur mehr die Höhe des Zwangsgeldes. Das Gericht hielt an der Summe (inzwischen $8 \times 61.000$ SEK = 488.000 SEK!) fest, da kein Gewinn aus dem Hinausschieben pflichtgemäßen Handelns gezogen werden dürfe. Die Kommune ging in Berufung mit dem Antrag, das Urteil aufzuheben und das Zwangsgeld zurück zu erstatten. Mölndal argumentierte formal und stellte die Zuständigkeit der Bezirksregierung in Frage sowie die Rechtmäßigkeit von deren Vorgehen, ein Zwangsgeld tuell in SOU 2007:103, Bo för at leva - seniorbostäder och trygghetsbostäder.

20 Regeringsrätten, Urt. vom 23.07.2007, S. 5: „funktionshinder i form av utvecklings-störning...“

21 Ebda., S. 1: ,..oskäligt dröjsmål...” 
zu verhängen, ohne die Kommune anzuhören. Materiell wurde angeführt, dass die Umstände, die zu der Verzögerung geführt hätten, außerhalb der Einflussmöglichkeiten der Gemeinde gelegen hätten. Man habe für die Periode 2001 - 2010 ca. 50 neue Wohnungen für Behinderte geplant, der Bedarf sei aber unerwartet über diese Planung hinaus angestiegen. Obwohl man deshalb die Mittel dafür um 3,5 Millionen SEK aufgestockt habe, sei es in dem kurzen Zeitraum von drei Monaten nach Rechtskraft des Urteils nicht möglich gewesen, der Antragstellerin eine entsprechende Wohnung für sie und ihre Eltern zuzuweisen. Eine Einzelwohnung hätte angeboten werden können, wäre aber in diesem Fall nicht zumutbar gewesen. Das Kammergericht sah den Zwangsgeldbeschluss formal als korrekt ergangen an, insbesondere konnte die in der Gemeinde für die Durchführung des LSS zuständige Behörde verbindlich für diese handeln. Allerdings verlange die Beurteilung, ob ein rechtfertigender Grund zur Verzögerung vorliege, der Einzelfallabwägung. Hier habe die Gemeinde eine größere Neubauplanung in Angriff genommen. Das berechtige aber nicht gleichsam automatisch zu einem Leistungsaufschub, sonst liefe das System der „Sanktionsabgabe“ ins Leere. Bei größeren Bauvorhaben erscheine jedoch die übliche Dreimonatsfrist zu kurz, hier müsse ein Jahr Verzögerung hingenommen werden. Danach sei das Zwangsgeld entsprechend auf ein Monat, also 61.000 SEK zu kürzen.

Gegen dieses Urteil rief die Bezirksregierung das Oberste Gericht an. Hauptargument der Revision war, dass die Kommune den steigenden Bedarf an Servicewohnungen schon lange vor Planungsbeginn der Neubauten bekannt war. Im darauf ergangenen Urteil des Regeringsrätt heißt es, dass gesetzlich zwar nicht geregelt sei, ab wann eine Verzögerung rechtswidrig sei. Verwaltungsgerichtsurteile betr. Leistungen aus dem LSS gelten aber unmittelbar, weshalb grundsätzlich überhaupt kein Aufschub für die Befolgung des Urteils in Betracht komme. Dies sei offensichtlich, wenn es um persönliche Dienstleistungen oder Hilfen ginge. Bei Wohnungen könne es aber vertretbar sein, eine gewisse Verzögerung hinzunehmen. Hier war der Kommune aber lange bekannt, dass die Antragstellerin auf Hilfen angewiesen war, ein Bedarf an einer Servicewohnung für sie und ihre Eltern war schon vor Antragstellung absehbar. Die Kommune hätte also Zeit gehabt, ihre Leistungspflicht vorzubereiten. Wegen dieser Vorhersehbarkeit sei trotz der Neubauplanung die Verzögerung nicht zu rechtfertigen und eine Sanktionsabgabe von 200.000 SEK angemessen.

Für die beiden anderen Urteile vom gleichen Tage sei hier nur auf die spezifischen Nuancen der Fallgestaltung hingewiesen:

Die Gemeinde Kungälvs hatte ebenfalls trotz Gerichtsurteil die Bereitstellung einer Service-Wohnung mit dem Verweis auf mangelnde Ressourcen ${ }^{22}$ hinausgeschoben. Für den Antragsteller, einem 1978 geborenen, wegen Autismus in seiner Entwicklung gestörten Mann, hatte sich aber dessen Vater mit der Verzögerung einer Wohnungszuweisung einverstanden erklärt, weil sein Sohn, mit dem er zusammen- 
lebte, ohnehin eine längere Umstellungszeit benötige. Den Rechtstreit, der um die Berechtigung eines Zwangsgelds in Höhe von 50.000 SEK/Monat der Verzögerung ging, entschied das Regeringsrätt unter Verweis auf das erste Urteil vom gleichen Tage: Da Urteile der Verwaltungsgerichte in LSS-Sachen unmittelbar rechtskräftig sind, hat der Leistungsträger sie auch sofort umzusetzen. Ein „Einverständnis“ zu einem Leistungsaufschub kommt nicht in Betracht. Es könne, wenn überhaupt, nur bei der Entscheidung über die Höhe des Zwangsgeldes berücksichtigt werden. Das Gericht senkte deshalb das Zwangsgeld gegen Kungälvs auf insgesamt 100.000 SEK.

Die Gemeinde Markaryd schließlich wurde mit einem Zwangsgeld belegt, weil sie dem Antrag einer 1966 geborenen Frau, die 5 bis 19 Stunden/Woche persönliche Hilfen aufgrund Behinderung in ihrer Wohnung erhielt, auf eine „Gruppenwohnung ${ }^{\text {‘23 }}$ trotz Urteilsspruchs nicht entsprechen konnte. Die Kommune führte in der Klage gegen das deshalb verhängte Zwangsgeld an, dass sie den Bau passender Wohnanlagen nicht innerhalb von drei Monaten realsieren könne, ohne andere Gesetz zu verletzen. Im Übrigen hätte sie der Antragstellerin während der Wartezeit interimistisch diverse andere Wohnmöglichkeiten angeboten. Das Kammergericht hob das Ersturteil und den Zwangsgeldbeschluss auf. Es begründete dies damit, dass selbst wenn die Kommunen eine „,fortlaufende Verantwortung “24 für kurz- und langfristige Planung ihre Leistungspflichten aus dem LSS haben, sie doch nicht jede Situation vorhersehen könnten, etwa den Zuzug neuer Gemeindemitglieder. Dem schloss sich das Oberste Gericht nicht an: Die angeführten Schwierigkeiten befreiten die Kommunen nicht von ihrer Planungsverantwortung. Regeringsrätten verurteilte deshalb die Gemeinde Markaryd wieder zu der Sanktionsabgabe an den Staat von 40.000 SEK.

Die Sanktionierung der Leistungspflichten aus dem Sozialdienstgesetz zeigte ebenfalls schnell erhebliche praktische Wirkung: Seit der erwähnten Reform im Jahr 2006 wurde gegen 53 Kommunen aus 15 Regierungsbezirken Zwangsgelder in Höhe von insgesamt über 26 Millionen SEK verhängt ${ }^{25}$. Es kam bislang zu 166 Urteilen erster Instanz, deren genereller Tenor dahin lautet, dass mangelhafte ökonomische Ressourcen der Kommunen für diese im Bereich gesetzlich statuierter sozialer Dienste nicht pflichtbefreiend wirken. 71 der Urteile betrafen Hilfsansprüche von Kindern und Jugendlichen, es gab aber auch zahlreiche Urteile betr. der Ansprüche von erwachsenen Behinderten, darunter das gegen die Stadtgemeinde Biskopsgården in Göteborg mit dem bisher höchsten Zwangsgeld von 787.500 SEK. Fast 100 Urteile senkten die zuvor verhängten Zwangsgelder und einige hoben die Zwangsgeldbeschlüsse auf.

23 Das sind Wohnanlagen mit entsprechenden Fazilitäten und Serviceangeboten, in denen unterschiedlich Funktionsbehinderte und Nichtbehinderte möglichst komplikationslos zusammen leben können.

24 Regringsrätten, Urt. vom 23.07.2007, S. 11, "...kommunen har ett fortlöpande ansvar för planering av LSS-verksamheten..."

25 S. Norström, Miljonböter för uteblivet stöd, in: Dagens Samhälle, Nr. 1/2008, S. 4. 


\subsection{Die Gründe der Verzögerungen bei der Rechtsgewährung}

Nun fasst man auch in Schweden seit jeher den Rechtsstaatsgrundsatz, dass öffentlichrechtliche Körperschaften Gesetz und Gerichtsurteile unverzüglich zu befolgen haben, als selbstverständlich auf. Über Jahrzehnte lag auch außerhalb jeder Diskussion, dass etwa die Gemeinden diesem Grundsatz in der Praxis auch tatsächlich entsprachen. Deshalb bezeichnete es der Gesetzgeber in der Proposition zum „Sanktionsavgift-Gesetz" als vollkommen unakzeptabel, dass Gemeinden den Vollzug von Gerichtsurteilen verzögerten ${ }^{26}$. Das Phänomen „,domstolstrots“ war insofern wohl weniger eine Abkehr vom Rechtsstaatsprinzip, als vielmehr die Konsequenz der vermeintlichen Dialektik sozialer Rechte. Als Grundproblem der sozialen (Grundund Menschen-) Rechte wird allgemein „erlebt ${ }^{\text {‘27 }}$, dass ihre Verwirklichung beides erfordert: Das Vorhandensein (und den Willen zum Einsatz) ökonomischer Ressourcen zur Rechtsverwirklichung der Berechtigten und die Einschränkungen der Freiheitsrechte anderer ${ }^{28}$. Die das Gesetzgebungsverfahren zur Sanktionsabgabe im Sozialdienstgesetz vorbereitende Untersuchung mit dem Titel „Bewilligt aber nicht bekommen“'29 ergab nun, dass zwar beim sozialpolitischen Komplex „ServiceWohnungsbau" die Gemeinden meist den Mangel an ökonomischen Ressourcen als Ursache der Verzögerungen nannten, dass in diesem wie in anderen Bereichen Geldmangel aber nicht die eigentliche „Bremse“ war: Sehr häufig ergaben sich

- platsbrist / Mangel an Räumen,

- planeringsbrist / Planungsmängel,

- samordningsbrist / Koordinationsmängel,

- kunskapsbrist / Mangel an Sachkenntnis,

- informationsbrist / Informationsmängel,

- personalbrist / Personalmangel und

- uppföljningsbrist / Mängel bei der Sachbearbeitung ${ }^{30}$

als die eigentlichen Gründe dafür, dass die Berechtigten auf die Gewährung ihres Rechts warten mussten. Dieses Ergebnis deckt sich mit dem einer Untersuchung des „Socialstyrelsen“31 aus dem Jahr 2002, wonach ebenfalls ,unzureichende Koordination vorhandener Mittel durch die kommunalen Entscheidungsträger" als Hauptgrund dafür genannt wird, dass von Gesetz festgesetzte und vom Gericht bestätigte

26 Reg. Prop. 2001/02:122, S. 13 ff.

27 So Gustafsson, Rättens polyvalens. En rättsvetenskaplig studie av sociala rättigheter och rättssäkerhet. Lund 2002, S. 23.

28 Daher auch das Problem, etwa aus dem Sozialstaatsprinzip des Grundgesetzes unmittelbar bestimmte subjektive Rechte abzuleiten, s. statt vieler Jarass/Pieroth, Grundgesetz für die Bundesrepublik Deutschland, 9. Aufl., München 2007, Rn.112 ff. zu Art.20.

29 SOU 2004:118, Beviljats men inte fått. Einen Überblick über die aktuelle Entwicklung gibt Gustafsson, Taking Social Rights Seriously: Den handikappade lagen, in: Retfærd. 30. Årgang 2007. nr 1/116, S. $38-84$, insbes. S. $52-55$.

30 S. SOU ebenda, S. $157 \mathrm{ff}$.

31 Socialstyrelsen, Icke verkställda beslut och domar enligt LSS och Sol. Stockholm 2002. Socialstyrelsen ist die zentrale „Leitungsbehörde für das Sozialwesen“, die neben der Funk-tion als Rechtsund Fachaufsicht auch zuständig für Bürgerberatung und Untersuchungen im Sozialbereich ist. 
sozialrechtliche Ansprüche durch zögerliche Umsetzung rechtlicher Pflichten relativiert werden.

Vor dem Hintergrund dieser Ergebnisse kann sowohl das rigorose Vorgehen der Aufsichtsbehörden bei der Auferlegung von Zwangsgeldern, wie auch die Unnachgiebigkeit der Gerichte bei der Beurteilung eines triftigen Grunds für Verzögerungen bei der Leistungsgewährung nicht eigentlich erstaunen. Erstaunlich ist vielmehr, dass der Gesetzgeber den Mut gefunden hat, auf empirische Befunde sachgerecht zu reagieren - obwohl dies mit Ausgaben verbunden ist, die über das Steueraufkommen zu finanzieren sind.

\section{Behindertenrecht und Praxis: Ombudsman, Koordination und Hilfsmitteloptimierung}

Einen weiteren Blick auf die Rechtswirklichkeit lässt sich den Tätigkeitsberichten von Institutionen entnehmen, die im Bereich des Behindertenrechts engagiert sind.

\subsection{Der Handikappsombudsman, HO}

Besonders hervorzuheben sind dabei die Jahresberichte des „Ombudsmannes für Behinderte“, des „Handikapsombudsman“ $(\mathrm{HO})^{32}$. Nach der gesetzlichen Vorgabe und der dazu ergangenen Verordnung ${ }^{33}$ soll der HO

- auf eine Gesellschaft hinwirken, die auf Mannigfaltigkeit gegründet ist,

- die so gestaltet ist, dass Menschen mit Behinderung in jedem Alter am gesellschaftlichen Leben teilhaben können und

- die Gleichheit der Lebensbedingungen für Knaben und Mädchen, Frauen und Männer mit Behinderung gewährleistet.

Die Praxis der Arbeit des HO gilt der dabei auch der Überwachung der Einhaltung folgender Gesetze:

- Ges. betr. Verbot der Diskriminierung im Arbeitsleben aufgrund Behinderung ${ }^{34}$,

- Ges. betr. Gleichbehandlung von Studenten auf den Hochschulen ${ }^{35}$,

- Ges. betr. Verbot von Diskriminierung ${ }^{36}$, und

- Ges. betr. Verbot von Diskriminierung und anderer verletzender Behandlung von Kindern und Schülern ${ }^{37}$. 
Diese in schneller Folge 1999, 2001, 2003 und 2006 ergangenen Gesetze reflektieren zugleich den Ausbau der Antidiskriminierungsgesetzgebung in den letzten Jahren, die längst über ein bloßes Behindertenhilferecht hinaus ist. Auf diesem gesetzlich abgesteckten Feld ${ }^{38}$ nimmt der $\mathrm{HO}$ individuelle Beschwerden entgegen und wirkt generell auf die Überwindung oder Beseitigung von Hindernissen durch Kommunikation mit den Beteiligten oder Fallanalysen hin. Die Statistik der 2006 beim $\mathrm{HO}$ eingegangenen Individualbeschwerden zeigt, dass die weitaus meisten Beschwerden aus dem Zuständigkeitsbereich „Behinderung“ kommen ${ }^{39}$ :

\begin{tabular}{|l|l|}
\hline Aufgrund HO-Gesetz & 308 \\
\hline Diskriminierung im Arbeitsleben & 97 \\
\hline Gleichbehandlung von Studenten & 9 \\
\hline Diskriminierungsverbot & 86 \\
\hline Kinder- und Schülerschutz & 24 \\
\hline Summe & 524 \\
\hline
\end{tabular}

Von den 86 Beschwerden aufgrund des Gesetzes betr. Diskriminierungsverbot wurden 43 von Frauen, 41 von Männern ${ }^{40}$ und zwei von Organisationen eingelegt. Für 162 der insgesamt 524 Beschwerden konnte der HO mit juristischem Rat helfen. Allgemein versucht der $\mathrm{HO}$ den Beschwerden dadurch abzuhelfen, dass er auf die Beteiligten einwirkt, die Gesetz einzuhalten.

Dabei nennt der HO ein Problem, das auf den erwähnten „domstolstrots“ zurückverweist und wohl nicht nur auf Schweden beschränkt ist: In der Praxis ist oft nicht leicht $\mathrm{zu}$ entscheiden, ob eine Beschwerde deshalb begründet ist, weil eine vorgeschriebene Hilfe- oder Unterstützungsleistung nicht (rechtzeitig) erbracht worden ist, oder ob tatsächlich ein Fall von Diskriminierung vorliegt. Beeindruckend ist, dass 2006 den weitaus meisten Beschwerden durch Einwirken des HO unmittelbar abgeholfen werden konnte. Nur drei Mal musste ein förmlicher Vergleich geschlossen werden, wobei in einem Fall später dennoch der Rechtsweg eingeschlagen wurde. Nur in zwei Fällen konnte auch der HO einen Rechtsstreit nicht verhindern. Einer dieser beiden Fälle wird derzeit noch vom Statistischen Zentralbüro untersucht. Es geht dabei um einen hörgeschädigten 34-jährigen Mann, der aber voll arbeitsfähig ist und den eine privatrechtliche Arbeitsunfähigkeitsversicherung wegen seiner Hörschädigung als ein zu hohes Risiko einstufte. Es bestünde wegen der Hörschädigung eine höhere Wahrscheinlichkeit, dass er durch einen Unfall oder Erkrankung arbeitsunfähig werde.

38 Das bedeutet keine Kompetenzbegrenzung, sondern nur die Beschreibung eines Arbeitsschwerpunktes; der HO ist zugleich auch für jede Art Individualbeschwerde oder Hinweisen von Behindertenverbänden auf Missstände etc. zuständig.

39 S. hierzu und zum folgenden Handikappsombudsmannen, Årsredovisning 2006, Dnr. 2007/0229.

40 Dies belegt eine in Bezug auf Diskriminierung fast erreichte Gleichheit zwischen den Geschlechtern! 
Im anderen Fall ging es um einen Rollstuhlfahrer, der wegen (leichter) Trunkenheit aus einem Restaurant verwiesen wurde. Durch Einschaltung des HO kam die Sache vor Gericht, das feststellte, dass der Kläger schlechter behandelt wurde, als nicht behinderte Betrunkene und das ihm deshalb ein Schmerzensgeld zusprach ${ }^{41}$.

Zur Jahresmitte 2007 lässt sich eine Häufung der beim HO eingegangenen Beschwerden aus dem Bereich Wohnungsmarkt bzw. -ausbau feststellen ${ }^{42}$ sowie nach dem neuen Schutzgesetz für Kinder und Jugendliche. Gerade auf dem zuletzt genannten Feld sieht der HO hohen Informationsbedarf ${ }^{43}$.

Im Rahmen seiner Funktion, Informationen unter allen Beteiligten zu kommunizieren, nahm der HO auch an Konferenzen von „Handisam“ teil.

\section{2. „Handisam“ - die Koordinationsbehörde der Behindertenpolitik}

„Handisam“444, ist eine noch junge Behörde mit dem Auftrag, die Behindertenpolitik zu koordinieren. Sie ,soll darauf hinwirken, dass sich eine Gesellschaft entwickelt, in der alle gleichermaßen teilhaben können, unabhängig vom individuellen ,Funktionsvermögen'“45. Sie ist eines der Ergebnisse des „Nationalen Handlungsplans zur Behindertenpolitik ${ }^{\star 46}$, der die Behindertenpolitik insgesamt steuern soll.

Dieser Ansatz enthält eine Änderung der Perspektive der Behinderungspolitik, weg vom Handicap des Individuums, um durch die Hinwendung zu dessen Milieu und Umgebung dessen Lebensbedingungen zu verbessern. Es gilt nun primär ${ }^{47}$, Hindernisse zu beseitigen, dem Entstehen von Hindernissen vorzubeugen und Diskriminierung zu bekämpfen sowie die (technisch machbaren) Voraussetzungen für Selbständigkeit und Selbstbestimmung zu schaffen. Der politische Ansatz besteht jetzt darin, generelle Lösungen zu wählen, die sich für so viele Menschen wie möglich positiv auswirken. Wenn individuelle Behinderungen durch individuelle Unterstützung oder spezielle Sonderlösungen kompensiert werden können, so soll dies komplementär neben der Hauptlinie durchgeführt werden. Um Diskriminierung von vorneherein zu vermeiden, geht die schwedische Behindertenpolitik davon aus, dass die Menschen generell unterschiedliche Voraussetzungen mitbringen und dass Behinderungen diverser Funktionen eine von vielen natürlichen Variationen der individuellen Zusammensetzung der Bevölkerung darstellen. Aus dieser Perspektive folgt

41 Der Rechtstreit ist allerdings in die Berufung gegangen und bisher noch nicht entschieden.

42 HO Årsredovisning... S. 22.

43 S. ebenda.

44 Die Abkürzung folgt aus Myndighet för handikappolitisk samordning = Behörde zur Koordinierung der Behindertenpolitik.

45 So die Gründungsverordnung, Förordning 2001:526 om statliga myndigheters ansvar fö genomförande av handikappolitiken, $\S 1$.

46 Zum Handlungsplan "Från patient till medborgare - en nationell handlingsplan för handikappolitiken,

Prop. 1999/2000:79“, s. ausf. Köhler, Landesbericht Schweden, Anm. 1.

47 S. dazu und zum folgenden: Handisam, Riv Hindren - Riktlinjer för tillgänglighet. (= "Beseitigt die Hindernisse - Richtlinien zur Zugänglichkeit”), Stockholm 2007. 
eine Strategie und Arbeitsmethode, die davon ausgeht, Hindernisse als solche zu identifizieren, sie zu beseitigen und / oder Unterstützung zu ihrer Überwindung anzubieten, wenn dafür Bedarf besteht. Man strebt danach, jeden Sektor des gesellschaftlichen Lebens so auszugestalten, dass er für alle Mitbürger zugänglich ist. Die dafür anfallenden Kosten sollen in den normalen Haushalten enthalten sein und nicht als „Sonderkosten“ ausgewiesen werden. Dies wird „Verantwortungs- und Finanzierungsprinzip“ genannt.

Im Wesentlichen wird in drei Bereichen die Möglichkeit gesehen, das Hauptziel zu erreichen:

- auf lange Sicht soll die Entwicklung der Gesellschaft dahin gehen, Umwelt, Gebäude, Warenproduktion und Dienstleistungen so auszuformen, dass sie der Verschiedenheit der Menschen entspricht. Mit dem Ausgangspunkt „Design för Alla“ (= „Design für Alle") wird Zugänglichkeit und Anwendungsmöglichkeit für so viele Menschen wie möglich angestrebt, kostentreibende Korrekturen und Anpassungen im Nachhinein können so vermieden werden.

- Die bereits bestehenden technischen Lösungen sollen kontinuierlich verbessert und auf bessere Anwendbarkeit überprüft werden.

- Die Selbständigkeit und die Möglichkeiten zur Selbstbestimmung sollen für behinderte Menschen vergrößert werden.

Dabei ist wichtig, dass in den Entscheidungsprozessen der Einfluss derjenigen wächst, die ein Recht auf die Leistungen, Unterstützungen oder Dienste haben, die von der Gesellschaft angeboten werden. Dabei soll das Risiko vermieden werden, dass individuelle Unterstützung und am Einzelnen ausgerichtete Sonderlösungen zur Hauptalternative (mangelnder) öffentlich bereitgestellter Fazilitäten werden.

Die von HANDISAM herausgegebenen Richtlinien enthalten die Definition grundlegender Begriffe: So bedeutet „Zugänglichkeit“ sowohl allgemeine Zugänglichkeit von Einrichtungen, Gebäuden etc., wie auch Anwendbarkeit der Dinge des täglichen Lebens. Der Begriff steht für die Beschreibung der Voraussetzungen, die erforderlich sind, dass Personen mit Behinderung auf gleiche Weise am Leben teilnehmen können wie andere. „Zugänglichkeit“ und „Design för Alla“ komplettieren einander: Zugänglichkeit ist primär ein Begriff der in der Gesetzessprache und bei der Standardisierung angewandt wird. „Design för Alla“ hat einen breiteren Ansatz. Es schreibt in gewisser Weise die Idee des (skandinavischen) Funktionalismus ${ }^{48}$ fort. Schon bei der Planung (Design) von z. B: Bauten oder bei der Warenproduktion soll berücksichtigt werden, dass das Ergebnis in dem Sinne „funktioniert“, dass alle Menschen, unabhängig vom individuellen Funktionsvermögen es nutzen bzw. anwenden können.

$48 \mathrm{Zu}$ diesem s. etwa Widenheim, [Red.] : Utopi \& verklighet. - Stockholm : Moderna Museet [u.a.], 2000: Köhler, Det svenska folkhem, in: Zeitschrift für ausländisches und internationales Arbeits- und Sozialrecht (ZIAS) 1 (1987) 2. S. 203-209. 


\subsection{Das Hjälpmedelsinstitut (HI) / Institut für Hilfsmittel}

Eine erste Institution zur praktischen Hilfe für Behinderte, damals im Wesentlichen Bewegungsbehinderte, wurde 1911 gegründet $^{49} .1950$ schlossen sich die zahlreichen inzwischen entstandenen Behindertenorganisationen zusammen und errichteten das „Behindertenhilfezentralkomitee“. 1965 öffnete sich dieses für alle Arten der Behinderung. 1977 traten der nun „Handikapinstitut“ genannten Organisation der Verband der Regionalverwaltungen und der Staat bei. Das Institut wurde das zentrale Organ für alle Fragen der Behindertenhilfen. 1999 schloss sich der schwedische Gemeindeverband als dritte Kraft an. Der Name wurde in Hjälpmedelsinstitut (HI, Hilfsmittelinstitut) geändert.

Das HI arbeitet dafür, volle Teilnahme und Gleichheit von Personen mit Behinderungen dadurch zu erreichen, dass es bei der Entwicklung und Anwendung von guten und sicheren Hilfsmitteln mitwirkt und für eine effektive Hilfsmittelversorgung sorgt. Das HI soll die Regionalregierungen und die Kommunen sowie die anderen staatlichen Organen in diesem Wirksamkeitsbereich unterstützen. Ein Hauptarbeitspunkt des HI ist der nahe Kontakt zu den „Verbrauchern“. Deren Einfluss wird durch einen „Verbraucherrat“ gesichert und durch die kontinuierliche Zusammenarbeit mit den Betroffenen in einer Vielzahl von Aktivitäten und Projekten.

Die Arbeit des HI reflektiert wiederum die Erkenntnis, dass die Praxis eine Behindertenpolitik erfordert, die zwei Strategien verfolgt. Die eine zielt darauf, allgemein Hindernisse zu beseitigen und Zugänglichkeit für alle gesellschaftlichen Sektoren zu schaffen, die andere zielt auf spezielle Leistungen für den Bedarf des einzelnen Menschen. Das kann durch Rehabilitierung, Hilfsmittel, persönliche Hilfe, Arbeitstraining etc. erfolgen. Beide Strategien komplettieren im Idealfall einander. Denn wenn die Umwelt entsprechend ausgerichtet ist, sinkt der Bedarf an individueller Unterstützung. Eine zukunftgerichtete Behindertenpolitik berücksichtigt die Zusammenhänge zwischen Umweltgestaltung und Hilfsmittel.

Das HI verfolgte zuletzt mittelfristige angelegte Projekte mit folgenden Arbeitsschwerpunkten:

- ältere Menschen mit Behinderung, 2003 - 2009

- kognitive Behinderungen, $2003-2007$

- behindertengerechtes Wohnen, $2006-2008$.

Die Ausrichtung auf die Gruppe der älteren Menschen macht die Realitäten der Behindertenpolitik in Schweden sichtbar. So war die wichtigste Ursache für die ansteigende Nachfrage nach individuellen Hilfsmitteln der wachsende Anteil älterer Menschen, insbesondere der über 80-jährigen. Etwa 70 \% aller Hilfsmittel für Funktionsstörungen werden für Personen über 65 Jahre angewandt.

49 Die Darstellung folgt dem Tätigkeitsbericht 2007 des Instituts, i. e. HI, Verksamhetsprogramm 2007. Stockholm 2008. Die Rechtsgrundlage des HI beruht auf Verordnung, zuletzt Förordning (2007:1134) med instruktion för Myndigheten för handikappolitisk samordning. 
Die alternde Bevölkerung, der synchron damit wachsende Bedarf an Hilfsmitteln, neue Behandlungsmethoden und die von der Haushaltssanierung allgemein erzwungene Mittelbegrenzungen bei den Kommunen hatten zur Folge, dass die Ressourcen heute mehr denn je so effektiv wie möglich eingesetzt werden müssen. Auch im Bereich des Hilfsmittelangebots werden deshalb empirische Kosten-Nutzen-Studien durchgeführt. Dabei kommen zunehmend vorbeugenden Leistungen in den Blick. Die Idee des „Design för Alla“ wird so zu einem immer wichtigeren Konzept. Wenn alle Produkte und Dienstleistungen so standardisiert werden, dass sie von allen benutzt werden können, wird der Bedarf an individuell spezialisierten Hilfsmitteln reduziert. Als ein positiver Nebeneffekt wird dabei begrüßt, dass der wachsende Anteil der älteren Menschen von der Nachfrageseite her die Entwicklung von Produkten und Dienstleistungen nach der Idee des „Design för Alla“ zunehmend rentabel macht ${ }^{50}$.

\section{Behindertenleistungen in der Praxis: Ergebnisse eines empirischen Forschungsprojekts}

Die Forderung nach Gleichheit ist für die schwedische Wohlfahrtspolitik insgesamt grundlegend $^{51}$. In der Behindertenpolitik, die unter der ehrgeizigen Zielvorgabe steht, dass es für die Betroffenen keinen Unterschied machen darf, in welchen Kommunen oder in welchem Teil des Landes sie leben, mussten deshalb schon die zahlreichen zur „Sanktionsabgabe“ ergangenen Gerichtsurteile Aufsehen erregen.

Umso mehr Beachtung fanden die (vorab veröffentlichten ${ }^{52}$ ) Ergebnisse einer aktuellen empirischen Untersuchung einer Forschergruppe an der Universität Uppsala unter Leitung von Leif Lewin über das Leistungsniveau für Behinderte auf kommunaler Ebene. Deren Hauptergebnis löste allein schon deshalb eine intensive Diskussion aus, als man zur Kenntnis nehmen musste, dass es einen erheblichen Unterschied bedeutet, in welcher Kommune ein Behinderter lebt.

50 S. die statistischen Nachweise in HI, Verksamhetsprogramm 2007, S. $24 \mathrm{ff}$.

51 S. dazu (auf Deutsch) immer noch informativ Henningsen, Der Wohlfahrtsstaat Schweden, BadenBaden 1988, sowie die Beiträge in Graubard (Hrsg.), Die Leidenschaft für Gleichheit und Gerechtigkeit. Essays über den nordischen Wohlfahrtsstaat, Baden-Baden 1988 oder z. B. Moeller, Kontrollen im öffentlichen Recht und Demokratiegebot in Schweden - eine Besonderheit in Europa. (Diss. Berlin), Münster 2004, S. 32 ff.

52 Lewin, Vänsterstyrda kommuner ger mer stöd till handikappade, in: DN , Debatt, S. 6, 20.12.2007. In diesem Artikel beschreibt Lewin vorab die Ergebnisse eines Forschungsprojekts über Leistungsunterschiede für Behinderte, das demnächst veröffentlicht werden soll. Das Projekt wurde vom Forskingsråd för arbetsliv och socialvetenskap (FAS) (mit-) finanziert; die folgende Darstellung der Ergebnisse folgt dem Artikel von Lewin in DN, ebenda. 
Zunächst bereitete man für die Studie das reichhaltig vorhandene ${ }^{53}$ statistische Material auf. Immerhin gehen 70 \% der öffentlichen Ausgaben zur Finanzierung der ihnen gestellten Aufgabe an die Kommunen. Wie sie diese Kommunlaufgaben in der Praxis ausführen, ist ein bedeutender Teil der kommunalen Selbstverwaltung ${ }^{54}$, was auch zu dem Ergebnis führt, dass die Aufwendungen für Behindertenhilfen von einer Kommune zur anderen bei gleicher Gesetzeslage um bis zum 10-fachen schwanken können. Eine erste Erklärung für dieses Phänomen war die Auskunft der Kommunen, dass eben auch der Bedarf an Hilfeleistungen höchst unterschiedlich ist. Das konnte der Untersuchung aber nicht genügen, denn tatsächlich konnte niemand belegen, wie hoch ein Bedarf objektiv einzuschätzen ist. Lewin bezeichnet es als ein häufiges Problem der Sozialwissenschaften, dass oft „Bedarf“ mit „Nachfrage“ oder mit der ,zugeteilten Hilfeleistung“ gleichgesetzt wird, was natürlich ein Trugschluss ist. Eine weitere Schwierigkeit folgte unmittelbar aus dem Gesetz: Leistungen nach dem LSS müssen beantragt werden, doch sind viele Behinderte nicht in der Lage oder willens, einen Antrag zu stellen. Man weiß seit langem, dass die Zahl der objektiv Berechtigten etwa doppelt so hoch ist wie die derjenigen, die tatsächlich Hilfen beziehen ${ }^{55}$. Man musste also eine eigene Methode erarbeiten, um die „verpackte und sich verflüchtigende Wirklichkeit“56 erfassen zu können.

Ein Faktor, dem bei einem ,indirekten“ Vorgehen Bedeutung zukommt, ist die auf den ersten Blick nicht zu erklärende höchst unterschiedliche Zahl von Behinderten, die in einer Kommune wohnen. Es fiel auf, dass dort, wo früher (die inzwischen abgeschafften) geschlossenen Pflegeheime für Behinderte gelegen waren, die Zahl der Behinderten besonders hoch ist. Dies hatte den einfachen Grund, dass diese Menschen nach Schließung der Pflegeheime ja in der Kommune weiter wohnen blieben. Eine Erklärung der unterschiedlichen Aufwendungen für LSS-Leistungen liegt also ganz trivial darin, dass sie dort besonders hoch sind, wo früher Pflegeheime bestanden.

Ein ähnlicher Zusammenhang lässt sich für die hohen Kosten der im dünn besiedelten Norden des Landes gelegenen Gemeinden (,glesbygdskommuner") feststellen sowie für die sog. „ofärdskommuner“, das sind Kommunen in Regionen mit

53 Die statistische Aufbereitung ist in Schweden außerordentlich umfassend, s. etwa den jährlichen Bericht des Socialstyrelsen, Handikappomsorg - Lägesrapporter, zuletzt Stockholm 2007, oder die vom Socialstyrelsen zusammen mit dem Statistischen Zentralbüro erarbeiteten Berichte, z.B. Socialstyrelsen/ Statistiska centralbyrån (Hrsg.), Funktionshindrade personer år 2006. Kommunala insatser enligt socialtjänstlagen samt hälso- och sjukvårdslagen. Stockholm 2007 oder Arbetsmarknadsstyrelsen/ Statistiska centralbyrån (Hrsg.), Funktionshindrades situation på arbetsmarknaden - 4:e kvartalet 2006. Stockholm 2007.

54 Auf die (ungelöste) rechtliche Problematik, die entstehen kann, wenn das Prinzip der Selbstverwaltung mit einer Verurteilung der Gemeinde zur „Sanktionsabgabe“ kollidiert, weist eingehend Gustafsson, Taking Social Rights Seriously.... S. 58 ff. hin.

55 Unter Verweis auf amtliche Quellen gibt Lewin, Vänsterstyrda kommuner... an, dass im Jahr 2006 an 55.000 Hilfsbedürftige Leistungen bewilligt wurden, dass aber wohl mehr als 100.000 anspruchsberechtigt gewesen wären.

56 Lewin, ebda: „... en invecklad och undflyende verklighet, som man endast kan fånga in genom mera indirekta beräkningar." 
schwierigem Arbeitsmarkt, überdurchschnittlich hohem Krankenstand und/oder altem Bevölkerungsanteil. Hier summieren sich Faktoren, die es den Familien erschweren, sich selbst um behinderte Angehörige zu kümmern, was wiederum die Nachfrage nach öffentlichen Hilfen erhöht. Zudem lässt sich ein „Norrlandsfaktor“' ${ }^{\text {“57 }}$ feststellen, d. h., dass im Norden des Landes sich die Menschen zwecks Unterstützungsleistungen sehr viel eher an ihre Gemeinde wenden, als dies im Süden zu konstatieren ist. Daneben wirken sich auch einfache Zugangsschwierigkeiten auf die Antragshäufigkeit aus: So wenden vom Gemeindegebiet her kleine Kommunen erheblich mehr Mittel auf, als die großen Flächengemeinden, wo z. B. das Sozialamt für viele Bürger nur nach längerer Fahrt mit Bus oder PKW erreichbar, die Antragstellung also faktisch erschwert ist.

Hervorgehoben wird, dass den ökonomischen Ressourcen der Kommunen keine generell entscheidende Rolle zukommt: Reiche Gemeinden finanzieren nicht Leistungen für mehr Behinderte, doch sind dort die Leistungen allerdings im Einzelfall höher.

Schließlich blieb noch die Frage zu klären, welchen Einfluss die lokale politische Konstellation auf die Praxis der Behindertenpolitik der Gemeinden hat. Dabei ging man von der These Arend Lijpharts ${ }^{58}$ aus, dass Demokratie allgemein nicht „wie im Lehrbuch" funktioniert, wo Mehrheiten regieren und Minderheiten opponieren, sondern meist so, dass die konkurrierenden Parteien zusammen arbeiten, um (Sach-) Ergebnisse zu erzielen. Dies führe für ausgegrenzte Gruppen wie Kranke, Arbeitslose oder Behinderte oft zu besseren Lösungen. Die Forschergruppe um Lewin überprüfte diese Annahme unter der Fragestellung, ob Behindertenpolitik in Gemeinden, in denen die politischen Parteien zusammenarbeiten, generöser ist als in solchen, die nach dem parlamentarischen Idealtypus geführt werden. Im Ergebnis konnte die These Lijpharts nicht bestätigt werden: Bei kurzfristiger Betrachtung zeigten sich nur geringfügige Unterschiede danach, ob ein Gemeinderat von einer linken oder rechten Mehrheit oder von einer parteienübergreifenden Koalition bestimmt wird. Es zeigte sich aber, ,dass man den Ideologien Zeit geben muss, um sich auszuwirken ${ }^{، 59}$ - denn je länger eine Gemeinde von einer stabil etablierten linken Partei geführt wird, desto höher werden die Ausgaben, die man für die Behindertenhilfe bewilligt.

Es versteht sich von selbst, dass dieser Aspekt des Untersuchungsergebnisses in Schweden noch mehr Diskussionen angefacht hat, als das beim Nachweis unterschiedlicher Leistungsangebote allein schon der Fall war.

57 Unter Bezug auf die Befunde von Putnam, Gesellschaft und Gemeinsinn: Sozialkapital im internationalen Vergleich, Gütersloh 2001, betr. des Nord-Süd-Gefälles in Italien meint Lewin, dass auch in Schweden im Süden das ,,soziale Kapital“ besser entwickelt ist.

58 S. Lijphart, Patterns of Democracy, Yale University Press 1999.

59 Lewin, Vänsterstyrda kommuner.... ,men ideologier måste ges tid för att ha verka...” 


\section{Ausblick: Aktuelle Reformdiskussion}

Die auf hohem Niveau geführte Debatte um Wege zur Effizienzsteigerung des Leistungsangebots zusammen mit dem Bestreben, vermeidbare Ungleichheiten beim Leistungsbezug zu erkennen und abzubauen, gingen auch in die Arbeit der seit einigen Jahren eingesetzten Expertengruppe zur Vorbereitung diverser Gesetzesänderungen im LSS $^{60}$ ein. Dabei steht die Einführung eines ,individuellen Erstattungssystems" (= individuellt erstattningssystem) für das Leistungsangebot nach dem LSS und der gesetzlichen Pflegekostenerstattung $\left(\mathrm{LASS}^{61}\right.$ ) im Vordergrund. Des Weiteren ist eine Umstrukturierung der Finanzierung von LSS und LASS geplant.

In der Kommission verweist man nicht ohne Stolz darauf, dass Schweden 1994 mit der Verabschiedung des LSS und des LASS behindertenpolitisches Pionierland war. Aktuell sieht man sich aber von Ländern wie Großbritannien und der Bundesrepublik „überholt“, zumindest was die gesetzliche Garantie einer individuellen Wahlfreiheit von Behinderten hinsichtlich der von ihnen benötigten Leistungen anbetrifft ${ }^{62}$. Gemeint ist mit dem Beispiel Deutschland die Einführung eines Rechtsanspruchs auf ein persönliches Budget für Menschen mit Behinderung zum $01.01 .2008^{63}$. Damit können Leistungsempfänger von Rehabilitationsleistungen auf Antrag anstelle von Dienst- und Sachleistungen zur Teilhabe eine Geldleistung wählen. Sie sollen damit in die Lage gesetzt werden, Aufwendungen, die zur Deckung ihres persönlichen Hilfebedarfs erforderlich sind, selbst zu bezahlen. Dieses „Persönliche Budget“ löst das bisherige „Dreieck zwischen Leistungsträger, Leistungsempfänger und Leistungserbringer"664 auf. Sachleistungen werden durch Geldleistungen oder Gutscheine ersetzt.

In Schweden will man aber noch mehr als das. Der Verband der Kommunen und Landkreise (Sveriges Kommuner och Landsting, SKL) nannte in der Kommission im Konsens mit Experten und Parteivertretern folgende Argumente für die Einführung persönlicher Budgets, die zugleich die Forderung nach einer Änderung der Finanzierung tragen sollen ${ }^{65}$ :

1. Ein staatlich finanziertes System persönlicher Budgets mit unterschiedlichen Leistungsniveaus ist besser geeignet, dem Einzelbedarf zu genügen und dabei landesweite Gleichbehandlung zu sichern.

60 Ein Bericht über die Arbeit des „LSS-kommittén“, deren Ergebnis als SOU für den Sommer angekündigt wird, findet sich in Knape/Andén u. a., Inför en statlig „servicepeng“ i LSS-systemet, in: Dagens Samhälle, 2/2008, S. 21.

61 i. e. Lag (1993:389) om statlig assistansersättning, LASS. Wer zu einer der drei Gruppen des LSS gehört, nicht in einer Behindertenwohnung lebt, unter 65 Jahre alt ist und für seine grundlegenden Bedürfnisse persönliche Assistenz über mehr als 20 Stunden wöchentlich benötigt und, hat bis zu einer Obergrenze Anspruch auf Kostenersatz nach dem LASS, s. dazu ausführlich Köhler, Anm. 1.

62 S. das Zitat der deutschen Gesetzgebung bei Knape/Andén, „,servicepeng“... und deren Hinweis auf ähnliche Entwicklungen in anderen Ländern ebda.

63 S. $\S 159$ Abs. 5 SGB IX und VO zur Durchführung des $\S 17$ Abs. 2 bis 4 SGB IX (Budgetverordnung) vom 27.05.2004.

64 S. BMin für Arbeit und Soziales, Rechtsanspruch auf Persönliches Budget. www.budget.bmas.de.

65 S. Knape/Andén, ,servicepeng“... 
2. Der Staat hat dazu die Finanzkraft, ohne wie die Kommunen über Prioritäten bezüglich unterschiedlicher Bedürfnisse entscheiden zu müssen.

3. Ein persönliches Budget der Leistungsberechtigten führt zu mehr Rechtssicherheit bei den Leistungserbringern; Differenzen zwischen Kommunen über Zuständigkeiten würden entfallen.

4. Die Wahlmöglichkeiten, die andere Verbraucher bei Dienstleistungen haben, sollten gerechterweise auch Leistungsbeziehern nach dem LSS zustehen. Dies umso mehr, als es sich hier oft um Dienste im persönlichen (Intim-) Bereich handelt wie etwa Hilfen beim Duschen, Gang zur Toilette etc.

5. Diese Wahlmöglichkeit muss auch die Entscheidung für einen Dienstleister außerhalb der Kommune des Berechtigten umfassen, allein schon wegen der vielen Funktionsbehinderten, deren Arbeitsplatz nicht in der gleichen Kommune liegt, in der sie wohnen. Bei Wohnsitzwechsel muss der Bedarf nicht von der Zuzugskommune neu überprüft und die Leistung nicht neu organisiert werden. Die Wahlmöglichkeit sorgt für Konkurrenz unter den Anbietern und kann so zu einer Qualitätsverbesserung des Leistungsangebots führen.

6. Eine staatliche ,serviceersättning“ kann auf doppelte Weise die Integration Behinderter fördern. Da durch die Konnotation mit dem Berechtigten z.B. täglich erforderliche Hilfsleistungen zum Arbeitgeber „mitgenommen“ werden können, wird nicht nur der Behinderte flexibler, sondern die Arbeitgeber sparen auch Kosten für die behindertengerechte Arbeitsplatzgestaltung.

Der Verband SKL hebt in eigenen Informationen zum Thema LSS-Reform ${ }^{66}$ den Schwerpunkt auf den Aspekt Finanzierung: man führt an, dass seit den 90er Jahren die Kosten für die von den Kommunen vorzuhaltenden LSS-Leistungen um $141 \%$ gestiegen seien, ohne dass die Kommunen dafür vom Staat einen Ausgleich erhalten hätten. Man will dies nicht als Kritik am LSS verstanden wissen, sondern will den Grundsatz durchsetzen, dass die Instanz, die Reformen beschließt, auch deren Kosten tragen sollte. Übernähme der Staat die Kosten für eine persönliche Budgetierung, so würden sich zugleich die allgemein den Kommunen zu erstattenden Ausgaben verringern.

Schließlich wird noch das demografische Faktum der alternden Bevölkerung angeführt: Dadurch stiegen die Aufwendungen der Kommunen für Funktionsbehinderte von 1997 bis Ende 2006 von 17,6 Milliarden SEK auf knapp 45 Milliarden keine andere der kommunalen Aufgaben hatte $\mathrm{zu}$ einer auch nur vergleichbaren Kostensteigerung geführt.

Man sieht: Die vom Verband der Gebietskörperschaften geforderte Reform hat neben möglichen Verbesserungen des individuellen Status des Leistungsberechtigten auch einen handfesten finanzpolitischen Hintergrund. Hinzu kommt die Hoffnung, dass man dann, wenn man durch persönliche Budgets den Behinderten selbst für den „Kauf“ und damit in der Praxis eben auch für die Organisation der von ihm 
gebrauchten Dienste in die individuelle Verantwortung stellt, „Sanktionsabgaben“ nach Gerichtsurteilen für die Gemeinden im Bereich der persönlichen Dienste nicht mehr so häufig zu erwarten sein dürften.

Bei den Behindertenorganisationen ${ }^{67}$ überwiegt bislang die Skepsis: Sie kritisieren den Reformvorschlag, weil er nach ihrer Ansicht die Verantwortung für die am meisten hilfsbedürftigen Gemeindemitglieder von der Gemeinde auf einen staatlich finanzierten „LSS-Markt“ verschiebt. Diese Abkehr von der vom Reichstag beschlossenen generell gültigen Zielsetzung der Behindertenpolitik „Vom Patienten zum Mitbürger" sei der Versuch, „Vom Patienten zum unfreiwilligen Marktakteur“ zu wechseln.

Die Diskussion hält an. Somit wird erst die endgültige Gesetzesfassung zeigen, welcher Aspekt der geplanten Reform überwiegt: Die Stärkung der „Marktposition“ der Behinderten oder die Entlastung der Gemeinden.

67 S. den gemeinsamen Beitrag der Vorstände zahlreicher Behindertenorganisationen „Från patient till ofriwillig marknadsaktör“, in: Dagens Samhälle 5/2008, S. 23. 


\title{
5.4. Die Rolle der behinderten Menschen in der Zivilgesell- schaft der Tschechischen Republik
}

\author{
Von Petr Tröster
}

1. Einleitung

230

2. Systemorientierte Grundsatzcharakteristik der Lage behinderter Personen

3. Die Rolle der behinderten Personen in einzelnen Entwicklungsetappen der Tschechischen Republik nach dem Jahr 1989

4. Die Partizipation allgemein

5. Einschätzungen der Effektivität der bisherigen Nationalpläne und die Einwirkung der verwirklichten Maßnahmen auf diesem Gebiet

6. Weitere Aspekte, welche die Lage der behinderten Personen in der Gesellschaft beeinflussen

7. Analysen der Wirkung von Organisationen und Bürgergruppen der behinderten Menschen

8. Die Partizipation der Bürger mit Behinderung und deren Organisationen an der Verwaltung öffentlicher Angelegenheiten - Ziele, Aufgaben und Maßnahmen für die Jahre 2004-2009

9. Koordinierung und Untersuchung der Erfüllung des Nationalplans

10. System der Zuwendung staatlicher Dotationen

10.1. Programm der Grantunterstützung

10.2. Programm des Nachteilsausgleichs für behinderte Bürger 


\section{Einleitung}

Behinderung ist ein bedeutendes soziales Phänomen, das nicht ohne weiteres mit anderen von menschlicher Tätigkeit beeinflussten Situationen zu vergleichen ist. Behinderte Staatsbürger und andere behinderte Bewohner der Tschechischen Republik müssen eine Reihe von Hindernissen überwinden, die sich zum Teil aus der Behinderung und zum Teil infolge ihrer Beschränkungen ergeben - es handelt sich um Grenzen, welche ihnen die Organisation der menschlichen Gesellschaft im Laufe ihrer Entwicklung in den Weg stellt.

Die Tschechische Republik (ČR) ist in den letzten Jahren ebenfalls zu einem Staat geworden, der sich der erhöhten Verantwortung für die Beseitigung der Barrieren, welche den behinderten Bürgern zur vollständigen Beteiligung am gesellschaftlichen Leben im Wege stehen, bewusst geworden ist. Deswegen wurden in den vergangenen Jahren vier Nationalpläne angenommen, die zu einer Besserung der Rolle der behinderten Menschen in der Gesellschaft beitragen sollten. Der Nationalplan zur Hilfe von behinderten Bürgern wurde durch den Regierungsbeschluss Nr. 466 im Jahr 1992 genehmigt. Zu seinen Zielen gehörten u. a. insbesondere die Beseitigung der schwerwiegendsten Diskriminierungsfälle und die Einleitung systemorientierter Änderungen auf dem Gebiet der Unterstützung behinderter Bürger. 1993 wurde eine Aktualisierung des genannten Dokuments mit dem Titel „Nationalplan der Maßnahmen zur Reduzierung negativer Auswirkungen der Behinderung" vorgenommen, die durch Beschluss der Regierung der ČR Nr. 493 genehmigt wurde (08.09.1993). Einen weiteren Akt dieser Art hat die Regierung der ČR am 14. April 1998 durch ihren Beschluss Nr. 256 mit dem Namen „Nationalplan des Nachteilsausgleichs für behinderte Bürger" genehmigt.

Am 16. Juni 2004 genehmigte die Regierung der ČR durch ihren Beschluss Nr. 605 „Das mittelfristige Konzept der staatlichen Politik zugunsten von Bürgern mit Behinderung“ (im Weiteren nur „mittelfristiges Konzept“) und beauftragte die Regierungsmitglieder, dass sie in ihren Aktivitäten auf dem Gebiet der Legislative, Leitung, Methodik und Organisation, die mit den Bedürfnissen der behinderten Bürger zusammenhängen, vom mittelfristigen Konzept auszugehen und einzelne Konzeptmaßnahmen zu realisieren hätten. Parallel dazu beschied die Regierung, dass bis zum 30. Juni 2005 ein neuer Nationalplan für die Unterstützung und Integration behinderter Bürger für den Zeitraum 2006-2009 (im Weiteren auch „Nationalplan") ausgearbeitet und vorgelegt werden sollte, wobei der Plan von den Zielen und Aufgaben des mittelfristigen Konzepts ausgehen sollte. Der Nationalplan wurde durch Regierungsbeschluss vom 17.08.2005 unter Nr. 1004 genehmigt. Eine ausführlichere Behandlung dieses Themas erfolgt später. 


\section{Systemorientierte Grundsatzcharakteristik der Lage behinderter Personen}

Die Lage der Behinderten in der Tschechischen Republik kann man infolge der Existenz eines soliden rechtlichen Rahmens und dank der überwiegenden positiven Einstellung der Mehrheit der Gesellschaft als stabilisiert bezeichnen. Die in Frage kommenden potenziellen Diskriminierungs- bzw. Beschränkungstendenzen sind meist nicht die Folge einer bewussten Neigung zur Schädigung oder des Unwillens zur Lösung der Probleme von behinderten Menschen, sondern sind „nur“ ein Resultat relativ niedriger Kenntnisse der wirklichen Probleme der Behinderten als Bürgergruppe sowie als Individuen.

Die behinderten Bürger und deren Familien sind eine Bürgergruppe, deren Leben infolge eines prinzipiellen Ereignisses beeinflusst wurde - dem Bestehen einer Behinderung und deren Auswirkungen. Insbesondere im Fall einer schweren Behinderung kommt es zu merklichen Änderungen in den Chancen zur vollwertigen Teilnahme sowohl am gesellschaftlichen Leben als auch an privaten Aktivitäten. Auf diese Weise stellt also die Behinderung eine der schwierigsten Lebensprüfungen dar. Im Unterschied zu einer Reihe von anderen Gegebenheiten, auf die der Betroffene Einfluss hat, handelt es sich dabei um Ereignisse, die meist unabhängig vom Willen des Individuums geschehen. Die Behinderung und die davon Betroffenen werden mit Recht Objekte gezielter Solidarität der Gesellschaft. Darüber hinaus, insbesondere aus der Sicht der Organe der öffentlichen Verwaltung, sind die Behinderten eine Gruppe, die direkt und auch indirekt einen bedeutenden Teil des nationalen Bruttoeinkommens beeinflusst. Mit Hilfe effektiver, auf die Entwicklung der menschlichen Ressourcen gerichteter Maßnahmen, kann man auch auf diesem Gebiet eine erhöhte Teilnahme der behinderten Bürger an der Bildung (und auch Nutznießung) des gesellschaftlichen Wohlstands in der Zukunft erzielen.

Die schrittweise Offenlegung der Problematik der Behinderten für die Öffentlichkeit gehörte zu den charakteristischen Zügen der vergangenen Zeit, inklusive einer teilweisen Umstellung der Beziehungen der Öffentlichkeit zu dieser Bürgergruppe. Die häufige Präsenz von Behinderten in den Medien und in der Öffentlichkeit hat zwar keine unmittelbare Einwirkung auf die Lösung der einzelnen Fragen ihres Lebens. Aus langfristiger Sicht tragen sie aber zur Bildung des richtigen Auftritts und zum respektvollen Benehmen bei, die ihre Rückwirkung auch in den Äußerungen der Vertreter staatlicher Organe und Organisationen, welche sich auf dem Gebiet des Lebens der Behinderten beschäftigen, finden. Von Seiten der Behinderten und deren Organisationen wird z. B. die Kampagne zur Vermittlung der Erfolge behinderter Sportler/Sportlerinnen positiv gesehen. 


\section{Die Rolle der behinderten Personen in einzelnen Entwicklungs- etappen der Tschechischen Republik nach dem Jahr 1989}

Die grundsätzlichen Tendenzen dieses Zeitraums findet man in den folgenden Etappen:

- Die Anfangsetappe (1990-1992/1993): Es handelt sich um den Zeitraum grundsätzlicher gesellschaftlicher Änderungen, die auch eine qualitative Lageumstellung der behinderten Bürger mit sich brachte. Es entstehen Hunderte von nichtstaatlichen Bürgervereinen auf einer Zivilgruppenbasis. Hunderte neuer Einrichtungen zur Unterstützung von Behinderten, primär auf dem Gebiet des Schulwesens und der sozialen Versorgung, werden gegründet. Es kommt zur Entstehung des Regierungsausschusses für behinderte Bürger (aufgrund des Beschlusses der Regierung der ČR Nr. 151 vom 08.05.1991). Der erste Nationalplan der Hilfe wird angenommen. Es setzt sich ein hoher Grad von Entgegenkommen bei der Lösung der Anrechte und Bedürfnisse der Behinderten (sowohl auf politischer als auch auf der verwaltungsorientierten Ebene) durch. Die Vertretung der Behinderten ist noch nicht vereinigt. Es gibt zwei dominierende Gruppen nebeneinander: den neu errichteten Verein der Behindertenvertreter und den transformierten Invalidenverband (die Organisationen passen sich der föderativen Ordnung der Republik an; die Anzahl der Behindertenvereine steigt). Die ersten spezifischen Charakterzüge von den als Angelegenheiten behinderter Bürger respektierenden Rechtsnormen werden angenommen.

- Neunziger Jahre (1993-1998/1999): Es kommt zu einer Konsolidierung der Behindertenorganisationen und $\mathrm{zu}$ einer Professionalisierung der Staatsverwaltung auf diesem Gebiet. Die Entwicklung des so genannten non-profit-Sektors schreitet voran, es entstehen neue Formen und Methoden der Arbeit mit Behinderten oder Aktivitäten zu deren Gunsten. Zum Abschluss dieser Etappe wird ein Nationalplan des Nachteilsausgleichs für die behinderten Bürger vorbereitet. Es werden rechtliche Grundnormen mit unmittelbarem Bezug zur Behinderung (Sozialversicherung, Schulwesen, Transport) angenommen oder novelliert.

- Die Endphase der 90er Jahren bis zur Gegenwart: Die Anzahl der Probleme, welche die wichtigsten Partner - die politische und verwaltungstechnische Repräsentation auf der einen und die Vertreter der Behindertenorganisationen auf der anderen Seite - als Kollisionsereignisse zur Kenntnis nehmen, steigt. Die Dynamik der Lösungen verringert sich. Diese Behandlungsart, gestützt auf Änderungen und Korrekturen früher erstellter Systeme, überwiegt, wobei oft deren gründliche Analyse und sorgfältige Einschätzung der Auswirkungen fehlen. Es fehlt auch der Wille oder die Kraft, einige Fragen zu lösen, dies auch auf Kosten eines Ausfalls traditioneller Behandlungsmethoden und deren Ersatz durch moderne Unterstützungsmethoden. Die Aufgaben des Nationalplans aus dem Jahre 1998 bleiben auch nach sechs Jahren nicht voll erfüllt. Die Probleme der behinderten Bürger werden auf verschiedenen Ebenen weiter diskutiert und gelöst. Es herrscht überwiegend die subjektive, auf ad-hoc Lösungen gestützte Behandlungsart. Dafür gibt es aber auch objektive Ursachen, welche die Notwendigkeit 
von Änderungen innerhalb einer Reihe gesellschaftlicher, mit der Behinderung zusammenhängender Systeme verlangt. Auf dem Gebiet der öffentlichen Verwaltung tritt als Novum das lokale Element zu Tage - die souveränen Kreisorgane. Beide Seiten bejahen die Notwendigkeit zu (oft grundlegenden) Änderungen im Sozialversicherungssystem, in der Beschäftigungssphäre und auch auf dem Gebiet der Bildung und des Schulwesens. Die Änderungen in der Politik zugunsten der Behinderten werden Bestandteil einer breiteren Reform der öffentlichen Verwaltung und der eingeleiteten Reform der öffentlichen Haushalte der Tschechischen Republik. Es entsteht eine einheitliche Vertretungsorganisation der Bürger mit Behinderung in der $\breve{C} \mathrm{R}$ - der Nationalbeirat der Behinderten.

\section{Die Partizipation allgemein}

Einen bedeutenden Anteil an und eine merkliche Initiative zur Lösung dieser Probleme haben die Organisationen der Behinderten. Alle wichtigen internationalen Dokumente, Empfehlungen und Beschlüsse internationaler Regierungs- sowie Nichtregierungsorganisationen betonen in den letzten Jahren die Notwendigkeit der Einhaltung der Regel von Konsultations- und Partizipationsrechten der Behindertenorganisationen bei den Tätigkeiten der Verwaltungsorgane und bei der Lösung der Angelegenheiten der Behinderten. Die behinderten Bürger, unter anderem auch dank ihrer Lebenserfahrung, sind imstande, Vorgaben für die Politik zu formulieren und praktische Maßnahmen vorzuschlagen, um ihre Eingliederung in das gesellschaftliche Leben leichter zu machen. Im Falle der behinderten Bürger (deren Organisationen) erfolgte im letzten Jahrzehnt eine Entwicklung von der in Dutzende von Organisationen zersplitterten Bewegung hin zur Entstehung einer einzigen Dachorganisation, die qualifiziert ist, als Partner für den Staat und seine Organe zu dienen. Der Nationalbeirat der Behinderten der ČR und dessen Mitgliedsorganisationen vereinen mehr als 250.000 Mitglieder, also mehr als ein Viertel der Gesamtzahl aller Personen mit Behinderung in der ČR und mehr als die Hälfte aller Personen mit schwerwiegender Behinderung. In der ersten Phase nach dem Entstehen konzentrierten sich die Behindertenorganisationen (oft nur allein) insbesondere auf die bislang fehlenden Dienstleistungen für diese Bürger. Später begannen auch andere Dienstlieferanten diese Rolle zu spielen. Immer größere Bedeutung im Rahmen dieser Organisationen der Behinderten erlangt deren Beteiligung an der Formulierung von Vorgaben und der praktischen Umsetzung einer Politik zugunsten dieser Bürgergruppe.

\section{Einschätzungen der Effektivität der bisherigen Nationalpläne und die Einwirkung der verwirklichten Maßnahmen auf diesem Gebiet}

Im Zeitraum seit den 90er Jahren des vergangenen Jahrhunderts bis zur Gegenwart wurden folgende „Unterstützungspläne“ angenommen: 
- Der Nationalplan zur Hilfe behinderter Bürger, der durch den Beschluss der Regierung der ČR Nr. 466 vom 29. Juni 1992 genehmigt wurde.

- Der Nationalplan der Maßnahmen zur Reduzierung negativer Auswirkungen der Behinderung, genehmigt durch den Beschluss der Regierung der ČR Nr. 493 vom 8. September 1993.

- Der Nationalplan des Nachteilsausgleichs für behinderte Bürger, der durch den Beschluss der Regierung der ČR Nr. 256 vom 14. April 1998 genehmigt wurde.

- Der Nationalplan der Unterstützung und Integration der Bürger mit Behinderung für den Zeitraum 2006-2009, genehmigt durch den Beschluss der Regierung der ČR Nr. 1004 vom 17. August 2005.

Die Nationalpläne bedeuteten einen Beitrag zur Behandlungsverbesserung des Staates für die behinderten Bürger. Es ist ohne Zweifel, dass sie dazu beigetragen haben, einen Schwerpunkt der Regierungstätigkeit sowie anderer Zentralorgane der Staatsverwaltung auf eine Reihe von die behinderten Bürger betreffenden Problemen zu legen. Positiv wird auch die Tatsache beurteilt, dass die Behinderten immer Anteil an der Vorbereitung der Aufgaben in den Nationalplänen genommen haben, sodass die Bestimmungen der Maßnahmen in der Regel einen Kompromiss darstellen und zum „Vertrag“ eigener Art zwischen den Repräsentationen der behinderten Bürger und dem zuständigen Zentralorgan der Staatsverwaltung werden können.

Die Mehrheit der Verfassungsorgane der Tschechischen Republik schenkt den Angelegenheiten behinderter Bürger angemessene Beachtung.

- Parlament der ČR: Im Rahmen dieses gesetzgebenden Körpers gibt es spezialisierte, auf die Problematik der Behinderung orientierte Organe. Im Rahmen des Abgeordnetenhauses arbeitet der Subausschuss für behinderte Bürger mit verringerter Arbeitsfähigkeit beim Ausschuss für die Sozialpolitik und das Gesundheitswesen. Ein ähnlicher Subausschuss wurde auch beim Senat eingerichtet. Die genannten Organe dienen als Beratungsgremien für beide Kammern des Abgeordnetenhauses.

- Regierung der ČR: Ein Bestandteil der Exekutive ist der Regierungsausschuss für behinderte Bürger (im weiteren nur „RABB“ genannt), der als Beratungsstelle dient. Im Rahmen des RABB gilt seit seiner Errichtung das Repräsentationsprinzip bezüglich der Zusammensetzung (Mitglieder sind Premier, Vizepremiere und eine Reihe von Ministern), wobei dadurch ermöglicht wird, laufend eine Reihe von Problemen auf sehr hoher Ebene zu diskutieren. Positiv findet man auch, dass die dominierenden Organisationen der behinderten Bürger stetigen Anteil an der Diskussion haben.

Die Behinderten befürworten in der Regel die Existenz des RABB sowie die Tätigkeit seines Sekretariats.

Die zehn Jahre lange Tätigkeit des RABB ist von Nutzen eines solchen Organs innerhalb der Staatsverwaltung gewesen. 


\section{Weitere Aspekte, welche die Lage der behinderten Personen in der Gesellschaft beeinflussen}

Ein Begleitphänomen der Transformation der tschechischen Gesellschaft im vorigen Jahrhundert stellte ein spenderunfreundliches Milieu dar, u.zw. infolge einer nichtexistierenden Spendertradition, der allgemein nicht etablierten Eigentumsverhältnisse und der daraus resultierenden (nicht nur kapitalorientierten) Werte. Mittels Regelung des Spendenrechts zugunsten „karitativer“ Zwecke zu Beginn der 90er Jahre wurde das Modell einer möglichen Schenkung eines Teils des Einkommens auf Steuerbasis entwickelt, wobei natürliche Personen einen anderen Prozentsatz als juristische Personen nutzen können.

In der Tschechischen Republik wurde kein Modell einer gezielten Allokation eines Teils der Steuerpflicht (1-2\%) zugunsten eines konkreten karitativen oder ähnlichen Zwecks aus anderen Ländern (z. B. neu in der Slowakei) übernommen. Anstelle der Annahme von karitativen Gaben als eine ausnahmsweise genutzte Gelegenheit zur Lösung „oberstandardmäßiger“ oder bislang nicht existierender Dienste, Bedürfnisse und Mittel für die Behinderten nutzt man oft die Spender, um mit deren Hilfe ganz legitime standardmäßige Bedürfnisse und Dienste zu decken, welche sonst aus öffentlichen Ressourcen gedeckt werden sollten.

Die Rolle der Organe des Staates auf dem Gebiet der Prävention der sozialen Benachteiligung, der Bildung einer „offenen und freundlichen Gesellschaft“, bleibt eher vermittelnd. Der Staat kann mehr als bisher mittels der legislativen Instrumente, der Zielsetzung und Konzeptbildung, der Unterstützung von Änderungen des Verständnisses in der Öffentlichkeit zum Aufbau einer offenen Zivilgesellschaft, deren Bestandteil auch die Unterstützung und das Verständnis gegenüber Behinderten als wichtiges Element der verschiedenartigen, sich entwickelnden Gesellschaftskulturen eines modernen Staats, sein soll, beitragen.

Der vergangene Zeitraum hat aber keine präzise Antwort auf die Frage gegeben, wie viele Bürger mit einer Behinderung in der Tschechischen Republik leben. Die Beantwortung dieser Frage ist in einer jeden Gesellschaft immer von Klassifizierungskriterien abhängig. In der Tschechischen Republik gibt es ein Dutzend Klassifizierungsmerkmale, die in einigen Fällen nicht zusammengehörige oder inkompatible Eigenschaften aufweisen.

Man kann feststellen, sich einer gewissen Ungenauigkeit bewusst seiend, dass in der ČR etwa 1.000.000 Personen mit einer Behinderung leben - dies entspricht ca. 10\% der Einwohner. Diese Größenordnung entspricht der Situation in anderen europäischen Ländern. Der Prozentsatz der Behinderten variiert in der Regel von 5 bis hin zu 19\%. Praktisch handelt es sich jedoch nicht um einen großen Unterschied des Anteils der Behinderten an der Gesamtbevölkerung, sondern um eine Verschiedenartigkeit der Beurteilungskriterien. Nach dem Begleitbericht der Europäischen Union zur Annahme des Beschlusses des Europarates über das Europäische Jahr der behinderten Personen 2003 sind rund 38 Millionen Menschen von einer Behinderung betroffen, d.h. jeder zehnte Europäer aller Alterskategorien. 
Die Statistiken über die Anzahl der behinderten Bürger sind ohne Zweifel wichtig, man sollte sie jedoch nicht überschätzen.

\section{Analysen der Wirkung von Organisationen und Bürgergruppen der behinderten Menschen}

\section{Starke Seiten}

- die Behinderung ist ein so gravierendes Sozialereignis, dass dies in der Regel die Betroffenen zur Teilnahme an der Lösung ihrer Situation und zur Eingliederung in eine Behindertenorganisation motiviert

- die Behindertenorganisationen decken ein breites Spektrum individueller Behinderungen $\mathrm{ab}$ und werden deshalb $\mathrm{zu}$ einem wichtigen sowie anerkannten Repräsentanten der Ansichten und Bedürfnisse dieser Bürgergruppe

- zum Ende des genannten Zeitraums kam es zum Entstehen einer einheitlichen Repräsentation der Betroffenen - des Nationalbeirats der Behinderten

- $\quad$ seit 2001 entstehen, im Zusammenhang mit der Reform der öffentlichen Verwaltung, auch die Kreisrepräsentationen der Behinderten

- eine Reihe von Behindertenorganisationen verfügt über ein solides Potenzial, das bei der Lösung der Probleme nutzbar ist

- die wichtigsten Behindertenorganisationen in der ČR sind direkt oder mittels des Nationalbeirats aktiv an der Tätigkeit internationaler Behindertenorganisationen beteiligt, insbesondere an denen aus Europa und der Europäischen Union

- $\quad$ einige Behindertenorganisationen haben den Beweis geliefert, dass sie fähig sind, als Partner der Staatsverwaltung zu handeln und auch vielfältige Unterstützungssysteme zu bilden, welche in einigen anderen Ländern durch die Staatsorgane verwirklicht werden,

\section{Schwache Seiten}

- die Behinderten und deren Organisationen haben ihre Tätigkeiten ,auf der grünen Wiese“ begonnen und deshalb war es für sie unmöglich, an in Jahrzehnten gesammelte Erfahrungen und an die Entwicklung der Tätigkeitsprinzipien wie es in den fortgeschrittenen Ländern üblich ist, anzuknüpfen

- beim bestehenden Spendenklima in der ČR ist die Tätigkeit der Behindertenorganisationen nicht so gesichert wie dies in fortgeschrittenen Ländern üblich ist

- $\quad$ zwischen den einzelnen Bürgergruppen der Behinderten gibt es große Unterschiede (alters- und bildungsbedingt sowie im Hinblick auf die Behinderung) im Hinblick auf die gesellschaftlichen Aktivitäten und die Kompetenz zur Vertretung eigener Interessen

- bei einer Reihe von behinderten Bürgern kam es infolge verschiedener Umstände sowohl objektiver als auch subjektiver Natur zur Bildung einer passiven Einstellung zur Lösung der eigenen Rolle in der Gesellschaft und zur Bestrebung nach passivem Empfang der Hilfe 
- in grundsätzlichen Lebensbereichen (Bildung, Beschäftigung, Art der Freizeitgestaltung, Familienleben, Mobilität) sind behinderte Bürger immer benachteiligt, auch wenn die Ursachen einerseits zwar objektiver Art sind aber andererseits unabhängig vom Willen des Staats aus Ressourcen auf der Ebene der Gesellschaft lösbar wären

- die Tätigkeit der Behindertenorganisationen ist von Natur aus freiwillig und spiegelt die Prinzipien der Zivilgesellschaft wider, was in einigen Fällen zu einer niedrigen Professionalität führt

- Kreisrepräsentationen der Behinderten werden nur langsam zu vollwertigen Partnern der Kreisverwaltungsvertreter und erringen nur schrittweise die Kompetenz zur Partnerschaft bei der Lösung einzelner Aufgaben und der Abdeckung der entsprechenden Gebiete.

\section{Chancen - der Staat und die Behindertenorganisationen}

- das Prinzip der Partizipation kann in einem breiten Ausmaß zu einer Einordnung der Beziehungen zwischen Staat inkl. seiner Organe auf der einen und den Behindertenorganisationen auf der anderen Seite führen

- die Bürger mit Behinderung und deren Organisationen stellen einen der aktivsten Teile der Zivilgesellschaft dar, die im Prinzip Interesse zeigen, eigene Probleme zu lösen; aus dieser Tatsache können die Organe der staatlichen Verwaltung in breiterem Ausmaß als bisher Nutzen ziehen.

Wenn man unsere Gesellschaft als eine Gemeinschaft von Menschen mit gleichen Chancen charakterisiert, dann gehören die Behinderten an sich zu einer Gruppe, bei der die ungleiche Chancennutzung in der Regel als immanente Charakteristik kennzeichnend ist.

Die Annahme des einheitlichen Konzepts der Staatspolitik gegenüber den Bürgern mit Behinderung ist auch für die Staatsorgane von Bedeutung, für die damit ein nutzbringendes Instrument zur Planung, Entscheidung, Konzeptbildung sowie für operative Tätigkeiten gegeben ist.

\section{Die Partizipation der Bürger mit Behinderung und deren Organisa- tionen an der Verwaltung öffentlicher Angelegenheiten - Ziele, Aufgaben und Maßnahmen für die Jahre 2004-2009}

\section{Allgemeine Aufgaben}

In genanntem Zeitraum ist es wichtig, im Interesse sowohl des Staats als auch der behinderten Bürger, durch eine weitere Stärkung der Fähigkeiten der Behinderten zu einem Prozess der weiteren Entfaltung der politischen Lösungen sowie der Programme zugunsten dieser Bürgergruppe beizutragen. Insbesondere ist es nötig, die Partnerschaft zwischen den Organisationen der behinderten Bürger und anderen Sozialpartnern zu unterstützen. Es ist notwendig, die Mechanismen zur Unterstüt- 
zung einer breiteren Partizipation der behinderten Bürger an den Konsultationsprozessen zu vervollkommnen.

Die bisherige Praxis zeigt, dass die Schlüsselrolle bei der Beeinflussung der Politik zugunsten der Behinderten der Nationalbeirat der Behinderten der ČR $=$ NRZP ČR spielt (früher der Verein der Behindertenvertreter und der transformierte Invalidenverband der ČR). Es ist ebenfalls offensichtlich, dass es jetzt notwendig ist, diese unvertretbare Rolle um ein Element der fachlichen Tätigkeit bei der Formulierung und Verwirklichung der Konzeptdokumente auf diesem Gebiet zu ergänzen. Diese Entwicklung steht im Einklang mit dem Konzept der Kommission der Europäischen Gemeinschaften. Diese hat in ihrer Mitteilung $\operatorname{COM}(2000) 284$ final u.a. die Notwendigkeit von Forschungen, die auf diesem Gebiet die Nichtregierungsorganisationen unternehmen, betont.

Der NRZP ČR stellt zurzeit eine Koalition von 87 Behindertenorganisationen dar und ist der legitime Vertreter von mehr als 250.000 Mitgliedern. Der NRZP C̆R spielt nicht nur eine Rolle als Repräsentant der Interessen und Bedürfnisse der behinderten Bürger, sondern funktioniert auch als Initiator und Verwirklichter konkreter Programme zur Unterstützung der Behinderten. Bei der Verwirklichung der Aufgaben des NRZP ČR arbeitet dieser Beirat mit einer Reihe internationaler Organisationen und Institutionen, Organen der staatlichen und der lokalen Verwaltung, der Wirtschaftsphäre, Gewerkschaftsorganisationen, Forschungsanstalten, Medien und Nichtregierungsorganisationen zusammen.

Die Sicherung der Rahmenbedingungen für eine Professionalisierung der Behindertenorganisationen bei der Teilnahme an der Politikgestaltung zugunsten der behinderten Bürger ist deshalb zu einer Grundaufgabe geworden. Der Aktionsplan erstrebt gleichfalls eine maximale Eingliederung der behinderten Personen und deren Organisationen.

Im Rahmen des Nationalplans findet man folgende Maßnahmen für diesen Bereich:

1. Sicherung der Teilnahme der Organisationen der behinderten Bürger am Legislativprozess.

2. Verwirklichung von Forschungstätigkeiten auf dem Gebiet der Soziologie der Behinderung und der Lebensqualität der behinderten Bürger, die zur Vorbereitung der Konzeptdokumente und für die Entwürfe der Legislativänderungen unentbehrlich sind.

3. Stiftung eines Preises des Regierungsausschusses für sich mit den Problemen der Behinderten beschäftigenden publizistischen Arbeiten.

4. Auflegung einer Informationskampagne über effektive Formen der Unterstützung von behinderten Bürgern.

5. Teilnahme der Vertretung der $\breve{C} R$ am European Disability Forum und an Rehabilitation International.

Ausgangspunkt für die Erfüllung dieser Politik in der ČR soll die Revision der Dotationspolitik gegenüber den Behindertenorganisationen werden. Es handelt sich hierbei insbesondere um die deutliche Trennung der Finanzierung von Dienstleis- 
tungen auf der einen und der Finanzierung der politischen Teilnahme zugunsten der Behinderten auf der anderen Seite. Die Verwirklichung dieser Aufgabe wird keine Erhöhung der Ausgaben aus dem Staatshaushalt mit sich bringen. Zur Finanzierung der Teilnahme an der Gestaltung der Politik zugunsten der Behinderten ist es möglich, auch weiterhin ausreichende Mittel aus dem bestehenden Unterstützungsprogramm des Gesundheitsministeriums der ČR „Öffentliche zielbewusste Aktivitäten der Bürgervereinigungen der Behinderten“ zu schöpfen. Die Finanzierung sollte stabil bleiben.

Auch ist es aus der bisherigen Praxis ersichtlich, dass der Regierungsausschuss für behinderte Bürger eine Schlüsselrolle bei der Koordinierung der Gestaltung der Politik zugunsten der Behinderten spielt. Es wird jedoch nötig sein, seinen Tätigkeitsumfang zu erweitern, u.zw. im Hinblick auf die geplante Errichtung einer Kommission für Bürger mit Behinderung unter Teilnahme der Kreise. Der Regierungsausschuss wird nach dem Beschluss der Regierung der ČR Nr. 596/2003 regelmäßig im allgemeinen Sinne mit den Kreisvertretern über die aktuellen Probleme der behinderten Bürger und über die Gestaltung sowie Verwirklichung der Kreispläne des Nachteilsausgleichs der behinderten Bürger beraten.

Eine weitere bedeutende Aufgabe des Staats ist es, bei der Verwirklichung der Partizipationspolitik sowohl den National- als auch den Regionalprogrammen zur Unterstützung der behinderten Bürger Unterstützung zu gewähren. Die Prinzipien zur Verwirklichung stellt die Resolution der Vollversammlung der UNO Nr. 48/96 vom 20.12.1993 über Standardregeln des Nachteilsausgleichs für behinderte Bürger auf. Die Behindertenorganisationen sollen nach dieser Resolution in weitem Ausmaß ihren Einfluss in den zu diesem Zweck in den einzelnen Staaten errichteten Koordinierungs- und Untersuchungsausschüssen ausüben. Die Ausschüsse selbst sollen dauerhaft und weitestgehend unabhängig sein sowie mit den notwendigen Mitteln zur Erfüllung ihren Aufgaben ausgestattet werden, wobei ihre Tätigkeit legislativ sowie administrativ geregelt werden muss. Diese Prinzipien wurden sowohl bei der Vorbereitung als auch bei der Durchführung aller bisherigen Nationalpläne zum Nachteilsausgleich für behinderte Bürger eingehalten. Auch in Zukunft sollten sie als Instrumente für die Lösung einzelner Aufgaben des mittelfristigen Konzepts der Staatspolitik gegenüber behinderten Bürgern respektiert werden.

In Anknüpfung an diese Pläne ist es notwendig, schrittweise zur Annahme und Verwirklichung der Kreispläne des Nachteilsausgleichs für behinderte Bürger zu kommen. Ein besonderes Augenmerk sollte den Tätigkeiten der spezialisierten Initiativen, untersuchungsorientierten und koordinierenden Organen eines jeden Kreises im Hinblick auf die Ausarbeitung und Verwirklichung der Kreispläne gewidmet werden.

\section{Kurze Übersicht von Aufgaben und Maßnahmen:}

- Umgestaltung der Methodik für die Zwecke der Dotationenzuteilungen aus dem Haushaltskapitel des Gesundheitsministeriums im Rahmen des Programms öffentlicher zielbewusster Aktivitäten der Bürgervereinigungen der Behinderten. 
- Stetige Aktualisierung des Nationalplans zum Nachteilsausgleich für Bürger mit Behinderung.

- Annahme und Verwirklichung der Pläne des Nachteilsausgleichs der Bürger mit Behinderung in allen Kreisen der ČR.

- Verwirklichung von Änderungen in der Verhandlungsordnung und der Zusammensetzung des Sekretariats des Regierungsausschusses gemäss der aktualisierten Evidenz der existierenden Kreiskommissionen für die behinderten Bürger und nach dem Stand der Verwirklichung der Kreispläne zum Nachteilsausgleich für behinderte Bürger.

\section{Koordinierung und Untersuchung der Erfüllung des Nationalplans}

Die Ausführung des Nationalplans ist ein komplizierter, verknüpfter Prozess, an dem nicht nur die zentralen Verwaltungsorgane, sondern auch die Kreisorgane sowie die Behindertenorganisationen selbst beteiligt sind. Aus den Erfahrungen mit der Koordinierung aller bisherigen Nationalpläne entsteht das Bedürfnis nach präziseren und detaillierten Regeln für diesen Prozess.

Aus den Maßnahmen des Nationalplans können sich keine Aufgaben für die einzelnen Kreise ergeben. Stattdessen ist es aber möglich und zweckmäßig, den Kreisorganen dauerhaft methodische Hilfe zu leisten und zwischen ihnen einen Dialog über die aktuellen Probleme der Kreispolitik zugunsten von behinderten Bürgern in Gang zu setzen.

Für diese Zwecke beinhaltet der Nationalplan folgende Maßnahmen:

1. Ausarbeitung der Regeln für Untersuchung und Einschätzung der Aufgabenerfüllung aus dem Nationalplan.

2. Jährliche Aktualisierung des Nationalplans.

3. Vorlage von Jahresberichten aller relevanten Ressorts über die entsprechende Maßnahmenerfüllung aus dem Nationalplan im abgelaufenen Jahr.

4. Die Vorbereitung, die Verwirklichung und die laufende Untersuchung der Kreispläne des Nachteilsausgleichs für behinderte Bürger methodisch zu unterstützen.

\section{System der Zuwendung staatlicher Dotationen}

\subsection{Programm der Grantunterstützung}

Der Zweck des Programms: Das Programm ist zur Unterstützung von Projekten nichtstaatlicher, non- profit Organisationen mit ganzstaatlichem Wirkungsfeld, die sich mit der Pflege der Behinderten und der chronisch Kranken sowie mit Senioren in der Tschechischen Republik beschäftigen, bestimmt.

Mit Hilfe dieses Programms unterstützt das Gesundheitsministerium der ČR Projekte, welche auf eine Intensivierung der Betreuung der Behinderten und der chro- 
nisch Kranken im Hinblick auf ihren Gesundheitszustand gerichtet sind. Aus der Sicht des Gesundheitssystems stellen diese Bürger eine Gruppe dauerhafter Leistungsbezieher von Gesundheitsdiensten dar. Bei der Deckung dieser Bedürfnisse ist nicht nur deren Qualität und Zugänglichkeit von großer Wichtigkeit; ebenso spielt die Effektivität der Wirkung eine wichtige Rolle.

$\mathrm{Zu}$ diesem Zweck werden Voraussetzungen geschaffen, um die Ausbildung zugunsten des Nachteilsausgleichs der behinderten Bürger zu ermöglichen und zur Verwirklichung dieses Prozesses beizutragen.

Im Zusammenhang mit dem Gesetz Nr. 218/2000 Slg über die Haushaltsregeln veröffentlicht das Gesundheitsministerium der ČR die Bedingungen für die Ausarbeitung und Registrierung der Projekte (Anträge um die staatliche Dotation für 2008) von nichtstaatlichen, non-profit Organisationen. Unter einer nichtstaatlichen, non-profit Organisation versteht man eine Bürgervereinigung im Sinne des Gesetzes Nr. 83/1990 Slg über die Vereinigungen der Bürger, eine öffentlich nutzbringende Gesellschaft im Sinne des Gesetzes Nr. 248/1995 Slg oder ein nach dem Gesetz über die Kirchen und religiöse Gemeinschaften registriertes Rechtssubjekt.

Es ist möglich, nur den Bürgervereinigungen der Behinderten mit ganzstaatlichem Wirkungsbereich oder den Bürgervereinigungen, zu deren Unterstützung sich der Staat durch ein besonderes Gesetz verpflichtet hat, Zuwendungen zu gewähren. Für eine Bürgervereinigung der Behinderten wird für das Dotationsprogramm ein Rechtssubjekt angesehen, das nach dem Gesetz Nr. 83/1990 Slg registriert worden ist und bei dem mindestens 50\% der Mitglieder behinderte Bürger sind. Für dieses Dotationsprogramm werden als behinderte Bürger auch chronisch kranke Bürger angesehen.

\section{Thematische Gebiete der Anträge um mögliche Dotationen}

- Rekonditions- und Bildungsaufenthalte mit gesundheitlichem Programm

- Frequentierte Bildungsveranstaltungen (Kurse und Schulungen)

- Editionstätigkeit

- Auf die Unterstützung der Kommunikationszentren zielende Projekte für demenzbetroffene Bürger und deren Familien

- Organisations- und administrativer Service für Bürgervereinigungen

\subsection{Programm des Nachteilsausgleichs für behinderte Bürger}

Der Zweck des Programms: Im Jahr 2008 wird das Gesundheitsministerium der ČR bei den Dotationsverfahren vom Regierungsbeschluss vom 17.08.2005 Nr. 1004 ausgehen, in dem der Nationalplan zur Unterstützung und Integration der behinderten Bürger für den Zeitraum 2006-2009 beschlossen wurde. Diese Form des Nationalplans entspricht den Standardregeln des Nachteilsausgleichs für behinderte Bürger, welche die UNO am 20.12.1993 beschlossen hat.

Die Dotationsverfahren im Jahr 2008 werden den Regierungsbeschluss vom 7. Februar 2001 Nr. 114 über die Grundsätze der Regierung für die Dotations- 
zuwendungen aus dem Staatshaushalt der Tschechischen Republik für die nichtstaatlichen, non-profit Organisationen durch die Zentralorgane der Staatsverwaltung sowie den Regierungsbeschluss Nr. 631 vom 11. Juni 2007 über die Hauptgebiete der staatlichen Dotationspolitik gegenüber nichtstaatlichen, non-profit Organisationen für das Jahr 2008, beachten.

Die Dotationsverfahren 2008 werden die sich für das Gesundheitsministerium ergebenden Aufgaben aus dem Regierungsbeschluss Nr. 605 vom 16. Juni 2004 über das mittelfristige Konzept der Staatspolitik zugunsten der behinderten Bürger berücksichtigen.

Die Zuwendung von Dotationen für ausgewählte Projekte im Rahmen des „Programms“ stellt eine konkrete Unterstützungsform zugunsten verschiedener Typen staatlicher und nichtstaatlicher Organisationen, z.B. von Bürgervereinen, humanitären Organisationen der Kirchen und anderer definierter Rechtssubjekte für deren Aktivitäten und Tätigkeiten, welche die Vorgaben des „Programms“ erfüllen, dar.

Die Erfahrungen bestätigen, dass dieses System der staatlichen finanziellen Dotation ein unentbehrliches Zusatzelement bei der Lösung der Probleme auf dem Gebiet der Betreuung der behinderten Bürger darstellt. Die zweckmäßig an konkrete Projekte gebundenen Dotationen, die auf die aktuellen Probleme auf lokaler, regionaler und staatlicher Ebene eine entsprechende Reaktion ergeben, helfen in bedeutendem Maße, die Aufgaben des Gesundheitsministeriums im Rahmen der Verwirklichung der Regierungsbeschlüsse in die Tat umzusetzen. Für die Antragsteller ist von Bedeutung, dass sie die vom Gesundheitsministerium aufgestellten Bedingungen erfüllen müssen und im Falle der Projektbilligung auch den im Beschluss über die $\mathrm{Zu}$ wendung von Investitionsdotationen aus dem Staatshaushalt der C̆R festgelegten Bedingungen gerecht werden müssen.

Kreis möglicher Antragsteller: In Übereinstimmung mit dem Beschluss der Regierung der ČR vom 11. Juni 2007 Nr. 631 über die Hauptgebiete der staatlichen Dotationspolitik gegenüber nichtstaatlichen, non-profit Organisationen für das Jahr 2008 können folgende Gruppen Anträge auf Dotationen aus dem Staatshaushalt der ČR stellen:

- Organisationen in direkter Wirksamkeit des Gesundheitsministeriums

- Organisationen in direkter Wirksamkeit anderer Ressorts

- Organisationen in direkter Wirksamkeit der Kreisorgane

- Verwaltungsorgane (der Städte und Gemeinden) und Organisationen in direkter Kontrolle der Städte und Gemeinden

- Nichtstaatliche, non-profit Organisationen - Bürgervereinigungen und öffentlich nutzbringende Gemeinschaften

- Nach dem Gesetz über die Kirchen und religiöse Gemeinschaften registrierte Rechtssubjekte

- Öffentliche non-profit Gesundheitsanstalten

- Andere Rechtssubjekte d.h. juristische und natürliche Personen, welche eine unternehmerische, durch Handels-, Bürgerliches und Gewerbegesetzbuch geregelte Tätigkeit ausüben. 
Als nichtstaatliche, non-profit Organisationen sind hier die Bürgervereinigungen und öffentlich nutzbringenden Gemeinschaften für körperlich oder geistig behinderte Menschen mit verschiedenen Formen der Behinderung, wie z. B. Blindheit, Teilblindheit, orthopädische Behinderung, Hörbehinderung, Geisteskrankheit oder Sprechbehinderung gemeint.

Die Bedingungen für die Antragstellung: Nach dem Gesetz Nr. 218/2000 Slg über die Haushaltsregeln gibt es kein Anrecht auf Dotation.

Eine Zuwendung von Dotationen aus diesem Programm ist sowohl für die aus der Gesundheitsversicherung finanzierte Gesundheitspflege im Sinne des Gesetzes Nr. 48/1997 Slg über die öffentliche Gesundheitsversicherung im Wortlaut späterer Vorschriften als auch für die Tätigkeiten, die im Gesetz Nr. 108/2006 Slg über die Sozialdiensten beschrieben sind, nicht erlaubt. 


\title{
5.5. Behinderte Menschen im türkischen Recht
}

\author{
Von Yasemin Körtek
}

1. Einleitung 246

2. Begriffsbestimmung: Behinderung 249

2.1. Der Behindertenbegriff des Behindertengesetzes, Gesetz Nr. $5378 \quad 249$

2.2. Der Behindertenbegriff im Arbeits-, Beamten- und Steuerrecht 250

2.3. Der Behindertenbegriff im Sozialversicherungsrecht 251

2.4. Der Behindertenbegriff im Bereich der Sozialhilfe und sozialen Fürsorge

2.5. Würdigung 254

3. Die Stellung behinderter Menschen im Arbeitsleben 255

4. Behinderte Menschen im System der sozialen Sicherheit 258

4.1. Bezug von Altersrente unter erleichterten Bedingungen 258

4.2. Bezug von Leistungen der Sozialhilfe und der sozialen Fürsorge $\quad 260$

5. Ausblick

Literaturverzeichnis 


\section{Einleitung}

Das System der sozialen Sicherheit in der Türkei befindet sich seit den 1990er Jahren in einem Reformprozess. Im Zuge der Reformen hat der türkische Gesetzgeber am 31.05.2006 das neue Sozialversicherungs- und allgemeine Krankenversicherungsgesetz, Gesetz Nr. 5510, verabschiedet, das zum 01.10.2008 in Kraft getreten ist. ${ }^{1}$ Das Recht der Menschen mit Behinderung, das nicht Gegenstand der Reformen ist, steht weniger im Fokus des Interesses der Öffentlichkeit als das Renten- oder Krankenversicherungsrecht. Doch sind in den letzten Jahren wesentliche Entwicklungen auf diesem Gebiet zu verzeichnen.

Im Jahr 2002 wurde in der Türkei eine Untersuchung durchgeführt, deren Gegenstand Menschen mit Behinderung bildeten. Insbesondere sollten die Anzahl der Menschen mit Behinderung, ihre Erwartungen und sozio-ökonomischen Probleme, die Art der Behinderung sowie etwaige regionale Unterschiede ermittelt werden. ${ }^{2}$ Diese Untersuchung brachte zu Tage, dass 12,29\% der Gesamtbevölkerung somit ca. 8,4 Millionen Menschen eine Behinderung haben. ${ }^{3}$ Dabei sind lediglich 21,7\% der Gesamtzahl der Menschen mit Behinderung in den Arbeitsmarkt eingegliedert, sodass die Teilhabe Behinderter am Arbeitsleben ein Hauptproblem darstellt. ${ }^{4}$ An diese Untersuchung aus dem Jahr 2002 schloss sich ein weiteres Projekt an, mit welchem die objektiven Lebensbedingungen von behinderten Menschen, wie z. B. die Inanspruchnahme angebotener Leistungen, näher erforscht wurden. ${ }^{5}$ Es stellte sich u. a. heraus, dass 55,7\% der Menschen mit Behinderung Gesundheitsleistungen, dagegen lediglich 12,29\% Ausbildungs- und nur 5,9\% Pflegedienste in Anspruch

1 Sosyal Sigortalar ve Genel Sağlık Sigortası Kanunu, RG vom 16.06.2006, Nr. 26200.

Nachdem das Verfassungsgericht mit Urteil vom 15.12.2006 mehrere Vorschriften des Gesetzes Nr. 5510 im Rahmen einer abstrakten Normenkontrolle für verfassungswidrig erklärt hatte (E. 2006/111, K. 2006/112, RG vom 30.12.2006, Nr. 26392), verschob der türkische Gesetzgeber das Datum des Inkrafttretens des gegenständlichen Gesetzes ursprünglich zum 01.01.2007 mittlerweile zum 01.06.2008 (Art. 28 Abs. 16 Gesetz Nr. 5724 vom 14.12.2007, RG vom 28.12.2007, Nr. 26740 mükerrer). Die Große Nationalversammlung hat am 17.04.2008 das Gesetz Nr. 5754 zur Änderung des Gesetzes Nr. 5510 verabschiedet (Sosyal Sigortalar ve Genel Sağlık Sigortası Kanunu ile bazı Kanun ve Kanun Hükmünde Kararnamelerde degişiklik yapılmasına dair Kanun; RG vom 08.05.2008, Nr. 26870), das für bestimmte Vorschriften verschiedene Zeiten betreffend des Inkrafttretens regelt (Art. 74 Gesetz Nr. 5754). Das Gesetz Nr. 5510 ist in seiner Gesamtheit zum 01.10.2008 in Kraft getreten.

2 Die Ergebnisse dieser umfangreichen Untersuchung (Türkiye Özürlüler Araştırması Temel Göstergeleri) können entnommen werden unter: http://www.ozida.gov.tr

3 Im Vergleich hierzu lag die Anzahl schwerbehinderter Menschen in der Bundesrepublik zum 31.12.2005 bei 6,8 Millionen und somit bei 8,2\% der Gesamtbevölkerung. Quelle: Statistisches Bundesamt, Statistik der schwerbehinderten Menschen, 2005. Vgl. T.C. Başbakanlık, Özürlüler İdaresi Başkanlığı, Stratejik Plan (2008-2012), S. 8, zu entnehmen unter: http://www.ozida.gov.tr

5 Türkiye Bilimsel ve Teknik Araştırma Kurumu (TÜBİTAK), Türkiye Özürlüler Araştırması 2002 İkincil Analizi, 2006, zu entnehmen unter: http://www.ozida.gov.tr 
nehmen. Demzufolge steht die Behandlung der die Behinderung verursachenden Gesundheitsbeeinträchtigungen im Vordergrund. ${ }^{6}$

Menschen mit Behinderung sind besonders schutzbedürftig, insbesondere, wenn ihre Teilhabe am gesellschaftlichen Leben und ihre soziale Absicherung betroffen sind. Die türkische Verfassung statuiert in ihrem Art. 60 das Recht eines Jeden auf soziale Sicherheit. Zugleich wird der türkische Staat im Allgemeinen verpflichtet, alle Maßnahmen zur Gewährleistung dieser Sicherheit zu ergreifen und die notwendigen Institutionen $\mathrm{zu}$ schaffen. ${ }^{7}$ In diesem Zusammenhang erteilt die türkische Verfassung dem Staat den konkreten Auftrag, alle Maßnahmen zur Gewährleistung des Schutzes von Behinderten sowie ihrer Eingliederung in das Gemeinschaftsleben zu treffen (Art. 61 Abs. 2 TV). Der türkische Gesetzgeber hat sich, ausgehend von seiner verfassungsrechtlichen Verpflichtung, der behinderten Menschen angenommen und gesetzliche Regelungen geschaffen. Neben der verfassungsrechtlichen Vorgabe darf nicht aus dem Blick verloren werden, dass die Türkei auf Grund internationaler Verpflichtungen zur Regelung der Rechte von Menschen mit Behinderung angehalten ist: Die Europäische Sozialcharta wurde mit Gesetz Nr. 3581 vom 16.06.1989 und ihre revidierte Fassung mit Gesetz Nr. 5547 vom 27.09.2006 ratifiziert $^{8}$ - ohne dass ein Vorbehalt im Hinblick auf deren Art. 15 erklärt wurde. ${ }^{9}$ Schließlich ist anzumerken, dass die Türkei das Internationale Übereinkommen über die Rechte von Menschen mit Behinderungen ${ }^{10}$ am 30.03.2007 unterzeichnet hat. Die Ratifizierung steht noch aus.

Rechte von Menschen mit Behinderung werden durch verschiedene Normen gewährleistet. ${ }^{11}$ Das Präsidium für Angelegenheiten behinderter Menschen (Özürlü İdaresi Başkanlığı) wurde mit der Rechtsverordnung mit Gesetzeskraft Nr. 571 vom 25.03.1997 $7^{12}$ errichtet, um die ordnungsgemäße Durchführung der vorgesehenen Dienste für Menschen mit Behinderung zu gewährleisten, für die Zusammenarbeit und Koordination zwischen nationalen ${ }^{13}$ und internationalen Institutionen zu sorgen,

6 Türkiye Bilimsel ve Teknik Araştırma Kurumu (TÜBİTAK), Türkiye Özürlüler Araştırması 2002 İkincil Analizi, 2006, S. 34, 55, zu entnehmen unter: http://www.ozida.gov.tr.

7 Art. $60 \mathrm{TV}$ ist eine Ausprägung des in Art. 2 TV niedergelegten Sozialstaatsprinzips. Vgl. Entscheidung des Verfassungsgerichts vom 06.01.2005, E. 2001/479, K. 2005/1, RG vom 18.10.2005, Nr. 25970; Wedekind, Die türkische Verfassung 1982, Art. 60.

8 RG vom 16.06.1989, Nr. 20215; Ministerratsbeschluss 89/14434 vom 01.08.1989, RG vom 14.10.1989; RG vom 03.10.2006, Nr. 26308; Ministerratsbeschluss 2007/11907 vom 22.03.2007, RG vom 09.04.2007, Nr. 26488.

9 Diese Norm regelt die Rechte von Menschen mit Behinderung betreffend die Ausbildungsförderung, Teilhabe am Arbeitsleben und Teilhabe am Leben in der Gemeinschaft.

10 Hierzu Welke, Archiv 4/2007, S. 60 ff.

11 Eine Aufzählung aller relevanten Gesetze, Verordnungen mit Gesetzeskraft, Durchführungsverordnungen, Runderlasse und Bekanntmachungen findet sich in T.C. Başbakanlık, Özürlüler İdaresi Başkanlığı, Stratejik Plan (2008-2012), S. 16-20, zu entnehmen unter: http://www.ozida.gov.tr.

12 Özürlüler İdaresi Başkanlığı Teşkilat ve Görevleri Hakkında Kanun Hükmünde Kararname, RG vom 30.05.1997, Nr. 23004 mükerrer.

13 Dienste/Leistungen an Menschen mit Behinderung werden durch verschiedene Institutionen erbracht. Die zuständigen Ministerien sind insbesondere das Gesundheitsministerium sowie das Ministerium für Arbeit und soziale Sicherheit. 
zur Erarbeitung nationaler Politiken für Menschen mit Behinderung beizutragen sowie bestehende Probleme einschließlich Lösungsansätze hierfür zu ermitteln (Art. 1 Abs. 1). Das höchste Beratungsorgan ist der Behindertenrat (Özürlüler Şûrası, Art. 20), der erstmals in der Zeit vom 29.11.1999-02.12.1999 tagte. Der Behindertenrat entschied, dass ein Rahmengesetz zu erlassen war. Die wesentlichen Inhalte dieses Rahmengesetzes wurden durch weitere Beschlüsse festgelegt, auf Grundlage derer das Präsidium ein Behindertengesetz ausarbeitete. ${ }^{14}$ Dieses Behindertengesetz, Gesetz Nr. 5378, ${ }^{15}$ wurde am 01.07.2005 von der Großen Nationalversammlung erlassen. Primärzweck ist es, den Eintritt von Behinderungen zu verhindern, Angelegenheiten von Menschen mit Behinderung hinsichtlich Gesundheit, Bildung ${ }^{16}$, Rehabilitation, Beschäftigung ${ }^{17}$, Pflege und sozialer Sicherheit zu regeln sowie Maßnahmen zur Förderung und Behebung von Hindernissen zu ergreifen, damit die Teilhabe am gesellschaftlichen Leben gewährleistet ist. Zur Durchführung des Behindertengesetzes wurden mehrere Verordnungen erlassen, wie die Verwaltungsverordnung betreffend den Grad der Behinderung, der Klassifikation und der, dem behinderten Menschen vom Gesundheitsausschuss auszustellenden, Atteste ${ }^{18}$, die an die Stelle der Vorgängerregelung ${ }^{19}$ getreten ist.

Neben dem Behindertengesetz enthalten das Arbeitsgesetz, die Sozialversicherungsgesetze sowie die Gesetze bezüglich der Sozialhilfe und sozialen Fürsorge Vorschriften, die Leistungen für Menschen mit Behinderung normieren.

Der vorliegende Beitrag unternimmt den Versuch, in das Recht der Menschen mit Behinderung in der Türkei, das sich nach Erlass des Behindertengesetzes im Jahr 2005 noch in Entwicklung befindet, einzuführen. Im Folgenden wird zunächst der Behindertenbegriff, der in verschiedenen Normen niedergelegt ist, näher erläutert. Sodann wird die Stellung behinderter Menschen im Arbeitsleben und im System der sozialen Sicherung einschließlich der vorgesehenen Leistungen im Überblick dargestellt.

14 I. Özürlüler Şurası' nda alınan kararların değerlendirilmesi, zu entnehmen unter: http://www.ozida.gov.tr.

15 Özürlüler ve bazı Kanun ve Kanun Hükmünde Kararnamelerde değişiklik yapılması hakkında Kanun, RG vom 07.07.2005, Nr. 25868.

16 Menschen mit Behinderung sind, unter Berücksichtigung ihrer Besonderheiten, gleiche Ausbildungsmöglichkeiten zu gewähren wie Menschen ohne Behinderung (Art. 15 Abs. 1).

17 In diesem Zusammenhang ist auf die staatliche Unterstützung für Betriebe hinzuweisen, die vermehrt Menschen mit Behinderung beschäftigen (Art. 14 Behindertengesetz i. V. m. Durchführungsverordnung / Korumalı İşyerleri Hakkında Yönetmelik, RG vom 30.05.2006, Nr. 26183).

18 Özürlülük Ölçütü, Sınıflandırılması ve Özürlülere verilecek Sağlık Kurulu Raporları hakkında Yönetmelik, RG vom 16.07.2006, Nr. 26230.

19 Özürlülere Verilecek Sağlık Kurulu Raporları Hakkında Yönetmelik, RG vom 18.03.1998, Nr. 23290. 


\section{Begriffsbestimmung: Behinderung}

\subsection{Der Behindertenbegriff des Behindertengesetzes, Gesetz Nr. 5378}

\section{Gemäß Art. 3 Buchst. a des Behindertengesetzes sind Menschen behindert,}

„wenn sie von Geburt an oder später aus einem beliebigen Grund ihre körperlichen, geistigen, seelischen, emotionalen und sozialen Fähigkeiten in verschiedenen Graden verloren haben und aus diesem Grund Schwierigkeiten bei der Teilnahme am gesellschaftlichen Leben sowie Erfüllung der alltäglichen Bedürfnisse haben und der Schutz-, Pflege-, Rehabilitations-, Beratungs- und Unterstützungsleistungen bedürfen“. ${ }^{20}$

Der türkische Gesetzgeber hat mit der Definition der Behinderung, wie der deutsche Gesetzgeber auch ${ }^{21}$, an den Behinderungsbegriff der Weltgesundheitsorganisation angeknüpft. Anders als nach deutschem Recht fehlt aber die Normierung einer bestimmten Dauer der Funktionsstörungen, um die vorübergehende Beeinträchtigung von der Teilhabe auszuschließen: § 2 Abs. $1 \mathrm{~S}$. 1 SGB IX verlangt für das Vorliegen einer Behinderung eine voraussichtliche Dauer der Funktionsbeeinträchtigung von mehr als sechs Monaten. Nach türkischem Recht wird im Vergleich mit § 2 Abs. 1 S. 1 SGB IX zudem eine Abweichung von dem für das Lebensalter typischen $\mathrm{Zu}-$ stand nicht verlangt. Dies bedeutet aber nicht, dass vorübergehende Funktionsstörungen und alterstypische Gesundheitsbeeinträchtigungen eine Behinderung begründen können, wie aus den Vorgaben zur Beurteilung des Funktionsverlustes entnommen werden kann: Ausgehend vom (alterstypischen) Normalzustand sind, abgesehen von einigen Ausnahmen, dauerhafte Beschränkungen zu beurteilen. ${ }^{22}$ Unterschieden wird des Weiteren, je nach dem festgestellten Behinderungsgrad, zwischen geringer und schwerer Behinderung sowie Behinderten, die der Pflege bedürfen (Art. 3 Buchst. c-e Behindertengesetz). Nähere Regelungen erfolgen in den auf Grundlage des Behindertengesetzes erlassenen Durchführungsverordnungen.

So wird der Behindertengrad eigens durch die hierzu ermächtigten Gesundheitsausschüsse (Özürlü Sağlık Kurulu) ${ }^{23}$ ermittelt und verbindlich festgelegt. ${ }^{24}$ Hierbei

20 “Özürlü: Doğuştan veya sonradan herhangi bir nedenle bedensel, zihinsel, ruhsal, duyusal ve sosyal yeteneklerini çeşitli derecelerde kaybetmesi nedeniyle toplumsal yaşama uyum sağlama ve günlük gereksinimlerini karşılama güçlükleri olan ve korunma, bakım, rehabilitasyon, danışmanlık ve destek hizmetlerine ihtiyaç duyan kişiyi,".

21 Götz, in: Kossens/von der Heide/Maaß (Hrsg.), SGB IX, 2. Aufl., § 2 Rn. 5.

22 Siehe Anlage 4 zur Verwaltungsverordnung betreffend den Grad der Behinderung, der Klassifikation und der den behinderten Menschen vom Gesundheitsausschuss auszustellenden Atteste (Özür Durumuna Göre Tüm Vücut Fonksiyon Kaybı Oranları Cetveli ve Hesaplama Tablosu).

23 Die ermächtigten und zuständigen Stellen sind in der Anlage 1 Verwaltungsverordnung betreffend den Grad der Behinderung, der Klassifikation und der den behinderten Menschen vom Gesundheitsausschuss auszustellenden Atteste aufgeführt (Özürlü Sağlık Kurulu Raporu Vermeye Yetkili Sağlık Kuruluşları).

24 Der Betroffene kann gegen den Bericht des Gesundheitsausschusses beim Gesundheitsamt seines Regierungsbezirks (il sağlık müdürlüğ̈̈) Widerspruch einlegen. Der Rechtsweg zu den Gerichten nach Abschluss des Widerspruchverfahrens ist jedoch nicht eröffnet (vgl. Art. 10 Verwaltungsverordnung betreffend den Grad der Behinderung, der Klassifikation und der den behinderten Men- 
ist die von der Weltgesundheitsorganisation (WHO) herausgegebene medizinische Klassifikation der Behinderung ${ }^{25}$ zugrunde zu legen. ${ }^{26}$ Entscheidend ist der Funktionsverlust als Folge der Behinderung und deren Auswirkung auf alle Lebensbereiche. ${ }^{27}$ Eine Schwerbehinderung liegt vor, wenn ein Verlust der gesamten Körperfunktionen von mehr als 50\% medizinisch festgestellt wurde und die eigenständige Vornahme der wesentlichen Verrichtungen im Bereich der Körperpflege, Ernährung, Mobilität und Teilnahme erschwert oder der Betroffene der Fähigkeiten zur Vornahme dieser beraubt und auf Hilfe von dritter Seite angewiesen ist. ${ }^{28}$ Diese Umschreibung verleitet zu der Folgerung, dass die Eigenschaft der Schwerbehinderung neben dem Verlust der gesamten Körperfunktionen von mehr als 50\% zusätzlich das Vorliegen der Pflegebedürftigkeit zur Voraussetzung hat. Diese Folgerung ist jedoch nicht richtig, weil das Behindertengesetz selbst zwischen Schwerbehinderten und pflegebedürftigen Behinderten unterscheidet, daher: Die Eigenschaft der Schwerbehinderung erfordert das Vorliegen eines Grades der Behinderung von mindestens $50 \%$. Pflegebedürftigkeit ist zu bejahen, wenn neben der medizinisch festgestellten Schwerbehinderung der Betroffene die Fähigkeit zur Vornahme der gewöhnlichen und wiederkehrenden Verrichtungen im alltäglichen Leben in erheblichem Maße verloren hat und daher auf Hilfe sowie Pflege Dritter angewiesen ist. ${ }^{29}$

\subsection{Der Behindertenbegriff im Arbeits-, Beamten- und Steuerrecht}

Im Bereich des Arbeits- und Beamtenrechts sind mehrere Regelungen vorhanden, die die Eingliederung behinderter Menschen in den Arbeitsmarkt fördern sollen. Auch enthält das Steuerrecht Vergünstigungen, die an die Behinderteneigenschaft anknüpfen. Der Tatbestand der Behinderung wird in den einschlägigen rechtlichen Vorschriften jeweils näher beschrieben.

Das Arbeitsrecht verlangt den Verlust der Arbeitskraft (çalışma gücü). Demzufolge ist eine Behinderung eines Menschen i.S.d. Arbeitsrechts zu bejahen, wenn

„dessen Arbeitsfähigkeit ${ }^{30}$ wegen seiner körperlichen, geistigen, seelischen, emotionalen und sozialen Beeinträchtigungen um mindestens $40 \%$ gemindert und dies durch ein Attest des Gesundheitsausschusses nachgewiesen ist " ${ }^{31}$

schen vom Gesundheitsausschuss auszustellenden Atteste). In diesem Zusammenhang ist zu erwähnen, dass das türkische Rechtssystem keine gesonderte Sozialgerichtsbarkeit kennt.

25 International Classification of Functioning, Disability and Health (ICF). Die deutsche Version kann entnommen werden unter: http://www.dimdi.de.

26 Siehe Art.5, 6 ff. der Verwaltungsverordnung betreffend den Grad der Behinderung, die Klassifikation und die den behinderten Menschen vom Gesundheitsausschuss auszustellenden Atteste.

27 Hierzu Anlage 4 zur Verwaltungsverordnung betreffend den Grad der Behinderung, die Klassifikation und die den behinderten Menschen vom Gesundheitsausschuss auszustellenden Atteste.

28 Art. 4 Buchst. a der Verwaltungsverordnung betreffend den Grad der Behinderung, die Klassifikation und die den behinderten Menschen vom Gesundheitsausschuss auszustellenden Atteste.

29 Die Schwerbehinderteneigenschaft erfordert auch nach deutschem Recht die Feststellung eines Grades der Behinderung von 50 oder mehr, § 2 Abs. 2 SGB IX.

30 Die Begriffe, Arbeitskraft' und ,Arbeitsfähigkeit' werden synonym verwendet. 
Der beamtenrechtliche Behindertenbegriff knüpft an den Behindertenbegriff des Behindertengesetzes an: Nicht der Verlust der Arbeitsfähigkeit in einem bestimmten Grad als Folge der Gesundheitsstörungen, sondern der Verlust von Körperfunktionen von mindestens $40 \%$ wird verlangt. ${ }^{32}$ Die Beeinträchtigung der Arbeitskraft um mindestens $40 \%$ ist zudem Voraussetzung für Vergünstigungen bei der Einkommensteuererhebung: Bei Erwerbstätigen, deren Arbeitsfähigkeit um mindestens 80\% gemindert ist, liegt eine Behinderung ersten Grades, bei Minderung der Arbeitsfähigkeit um mindestens $60 \%$ dagegen liegt eine Behinderung zweiten Grades, und schließlich bei Erwerbstätigen, deren Arbeitsfähigkeit um mindestens $40 \%$ gemindert ist, liegt eine Behinderung dritten Grades vor. ${ }^{33} \mathrm{Ab}$ dem 01.01 .2008 beträgt die monatliche Steuerermäßigung für Behinderte ersten Grades 600 YTL, für Behinderte zweiten Grades 300 YTL und für Behinderte dritten Grades 150 YTL. $^{34}$

\subsection{Der Behindertenbegriff im Sozialversicherungsrecht}

Das Sozialversicherungssystem in der Türkei befindet sich im Umbruch. Mit dem Inkrafttreten des Gesetzes Nr. 5510 zum 01.10.2008 sind die bisher bestehenden fünf Sozialversicherungsgesetze, deren Regelungen auf jeweils fünf Versichertengruppen - nämlich abhängig Beschäftigte, abhängig Beschäftigte in der Landwirtschaft, selbständig Beschäftigte und selbständig Beschäftigte in der Landwirtschaft sowie die Beamten und sonstigen Beschäftigten des öffentlichen Dienstes - Anwendung finden ${ }^{35}$ in einem Gesetzeswerk mit dem Ziel zusammengefasst, einheitliche Regelungen für alle Versichertengruppen zu schaffen. Da die bisherigen Sozialversicherungsgesetze als auch das neue Gesetz Nr. 5510 Leistungen für Behinderte vorsehen, ist der Frage nachzugehen, welcher Behindertenbegriff den einschlägigen Regelungen jeweils zur Grunde gelegt wird.

31 „Özürlü: Bedensel, zihinsel, ruhsal, duygusal ve sosyal yeteneklerindeki engelleri nedeniyle çalışma gücünün en az yüzde 40 indan yoksun olduğu sağlık kurulu raporuyla belgelenenleri,“ Art. 3 Verwaltungsverordnung betreffend die Beschäftigung von Behinderten, Strafentlassenen und Terrorgeschädigten (Özürlü, Eski Hükümlü ve Terör Mağduru İstihdamı Hakkında Yönetmelik, RG vom 24.03.2004, Nr. 25412).

32 Art. 3 Buchst. c Verwaltungsverordnung hinsichtlich der Bedingungen für die Aufnahme von Behinderten in den Beamtendienst und der vorzunehmenden Prüfungen (Özürlülerin Devlet Memurluğuna Alınma Şartları ile Yapılacak Yarışma Sınavları hakkında Yönetmelik, RG vom 16.09.2004, Nr. 25585).

Die Vorgängerregelungen verlangten den dauerhaften Verlust der Arbeitsfähigkeit um mindestens 40\% (Art. 2, 3 Verwaltungsverordnung betreffend die Aufnahme von Behinderten in den Beamtendienst und die Dienste, für die sie eingesetzt werden / Sakatların Devlet Memurluğuna Alınma Şartları İle Hangi İşlerde Çalıştırılacakları Hakkında Yönetmelik, RG vom 27.07.1983, Nr. 18111).

33 Art. 31 Einkommenssteuergesetz, Gesetz Nr. 193 (Gelir Vergisi Kanunu).

34 Nach deutschem Steuerrecht erhalten Menschen mit Behinderung ab einem Behindertengrad von mindestens 25\% zur Minderung der Steuerbelastung einen jährlichen Pauschbetrag, dessen Höhe vom festgestellten Grad der Behinderung abhängt (§ 32b EStG).

Ausführlich hierzu Aşkın, Das Sozialversicherungssystem der Türkei, 2004. 
Zunächst ist festzuhalten, dass die Sozialversicherungsgesetze für selbständig Erwerbstätige, Gesetz Nr. 1479 vom 02.09.1971 und Gesetz Nr. 2926 vom $17.10 .1983,{ }^{36}$ und das Sozialversicherungsgesetz für abhängig Beschäftigte in der Landwirtschaft, Gesetz Nr. 2925 vom 17.10.1983, ${ }^{37}$ keine Versicherungsleistungen bei Vorliegen einer Behinderung vorsehen. Dagegen können abhängig Beschäftigte und Beamte sowie sonstige Beschäftigte des öffentlichen Dienstes bei Vorliegen einer Behinderung Altersrente unter erleichterten Bedingungen beziehen.

Das Sozialversicherungsgesetz, Gesetz Nr. 506 vom $17.10 .1964,{ }^{38}$ mit dessen Regelungen die soziale Absicherung von abhängig Erwerbstätigen gegen die sozialen Risiken Arbeitsunfall und Berufskrankheit, Krankheit, Mutterschaft, Invalidität sowie im Alter und im Todesfall gewährleistet wird, knüpft den erleichterten Bezug einer Altersrente für abhängig beschäftigte Menschen mit Behinderung an die Bewilligung von Steuervergünstigungen gemäß dem Einkommenssteuergesetz: Die Bezugsvoraussetzungen sind abhängig von der für die Steuervergünstigung relevanten Einordnung in eine der vorgesehenen Behindertengrade. Eine nähere Definition der Behinderung enthält das Sozialversicherungsgesetz dagegen nicht. Auch das Pensionsversicherungsgesetz, Gesetz Nr. 5434 vom 08.06.1949, ${ }^{39}$ dessen Regelungen zur sozialen Absicherung von Beamten und sonstigen Beschäftigten des öffentlichen Dienstes dienen, sieht die Möglichkeit für Versicherte mit Behinderung vor, bei Erfüllung bestimmter versicherungsrechtlicher Voraussetzungen vorzeitig Altersrente zu beziehen. Erforderlich ist, dass eine Behinderung von mindestens $40 \%$ festgestellt wurde. Es wird auf den Behindertenbegriff des Behindertenrechts Bezug genommen. Das neue Sozialversicherungs- und allgemeine Krankenversicherungsgesetz, Gesetz Nr. 5510, verfügt über keine nähere Umschreibung einer Behinderung. Ebenso wird die Verknüpfung mit dem Steuerrecht aufgegeben. Je nachdem, ob eine Behinderung zum Verlust der Arbeitsfähigkeit von mehr als 60\%, von $59 \%$ - 50\% oder von $49 \%$ - 40\% geführt hat, werden die Voraussetzungen für den erleichterten Bezug einer Altersrente neu geregelt.

\subsection{Der Behindertenbegriff im Bereich der Sozialhilfe und sozialen Fürsorge}

Das Behindertengesetz schreibt in seinem Art. 6 vor, dass Menschen mit Behinderung Pflege- und Rehabilitationsleistungen zu erbringen sind, damit diese ein gesundes, zufriedenes und selbständiges Leben führen können. In diesem Zusammenhang ist zu erwähnen, dass das türkische Sozialversicherungssystem keine Pflegeversiche20.10.1983, Nr. 18197.

37 Tarım İşçileri Sosyal Sigortalar Kanunu, RG vom 20.10.1983, Nr. 18197.

38 Sosyal Sigortalar Kanunu, RG vom 29., 30., 31.07.1964, Nr. 11766-11779.

T. C. Emekli Sandığı Kanunu, RG vom 17.06.1949, Nr. 7235. 
rung kennt. ${ }^{40}$ Die Erbringung sowie Finanzierung von Pflegeleistungen fällt vorwiegend in die Zuständigkeit der Anstalt für soziale Dienste und Kinderfürsorge. In der Folge ist der Bereich der Pflege im System der Sozialhilfe und der sozialen Fürsorge/Dienste ${ }^{41}$ verankert, d.h. zur Erbringung von Pflegediensten an pflegebedürftige Menschen mit Behinderung ist die Bedürftigkeit zusätzliche Voraussetzung. ${ }^{42} \mathrm{Im}$ Jahr 2006 wurden für insgesamt 4381 Behinderte in insgesamt 66 (= 41 stationäre und 25 ambulante) Pflegeeinrichtungen Pflegedienste erbracht. ${ }^{43}$ Die Definition des Behindertengesetzes für pflegebedürftige Menschen mit Behinderung ist hierbei übernommen worden, während der Begriff der Behinderung eine Konkretisierung dahingehend erhalten hat, dass die Funktionsbeeinträchtigung Folge einer Krankheit oder eines Unfallereignisses sein muss. ${ }^{44}$ Die Feststellung der Pflegebedürftigkeit liegt hierbei in dem Zuständigkeitsbereich der Regierungs-/Provinzbezirkverwaltungen: Auf Grundlage eines Untersuchungsberichts (sosyal inceleme raporu) des sachbearbeitenden Sozialarbeiters erstellt der eingesetzte Ausschuss zur Beurteilung der Pflegedienste (Bakım Hizmeti Değerlendirme Heyeti) ein Pflegegutachten (bakım raporu). ${ }^{45}$

Menschen mit Behinderung erhalten aus dem System der Sozialhilfe und sozialen Fürsorge zudem eine monatliche Rente gemäß den Vorschriften des Gesetzes zur Gewährung einer Rente an bedürftige Bürger, die das 65. Lebensjahr vollendet ha-

40 Zur Einführung einer Pflegeversicherung für Menschen mit Behinderung ausführlich Kahramanoğlu, Özürlüler için bakım sigortası önerisi (Vorschlag für eine Pflegeversicherung für Menschen mit Behinderung), zu entnehmen unter: http://www.sosyalhizmetuzmani. org/bakimsigortasi.htm; Seyyar, Dünya' da ve Türkiye' de Yaşlılığa Bağlı Bakım Sorununa Karşı Güvence Sistemleri (Systeme zur Absicherung des altersbedingten Pflegerisikos in der Welt und der Türkei), Öz-Veri, 2005, C. 2 S.1, zu entnehmen unter: http://www.ozida.gov.tr/ozveri/ ov3/ov3yaslibakim.htm.

41 Den Begriff der sozialen Dienste (sosyal hizmetler) definiert das Gesetz über die Anstalt für soziale Dienste und Kinderfürsorge als Gesamtheit von Leistungen, die mit dem Ziel erfolgen, materielle, immaterielle und soziale Entbehrungen zu beheben und Bedürfnisse zu decken, die Entstehung sozialer Probleme verhindern und lösen zu helfen sowie den Lebensstandard zu verbessern (Art. 3).

42 Anzumerken ist, dass die in den Gesetzen zur Sozialhilfe und sozialen Fürsorge normierten Leistungen nicht als Rechte des Einzelnen konzipiert sind. Darin liegt ein Verstoß gegen Art. 13, 14 der Europäischen Sozialcharta sowie ihrer revidierten Fassung.

Näheres hinsichtlich der Pflegedienste wird in den Verwaltungsverordnungen betreffend die öffentlichen und privaten Pflegeeinrichtungen sowie der Verwaltungsverordnung zur Feststellung der pflegebedürftigen Behinderten und der Ausgestaltung der Pflegedienste geregelt (Bakıma Muhtaç Özürlülere Yönelik Resmî Kurum ve Kuruluşlar Bakım Merkezleri Yönetmeliği, Bakıma Muhtaç Özürlülere Yönelik Özel Bakım Merkezleri Yönetmeliği, Bakıma Muhtaç Özürlülerin Tespiti ve Bakım Hizmeti Esaslarının Belirlenmesine İlişkin Yönetmelik; RG vom 30.07.2006, Nr. 26444).

43 Weitere 3729 Menschen mit Behinderung warteten auf die Gewährung von stationären Pflegeleistungen und Rehabilitationsleistungen. Vgl. Tätigkeitsbericht der Anstalt für soziale Dienste und Kinderfürsorge für 2006, S. 41 ff., zu entnehmen unter: http://www.shcek.gov.tr/duyuru/apk/2006 Faaliyet_Raporu/2006FaaliyetRaporu.pdf.

44 Art. 1, $\overline{3}$ Buchst. c, d Gesetz über die Anstalt für soziale Dienste und Kinderfürsorge, Gesetz Nr. 2828 (Sosyal Hizmetler ve Çocuk Esirgeme Kurumu Kanunu), Art. 28 Behindertengesetz.

45 Gegen das Pflegegutachten kann innerhalb von 30 Tagen nach Bekanntgabe Widerspruch eingelegt werden. Vgl. Art. 7 Verwaltungsverordnung betreffend die Feststellung der pflegebedürftigen Behinderten und der Ausgestaltung der Pflegedienste n. F. 
ben, Gesetz Nr. 2022. ${ }^{46}$ Als Behinderte im Sinne des Gesetzes sind Menschen anzusehen, die ihre Arbeitskraft um mindestens $40 \%$ bis $69 \%$ verloren haben. Menschen, die ihre Arbeitsfähigkeit dagegen um mehr als 70\% verloren haben, sind als Behinderte anzusehen, die ihr Leben ohne Hilfe von anderen nicht fortführen können. ${ }^{47}$

Schließlich leistet die Generaldirektion für (religiöse) Stiftungen ${ }^{48}$ an bedürftige Menschen mit Behinderung eine monatliche Rente, wenn diese infolge ihrer körperlichen, geistigen und seelischen Verfassung einen Funktionsverlust von mindestens $40 \%$ haben. $^{49}$

\subsection{Würdigung}

Es lässt sich festhalten, dass in den verschiedenen Vorschriften, die Rechte der Menschen mit Behinderung normieren, der Begriff der Behinderung gesondert umschrieben wird, indem für die Anwendung der jeweiligen Vorschriften das Vorliegen eines bestimmten Behindertengrades verlangt wird. Es besteht jedoch kein Zweifel daran, dass in jedem Rechtsgebiet der funktionale Behinderungsbegriff des Behindertengesetzes Geltung hat, zumal der Grad der Behinderung durch Vorlage der Atteste des jeweiligen, für die Feststellung der Behinderung zuständigen Gesundheitsausschusses, die auf Grundlage der gegenständlichen Verwaltungsverordnung zu erstellen sind, nachzuweisen ist.

Das Erfordernis in einigen Vorschriften dahingehend, dass die vorhandene physische und/oder psychische Funktionsbeeinträchtigung zugleich den Verlust der Arbeitsfähigkeit zu einem bestimmten Grad zur Folge haben muss, trägt in diesem Zusammenhang zur Verwirrung bei. Insbesondere im Bereich des Sozialversicherungsrechts ist eine Abgrenzung zur Erwerbsminderung/Invalidität erforderlich. Denn die bisher in Kraft befindlichen Sozialversicherungsgesetze als auch das neue Sozialversicherungs- und allgemeine Krankenversicherungsgesetz, welches zum 01.10.2008 in Kraft getreten ist, sehen bei Minderung der Arbeitskraft ab einem bestimmten Grad und zugleich Erfüllung bestimmter versicherungsrechtlicher Voraussetzungen die Gewährung einer Invaliditätsrente vor. Die Minderung der Erwerbs-/Arbeitsfähigkeit jedoch ist nicht gleichzusetzen mit dem Vorliegen einer Behinderung und umgekehrt. Vielmehr ist der Begriff der Behinderung weit auszu-

Zusatzartikel 1 Gesetz Nr. 2022 (65 Yaşını Doldurmuş Muhtaç, Güçsüz ve Kimsesiz Türk Vatandaşlarına Aylık Bağlanması Hakkında Kanun).

7 Art. 10 Verwaltungsverordnung zur Durchführung des Gesetzes Nr. 2022 (65 Yaşını Doldurmuş Muhtaç, Güçsüz ve Kimsesiz Türk Vatandaşları ile Özürlü ve Muhtaç Türk Vatandaşlarına Aylık Bağlanması Hakkında Yönetmelik).

48 Die Generaldirektion wurde mit der Rechtsverordnung mit Gesetzeskraft Nr. 227 vom 08.06.1984 gegründet (Vakıflar Genel Müdürlüğü' nün teşkilat ve görevleri hakkında kanun hükmünde kararname, RG vom 18.04.1984, Nr. 18435). Die religiösen Stiftungen nehmen traditionell eine beson-

dere Rolle auf dem Gebiet der Sozialhilfe und sozialen Dienste ein.
Art. 4, 5 Verwaltungsverordnung betreffend die von Stiftungen zu zahlenden Bedürftigkeitsrenten und $\mathrm{zu}$ betreibenden Armenküchen (Muhtaç Aylığı ve Vakıf İmaret Yönetmeliği, RG vom 28.12.1989, Nr. 20386). 
legen.$^{50}$ Die Beeinträchtigung der Funktionsfähigkeit und die damit einhergehende Beeinträchtigung der Teilhabe am gesellschaftlichen Leben sowie die bedingte Hilfebedürftigkeit, welche für die Feststellung des Grades der Behinderung von Bedeutung ist, erfolgt, wie bereits erwähnt, gemäß den Regelungen der gegenständlichen Durchführungsverordnung unter Zugrundelegung der ICF durch die hierfür eingesetzten und zuständigen Gesundheitsausschüsse. Zusammenfassend dargestellt werden bei der Feststellung des Funktionsverlustes nicht nur die physischen und psychischen Beeinträchtigungen beurteilt, sondern zudem die Beeinträchtigung der Teilhabe und Aktivitäten, also die Leistung und Leistungsfähigkeit in verschiedenen Lebensbereichen, unter Berücksichtigung von Umwelt- und personenbezogenen Faktoren. Bezugspunkt für den Verlust der Arbeitsfähigkeit ist dagegen nur das Leistungsvermögen des Betroffenen auf dem allgemeinen Arbeitsmarkt. ${ }^{51}$ Die Teilhabe am Erwerbsleben ist bereits Teil der Prüfungen im Rahmen der Beurteilung des Behindertengrades. Auf den Verlust der Arbeitskraft gesondert abzustellen, ist als Anknüpfungspunkt der den Behinderten gewährten Rechte nicht erforderlich. Eine Bezugnahme auf den Behindertengrad als solches ist ausreichend.

\section{Die Stellung behinderter Menschen im Arbeitsleben}

Art. 30 des Arbeitsgesetzes, Gesetz Nr. 4857, verpflichtet private und öffentliche Arbeitgeber in Betrieben, in denen 50 und mehr Arbeitnehmer beschäftigt werden, Behinderte, Verurteilte und Terroropfer entsprechend ihrer beruflichen, körperlichen und seelischen Konditionen auf geeigneten Arbeitsplätzen ${ }^{52}$ zu beschäftigen. ${ }^{53}$ Die diesbezügliche Beschäftigungsquote wird jährlich durch den Ministerrat bestimmt. ${ }^{54}$ Das Gesetz normiert eine Gesamtquote von $6 \%$ der Gesamtzahl der beschäftigten Arbeitnehmer, wobei der Prozentsatz der zu beschäftigenden Behinderten mindes-

50 Canıklığlu, Kamu-İş, C. 6, S. 4/2002, S. 251.

51 Jörg, in: Kreikebohm (Hrsg.), SGB VI, 2. Aufl., Art. 43 Rn. 5.

52 Die Verwaltungsverordnung betreffend die Beschäftigung von Behinderten, Strafentlassenen und Terrorgeschädigten enthält in ihrem Anhang 1 eine Tabelle der Berufe, die von Behinderten je nach Art der Behinderung ausgeübt werden können (Özürlülerin Çalıştırılabilecekleri İşler). Grundsätzlich müssen Arbeitgeber innerhalb der betrieblichen Möglichkeiten die Arbeitplätze, auf denen Arbeitnehmer mit Behinderung beschäftigt werden, behindertengerecht gestalten (Art. 14).

53 Hierzu ausführlich Tezel, S. 106 ff.; Koç, E-Yaklaşım, Mai 2005; Kılıç, E-Yaklaşım, Juni 2006.

54 Ausgehend von der Gesamtzahl der beschäftigten Arbeitnehmer sind ab dem 01.01.2006 4\% (öffentliche Arbeitgeber) bzw. 3\% (private Arbeitgeber) der Arbeitsplätze mit Behinderten zu besetzen (Ministerratsbeschluss 2005/9077, RG vom 08.07.2005, Nr. 25869). Die festgesetzten Quoten haben weiterhin Geltung im Zeitraum vom 01.01.2008-31.12.2008 (Ministerratsbeschluss 2008/13195, RG vom 13.02.2008, Nr. 26786).

Derzeit liegt ein Gesetzesentwurf zur Änderung des Arbeitsgesetzes vor (İş kanunu ve bazı Kanunlarda degişiklik yapılması hakkında Kanun Tasarısı). Demzufolge soll die Verpflichtung von Arbeitgebern des privaten Sektors, $3 \%$ der vorhandenen Arbeitsplätze mit behinderten Arbeitnehmern zu besetzen, gesetzlich festgeschrieben werden (Art. 2). 
tens die Hälfte betragen muss. ${ }^{55}$ Arbeitgeber stellen die von ihnen zu beschäftigenden Arbeitnehmer mit Behinderung in Zusammenarbeit mit der Anstalt für Arbeit ${ }^{56}$ ein. ${ }^{57}$ Als Anreiz zur Förderung der Eingliederung von Menschen mit Behinderung in den Arbeitsmarkt wurde die Ermäßigung der abzuführenden Sozialversicherungsbeiträge geschaffen: Arbeitgeber, die über die festgesetzte Quote hinaus oder ohne eine gesetzliche Verpflichtung Behinderte einstellen oder Behinderte mit der Minderung der Arbeitsfähigkeit von mehr als $80 \%$ beschäftigen, müssen für jeden der auf diese Weise beschäftigten behinderten Arbeitnehmer lediglich 50\% der auf den Arbeitgeber anfallenden Sozialversicherungsbeiträge entrichten. Der restliche Teil wird von der Staatskasse übernommen. ${ }^{58}$ Verstöße gegen die Beschäftigungspflicht sind mit der Verhängung einer Geldbuße zu ahnden.

Mit Gesetz Nr. 5763 vom 15.05.2008 ${ }^{60}$ sind die Begünstigungen bei Beschäftigung von Menschen mit Behinderung neu geregelt worden. Seit dem 01.07.2008 trägt der Staat die Sozialversicherungsbeiträge, die der Arbeitgeber für jeden der von ihm zu beschäftigenden Arbeitnehmer mit Behinderung abführen muss. ${ }^{61}$ In Fällen, in denen Arbeitgeber Arbeitnehmer mit Behinderung beschäftigen, ohne hierzu gesetzlich verpflichtet zu sein, oder die normierte Pflichtquote von 3\% überschreiten übernimmt der Staat die Arbeitgeberbeiträge zur Sozialversicherung, die für diese Arbeitnehmer abzuführen sind, i.H.v. 50\% (vgl. Art. 30 n.F.). ${ }^{62}$

Arbeitsgesetz Nr. 1475 vom 25.08.1971 (RG vom 01.09.1971), welches nach dem Inkrafttreten des Gesetzes Nr. 4857 (RG vom 10.06.2003, Nr. 25134) mit Ausnahme seines Art. 14 aufgehoben wurde, sah bereits eine Beschäftigungspflicht für Menschen mit Behinderung von 3\% vor (Art. 25).

56 Die Anstalt für Arbeit hat in diesem Zusammenhang u. a. die Aufgabe, neue Beschäftigungsfelder für Menschen mit Behinderung zu erforschen und die Eingliederung von Behinderten in den Arbeitsmarkt durch Ausbildungs- und Rehabilitationsmaßnahmen zu erleichtern (Art. 12, 13). Im Jahr 2006 betrugen die diesbezüglichen Ausgaben 3.534.754,59 YTL. Vgl. Anstalt für Arbeit, Tätigkeitsbericht 2006, S. 97, zu entnehmen unter: http://www.iskur.gov.tr/mydocu/2006_y\%FDl\% FD_faaliyet_raporu.pdf.

57 Private Arbeitgeber, die ohne die Mitwirkung der Anstalt für Arbeit behinderte Arbeitnehmer eingestellt haben, müssen dies innerhalb von einem Monat bei der Anstalt für Arbeit anzeigen (Art. 5 Abs. 2). Im Jahr 2006 waren insgesamt 28.236 Menschen mit Behinderung auf Arbeitssuche. Hiervon wurden insgesamt 23.781 in den Arbeitsmarkt eingegliedert. Zudem wurden 94 Berufsbildungs- und Rehabilitationsprogramme durchgeführt, an denen insgesamt 3.047 Menschen mit Behinderung teilnahmen. Vgl. Anstalt für Arbeit, Tätigkeitsbericht 2006, S. 24, 30.

58 Art. 30 Abs. 10 Arbeitsgesetz; Art. 25 Verwaltungsverordnung betreffend die Beschäftigung von Behinderten, Strafentlassenen und Terrorgeschädigten.

59 Art. 101 Arbeitsgesetz; Art. 27 Verwaltungsverordnung betreffend die Beschäftigung von Behinderten, Strafentlassenen und Terrorgeschädigten.

Für das Jahr 2008 beträgt die für jeden nichtbeschäftigten Behinderten zu zahlende Geldbuße 1.357 YTL. Die erhobenen Geldbußen fließen in den Etat der Anstalt für Arbeit ein. Im Jahr 2006 wurden seitens der Arbeitgeber Geldbußen in Höhe von insgesamt 3.910.934,68 YTL gezahlt. Vgl. Anstalt für Arbeit, Tätigkeitsbericht 2006, S. 86.

60 İş Kanunu ve bazı Kanunlarda değişiklik yapılması hakkında Kanun, RG vom 26.5.2008, Nr. 26887. Hierzu Körtek, ZESAR 11-12/08, 483 ff.

61 Berechnungsgrundlage ist die unterste Grenze des bei der Bemessung der Sozialversicherungsbeiträge zugrunde zu legenden relevanten Einkommens.

62 Näheres regelt Runderlass 2008/77 vom 19.8.2008, Nr. B.13.2.SSK.5.01.08.00/ 73-0310- 575886. 
Zudem sind Menschen mit Behinderung in den Beamtendienst zu übernehmen. Das Beamtengesetz legt eine Quote von 3\% des gesamten Personals fest (vgl. Art. 53). ${ }^{63}$

Menschen mit Behinderung dürfen im Arbeitsleben - sowohl bei der Einstellung als auch während des Arbeitsverhältnisses - nicht wegen ihrer Behinderung diskriminiert werden (Art. 14 Abs. 1, 2 Behindertengesetz) ${ }^{64}$ Der Charakter dieses behindertenrechtlichen Diskriminierungsverbots als subjektives Recht des Einzelnen ist fraglich. So sind etwaige Rechtsfolgen, die aus dem Verstoß gegen das Diskriminierungsverbot resultieren, nicht vorgesehen. Die gleichen Erwägungen gelten für das allgemeine Diskriminierungsverbot in Art. 4 Buchst. a: Diese Vorschrift verpflichtet den Staat, soziale Politiken zu entwickeln, um die Ausbeutung der Menschen mit Behinderung zu verhindern. Diskriminierungen sind verboten, vielmehr ist die Bekämpfung von Diskriminierungen die Grundlage der Behindertenpolitik. Anders dagegen das Diskriminierungsverbot in Art. 5 des Arbeitsgesetzes: Im Rahmen eines bestehenden Arbeitsverhältnisses darf ein Arbeitnehmer wegen der Sprache, Rasse, Geschlecht, politischen Auffassung, philosophischen Glaubens, Religion und Konfession und ähnlicher Gründe nicht diskriminiert werden. Zwar wird der Zustand der Behinderung nicht gesondert aufgeführt. Jedoch kann eine Behinderung unter dem Tatbestand des ähnlichen Grundes subsumiert werden. Bei nachgewiesenem Verstoß gegen das arbeitsrechtliche Diskriminierungsverbot kann ein Arbeitnehmer - neben der als Folge der Diskriminierung vorenthaltenen Behandlung - Schadensersatz bis zu vier Monatsgehältern beanspruchen. Gleichzeitig wird gegen den Arbeitgeber eine Geldbuße verhängt (Art. 5, 99 a Arbeitsgesetz). ${ }^{65}$ Besonderen Kündigungsschutz für Arbeitnehmer mit Behinderung kennt das türkische Recht im Gegensatz zum deutschen Recht nicht ${ }^{66}$, sodass dem Diskriminierungsverbot im Rahmen der Beendigung von Arbeitsverhältnissen ein besonderes Gewicht zukommt. ${ }^{67}$

63 Zur Durchführung der Beschäftigungsverpflichtung wurde die Verwaltungsverordnung betreffend die Bedingungen für die Aufnahme von Behinderten in den Beamtendienst und der vorzunehmenden Prüfungen erlassen (Özürlülerin Devlet Memurluğuna Alınma Şartları İle Yapılacak Yarışma Sinavları Hakkında Yönetmelik), in welcher das Verfahren und die Einstellungsvoraussetzungen eine Regelung erfahren haben. Unter Berücksichtigung der Tatsache, dass die Kontingente der in den Beamtendienst zu übernehmenden Behinderten nicht ausgeschöpft werden, wurde die Festlegung einer oberen Altersgrenze bei der Einstellung mit Runderlass des Ministerpräsidenten, Nr. 2006/15, aufgehoben (RG vom 24.06.2006, Nr. 26208).

64 Das allgemeine Gleichbehandlungsgebot ist in Art. 10 der türkischen Verfassung (TV) niedergelegt.

65 Die Höhe der zu verhängenden Geldbuße ist für das Jahr 2008 auf 88 YTL für jeden diskriminierten Arbeitnehmer festgesetzt.

66 Siehe $\S \S 85$ ff. SGB IX, die einen besonderen Kündigungsschutz für Schwerbehinderte und diesen gleichgestellte Menschen ( $\S 2$ Abs. 2, 3, 68 SGB IX) vorsehen.

67 Anzumerken ist, dass ein Kündigungsschutzrecht für Arbeitnehmer in der Türkei im Jahre 2003 geschaffen wurde. Hierzu Yarayan, ZIAS 2/2005, S. 194 ff. m. w. N. 


\section{Behinderte Menschen im System der sozialen Sicherheit}

\subsection{Bezug von Altersrente unter erleichterten Bedingungen ${ }^{68}$}

Die soziale Absicherung von Erwerbstätigen gegen bestehende soziale Risiken wird in der Türkei, wie bereits ausgeführt, durch verschiedene Sozialversicherungsgesetze geregelt. Die Abgrenzung erfolgt jeweils durch die Art der ausgeübten Erwerbstätigkeit. Die Intention des türkischen Gesetzgebers ist es, mit dem neuen Sozialversicherungs- und allgemeine Krankenversicherungsgesetz, Gesetz Nr. 5510, ein einheitliches System der sozialen Sicherheit für alle Versichertengruppen zu schaffen. Das Gesetz Nr. 5510 ist zum 01.10.2008 in Kraft getreten.

Der Bezug von Invaliditätsrente gemäß den Vorschriften des Sozialversicherungsgesetzes Nr. 506, ${ }^{69} \mathrm{zu}$ dessen Versichertenkreis abhängig beschäftigte Arbeitnehmer gehören, ist ausgeschlossen, wenn der Versicherte zu Beginn der erstmaligen versicherungspflichtigen Beschäftigung auf Grund gesundheitlicher Beeinträchtigungen bereits als erwerbsgemindert einzustufen war (Art. 53 B Gesetz Nr. 506). ${ }^{70}$ Invalidität in diesem Sinne liegt vor, wenn der Versicherte seine Arbeitskraft um mindestens $2 / 3$ verloren hat. Ist der Verlust der Arbeitskraft die Folge eines Arbeitsunfalls oder einer Berufskrankheit, dann genügt ein Verlustgrad von $60 \%$ für das Vorliegen des Versicherungsfalles der Invalidität (Art. 53 A, 1 Gesetz Nr. 506). Doch können Behinderte, deren gesundheitliche Funktionseinschränkungen bereits im Zeitpunkt der erstmaligen Aufnahme einer Erwerbstätigkeit ihre Invalidität im Sinne des Sozialversicherungsrechts begründen, unter erleichterten Bedingungen Altersrente beziehen. Neben der festgestellten Invalidität ${ }^{71}$ muss der Versicherte eine Versicherungszeit von 15 Jahren aufweisen und für 3600 Tage Beiträge zur Invaliditäts-, Alters- und Hinterbliebenenversicherung entrichtet haben (Art. $60 \mathrm{C}$, a Gesetz Nr. 506). Versicherte mit Behinderung, die zugleich Anspruch auf Steuerermäßigung nach den Vorschriften des Einkommensteuergesetzes haben, konnten unter den gleichen versicherungsrechtlichen Voraussetzungen vorzeitig Altersrente beziehen (Art. 60 C, b Gesetz Nr. 506 a. F.). Mit Gesetz Nr. 4958 vom 29.07.200372 wurde der Bezug von Altersrente für Menschen mit Behinderung, die einen Anspruch auf Steuerermäßigung haben, neu geregelt: Versicherte, die als Behinderte ersten Grades einzustufen sind, müssen eine Versicherungszeit von 15 Jahren aufweisen und mindestens 3600 Tage Beiträge zur Invaliditäts-, Alters- und Hinterblie-

Ausführlich Canıklıoğlu, a. a. O.; Başterzi, S. 157 ff., S. 179 ff.

Hierzu Hänlein, Deutsche Rentenversicherung 6-7-8/98, S. 556 ff.

Nach deutschem Recht ist der Bezug einer vollen Erwerbsminderungsrente u. a. abhängig von der Erfüllung der allgemeinen Wartezeit von fünf Jahren (§§ 43 Abs. 1, 50 Abs. 1 SGB VI). Tritt dagegen die volle Erwerbsminderung vor Ablauf der allgemeinen Wartezeit ein, muss der Versicherte für den Bezug der vollen Erwerbsminderungsrente eine Wartezeit von 20 Jahren erfüllen $(\S 43$ Abs. 6 SGB VI). Zur vorzeitigen Wartezeiterfüllung siehe $\S \S 53,245$ SGB VI.

1 Die Minderung der Arbeitskraft wird anhand der Regelungen der Rechtsverordnung über Gesundheitsverfahren in der Sozialversicherung beurteilt (Sosyal Sigorta Sağlık İşlemleri Tüzüğü).

RG vom 06.08.2003, Nr. 25191. 
benenversicherung entrichtet haben. Versicherte mit einer Behinderung zweiten Grades müssen dagegen 18 Jahre versichert gewesen sein und mindestens für 4000 Tage Beiträge entrichtet haben. Schließlich haben Versicherte mit einer Behinderung dritten Grades Anspruch auf Altersrente, wenn das Vorliegen einer Versicherungszeit von 20 Jahren und Beitragstagen von 4400 bejaht werden kann (Art. $60 \mathrm{C}$, b Gesetz Nr. 506). ${ }^{73}$

Menschen mit Behinderung, die als Beamte in den Versichertenkreis des Pensionsversicherungsgesetzes Nr. 5434 fallen, können nach einer Dienstzeit von 15 Jahren Altersrente beziehen. Entsprechend der Regelungen des Gesetzes Nr. 506 ist das Erreichen eines bestimmten Alters nicht erforderlich. ${ }^{74}$ Voraussetzung gemäß Art. 39 Buchst. $\mathrm{j}$ ist neben dem Vorliegen eines Behindertengrades von $40 \%{ }^{75}$, dass der Versicherte in den öffentlichen Dienst als Behinderter gemäß den diesbezüglichen Regelungen übernommen wurde (Art. 53 Abs. 1, 2 Beamtengesetz). In der Folge kann der Bezug einer vorzeitigen Altersrente wegen Fehlens der rein formellen Voraussetzung der Einstellung in den Beamtendienst als Behinderter versperrt sein. Um dies zu verhindern, hat der Gesetzgeber den Zusatzübergangsartikel 22 normiert: ${ }^{76}$ Versicherte, die nicht als Behinderte auf Grundlage der diesbezüglichen Vorschriften des Beamtenrechts in den öffentlichen Dienst aufgenommen wurden, können bei Vorliegen der versicherungsrechtlichen Voraussetzung, nämlich 15 Jahre Dienstzeit und einem Behinderungsgrad von mindestens $40 \%$, vorzeitig Altersrente beziehen.

Das neue Sozialversicherungs- und allgemeine Krankenversicherungsgesetz, Gesetz Nr. 5510, hat den Bezug von vorzeitiger Altersrente für Menschen mit Behinderung neu geregelt. ${ }^{77}$ Versicherte, die keinen Anspruch auf Invaliditätsrente haben, weil sie bereits vor der erstmaligen Aufnahme einer versicherungspflichtigen Erwerbstätigkeit auf Grund einer Krankheit oder Behinderung als invalide im Sinne des Gesetzes einzustufen waren, können Altersrente beziehen, wenn sie eine Versicherungszeit von mindestens 15 Jahren aufweisen sowie für 3960 Tage Beiträge zur Invaliditäts-, Alters- und Hinterbliebenenversicherung entrichtet haben (Art. 28 Abs. 4). Als Invalide ist ein Versicherter einzustufen, der seine Arbeitskraft mindestens in Höhe von 60\% verloren hat (Art. 25 Abs. 1). Des Weiteren können Versicherte, deren Arbeitsfähigkeit zwischen 50\% und 59\% gemindert ist, vorzeitig Altersrente beziehen, wenn sie eine Versicherungszeit von 16 Jahren vorweisen und für 4320 Tage Sozialversicherungsbeiträge entrichtet haben. Schließlich können Versicherte,

73 Vgl. Übergangsartikel 87 Gesetz Nr. 506 hinsichtlich der stufenweisen Anhebung der versicherungsrechtlichen Voraussetzungen. Hierzu Demirci, E-Yaklaşım, August 2007.

74 In diesem Zusammenhang ist darauf hinzuweisen, dass mit Reformgesetz Nr. 4447 vom 25.08.1999 (RG vom 08.09.1999, Nr. 23810) das Eintrittsalter für den Bezug von Altersrente stufenweise für Frauen auf 58 und für Männer auf 60 angehoben wurde. Mit Gesetz Nr. 5510 ist eine weitere Anhebung des Bezugsalters für beide Geschlechter stufenweise auf 65 bis zum Jahr 2048 vorgesehen.

75 Der Grad der Behinderung ist gemäß den diesbezüglichen Regelungen des Behindertenrechts durch den Gesundheitsausschuss festzustellen.

76 Başterzi, S. 180.

77 Hierzu Tezel, E-Yaklaşım, Januar 2007; Yıldız, Yaklaşım Dergisi, September 2006. 
deren Arbeitsfähigkeit zwischen $40 \%$ und 49\% gemindert ist, Altersrente beziehen, wenn eine Versicherungszeit von 18 Jahren und Beitragstage von 4680 festgestellt werden können (vgl. Art. 28 Abs. 5). ${ }^{78}$

Im Vergleich mit den bisherigen Regelungen wird die neue Rechtslage die Situation behinderter Erwerbstätiger teilweise verbessern und teilweise verschlechtern. So ist die Anknüpfung an die Steuervorteile, abhängig von dem festgestellten Grad der Behinderung, aufgehoben worden. Auch ist das Vorliegen der Invalidität im Sinne des Rentenversicherungsrechts bereits bei Verlust der Arbeitskraft von mindestens $60 \%$ zu bejahen. Während Beamte mit einem Grad der Behinderung von mindestens $40 \%$ nach gegenwärtiger Rechtslage bereits nach 15 Dienstjahren vorzeitige Altersrente beanspruchen können, werden sie nach neuer Rechtslage eine Versicherungszeit von 16 bzw. 18 Jahren vorweisen müssen. Ein bestimmtes Alter für den Bezug von Altersrente auf Grund einer Behinderung wird weiterhin nicht verlangt. ${ }^{79}$

\subsection{Bezug von Leistungen der Sozialhilfe und der sozialen Fürsorge}

Das türkische Sozialversicherungssystem deckt das Risiko der Pflegebedürftigkeit nicht ab. Die Erbringung von Pflegediensten für Menschen mit Behinderung wird vorwiegend durch die Anstalt für soziale Dienste und Kinderfürsorge gewährleistet. So müssen Pflegeeinrichtungen durch die Anstalt für soziale Dienste und Kinderfürsorge zugelassen werden (Art. 7 Behindertengesetz). Da die Pflege dem Bereich der Sozialhilfe und sozialer Fürsorge zuzuordnen ist, erfordert der Bezug von Pflegediensten neben der Pflegebedürftigkeit zusätzlich die Hilfebedürftigkeit des Betroffenen. Diese ist zu bejahen, wenn das Gesamteinkommen des Anspruchstellers oder bei Zusammenleben mit seiner Familie das Gesamteinkommen jedes Familienmitglieds $2 / 3$ des gesetzlichen Nettomindestlohnes nicht überschreitet. ${ }^{80}$ Die obere Grenze für das zu bewilligende Pflegegeld für die stationäre und die häusliche Pflege zieht das zweifache gesetzliche Nettomindestgehalt. ${ }^{81}$ Voraussetzung für die

78 Vgl. die stufenweise Anhebung der versicherungsrechtlichen Voraussetzungen in Übergangsartikel 6 Abs. 7 c, d, e.

79 In diesem Zusammenhang ist darauf hinzuweisen, dass nach deutschem Rentenversicherungsrecht für den Bezug einer vorzeitigen Altersrente neben der Anerkennung als Schwerbehinderter i. S. d. $\S 2$ Abs. 2 SGB IX und der Erfüllung der Wartezeit von 35 Jahren das 63. Lebensjahr vollendet werden muss ( $\$ 37 \mathrm{SGB}$ VI). Vgl. hierzu § 236a SGB VI i. V. m. Anlage 22.

80 Das Erfordernis der Bedürftigkeit wurde mit der jüngsten Änderung der Verwaltungsverordnung betreffend die Feststellung der pflegebedürftigen Behinderten und der Ausgestaltung der Pflegedienste konkretisiert (Bakıma Muhtaç Özürlülerin Tespiti ve Bakım Hizmeti Esaslarının Belirlenmesine İlişkin Yönetmelikte Değişiklik Yapılmasına Dair Yönetmelik, RG vom 23.10.2007, Nr. 26679).

81 Vgl. Zusatzartikel 7 Gesetz über die Anstalt für soziale Dienste und Kinderfürsorge.

In dem Zeitraum 01.01.2008-30.06.2008 beträgt das gesetzliche Nettomindestgehalt für einen alleinstehenden Erwerbstätigen über 16 Jahren (unter Berücksichtigung der neuen steuerrechtlichen Regelungen) bei 481,55 YTL. Die Bedürftigkeitsgrenze liegt demzufolge bei 321 YTL. 
Inanspruchnahme von Pflegeleistungen ist, dass der betroffene pflegebedürftige Mensch mit Behinderung nicht vom System der Sozialversicherung umfasst wird.

Darüber hinaus erhalten Menschen mit Behinderung gemäß den Regelungen des Gesetzes Nr. 2022 eine monatliche Rente, sofern sie über kein Einkommen verfügen, das über der Bedürftigkeitsgrenze liegt. Die Bedürftigkeitsgrenze errechnet sich aus der Vervielfältigung des in Art. 1 wiedergegebenen Indikators mit dem Koeffizienten für die Beamtenbezüge. In dem Zeitraum ab dem 01.01.2008 liegt die Bedürftigkeitsgrenze bei 79,18 YTL. ${ }^{82}$ Dieser Betrag kann zugleich als Regelleistung angesehen werden. Behinderte Menschen über 18 Jahren, für deren Unterhalt keine unterhaltsverpflichtete Person aufkommt und deren Verlust der Arbeitskraft bei mindestens 70\% liegt, erhalten eine monatliche Rente in Höhe der dreifachen Regelleistung. Menschen mit Behinderung, deren Verlust der Arbeitskraft zwischen 40\% und $69 \%$ liegt, erhalten eine monatliche Rente in Höhe der zweifachen Regelleistung. Schließlich erhalten Bedürftige, die für die Pflege von behinderten Angehörigen unter 18 Jahren sorgen, eine monatliche Rente in Höhe der zweifachen Regelleistung (vgl. Zusatzartikel 1). Die gesundheitliche Versorgung von Empfängern von Sozialleistungen gemäß den Regelungen des Gesetzes Nr. 2022 wird zudem über die sog. Grüne Karte, die nach den Vorschriften des Gesetzes Nr. 3816 erteilt wird ${ }^{83}$, sichergestellt (Art. 7). Zuständig für die Auszahlung der monatlichen Sozialhilfeleistungen war die Pensionsversicherungskasse. Im Rahmen der Reformen des Systems der sozialen Sicherheit ist die Pensionskasse, die zugleich für die Anwendung und Durchsetzung der Regelungen des Gesetzes Nr. 5434 zuständig war, auf Grundlage des Gesetzes Nr. 5502 ${ }^{84}$ samt ihren Aufgaben und Kompetenzen auf die Anstalt für soziale Sicherheit übergegangen, sodass nunmehr die Zuständigkeit der Anstalt für soziale Sicherheit gegeben ist (Übergangsartikel 1). ${ }^{85}$

Die Generaldirektion für Stiftungen leistet zudem monatliche Zahlungen an Menschen mit Behinderung, die nicht dem Sozialversicherungssystem unterfallen und bedürftig sind. Die Gesamtzahl der Bedürftigen einschließlich Menschen mit Behinderung, die eine monatliche Zahlung erhalten, wird jährlich nach Einholung der Stellungnahme des Finanzministeriums bestimmt. Im Jahr 2007 wurden monatliche

Nicht nur die Einrichtungen der Anstalt für soziale Dienste und Kinderfürsorge erbringen Pflegeleistungen, sondern auch private Organisationen. Es kann keine Rede davon sein, dass die Ausgaben einer privaten Einrichtung durch das für den Aufenthalt eines Pflegebedürftigen zu zahlende Pflegegeld gedeckt werden können.

82 Dieser Betrag errechnet sich aus der Multiplikation des Indikators 1600 mit dem Koeffizienten 0,049486 für die Beamtenbezüge (http://www.emekli.gov.tr/sgk/2022Aciklamalar.html).

83 Ödeme Gücü Olmayan Vatandaşların Tedavi Giderlerinin Devlet Tarafından Karşılanması ve Yeşil Kart Uygulaması Hakkında Kanun, RG vom 03.07.1992, Nr. 21273.

Voraussetzung für die Übernahme der Behandlungskosten durch den Staat ist, dass der Betroffene nicht durch das bestehende Sozialversicherungssystem gegen das Risiko der Krankheit abgesichert ist. Mit dem Inkrafttreten des Gesetzes 5510 und der damit verbundenen Einführung einer allgemeinen Krankenversicherung werden Inhaber der sog. Grünen Karte in den Versichertenkreis der allgemeinen Krankenversicherung fallen (Art. 60 Abs. 1 Buchst. c Nr. 1 Gesetz Nr. 5510).

84 Sosyal Güvenlik Kurumu Kanunu, RG vom 20.05.2006, Nr. 26173.

85 Hierfür wurde die Generaldirektion für prämienunabhängige Zahlungen geschaffen (Primsiz Ödemeler Genel Müdürlüğü). 
Bedürftigkeitsrenten an insgesamt 5000 Bedürftige ausgezahlt. Die Höhe dieser Renten lag bei 239,24 YTL. ${ }^{86}$ Dieser Betrag bildet zugleich die Bedürftigkeitsgrenze, d.h. bei Einkommen oder Vermögen, das über diesem Betrag liegt, werden keine monatlichen Zahlungen geleistet. ${ }^{87}$

Zur Ergänzung ist auszuführen, dass über den Fonds zur Förderung der gegenseitigen sozialen Hilfe und Solidarität, der mit gleichlautendem Gesetz Nr. 3294 vom 29.05.1986 gegründet wurde ${ }^{88}$ Hilfeleistungen an Behinderte erbracht werden. So wurden im Rahmen der Ausbildungsförderung im Schuljahr 2005/2006 27.000 Schüler mit Behinderung kostenlos in ihre Schulen befördert. Im Rahmen der Heilbehandlung wurden die Kosten für medizinische Hilfsgeräte für 1.435 Menschen mit Behinderung übernommen. ${ }^{89}$

\section{Ausblick}

Der Erlass des Behindertengesetzes im Jahr 2005 nebst zahlreicher (Durchführung-) Verordnungen ist ein wichtiger Schritt zur Verbesserung der rechtlichen Stellung behinderter Menschen, auch wenn die vorgesehenen Instrumentarien zur Eingliederung und Teilhabe nicht als durchsetzbare Rechte konzipiert sind. Die Praxis zeigt jedoch, dass die Betroffenen einschließlich ihrer Familien und auch die Stellen, die für die Durchsetzung der Behindertenrechte und Gewährung der geschaffenen Leistungen zuständig sind, das Behindertenrecht nicht ausreichend kennen. Insbesondere die Lokalverwaltungen sind betroffen: Durch die Umgestaltung der öffentlichen Verwaltung im Zuge der Bemühungen um eine EU-Mitgliedschaft wurden den Lokalverwaltungen (Großstadt, Stadtgemeinde) neue Aufgaben, auch Menschen mit Behinderung betreffend, zugewiesen. ${ }^{90}$ Um für die bestehenden Kenntnislücken einschließlich der hiermit verbundenen Schwierigkeiten der Betroffenen, von den angebotenen Leistungen zu profitieren, ${ }^{91}$ Abhilfe zu schaffen, ist im Juni 2007 ein umfangreiches Projekt unter Beteiligung u.a. des Innen-, Kultus- und Gesundheitsministeriums, des Ministeriums für Arbeit und soziale Sicherheit sowie der Lokal-

Siehe http://www.vgm.gov.tr/sosyal/muhtac_ayligi.cfm.

Art. 5 Verwaltungsverordnung betreffend die von Stiftungen zu zahlenden Bedürftigkeitsrenten und zu betreibenden Armenküchen.

Sosyal Yardımlaşma ve Dayanışmayı Teşvik Kanunu, 14.06.1986, Nr. 19134.

9 Tätigkeitsbericht der Generaldirektion für gegenseitige soziale Hilfe und Solidarität für das Jahr 2006, S. 76, 79/80, zu entnehmen unter:

http://www.sydgm.gov.tr/sydtf/web/duyurular/SYDGM2006Y\%C4\%B11\%C4\%B1FaaliyetRaporu. pdf.

Schwerpunkt der zweiten Tagung des Behindertenrates in der Zeit vom 26.-28.09.2005 waren die Lokalverwaltungen (Yerel Yönetimler ve Özürlüler). Die Tagungsberichte einschließlich der Beschlüsse können entnommen werden unter: http://www.ozida.gov.tr

1 Hervorzuheben sind der Zugang zu den Pflegedienst- und Sozialhilfeleistungen, die Dauer der Erteilung von medizinischen Attesten zur Beurteilung des Behindertengrades und die Inanspruchnahme von Ausbildungs- und Arbeitsbeschaffungsmaßnahmen. 
verwaltungen gestartet. ${ }^{92}$ Die Koordination innerhalb der Projektpartner obliegt dem Präsidium für Angelegenheiten Behinderter. Zum jetzigen Zeitpunkt wurden bereits in 27 von 81 Regierungsprovinzen Konferenzen veranstaltet. Dieses ambitionierte Projekt wird einen wesentlichen Beitrag dazu leisten, das Bewusstsein der Betroffenen und ihrer Angehörigen ob ihrer Rechte sowie der staatlichen Stellen ob ihrer Verantwortung und Aufgaben zu stärken.

Der Bereich der Pflegedienste, insbesondere die häusliche Pflege, steht gegenwärtig im Fokus des Interesses, welcher Thema der dritten Tagung des Behindertenrates in der Zeit vom 19.-23.11.2007 war. ${ }^{93}$ Nach einer Bestandsaufnahme wurden die bestehenden Schwierigkeiten bei der Gewährung von Pflegeleistungen an Menschen mit Behinderung festgehalten und Lösungsvorschläge formuliert. Insbesondere ist nicht das Fehlen von Regelungen zu beklagen, sondern deren Durchführung und Überwachung. Ein besonderer Schwerpunkt muss u.a. auf die Ausbildung und den Einsatz von qualifiziertem Pflegepersonal sowie dem Nebeneinander von Pflege und medizinischer Versorgung gesetzt werden. Die Entwicklungen in naher Zukunft sind mit Interesse zu verfolgen. unter: http://www.ozida.gov.tr. 


\section{Literaturverzeichnis}

Basri Aşkın, Das Sozialversicherungssystem der Türkei, Marburg, 2004

Fatma Başterzi, Yaşlılık Sigortası (Altersversicherung), Istanbul, 2006

Nurşen Canıklıoğlu, Türk Sosyal Güvenlik Hukukunda sakatlara emekli-yaşlılık aylığı bağlanmasına hak kazanma bakımından bir karşılaştırma (Voraussetzungen einer Altersrente für Menschen mit Behinderung im Recht der sozialen Sicherheit im Vergleich), Kamu-İş, C. 6, S. 4/2002, zu entnehmen unter: http://www.kamu-is.org.tr/pdf/6411.pdf

Hakkı Demirci, Sosyal Güvenlik sisteminde sakatlara tanınan vergi avantajına bağlı erken emeklilik uygulaması (Durch Steuervergünstigungen bedingte vorzeitige Altersrente für Menschen mit Behinderung im System der sozialen Sicherheit), E-Yaklaşım, August 2007, zu entnehmen unter http://www.yaklasim.com

Andreas Hänlein, Die soziale Sicherung für den Fall der Invalidität in der Türkei, Deutsche Rentenversicherung, 6-7-8/98, S. 556 ff.

Ertan Kahramanoğlu, Özürlüler için bakım sigortası önerisi (Vorschlag für eine Pflegeversicherung für Menschen mit Behinderung), zu entnehmen unter: http://www.sosyalhizmetuzmani.org/bakimsigortasi.htm

Taki Kllıç, Özürlü, Eski Hükümlü ve Terör Mağduru çalıştırma esasları ve uygulamada karşılaşılan zorluklar (Grundsätze und Durchführungsschwierigkeiten bei Beschäftigung von Menschen mit Behinderung, Strafentlassenen und Terroropfer), E-Yaklaşım, Juni 2006, zu entnehmen unter http://www.yaklasim.com

Muzaffer Koç, Özürlü İstihdamı (Beschäftigung von Menschen mit Behinderung), E-Yaklaşım, Mai 2005, zu entnehmen unter http://www.yaklasim.com

Yasemin Körtek, Wege zu mehr Beschäftigung in der Türkei - vergleichende Betrachtungen zum deutschen Arbeits- und Sozialrecht, ZESAR 11./12./08, S. 483 ff.

Ali Seyyar, Dünya' da ve Türkiye' de Yaşlılığa Bağlı Bakım Sorununa Karşı Güvence Sistemleri (Systeme zur Absicherung des altersbedingten Pflegerisikos in der Welt und der Türkei), Öz-Veri, 2005, zu entnehmen unter: http://www.ozida.gov.tr/ozveri/ov3/ov3yaslibakim.htm

Şevket Tezel, İş ve Sosyal Güvenlik Hukuku El Kitabı (Handbuch Arbeitsrecht und Recht der sozialen Sicherheit), 2. Auflage, Ankara, 2007

Şevket Tezel, 5510 Sayılı Kanun özürlü statüsü açısından çalışanlara ne getiriyor? (Welche Änderungen bringt das Gesetz Nr. 5510 für Erwerbstätige mit Behinderung?), E-Yaklaşım, Januar 2007, zu entnehmen unter http://www.yaklasim.com

Rudolf Wedekind, Die türkische Verfassung 1982, Hannover, 1984

Antje Welke, Das Internationale Übereinkommen über die Rechte von Menschen mit Behinderungen, Archiv 4/2007, $60 \mathrm{ff}$.

Ali Yarayan, Der neue Kündigungsschutz in der Türkei, ZIAS 2/2005, $194 \mathrm{ff}$.

Ramazan Ylldız, Yeni Sosyal Güvenlik Kanunu'nunda özürlülelerin emekliliği (Behindertenrente im neuen Gesetz zur sozialen Sicherheit), Yaklaşım Dergisi, September 2006, zu entnehmen unter http://www.yaklasim.com 


\title{
5.6. Die Rechte der behinderten Menschen in Russland: Zustandsanalyse unter Berücksichtigung der aktuellen Entwicklungen
}

\author{
Von Maksat Kachkeev
}

1. Einleitung 266

2. Allgemeine rechtliche Bedingungen 266

2.1. Verfassung der Russischen Föderation von 1993

2.2. Gesetz „Über den sozialen Schutz der Behinderten“ 266

2.2.1. Definition der Behinderung 266

2.2.2. Einstufung gemäß der Behinderungsgruppe 267

2.2.3. Einstufung gemäß der Einschränkung der Berufstätigkeit 268

3. Finanzielle Leistungen 269

3.1. Monatliche Geldzahlung $\quad 269$

3.2. Paket von sozialen Leistungen 269

3.3. Arbeitsrente bzw. Behindertenrente 270

4. Arbeitsrechtliche Bedingungen 271

4.1. Gewährleistung der Beschäftigung 272

4.2. Arbeitsbedingungen 272

4.3. Quotierungsvorschriften 272

5. Rehabilitierung 273

5.1. Individuelles Programm der Rehabilitierung 274

5.2. Technische Mittel der Rehabilitierung 274

$\begin{array}{ll}\text { 6. Zusammenfassung } & 275\end{array}$ 


\section{Einleitung}

Russland steht derzeit aufgrund seiner neuerrungenen Rolle als „Energiesupermacht" und der damit verbundenen außenpolitischen Bedeutung im Rampenlicht der Medien. Themen wie beispielsweise die Rechte behinderter Menschen sind angesichts der bevorstehenden Dumawahlen Ende dieses Jahres sowie den Präsidentschaftswahlen am Anfang des Jahres 2008 aktueller denn je. In diesem Bericht wird von den großen politischen Themen Abstand genommen und der Schwerpunkt auf die Lage der behinderten Menschen in Russland gelegt. Die These, dass gerade die Stellung benachteiligter Menschen einen "Lackmustest" für die Funktionsfähigkeit des jeweiligen Staates darstellt, ist nicht neu. Mit dieser Studie werden in erster Linie bestimmte Aspekte der rechtlichen Situation behinderter Menschen in Russland untersucht, sowohl auf Verfassungs- und Gesetzesebene als auch anhand von Verwaltungsvorschriften. Dabei werden sowohl die allgemeinen rechtlichen Rahmenbedingungen als auch die finanziellen und arbeitsrechtlichen Besonderheiten bezüglich der behinderten Menschen ins Auge gefasst.

\section{Allgemeine rechtliche Bedingungen}

\subsection{Verfassung der Russischen Föderation von 1993}

Die Verfassung der Russischen Föderation enthält nicht ausdrücklich (wie z.B. in Art. 3 Abs. 3 des deutschen Grundgesetzes: „Niemand darf wegen seiner Behinderung benachteiligt werden“) Rechte behinderter Menschen. Eine implizite Verweisung finden wir in Art. 7, der lautet: „Die Russische Föderation ist ein Sozialstaat, dessen Politik auf die Schaffung von Vorraussetzungen gerichtet ist, welche ein Leben und eine freie Entfaltung des Menschen gewährleistet" ${ }^{\text {“1 }}$. Weiterhin ist Art. 39 zu erwähnen: „1. Jedem wird die soziale Sicherung im Alter, bei Krankheit, Invalidität und Verlust des Ernährers, für die Kindererziehung und in anderen gesetzlich festgelegten Fällen garantiert. 2. Staatliche Renten und Sozialunterstützungen werden durch Gesetz festgelegt.“

\subsection{Gesetz „Über den sozialen Schutz der Behinderten“}

\subsubsection{Definition der Behinderung}

Die rechtliche Situation der Behinderten wird in großem Maße durch das Gesetz „Über den sozialen Schutz der Behinderten“2 bestimmt (im folgendem „Behinder-

$1 \quad$ Verfassung der Russischen Föderation vom 12.12.1993.

2 Gesetz der Russischen Föderation vom 24.11.1995 „Über den sozialen Schutz der Behinderten“ 
tengesetz" genannt). Dieses wurde im Jahre 1995 verabschiedet. Das Behindertengesetz galt seinerzeit als sehr fortschrittlich und entsprach durchaus dem Zeitgeist der Demokratisierung, der damals in Russland herrschte und die russische Rechtsordnung stark beeinflusste ${ }^{3}$. Das Gesetz enthält und erörtert beispielsweise solche Begriffe wie „Rehabilitierung“, welche „als ein Prozess der völligen oder partiellen Wiederherstellung der Fähigkeiten zur gesellschaftlichen oder beruflichen Tätigkeit“4 definiert wird. Der Begriff „Barrierefreiheit“ wurde zwar nicht ausdrücklich erwähnt, ist aber implizit unter der Überschrift „Gewährleistung des freien Zuganges zu den Einrichtungen der sozialen Infrastruktur" - enthalten, so dass sowohl staatliche Behörden als auch private Unternehmen dazu verpflichtet sind, den Behinderten freien Zugang zu Einrichtungen en der sozialen Infrastruktur zu gewährleisten ${ }^{5}$.

Gemäß diesem Gesetz ist eine Person behindert, wenn eine dauerhafte (andauernde) Schädigung ihrer Gesundheit vorliegt, welche durch eine Krankheit, die Folgen eines Unfalles oder andere Umstände bedingt ist, die zur Einschränkung des Alltagslebens führt und die Notwendigkeit des sozialen Schutzes hervorruft ${ }^{6}$. Die Einschränkung des Alltagslebens ist gemäß diesem Gesetz ein völliger oder partieller Verlust der Fähigkeit oder der Möglichkeit (der betroffenen Person), sich zu versorgen, zu bewegen, zu orientieren, zu kommunizieren, das Verhalten zu kontrollieren, zu lernen oder eine berufliche Tätigkeit auszuüben? ${ }^{7}$.

\subsubsection{Einstufung gemäß der Behinderungsgruppe}

Gemäß dem Behindertengesetz wird das Feststellungsverfahren bezüglich der Behinderung durch die Föderale Staatliche Behörde der Ärztlichen und Sozialen Begutachtung, die in das Gesundheitsministerium der Russischen Föderation eingegliedert ist, durchgeführt ${ }^{8}$. Bei der Feststellung der Behinderung sind die folgenden Kriterien festzustellen, die sich aus dem Begriff der Behinderung gemäß dem Behindertengesetz herleiten lassen":

- ein dauerhaft schlechter gesundheitlicher Zustand;

- eine Einschränkung des Alltagslebens;

- die Notwendigkeit des sozialen Schutzes.

3 Sacharov/Tučkova, Pravo sozialnogo obespeenija v Rossii (Recht der sozialen Versorgung in Russland), Moskau 2005, S. 266.

4 Art. 9 des Behindertengesetzes.

5 Art. 15 Abs. 1 des Behindertengesetzes.

6 Art. 1 Abs. 1 des Behindertengesetzes.

7 Art. 1 Abs .2 des Behindertengesetzes.

8 Art. 8 Abs. 1 des Behindertengesetzes; siehe auch die Verordnung der Regierung der Russischen Föderation Nr. 805 vom 16.12.2004 „Über die Organisation und Tätigkeit der föderalen staatlichen Behörden der ärztlichen Begutachtung". Art. 1 Abs. 1 des Behindertengesetzes. 
Dabei ist zu bemerken, dass das Eintreten nur eines dieser drei Kriterien nicht automatisch zur Anerkennung des Betroffenen als behinderte Person führt ${ }^{10}$. Je nach Einschränkung des Alltagslebens, die durch eine Gesundheitsstörung bedingt ist, wird der Behinderte in die I., II. oder III. Gruppe der Behinderung eingestuft. Bei der Einteilung der Behinderten in diese drei Gruppen aufgrund der Einschätzung des Alltagslebens sind die folgenden Kriterien zu nennen: die Fähigkeit sich zu versorgen (1), zu bewegen (2), zu orientieren (3), zu kommunizieren (4), das Verhalten zu kontrollieren (5), zu lernen (6) und eine berufliche Tätigkeit auszuüben (7) ${ }^{11}$. Die I. Gruppe enthält die schwersten Formen der Behinderung und zeichnet sich durch die völlige Unfähigkeit des Behinderten aus, die vorgenannten Tätigkeiten auszuüben. Die II. und III. Gruppe enthalten entsprechend die leichteren Fälle der Behinderung.

Die Einstufung in die I. Gruppe wird für 2 Jahre festgesetzt, die Einstufung in die II. und III. Gruppe für jeweils ein Jahr. D.h., dass die Betroffenen sich nach Ablauf untersuchen lassen müssen, um die Einstufung verlängern lassen zu können ${ }^{12}$.

\subsubsection{Einstufung gemäß der Einschränkung der Berufstätigkeit}

Besonders ist die folgende Regelung zu erwähnen, die erst seit dem Jahr 2004 existiert: In das Behindertengesetz wurde eine Novelle eingefügt, die neben der Verleihung des Behindertengrades zusätzlich einen Grad der Einschränkung der Berufstätigkeit einführt. Dieser gliedert sich ebenfalls in drei Stufen bzw. drei Grade: der III. Grad der Einschränkung der Berufstätigkeit entspricht etwa dem I. Grad der Behinderung, der II. Grad der Einschränkung dem II. Grad der Behinderung und der I. Grad der Einschränkung der Berufstätigkeit entspricht dem III. Grad der Behinderung $^{13}$.

Es stellt sich die Frage, warum der Gesetzgeber neben der üblichen, seit Jahrzehnten etablierten Eingliederung in Behindertengruppen noch eine zusätzliche Eingliederung aufgrund des Grades der Einschränkung der Berufstätigkeit eingeführt hat. Zur Beantwortung dieser Frage wird eine kurze Analyse der sozialen Leistungen benötigt, die den Behinderten zu Verfügung stehen.

10 Art. 6 der Verordnung der Regierung der Russischen Föderation Nr. 95 „Über die Regelungen und Bedingungen der Feststellung einer Person als behindert" vom 20.02.2006

11 siehe die Verordnung N.535 des Gesundheitsministeriums der Russischen Föderation vom 20.08.2005 „Über die Verabschiedung der Klassifikationen und Kriterien, die von föderalen staatlichen Behörden der sozial-ärztlichen Begutachtung während der sozial-ärztlichen Begutachtung ausgewertet werden“.

12 Art. 9 der Verordnung der Regierung der Russischen Föderation Nr. 95 „Über die Regelungen und Bedingungen der Feststellung einer Person als behindert" vom 20.02.2006.

13 Art. 28.1. des Behindertengesetzes, Art. 8 der Verordnung der Regierung der Russischen Föderation Nr. 95 „Über die Regelungen und Bedingungen der Feststellung einer Person als behindert“ vom 20.02.2006; Sacharov/Tučkova, Pravo sozialnogo obespeenija v Rossii (Recht der sozialen Versorgung in Russland), Moskau 2005, S. 267. 


\section{Finanzielle Leistungen}

Die materielle Versorgung der behinderten Menschen umfasst Geldzahlungen auf unterschiedlicher Basis ${ }^{14}$. In diesem Zusammenhang kommen unterschiedliche normative Quellen in Betracht: In erster Linie sind dies das oben genannte Behindertengesetz sowie die föderalen Gesetze „Über die staatliche Sozialhilfe“ und „Über die Arbeitsrente in der Russischen Föderation“. Darüber hinaus bestehen unterschiedliche Arten der finanziellen Leistungen je nach rechtlicher Grundlage: die monatliche Geldzahlung, das Paket der sozialen Leistungen sowie die Arbeitsrente.

\subsection{Monatliche Geldzahlung}

Je nach Grad der Einschränkung der Berufstätigkeit wird die dementsprechende sogenannte "Monatliche Geldzahlung" gezahlt ${ }^{15}$ :

- für den Behinderten mit der Einschränkung der Berufstätigkeit III. Grades 1400 Rubel (ca. 40 Euro $^{16}$ );

- für die Behinderten mit der Einschränkung der Berufstätigkeit II. Grades 1000 Rubel (ca. 28 Euro);

- und für die Behinderten mit der Einschränkung der Berufstätigkeit I. Grades 800 Rubel (ca. 22 Euro);

- Behinderte, denen kein Grad der Einschränkung der Berufstätigkeit zugebilligt wurde, bekommen 500 Rubel (ca. 14 Euro);

\subsection{Paket von sozialen Leistungen}

Außerdem stehen den Behinderten gemäß dem Gesetz „Über die staatliche Sozialhilfe“17 andere Fördermöglichkeiten zu. Sie werden „Paket von sozialen Leistungen“ genannt ${ }^{18}$. Darunter fallen vor allem ${ }^{19}$ :

- die kostenlose ärztliche Versorgung einschließlich der notwendigen Medikamente sowie, sofern dies durch einen Arzt verschrieben wurde, eine Kurbehandlung;

- die kostenlose Fahrt mit dem öffentlichen Nahverkehr zum Ort der Behandlung und zurück. Den Behinderten mit der Einschränkung der Berufstätigkeit III. Grades sowie einer begleitenden Person wird die Fahrt mit anderen Trans-

14 Art. 27 des Behindertengesetzes.

15 Art. 28.1 des Behindertengesetzes.

16 Stand 2.1.2008.

17 Das föderale Gesetz Nr. 178-Ф3 „Über die staatliche Sozialhilfe“ vom 17.7.1999

18 Art. 6.1. des föderalen Gesetzes „Über die staatliche Sozialhilfe“.

19 Art. 6.2. des föderalen Gesetzes „Über die staatliche Sozialhilfe“. 
portmitteln (z.B. mit einem Flugzeug) zum Ort der Kurbehandlung übernommen.

Der Sinn und Zweck der Einführung der Einteilung aller Behinderten aufgrund des Grades der Einschränkung der Berufstätigkeit wird außerdem in einem Schreiben des Gesundheitsministeriums der Russischen Föderation evident:

\begin{abstract}
„Der Grad der Einschränkung der Berufstätigkeit entspricht nicht immer der Behindertengruppe. So kann z.B. einem Blinden, dem eine Arbeit unter speziell für ihn geschaffenen Bedingungen ermöglicht wird, der II. Grad der Einschränkung der Berufstätigkeit und die I. Behindertengruppe verliehen werden.“ Und weiter: „Eine Lehrerin, der eine Körperglied fehlt, wird in die II. Gruppe der Behinderung eingeteilt. Da dies aber kein Hindernis ist, einen Beruf auszuüben, kann ihr aufgrund dessen keine Einschränkung der Berufstätigkeit zugebilligt werden ${ }^{\text {‘20 }}$.
\end{abstract}

An diesem Brief wird die Intention der russischen Regierung klar, durch die Einführung dieser Novelle die Kosten für die Sozialleistung für die Behinderten zu senken, welche gerade von dem Grad der Einschränkung der Berufstätigkeit abhängt.

\title{
3.3. Arbeitsrente bzw. Behindertenrente
}

Schließlich ist die Einteilung nach dem Grad der Einschränkung der Berufstätigkeit für die Festsetzung der Arbeitsrente entscheidend.

Diese Art der Sozialleistung entsteht beim Eintritt der Behinderung und der daraus folgenden Einschränkung der Berufstätigkeit des III., II. oder I. Grades. Dies wird im Gesetz „Über die Arbeitsrente in der Russischen Föderation“'21 geregelt. Mit der Problematik der Wirkung der Beschränkung der Arbeitstätigkeit auf die Höhe der Rente befasste sich auch kurz das Verfassungsgericht der Russischen Föderation $^{22}$.

Herr Konin, der eine Sehbehinderung der I. Gruppe hat, wurde nach der ärztlichen Begutachtung hinsichtlich der Einschränkung der Berufstätigkeit als II. Grad eingestuft, was zur Folge hatte, dass der Basisumfang seiner Rente um mehr als die Hälfte (von 1320 Rubel auf 660) herabgesetzt wurde. Herr Konin legte Verfassungsbeschwerde mit der Begründung ein, dass das Gesetz „Über die Arbeitsrente“ nicht verfassungskonform sei. Verletzt seien unter anderem Art. 7 (Sozialstaatsprinzip und Gewährleistung des Sozialschutzes den Behinderten), Art. 19 (Gleichheit vor dem Gesetz), Art. 39 (Gewährleistung der Sozialvorsorgung der Behinderten)

Zit nach: Sacharov/Tučkova, Pravo sozialnogo obespeenija v Rossii (Recht der sozialen Versorgung in Russland), Moskau 2005, S. 267.

21 Das föderale Gesetz Nr. 173-Ф3 «Über die Arbeitsrente in der Russischen Föderation» vom17.12.2001 - Art. 4 Abs. 3 Satz 1, Art. 8 und Art. 15 dieses Gesetzes. Beschluss des Verfassungsgerichts N. 602-O vom 21.12.2006. 
der Verfassung der Russischen Föderation. Das Verfassungsgericht betonte in seinem Beschluss folgendes:

„Im Föderalgesetz „Über die Arbeitsrente“ veränderte der Gesetzgeber nicht - im Vergleich zu früher geltendem Recht - das Kriterium der Berechnung (der Basis) der Arbeitsrente. Diese Veränderung führt nicht zur Verletzung der Menschenrechte, wenn die Verfassungsprinzipien der Gleichheit sowie der Aufrechterhaltung des Vertrauens der Bürger in das Gesetz und der Handlung des Staates nicht verletzt werden ${ }^{23}$ “. Weiter stellt das Verfassungsgericht Folgendes fest: „Die Differenzierung der Höhe (der Basis) der Behindertenrente je nach dem Grad der Einschränkung der Berufstätigkeit hat den Zweck, ein höheres Niveau der Rentenversorgung für diejenige Personen zu bestimmen, die die Arbeitsfähigkeit vollkommen verloren haben und folglich nicht imstande sind, eigenes Einkommen zu verdienen. Den Personen, die ihre Arbeitsfähigkeit ganz oder zum Teil behalten haben, wird die Basisrente in einem geringeren Umfang zugebilligt - in Anbetracht der Möglichkeit der Fortsetzung der Berufstätigkeit.“24.

Der Grund der Zurückweisung der Verfassungsbeschwerde war, dass der Kläger in seiner Beschwerde mit der ärztlichen Begutachtung, die ihn als II. Grad der Einschränkung der Berufstätigkeit eingestuft hat, faktisch nicht einverstanden war $^{25}$. Die Überprüfung der Gesetzmäßigkeit der Entscheidungen der Staatsorgane liege nicht in der Kompetenz des Verfassungsgerichts ${ }^{26}$. Somit wurde die Verfassungsbeschwerde zurückgewiesen.

Der Wunsch der russischen Regierung, den Zugang zu den Rentenkasse zu verengen, ist vor allem in Anbetracht der statistischen Daten verständlich: Die Zahl der Empfänger der Behindertenrente lag im Jahre 2005 bei ca. 4, 5 Mio. Menschen; ihre Zahl wächst kontinuierlich. In den Jahren 2001 bis 2005 ist sie um 25\% gestiegen ${ }^{27}$.

\section{Arbeitsrechtliche Bedingungen}

Wenn die russische Regierung durch solche Schritte die Behinderten zwingt, eine berufliche Tätigkeit anzustreben, stellt sich die Frage, welche Maßnahmen von der russischen Regierung in Richtung der Arbeitsbeschaffung für behinderte Menschen ergriffen wurden. Die Lebensqualität der behinderten Menschen hängt in erster Linie von ihrer gesellschaftlichen Anerkennung ab, welche diese vor allem im Berufsleben erhalten.

Ziff. 2 Abs. 4 des Beschlusses des Verfassungsgerichts N. 602-O vom 21.12.2006.

Ziff. 2 Abs. 5 des Beschlusses des Verfassungsgerichts N. 602-O vom 21.12.2006.

Ziff. 3 Abs. 1 des Beschlusses des Verfassungsgerichts N. 602-O vom 21.12.2006.

Ziff. 3 Abs. 2 des Beschlusses des Verfassungsgerichts N. 602-O vom 21.12.2006.

Sacharov/Tučkova, Pravo sozialnogo obespeenija v Rossii (Recht der sozialen Versorgung in Russland), Moskau 2005, S. 262. 


\subsection{Gewährleistung der Beschäftigung}

Die Vorschriften des Behindertengesetzes in diesem Zusammenhang bestimmen, dass die föderalen Organe der staatlichen Gewalt die Arbeitsbeschäftigung der Behinderten gewährleisten sollen ${ }^{28}$. Dies wird unter anderem durch (1) die Quotierung der minimalen Anzahl von Arbeitsplätze für die Behinderten, (2) die Reservierung der Arbeitsplätze in Berufen, die für die Behinderten am geeignetsten sind, (3) die Förderung von Unternehmen bei der Schaffung von zusätzlichen Arbeitsplätzen für die Behinderten, (4) die Schaffung von speziellen Arbeitsbedingungen gemäß der individuellen Programme der Rehabilitierung der Behinderten, (5) die Schaffung der Bedingungen für eine unternehmerische Tätigkeit der Behinderten sowie durch (6) die Schaffung von Lernprogrammen mit dem Zweck, neue berufliche Tätigkeiten für behinderte Menschen zu eröffnen, erreicht ${ }^{29}$.

\subsection{Arbeitsbedingungen}

Sowohl die staatlichen als auch die privaten Arbeitgeber sind gemäß dem Behindertengesetz verpflichtet, notwendige Arbeitsbedingungen, die im Einklang mit dem individuellen Programm der Rehabilitierung der jeweils betroffenen Personen stehen, zu schaffen ${ }^{30}$. Darüber hinaus ist die Vereinbarung von Arbeitsbedingungen, die die Lage der behinderten Arbeitnehmer im Vergleich zu nicht-behinderten Mitarbeitern verschlechtern, in Arbeitsverträgen unzulässig ${ }^{31}$. Für die Behinderten der II. Behinderungsgruppe ist eine verkürzte Arbeitszeit mit nicht mehr als 35 Stunden pro Woche unter der Zahlung der vollen Bezüge vorgesehen ${ }^{32}$. Außerdem sind die Ableistung von Überstunden sowie Nachtarbeit nur mit dem Einverständnis der betroffenen Person sowie unter der Bedingung, dass dies keine Gefahr für deren Gesundheit darstellt, erlaubt ${ }^{33}$. Die Zahl der Urlaubstage beträgt nicht weniger als 30 Tage ${ }^{34}$.

\subsection{Quotierungsvorschriften}

Das Behindertengesetz schreibt zwar den Unternehmen, die mehr als 100 Mitarbeiter beschäftigen, vor, behinderte Menschen bis zum Erreichen einer Quote von 2 bis 
4\% der Gesamtzahl der Arbeitnehmer einstellen zu müssen ${ }^{35}$. Jedoch ist die Wirkung dieser Quotenregelung in Anbetracht der Tatsache, dass in Russland die Zahl der kleineren und mittleren Unternehmen mit weniger als 100 Mitarbeitern überwiegt, äußerst gering. Keine positive Wirkung zeigt auch die Abschaffung der Norm, nach der im Falle der Nichterfüllung der oben genannten Behindertenquote eine bestimmte Summe in das regionale Budget zu zahlen ist ${ }^{36}$. Als Folge ist mit der Senkung der finanziellen Mittel für die verschiedenen Rehabilitations- und Teilhabeprogramme zu rechnen. Im russischen Parlament liegen bereits unterschiedliche Vorschläge vor, die eine Senkung der Mitarbeiterzahl des Unternehmens auf 50 oder sogar 30 Mitarbeiter vorsehen. Es ist aber bis jetzt unklar, wann diese Gesetzesvorlagen von der Duma verabschiedet werden.

Nach dieser kurzen Erörterung stellt sich die Frage, ob effektive Fortschritte bei der Schaffung von Arbeitsplätzen für die behinderten Menschen bzw. bei der Durchsetzung ihrer Rehabilitierung mit oben genannten Maßnahmen erreicht wurden. Hier aber sieht die Lage eher negativ aus: Die Arbeitslosenquote ist extrem hoch: nur 10 bis $15 \%$ der behinderten Menschen haben die Möglichkeit ihren Beruf auszuüben. Daraus folgt, dass man die oben gestellte Fragen eher mit „nein“ beantworten muss.

\section{Rehabilitierung}

Das Behindertengesetz umfasst auch die notwendigen Rehabilitierungsmaßnahmen, die in jeweiligen Fällen der Behinderung entstehen ${ }^{37}$. Darunter versteht man „das System und den Prozess der völligen oder partiellen Wiederherstellung der Fähigkeit der behinderten Menschen zur alltäglichen, gesellschaftlichen und professionellen Tätigkeit ${ }^{\text {‘38 }}$. In diesem Sinne gewährleistet der Staat die Durchführung der Rehabilitierungsmaßnahmen, den Zugang zu den notwendigen technischen Mitteln und Dienstleistungen, die durch die föderale Liste der Rehabilitierungsmaßnahmen, technischen Mitteln der Rehabilitierung und Dienstleistungen vorgesehen $\operatorname{sind}^{39}$. Diese Liste wird von der Regierung der Russischen Föderation verabschiedet ${ }^{40}$.

Art. 21 Abs. 1 des Behindertengesetzes.

Empfehlungen des Rates für Behindertetenangelegenheiten beim Vorsitzenden des Föderationsrates der Föderationsversammlung der Russischen Föderation, Abgedruckt in: Voprosy sozial'nogo obespečenia, Nr. 14 2007, S. 11.

Kap. III. (Art. 9-12) des Behindertengesetzes.

Art. 9 Abs. 1 des Behindertengesetzes.

Art. 10 Abs. 1 des Behindertengesetzes.

Art. 10 Abs. 12 des Behindertengesetzes, sie auch die Verordnung der Regierung der Russischen Föderation Nr. 2347-p vom 30.11.2005. 


\subsection{Individuelles Programm der Rehabilitierung}

Weiterhin wird in jedem einzelnen Fall ein sogenanntes individuelles Programm der Rehabilitierung erstellt, das durch die oben genannte Behörde der ärztlichen Begutachtung bestimmt wird. Das Programm umfasst die einzelnen in jedem individuellen Fall die erforderliche Art, den Umfang und die Frist der Durchführung der ärztlichen, beruflichen und anderer Rehabilitierungsmaßnahmen ${ }^{41}$. Sowohl die staatlichen Verwaltungsorgane als auch die privaten Organisationen sind verpflichtet, den in diesem Programm erhaltenen Maßnahmenkatalog durchzusetzen ${ }^{42}$. Das individuelle Programm hat einen empfehlenden Charakter, d.h., dass die jeweilige Person das Recht hat, auf eine oder mehrere Rehabilitierungsmaßnahmen oder auch auf das ganze Programm zu verzichten ${ }^{43}$. Dabei wird den betroffenen behinderten Personen ein gewisser Spielraum bei der Wahl der notwendigen technischen Mittel eingeräumt ${ }^{44}$. Falls das notwendige technische Mittel oder die Dienstleistung nicht zur Verfügung gestellt werden kann, wird eine Kompensation in Höhe der Kosten des fehlenden technischen Mittels ausgezahlt ${ }^{45}$.

\subsection{Technische Mittel der Rehabilitierung}

Unter „technischen Mitteln“ versteht man Ausstattungen, die eine technische Lösung enthalten, zu denen unter anderem spezielle, für die Kompensierung oder Beseitigung der standhaften Störungen benutzten, technische Geräte zu zählen sind ${ }^{46}$. In diesem Sinne sind die folgenden technischen Mittel zu nennen ${ }^{47}$ :

- $\quad$ spezielle Mittel für die Eigenversorgung;

- $\quad$ spezielle Mittel für die Pflege;

- $\quad$ spezielle Mittel zur Orientierung (einschließlich eines Blindenhundes mit Ausrüstung), Unterhaltung und Informationsaustausch;

- $\quad$ spezielle Mittel für das Erlernen, die Ausbildung (einschließlich der Literatur für Blinde) und die Ausübung der beruflichen Tätigkeit;

- Prothesen (unter anderem auch Hörgeräte);

- $\quad$ spezielle Sportausrüstung.

Die Entscheidung über die Versorgung mit den technischen Mitteln wird aufgrund der ärztlichen Begutachtung getroffen ${ }^{48}$. Die Kosten für die Finanzierung der 
notwendigen technischen Mittel trägt dabei das föderale Budget und der Fond der Sozialversicherung ${ }^{49}$.

Die tatsächliche Lage der Versorgung mit den technischen Mitteln sieht indes kritisch aus ${ }^{50}$. Das liegt in erster Linie daran, dass der Umfang des zu diesem Zweck bestimmten Anteils des föderalen Budgets unzureichend ist und dementsprechend nicht alle Bedürfnisse befriedigen kann. Außerdem gibt es eine ganze Reihe von Unstimmigkeiten zwischen den zuständigen Behörden mit der Folge, dass die Budgetmittel ungleich verteilt werden, weil es vor allem an einer einheitlichen normativen Grundlage der zwischenbehördlichen Zusammenarbeit fehlt ${ }^{51}$. Daraus entstehen gewisse Ungleichheiten, z.B. bei der Versorgung mit den orthopädischen Prothesen, wo nur eine geringere Zahl der Behinderten ausgestattet ist, während die Mehrheit keinen Zugang zu den notwendigen Hilfsmitteln hat. Im Endeffekt leiden darunter die Personen, für die das System eigentlich geschaffen wurde ${ }^{52}$.

\section{Zusammenfassung}

Vor allem ist die Tatsache besorgniserregend, dass die russische Verfassung die Diskriminierung aufgrund der Behinderung nicht explizit verbietet, sondern lediglich auf die allgemeinen rechtlichen Bedingungen im Rahmen des Sozialstaates verweist ${ }^{53}$. Wenig hilfreich sind in diesem Zusammenhang die entsprechenden Vorschriften bezüglich der sozialen Sicherung aufgrund der Behinderung. Das Behindertengesetz enthält zumeist weitgehend vage und generell-formulierte Vorschriften. Das Hauptproblem besteht darin, dass solch wichtige Angelegenheiten wie die ärztliche Beurteilung oder die Einstufung in die Behinderungsgruppen bzw. gemäß des Grades der Einschränkung der Berufstätigkeit ausschließlich auf der Ebene der Regierungs- ${ }^{54}$ oder der ministerialen ${ }^{55}$ Verordnungen erfolgen. Dies ist umso wichtiger, weil sie ausschlaggebend und grundlegend für die Bestimmung der finanziellen Leistungen seitens des Staates sind und somit die Lebensgrundlage der betroffenen behinderten Personen darstellen. Mangelhaft ist in diesem Zusammenhang ebenfalls die Praxis des Ausschlusses breiter Schichten behinderter Personen von den sozialen Leistungen aufgrund der Einschränkung der Berufstätigkeit.

Art. 11.1 Abs. 5 des Behindertengesetzes.

Siehe z.B. die Empfehlungen des Rates für Behindertetenangelegenheiten beim Vorsitzenden des Föderationsrates der Föderationsversammlung der Russischen Föderation, Abgedruckt in: Voprosy sozial'nogo obespečenia, Nr. 14 2007, S. 12.

Ebenda.

Ebenda.

Art 7 der Verfassung der Russischen Föderation.

Z.B. die Verordnung der Regierung der Russischen Föderation Nr. 805 vom 16.12.2004 „Über die Organisation und Tätigkeit der föderalen staatlichen Behörden der ärztlichen Begutachtung“.

die Verordnung N.535 des Gesundheitsministeriums der Russischen Föderation vom 20.08.2005 „Über die Verabschiedung der Klassifikationen und Kriterien, die von föderalen staatlichen Behörden der sozial-ärztlichen Begutachtung während der sozial-ärztlichen Begutachtung ausgewertet werden“. 
Besorgniserregend ist ebenfalls die Statistik. Im Jahre 2006 betrug die Anzahl der behinderten Menschen in Russland 11,4 Mio., was ungefähr 7\% der Gesamtbevölkerung darstellt ${ }^{56}$. Diese Quote wächst drastisch: im Jahre 1992 zählte man in Russland 4,7 Mio. behinderte Menschen und ihre Zahl hat sich nur in den letzten sechs Jahren mehr als verdoppelt. Jährlich werden in Russland weitere 1,2 Mio. Menschen als behindert eingestuft, davon 95 bis $97 \%$ auf Lebenszeit ${ }^{57}$. Aber auch die Zahl der Bevölkerung in Russland schrumpft insgesamt drastisch: Laut einiger Prognosen werden im Jahre 2050 in Russland zwischen 35 bis 50 Mio. Menschen weniger leben als jetzt ${ }^{58}$. Die Situation der behinderten Menschen ist nur ein Teil der desolaten sozial-wirtschaftlichen Lage Russlands und spiegelt die drastische Senkung der Lebensqualität der Bevölkerung sowie den degradierenden Zustand des Gesundheitswesens wieder.

56 Van 'šin, Komu nušna reabilitazia invalidov, in: Voprosy sozial'nogo obespečenia, Nr.10 2006, S.9.

57 Fedorov, V Rossii bolee 11 millionov invalidov, in: Voprosy sozial'nogo obespečenia, Nr.21 2007, S.9

58 Van'šin, Komu nušna reabilitazia invalidov, in: Voprosy sozial'nogo obespečenia, Nr.10 2006, S. 9. 


\subsection{Teilhaberechte behinderter Menschen in den Mitgliedstaaten der Europäischen Union}

Bericht über Leitbilder und Modelle

Von Peter Trenk-Hinterberger

1. Vorbemerkung

2. Struktur des Berichts

2.1. Leitbilder und Modelle

2.2. Ausgewählte Teilhabebereiche und Teilhabeziele

2.2.1 Inklusion in das Bildungssystem

2.2.1.1. Systeme der Inklusion

2.2.1.2. Eingleisige Systeme

2.2.1.3. Zweigleisige Systeme

2.2.2. Inklusion in das Arbeitsleben 284

2.2.2.1. Ebenen der Inklusion $\quad 284$

2.2.2.2. Allgemeiner Arbeitsmarkt 284

2.2.2.1. Erschließung und Schaffung regulärer Beschäftigung

2.2.2.2. Geldleistungen für eine Existenzgründung

2.2.2.2.3. Schutzinstrumente zur Erhaltung von Arbeitsplätzen

2.2.2.4. Berufliche Rehabilitation

2.2.2.5. Nicht-kategoriale Leistungen der Beschäftigungsförderung

2.2.2.3. Besonderer (,geschützter“) Arbeitsmarkt 289

2.2.2.4. Therapeutischer Beschäftigungsmarkt 292

2.2.3. Inklusion in die Einkommenssicherung 292

2.2.3.1. Beitragfinanzierte und beitragsunabhängige Leistungen 292

2.2.3.2. Beitragsfinanzierte kategoriale Systeme 293

2.2.3.2.1. Methode „Rehabilitation vor Rente“ 293

2.2.3.2.2. Methode des aktuellen Fähigkeitsprofils 294

2.2.3.2.3. Methode der Schädigungsorientierung 295

2.2.3.3. Nicht beitragsfinanzierte kategoriale oder nicht kategoriale Systeme

2.2.4 Inklusion durch Unterstützung bei grundlegenden Alltagsverrichtungen 
2.2.4.4. Bedürftigkeitsprüfung

2.2.5. Inklusion durch Selbstbestimmungsrechte

2.2.6. Inklusion durch Recht auf Barrierefreiheit

2.2.7 Inklusion durch Antidiskriminierungs- und Nachteilsausgleichsrechte

2.2.7.1. Antidiskriminierungsrechte 


\section{Vorbemerkung}

Eine detaillierte, nach allen einzelnen Mitgliedstaaten der Europäischen Union (EU) aufgeschlüsselte Untersuchung über sämtliche rechtlich ausgestaltete Bereiche der Teilhabe behinderter Menschen am Leben in der Gesellschaft liegt bislang nicht vor. ${ }^{1}$ Die immensen Schwierigkeiten einer solchen breit angelegten Untersuchung nationaler rechtlicher Konzepte und Maßnahmen, die das Thema „Behinderung“"2 betreffen, Menschen mit Behinderungen umfassen und sich auf diese und andere Betroffene (z.B. Angehörige oder Arbeitgeber) auswirken, sind vielfältig: So unterscheiden sich die einzelnen nationalen „Modelle“3 ${ }^{“ 3}$ er Teilhaberechte für behinderte Menschen unter anderem durch Leitbilder, ${ }^{4}$ Rechts- und Politiktraditionen, Regelungsebenen und Zuständigkeiten (z.B. zentral, regional, kommunal), uneinheitliche Begriffe der Behinderung, ${ }^{5}$ Vielfalt der Regelungsbereiche (unter anderem Verfassungsrecht, Sozialrecht, Arbeitsrecht, Zivilrecht, Steuerrecht) verschiedene Anknüp-

1 Zumeist handelt es sich um überblicksartige Kompendien oder um Untersuchungen, die sich auf einzelne Bereiche (z.B. das Schulwesen bzw. den Zugang zum Arbeitsmarkt) oder ausgewählte Staaten beschränken. Vgl. etwa Degener/Quinn: A Survey of International, Comparative and Regional Disability Law Reform, in: Breslin/Yee (ed.), Disability Rights Law and Policy: International and National Perspectives, New York 2002, S. 3 ff.; Steinmeyer, Europäisches Behindertenrecht, in: Hanau/Steinmeyer/Wank, Handbuch des europäischen Arbeits- und Sozialrechts, München 2002, S. 1118 ff.; Council of Europe (ed.), Rehabilitation and integration of people with disabilities: policy and legislation, 7th ed., Strasbourg 2003; Europäische Kommission (Hrsg.), Soziale Sicherung für Menschen mit Behinderung - MISSOC-Info 1/2003, Brüssel 2003; Hvindén, Nordic Disability Policies in a Changing Europe: Is there Still a Distinct Nordic Model?, Social Law and Administration 2004, S. 170 ff.; Marin/Prinz/Queisser (eds.), Transforming Disability Welfare Policies? Towards Work and Equal Opportunities, Aldershot 2004; Lawson/Gooding (ed.), Disability Rights in Europe: From Theory to Practice, Oxford/Portland 2005.

2 Im Folgenden soll „Behinderung“ Menschen mit langfristigen körperlichen, geistigen, seelischen oder Sinnesschädigungen umfassen, die sie im Zusammenwirken mit verschiedenen Barrieren daran hindern können, gleichberechtigt mit anderen uneingeschränkt und wirksam an der Gesellschaft teilzunehmen (in Anlehnung an Art. 1 Abs. 2 des UN-Übereinkommens über die Rechte von Menschen mit Behinderungen, das am 3.3.2008 in Kraft trat). Dazu z.B. Bielefeldt, Zum Innovationspotenzial der UN-Behindertenkonvention, Deutsches Institut für Menschenrechte, Berlin 2006.

3 Mit „Modell“ wird hier die vereinfachende und abstrahierende Beschreibung der jeweiligen rechtlichen Gestaltungen von Konzepten und Maßnahmen verstanden.

4 Mit „Leitbild“ wird nachfolgend die Zielvorstellung für eine angestrebte Zukunft bezeichnet, die Konzepte und Maßnahmen auf ein bestimmtes Ziel hin koordiniert.

5 Zur „Behinderung“, deren Ursachen und Erscheinungsformen überaus vielgestaltig sind und die in den einzelnen Staaten der EU auch nicht durchweg einheitlich umschrieben wird umfassend Europäische Kommission (Hrsg.), Definition des Begriffs „Behinderung“ in Europa: Eine vergleichende Analyse - Eine Studie der Universität Brunel, Brüssel 2003. Nur hingewiesen werden kann auf die lebhafte Diskussion um das Konstrukt „Behinderung“: Teilweise wird in den Staaten der EU am medizinischen Modell der Behinderung festgehalten, das auf die funktionale Fähigkeit einer Person abstellt, während in anderen Staaten auf der Grundlage eines sozialen Modells die Behinderung als Folge des Zusammenspiels eines Individuums (mit seinen Beeinträchtigungen) mit seinem Umfeld verstanden wird. $\mathrm{Zu}$ dieser Diskussion vgl. z.B. Cloerkes (Hrsg.), Wie man behindert wird: Texte zur Konstruktion einer sozialen Rolle und zur Lebenssituation betroffener Menschen, Heidelberg 2003; Degener, Behinderung als rechtliche Konstruktion, in: Lutz (Hrsg.), Der (im-perfekte) Mensch - Metamorphosen von Normalität und Abweichung, Köln 2003, S. 448 ff.; aus deutscher Sicht: Welti, Behinderung und Rehabilitation im sozialen Rechtsstaat, Tübingen 2005, S. $10 \mathrm{ff}$. 
fungspunkte für Teilhaberechte (z.B. Arten der Behinderung und Ursachen einer Behinderung $^{6}$ ), die an der Realisierung von Teilhaberechten beteiligten (z.B. staatlichen und nicht-staatlichen gemeinnützigen oder gewinnorientierten) Akteure, uneinheitliche Regelungstechniken (z.B. weit gefaßte Rahmenregelungen, die sehr flexible Aktivitäten ermöglichen, und eng gefaßte Detailregelungen, die keine Spielräume eröffnen), verschiedene Leistungsarten und Leistungserbringungssysteme (z.B. Dienstleistungen und Geldleistungen, Sachleistungssysteme und Kostenerstattungssysteme oder Kostenvorschusssysteme), differenzierte Finanzierungssysteme (je nach Systemart und Zuständigkeiten z.B. Beitragsfinanzierung, Steuerfinanzierung, Mischfinanzierung) sowie unterschiedliche Techniken der Kooperation, Koordination und übergreifenden Teilhabeplanung (z.B. zwischen den beteiligten Trägern der sozialen Sicherung). Insofern kann von den nachfolgenden Ausführungen eine solche umfassende vergleichende Untersuchung weder geleistet noch erwartet werden. Die Zielsetzung der hier vorgestellten Befunde ist vielmehr eine wesentlich bescheidenere: Es kann lediglich auf einige dominierende Leitideen der Teilhaberechte behinderter Menschen, auf typische Modelle und auf ausgewählte Aspekte einzelner Teilhabebereiche in den Staaten der $\mathrm{EU}^{7}$ eingegangen werden, für die bereits mehr oder weniger intensive Forschungsarbeiten vorliegen. ${ }^{8}$

So sehen die Staaten der EU z.B. für die Folgen einer Behinderung, deren Ursache ein Arbeitsunfall oder eine Berufskrankheit sind, besondere Systeme der Kompensation und Restitution vor; dies gilt unter anderem auch für Behinderungen, deren Ursache Schädigungen bei militärischen Verrichtungen sind (veterans' disablity compensation).

Im Rahmen der EU und für einzelne Staaten der EU werden -freilich nur für einzelne Teilbereicheschon seit längerem rechtvergleichende Untersuchungen zur Lage behinderter Menschen für einzelne Teilbereiche durchgeführt; vgl. die in Fn. 1 genannten Untersuchungen des Europarats und der Europäischen Kommission; ferner etwa Aarts, European Experiences with Disability Policy, in: Marshaw/Reno/Burkhauser/Berkowitz (ed.), Disability Cash Work Benefits, W.E. Upjohn Institute of Employmnent Research, 1996, S. 129 ff.; Europäische Kommission (Hrsg.), Kompendium der von den einzelnen Mitgliedstaaten verfolgten Chancengleichheitspolitik zugunsten behinderter Menschen, Brüssel, 1998.

Die folgenden Ausführungen stützen sich vor allem auf die in Fn. 1,5 und 7 genannten Arbeiten, ferner auf Pitschas/v. Maydell/Schulte (Hrsg.), Teilhabe behinderter Menschen an der Bürgergesellschaft in Asien und Europa - Eingliederung im Sozial- und Rechtsvergleich, Speyer 2002 (Landesberichte zu Belgien -von van Langendonck-, Italien -von Hohnerlein-, Tschechische Republik von Tröster-, Slowakische Republik -von Barancova- und Ungarn -von Fazekas-); v. Maydell/Pitschas/Schulte (Hrsg.), Behinderung in Asien und Europa im Politik- und Rechtsvergleich Mit einem Beitrag zu den USA, Baden-Baden 2003 (Landesberichte zu Schweden -von Köhler-, Belgien -von van Langendonck-, Niederlande -von Kötter- Italien -von Hohnerlein-, Spanien -von Ferreras Alonso-, Ungarn -von Fazekas-, Tschechien -von Tröster), ferner auf die Landesberichte auf dem Workshop „Kulturwissenschaftliche Grundlegung und Erklärungshypothesen divergenter Politiken, sowie Rechtsetzung für Menschen mit Behinderung in Europa und Asien unter den Bedingungen des demographischen Wandels“ (20.-24.10.2007 in München, veranstaltet durch von Maydell u.a.) sowie schließlich auf die in den weiteren Fußnoten zitierten Publikationen. 


\section{Struktur des Berichts}

\subsection{Leitbilder und Modelle}

Über etliche Jahrzehnte beherrschten in den Staaten der EU Leitbilder wie „Normalisierung“ und „Integration“ die Diskussion um Teilhabe und Anerkennung behinderter Menschen. Zunehmend werden diese Leitbilder jedoch ergänzt, überlagert und sogar verdrängt von Leitbildern wie „Empowerment“, „Selbstbestimmung“ und „Inklusion“. Insbesondere das letztgenannte Leitbild der „Inklusion“ prägt zunehmend die Diskussion um die Ausgestaltung und Implementation von Rechtsnormen mit Bezug zu behinderten Menschen und ihrer Teilhabe am Leben in der Gesellschaft. Der Begriff der (sozialen) Inklusion formuliert in diesem Zusammenhang das Postulat, dass jeder Mensch in seiner Individualität akzeptiert wird und die Möglichkeit hat, in vollem Umfang an der Gesellschaft teilzuhaben, wobei Unterscheide und Abweichungen im Rahmen der Inklusion von der Gesellschaft weder in Frage gestellt noch als Besonderheit gesehen werden, vielmehr das Recht auf Teilhabe sich auf sämtliche Lebensbereiche bezieht, die grundsätzlich von allen - unabhängig von spezifischen Bedürfnissen und Lebensmöglichkeiten - uneingeschränkt genutzt werden können. ${ }^{9}$ Dieses Leitbild der (sozialen) „Inklusion“ wird nachfolgend zur Strukturierung der exemplarisch aufgegriffenen Bereiche der Teilhaberechte behinderter Menschen genutzt; innerhalb dieser Bereiche werden dann einzelne typischen Modelle der Gestaltung von Teilhaberechten skizziert.

Damit soll nicht behauptet werden, dass Inklusion behinderter Menschen in den Staaten der EU ein bereits erreichter Zustand wäre. Es soll vielmehr lediglich anhand dieses Leitbilds versucht werden, Dimensionen, Stand und Probleme der Umsetzung von Inklusion zu veranschaulichen, und zwar zum einen anhand von vier ausgewählten zentralen Teilhabebereichen (Bildungswesen, Arbeitsleben, Einkommenssicherung und grundlegende Alltagsverrichtungen), zum anderen anhand von vier wichtigen Teilhabezielen (Selbstbestimmung, Barrierefreiheit, Antidiskriminierung und Nachteilsausgleich).

9 Vgl. nur Wansing, Teilhabe an der Gesellschaft: Menschen mit Behinderung zwischen Inklusion und Exklusion, Wiesbaden 2005. Vom Leitbild der Inklusion wird auch das in Fn. 2 genannte UNÜbereinkommen beherrscht. Umfrageergebnisse in der EU lassen im Übrigen einen breiten Konsens über die Inklusion behinderter Menschen in die Gesellschaft erkennen, vgl. Europäische Kommission (Hrsg.), Eurobarometer Spezial: Diskriminierung in der Europäischen Union, Brüssel 2007, S. 47 ff. 


\subsubsection{Inklusion in das Bildungssystem}

\subsubsection{Systeme der Inklusion}

In einigen Staaten der EU läßt sich - teils seit längerem, teils in neuerer Zeit - die Tendenz beobachten, behinderten Kindern und Jugendlichen ${ }^{10}$ die Teilhabe am allgemeinen Bildungssystem - und insbesondere am Unterricht in allgemeinen Schulen (mainstream schools, Regelschulen) zu ermöglichen. ${ }^{11}$ Dabei werden im Wesentlichen zwei Wege beschritten (in der Regel: bei grundsätzlicher Schulpflicht für alle behinderten Kinder und Jugendlichen): Zum einen eine weitgehende Inklusion behinderter Schüler in das allgemeine Schulsystem (unten 2.2.1.2.) und zum anderen eine Trennung in ein allgemeines und ein besonderes (für behinderte Schüler konzipiertes) Schulsystem (unten 2.2.1.3.). ${ }^{12}$

\subsubsection{Eingleisige Systeme}

Zum Teil wird nach dem Grundsatz verfahren, dass alle Kinder allgemeine Schulen besuchen und lediglich besondere Maßnahmen im Rahmen des allgemeinen Schulbesuchs angeboten und durchgeführt werden, um diesen Schulbesuch zu ermöglichen. Bei solchen besonderen Maßnahmen kann es sich z.B. um speziell ausgebildete und eingesetzte Lehrer oder um begleitende Dienste und Personen handeln, die als „Integrationshelfer“ - etwa für alltägliche Verrichtungen während des Schulbesuchs - zur Verfügung stehen. ${ }^{13}$ Allerdings ist dieses Modell der Inklusion in den einzelnen Staaten zwar weitgehend für die Primarstufe verwirklicht; bei der Umset-

10 Zumeist als ,pupils with special educational needs“ bezeichnet.

11 Zum Bereich Universitäten und behinderte Menschen (z.B. bestimmte Quote von Studienplätzen für behinderte Studierende ab einem bestimmten Schweregrad der Behinderung, spezielle Stipendien, Prüfungserleichterungen) vgl. Steinmeyer (oben Fn. 1), Rz. 41 ff.; ferner OECD Publishing (Hrsg.), Students with Disabilities, Learning Difficulties and Disadvantages: Policies, Statistics and Indicators, Bertram Print on Demand, 2008 (zu 12 EU-Staaten sowie Japan, Korea, Mexiko, Schweiz, Türkei und USA).

12 Zum Ganzen eingehend Meijer, Integration in Europe: Provisions for Pupils with Special Education Needs - Trends in 14 European Countries, Middelfart 1998; Hans/Ginnold (Hrsg.), Integration von Menschen mit Behinderung - Entwicklungen in Europa, Neuwied 2000 (überwiegend zur Inklusion in das Schulsystem); Mittler, Towards Inclusive Education, London 2000; Vislie, From integration to inclusion: focusing global trends and changes in the western Europe societies, European Journal of Special Needs Education 2003, S. 17 ff.; Meijer, Special Needs Education in Europe: Inclusive Policies and Practices, 2007 (unveröffentlichtes Manuskript eines Vortrags auf der Europäischen Konferenz zur Integration behinderter Menschen am 11. und 12.6.2007 in Berlin); ferner umfassend zu behinderten Schülern OECD Publishing (Hrsg.) -oben Fn. 11-.

13 Exemplarisch dazu Kailer, „Inclusion in schools“ - am Beispiel England, Diplomarbeit, Universität Innsbruck, Fakultät für Bildungswissenschaften, 2006. 
zung auf der Ebene der Sekundarstufe scheint es hingegen organisatorische und finanzielle Schwierigkeiten zu geben. ${ }^{14}$ Bemerkenswert erscheint hier zudem der Befund, dass es nicht nur die Leitidee der Inklusion - im Sinne einer Überwindung von Sonderinstitutionen für behinderte Menschen - ist, die eine solche Eingleisigkeit der Schulbildung bedingt, sondern dass es teilweise ganz pragmatische Überlegungen sind, die zu einer solchen Eingleisigkeit beitragen: So zeigen Befunde aus Staaten, die eine niedrige Bevölkerungsdichte aufweisen, eine deutliche Tendenz zur Eingleisigkeit, weil in solchen Staaten das Vorhalten von besonderen Schulen für behinderte Kinder und Jugendliche, die - eben wegen der niedrigen Bevölkerungsdichte - nur für regional große Einzugsbereiche geschaffen werden müssten, nicht nur beträchtlich höhere Kosten verursachen (auch Transportkosten), sondern auch zu einer Entfremdung der behinderten Kinder und Jugendlichen von ihrem heimatlichen sozialen Umfeld bedeuten würde. ${ }^{15}$

\subsubsection{Zweigleisige Systeme}

Andere Staaten hingegen favorisieren ein zweigleisiges System, also die Trennung in allgemeine Schulen und besondere Schulen für behinderte Kinder und Jugendliche (zum Teil auch differenziert nach Behinderungsarten). In solchen zweigleisigen Systemen wird die Beteiligung behinderter Schüler am Bildungssystem im Wesentlichen nicht durch Inklusion, sondern durch (mehr oder weniger konsequente) Selektion bearbeitet. Allerdings lassen sich innerhalb dieser Gruppe von Staaten unterschiedliche Tendenzen beobachten: So erfolgt teilweise eine Ausweitung der Möglichkeiten inklusiver Schulbildung, die unter anderem begünstigt wird durch politischen Druck der betroffenen Eltern, aber auch durch ein gesetzlich eingeräumtes Wunschrecht der Eltern (das - wenn es zugunsten einer allgemeinen Schulbildung ihrer behinderten Kinder ausgeübt wird - zu einer Modifikation des allgemeinen Schulsystems zwingt). Ferner lassen sich eine zunehmende Kooperation und Durchlässigkeit zwischen den beiden - grundsätzlich voneinander getrennten - Systemen der Schulbildung feststellen, indem jedenfalls einzelne behinderte Schüler unter bestimmten Voraussetzungen in das allgemeine Schulsystem übernommen werden können. Diese Tendenz wird zum Teil auch dadurch begünstigt, dass den in den allgemeinen Schulen Lehrenden die bislang fehlende fachliche Kompetenz durch externen Sachverstand (z.B. durch „Sonderpägagogische Zentren“) begleitend vermittelt wird, oder dass der inklusive Schulbesuch durch persönliche Assistenz flankiert wird (wobei diese Assistenz teils schulrechtlich, teils sozialrechtlich ausgestaltet sein kann). Allerdings scheinen in den Staaten des zweigleisigen Systems, in denen eine Tendenz zur Durchlässigkeit zwischen den beiden Systemen und zur Inklusion in das allgemeine Schulsystem vorherrscht, auch erhebliche Widerstände 
gegen eine solche Inklusion zu bestehen. So fürchten etwa allgemeine Schulen - im immer häufigeren Wettbewerb um Schüler - um die Effektivität ihrer Ausbildung und damit um ihren Ruf auf dem „Markt“ der Schulbildung; und Eltern befürchten Nachteile für ihre nicht behinderter Kinder in der Annahme, diese würden durch die gemeinsame Erziehung mit behinderten Kindern und die Rücksichtnahme auf deren vermeintlich bestehenden Lernprobleme in der schulischen Entwicklung und damit letztlich auch auf dem Berufsausbildungs- und Arbeitsmarkt benachteiligt.

\subsubsection{Inklusion in das Arbeitsleben}

\subsubsection{Ebenen der Inklusion}

Im Hinblick auf die Inklusion in das Arbeitsleben unterscheiden die Staaten der EU im Wesentlichen drei Ebenen des Arbeitslebens: Die erste Ebene bildet der allgemeine (,erste“) Arbeitsmarkt, ${ }^{16}$ die zweite Ebene ein besonderer (,geschützter") Arbeitsmarkt mit einem Mindestmaß wirtschaftlich verwertbarer Arbeitsleistung der dort Beschäftigten und die dritte Ebene ein „therapeutischer“ Beschäftigungssektor. ${ }^{17}$

\subsubsection{Allgemeiner Arbeitsmarkt}

Soweit einzelne Staaten die Teilhabe behinderter Menschen am Arbeitsleben des allgemeinen Arbeitsmarkts rechtlich regeln, sehen sie eine breite Palette an Instrumenten vor, die eine solche Teilhabe ermöglichen sollen. ${ }^{18}$ Diese unterschiedlichen Instrumente kann man systematisch unterscheiden in solche, die Arbeitsplätze auf dem allgemeinen Arbeitsmarkt - also in „regulärer“ abhängiger Beschäftigung erschließen (unten 2.2.2.2.1.) oder die Gründung einer selbständigen Existenz för-

16 Die Arbeitslosenquote auf dem allgemeinen Arbeitsmarkt ist bei behinderten und andauernd gesundheitlich beeinträchtigten Personen ist in den Mitgliedstaaten der EU immer noch hoch. Vgl. dazu European Commission (ed.), The employment situation of people with disabilities in the European Union - A study prepared by EIM Business and Policy Research, Brüssel 2002; ferner die Zahlen in: Ward/Grammenos (ed.), Men and Women with Disabilities in the EU Final Report, 2007 (www.ec.europa.eu/employment_social).

17 Allgemein zur Inklusion in das Arbeitsleben z.B. Bloch/Prins (ed.), Who returns to Work and Why? A Six-Country Study on Work Incapacitiy and Reintegration, New Bunswick 2001; Marin/Prinz/Queisser (eds.), Transforming Disability Welfare Policies? Towards Work and Equal Opportunities, Aldershot 2004.

18 Dazu z.B. Thornton/Lunt, Employment Policies for Desabled People in Eighteen Countries: A Review, University of York 1997; European Commision (ed.), Benchmaking employment policies for people with disabilities - A study prepared by ECOTEC Research and Consulting Ltd., Brüssel 2000; European Commission (ed.), Access to Assistive Technology in the European Union - A Study prepared by Deloitte \& Touche, Brüssel 2003, dort inbesondere S. 21 ff. (Legislative and regulatory framework). 
dern sollen (unten 2.2.2.2.2.) und Schutzinstrumente, mit denen der Erhalt bestehender Beschäftigungsverhältnisse bezweckt wird (unten 2.2.2.2.3.). Darüber hinaus ist zu berücksichtigen, dass der Inklusion in den Arbeitsmarkt eine Phase der beruflichen Rehabilitation vorgeschaltet sein kann (unten 2.2.2.2.4.), und dass die meisten Staaten auch nicht-kategoriale Leistungen zur Integration in den allgemeinen Arbeitsmarkt vorsehen, also Leistungen, die nicht unbedingt mit einer Behinderung oder einer gesundheitlichen Beeinträchtigung zu tun haben müssen, aber auch behinderten Menschen offenstehen (unten 2.2.2.2.5.). ${ }^{19}$

\subsection{Erschließung und Schaffung regulärer Beschäftigung}

Beschäftigungspflichten der Arbeitgeber: Weit verbreitet sind in den einzelnen Staaten Regelungen zu Beschäftigungspflichten der privaten und öffentlichen Arbeitgeber. Dabei beruhen solche Pflichten in der Regel gegenüber einem Kreis von Personen, die einen bestimmten Status (z.B. einen bestimmten „Grad der Behinderung") aufweisen, der seinerseits anhand von Schädigungstabellen festgestellt wird und ab einem - anhand solcher Tabellen - bestimmten Maß der Behinderung die grundsätzliche Beschäftigungspflicht der privaten oder öffentlichen Arbeitgeber begründet. Die Feststellung eines solchen Status (z.B. als „Schwerbehinderter“) wird in den meisten Staaten seit langem von medizinischen Feststellungen und Daten dominiert, während in einigen Staaten auch anderen Fachleuten (z.B. Arbeitsmarktexperten) ein größerer Einfluss auf die Statusfeststellung eingeräumt wird. Der genannte Status kann dann im Hinblick auf die genannten Arbeitgeber unter anderem eine Rolle spielen bei der Erfüllung festgelegter Quoten, mit denen die Beschäftigung einer bestimmten Anzahl von behinderten Arbeitnehmern erreicht werden soll (,Pflichtquoten“). ${ }^{20}$

Die Sanktionen bei Nichterfüllung dieser Quoten sind freilich unterschiedlich ausgestaltet. Eine typische Sanktion besteht in der Verpflichtung der Arbeitgeber, einen Geldbetrag (,Ausgleichsabgabe“ usw.) an einen staatlich verwalteten Fonds zu zahlen, aus dem dann Leistungen (z.B. an Arbeitgeber) vor allem zur Einrichtung und Gestaltung von behinderungsgerechten Arbeitsplätzen erbracht werden können.

19 Trotz eines vielfältigen Instrumentariums von Beschäftigungsmaßnahmen für behinderte Menschen lassen sich freilich Defizite bei der Umsetzung dieses Instrumentariums nicht leugnen. Bezeichnend für diesen Befund ist z.B. der von der Europäischen Kommission veröffentlichte Bericht „Disability Mainstreaming in the European Employment Strategy“ (vom 1.7.2005, EMCO/11/290605), der eine Fülle solcher Defizite benennt. Vgl. ferner Waddington, Legislating to Employ People with Disabilities: The European and American Way, Maastricht Law Journal 1994, S. 376 ff.; Waddington/Diller, Tensions and Coherence in Disability Policy: The Uneasy Relationship between Social Welfare and Civil Rights Models of Disability in American, European and International Employment Law, in: Breslin/Yee (eds.), Disability Rights Law and Policy: International and National Perspectives, New York 2002, S. 241 ff.; Steinmeyer (oben Fn. 1), Rz. 55 f.

20 Vgl. Waddington, Legislating to Employ People with Disabilities: The European and American Way, Maastricht Law Journal 1994, 376 ff.; Thornton/Lunt (oben Fn. 18); mit Hinweisen zu einzelnen Staaten; ferener Waddington/Diller (oben Fn. 19); Steinmeyer (oben Fn. 1), Rz. 55 f. 
In einigen Staaten wird zudem die Förderung von (regulärer) Beschäftigung behinderter Frauen besonders betont, um die notwendige geschlechtsspezifische Betrachtung von Behinderung und Teilhabe - insbesondere auf dem Arbeitsmarkt - nicht zu vernachlässigen (etwa im Hinblick auf die Vereinbarkeit von Erziehungsaufgaben und Erwerbsarbeit durch wohnortnahe Beschäftigungsmöglichkeiten oder durch in Teilzeit nutzbare Angebote).

Geldleistungen für reguläre abhängige Beschäftigung: ${ }^{21}$ Vielfach sind Leistungen zur Integration behinderter Menschen in den allgemeinen Arbeitsmarkt vorgesehen, die entweder ganz oder zumindest teilweise aus Mitteln (Fonds) finanziert werden, die Arbeitgeber aufbringen müssen, weil sie ihre Beschäftigungspflicht nicht erfüllen (vgl. oben). Dazu gehören, - um nur einige typische Beispiele zu nennen - Geldleistungen

- an Arbeitgeber (z.B. Unternehmen oder nicht-staatliche Wohlfahrtsorganisationen), um behinderungsgerechte Arbeitsplätze (z.B. durch Umbau von Maschinen oder Räumen) einzurichten, oder um einen Anreiz für eine Ausbildung behinderter Menschen, für eine Probebeschäftigung oder für eine befristete Beschäftigung zu schaffen (z.B. durch Ausbildungszuschüsse, durch Einarbeitungszuschüsse, durch Zuschüsse zum Ausgleich von Minderleistung, durch Ermäßigung oder Befreiung von Sozialversicherungsbeiträgen für die beschäftigten behinderten Menschen);

- an behinderte Menschen, um mit bestimmten angepassten Hilfsmitteln (z.B. speziellen Werkzeugen) eine behinderungsgerechte Tätigkeit ausüben oder um mit einem behinderungsgerecht umgebauten Kraftfahrzeug die Arbeitsstelle erreichen zu können;

- an Arbeitgeber, an behinderte Menschen oder an Dritte zur Finanzierung einer persönlichen Assistenz, die als individuelle Begleitung des behinderten Beschäftigten im Arbeitsleben zur Erlangung oder zum Erhalt eines bestehenden Arbeitsplatzes beitragen soll.

Für diesen Bereich lässt sich freilich eine Tendenz feststellen, stärkere Rechtspositionen für bereits Beschäftigte als für künftig zu Beschäftigende festzuschreiben, indem bereits Beschäftigten Rechte auf spezielle Vorkehrungen an ihrem bisherigen Arbeitsplatz und bei demselben Arbeitgeber eingeräumt werden. Diese Tendenz könnte vor allem damit zusammenhängen, dass das europäische Sozialmodell ein Modell weitreichender Arbeitnehmerrechte auch und gerade im Hinblick auf behinderte Arbeitnehmer ist (z.B. im Hinblick auf besondere Pflichten der Arbeitgeber gegenüber behinderten Arbeitnehmern sowie auf einen besonderen Schutz bei Kündigungen) und damit beschäftigungsfördernde Maßnahmen stärker auf diejenigen ausgerichtet sind, die schon im Arbeitsleben stehen („Insider“), während diejenigen,

21 Dazu aus ökonomischer Sicht Verick, Do Financial Incentives Promote the Employment of the Disabled? IZA Discussion Paper No. 1256 (Forschungsinstitut zur Zukunft der Arbeit), Bonn 2004 (http://ssm.com/abstract=579705). 
die erst den Zugang zu einer Beschäftigung suchen („Outsider“) eher weniger Beachtung finden.

Einzelne Staaten der EU verknüpfen ihre Quotensysteme zudem mit Managementplänen zur Förderung der bereits im Betrieb oder Unternehmen beschäftigten behinderten Menschen. Ein solches betrieblichen Eingliederungsmanagement (nach dem Prinzip „Eingliederung statt Entlassung“) ist -mit unterschiedlicher Ausgestaltung- im Wesentlichen ein Verfahren zur möglichst frühzeitigen Beendigung von längerfristiger Arbeitsunfähigkeit (infolge Krankheit oder Behinderung) und zur Sicherung des Arbeitsplatzes (z.B. durch behinderungsgerechte Umgestaltung oder durch Reduzierung der Arbeitszeit). Typisch für ein solches betriebliches Eingliederungsmanagement sind Pflichten des Arbeitgebers zur Kooperation und zum Dialog insbesondere mit dem betroffenen Arbeitnehmer, den Kollektivvertretungen der Arbeitnehmer, Betriebsärzten, Sicherheitsfachkräften und Arbeitsschutzbeauftragten.

\subsection{Geldleistungen für eine Existenzgründung}

Weniger ausgeprägt sind in den einzelnen Staaten der EU Förderleistungen für behinderte Menschen zur Gründung einer selbständigen Existenz (z.B. durch günstige Darlehen, durch Befreiung von Sozialversicherungsbeiträgen oder durch die Übernahme der Kosten für eine logistische Unterstützung bei der Existenzgründung). Die Gründe für dieser eher vernachlässigte Strategie dürften vor allem darin liegen, dass der wirtschaftlichen Erfolg einer solchen Existenzgründung von vielen (vor allem konjunkturellen) Faktoren abhängt, auf die der behinderte Mensch keinen Einfluss hat, und deshalb der Einsatz von finanziellen Mitteln für prognostisch unsichere Unternehmungen (auch rechtlich) gescheut wird.

\subsection{Schutzinstrumente zur Erhaltung von Arbeitsplätzen}

Verbreitet sind in den Staaten der EU Instrumente zum Schutz behinderter Beschäftigter vor Verlust des Arbeitsplatzes, wobei diese Instrumente in sehr unterschiedlicher Ausprägung sowie Intensität vorzufinden sind und die

- von Rechtspflichten der Arbeitgeber zur Beschäftigung (bereits in einem Arbeitsverhältnis stehender) behinderter Arbeitnehmer auf Arbeitsplätzen, auf denen sie ihre Fähigkeiten und Kenntnissen möglichst voll verwerten und weiterentwickeln können,

- über die bevorzugte Berücksichtigung bei innerbetrieblichen Maßnahmen der beruflichen Bildung (Fortbildung, Umschulung) oder die behinderungsgerechte Einrichtung und Ausgestaltung von Arbeitsstätten

- bis hin zu Schutzinstrumenten im Zusammenhang mit beabsichtigten Entlassungen behinderter Beschäftigter (im Sinne eines erhöhten Kündigungschutzes) reichen. In diesem Bereich wird der besondere Schutz behinderter Beschäftigter 
entweder dadurch zu erreichen versucht, dass eine staatliche Behörde der Kündigung zustimmen muss, oder aber dass die Kündigung einer besonderen Rechtfertigung bedarf (die gerichtlich streng überprüft wird). ${ }^{22}$ Allerdings scheint in den Staaten, die einen solchen besonderen Kündigungsschutz vorsehen, die Wirkung dieses Schutzes mehr den bereits beschäftigten Behinderten als den arbeitsuchenden behinderten Beschäftigten zugute zu kommen (weil er sich für die letztgenannten eher als ein Einstellungshindernis erweisen kann).

\subsubsection{Berufliche Rehabilitation}

Spezifische behinderungsbezogene Beschäftigungsmaßnahmen können -als Vorphase der Inklusion in den allgemeinen Arbeitsmarkt- auch Maßnahmen der beruflichen Rehabilitation sein. Solche Maßnahmen der beruflichen Rehabilitation zur Eingliederung in den allgemeinen Arbeitsmarkt umfassen in den Staaten der EU ein breites Spektrum, das von der Berufsvorbereitung, über die berufliche Ausbildung, berufliche Anpassung und Weiterbildung, stufenweiser Wiedereingliederung, Arbeitsassistenz als Hilfe zur Erlangung eines Arbeitsplatzes, zur Berufsausübung erforderliche (technische) Hilfsmittel bis hin zur Übernahme der Kosten für die Ausstattung und Erhaltung einer behinderungsgerechten Wohnung reichen können. Dabei sind die in Betracht kommenden Leistungen zur Teilhabe am Arbeitsleben - unter Umständen erst nach Durchlaufen einer Phase der medizinischen Rehabilitation ${ }^{23}$ - zum Teil den Sozialversicherungssystemen (typischerweise der Rentenversicherung oder der Arbeitslosenversicherung) und zum Teil ausschließlich oder nachrangig bedürftigkeitsabhängigen Leistungssystemen zugeordnet; vereinzelt sind die Leistungen auch speziell auf die betreffende Schädigung ausgerichtet (z.B. für blinde Menschen). Mitgliedstaaten der EU liegen -soweit ersichtlich- bislang nicht vor. Vereinfachend kann man -bei aller Vorsicht- sagen, dass erstens der medizinischen Rehabilitation ein ganz unterschiedlicher Stellenwert zugemessen wird, zweitens sich Einrichtungen der medizinischen Rehabilitation zumeist im Bereich von Ballungsgebieten und Großstädten befinden und drittens medizinische Rehabilitationsleistungen überwiegend ambulant und durch Abteilungen angeboten werden, die Akutkrankenhäusern angegliedert sind. Dazu z.B. Gobrecht, Investitionen in grenzüberschreitende Gesundheitsversorgung in Europa: Das Beispiel der medizinischen Rehabilitation, Genf (WHO) 2006 (unveröffentlichtes Vortragsmanuskript;www.sozialbank.de/finale/inhalt/service/fachbeitraege29112.shtml). Überblicke (auch zur medizinischen Rehabilitation) zu einzelnen Ländern bei Lewerenz/Köhler, Rehabilitation in Frankreich, DAngVers 2000, 244 ff.; dies., Rehabilitation in Österreich, DAngVers 2000, 343 ff.; dies., Rehabilitation in Spanien, DAngVers 2001, 83 ff.; Köhler/Lewerenz, Rehabilitation in den Niederlanden, DAngVers 2001, 379 ff; Lewerenz, Rehabilitation in Belgien, DAngVers 2002, 298 ff.; ders., Rehabilitation in der Schweiz, DAngVers 2002, 423 ff.; ders., Rehabilitation in Finnland, DAngVers 2003, 439 ff.; ders., Rehabilitation in Schweden, DAngVers 2003, 542 ff.; ders., Rehabilitation in Tschechien, DAngVers 2004, 517 ff.; ders., Rehabilitation in Polen, RVaktuell 2006, $104 \mathrm{ff}$. 


\subsection{Nicht-kategoriale Leistungen der Beschäftigungsförderung}

Ein Überblick über Beschäftigungsmaßnahmen für behinderte Menschen in den Staaten der EU muss auch berücksichtigen, dass es einerseits kategoriale, also speziell für behinderte Menschen vorgesehene Maßnahmen der Beschäftigungsförderung gibt, dass aber andererseits auch Beschäftigungsmaßnahmen für einen breiteren Personenkreis vorgesehen sind, der im Hinblick auf eine Beschäftigung Probleme hat, die nicht unbedingt mit einer Behinderung oder einer gesundheitlichen Beeinträchtigung zu tun haben müssen (so z.B. der Personenkreis der Langzeitarbeitslosen). Bei solchen allgemeinen Beschäftigungsmaßnahmen konzentrieren in einigen Staaten die zuständigen Sozialleistungsträger ihre Bemühungen auf Gruppen, die als ,schwer vermittelbar" charakterisiert werden (weil sie z.B. ein geringes Bildungs- und Ausbildungsniveau aufweisen). Im Rahmen solcher allgemeiner Beschäftigungsmaßnahmen kann eine Behinderung insofern eine Rolle spielen, als die Einstufung als behinderter Mensch entweder für die Zuteilung zu bestimmten beschäftigungsfördernden Sondermaßnahmen eingesetzt werden kann oder eine leichtere und schnellere Inaspruchnahme derjenigen beschäftigungsfördernden Maßnahmen möglich ist, die in der Regel den „nur“ schwer vermittelbaren Personen (z.B. also den Langzeitarbeitslosen) vorbehalten sind. Allerdings geraten in diesem Bereich die schwer vermittelbaren behinderten Menschen in Konkurrenz zu den anderen Gruppen schwer vermittelbarer Personen (zunehmend z.B. älterer und junger Langzeitarbeitsloser), so dass bei einer Verallgemeinerung von Beschäftigungsmaßnahmen die finanziellen und personellen Ressourcen der Sozialleistungsträger von den behinderten Menschen zugunsten derjenigen Gruppen geschmälert oder teilweise umgelenkt werden können, die Probleme nicht wegen der Behinderung, sondern wegen anderer Faktoren haben. Umgekehrt kann es aber auch sein, dass bei einer - wie auch immer gearteten - besonderen Beachtung behinderter Menschen im Rahmen allgemeiner Beschäftigungsmaßnahmen die Betroffenen eine Zuteilung des Behindertenstatus anstreben, um in den Genuß einer Bevorzugung als behinderter Mensch zu gelangen (und dies von den Sozialleistungsträgern auch gefördert wird, weil z.B. ein gut ausgebildeter Rollstuhlfahrer in ein bestimmtes Arbeitsumfeld leichter zu integrieren ist, als jemand, der nicht behindert, aber unqualifiziert ist).

\subsubsection{Besonderer (,geschützter“) Arbeitsmarkt}

Soweit eine Inklusion in den allgemeinen Arbeitsmarkt aus Gründen in der Person des behinderten Menschen nicht möglich ist, sehen die meisten Staaten der EU unterschiedliche Arten der Inklusion durch Beschäftigung auf einen besonderen (,geschützten") Arbeitsmarkt vor (sheltered employment). ${ }^{24}$ Dabei wird in aller Regel ein bestimmtes Maß an Unterstützungs- und Förderbedarf sowie ein Mindestmaß 
wirtschaftlich verwertbarer Arbeitsleistung des behinderten Menschen vorausgesetzt (wobei die „Messung“ dieses Mindestmaßes nicht einheitlich erfolgt und in der Sache nicht unproblematisch ist). Was die Orte einer solchen ,geschützten“ Beschäftigung betrifft, so kann man wie folgt unterscheiden:

- Typisch ist die Beschäftigung in besonderen Werkstätten oder Beschäftigungszentren im Sinne eines geschlossenen Sonderarbeitsmarkts, der ausschließlich behinderten Menschen vorbehalten ist, der ein bestimmtes Mindestmaß wirtschaftlich verwertbarer Arbeitsleistung erbringen kann. ${ }^{25}$ Dabei soll - jedenfalls von der Konzeption dieser Einrichtungen - nicht ausgeschlossen sein, dass geeignete behinderte Menschen aus diesem Sonderarbeitsmarkt auf den allgemeinen Arbeitsmarkt wechseln können (auch wenn dies in der Realität - nach den vorliegenden Daten - selten der Fall zu sein scheint). Soweit ersichtlich haben die in den genannten Einrichtungen des Sonderarbeitsmarktes beschäftigten behinderten Menschen in aller Regel nicht den Status regulärer Arbeitnehmer, sondern - vor allem zu ihrem Schutz - einen speziellen, modifizierten Status (unter anderem hinsichtlich ihrer Pflichten im Zusammenhang mit der Erbringung der Arbeitsleistung). Ob freilich eine solche Beschäftigung in besonderen Einrichtungen - wie „beschützenden Werkstätten - tatsächlich dem Konzept der Inklusion entspricht, wird in einzelnen Staaten der EU durchaus kontrovers diskutiert.

- Weniger verbreitet ist die Beschäftigung auf Arbeitsplätzen, die zwar finanziell und organisatorisch den oben genannten besonderen Werkstätten oder Beschäftigungszentren zugeordnet sind, die aber räumlich in Betrieben des allgemeinen Arbeitsmarkts eingerichtet werden und eine Zwischenstufe für den Übergang von der beschützten Beschäftigung auf den allgemeinen Arbeitsmarkt darstellen sollen. Ergänzt werden solche ausgelagerten beschützten Arbeitsplätze zum Teil durch eine begleitende Assistenz, die Hilfestellung bei Schwierigkeiten am Arbeitsplatz anbietet (z.B. bei individuellen Problemen des behinderten Beschäftigten mit dem Arbeitgeber oder mit anderen - nichtbehinderten - Beschäftigten des Betriebs). Bemerkenswert ist in diesem Bereich der Beschäftigung, dass der arbeitsrechtliche Status der behinderten Beschäftigten wegen der an den allgemeinen Arbeitsmarkt angenäherten Beschäftigungssituation nach dem jeweiligen Stand und Ausmaß der Integration flexibel gehandhabt werden kann (,disability flexicurity").

- Zunehmend findet sich in einzelnen Staaten der EU die Beschäftigung behinderter Menschen (zum Teil erst ab einem bestimmen Schweregrad der Behinderung) in besonderen - eigens für diese behinderten Menschen geschaffenen Unternehmen oder Betrieben. Vorzufinden sind hier z.B. rechtlich und wirtschaftlich selbständigen Selbsthilfefirmen und Integrationsunternehmen bzw. „social enterprises“, ferner rechtlich und wirtschaftlich unselbständige Integrationsabteilungen in Beschäftigungsstellen öffentlicher Arbeitgeber oder in Wirt- 
schaftsunternehmen privat-gewerblicher Arbeitgeber (z.B. als innerbetriebliche Alternative zur Beschäftigung behinderter Arbeitnehmer, die auf ihrem bisherigen Arbeitsplatz im Unternehmen nicht mehr weiterbeschäftigt werden können). Es handelt sich bei dieser Form der Beschäftigung entweder um eine solche auf dem allgemeinen Arbeitsmarkt oder um eine solche, die einer Beschäftigung auf dem allgemeinen Arbeitsmarkt weitgehend angenähert ist. Dementsprechend sind in solchen besonderen Unternehmen oder Betrieben behinderte Menschen dann entweder in regulären Beschäftigungsverhältnissen oder in Beschäftigungsverhältnissen mit begrenzter arbeitsrechtlicher Modifizierung (,disability flexicurity“) tätig. Verbreitet sind derartige Beschäftigungsverhältnisse im Bereich der Güterproduktion oder der Dienstleistungen (sei es als unmittelbar Beschäftigte, sei es im Rahmen von Zeitarbeit). Adressat der Beschäftigungsmöglichkeiten sind zumeist behinderte Menschen, deren Teilhabe an einer sonstigen Beschäftigung auf dem allgemeinen Arbeitsmarkt aufgrund von Art und Schwere der Behinderung (z.B. psychischer Krankheit) oder wegen sonstiger Umstände (z.B. Mangel an Arbeitsplätzen für psychisch kranke Menschen) auf besondere Schwierigkeiten stößt. Ziel solcher Beschäftigungen ist es, eine Brücke zur Eingliederung auf dem (völlig ,ungeschützten“) allgemeinen Arbeitsmarkt zu bilden.

Allerdings darf in diesem Bereich der besonderen Unternehmen oder Betrieben - aber auch im Bereich der zuvor angesprochenen besonderen Werkstätten und Beschäftigungszentren (oben 2.2.2.1. und 2.2.2.2.) - nicht übersehen werden, dass es für bestimmte Beschäftigungsorte eine Dominanz bestimmter Behinderungsarten geben (z.B. der körperlichen und der geistigen Behinderung) und damit eine Auslese sowie Ausgrenzung in dem Sinne erfolgen kann, dass die Inklusion in das Arbeitsleben auf Grund bestimmter „Behindertenhierarchien“ erfolgt und sich z.B. nach der Rangordnung von Produktivität und Ertrag der Beschäftigung eines behinderten Menschen richtet. Hinzu kommt, dass der ,geschützte“ Arbeitsmarkt in den einzelnen Staaten der EU unter beträchtlichem Wettbewerbsdruck steht, der zur Auslese der leistungsfähigsten behinderten Beschäftigten tendiert (zu Lasten der wenig oder weniger Leistungsfähigen, die überhaupt keine Inklusion in das Arbeitsleben erfahren oder für die dann allenfalls der unten bei 4. skizzierte therapeutische Arbeitsmarkt verbleibt). Ferner ist auch auf den Finanzierungsdruck hinzuweisen, dem die - in der Regel - von öffentlichen Zuweisungen abhängigen Einrichtungen des ,geschützten" Arbeitsmarkts in Zeiten knapper öffentlicher finanzieller Ressourcen ausgesetzt sind. Schließlich ist offenbar auch zu beobachten, dass behinderte Menschen, die - insbesondere im Fall hoher allgemeiner Arbeitslosigkeit - auf dem ersten Arbeitsmarkt keine Beschäftigung finden, in geschützte Einrichtungen aufgenommen werden, obwohl sie dort (z.B. aufgrund ihrer Qualifikation und Leistungsfähigkeit) fehlplaziert sind (gleichwohl aber dort aus der Sicht der Träger der beschützten Arbeitseinrichtungen gern beschäftigt werden, weil sie zur Steigerung des Leistungsniveaus und der Produktivität beitragen). 


\subsubsection{Therapeutischer Beschäftigungsmarkt}

Vereinzelt finden sich für Menschen mit schwerer und mehrfacher Behinderung sowie für (auch alte) behinderte Menschen mit erheblichem Unterstützungsbedarf Beschäftigungsstellen auf einem „therapeutischen“ Beschäftigungsmarkt, auf dem nicht an ein Mindestmaß wirtschaftlich verwertbarer Arbeitsleistung vorausgesetzt wird, sondern auf dem es um eine Tätigkeit im Sinne einer ,sinnvollen“, tagesstrukturierenden Beschäftigung geht. Solche Beschäftigungsstellen sind teilweise unter dem „verlängerten Dach“ der besonderen ("beschützenden“) Werkstätten oder Beschäftigungszentren angesiedelt, allerdings in aller Regel als eigenständiger Beschäftigungsbereich. In diesem Zusammenhang fällt besonders auf, dass der Status der auf dem therapeutischen Beschäftigungsmarkt tätigen behinderten Menschen weitgehend ungeregelt ist und eher als rechtliches Experimentierfeld denn als rechtlich konsolidierter Beschäftigungsmarkt bezeichnet werden kann.

\subsubsection{Inklusion in die Einkommenssicherung}

\subsubsection{Beitragfinanzierte und beitragsunabhängige Leistungen}

In allen Staaten der EU sind Geldleistungen für Menschen vorgesehen, die aufgrund einer Behinderung erwerbsgemindert oder erwerbsunfähig sind. Dabei kann unterschieden werden zwischen

- $\quad$ einer beitragsfinanzierten kategorialen Versicherungsleistung, die als Kategorie eine Erwerbsminderung oder eine Erwerbsunfähigkeit voraussetzt und deren Höhe sich vor allem nach der Höhe des während des Versicherungslebens durch Beiträge versicherten Arbeitseinkommens richtet (unten 2.2.3.2.), und

- einer beitragsunabhängigen kategorialen (Erwerbsminderung oder Erwerbsunfähigkeit voraussetzenden) oder nicht kategorialen (also nicht von Erwerbsminderung oder Erwerbsunfähigkeit abhängenden) Leistung, die eine Grundsicherung garantieren soll und die in einzelnen Staaten auf einer Bedürftigkeitsprüfung beruht, in anderen hingegen ohne eine solche Prüfung gewährt wird (unten 2.2.3.3.).

Zudem wird in einzelnen Staaten unterschieden zwischen Personen,

- die anspruchsberechtigt sind, weil sie (z.B. infolge einer Behinderung) überhaupt nicht mehr erwerbstätig sein können - sie also erwerbsunfähig sind -, wobei dann noch festgelegt wird, welche Tätigkeiten für die Anspruchsberechtigung nicht schädlich sind (z.B. therapeutische Tätigkeiten oder Tätigkeiten auf dem allgemeinen Arbeitsmarkt von weniger als drei Stunden täglich), und

- bei denen zwar nicht eine Erwerbsunfähigkeit, sondern „nur“ eine geminderte Erwerbsfähigkeit vorliegt, die mit einer Teilzeitbeschäftigung auf dem allgemeinen Arbeitsmarkt vereinbar ist. Sind nur wenige Bezieher einer solchen anteiligen Geldleistung aber tatsächlich in Teilzeit erwerbstätig, stellt ein solches 
System eher die Möglichkeit dar, geringere Leistungen an weniger behinderte Menschen zu zahlen. Zum Teil werden dann, wenn diese geringeren (in der Regel: Versicherungs-)Leistungen nicht einen bestimmten Existenzmindeststandard decken durch Leistungen ergänzender - bedürftigkeitsabhängiger - Systeme aufgestockt.

\subsubsection{Beitragsfinanzierte kategoriale Systeme}

Die in Bezug auf diese Leistungen der Einkommenssicherung im Bereich der Sozialversicherung verwendeten Bedingungen setzen eine Feststellung der geminderten Erwerbsfähigkeit bzw. der Erwerbsunfähigkeit voraus. Für diese Feststellung lassen sich in den einzelnen Staaten der EU im Wesentlichen drei Methoden unterscheiden, nämlich die Methode der „Rehabilitation vor Rente“ (unten 2.2.3.2.1.), die Methode des aktuellen Fähigkeitsprofils (unten 2.2.3.2.2.) und die Methode der Schädigungsorientierung (unten 2.2.3.2.3.), wobei die hier vorgenommene Kategorisierung für einige Staaten nicht trennscharf durchgehalten werden kann, weil die eine der genannten Methoden mit Elementen einer anderen Methode vermischt ist.

\subsection{Methode „Rehabilitation vor Rente“}

In einer Reihe von Staaten werden bei der Bewertung und Feststellung der Erwerbsminderung/Erwerbsunfähigkeit die vorrangigen Möglichkeiten einer medizinischen und/oder beruflichen Rehabilitation berücksichtigt. Typisch ist dieser Ansatz für Sozialversicherungssysteme, die dem Grundsatz "Rehabilitation vor Rente“ folgen und einen differenzierten Katalog von Rehabilitationsmaßnahmen vorsehen. Dabei steht die Einstufung als „behinderter Mensch“ nicht notwendig am Beginn des Prüfungsverfahrens. Davor gibt es teilweise die Möglichkeit, als von einer Behinderung bedrohter oder als kranker Mensch eingestuft zu werden. Kennzeichnend für diesen Ansatz ist ein (detaillierter) Begutachtungsprozess

- sowohl im Hinblick auf die medizinische Rehabilitation (in der Regel dominiert von medizinischen Untersuchungen, sei es von Ärzten des Sozialversicherungsträgers selbst, sei es von externen Ärzten, z.B. den behandelnden Ärzten des Antragstellers) ${ }^{26}$,

- als auch im Hinblick auf die berufliche Rehabilitation (wobei hier die Untersuchungen wegen der vielfältigen Aspekte des Arbeitslebens nicht nur medizinische, sondern z.B. auch psychologische, arbeitstechnische und arbeitsmarktpolitische Gesichtspunkte berücksichtigen, insbesondere die Eignung und Neigung des Betroffenen sowie die Aussichten einer angestrebten beruflichen Ausbildung auf dem allgemeinen Arbeitsmarkt). 
Insgesamt betrachtet, besteht für die Sozialversicherungsträger bei diesem Verfahren ein hohes $\mathrm{Ma} ß$ an individueller Beurteilung und eine begrenzte Ermessensfreiheit für die Entscheidung im Einzelfall über die Gewährung von Leistungen zur Rehabilitation. Führen die Leistungen zur Rehabilitation nicht zum gewünschten Erfolg, endet der Prozess in der Regel mit der Berentung wegen teilweiser Erwerbsminderung (zumeist mit der Folge der Arbeitslosigkeit oder Teilarbeitslosigkeit) oder wegen voller Erwerbsminderung (zum Teil ergänzt um Vorruhestandsregelungen wegen gesundheitlicher Beeinträchtigungen).

\subsection{Methode des aktuellen Fähigkeitsprofils}

In einigen Staaten wird -als Voraussetzung für Leistungen der Einkommenssicherung für behinderte Menschen- mit Hilfe einer „Momentaufnahme“ festgestellt, ob eine Person zu einem bestimmten Zeitpunkt - z.B. nach dem Ende der Krankschreibung - erwerbsfähig, erwerbsgemindert oder erwerbsunfähig ist. Bei diesem Ansatz erfolgt die Konzentration auf die Frage, weshalb eine Person nicht in der Lage ist, eine Arbeit auszuüben bzw. aufzunehmen. Damit erfolgt - anhand eines Fähigkeitsprofils - eine Reduktion der Perspektive auf die arbeitsplatzrelevanten aktuellen Fähigkeiten des Betroffenen. Hierbei wird das individuelle Fähigkeitsprofil (z.B. anhand standardisierter Skalen - „Personal Capability Assessment ${ }^{\star 27}$ ) ermittelt und (z.B. mit Hilfe einer Punktzahl) bewertet. Die Entscheidung darüber, ob jemand erwerbsgemindert oder erwerbsunfähig ist, wird dann vom Erreichen einer bestimmten Gesamtzahl von akkumulierten Punkten abhängig gemacht.

Demgegenüber wird in anderen Staaten zwar auch ein individuelles Fähigkeitsprofil erstellt, dieses aber mit den Anforderungsprofilen einer Vielzahl von konkreten Arbeitsplätzen abgeglichen, die auf dem jeweiligen Arbeitsmarkt angeboten werden. Durch diesen Abgleich wird dann ermittelt, ob und welche Arbeitsplätze für den Betroffenen noch in Betracht kommen, welcher „Abstieg“ ihm in der Hierarchie des Arbeitsplatzniveaus zuzumuten ist oder ob er (in einer „Alles-oder-nichts“Variante) „erwerbsfähig“ oder „nicht erwerbsfähig“ ist (also ohne die Möglichkeit einer nur teilweisen Erwerbsfähigkeit). Im Wesentlichen steht bei beiden Fällen dieser Methode des Fähigkeitsprofils weniger die Verbesserung der Aussichten auf eine Teilhabe am Arbeitsleben im Vordergrund, als die Aussage darüber, ob und in welchem Umfang eine Person in ihrer aktuellen Gesamtverfassung dem Arbeitsmarkt zur Verfügung steht. 


\subsection{Methode der Schädigungsorientierung}

In einigen Staaten werden der Bewertung der Erwerbsfähigkeit Schädigungstabellen zugrunde gelegt, die bestimmte Werte (z.B. Grade) zur Beschreibung des auf eine Schädigung bezogenen medizinischen Zustands einer Person enthalten (die also z.B. den Grad des Hörverlustes mit anerkannten technischen Geräten messen und mit einem bestimmten Behinderungsgrad bewerten). Die Besonderheit einer solchen „direkten“ Messung, die eindeutig von Ärzten dominiert wird, liegt darin, dass in der Regel die Folgen für wichtige Aktivitäten des Lebens, wie insbesondere das Arbeitsleben, nicht oder nicht durchweg berücksichtigt werden. Dies kann freilich auch den Vorteil haben, dass die Bearbeitungskosten im Zusammenhang mit der Feststellung einer Erwerbsunfähigkeit relativ niedrig sind und die Feststellung des Grades der Schädigung zugleich eine Ausstrahlung auch auf andere Akteure außerhalb des Bereichs der Einkommenssicherung (z.B. im Hinblick auf bestimmte Nachteilsausgleiche - wie Steuerermäßigungen oder Gebührenbefreiungen - haben kann).

\subsubsection{Nicht beitragsfinanzierte kategoriale oder nicht kategoriale Systeme}

Soweit einzelne Staaten eine Einkommenssicherung ohne kategoriale Voraussetzungen (wie z.B. Behinderung oder Erwerbsunfähigkeit) vorsehen, handelt es sich um beitragsfreie Leistungen einer Grundsicherung für Personen, die nicht arbeiten können, unabhängig davon, ob der Grund dafür (bloße) Arbeitslosigkeit, Behinderung oder ein anderer Faktor (z.B. die Pflege von Angehörigen) ist. Diese Gewährung solcher beitragsfreier - nicht kategorialer - Leistungen setzt entweder in bestimmten Grenzen eine individualisierende Bewertung des Einzelfalles voraus oder aber erfolgt in Form pauschalierender und standardisierender Verfahren zur Leistungsbestimmung. Einzelne Staaten wiederum sehen eine (im Vergleich zu den nicht kategorialen Leistungen großzügigere) beitragfreie Leistungen einer Grundsicherung speziell für solche Personen vor, die der Kategorie „erwerbsunfähig“ angehören (also nicht „nur“ z.B. arbeitslos sind). Zumeist (also nicht durchweg in allen betroffenen Staaten) setzen beide Varianten der nicht beitragsfinanzierten Leistungen eine Bedürftigkeitsprüfung voraus, die bei einem kategorialen Ansatz (wegen der besonderen Lage und den speziellen Bedürfnissen behinderter oder erwerbsunfähiger Menschen) weniger streng sein kann als bei einem nicht kategorialen Ansatz.

\subsubsection{Inklusion durch Unterstützung bei grundlegenden Alltagsverrichtungen}

\subsubsection{Variationsbreite}

Die Struktur von Ansprüchen auf unterstützende Leistungen und Hilfen bei grundlegenden Alltagsverrichtungen (Nahrungsaufnahme, Körperpflege, Mobilität, haus- 
wirtschaftliche Versorgung) variiert in den einzelnen Mitgliedstaaten der EU in mehrfacher Hinsicht. ${ }^{28}$ Typisch sind hier zum einen die unterschiedliche Abhängigkeit der unterstützenden Leistungen (zum Teil als Leistungen zur Pflege bezeichnet) von der Erwerbsunfähigkeit oder vom Alter des Betroffenen (unten 2.2.4.2.), die verschiedenen Dimensionen der grundlegenden Alltagsverrichtungen (unten 2.2.4.3.) und die unterschiedliche Rolle der Bedürftigkeitsprüfung (unten 2.2.4.4.).

\subsubsection{Erwerbsunfähigkeit und Altersgrenzen}

In einigen Staaten wird die Unterstützung bei grundlegenden Alltagsverrichtungen nur Personen gewährt, die nicht erwerbstätig sind, weil sie Bezieher einer - wegen einer Behinderung - gezahlten einkommenssichernden Leistung wegen Erwerbsunfähigkeit sind. Diese Konzeption beruht offensichtlich auf der Annahme, dass derjenige, der die grundlegenden Alltagsverrichtungen nicht ausüben kann, zwangsläufig auch zu behindert ist, um die komplexeren, schwierigeren und anspruchsvolleren Tätigkeiten auf einem Arbeitsplatz auszuüben. Dass eine solche Annahme nicht unproblematisch ist, ergibt sich schon aus der einfachen Überlegung, dass auf einem Arbeitsplatz spezielle Fähigkeiten und Tätigkeiten erforderlich sein können, die durchaus damit in Einklang stehen können, dass der Betreffende nicht in der Lage ist, bestimmte grundlegende Alltagsverrichtungen selbständig zu verrichten.

Teilweise wird die Unterstützung bei grundlegenden Alltagsverrichtungen vom Erreichen eines bestimmten Lebensalters (und des Bezugs einer Altersrente) abhängig gemacht, offensichtlich in der Annahme, dass erst ab Erreichen eines höheren Lebensalters typischerweise ein Bedarf an Unterstützung bei Alltagsverrichtungen besteht. Zum Teil finden sich aber auch Regelungen, mit deren Hilfe die Bedürfnisse älterer behinderter Menschen in geringerem Maße berücksichtigt werden als die Bedürfnisse jüngerer behinderter Menschen, indem zwar nicht Unterschiede bei den Bedürfnissen gemacht, sondern für dieselben Bedürfnisse geringere Leistungen an ältere behinderte Menschen gezahlt werden. Gerechtfertigt wird dies vor allem damit, dass das Aktivitätsspektrum und damit auch einzelne grundlegende Alltagsverrichtungen in unterschiedlichen Altersphasen auch unterschiedlich sind, nämlich ältere Menschen (etwa solche über 65 Jahre) typischerweise nicht mehr erwerbstätig sind und damit der Unterstützungsbedarf z.B. bei der Mobilität geringer ausfällt. In Staaten freilich, in denen eine solche unterschiedliche Bewertung von Bedürfnissen älterer und jüngerer behinderter Menschen nicht gemacht wird, kann es dazu kom-

Vgl. dazu z.B. - freilich durch die Rechtsentwicklung teilweise überholt - Gabanyi/May/Schneider, Absicherung des Pflegerisikos am Beispiel ausgewählter europäischer Länder, Forschungsbericht des Bundesministers für Arbeit und Sozialordnung, Bd. 218, Bonn 1992; Europäische Kommission (Hrsg.), Sozialschutz bei Pflegebedürftigekit im Alter in den 15 EU-Mitgliedstaaten und in Norwegen, Brüssel 1998; Eisen/Mager (Hrsg.), Pflegebedürftigekit und Pflegesicherung in ausgewählten Ländern, Opladen 1999; Österle/Meichenitsch, Pflegeversicherung in Europa - Neuorganisation der Pflege, SozSich (Österreich) 2007, 536 ff. 
men, dass mit der Zunahme älterer behinderter Menschen (die in allen Staaten der EU festzustellen ist) die zur Verfügung stehenden Mittel zu Lasten der jüngeren behinderten Menschen eingesetzt werden. Für beide Modelle (Leistungen erst ab einem bestimmten Alter bzw. Gleichbehandlung der Bedürfnisse ohne Rücksicht auf das Alter) stellt sich das Problem, zwischen Bedürfnissen zu unterscheiden, die mit dem ,normalen“ Alter zu tun haben, und jenen, die aufgrund spezifischer Umstände - die z.B. einer „Behinderung“ geschuldet sind - zusätzlich bestehen. Für diese Unterscheidung wird zumeist mit Hilfe medizinischer Indikatoren eine Bewertung der zusätzlichen (spezifisch behinderungsbedingten) Bedürfnisse vorgenommen.

Beide Konzeptionen, also sowohl die Koppelung an die Erwerbsunfähigkeit als auch die Koppelung an das Erreichen eines bestimmten Lebensalters, müssen zudem im Zusammenhang gesehen werden mit der institutionellen Zuständigkeit für die Gewährung von Unterstützung bei grundlegenden Alltagshandlungen, nämlich der Zuständigkeit von Systemen der Erwerbsunfähigkeits- oder Altersrentenversicherung.

\subsubsection{Dimensionen der grundlegenden Alltagsverrichtungen}

Sowohl in den Staaten, in denen eine Unterstützung bei grundlegenden Alltagsverrichtungen unabhängig von einer Erwerbsunfähigkeit oder unabhängig vom Erreichen eines bestimmten Lebensalters gewährt wird, als auch in den Staaten, die abhängig von den genannten Voraussetzungen eine Unterstützung bei grundlegenden Alltagsverrichtungen gewähren, findet man sehr unterschiedliche Ausgestaltungen einer solchen Unterstützung:

- So berücksichtigen die Mitgliedstaaten nicht alle die gleichen grundlegenden Alltagsverrichtungen. Zwar ist ein gewisser Kernbereich (z.B. Körperpflege) in den meisten Staaten vorzufinden; bestimmte grundlegende Alltagsverrichtungen bleiben hingegen teilweise außer Betracht, so unter anderem die Mobilität (z.B. werden nur interne, also innerhalb der Wohnung stattfindende, nicht aber externe Mobilität berücksichtigt), die „sozialen“ Aktivitäten wie Kommunikation und Unterhaltung sowie die hauswirtschaftlichen Verrichtungen. Es erfolgt also eine Gewichtung der Bedürfnisse in solche, die zu befriedigen sind und solche, die unberücksichtigt bleiben.

- Werden Geldleistungen für die Unterstützung bei grundlegenden Alltagsverrichtungen gewährt, so wird das Problem, wie die Verwendung der Geldleistung $\mathrm{zu}$ kontrollieren ist - jeweils nach der dem Betroffenen rechtlich eingeräumten Autonomie - unterschiedlich geregelt: Die Lösung des Problems reicht von einem „Laissez-faire“-Ansatz über eine strukturierte Möglichkeit der autonom organisierten Hilfe (z.B. der behinderte Mensch als Arbeitgeber einer Hilfeperson für die grundlegenden Alltagsverrichtungen) bis hin zu einer mehr oder weniger minutiösen Kontrolle der zweckbestimmten Verwendung der Geldleistung. 


\subsubsection{Bedürftigkeitsprüfung}

In einigen Staaten ist die Gewährung von Unterstützung bei grundlegenden Alltagsverrichtungen (zum Teil auch gekoppelt an Altersgrenzen) mit einer Bedürftigkeitsprüfung verbunden, also nicht nur an kategoriale Kriterien (wie Behinderung oder Lebensalter), sondern an eine Prüfung (im Sinne einer nicht-kategorialen Maßnahme), die nur denjenigen den Weg zu Leistungen eröffnet, die nicht mit eigenen Mitteln (insbesondere mit ihrem Einkommen und Vermögen) selbst den vorhandenen Bedarf an Unterstützung bei grundlegenden Alltagsverrichtungen decken können.

\subsubsection{Inklusion durch Selbstbestimmungsrechte}

Zunehmend ist zu beobachten, dass sich in den Rechtsordnungen einzelner Mitgliedstaaten der EU die Erkenntnis durchsetzt, wie wichtig die individuelle Autonomie für behinderte Menschen ist, insbesondere die Freiheit, eigene Entscheidungen zu treffen. ${ }^{29}$ Die für ein selbstbestimmtes Handeln behinderter Menschen erforderlichen rechtlichen Instrumente sind bislang freilich nur für Teilbereiche und auch dort nur in bestimmten Ansätzen entwickelt worden. Nach wie vor scheint in den Mitgliedstaaten der EU nicht durchgängig und dominant ein „SelbstbestimmungsModell“, sondern überwiegend (noch) ein „Fürsorge-Modell“ vorherrschend zu sein.

Exemplarisch für Elemente eines solchen „Selbstbestimmungs-Modells“ ist ein (unterschiedlich ausgeprägtes) Wunsch- und Wahlrecht des behinderten Menschen (etwa im Hinblick auf die Art oder die Ausführung einer Leistung), das freilich unter anderem durch Maximen wie „Berechtigung“ eines Wunsches oder „Wirtschaftlichkeit“ und „Sparsamkeit“ bei der Leistungserbringung begrenzt sein kann. In diesem Zusammenhang lässt sich unter anderem das Bestreben feststellen, Hilfeleistungen für behinderte Menschen so zu organisieren, dass die behinderte Person so weit wie möglich selbst bestimmen kann, z.B. welche Dienste sie in Anspruch nehmen möchte und in welcher Form diese bereitgestellt werden, um ihre persönliche Wünsche und Vorlieben zu berücksichtigen (etwa bei der Wahl zwischen der Versorgung in Großeinrichtungen oder unabhängigem, von gemeindeintegrierten Diensten unterstütztem Wohnen). ${ }^{30}$

Vgl. nur Waldschmidt, Selbstbestimmung als behindertenpolitisches Paradigma - Perspektiven der Disability Studies, Aus Politik und Zeitgeschichte B 8/2003, S. 13 ff.

30 Dazu Mansell/Knapp/Beadle-Brown/Beecham, Übergang von Großeinrichtungen zu selbstbestimmtem Leben in der Gemeinde - Ergebnisse und Kosten: Bericht einer europäischen Studie, Canterbury 2007, die den entsprechenden Veränderungsprozess in drei Ländern (England, Deutschland und Italien) untersuchen. Vgl. auch Horn, Gemeindenah betreut statt stationär versorgt, Sozialrecht und Praxis 2008, S. 226 ff. Ferner: Inclusion Europe u.a. (Hrsg.), Included in Society - Ergebnisse und Empfehlungen der europäischen Studie über gemeindenahe Wohnangebote für Menschen mit Behinderungen, Brüssel 2004 (dort insbesondere in Anhang I: „Studie zu rechtlichen und finanziellen Grundlagen von Wohnangeboten für behinderte Menschen in Europa"). 
Anzutreffen sind ferner - insbesondere als Ausfluss des „SelbstbestimmungsModells“ -

- unterschiedlich ausgeprägte Mitwirkungs- und Mitspracherechte behinderter Menschen (z.B. am Arbeitsplatz in Gestalt von Interessenvertretungen behinderter Beschäftigter oder in Einrichtungen in Gestalt von Beiräten behinderter Bewohner einer solchen Einrichtung); ${ }^{31}$

- die Erbringung von Leistungen für behinderte Menschen in Form von Persönlichen Budgets ansehen, die eine Alternative zur fremdbestimmten Leistungszuweisung darstellen und dem behinderten Menschen die Möglichkeit eröffnen kann, in bestimmtem Umfang autonom über die Art der Leistung und den Leistungserbringer $\mathrm{zu}$ entscheiden. ${ }^{32}$ Allerdings wirft diese Form der Leistungserbringung eine Fülle von (auch rechtlichen und finanziellen) Problemen auf, die in den Rechtsordnungen, in denen das Persönliche Budget vorgesehen ist, noch nicht in vollem Umfang gelöst sind. ${ }^{33}$

- die Abschaffung oder zumindest Abschwächung von Entmündigung oder sonstiger Einschränkung der rechtlichen Handlungsfähigkeit behinderter Menschen (z.B. des Rechts zu heiraten oder an Wahlen teilzunehmen), ${ }^{33}$

- die unterschiedlichen Formen der autonomen Gestaltung der Phase des „Ruhestandes" älterer behinderter Menschen.

\subsubsection{Inklusion durch Recht auf Barrierefreiheit}

Um behinderten Menschen ein unabhängiges Leben und die volle Teilhabe in allen relevanten Politikbereichen und an allen Aspekten des Lebens zu ermöglichen, sind in einzelnen Staaten der EU mehr oder weniger intensive Bemühungen festzustellen, rechtliche Regelungen und Maßnahmen zu schaffen, um Hindernisse, die der Chancengleichheit von Menschen mit Behinderungen immer noch entgegenstehen, weiter abzubauen (disability mainstreaming). ${ }^{34}$

31 Vgl. dazu aus deutscher Sicht Schlummer/Schütte, Mitwirkung von Menschen mit geistiger Behinderung: Schule, Arbeit, Wohnen, München 2006.

32 Zur rechtlichen Konstruktion von Persönlichen Budgets und den Erfahrungen mit dieser Leistungsform in den Niederlanden, in Großbritannien und in Schweden vgl. Hajen, Persönliche Budgets in der Behindertenpolitik, NDV 2001, 113 ff; Loeken, Persönliches Budget für behinderte und pflegebedürftige Menschen im europäischen Vergleich, in: AHA e.V./Windisch (Hrsg.), Persönliches Budget. Neue Form sozialer Leistung in der Behindertenhilfe und Pflege. Nutzerorientierung oder Sparzwang?, Neu-Ulm 2006, S. 30 ff. (Materialien der AG SPAK, M 192); Bundestags-Drucksache $16 / 3983$ (vom 21.12.2006), S. 19 ff.

33 Exemplarisch (zu Niederlande, Schweden und Großbritannien) Schmidt, Das Persönliche Budget Mehr Selbstbestimmung und Teilhabe für Menschen mit einer Behinderung oder eine neue Form der Einsparpolitik?, Oldenburg 2005; Loeken (oben Fn. 32).

33 Vgl die Beispiele bei Hellmann, Rechtliche Unterstützung und Vertretung für Menschen mit geistiger Behinderung in den EU-Staaten, BtPrax 2006, $87 \mathrm{ff}$.

34 Disability Mainstreaming bedeutet in diesem Zusammenhang, dass das Anliegen von Menschen mit Behinderung zu einem wichtigen Bestandteil von Prozessen in Politik, Verwaltung und Gesellschaft wird, und zwar von Beginn an und nicht erst, nachdem Entscheidungen gefallen sind. Des- 
Ein Schwerpunkt liegt dabei auf der Herstellung von Barrierefreiheit, indem etwa Produkte (z.B. Telefone, Geldautomaten), die physische Umgebung (z.B. Gebäude), Transportmittel (z.B. Eisenbahnen), Informations- und Kommunikationstechniken (eAccessibility, z.B. barrierefreie Webseiten für sehbehinderte und blinde Menschen) sowie andere Einrichtungen und Dienstleistungen, die der Öffentlichkeit zur Verfügung stehen, in einer für alle Menschen - unabhängig von einer Behinderung gleichermaßen nutzbaren und zugänglichen Art und Weise gestaltet werden. In diesem Bereich der Barrierefreiheit (accessibility) ist europaweit eine Zunahme von rechtlichen Regelungen (z.B. in Gestalt von Behindertengleichstellungsgesetzen) zu beobachten, die auf den Abbau von Barrieren und auf die Herstellung von Barrierefreiheit zielen, und zwar nicht nur im Hinblick auf die physische Umwelt (wie z.B. Gebäude), sondern - im Sinne einer weit verstandenen Barrierefreiheit - unter anderem auch auf den chancengleichen Zugang zu Recht, Rechtsschutz und Rechtsetzung. Exemplarisch ist hinzuweisen auf die rechtliche Verankerung

- von Nutzungsrechten behinderter Menschen (dies bedeutet unter anderem, dass eine Beförderung - z.B. im Flug-, Eisenbahn- oder Busverkehr - nicht allein wegen einer Behinderung ausgeschlossen werden darf); ${ }^{35}$

- von Mindeststandards bei der Gestaltung von Verkehrsmitteln (z.B. Einstiegshilfen) oder Gebäuden (z.B. Rampen für Rollstuhlfahrer);

- von Klagerechten der Verbände behinderter Menschen (um die Möglichkeiten zur Durchsetzung von Barrierefreiheit im Interesse der einzelnen behinderten Menschen zu stärken);

- $\quad$ eines erleichterten Zugangs zu Auskunft und Beratung - speziell zu Leistungen für behinderte Menschen - durch staatliche Stellen oder (mit Hilfe staatlicher finanzieller Unterstützung) durch Selbsthilfeorganisationen oder Selbsthilfegruppen behinderter Menschen;

- von speziellen Beschwerdestellen, z.B. in Gestalt von „Behindertenbeauftragten“ oder von „Ombudsmännern“, an die sich behinderte Menschen im Falle einer (wenn auch nur vermeintlichen) Verletzung ihrer Rechte wenden und dann Unterstützung bei der Durchsetzung ihrer Rechte erfahren können;

- eines Einbezugs von Organisationen behinderter Menschen in den Prozess der Rechtsetzung und Rechtsumsetzung.

Allerdings darf nicht übersehen werden, dass einzelne der genannten Regelungen zur Herstellung von Barrierefreiheit mit etlichen rechtlichen Vorbehalten versehen sind (z.B. Abhängigkeit der barrierefreien Gestaltung bestimmter Gebäude - wie

halb ist Disability Mainstreaming einerseits ein Instrument zur Umsetzung von Barrierefreiheit und Gleichstellung behinderter Menschen und andererseits ein Konzept, weil es eine andere Vorgehensweise und ein grundlegendes Umdenken bzw. einen Perspektivwechsel erfordert. Dazu z.B. Grüber, „Disabiltiy Mainstreaming“ als Gesellschaftskonzept - Annäherungen an einen vielversprechenden Begriff, Sozialrecht und Praxis 2007, S. $437 \mathrm{ff}$.

35 Eingehend zum dieser Entwicklung -auch unter Berücksichtigung der europarechtlichen Entwicklungen- Sieger, Mobilität für alle - Herausforderung für ganz Europa, Sozialrecht und Praxis 2007, S. $635 \mathrm{ff}$. 
etwa der Bahnhöfe - von der Zahl der potenziellen behinderten Nutzer), und dass nicht wenige der Regelungen lediglich Programme formulieren, deren Missachtung keinerlei rechtliche Sanktionen zur Folge haben und deshalb eher symbolische Politik darstellen (deren Wirkung freilich indes nicht unterschätzt werden darf).

\subsubsection{Inklusion durch Antidiskriminierungs- und Nachteilsausgleichsrechte}

\subsubsection{Antidiskriminierungsrechte}

Ungeachtet unterschiedlicher Ansätze in den verschiedenen Staaten der EU sehen die meisten dieser Staaten in irgendeiner Weise in ihren Verfassungen Regelungen vor, die eine Diskriminierung von Menschen mit Behinderungen verbieten. ${ }^{36}$

Festzustellen ist dabei, dass eine Reihe von Verfassungen ausdrückliche Regelungen vorsieht, welche die Diskriminierung behinderter Menschen verbieten, während in anderen Ländern die Rechtsgrundlage für das Verbot einer Benachteiligung behinderter Menschen das allgemeine Diskriminierungsverbot ist. ${ }^{37}$ Hinzu kommt, dass in allen Staaten der EU inzwischen Vorschriften bestehen, die eine (unmittelbare oder mittelbare) Benachteiligung bzw. die Anweisung zu einer Benachteiligung aus Gründen einer Behinderung im einfachen Recht, insbesondere im Zivil- und Arbeitsrecht ${ }^{38}$ grundsätzlich verbieten und nur in engen Ausnahmen eine unterschiedliche Behandlung - z.B. im Arbeitsrecht wegen beruflicher Anforderungen gestatten. So dürfen nach den Antidiskriminierungsvorschriften dieser Staaten z.B. Bewerber aufgrund einer Behinderung nur dann abgelehnt werden, wenn ein bestimmter gesundheitlicher Zustand wegen der Art der auszuübenden Tätigkeit oder der Bedingung ihrer Ausübung eine wesentliche und entscheidende berufliche Anforderung darstellt, der Zweck rechtmäßig und die Anforderung angemessen ist.

36 Allgemein zum Diskriminierungsverbot wegen einer Behinderung in den Staaten der EU u.a. Breslin/Yee, Disability Rights and Policy: International and National Perspectives, New York 2002; EU Network of Independent Experts on Disability Discrimination - Baseline Study: Disability Discrimination Law in the EU Member States, Utrecht 2004; Cormack/Bell, Entwicklung des Antidiskriminierungsrechts in Europa - Ein Vergleich in den 25 EU-Mitgliedstaaten, Utrecht 2005, S. 24 ff.; Degener, Antidiskriminierungsrechte für Behinderte: Ein globaler Überblick, ZaöRV 2005, S. 887 ff; dies., Disability Discrimination Law: A Global Comparative Approach, in: Lawson/Gooding (ed.), Disability Rights in Europe: From Theory to Practice, Oxford/Portland 2005, S. 87 ff.; McColgan/Niessen/Palmer, Comparative analysis on national measures to combat discrimination outside employment and occupation, Utrecht 2006; Schiek (Hrsg.), Allgemeines Gleichbehandlungsgesetz (AGG), München 2007, § 1 Rz 1 ff. Zum europarechtlichen Hintergrund der Antidiskriminierungsesetzgebung in der EU-Staaten vgl. Bell, Anti-Discrimination Law and the EU, Oxford 2002; Leder, Das Diskriminierungsverbot wegen einer Behinderung, Berlin 2006; Schiek/Waddington/Bell (ed.), Cases, Materials and Text on National, Supranational and International Non-Discrimination Law, Oxford 2007.

37 Zum Ganzen vgl. Steinmeyer (oben Fn. 1), Rz. 14 ff. mit rechtsvergleichenden Hinweisen.

38 Zu Regelungen im Strafrecht vgl. Steinmeyer (oben Fn. 1), Rz. 24 ff. 
Zum Teil sind die Vorschriften zum Verbot der Benachteiligung wegen einer Behinderung sowie die Rechtsfolgen einer solchen Benachteiligung über viele Rechtsvorschriften verstreut, zum Teil haben aber die Staaten das Benachteiligungsverbot wegen einer Behinderung in spezifische Antidiskriminierungsgesetze aufgenommen. ${ }^{39}$ Bemerkenswert erscheint hier, dass in den Antidiskriminierungsvorschriften teilweise keine eigene Bestimmung des Begriffs Behinderung erfolgt. Soweit allerdings der Begriff der Behinderung definiert wird, gibt es eine Reihe gemeinsamer Elemente, nämlich zeitliche Anforderungen (so muss die Beeinträchtigung länger andauern oder sogar von Dauer sein), verschiedene Grade der Beeinträchtigung und tatsächlicher Status (in dem Sinne, dass unterscheiden wird, ob zu einer Beeinträchtigung nur bestehende oder auch künftige Beeinträchtigungen - im Sinne einer drohenden Behinderung - zählen). ${ }^{40}$

Typisch für die Rechtsfolgen einer unzulässigen Benachteiligung wegen einer Behinderung sind unter anderem Vorschriften zur Beweislast und zu finanziellen Sanktionen gegenüber dem Arbeitgeber: Fühlt sich nämlich z.B. Stellenbewerber benachteiligt, so reicht im Rahmen eines von ihm angestrengten gerichtlichen Verfahrens die Vorlage von Indizien, die eine Benachteiligung wegen einer Behinderung vermuten lassen. Gelingt ihm dies, ist es am Arbeitgeber zu beweisen, dass keine unzulässige Diskriminierung vorgelegen hat. Kann der Arbeitgeber die Vorwürfe nicht entkräften, drohen ihm Ansprüche auf Schadensersatz (wegen eines konkreten Vermögensschadens) und auf Entschädigung (für den Schaden, der nicht Vermögensschaden ist), die der Höhe nach grundsätzlich unbegrenzt ist. Um die Akzeptanz von Antidiskriminierungsvorschriften bei den Arbeitgebern zu erhöhen, sind teilweise finanzielle Unterstützung seitens des Staates bzw. der Sozialleistungsträger vorgesehen. Insgesamt betrachtet scheint freilich die Erfüllung der den Arbeitgebern obliegenden Verpflichtungen zur Förderung der Gleichbehandlung eher durch staatliche Maßnahmen zur Förderung der Chancengleichheit durchgesetzt zu werden als durch die Begründung individueller Klagerechte.

Schließlich lassen sich - um ein weiteres Beispiel anzuführen -, in den einzelnen Staaten der EU unterschiedliche Ausgestaltungen von Antidiskriminierungs- bzw. Gleichbehandlungsstellen erkennen, an die sich Menschen wenden können, die von Diskriminierung wegen einer Behinderung betroffen sind: So z.B. Ombudsstellen, ferner Stellen, die nur für einzelne Diskriminierungsmerkmale zuständig sind sowie schließlich Stellen, die für verschiedene Diskriminierungsmerkmale zuständig sind und sich die Aufgaben teilen. ${ }^{41}$

39 Dazu die oben in Fn. 36 genannten Publikationen; ferner z.B. für Großbritannien: Disability Discrimination Act 1995 (erweitert duch Disability Discrimination Act 2005); für Deutschland: Allgemeines Gleichbehandlungsgesetz (von 2006).

40 Vgl. Degener, The Definition of Disability in German and International Discrimination Law, Disability Studies Quarterly, Vol. 26 No. 2 Spring 2006 (online).

41 Nachweise bei Laskowski, in: Schiek (Hrsg.) -oben Fn. 36-, Vorbemerkung zu $\S \S 25$ ff., Rz. 1 ff. 


\subsubsection{Nachteilsausgleichsrechte}

Mittelbar verknüpft mit Antidiskriminierungsrechten finden sich in den einzelnen Staaten der EU zahlreiche Regelungen über Nachteilsausgleiche, also über Maßnahmen, Leistungen und Hilfen für behinderte Menschen zum Ausgleich behinderungsbedingter Nachteile oder Mehraufwendungen $\mathrm{zu}$ gewähren. $\mathrm{Zu}$ diesen Nachteilsausgleichen, für die in der Regel bestimmte Voraussetzungen erfüllt sein müssen (z.B. ein bestimmten Schweregrad der Behinderung oder eine spezielle Art der Behinderung) gehören - um nur einige Beispiele zu nennen $-{ }^{42}$

- Ermäßigung bei oder Befreiung von bestimmten Steuern (z.B. von der Einkommensteuer absetzbare Pauschbeträge, Wegfall der Kraftfahrzeugsteuer), ${ }^{43}$

- Förderung im Bereich Beförderung und Verkehr (z.B. Fahrtkostenermäßigung, Parkerleichterungen),

- Unterstützung im Bereich Wohnen und Bauen (z.B. bevorzugte Vergabe von öffentlich finanziertem Wohnraum),

- Hilfen bei Kommunikation und Medien (z.B. Ermäßigung bei Telefongebühren, Befreiung von Rundfunk- und Fernsehgebührenpflicht).

Insgesamt gesehen handelt es sich um eine nur schwer überschaubare Fülle von Nachteilsausgleichen, die sich über die gesamte Breite einer Rechtsordnung erstrecken können, und deren Bedeutung für die betroffenen behinderten Menschen nicht unterschätzt werden darf, vor allem im Hinblick darauf, dass durch solche Nachteilsausgleiche eine Inklusion in die Gesellschaft befördert und damit eine Exklusion durch Diskriminierung wegen einer Behinderung möglichst weitgehend vermieden werden kann.

\section{Resümee}

Der vorstehende Bericht vermag nur einen ersten Einblick in die unterschiedlichen - mit Hilfe des Leitbildes der Inklusion strukturierten - Modelle von rechtlich verfassten Konzepten und Maßnahmen zur Teilhabe behinderter Menschen am Leben in der Gesellschaft vermitteln, die in den Staaten der EU vorzufinden sind. Deutlich wird bei dieser Übersicht aber immerhin zunächst, dass nach wie vor verschiedene Wege beschritten werden, deren Differenzierung in weitem Umfang pfadabhängig von den ungleichartigen kulturellen (politischen, sozialen, rechtlichen usw.) Besonderheiten der einzelnen Staaten sein dürfte. ${ }^{44}$ Zugleich wird aber auch deutlich, dass

42 Ausgewählte rechtsvergleichende Hinweise bei Steinmeyer (oben Fn. 1), Rz. 46 ff.

43 Rechtsvergleichende Hinweise bei Steinmeyer (oben Fn. 1), Rz. 69.

44 Diese Annahme bedürfte freilich einer intensiven interdisziplinären Untersuchung. Allgemein dazu Davy, Pfadabhängigkeit in der sozialen Sicherheit, in: Deutscher Sozialrechtsverband (Hrsg.), Sozialrechtsgeltung in der Zeit, Berlin 2007, S. 103 ff. 
ein Vergleich der unterschiedlichen Modelle bereits jetzt Diskussionen und Reformen in einzelnen Staaten angestoßen hat. Dass man z.B. in Deutschland über ein Persönliches Budget nicht nur diskutiert, sondern sogar einen Rechtsanspruch auf ein solches Persönliches Budget eingeführt hat, ist eine Konsequenz rechtsvergleichender Analysen. ${ }^{45}$ Und schließlich wird auch deutlich, dass es einen (ehedem) schroffen Gegensatz zwischen einer Konzeption der Förderung behinderter Menschen einerseits und einer Konzeption des Schutzes gegen Diskriminierung behinderter Menschen andererseits nicht (mehr) gibt, sondern dass beide Konzeptionen in mehr oder weniger prägnanter Ausprägung in jedem einzelnen Staat der EU nebeneinander bestehen und diese Konzeptionen sich zunehmend ergänzen und - jedenfalls in nicht wenigen Bereichen - zumindest im Ergebnis konvergieren. ${ }^{46}$

46 So die zutreffende Beobachtung von Steinmeyer (oben Fn. 1), Rz. 72 ff. 


\title{
5.8. Behindertenpolitik und Behindertenrecht in Europa: Behindertenpolitische und behindertenrechtliche Grundlegung: die Europäische Union
}

\author{
Von Bernd Schulte
}

1. Europäische Politik und Europäisches Recht

1.1. Europarat

1.2. Europäische Union/Europäische Gemeinschaft

2. Das Recht der Rehabilitation, der Eingliederung und der Teilhabe von

Menschen mit Behinderungen in den Mitgliedstaaten der Europäischen Union

3. Teilhabe und Inklusion von Menschen mit Behinderungen als Gegenstand

Europäischer Politik und Europäischen Rechts

3.1. Entstehung und Entwicklung einer Europäischen Politik für Menschen mit Behinderungen

3.2. Verbot der Diskriminierung wegen einer Behinderung

4. Aktuelle Rechtsentwicklungen - insbesondere das UN-Übereinkommen über die Rechte von Menschen mit Behinderungen - und Ausblick

Annex 1: UN-Konvention über Rechte von Menschen mit Behinderung (2006) (Auszug)

Annex 2:Richtlinie 2000/78/EG des Rates vom 27. November 2000 zur Festlegung eines allgemeinen Rahmens für die Verwirklichung der Gleichbehandlung in Beschäftigung und Beruf (Auszug) 
„Die Würde des Menschen ist unantastbar. Sie ist zu achten und zu schützen“ (Charta der Grundrechte der Europäischen Union - Artikel 1 Würde des Menschen -)

„Die Union anerkennt und achtet den Anspruch von Menschen mit Behinderung auf Maßnahmen zur Gewährleistung ihrer Eigenständigkeit, ihrer sozialen und beruflichen Eingliederung und ihrer Teilnahme am Leben der Gemeinschaft" (Charta der Grundrechte der Europäischen Union - Artikel 26 Integration von Menschen mit Behinderung -)

„...kann der Rat ... Vorkehrungen treffen, um Diskriminierungen aus Gründen ... einer Behinderung ... zu bekämpfen“ (Vertrag über die Gründung der Europäischen Gemeinschaft - Artikel 13 Antidiskriminierungsmaßnahmen -)

„Die Grundsätze dieses Übereinkommens sind: a) die Achtung der dem Menschen innewohnenden Würde, seiner individuellen Autonomie, einschließlich der Freiheit, eigene Entscheidungen zu treffen, sowie seiner Unabhängigkeit; b) die Nichtdiskriminierung; c) die volle und wirksame Teilhabe an der Gesellschaft und Einbeziehung in die Gesellschaft; d) die Achtung vor der Unterschiedlichkeit von Menschen mit Behinderungen und die Akzeptanz dieser Menschen als Teil der menschlichen Vielfalt und der Menschheit; e) die Chancengleichheit; f) die Zugänglichkeit; g) die Gleichberechtigung von Mann und Frau; h) die Achtung vor den sich entwickelnden Fähigkeiten von Kindern mit Behinderungen und die Achtung ihres Rechts auf Wahrung ihrer Identität.“ (Übereinkommen der Vereinten Nationen über die Rechte von Menschen mit Behinderung vom 13. Dezember 2006 - Artikel 3 Allgemeine Grundsätze -) (zitiert nach BR-Drucks. 760/08) 


\section{Europäische Politik und Europäisches Recht ${ }^{1}$}

Europarecht $i . w$. S. umfasst das Recht der europäischen internationalen Organisationen, d. h. sowohl das Recht des Europarats als auch des sich aus der Europäischen Union und der supranationlen Europäischen Gemeinschaft zusammensetzenden Europäischen Staatenverbundes mit seinen gegenwärtig 27 Mitgliedstaaten.

\subsection{Europarat}

Dem Europarat als der älteren und von Anfang an auch vor allem humanitären Zielsetzungsverpflichteten und sozial orientierten internationalen Organisation kommt für die Behindertenpolitik und das Behindertenrecht in Europa zumal auch für die Europäische Union und die Europäische Gemeinschaft als ihrem supranationalen Kern eine Vorläuferfunktion zu.

Die Konvention zum Schutze der Menschenrechte und Grundfreiheiten (Europäische Menschenrechtskonvention - EMRK ) vom 4. November 1950, die am 3. September 1953 in Kraft getreten ist, ${ }^{2}$ wirkt durch den Grundsatz der Völkerrechtstreue auf die Auslegung sonstigen nationalen Rechts einschließlich des Verfassungsrechts ein. Der Europäische Gerichtshof der EG/EU hat die EMRK als Erkenntnisquelle für das Europäische Gemeinschaftsrecht anerkannt. ${ }^{3}$ Die Konvention hat auch bei der Erarbeitung der Charta der Grundrechte der Europäischen Union Pate gestanden.

Eine weitere Grundlage für die Arbeit des Europarats im Sozialbereich ist die $E u$ ropäische Sozialcharta (ESC), in der auch soziale Rechte niedergelegt sind. Um Menschen mit Behinderung ungeachtet ihres Alters und der Art und Ursache ihrer Beeinträchtigung eine wirksame Ausübung des Rechts auf Eigenständigkeit, soziale

1 Vgl. zum Folgenden Schulte, Behindertenpolitik und Behindertenrecht in der Europäischen Union als Gemeinschaftsprojekt, in: von Maydell/ Pitschas/Schulte (Hrsg.), Behinderung in Asien und Europa im Politik- und Rechtsvergleich. Mit einem Beitrag zu den USA, Baden-Baden, 2003, S. 479 ff.; ders., Behindertenrecht und Behindertenpolitik in der Europäischen Union, in: Aus Politik und Zeitgeschichte (APUZ) 2003 B 8, S. 46 ff.; ders., Einordnung des Forschungsprojekts in das Recht der beteiligten europäischen Staaten und in das Europäische Sozialrecht und die Europäische Sozialpolitik, in: Pitschas/von Maydell/Schulte (Hrsg.), Teilhabe behinderter Menschen an der Bürgergesellschaft in Asien und Europa, Eingliederung im Sozial- und Rechtsvergleich, Speyer (Speyerer Arbeitsheft Nr. 41) 2002, S. 11 ff.; ders., Behindertenrecht und Behindertenpolitik in der Europäischen Union, in: Sozialrecht in Deutschland und Europa (ZFSH/SGB) 2008, S. 131 ff. - Teil $1-u$. S. 200 ff. -Teil 2 -. Auf diese Vorarbeiten - siehe dazu auch den Beitrag von von Maydell in diesem Band - wird verwiesen. -

Zum gesamten deutschen Behindertenrecht sehr umfassend und äußerst instruktiv Welti, Behinderung und Rehabilitation im sozialen Rechtsstaat, Tübingen 2005; zum Europäischen Behindertenrecht einschließlich des einschlägigen nationalen Rechts der Mitgliedstaaten vgl. Steinmeyer, Europäisches Behindertenrecht, in: Hanau/Steinmeyer/Wank (Hrsg.), Handbuch des europäischen Arbeits- und Sozialrechts, München 2002, S. 1118 ff., Siehe zum Behindertenrecht europäischer Länder auch Trenk-Hinterberger in diesem Band. 
Eingliederung und Teilhabe am Leben der Gemeinschaft zu gewährleisten, verpflichten sich die Vertragsparteien in Art. 15 ESC

\begin{abstract}
„1. die erforderlichen Maßnahmen zu ergreifen, um für behinderte Menschen Beratung, schulische und berufliche Bildung soweit wie möglich im Rahmen des allgemeinen Systems oder, sofern dies nicht möglich ist durch öffentliche oder private Sondereinrichtungen bereitzustellen; 2. ihren Zugang zur Beschäftigung durch alle Maßnahmen zu fördern, mit denen ein Anreiz für Arbeitgeber geschaffen werden kann, behinderte Menschen in der normalen Arbeitsumwelt einzustellen und weiterzubeschäftigen und die Arbeitsbedingungen an die Bedürfnisse dieser Menschen anzupassen, oder, wenn dies aufgrund der Behinderung nicht möglich ist, durch Gestaltung oder Schaffung von geschützten Arbeitsplätzen entsprechend dem Grad der Beeinträchtigung. Diese Maßnahmen können gegebenenfalls den Rückgriff auf besondere Arbeitsvermittlungs- und Betreuungsdienste rechtfertigen; 3. ihre vollständige soziale Eingliederung und volle Teilhabe am Leben der Gemeinschaft zu fördern, insbesondere durch Maßnahmen, einschließlich technischer Hilfen, die darauf gerichtet sind, Kommunikations- und Mobilitätshindernisse zu überwinden und ihnen den Zugang zu Beförderungsmitteln, Wohnraum, Freizeitmöglichkeiten und kulturellen Aktivitäten zu ermöglichen. “4
\end{abstract}

Bereits auf der Ebene des Europarates sind mithin zwei unterschiedliche Ansätze erkennbar, mit denen einzelne Staaten an die vorstehend aufgeworfenen Fragestellungen herangehen: Zumal in den angelsächsischen Ländern spielt das Verbot der Diskriminierung von Menschen mit Behinderung eine maßgebliche Rolle: Die Eingliederung Behinderter, die Gewährleistung von Chancengleichheit, Gleichbehandlung und Teilhabe sollen verwirklicht werden durch rechtliche Regelungen, die Diskriminierungen wegen einer Behinderung verbieten.

Diesem Ansatz steht herkömmlicherweise ein zumal in den kontinentaleuropäischen Ländern verbreitetes Konzept gegenüber, das auch in Deutschland dominiert und welches sein Hauptaugenmerk auf Defizit- bzw. Nachteilsausgleich und Fördermaßnahmen richtet, durch die gezielt die Lage der Menschen mit Behinderungen verbessert werden soll. Klassisches Beispiel dafür ist das Schwerbehindertenrecht, das u. a. mit Beschäftigungsquoten arbeitet, dem Arbeitgeber Behinderter Pflichten auferlegt, zugleich aber Fördermaßnahmen einschließlich Leistungen an Arbeitgeber vorsieht.

Der Vergleich zeigt, dass diese beiden Ansätze sich zwar deutlich unterscheiden, dass es aber zugleich - in wachsendem Maße - gewisse Konvergenzen gibt: So wird in den Ländern mit „Antidiskriminierungs-Ansatz“ auch verstärkt mit einem „Förderungsmaßnahmen-Ansatz" gearbeitet und wird anderswo - in jüngster Zeit vor allem aufgrund einschlägigen EG-rechtlichen Regelungen - der „Förder-Ansatz“ durch

Vgl. Steinmeyer, Die Rolle des Europarats im Sozialbereich am Beispiel der Behindertenpolitik, in: Becker/Boecken/Nußberge/Steinmeyer (Hrsg.), Reformen des deutschen Sozial- und Arbeitsrechts im Lichte supra- und internationaler Vorgaben, Baden-Baden 2005, S. 65 ff.; auch Schulte, ZfSH/SGB 2008, aaO (wie Fn. 1).-

Kritisch zur Rolle der EMRK im Hinblick auf die Verbesserung des Behindertenschutzes vgl. Clements/Read, The dog that didn't bark: The Issue of Access to Rights under the European Convention Human Rights by Disabled People, in: Lawson/Gooding (Hrsg.), Disability Rights in Europe. From Theory to Practice, Oxford 2005, die u. a. auf die geringe Spruchpraxis des Europäischen Gerichtshofs für Menschenrechte zu den Rechten behinderter Menschen hinweisen. 
Antidiskriminierungsvorschriften ergänzt, und man erkennt in wachsendem Maße, dass nur eine Kombination beider Ansätze zum Ziel führen kann.

„Schrittmacherdienste“ für das Europäische Behindertenrecht i. S. des Behindertenrechts der Europäischen Union/Europäischen Gemeinschaft (im Folgenden im Vorgriff auf die künftige Nomenklatur nach Inkrafttreten des Vertrags von Lissabon und zur Vereinfachung nurmehr: der Europäischen Union) hat der Europarat im Jahre 1992 auch geleistet mit seiner Entschließung über ,Eine kohärente Politik für behinderte Menschen, "5 die eine konzeptionelle Leitlinie vorgibt, welche Forderungen zugunsten von Menschen mit Behinderungen für unterschiedliche Lebens- und Politikbereiche formuliert und gleichsam eine Art „Drehbuch“ für eine zeitgemäße europaweite, d. h. für alle Mitgliedstaaten des Europarechts Geltung beanspruchende - freilich lediglich den Rang einer rechtlich unverbindlichen Empfehlung einnehmenden - Vorgabe für die Behindertenpolitik enthält.

\subsection{Europäische Union/Europäische Gemeinschaft}

Diese rechtlichen und politischen Vorgaben des Europarates - und im Übrigen auch solche der Vereinten Nationen wie die im Dezember 1993 von der Generalversammlung der Vereinten Nationen verabschiedeten Rahmenbedingungen für die Herstellung der Chancengleichheit für Behinderte und jüngst das Übereinkommen über die Rechte von Menschen mit Behinderung vom 6. Dezember 2006 (siehe Annex 1.) wie auch Rechtsinstrumente und Dokumente anderer internationaler Organisationen - Internationale Arbeitsorganisation, Weltgesundheitsorganisation, OECD u. a. - ${ }^{6}$ haben Recht und Politik der Europäischen Union stark geprägt, nachdem diese mit der Europäischen Gemeinschaft als supranationaler Kern zunächst ausschließlich und bis zum heutigen Tag überwiegend ökonomisch orientiert gewesen ist, war doch zentrales Ziel des Vertrags über die Gründung der Europäischen Wirtschaftsgemeinschaft (EWGV) die Errichtung eines Gemeinsamen Marktes, den das in den 1970er Jahren lancierte Binnenmarktprojekt zu vollenden trachtet. ${ }^{7}$

Die durch den Vertrag über die Europäische Union (,Vertrag von Maastricht") erfolgte Gründung der Europäischen Union stellte dann einen weiteren Schritt zur Vertiefung der Europäischen Integration dar: Die durch diesen Vertrag vorgenommene Umbenennung des Vertrags zur Gründung der Europäischen Wirtschaftsgemeinschaft in Vertrag zur Gründung der Europäischen Gemeinschaft spiegelt die Erweiterung der Zielsetzungen des „Europäischen Staatenverbundes ${ }^{68}$ wider, der

6 Vgl. dazu Maschke, Behindertenpolitik in der Europäischen Union, Lebenssituation behinderter Menschen und nationale Behindertenpolitik der 15 Mitgliedstaaten, Hamburg 2008, S. 195 ff.

7 Vgl. im einzelnen die Einheitliche Europäische Akte von 1987 als erste „Revision“ des EWGVertrages und „Blaupause“ des Binnenmarktprojekts

8 Vgl. zu diesem Begriff BVerfGE 89, 155 ff. (sog. „Maastricht-Urteil“) zur Verfassungsmäßigkeit des deutschen Zustimmungsgesetzes zum Vertrag über die Europäische Union („Vertrag von Maastricht") 
nicht mehr allein auf die Herstellung einer Wirtschaftsgemeinschaft gerichtet ist, sondern die Schaffung eines Staatenverbundes in umfassenderem Sinne anstrebt bis hin zu einer Wirtschafts- und Währungsunion, einer Politischen Union und last but not least einer Sozialgemeinschaft (während von einer Sozialunion zu sprechen beim gegenwärtigen Stand der Sozialintegration auf Europäischer (= EU) Ebene m. E. noch verfrüht ist).

Was die Entstehung und Entwicklung einer Europäischen Behindertenpolitik angeht, so war in der ersten Phase der Europäischen Integration Anfang der 1970er Jahre das Soziale lediglich ein „Annex“ zur Wirtschaftspolitik, d. h. soziale Zielsetzungen wurden nur insofern verfolgt, als es die Errichtung des Gemeinsamen Marktes erforderte. Erst allmählich seit Anfang der 1970er Jahre und dann verstärkt Ende der 1980er Jahre, beginnend mit der für die Herausbildung einer genuinen Europäischen Sozialpolitik sehr bedeutsamen, rechtlich unverbindlichen politischen Erklärung der Gemeinschaftscharta der sozialen Grundrechte der Arbeitnehmer von 1989 hat gleichsam als „soziale Flankierung“ des mit der Einheitlichen Europäischen Akte auf den Weg gebrachten Binnenmarktprojekts eine Stärkung der sozialen Dimension auf Gemeinschaftsebene eingesetzt, die seitdem kontinuierlich fortschreitet.

Die Gemeinschaftscharta enthält auch bereits ein „soziales Grundrecht“ zugunsten von Menschen mit Behinderung, auch wenn ansonsten Arbeitnehmerrechte im Vordergrund stehen:

„31. Im Hinblick auf die möglichst vollständige Eingliederung der Behinderten in das Erwerbsleben sind zusätzliche konkrete Maßnahmen zu treffen, die vor allem die berufliche Ausbildung, die Eingliederung und Rehabilitation sowie den Bereich der gesellschaftlichen Integration durch die Verbesserung des Zugangs zu Räumen, der Mobilität, der Verkehrsmittel und der Wohnraumversorgung betreffen." ${ }^{\circ 9}$

Auch heute noch sind die Mitgliedstaaten allerdings im Grundsatz allein für Aufund Ausbau sowie Umgestaltung ihrer Systeme der sozialen Sicherheit zuständig. Doch ist auch dabei das Europäische Gemeinschaftsrechts zu beachten:. So findet beispielsweise das Verbot der Diskriminierung wegen der Staatsangehörigkeit (Art. 12 EG-Vertrag) Anwendung, sind Frauen und Männer grundsätzlich gleich zu behandeln (Art. 141 EG-Vertrag mit dem Grundsatz des gleichen Entgelts für gleich(wertig)e Arbeit und sekundärrechtlich die EG-Gleichbehandlungsrichtlinien für Frauen und Männer in Beschäftigung und sozialer Sicherheit), gelten grundsätzlich auch die Vorschriften des Europäischen Wettbewerbs (Art. 81 - 89 EG-Vertrag) einschließlich Beihilfenrechts und des sekundärrechtlich geregelten Europäischen Vergaberechts, sofern, solange und soweit im Bereich der sozialen Sicherheit wirtschaftlich gehandelt wird (wobei es Ausnahmen gibt für Tätigkeiten von allgemeinem wirtschaftlichen Interesse, $d$. h. für die Wahrnehmung von Gemeinwohlaufgaben). Mit dieser gleichsam mittelbaren Einflussnahme auf die sozialpolitischen Akschaftscharta sozialer Grundrechte und soziales Aktionsprogramm der EG-Kommission - Zwischenbilanz und Ausblick -, in: ZfSH/SGB 1990, S. 225 ff. 
tivitäten der Mitgliedstaaten hat die Europäische Integration aber zugleich eine Einschränkung der faktischer Handlungsautonomie der EU-Staaten auch im Bereich der Sozialpolitik zur Folge, kann die Sozialpolitik einschließlich der Politik der sozialen Sicherheit in den wegen der Übertragung von Zuständigkeiten, z. B. für die Wirtschafts- und Währungspolitik - hierfür „steht“ u.a. der Euro -, auf die supranationale Europäische Gemeinschaft nurmehr postsouveränen Mitgliedstaaten der Europäischen Union nicht mehr als eine rein nationale Domänen betrachtet werden, sondern sie ist zwangsläufig trans- und z. T. supranational.

Von besonders aktueller Bedeutung ist das Zusammentreffen von Gemeinsamem Markt sowie Europäischem Binnenmarkts- und Wirtschaftsrecht einerseits und national gestalteter und auch künftig national verantworteter Sozialstaatlichkeit, nationaler sozialer Daseinsvorsorge und nationalem Sozialrecht einschließlich des Behindertenrechts andererseits: Sowohl die wirtschaftlichen Grundfreiheiten des Gemeinsamen Marktes - Kapitalverkehrsfreiheit und Warenverkehrsfreiheit sowie die Personenverkehrsfreiheiten, d. h. Freizügigkeit der Arbeitnehmer, Niederlassungsfreiheit der Selbstständigen und Dienstleistungsfreiheit - als auch die Wettbewerbsregeln des Europäischen Gemeinschaftsrechts - Verbot wirtschaftsbeschränkender Vereinbarungen und abgestimmter Verhaltensweisen, Verbot der missbräuchlichen Ausnutzung einer marktbeherrschenden Stellung sowie Beihilfenrecht, d. h. die Kontrolle staatlicher Zuwendungen an Unternehmen und Vergaberecht treten in zunehmendem Maße in ein Spannungsverhältnis zu den Institutionen der öffentlichen sozialen Sicherheit, zu den für die Behindertenhilfe traditionell besonders wichtigen „zwischen Staat und Markt“ angesiedelten freigemeinnützigen Institutionen und $\mathrm{zu}$ den privat-gewerblichen und gewinnorientierten Leistungsanbietern, deren Bedeutung ständig zunimmt. Die Sozial- und insbesondere Sozialstrukturpolitik unterliegt nämlich gegenwärtig allenthalben in Europa einer neuen Verantwortungsteilung zwischen Staat, Markt und auch Institutionen „Zwischen Staat und Markt“, z. B. den Verbänden der freien Wohlfahrtspflege, die gerade auch in der Behindertenpolitik eine wichtige Rolle spielen (ein „Paradigmenwechsel“, den man als Übergang vom sozialen Interventionsstaat bzw. Sozialleistungsstaat zum sozialen Gewährleistungsstaat auf den Begriff bringen kann:

Die Daseinsvorsorge in Gestalt der unmittelbaren Leistungserbringung durch den Staat - und das heißt insbesondere auch in kommunaler Regie durch Gemeinden und Gemeindeverbände ${ }^{10}$ sowie auch durch rechtlich eigenständige Körperschaften und Anstalten - wird in zunehmendem Maße durch ein neues Modell staatlicher Gewährleistung von durch private Dritte in staatlichem Auftrag erbrachte gemeinwohlorientierte Dienstleistungen ersetzt. Dieser Wandel entspricht dem Übergang vom sozial intervenierenden Wohlfahrtsstaat der zweiten Hälfte des 20. Jahrhunderts zum aktivierenden und kooperativen - und zugleich aber auch „distanzierteren“ und „schlankeren“ - Wohlfahrts- bzw. Sozialstaat von heute und ist nicht zuletzt auch auf Globalisierung und Kontinentalisierung - in Europa: „Europäisierung“ - zu- 
rückzuführen. $\mathrm{Zu}$ betonen ist in diesem Zusammenhang, dass nicht eine Ausweitung des Anwendungsbereichs des Europäischen Rechts, sondern die zunehmende Ökonomisierung und stärkere Marktorientierung der Sozialpolitik der Mitgliedstaaten der Europäischen Union diese Entwicklung befördert. Damit erlangt das Europäische Gemeinschaftsrecht wachsende Bedeutung auch für die soziale Daseinsvorsorge und damit zugleich für die Behindertenhilfe in den EU-Staaten.

Im Gefolge der Judikatur des Europäischen Gerichtshofs zur Geltung der wirtschaftlichen Grundfreiheiten, namentlich der Warenverkehrs- und Dienstleistungsfreiheit im Gesundheitsbereich ${ }^{11}$ ist diese Entwicklung offenkundig geworden. Bei den Gesundheitsleistungen, die diesbezüglich eine Vorreiterrolle spielen, hat diese „Entterritorialisierung“ bereits dazu geführt, dass es in zunehmendem Maße grenzüberschreitende Patienten- und Klientenmobilität gibt, zumal in Grenzregionen. Auch wenn die grenzüberschreitende Erbringung und Inanspruchnahme von Gesundheits- und Sozialleistungen, für die die Europäische Kommission jüngst einen Richtlinienvorschlag unterbreitet hat, bis heute noch keinen großen quantitativen Umfang erreicht - EU-weit lediglich rd. 1 v. H. der Gesundheitsausgaben -, wird sie in der Zukunft zunehmen, auch für Menschen mit Behinderungen, die beispielsweise Rehabilitationsleistungen im Grundsatz in allen EU-Mitgliedstaaten werden in Anspruch nehmen können, solange die geltend gemachten Kosten nicht den Betrag übersteigen, auf den sich die Leistungsinanspruchnahme im Heimatstaat belaufen würden. ${ }^{12}$

Art. 36 der Charta der Grundrechte der Europäischen Union (GRCh) von 2000, die mit Inkrafttreten des Vertrags von Lissabon ihrerseits gleichfalls Rechtsverbindlichkeit erhalten wird, gewährleistet in Kapitel IV. Solidarität neben anderen sozialen Grundrechten auch den für behinderte Menschen in besonderem Maße relevanten Zugang zu Dienstleistungen von allgemeinem wirtschaftlichem Interesse, wie er durch die einzelstaatlichen Rechtsvorschriften im Einklang mit dem Vertrag zur Gründung der Europäischen Gemeinschaft geregelt ist (siehe Annex 3).

Die Charta gibt die Richtung an, in welche die Sozialgrundrechtlichkeit auf Europäischer Ebene „gedacht“ und fortentwickelt werden wird. Zugleich illustriert die behindertenspezifische Vorschrift des Art. 26 (der diesem Beitrag in vollem Wortlaut vorangestellt ist ${ }^{13}$ ) den hohen Stellenwert des arbeits- und sozialrechtlichen

11 EuGH, Rs. 120/95 (Decker), Slg. 1998, I-1831; Rs. C-156/96 (Kohll). Slg. 1998, I-1931; Rs. C368/98 (Vanbraekel), Slg. 2001, I-5363; Rs. $57 / 99$ (Smits u. Peerbooms), Slg. 2001, I-5473; Rs. C56/01 (Inizan), Slg. 2003, I-12403; Rs. C-385/99 (Müller-Fauré/Van Riet), Slg. 2003, I-4509; Rs. 8/02 (Leichtle), Slg. 2004, I-2641; Rs. 120/95 (Watts), Slg. 2006, I-4325; Rs. C-444/05 (Stamatelaki) - in Slg. noch uv.

12 Vgl. Schulte, Zur Kur nach Abano Terme, zum Zahnarzt nach Antwerpen? Europäische Marktfreiheit und nationales Krankenversicherungsrecht, in: Sozialrecht in Deutschland und Europa (ZfSH/SGB) 1999, S. 269 ff. u. 347 ff.; ferner ders., Die Ausübung der Patientenrechte bei der grenzüberschreitenden Gesundheitsversorgung $-\mathrm{Zu}$ dem Richtlinienentwurf der Europäischen Kommission vom 2. Juli 2008 -, in: Gesundheits- und Sozialpolitik $(G+S)$ 2008, S. .. - Teil $1-$ u. 2009, S. ... - Teil 2 - (in Druck) 
Behindertenschutzes sowie des Diskriminierungsverbots wegen Behinderung im Europäischen Recht. ${ }^{14}$

In allen Mitgliedstaaten der Europäischen Union sind Menschen mit Behinderungen mit zahlreichen und vielfältigen Barrieren konfrontiert, nicht nur virtuell bei der Stellensuche und dem Erhalt des Arbeitsplatzes, sondern auch ganz konkret bei der Suche nach geeigneten Verkehrsmitteln, beim physischen Zugang zu Gebäuden und Einrichtungen im Kommunikationsbereich, bei der Wahrnehmung beruflicher Ausund Weiterbildungsmöglichkeiten und auch beim Zugang zur sozialen Sicherheit. Derartige Barrieren hindern Menschen mit Behinderungen häufig daran oder erschweren es ihnen doch zumindest, sich gleichberechtigt am Arbeitsleben und am gesellschaftlichen überhaupt Leben zu beteiligen. Aus diesem Grund sind Menschen mit Behinderungen auch sowohl häufiger ohne abhängige Beschäftigung als auch seltener selbständig erwerbstätig als nicht behinderten Menschen. (Die durchschnittliche Erwerbstätigkeitsquote für Menschen mit Behinderungen in der EU belief sich um die Jahrtausendwende auf rd. 44 v. H. und lag damit um rd. 17 v. H. unter der Quote für die gesamte Bevölkerung.)

Die Vorschrift des Art. 26 GRCh gewährt kein unmittelbares subjektives Recht, ist aber in verschiedener Hinsicht von Bedeutung: Es wird im Zusammenhang mit dem Diskriminierungsverbot wegen Behinderung klargestellt, dass Fördermaßnahmen dem genannten Ziel nicht entgegenstehen. Es wird auch deutlich, dass das Europäische Recht auf nationaler Ebene entsprechende Rechtsvorschriften und Maßnahmen voraussetzt. Das Recht auf Gleichbehandlung hat eine „Brückenfunktion“ zwischen den Freiheitsrechten und den Gleichheitssätzen einerseits sowie den im Kapitel 4. Solidarität der Charta aufgenommenen wirtschaftlichen und sozialen Grundrechten andererseits. Die Bedeutung des Europäischen Behindertengrundrechts für das nationale Sozialrecht der Mitgliedstaaten geht damit deutlich über eine lediglich symbolische Gesetzgebung hinaus.

Die Grundrechtecharta wird nach ihrem Inkrafttreten zeitgleich mit dem Vertrag von Lissabon, dem dadurch geänderten Vertrag über die Europäische Union und dem an die Stelle des EG-Vertrags tretenden Vertrags über die Arbeitsweise der Europäischen Union eine wichtige Rechtsgrundlage und rechtliche Vorgabe für das Europäische Behindertenrecht sein (siehe Annex 3.).

\section{Das Recht der Rehabilitation, der Eingliederung und der Teilhabe von Menschen mit Behinderungen in den Mitgliedstaaten der Europäischen Union}

Die medizinischen, sozialen und pädagogischen Wissenschaften haben neue Erkenntnisse gewonnen und Modelle vorgestellt darüber, was Behinderung ausmacht. 
In der jüngeren Vergangenheit haben behinderte Menschen selbst in zunehmendem Maße gesellschaftliche Defizite an Teilhabe und Gleichheit artikuliert. ${ }^{15}$

Die internationale Gemeinschaft hat im Rahmen der Vereinten Nationen - jüngst in Gestalt des Übereinkommens der Vereinten Nationen über die Rechte von Menschen mit Behinderung vom 6. Dezember 2006 (siehe Annex 1) -, der Weltgesundheitsorganisation, der Internationalen Arbeitsorganisation, des Europarats und in jüngerer Zeit auch in zunehmendem Maße eben auch des Europäischen Staatenverbundes vieles davon aufgegriffen und in rechtliche Normen und fachlichen Regelwerken gefasst sowie in rechtlich unverbindlichen Empfehlungen und Standards festgehalten und systematisiert.

Der Begriff der Behinderung selbst ist ein über Jahrzehnte gewachsener außerrechtlicher Begriff und mit seinem heutigen Inhalt erst später eingeführt worden.

Behinderungen gehen auf sehr unterschiedliche Gesundheitsstörungen zurück. Bis ins 20. Jahrhundert hinein waren vor allem Blindheit, Taubheit, Verkrüppelung, Geistesschwäche, Geisteskrankheit und Trunksucht verwendete Rechtsbegriffe. Hinzu kamen abstraktere Bezeichnungen für bestimmt Aspekte der gesellschaftlichen Folgen der Gesundheitsstörungen wie Invalidität, Hilflosigkeit und Gebrechen. Andere Begriffe wurden als Reaktionen auf Behinderung geprägt wie Pflegebedürftigkeit oder - z. B. sonderpädagogischer - Förderbedarf. Mit dem steigenden Bewusstsein für die gesellschaftliche Bedingtheit der Behinderung ist neuerlich der Begriff Barriere (Zugänglichkeit) hinzugetreten. Gesellschaftliche Konsequenz einer Behinderung kann Ausschließung von gesellschaftlichem Leben (Exklusion) sein.

Die medizinischen, beruflichen, sozialen und dann auch rechtlichen Bemühungen um den Umgang mit Behinderung und behinderten Menschen sind vor allem mit dem Begriff Rehabilitation bezeichnet worden. Dieser Begriff ist im Kontext von Recht, Gesundheitswesen, Bildungssystem und Arbeitswelt sowie von gesellschaftlichem Leben überhaupt mit jeweils Konnotationen belegt und in unterschiedliche institutionelle, fachliche und rechtliche Kontexte einbezogen worden.

Die „International Classification of Impairments, Disabilities and Handicaps“ der Weltgesundheitsorganisation betont den gesellschaftlichen Kontext, in dem Menschen mit Behinderungen leben, sowie ihre positiven Möglichkeiten (Funktionsfähigkeiten) zur Erreichung aktiver und selbstbestimmter Teilhabe als Ziel. Der Begriff Partizipation steht dabei nicht nur für die soziale Überwindung der negativen Befindlichkeit, des handicaps, sondern verkörpert nun eine klare sozialintegrative Zielsetzung. Diese deutliche Richtungsänderung hin zu behindertensoziologischem Denken entspricht weitgehend der Position, welche die „Soziologie der Behinderten" als Wissenschaft vom Zusammenleben der Menschen vertritt. ${ }^{16}$

Die im 19. und 20. Jahrhundert in Kontinentaleuropa entstandenen Rechts- und Sozialordnungen haben die Frage der Behinderung und der behinderten Menschen zum einen im Sozialrecht geregelt, zum anderen aber auch viele weitere Schutznor-

Vgl. zum Vorstehenden Welti, aaO (Fn. 1), m. w. N. 
men geschaffen, vorwiegend im sonstigen öffentlichen Recht einschließlich etwa des Steuerrechts, aber auch im Arbeitsrecht und im Zivilrecht. Dabei ist in der zweiten Hälfte des 20. Jahrhunderts der Begriff der Eingliederung bzw. Integration geprägt worden. Eingliederung soll die Möglichkeit dafür schaffen, dass behinderte Menschen menschenwürdig leben und am gesellschaftlichen Leben teilhaben können. Diese Zielsetzung weist über einen rein kompensatorischen Ansatz und zugleich über das Sozialrecht hinaus. Die Ziele der Rehabilitation können und müssen in allen Bereichen der Gesellschaft und des Rechts verfolgt werden, in denen persönliche Beeinträchtigungen und gesellschaftliche Barrieren sich zu Behinderungen ,verdichten. ${ }^{\text {‘17 }}$ So kann die Teilhabe behinderter Menschen am Arbeitsleben nicht allein mit sozialrechtlichen Mitteln gesichert werden, sondern sie bedarf auch arbeitsrechtlicher Regelungen. Die Mobilität von Rollstuhlfahrern oder Blinden wird nicht alleine durch sozialrechtlich bereitgestellte Hilfsmittel erreicht, sondern auch durch eine barrierefreie Gestaltung von Straßen und öffentlichen wie privaten Gebäuden. Nahezu alle Rechtsbereiche können somit für die Rehabilitation, Integration und Teilhabe behinderter Menschen relevant werden.

Aus soziologischer Sicht ist für das Erreichen des Ziels der Integration nicht zuletzt die Entstigmatisierung wesentlich; Integration ist danach ein auf Solidarität fußender und auf Emanzipation ausgerichteter gesellschaftlicher Interaktionsprozess, der von sozialen Zuschreibungen und Etikettierungen absieht und das Behindertsein als ,normal' belässt und nicht 'verbesondert'. Behinderte Menschen sollen unabhängig von Art und Schweregrad ihrer Beeinträchtigung vorbehaltlos, d. h. unabhängig von ihren Fähigkeiten und Unfähigkeiten in allen Lebensbereichen grundsätzlich die gleichen Zutritts-, Teilnahme- und Teilhabechancen haben wie nichtbehinderte. Ein solcher fundamentaler rechtlicher Ansatz verweist darauf, dass bereits der Terminus „Integration“ ein „Grundrecht im Zusammenleben der Menschen“ betont und zugleich dem international und auch auf Europäischer Ebene favorisierten Begriff von Inklusion entspricht. Für das Ziel der Integration sind nicht zuletzt vielfältige Möglichkeiten von Kontakten zwischen Behinderten und Nichtbehinderten ausschlaggebend, weil diese zum Abbau bestehender Vorurteile führen und behinderten Personen als solche respektierende Einstellungen und Verhaltensweisen hervorbringen können. Nicht zuletzt die Erkenntnis, dass alle Menschen im Laufe ihres Lebens behindert werden können, sollte geeignet sein, Solidarität zwischen Behinderten und Nichtbehinderten zu stiften.

Dies lässt sich dies am Beispiel des gemeinsamen Unterrichts behinderter und nicht behinderter Kinder illustrieren: ${ }^{18}$

17 So plastisch Welti, aaO (Fn. 1), S. 1.

18 Vgl. zu dieser Thematik exemplarisch die Beiträge in: Becker/Graser (Hrsg.), Perspektiven der schulischen Integration von Kindern mit Behinderung. Interdisziplinäre und vergleichende Betrachtungen, Baden-Baden 2004; siehe insbesondere auch Trenk-Hinterberger in diesem Band.-

$\mathrm{Zu}$ europäischen Fallstudien O'Harlon/Randoll (Hrsg.), A child with learnig difficulties in Europe. Nine Case Studies, Baden-Baden 1993; Bloemers, W. u. a. (Hrsg.), European Perspectives on Disabled People/Behinderte Menschen aus europäischen Blickwinkeln, Frankfurt/Main u.a. 2004 
In den Schlussfolgerungen des Rates und der im Rat vereinigten Minister für das Bildungswesen der Mitgliedstaaten vom 14.5.1987 heißt es bereits: ${ }^{19}$

„Die Integration behinderter Kinder in normalen Schulen sollte als wichtiger Teil der Aktion zur Eingliederung der Behinderten in die Gesellschaft gesehen werden. Sie kann für behinderte Kinder zu einem befriedigenderen Erwachsenen- und Erwerbsleben führen. Gleichzeitig ist es für normale Kinder gewinnbringend, gemeinsam mit behinderten Kindern aufzuwachsen, und die Integration behinderter kann zu allgemein wünschenswerten schulischen Reformen Anlass geben",

und in der Entschließung des Rates und der im Rat vereinigten Minister für das Bildungswesen vom 31.5.1990 über die Eingliederung von behinderten Kindern und Jugendlichen in allgemeine Bildungssysteme heißt es:

„1. Die Mitgliedstaaten sind übereingekommen, sich (...) um die Eingliederung (...) behinderter Schüler $(\ldots)$ in ihre allgemeinen Bildungssysteme zu bemühen.

2. Die völlige Eingliederung in das allgemeine Bildungssystem sollte in allen geeigneten Fällen als vorrangige Option gelten. “ ${ }^{20}$

Eine entsprechende Vorgabe (die möglicherweise zu Änderungen in der entsprechenden deutschen Rechtspraxis führen wird, was ihre Bedeutung für die Praxis auch in den EU-Staaten unterstreicht) enthält jetzt auch das UN-Übereinkommen von 2006 (siehe dazu Annex 1.) in Artikel 7 (Behinderte Kinder).

In der Studie „Transforming Disability into Ability“ der OECD ${ }^{21}$ werden Rechte und Pflichten behinderter Menschen zur Teilhabe am Arbeitsleben formuliert und wird Teilhabeleistungen Vorrang vor Geldleistungen gegeben. Insofern sind auch die behinderten Menschen Adressaten aktivierender Sozialpolitik, wie sie auf nationale wie auf Europäischer Ebene programmiert, propagiert und praktiziert wird, zumal im Rahmen einer auch Menschen mit Behinderung berücksichtigenden Beschäftigungspolitik. ${ }^{22}$ Der gegenüber „Integration“ umfassendere Begriff „Inklusion " bezeichnet auf der Europäischen Ebene diesen politischen Ansatz. ${ }^{23}$

Die Rechtsvorschriften, die spezielle Regelungen für Menschen mit Behinderung vorsehen, sind allenthalben nicht in einer einzigen einheitlichen gesetzlichen Regelung enthalten, sondern über verschiedene Rechtsbereiche verstreut - vom Arbeitsund Zivilrecht über das Sozial- und sonstige Besondere Verwaltungsrecht bis hin zum Steuerrecht und in zunehmendem Maße auch dem Verfassungsrecht. Weit gefasst kann der Begriff Behindertenrecht als Sammelbegriff die Gesamtheit aller rechtlichen Vorschriften bezeichnen, die Betroffenen helfen sollen, eine Behinderung bzw. deren Auswirkungen zu überwinden.

19 Vgl. ABl. EG 1987 Nr. C 211 vom 8. 8. 1987.

20 Vgl. ABl. EG 1990 Nr. C 162 vom 3. 7. 1990.

21 OECD (Hrsg.), Transforming Disability into Ability, Paris 2003; dazu auch Welti, Leistungen zur Teilhabe als Element aktivierender Intervention, in: Deutscher Sozialrechtsverband (Hrsg.), Jahrestagung 2003 in Berlin, Berlin 2004, S. 85 ff., 86

22 Vgl. dazu Eichhorst/Kaufmann/Konle-Seidl (Hrsg.), Bringing the Jobless into Work? Experiences with Activation Schemes in Europe and the US, Berlin 2008

23 Vgl. z. B. Europäische Kommission, Gemeinsamer Bericht über die soziale Eingliederung, soziale Sicherheit und soziale Integration, Brüssel 2004 
Das Recht hat die Funktion, getroffene politische Entscheidungen umzusetzen. Zu bewerten, wann Integration gelungen ist, ist herkömmlicherweise Sache der Medizin, der Pädagogik, der Soziologie und auch der Ökonomie. Das Recht kann für möglichst große Konsistenz der im Gesetz materialisierten normativen Vorgaben sorgen, Widersprüche aufzeigen sowie auf offene Fragen hinweisen. Es bestimmt ferner, wer welche Entscheidung in welchem Verfahren $\mathrm{zu}$ treffen hat und setzt dafür den verfahrensmäßigen Rahmen.

In welchem Ausmaß und auf welche Weise Menschen mit Behinderung in die jeweilige Gesellschaft eingegliedert werden und teilhaben können, hängt von zahlreichen Faktoren ab: Durch Kultur, Religion und gesellschaftliche und politische Anschauungen $u$. a. geprägte Leitbilder, die sich auch in der Verfassung und im sonstigen Recht widerspiegeln, sind ggf. ähnlich bedeutsam wie die wirtschaftlichen und sozialen Verhältnisse in einem Land. Auch diesbezüglich ist es nicht zuletzt das Recht, welches gesellschaftliche Leitbilder zu politische Zielvorgaben erhebt, Institutionen schafft, Rechtsansprüche auf Sozialleistungen begründet und überhaupt die Rechtsstellung von Menschen mit Behinderung konkretisiert, u. a. dadurch, dass Verbote und Gebote ausgesprochen, Leistungsansprüche eingeräumt und Institutionen für die Leistungsgewährung und damit Förderung der Integration in die Gesellschaft geschaffen werden. Insofern enthalten die Rechtsordnungen aller EUMitgliedstaaten Vielzahl auch die Behinderten betreffende Regelungen.

Das System der Leistungserbringung stellt sich i. d. R. sehr uneinheitlich dar, weil die Dienste und Einrichtungen von einer Vielzahl unterschiedlicher Träger unterhalten werden: ${ }^{24}$ So erbringen Rehabilitationsträger zwar auch Leistungen in eigenen Einrichtungen, doch bedienen sie sich auch der Hilfe sonstiger öffentlicher, wohlfahrtsverbandlich-freigemeinnütziger oder in zunehmendem Maße auch privatgewerblicher Leistungserbringer.

Es besteht heute von Rechts wegen auch in wachsendem Maße die Möglichkeit, die Wahl des konkreten Leistungserbringers der Entscheidung des behinderten Menschen selbst zu überlassen, indem ihm nach Vorbildern aus einzelnen europäischen Ländern - z. B. den Niederlanden - ein zweckgebundenes persönliches Budget eingeräumt wird, welches es ihm erlaubt, die benötigten sozialen Dienstleistungen selbst „einzukaufen“. Überhaupt kommt dem Wunsch- und Wahlrecht des Betreffenden zunehmende Bedeutung zu.

Im Jahre 2003 hat das Informationssystem der Europäischen Kommission zur sozialen Sicherheit MISSOC (= Mutual Information System on Social Security) in einer Sonderpublikation zum Thema „Soziale Sicherung für Menschen mit Behinderung ${ }^{625}$ in einem ersten Teil einen Überblick über die wichtigsten Entwicklungen in Europa in Bezug auf Menschen mit Behinderung sowie eine Darstellung der Kon-

24 Vgl. exemplarisch für Deutschland, als Problemaufriss und Raster auch für vergleichende Studien geeignet und auch heute noch in methodischer Hinsicht überzeugend Schulin, Die soziale Sicherung der Behinderten, Wiesbaden 1981

25 Europäische Kommission (Hrsg.), Soziale Sicherung für Menschen mit Behinderung. MISSOCInfo 1/2003, Brüssel 2003 
zepte und der Ausgestaltung von Rechten und Leistungen gegeben; ein zweiter Teil enthält Berichte aus den 15 ,alten“ EU-Mitgliedstaaten und den drei Ländern Island, Liechtenstein, Norwegen als neben den EU-Staaten erweiterten Mitgliedstaaten des Europäischen Wirtschaftsraums (EWR) sowie der Schweiz, ${ }^{26}$ die unterschiedene Politikansätze sowie Modelle und Leitbilder der einzelnen Länder widerspiegeln. ${ }^{27}$

Peter Trenk-Hinterberger ${ }^{28}$ führt in seinem Beitrag aus, wie sich die einzelnen nationalen „Modelle“ der Teilhaberechte für behinderte Menschen u. a. unterscheiden durch Leitbilder, Rechts- und Politiktraditionen, Regelungsebenen und Zuständigkeiten - zentral, regional, kommunal (und in der EU in wachsendem Maße eben auch „Europäisch“, d. h. trans- und auch supranational) -, Behinderungsbegriffe, Vielfalt der rechtlichen Regelungsbereiche (u. a. Verfassungsrecht, Sozialrecht, Arbeitsrecht, Zivilrecht, Steuerrecht), unterschiedliche Anknüpfungspunkte für Teilhaberechte (z. B. Art und Ursachen der Behinderung), die an der Realisierung von Teilhaberechten beteiligten (z. B. staatlichen und nicht-staatlichen - gemeinnützigen oder gewinnorientierten -) Akteure, Regelungstechniken (z. B. weit gefasste Rahmenregelungen, die sehr flexible Aktivitäten ermöglichen, und eng gefasste Detailregelungen), Leistungsarten und Leistungserbringungssysteme - z. B. Dienst-, Sach- und Geldleistungen -, soziale Sicherungssysteme - öffentliche oder private Versicherungssysteme mit Kostenerstattungs- oder Sachleistungsprinzip und staatliche Gesundheitsdienste -, unterschiedliche Finanzierungssysteme - z. B. Beitrags-, Steuer- oder Mischfinanzierung -,Techniken der Kooperation, Koordination und übergreifenden Planung und Steuerung - zwischen den beteiligten Trägern der sozialen Sicherheit, zwischen Leistungserbringern sowie zwischen Leistungsträgern und Leistungserbringern $-\mathrm{u}$. a.

Jahrzehntelang beherrschten in den EU-Staaten die Leitbilder Normalisierung und Integration die Diskussion um Teilhabe und Anerkennung behinderter Menschen. Zunehmend werden diese Leitbilder jedoch ergänzt, überlagert und sogar verdrängt von Leitbildern wie Empowerment, Autonomie, Selbstbestimmung und Inklusion. Namentlich das letztgenannte Leitbild der Inklusion prägt zunehmend die Diskussion um die Ausgestaltung und Umsetzung von Rechtsnormen mit Bezug zu

26 Belgien, Dänemark, Deutschland, Griechenland, Spanien, Frankreich, Irland, Island, Italien, Liechtenstein, Luxemburg, Niederlande, Norwegen, Österreich, Portugal, Schweiz, Finnland, Schweden, Vereinigtes Königreich

$27 \mathrm{Zu}$ einem Überblick und auch zum Folgenden vgl. Engel/Pfeuffer, Einführung in: Europäische Kommission (Hrsg.), aaO (Fn. 25), S. 1 ff. sowie die einzelnen Landesberichte.-

$\mathrm{Zu}$ einem aktuellen Überblick über die z. T. auch behindertenspezifischen Leistungen bei Invalidität und Pflegebedürftigkeit vgl. Europäische Kommission, MISSOC, Brüssel 2007. Die soziale Sicherheit in den Mitgliedstaaten der Europäischen Union, des Europäischen Wirtschaftsraums und der Schweiz, Brüssel 2007 (Tabelle II - Krankheit - Sachleistungen; VI: Invalidität und XII: Pflegebedürftigkeit.-

$\mathrm{Zu}$ Indikatoren und statistischen Daten zur Art und Ausmaß von Behinderung in der EU vgl. European Commission/Eurostat, Disability and social participation in Europe, Luxemburg 2001

28 Siehe Trenk-Hinterberger, Teilhaberecht behinderter Menschen in den Mitgliedstaaten der Europäischen Union - Bericht über Leitbilder und Modelle, in diesem Band. 
behinderten Menschen und ihrer Teilhabe an der Gesellschaft, zumal Europäischer Ebene. Es enthält in diesem Zusammenhang das Postulat, dass jeder Mensch in seiner Individualität akzeptiert wird und ihm das Recht zuerkannt wird, in vollem Umfang an den einzelnen Funktionsbereichen der Gesellschaft teilzuhaben, wobei das Recht auf Teilhabe sich auf sämtliche Lebensbereiche erstreckt, die grundsätzlich jedermann - unabhängig von spezifischen Bedürfnissen und Möglichkeiten offen stehen. Dieses Leitbild dient der Strukturierung einzelner Teilhabebereiche auch von Menschen mit Behinderung wie Bildungswesen, Erwerbsleben, Einkommenssicherung, grundlegende Alltagsverrichtungen, u.a. mit den Teilhabezielen Selbstbestimmung (Autonomie), Nachteilsausgleich, Verbot der Diskriminierung/Gleichbehandlung und Barrierefreiheit.

Am 20. Dezember 1993 sind von der Generalversammlung der Vereinten Nationen ,, Rahmenbedingungen für die Herstellung von Chancengleichheit für behinderte Menschen "verabschiedet worden. Danach (gemäß Regel 15) sind die Mitgliedstaaten der Vereinten Nationen gehalten, bestehende diskriminierende Rechtsvorschriften abzuschaffen und die rechtlichen Rahmenbedingungen für die Gleichberechtigung behinderter Menschen zu schaffen (siehe dazu unten 4.).

Im Vorfeld des „Europäischen Jahres“ 2003 ist auf dem Europäischen Kongress der Menschen mit Behinderungen im März 2002 in Madrid die sog. Deklaration von Madrid unter dem Titel „Non-discrimination plus positive action results in social inclusion" proklamiert worden.

Die Europäische Kommission versteht soziale Inklusion als „Prozess, durch den gewährleistet wird, dass Personen, die von Armut und sozialer Ausgrenzung (Exklusion) bedroht sind, die erforderlichen Chancen und Mittel erhalten, um am wirtschaftlichen, sozialen und kulturellen Geschehen voll teilzunehmen und in den Genuss des Lebensstandards und Wohlstands zu kommen, der in der Gesellschaft, in der sie leben, als normal gilt“", und sie definiert Exklusion als „Prozess, durch den bestimmte Personen an den Rand der Gesellschaft gedrängt und durch ihre Armut bzw. unzureichender Grundfertigkeiten oder fehlender Angebote für lebenslanges Lernen oder aber infolge von Diskriminierung an der vollwertigen Teilhabe gehindert werden". ${ }^{29}$

Nicht zufällig nennt sich ein Zusammenschluss von 47 Verbänden aus 34 Ländern, der eine umfassende und koordinierte Strategie zu entwickeln sucht, die darauf abzielt, den Zugang von Menschen mit geistiger Behinderung zu Verwaltung und Justiz zu verbessern, im Anschluss an das von der Europäischen Kommission geförderte Projekt „Inklusion. Recht und Gerechtigkeit für Menschen mit geistiger Behinderung" Inclusion Europe. ${ }^{30}$

29 Europäische Kommission, Gemeinsamer Bericht über die soziale Eingliederung, Soziale Sicherheit und soziale Integration, Brüssel/Luxemburg 2004, S. 12; dazu Schulte, Soziale Inklusion im europäisch-vergleichenden Zusammenhang aus juristischer Sicht, Düsseldorf 2008

30 Vgl. dazu Inclusion Europe, Armut und geistige Behinderung, Brüssel 2005; ders., Gleiche Rechte für alle! Zugang zu Rechten und Gerechtigkeit für Menschen mit geistiger Behinderung, Brüssel 2005 
Im Hinblick auf die Eingliederung in das Erwerbsleben unterscheiden die EUStaaten im Wesentlichen drei Ebenen: Den allgemeinen (,ersten“) Arbeitsmarkt, den besonderen („geschützten“) Arbeitsmarkt mit einem Mindestmaß wirtschaftlich verwertbarer Arbeitsleistung der dort Beschäftigten, und den „therapeutischen“ Beschäftigungssektor. ${ }^{31}$

Ansprüche auf unterstützende Leistungen und Hilfen bei grundlegenden Verrichtungen des täglichen Lebens betreffen vor allem die Nahrungsaufnahme, Körperpflege, Mobilität und die hauswirtschaftliche Versorgung. ${ }^{32}$

In zunehmendem Maße ist auch zu beobachten, dass sich in den Rechtsordnungen einzelner Mitgliedstaaten die Erkenntnis durchsetzt, wie wichtig die individuelle Autonomie für behinderte Menschen ist, mithin die Freiheit, eigene Entscheidungen treffen zu können. ${ }^{33}$ Die für ein selbstbestimmtes Handeln behinderter Menschen erforderlichen rechtlichen Instrumente sind bislang freilich nur Teilbereiche und auch dort nur in bestimmten Ansätzen entwickelt worden in Richtung auf ein „Selbstbestimmungs-Modell“, welches das früher dominierende „Fürsorge-Modell“ zu ersetzen sucht.

Ein Element eines solchen „Selbstbestimmungs-Modell“ ist ein Wunsch- und Wahlrecht des behinderten Menschen etwa im Hinblick auf die Art und/oder Ausgestaltung und Ausführung einer Leistung (welches freilich i. d. R. durch Maximen wie „Berechtigung“ eines Wunsches und „Wirtschaftlichkeit“ der Leistungserbringung u.a. begrenzt ist.

Ausprägungen des „Selbstbestimmungs-Modells“ sind Mitbestimmungs-, Mitwirkungs-, Mitsprache-, Anhörungs-, Informations- und sonstige Beteiligungsrechte etwa am Arbeitsplatz in Gestalt von Interessenvertretungen behinderter Arbeitnehmer oder in Einrichtungen in Gestalt von Beiräten behinderter Bewohner einer solchen Einrichtung.

Die bereits angesprochene Erbringung von Leistungen für behinderte Menschen in Form eines persönlichen Budgets (personal budget) ist eine Alternative zu fremdbestimmter Leistungsbestimmung und -zuweisung und ermöglicht es dem behinder-

31 Vgl. dazu Europäische Kommission (Hrsg.), Benchmarking employment policies for people with disabilities - A study prepared by ECOTEC Research and Consulting Ltd., Brüssel 2000; dies. (ed.), Access to Assistive Technology in the European Union - A Study prepared by Deloitte \& Touche, Brüssel 2003; dies., Disability Manstreaming in the European Employment Strategy, Brüssel 2005, (EMCO/11/290605); vgl. ferner Waddington, Legislation to Employ People with Disabilities: The European and American Way, in: Maastricht Law Journal 1994, S. 376 ff.; dies./Diller, Tensions and Coherence in Disability Policy: The Uneasy Relationship between Social Welfare and Civil Rights Models of Disability in American, European and International Employment Law, in: Breslin/Yee (Hrsg.), Disability Rights Law and Policy: International and National Perspectives, New York 2002, S. $241 \mathrm{ff}$.

32 Vgl. dazu z. B. Europäische Kommission (Hrsg.), Sozialschutz bei Pflegebedürftigkeit im Alter in den 15 EU-Mitgliedstaaten und in Norwegen, Brüssel 1998; dies., MISSOC 2007, Fn. 27); ferner Schulte, „Pflege in Europa“ - Die soziale Absicherung des Risikos der Pflegebedürftigkeit in den Mitgliedstaaten der Europäischen Union -, in: Sozialrecht in Deutschland und Europa (ZFSH/SGB) 2008, S. ...-Teil 1 -, 2009, S. ... - Teil 2 - u. S. ... - Teil 3 - (in Druck)

33 Vgl. Waldschmidt, Selbstbestimmung als behindertenpolitisches Paradigma - Perspektiven der Disability Studies, in: Aus Politik und Zeitgeschichte (APUZ) B 8/2003, S. 13 ff. 
ten Menschen, in bestimmtem Umfang autonom über die Art der Leistung und den Leistungserbringer zu entscheiden.

Auch die Abschaffung oder zumindest Abschwächung von Einschränkungen der rechtlichen Handlungsfähigkeit behinderter Menschen ist hier zu nennen, wie sie in den modernen Regelungen über Betreuung/Vormundschaft (Guardianship) über volljährige Personen ihren Niederschlag findet. ${ }^{34}$ Hier macht das UNÜbereinkommen von 2006 über die Rechte behinderter Menschen wichtige und weitreichende Vorgaben (in Artikel 12: Gleiche Anerkennung vor dem Recht (siehe dazu Annex 1) (die möglicherweise auch zu Änderungen des deutschen Betreuungsrechts nötigen werden). ${ }^{35}$

Schließlich ist die Ausformung eines spezifischen Altenrechts als Schutzrecht für ältere Menschen hier zu nennen.

Um behinderten Menschen ein unabhängiges Leben und volle Teilhabe in allen relevanten Politikbereichen und an allen Aspekten des Lebens zu ermöglichen, sind in einzelnen Mitgliedstaaten mehr oder weniger intensive Bemühungen festzustellen, in umfassender Weise durch rechtliche Regelungen und sonstige Maßnahmen dafür zu sorgen, dass Hindernisse, die der Chancengleichheit von Menschen mit Behinderung immer noch entgegenstehen, abgebaut werden. ${ }^{36}$ Solches sog. ,disability mainstreaming “ bedeutet, dass die Anliegen von Menschen mit Behinderungen $\mathrm{zu}$ einem wichtigen Bestandteil aller Prozesse in Politik, Recht, Verwaltung und Gesellschaft werden, bevor Entscheidungen fallen und Maßnahmen getroffen werden, und erfordert insofern einen Perspektivenwechsel.

Ein Schwerpunkt liegt dabei auf der Herstellung von Barrierefreiheit (bzw. Zugänglichkeit) dergestalt, dass die physische Umgebung (z. B. Gebäude und Straßen), Transportmittel (z. B.- Bahnen und Busse), Informations- und Kommunikationstechnologien sowie andere Einrichtungen und Dienstleistungen, die der Öffentlichkeit zur Verfügung stehen, in einer für alle Menschen - auch solche mit Behinderungen - gleichermaßen nutzbaren und zugänglichen Art und Weise gestaltet werden. In diesem Bereich der „accessibility“ ist eine Zunahme von rechtlichen Regelungen, z. B. in Gestalt von Behindertengleichstellungsgesetzen, zu registrieren im Sinne einer in weitem Sinne verstandenen Barrierefreiheit unter Einschluss des gleichberechtigten und gleich möglichen Zugangs zum Recht einschließlich Rechtsschutz und Rechtsdurchsetzung. Hierzu gehören Klagerechte der Betroffenen und ihrer Verbände, ein erleichterter Zugang zu Aufklärung, Auskunft und Beratung insbesondere auch in Bezug auf Leistungen für Behinderte sowohl durch staatliche aös auch durch nichtstaatliche und staatsunabhängige Hilfsorganisationen und Selbsthilfegruppen, spezielle Beschwerdeinstanzen, z. B. in Gestalt in von „Behin-

Vgl. dazu Schulte, Betreuungsrecht in internationaler Perspektive, München 2009 (Ms. Veröff. i. Vorb.)

35 Vgl. Lachwitz, Übereinkommen der Vereinten Nationen über die Rechte von Menschen mit Behinderung. Auswirkungen auf die Rechte von Menschen mit geistiger Behinderung und/oder psychosozialen Problemen, in: Betreuungsrechtliche Praxis (BtPrax) 2008, S. 143 ff.

36 Vgl. Disability Mainstreaming als Gesellschaftskonzept - Annäherungen an einen vielversprechenden Begriff, in: Sozialrecht und Praxis 2007, S. 437 ff. 
dertenbeauftragten“ oder „Ombudsmännern“ und -,frauen “, an die sich behinderte Menschen im Falle einer wirklichen oder vermeintlichen Verletzung ihrer Rechte wenden und wo sie dann Unterstützung bei der Durchsetzung ihrer Rechte erfahren können, sowie auch der Einbezug von Organisationen behinderter Menschen in den Prozess der Rechtsetzung und Rechtsumsetzung (wie dies exemplarisch bei der Erarbeitung des UN-Übereinkommens über die Rechte von Menschen mit Behinderungen der Fall gewesen ist) (siehe Annex 1). ${ }^{37}$

Auch sehen viele Mitgliedstaaten der EU mittlerweile nicht zuletzt aufgrund der EG-rechtlichen Vorgaben ${ }^{38}$ Regelungen vor, die eine Diskriminierung von Menschen mit Behinderungen verbieten. ${ }^{39}$ In einzelnen Mitgliedstaaten finden sich Regelungen über Nachteilsausgleiche, d.h. über Maßnahmen, Leistungen und Hilfen für behinderte Menschen zum Ausgleich behinderungsbedingter Nachteile oder Mehraufwendungen, wie z. B. Ermäßigung bei oder Befreiung von bestimmten Steuern, Förderung im Bereich Beförderung und Verkehr (z. B. Fahrtkostenermäßigung), Unterstützung im Bereich Wohnen und Bauen (z. B. bevorzugte Vergabe von Wohnungen), Hilfen bei Kommunikation und Information (z. B. Ermäßigung bei Telefongebühren, Befreiung von den Rundfunk- und Fernsehgebührenpflicht).

Deutlich wird bei diesem kursorischen Überblick, dass nach wie vor in den EUStaaten unterschiedliche Wege beschritten werden, deren Ausgestaltung in weitem Umfang ,pfadabhängig“ von den unterschiedlichen politischen, kulturellen sozialen, rechtlichen usw. Besonderheiten der einzelnen Staaten bestimmt wird. ${ }^{40}$ Zugleich wird aber auch deutlich, dass ein Vergleich der unterschiedlichen Modelle bereits jetzt Diskussionen und Reformen in einzelnen Staaten angestoßen hat.

Dass man z. B. in Deutschland über ein persönliches Budget nicht nur diskutiert, sondern sogar einen Rechtsanspruch auf ein solches persönliches Budget eingeführt hat, ist eine Konsequenz rechtsvergleichender Analysen und das Studium EUausländischer ,good practices“.

37 Vgl. dazu den Beitrag von Lachwitz in diesem Band m.w.N.

38 Siehe dazu unten 3.

39 Allgemein zum Diskriminierungsverbot wegen einer Behinderung in den Staaten der EU u. a. vgl. Breslin/Yee, Disability Rights and Policy, International and National Perspectives, New York 2002; EU Network of Independent Experts on Disability Discrimination, Baseline Study: Disability Discrimination Law in the EU Member States, Utrecht 2004; Cormack/Bell, Entwicklung des Antidiskriminierungsrechts in Europa - Ein Vergleich in den 25 EU-Mitgliedstaaten, Utrecht 2005, S. 24 ff.; Degener, Antidiskriminierungsrechte für Behinderte: Ein globaler Überblick, Zeitschrift für ausländisches und öffentliches Recht und Völkerrecht (ZaöRV) 2005, S. 887 ff., dies., Disability Discrimination Law: A Global Comparative Approach, in: Lawson/Gooding (Hrsg.), Disability Rights in Europe: From Theory to Practice, Oxford u.a. 2005, S. 87 ff. ; McColgan/Niessen/Palmer, Comparative analysis on national measures to combat discrimination outside employment and occupation, Utrecht 2006; Leder, Das Diskriminierungsverbot wegen einer Behinderung, Berlin 2006; Schiek/Waddington/Bell, Cases, Materials and Text on National, Supranational and International Non-Discrimination Law, Oxford 2007

40 Vgl. dazu Davy, Pfadabhängigkeit in der sozialen Sicherheit, in: Deutscher Sozialrechtsverband (Hrsg.), Sozialrechtsgeltung in der Zeit (Tagung 2006 des Deutschen Sozialrechtsverbandes), Ber$\operatorname{lin} 2007$, S. $103 \mathrm{ff}$. 
Schließlich wird auch deutlich, dass es einen (ehedem durchaus vorhandenen) schroffen Gegensatz zwischen einer Konzeption der Förderung behinderter Menschen einerseits und einer Konzeption des Schutzes gegen Diskriminierung behinderter Menschen andererseits nicht (mehr) gibt, sondern dass beide Konzeptionen in mehr oder weniger prägnanter Ausprägung in jedem einzelnen Mitgliedstaat nebeneinander bestehen und diese Konzeptionen sich zunehmend ergänzen und - jedenfalls in Bereichen - zumindest im Ergebnis konvergieren.

Seit den 1990er Jahren ist in rechtlicher Hinsicht in Europa insofern ein Paradigmenwechsel eingeleitet worden, als behinderte Menschen nicht mehr in erster Linie als Empfänger von Sozialleistungen angesehen werden, sondern als gleichberechtigte Bürger. Dies bedeutet nicht zuletzt eine Abwendung von einem überwiegend medizinischen Verständnis und eine stärkere Hinwendung zu einer sozialen Sehweise von Behinderung, dem es u. a. entspricht, die Probleme behinderter Menschen nicht als individuelles „Schicksal“ aufgrund von Defiziten einzuordnen, sondern als Umweltprobleme i.w.S. zu betrachten.

Diese veränderte Sichtweise auf die Behindertenproblematik tritt nicht nur auf Europäischer Ebene, sondern nahezu weltweit zutage, wie nicht zuletzt das Übereinkommen über die Rechte von Menschen mit Behinderungen von 2006 zeigt (siehe Annex 1.)

In jüngerer Zeit finden sich umfassende Beschreibungen von Behinderungen insbesondere im Zusammenhang mit Diskriminierungsverboten.

So wird beispielsweise ${ }^{41}$ im britischen Disability Discrimination Act eine umfassende Beschreibung gegeben, wobei hier darauf hingewiesen wird, dass aufgrund der jeweils unterschiedlichen Zielsetzungen eine Vereinheitlichung der verschiedenen Definitionen, die sich aus anderen Gesetzen ergeben, nicht geplant ist.

In der Schweiz ist in Bezug auf Maßnahmen zur Beseitigung von Ungleichheiten im Bundesgesetz über die Beseitigung von Benachteiligungen von Menschen mit Behinderungen eine umfassende Beschreibung von Behinderung aufgenommen. Danach liegt eine Behinderung bei Vorliegen einer Funktions- oder Aktivitätsbeeinträchtigung sowie einer Beeinträchtigung im Hinblick auf die gesellschaftliche Teilhabe der Menschen vor; hierbei wird auf das Alter und Geschlecht sowie nach den vorherrschenden sozialen und kulturellen Bedingungen „,normale Maß von Aktivität und Teilhabe abgestellt.

Entsprechend vielfältig sind die verwandten Kriterien für das Vorliegen einer Behinderung.

Die Dauer der Beeinträchtigung dient i.d. R. der Abgrenzung von Behinderung gegenüber einer Krankheit oder den Folgen eines Unfalls; in einigen Ländern werden hier explizit zeitliche Grenzen (i. d. R. sechs Monate) genannt.

Unterschiedliche Leistungsgesetze kommen auch je nach der Ursache oder der Art der Behinderung zur Anwendung (z. B. Frankreich, Luxemburg, Finnland). 
Weitere Kriterien sind der Grad der Behinderung sowie das Alter der Betroffenen, die Art und der Grad der Beeinträchtigung körperlicher und mentaler Fähigkeiten (z. B. Dänemark, Frankreich, Luxemburg) oder eingeschränkte Fähigkeiten bei der Ausübung der Verrichtungen des täglichen Lebens, wozu auch die eingeschränkte Mobilität oder ein Hilfebedarf in Gestalt einer Begleitung oder Assistenz gehört (z. B. Liechtenstein, Luxemburg, Vereinigtes Königreich).

Auch die Minderung der Erwerbsfähigkeit und das Vorliegen von Erwerbsunfähigkeit spielen häufig als Kriterien für Behinderungen eine wichtige Rolle (z. B. Dänemark, Frankreich, Liechtenstein, Luxemburg, Norwegen, Finnland, Vereinigtes Königreich, Schweiz). Einige Länder stellen in ihren Leistungsgesetzen explizit auf die Folgen einer Behinderung.

So lehnt sich z. B .das dänische System an ein „soziales Modell“ an und stellt umfassend die Lebenslage des behinderten Menschen in Rechnung und nicht verschiedene Kategorien von Behinderung.

Häufig wird hinsichtlich der Leistungen zwischen Renten für Erwerbsunfähige/Invaliden auf der einen Seite und Hilfen für erwerbsfähige behinderte Menschen auf der anderen Seite unterschieden (z. B. Dänemark, Norwegen, Frankreich, Liechtenstein).

Die Gewährleistung gesellschaftlicher Teilhabe und Chancengleichheit von Menschen mit Behinderung ist ein zentrales Thema. Häufig enthalten die Verfassungen der Mitgliedstaaten ein ausdrückliches Verbot von Diskriminierung u. a. aufgrund von Behinderung. Der Schutz behinderter Menschen vor Diskriminierung kann überdies durch besondere Gesetze zum Diskriminierungsverbot oder Gleichstellungsgesetze für Menschen mit Behinderungen erreicht werden. Inhaltlich geht es bei den gesetzlichen Regelungen vor allem darum, den Zugang zu Bildung, Arbeit, Diensten, Gütern, Gebäuden und Verkehr so zu gestalten, dass er möglichst auf keine Barrieren trifft, sondern für alle einschließlich behinderter Menschen gleichermaßen möglich ist. In diesem Zusammenhang ist auch auf den bereits angesprochenen Ansatz zu verweisen, Behinderung als Querschnittsthema in allen Bereichen zu berücksichtigen (,mainstreaming“). Besonderes Augenmerk wird des Weiteren nach wie vor auf den Ansatz der Normalisierung gelegt. Auch hier gibt es besondere Maßnahmen zur Eingliederung in den Arbeitsmarkt, zum Abbau von Barrieren im öffentliche Raum, zur Umsetzung des Grundsatzes ,ambulant“ vor „stationär“, zur Schaffung von Wohneinheiten sowie zur Verbesserung der Qualität von sozialen Dienstleistungen, z. B. durch Standardsetzung und Zertifizierung. 


\section{Teilhabe und Inklusion von Menschen mit Behinderungen als Gegenstand Europäischer Politik und Europäischen Rechts}

\subsection{Entstehung und Entwicklung einer Europäischen Politik für Menschen mit Behinderungen}

Von Bedeutung auch für Menschen mit Behinderungen ist seit jeher auch das Europäische koordinierende Sozialrecht, geregelt durch die auf Art. 42 EG (ex 51 EGV) gestützten Verordnung (EWG) Nr. 1408/71 und Nr. 574/72 über die soziale Sicherheit der Arbeitnehmer, Selbständige und Studenten, die in der Gemeinschaft zu- und abwandern (demnächst ersetzt durch die Verordnung (EG) 883 (2004) und ihre noch zu erlassende Durchführungsverordnung). ${ }^{42}$

Nach den Vorschriften über Leistungen bei Krankheit (Art. 18 - 36 VO 1408/71) werden Sachleistungen - z.B. ambulante und stationäre medizinische Behandlung und medizinische Rehabilitationsleistungen - grundsätzlich vom Träger des Wohnoder Aufenthaltsortes der von einem in einen anderen Mitgliedstaate wandernden versicherten Person" gewährt auf Kosten des für die soziale Sicherheit des Betroffenen zuständigen Staates.

Setzt das Vorliegen des Leistungsanspruchs die Erfüllung von Versicherungs- oder Beschäftigungszeiten voraus, so werden nach den Rechtsvorschriften anderer Mitgliedstaaten zurückgelegte Zeiten berücksichtigt (Grundsatz der Zusammenrechnung von Zeiten).

Auch Leistungen bei Pflegebedürftigkeit, die für Menschen mit Behinderung häufig von Bedeutung werden nach Maßgabe der Rechtsprechung des Europäischen Gerichtshofs als Leistungen bei Krankheit behandelt. Demgemäss muss z. B. Pflegegeld ggf. ins EU-Ausland gezahlt werden, d. h. es ist exportpflichtig. ${ }^{43}$ Auch Leistungen der beruflichen Rehabilitation werden von den Gemeinschaftsverordnungen über die soziale Sicherheit erfasst.

Betrachtet man die internationale Entwicklung, so war es eine Tat, ${ }^{44}$ den bereits bestehenden europarechtlichen Schutz vor Diskriminierungen ${ }^{45}$ auf andere Merkma-

42 Vgl. dazu Schulte, Die Geschichte der Reform der Verordnung (EWG) Nr. 1408/71, in: Deutsche Rentenversicherung Bund/Max-Planck-Institut für ausländisches und internationales Sozialrecht (Hrsg.), Die Reform des Europäischen koordinierenden Sozialrechts. Von der VO (EWG) Nr. 1408/71 zur VO (EG) Nr. 883/2004, Berlin 2007, S. 9 ff., m.w.n.; ferner Fuchs (Hrsg.), Europäisches Sozialrecht, Baden-Baden 2005; Marhold (Hrsg.), Das neue Europäische Sozialrecht Wien 2006: demnächst Eichenhofer (Hrsg.), 50 Jahre Europäische Sozialrechtskoordinierung (Jahrestagung des Europäischen Instituts für Soziale Sicherheit 2008 in Berlin) (Arbeitstitel), Berlin 2009 ( $i$. Vorb.)

43 Vgl. zu dieser Problematik die Beiträge in: Sieveking (Hrsg.), Soziale Sicherung bei Pflegebedürftigkeit in der Europäischen Union, Baden-Baden 1998; auch Schulte, aaO (Fn,. 12), Teil 1.

44 Thüsing, Handlungsbedarf im Diskriminierungsrecht. Die Umsetzungserfordernisse auf Grund der Richtlinien 2000/78/EG und 2000/43/EG, in: Neue Zeitschrift für Arbeitsrecht (NZA) 2001, S. 1061 f.; instruktiv auch Davy, aaO (Fn. 40)

45 Vgl. zum US-amerikanischen Recht, das eine ähnliche Entwicklung mit etwa 20-jährigem Vorsprung vor Europa genommen hat, Lewis, Employment Discrimination Law, London 1994. 
le als das Geschlecht auszudehnen. Ein entscheidender Schritt in diese Richtung ist im Jahre 2000 unternommen worden mit der Richtlinie 2000/78/EG, die eine Benachteiligung des Arbeitnehmers wegen der Religion oder der Weltanschauung, einer Behinderung, des Alters oder der sexuellen Identität untersagt.

Die Bedeutung dieser Europäischen Rechtsakte für die EU-Mitgliedstaaten ist außerordentlich groß, zumal für Rechtsordnungen, die wie z. B. die deutsche herkömmlicherweise dem Diskriminierungsschutz keine allzu große Bedeutung beimessen.

Die Politik der Europäischen Union für Menschen mit Behinderungen stand seit Ende der 1980er Jahre im Zeichen der Eingliederung bzw. Integration. Behinderte Menschen sollen die Möglichkeit haben, selbständig an der Gesellschaft in allen ihren Formen und Ausprägungen teilzuhaben auf der Grundlage gleicher Rechte wie alle anderen Bürger. Dieser Politikansatz verlangt, dass die Bedürfnisse von Menschen mit Behinderung grundsätzlich bei der Planung und Durchführung von Maßnahmen in alle relevanten Tätigkeitsbereichen der Gemeinschaft - Beschäftigung, Bildung, Binnenmarkt, soziale Eingliederung, Verkehr usw. - einbezogen werden; eine hervorragende Rolle spielt dabei die Eingliederung in das Erwerbsleben.

Darüber hinaus kommt der Verankerung des Rechts auf Chancengleichheit maßgebliche und wachsende Bedeutung zu. Dies heißt zugleich, dass sich die Politik der Europäischen Union, die in der Vergangenheit auch im sozialen Bereich zunächst ausschließlich und dann doch immer noch wesentlich von wirtschaftlichen Erwägungen bestimmt gewesen ist, sich verstärkt von humanitären und grundrechtlichen Maßstäben leiten lassen muss.

Ein Ausweis dieser politischen Neuorientierung war die von der Europäischen Kommission im Jahre 1996 vorgelegte Mitteilung über die Chancengleichheit für behinderte Menschen, die darauf abzielte, die wesentlichen Hindernisse, mit denen behinderte Menschen konfrontiert werden, zu ermitteln und zu beseitigen, und in der die Europäische Kommission sich darüber hinaus dazu verpflichtet hat, die Anliegen der Menschen mit Behinderung in die Gestaltung der Politik und der Gesetzgebung via ,mainstreaming“ allen Gebieten einzubringen, zugleich aber anerkannt hat, dass die Zuständigkeit für Maßnahmen in diesem Bereich nach wie vor bei den Mitgliedstaaten liegt und die Gemeinschaftsorgane im Wesentlichen auf die Unterstützung mitgliedstaatlicher Maßnahmen und die Förderung der Koordination und der Kooperation zwischen den Mitgliedstaaten beschränkt sind.

Durch Unterstützung der mit Behindertenfragen befassten Nichtregierungsorganisationen und durch das Europäische Behindertenforum ist diese Thematik zugleich in den - hier in Annäherung an den Begriff Sozialer Dialog für den Dialog mit den Sozialpartnern hier so genannten - Zivilen Dialog einbezogen worden.

Das Jahr 2003 ist seinerzeit von der Europäischen Union zum „Europäischen Jahr der Menschen mit Behinderungen" ausgerufen worden. Dadurch sollte nach Angaben der zuständigen Kommissarin allen Menschen speziell ins Bewusstsein gerufen werden, dass Personen mit Behinderungen das Recht auf Gleichbehandlung und auf Teilnahme und Teilhabe in allen Bereichen haben. Es geht darum die Barrieren anzugehen, mit denen behinderte Menschen immer wieder konfrontiert wer- 
den. Letztendlich sollte das Europäische Jahr das Bewusstsein dafür wecken, dass Behinderung ein Thema ist, welches alle angeht. ${ }^{46}$

\subsection{Verbot der Diskriminierung wegen einer Behinderung}

Die Vorschrift des Art. 13 EG, der durch den Vertrag von Amsterdam in den EGVertrag eingefügt worden ist, ergänzt das für die Europäische Integration seit jeher zentrale Verbote der Diskriminierung aus Gründen der Staatsangehörigkeit eines Mitgliedstaats ${ }^{47}$ und des Geschlechts? um eine Reihe weiterer Diskriminierungsverbote, u. a. die Behinderung. ${ }^{48}$ Nachdem durch Art. 13 EG-Vertrag die Europäische Gemeinschaft ermächtigt worden ist, Diskriminierungen aus Gründen des Geschlechts, der Rasse, der ethnischen Herkunft der Religion oder Weltanschauung, einer Behinderung, des Alters oder der sexuellen Ausrichtung zu bekämpfen, ist durch den Vertrag von Nizza dieser Vorschrift ein Absatz 2 angefügt worden, derzufolge der Rat im Verfahren der Mitentscheidung über gemeinschaftliche Fördermaßnahmen entscheidet, mit denen die Mitgliedstaaten zur Verwirklichung der in dieser Vorschrift genannten Ziele beitragen. Aus der Vorschrift des Art. 13 EG selbst lassen sich - im Gegensatz zur Vorschrift des Art. 141 EG (ex 119 EGV) hinsichtlich des Grundsatzes gleichen Entgelts für gleiche Arbeit für Männer und Frauen - keine unmittelbaren Rechte ableiten; sie kann aber unmittelbar die Auslegung des Gemeinschaftsrechts beeinflussen. Seither hat die Europäische Union aber eine genuine Kompetenz für Regelungen gegen Diskriminierung wegen einer Behinderung. Ihre Wahrnehmung setzt einen einstimmigen Beschluss des Ministerrates mit Zustimmung des Europäischen Parlaments voraus. Auf Art. 13 EG gestützt ist die für den Diskriminierungsgrund Behinderung einschlägige Richtlinie 2000/78/EG des Rates zu Festlegung eines allgemeinen Rahmens für die Verwirklichung der Gleichbehandlung in Beschäftigung und Beruf ergangen (siehe Annex 2.). ${ }^{49}$

Ein Bündel von Richtlinien („Europäischen Rahmengesetzen“) ${ }^{50}$ verpflichten u.a. dazu, Schutz vor Diskriminierung in Beschäftigung und Beruf hinsichtlich der

46 Vgl. zum Vorstehenden Schulte, aaO (wie Fn. 1), ZfSH/SGB 2008, S. 208 ff.

47 Vgl. dazu nur Becker, Die Bedeutung des gemeinschaftsrechtlichen Diskriminierungsverbots für die Gleichstellung von Sachverhalten im koordinierenden Sozialrecht, in: Vierteljahresschrift für Sozialrecht (VSSR) 2000, S. 221; Rossi, Das Diskriminierungsverbot nach Art. 12 EGV, in: Europarecht (EuR) 2000, S. $197 \mathrm{ff}$.

48 Vgl. Davy, Das Verbot der Diskriminierung wegen der Behinderung im deutschen Verfassungsrecht und im europäischen Gemeinschaftsrecht, in: Deutscher Sozialrechtsverband (Hrsg.), Die Behinderten in der sozialen Sicherung (Bundestagung 2001 des Deutschen Sozialrechtsverbundes in Schwerin), Wiesbaden 2002, S. $7 \mathrm{ff}$.

49 ABl. EG 2000 Nr. L 306/16

50 Richtlinie (RL) 2000/43/EG des Rates vom 29. Juni 2000 zur Anwendung des Gleichbehandlungsgrundsatzes ohne Unterschied der Rasse oder der ethnischen Herkunft,50 ABl. EG Nr. L 180 S. 22; RL 2000/78/EG des Rates vom 27. November 2000 zur Festlegung eines allgemeinen Rahmens für die Verwirklichung der Gleichbehandlung in Beschäftigung und Beruf, ABl. EG Nr. L 303 S. 16; RL 2002/73/EG des Europäischen Parlaments und des Rates vom 23. September 2002 zur Ände- 
Merkmale Rasse, ethnische Herkunft, Religion und Weltanschauung, Behinderung, Alter, sexuelle Identität und Geschlecht rechtlich insbesondere im Verhältnis zwischen Arbeitgebern und Arbeitnehmern umzusetzen.

Die Richtlinien definieren für ihren jeweiligen Geltungsbereich die unterschiedlichen Arten von Diskriminierung und verpflichten u.a. auch zu wirksamen Sanktionen bei Verstößen gegen das Gleichbehandlungsgebot und sehen Beweiserleichterungen für die Betroffenen vor. Um den Rechtsschutz effektiv zu gewährleisten, schreiben die Richtlinien ergänzend vor, dass Verbände das Recht erhalten sollen, sich zur Unterstützung der Betroffenen an den Verfahren zu beteiligen. Ferner muss eine nationale Stelle („Antidiskriminierungsstelle“) eingerichtet werden, deren Aufgabe es ist, die Verwirklichung des Grundsatzes der Gleichbehandlung aller Personen ohne Diskriminierung zu fördern.

Die $R L$ 2000/78/EG gilt in personeller Hinsicht für alle Personen im öffentlichen und privaten Bereich einschließlich öffentlicher Stellen in Bezug auf die Bedingungen für den Zugang zu selbständiger und unselbständiger Erwerbstätigkeit, zu allem Formen und Ebenen der Berufsberatung, der Berufsausbildung, der beruflichen Weiterbildung und Umschulung, der Beschäftigungs- und Arbeitsbedingungen, sachlicher Hinsicht und die Mitgliedschaft der Arbeitnehmer und Arbeitgeber in Berufsorganisationen (Art. 3 Abs. 1). Ausgeklammert bleiben Leistungen der sozialen Sicherheit und des sozialen Schutzes (Art. 3 Abs. 3). Die Mitgliedstaaten dürfen auch die Streitkräfte ausnehmen (Art. 3 Abs. 4).

Eine Diskriminierung kann verneint werden, wenn das betreffende Unterscheidungsmerkmal aufgrund der Art der Tätigkeit oder der Umstände ihrer Ausübung eine maßgebliche berufliche Anforderung darstellt (Art. 4 Abs. 1).

Arbeitgeber müssen angemessene Vorkehrungen treffen, um die Anwendung des Gleichbehandlungsgrundsatzes auf behinderte Menschen zu gewährleisten (Art. 5).

Der Gleichbehandlungsgrundsatz hindert die Mitgliedstaaten auch nicht daran, zur Herbeiführung tatsächlicher Gleichheit im Berufsleben spezifische Maßnahmen beizubehalten oder einzuführen, mit denen Benachteiligungen verhindert oder ausgeglichen werden können (Art. 7 Abs. 1).

Die Mitgliedstaaten müssen dafür sorgen, dass alle Personen, die sich durch die Nichtanwendung des Gleichbehandlungsgrundsatz in ihren Rechten verletzt fühlen, ihre Ansprüche auf die gerichtlich geltend machen und dabei auch von Verbänden; Organisationen oder anderen juristischen Personen unterstützen oder vertreten lassen können (Art. 9). Zur besseren Rechtsdurchsetzung können sich Personen, die sich diskriminiert fühlen und die Tatsachen glaubhaft machen, die das Vorliegen einer Diskriminierung vermuten lassen auf eine Beweislastumkehr berufen; der beklagten Person obliegt es dann zu beweisen, dass der Gleichbehandlungsgrundsatzes nicht

rung der Richtlinie 76/207/EWG des Rates zur Verwirklichung des Grundsatzes der Gleichbehandlung von Männern und Frauen hinsichtlich des Zugangs zur Beschäftigung, zur Berufsbildung und zum beruflichen Aufstieg sowie in Bezug auf die Arbeitsbedingungen, ABl. EG Nr. L 269 S. 15; RL 2004/113/EG des Rates vom 13. Dezember 2004 zur Verwirklichung des Grundsatzes der Gleichbehandlung von Männern und Frauen beim Zugang zu und bei der Versorgung mit Gütern und Dienstleistungen, ABl. EG Nr. L 
verletzt ist (Art. 10). Schließlich sollen die Mitgliedstaaten den sozialen Dialog zwischen Arbeitgebern und Arbeitnehmern sowie mit Nichtregierungsorganisationen fördern, mit dem Ziel, die rechtlichen Vorgaben der Richtlinien durchzusetzen (Art. 14). (Siehe zur RL 2000/78/EG Annex 2.)

Die Mitgliedstaaten haben die Richtlinie durch Erlass der erforderlichen nationalen Rechts- und Verwaltungsvorschriften umgesetzt. Auf diese Weise rücken - auf nationaler wie auf internationaler und zumal auch Europäischer Ebene - in jüngster Zeit zunehmend das Verbot von Diskriminierung und das Ziel der Chancengleichheit in den Mittelpunkt, und zwar sowohl in der öffentlichen Diskussion als auch in der politischen und rechtlichen Auseinandersetzung.

Damit einher geht ein Paradigmenwechsel im Grundansatz der Politik zugunsten von Menschen mit Behinderung: Den Ausgangspunkt bildet hier nicht mehr die „paternalistische“ oder auch „maternalistische“ sozialfürsorgerische Perspektive, welche die Behindertenpolitik auch noch in den vergangenen Jahrzehnten geprägt hat, sondern im Mittelpunkt der Befassung stehen die behinderten oder von Behinderung bedrohten Menschen als gleichberechtigte Träger der ihnen zustehenden Rechte. Zugleich sollen Hindernisse beseitigt werden, die trotz aller erreichten Fortschritte einer gleichwertigen Teilhabe behinderter Menschen am Leben in der Gesellschaft noch immer entgegenstehen.

In den Begründungserwägungen hat sich der Europäische Gesetzgeber u. a. bezogen auf den Schutz der Behinderten nach der Charta der sozialen Grundrechte der Arbeitnehmer von 1989, auf die Beschäftigungspolitischen Leitlinien aus dem Jahr 2000 auf die Notwendigkeit, einen Arbeitsmarkt zu schaffen, der die soziale Eingliederung fördert, sowie auf die Bedeutung der Bekämpfung von Diskriminierungen auch für die Erreichung der Ziele des EG-Vertrages, nämlich ein hohes Beschäftigungsniveau, ein hohes Maß an sozialem Schutz, die Hebung des Lebensstandards und der Lebensqualität für den wirtschaftlichen und sozialen Zusammenhalt, Solidarität und Freizügigkeit. Die Richtlinie strebt die Schaffung eines allgemeinen Rahmens zur Bekämpfung der Diskriminierung wegen Religion oder Weltanschauung, des Alters oder der sexuellen Ausrichtung und auch der Behinderung in Beschäftigung und Beruf für die Verwirklichung des Grundsatzes der Gleichbehandlung in den Mitgliedstaaten genannt.

Durch den Begriff der Gleichbehandlung wird im Vergleich zum Diskriminierungsverbot gleichsam insofern ein ,offensiverer Akzent“" gesetzt, als diese Formulierung auf ,positive“ statt nur auf ,defensive“ Maßnahmen hinweist. ${ }^{51}$

Sowohl das Aktionsprogramm zur Bekämpfung von Diskriminierungen ${ }^{52}$ als auch die Durchführung des Europäischen Jahres der Menschen mit Behinderungen $2003^{53}$ sind als Fördermaßnahmen auf Art. 13 EG gestützt worden.

51 So zurecht Welti, aaO (Fn. 1), S. 411

52 Beschluss des Rates vom 27. Dezember 2000 über ein Aktionsprogramm zur Bekämpfung von Diskriminierungen (2001-2006) (2000/750/EG), ABl. EG Nr. L 303/23 vom 2. Dezember 2000

53 Beschluss des Rates vom 3. Dezember 2001 über das Europäische Jahr der Menschen mit Behinderungen 2003 (2001/903/EG), ABl. EG Nr. L 335/15 vom 19. Dezember 2001 
Auf der Regierungskonferenz von Amsterdam ist seinerzeit auch eine Erklärung darüber aufgenommen, dass die Organe der Gemeinschaft bei allen Maßnahmen zur Angleichung von Rechts- und Verwaltungsvorschriften den Bedürfnissen von Personen mit Behinderung Rechnung tragen müssen ${ }^{54}$ Diese sog. Querschnittsklausel für die Bekämpfung von Diskriminierungen entspricht dem politischen Prinzip des aus Recht und Politik der Geschlechtergleichbehandlung bereits bekannten „Mainstreaming“: Sie soll dafür sorgen, dass bestimmte Anliegen in der Formulierung aller Gemeinschaftspolitiken umfassend und von Anfang an berücksichtigt werden. Das Gebot, bei staatlichem Handeln die Interessen auch behinderter Menschen zu berücksichtigen, wird so auf dem Wege einer umfassenden Anwendung des Diskriminierungsverbots verwirklicht. ${ }^{55}$

Die Richtlinie 2000/78/EG verbietet nicht nur unmittelbar und mittelbare Diskriminierungen u. a. wegen einer Behinderung, sondern verpflichtet auch Arbeitgeber, geeignete Maßnahmen zu ergreifen, um behinderten Menschen Zugang zur Beschäftigung, Ausübung eines Berufs und beruflichen Aufstieg zu ermöglichen. Die daraus erwachsende Belastung der Arbeitgeber wird durch Maßnahmen in Rahmen der Behindertenpolitik der Mitgliedstaaten zu kompensieren gesucht. Arbeitsrechtliche Gleichstellung und sozialrechtliche Förderung sind insofern keine konträren Konzepte, sondern komplementäre Strategien, die in unterschiedlicher Gewichtung in allen Mitgliedstaaten bereits heute zur Anwendung kommen.

Nach der Rechtsprechung des Europäischen Gerichtshofs zur Gleichbehandlung von Männern und Frauen, dem auch für andere Diskriminierungstatbestände eine Leitfunktion zukommt, ${ }^{56}$ liegt eine Diskriminierung vor, wenn unterschiedliche Vorschriften auf gleiche Sachverhalte angewandt werden oder wenn dieselbe Vorschrift auf ungleiche Sachverhalte angewandt wird.

Der Gleichbehandlungsgrundsatz kann auch nur dann als tatsächlich verwirklicht angesehen werden, wenn bei Verstößen gegen ihn, denjenigen, die Opfer einer Diskriminierung geworden sind, als Sanktion eine dem erlittenen Schaden angemessene Entschädigung zuerkannt wird.

Die Verwirklichung der Gleichbehandlung von Männern und Frauen, ausgehend von der Bestimmung des Art. 141 (ex 119) EG, der den Grundsatz des gleichen Entgelts für Männer und Frauen für gleich(wertig)e Arbeit festschreibt, und umgesetzt durch eine Reihe einschlägiger EG-Richtlinien, ${ }^{57}$ die auch den Bereich der

56 Vgl. z. B. EuGH, RS C-394/96 (Brown), Slg. 1998, I-4185; RS C-342/93 (Gillespiel), Slg. 1996, I475

57 Vgl. die sog. Entgeltgleichheitsrichtlinie RL 75/112/EWG, die sog. Gleichbehandlungsrichtlinie RL 76/207/EWG hinsichtlich der Beschäftigung und ihre Änderungsrichtlinie 2002/73/EG, RL 79/7/EWG in Bezug auf die soziale Sicherheit, RL 86/378/EWG zu den betrieblichen Systemen der sozialen Sicherheit, die Elternurlaubsrichtlinie RL 96/34/EG, die sogenannte Beweislastrichtlinie 80/EG über die Beweislast bei Diskriminierungen wegen des Geschlechts, die Teilzeitarbeitsrichtlinie RL 97/81/EG 
sozialen Sicherheit betreffen, stellt vielleicht das erfolgreichste Kapitel Europäischer Sozialpolitik dar.

Die ausgefeilte Dogmatik des geschlechtsspezifischen EG-Gleichbehandlungsgrundsatzes hat der Konzipierung des allgemeinen Antidiskriminierungsrechts Pate gestanden. Hier kann die Bedeutung der Judikatur des Europäischen Gerichtshofs nicht deutlich genug hervorgehoben werden und auch auf die künftige Rolle des Gerichtshofs im Zusammenhang mit der Auslegung des behindertenspefizischen Antidiskriminierungsrechts ist ausdrücklich hinzuweisen.

Nach der jüngsten Rechtsprechung des Europäischen Gerichtshofs ist eine Diskriminierung wegen einer Behinderung i. S. d. RL 200/78/EG ggf. auch dann gegeben, wenn eine Person nicht selbst behindert ist, sondern dadurch diskriminiert wird, dass eine Person, für die sie Pflegeleistungen erbringt, behindert ist (etwa das Kind einer Arbeitnehmerin). ${ }^{58}$

\section{Aktuelle Rechtsentwicklungen-insbesondere das UN- Übereinkommen über die Rechte von Menschen mit Behinderungen - und Ausblick}

Es gibt eine Europäische Politik für behinderte Menschen. Sie bündelt gleichsam Werte, Prinzipien, Ziele, rechtliche Regelungen und Politiken, die in den Mitgliedstaaten auch nach EU-rechtlichen Vorgaben praktiziert werden, namentlich soziale Leistungen für Menschen mit Behinderung und Maßnahmen gegen Diskriminierung. Hier gilt es auch den Beitrag des Europarates angemessen zu berücksichtigen.

Die sich seinerzeit formierende Behindertenpolitik der Europäischen Gemeinschaft stand in der zweiten Hälfte der 90er Jahre im Zeichen der Eingliederung bzw. Integration behinderter Menschen, sollten und sollen doch alle behinderten Menschen die Möglichkeit haben, selbstständig an der jeweiligen Gesellschaft ihres Heimatlandes in allen ihren Formen und Ausprägungen teilzuhaben auf der Grundlage gleicher Rechte und unter Inanspruchnahme der gleichen Vergünstigungen, wie sie alle übrigen Bürger genießen. Ein derartiger Politikansatz verlangt, dass die Bedürfnisse von Menschen mit Behinderung „gleichsam präventiv“ bereits bei der Planung und Durchführung von Maßnahmen in allen relevanten Tätigkeitsbereichen der Gemeinschaft - Beschäftigung, Bildung, Binnenmarkt, soziale Eingliederung, sozialer Schutz, Verkehr usw. - berücksichtigt werden; eine besonders wichtige Rolle spielt dabei die Eingliederung in das Erwerbsleben und damit dann auch die Beschäftigungspolitik.

Darüber hinaus gibt es eine Abstimmung der Sozialpolitiken der Mitgliedstaaten auf freiwilliger Basis, in jüngster Zeit mündend in die politische Strategie der sog. Offenen Methode der Koordinierung (OMK), die nach ihrer Anwendung in der Wirt- 
schafts- und - seit Ende der 90er Jahre - in der Beschäftigungspolitik seit dem Jahr 2000 auch im Bereich des Sozialschutzes praktiziert wird und die zwar nicht behindertenspezifisch ist, aber doch auch behindertenrelevant im Rahmen der Politik der Bekämpfung der sozialen Ausgrenzung, d. h. der „OMK Soziale Inklusion“, - der „OMK Alterssicherung“ sowie insbesondere auch - und für Menschen mit Behinderung besonders bedeutsam - der „OMK Gesundheitsversorgung und Langzeitpflege“. Tragendes Element dieser Strategie ist die Vereinbarung gemeinsamer Ziele, deren „An-,, und „Abnäherung“ seitens der Mitgliedstaaten anhand gemeinsam konsentierter Indikatoren in einem regelhaften Verfahren erfolgt, welches in einem festen zeitlichen Rahmen eingebettet ist. Dabei wird die jeweilige nationale Entwicklung in einem nationalen Bericht abgebildet, der mit den entsprechenden Berichten der anderen Mitgliedstaaten verglichen und in einem Europäischen Bericht bewertet wird. ${ }^{59}$

Sowohl die nationale - mitgliedstaatliche $-{ }^{60}$ als auch die Entwicklung auf Europäischer Ebene zeigen, dass die Behindertenthematik sowohl eine menschen- und grundrechtliche als auch eine sozialrechtliche Dimension hat. Dabei kommt der Verankerung des Rechts auf Chancengleichheit in allen maßgeblichen Gemeinschaftspolitiken Bedeutung zu.

Die Behindertenpolitik hat sich dahin gewandelt, dass die physischen oder geistigen Beeinträchtigungen eines Menschen nicht mehr i. S. eines „Defizit-Modells“ von Behindertenrecht und - politik thematisiert werden, sondern dass das Potenzial behinderter Menschen zur Beteiligung am gesellschaftlichen Leben und insbesondere am Erwerbsleben sowie die Achtung und Einlösung ihrer Grundrechte im Vordergrund stehen. Dementsprechend werden diese Rechte heute als Verpflichtungen der Regierungen verstanden, Menschen mit Behinderungen nicht zu diskriminieren oder auszugrenzen, rückt $\mathrm{m}$. a. W. der Gedanke der Chancengleichheit auch für Menschen mit Behinderung in den Vordergrund, Antidiskriminierungsvorschriften zu realisieren.

Die Behindertenpolitik der Europäischen Gemeinschaft wird nicht isoliert als eigenständiger Politikbereich betrieben, sondern notwendigerweise im Gesamtkontext der Europäischen Sozial-, Wirtschafts- und Beschäftigungspolitik. (von Maydell hat auf diese Komplexität des Behindertenrechts in seinem Einführungsbeitrag hingewiesen.) So lassen sich als aus diesen Politiken hervorgegangenes ,zur Norm gewordenes" Europäisches Behindertenrecht diejenigen Vorschriften des Europäischen Gemeinschaftsrechts auf den Begriff bringen, die auf Menschen mit Behinderungen Anwendung finden.

Eine Gruppe hochrangiger Experten für Behindertenfragen, bestehend aus Vertretern der Mitgliedstaaten, hat die Aufgabe, Prioritätensetzungen und Maßnahmen der Behindertenpolitiken in den Mitgliedstaaten zu beobachten, Informationen und Erfahrungen zu sammeln und die Europäische Kommission bei der Entwicklung von 
Methoden und bei der Berichterstattung über die Behindertenthematik in der Europäischen Union zu beraten.

Durch Unterstützung der mit Behindertenfragen befassten Nicht-Regierungsorganisationen und durch das Europäische Behindertenforum wird diese Thematik zugleich in den - hier in Annäherung an den Begriff des Sozialen Dialogs für den Umgang mit den Sozialpartnern hier so genannten - Zivilen Dialog einbezogen, in den die Europäische Kommission mit den Akteuren der Zivilgesellschaft in den Mitgliedstaaten eingetreten ist.

Auch der sog. Beschäftigungstitel (Art. 125 - 130 EG) des EG-Vertrags bietet rechtliche Ansatzpunkte für eine so konzipierte neue Behindertenpolitik der Europäischen Union. So hat der Rat im Jahre 1999 eine Entschließung über gleiche Beschäftigungschancen für behinderte Menschen verabschiedet. ${ }^{61}$

In ihrer Mitteilung „Auf dem Weg zu einem Europa ohne Hindernisse für Menschen mit Behinderungen "62 vom Mai 2000 verpflichtet die Europäische Kommission sich zur Entwicklung und Unterstützung einer umfassenden und integrierten Strategie zum Abbau baulicher und konstruktionsbedingter sowie gesellschaftlicher Hindernisse, die Menschen mit Behinderungen unnötigerweise in ihrem Zugang zu gesellschaftlichen und wirtschaftlichen Möglichkeiten einschränken. Die Mitgliedstaaten werden aufgefordert, die Behindertenperspektive bei der Festlegung von Maßnahmen in allein einschlägigen Bereichen einzubeziehen, behinderten Menschen durch den Abbau von Hindernissen eine uneingeschränkte Mitwirkung am gesellschaftlichen Leben zu ermöglichen und auf die öffentliche Meinung dahingehend einzuwirken, dass sie sowohl den Fähigkeiten von Menschen mit Behinderung als auch Strategien, die auf Chancengleichheit für behinderte Menschen abzielen, aufgeschlossen gegenüber steht.

Im letzten Jahrzehnt haben sich europaweit Organisationen von Menschen mit Behinderung für einen neuen Stellenwert der Behindertenthematik im breiteren Rahmen der Menschen- und Bürgerrechte eingesetzt.

Resümierend lässt sich mithin in der Tat die Feststellung treffen, dass es ein Europäisches Behindertenrecht und eine Europäische Politik für Menschen mit Behinderungen gibt. Sie bündeln gleichsam Werte, Prinzipien, Ziele, Rechtsregelungen und Politiken, die in den Mitgliedstaaten respektiert und praktiziert werden, namentlich Sozialleistungen für Menschen mit Behinderung sowie Maßnahmen gegen Diskriminierung, d. h. sie verfolgen Förderkonzeption und Antidiskriminierungsansatz, zwischen denen es aber keinen „Glaubenskrieg“ gibt, ${ }^{63}$ die aber aufeinander abgestimmt sein und verzahnt sein müssen. Ziel ist die Inklusion von Menschen mit Behinderungen.

Mit dem von der Generalversammlung der Vereinten Nationen verabschiedeten Übereinkommen über die Rechte von Menschen mit Behinderungen (siehe Annex 1),

61 Vgl. dazu im einzelnen Europäische Kommission/Generaldirektion, Beschäftigung und soziale Angelegenheiten, in: forum spezial: Fünf Jahre Sozialpolitik, Brüssel 1999, S. 51 ff.

62 KOM (2000) 284 endg.

63 So zurecht Steinmeyer, aaO (Fn. 1), S. 1118 ff. 
das in Deutschland 2007 in Kraft getreten ist,${ }^{64}$ liegt erstmals ein internationales Übereinkommen über die Rechte von Menschen mit Behinderungen für die 192 UNMitgliedstaaten vor, das den Schutz der Menschenrechte aus deren spezifischem Blickwinkel behinderter Menschen regelt. Alle Staaten, die diesen völkerrechtlichen Vertrag ratifizieren, sind verpflichtet, ihre Gesetzgebung zugunsten behinderter Menschen so auszurichten, dass die in der Konvention geregelten Rechte verwirklicht werden und eine gesellschaftliche Entwicklung in Gang gesetzt wird, die Menschen unabhängig von der Art und vom Schweregrad ihrer Behinderung als vollwertige und gleichberechtigte Bürger ihres Landes anerkennt.

Das Übereinkommen basiert auf den zentralen Menschenrechtsabkommen der Vereinten Nationen und konkretisiert die dort verankerten Menschenrechte für die Lebenssituation von Menschen mit Behinderungen. Es verbietet die Diskriminierung von Menschen mit Behinderungen in allen Lebensbereichen und garantiert ihnen die bürgerlichen, politischen und wirtschaftlichen, sozialen und kulturellen Menschenrechte. Das Übereinkommen ist am 3.Mai 2008 nach der zwanzigsten Ratifikation in Kraft getreten

Das ergänzende Fakultativprotokoll ist ein eigenständiger, völkerrechtlicher Vertrag. Es erweitert die Kompetenzen des Ausschusses für Menschen mit Behinderungen nach Artikel 34 des Übereinkommens um das Verfahren der Individualbeschwerde und das Untersuchungsverfahren. Beide Verfahren zielen darauf ab, die Umsetzung und Überwachung des Übereinkommens zu stärken.

Das Übereinkommen tritt als dritte Säule neben die zwei weiteren wichtigen Instrumente zur Förderung der Teilhabe behinderter Menschen auf der Ebene der Vereinten Nationen - das "Weltaktionsprogramm für Menschen mit Behinderungen“ (1982) und die „Rahmenbestimmungen für die Herstellung der Chancengleichheit von Menschen mit Behinderungen" (1993).

Das Weltaktionsprogramm wurde am 3. Dezember 1982 von der Generalversammlung der Vereinten Nationen angenommen (Res. 37/52); es stellt einen umfassenden politischen Rahmen dafür dar, die volle Teilhabe und Gleichbehandlung von behinderten Menschen im gesellschaftlichen Leben zu fördern. Es war ein Schritt, sich weltweit vom Ansatz zu lösen, Behinderung nur als Frage der Prävention und Rehabilitation zu begreifen, und stattdessen einen rechtebasierten Ansatz zu verankern.

Die Rahmenbestimmungen enthalten behindertenpolitische Empfehlungen für 22 Bereiche mit dem Ziel der vollen Teilhabe von behinderten Menschen an der Gesellschaft. Beim Weltaktionsplan und den Rahmenbestimmungen handelt es sich jedoch um rechtlich nicht verbindliche Instrumente. von Menschen mit Behinderungen sowie zu dem Fakultativprotokoll vom 13. Dezember 2006 zum Übereinkommen der Vereinten Nationen über die Rechte von Menschen mit Behinderungen, BGBl. 2008 Teil II Nr. 35 v. 31.12.2008, S. 1419

Vgl. dazu Petri/Stähler, Menschenrechte und Behinderung, in: ZESAR 2008, S. 167 ff.; Degener, Menschenrechtsschutz für behinderte Menschen, in: Vereinte Nationen 2006, S. 104 ff.; siehe dazu auch den Beitrag von Lachwitz in diesem Band. 
„Menschen mit Behinderungen“" sind nach dem Übereinkommen Menschen, die langfristige körperliche, seelische, geistige oder Sinnesbeeinträchtigungen haben, welche sie in Wechselwirkung mit verschiedenen Barrieren an der vollen, wirksamen und gleichberechtigten Teilhabe an der Gesellschaft hindern können. „Behinderung“ entsteht aus der Wechselwirkung zwischen Menschen mit Beeinträchtigungen und einstellungs- bzw. umweltbedingten Barrieren, die sie an der vollen, wirksamen und gleichberechtigten Teilhabe an der Gesellschaft hindern. Dies verdeutlicht, dass „Behinderung“ nicht als fest definiertes Konzept verstanden wird, sondern von gesellschaftlichen Entwicklungen abhängig ist.

Als „Diskriminierung“ aufgrund von Behinderung gilt jede Unterscheidung, Ausschließung oder Beschränkung aufgrund von Behinderung, die zum Ziel oder zur Folge hat, dass das auf die Gleichberechtigung mit anderen gegründete Anerkennen, Beanspruchen oder Ausüben aller Menschenrechte und Grundfreiheiten im politischen, wirtschaftlichen, sozialen, kulturellen, bürgerlichem oder jedem anderen Bereich beeinträchtigt oder vereitelt wird.

Subjektive Rechtsansprüche begründet auch das Übereinkommen nicht. Sie ergeben sich erst aufgrund innerstaatlicher Regelungen. Allerdings ist das Übereinkommen für die Staaten rechtlich verbindlich, die es ratifiziert haben.

„Barrierefreiheit" ist eine Zielvorgabe für die Gestaltung aller Lebensbereiche.

Die Bedeutung des Übereinkommens liegt vor allem darin, dass Problemlagen behinderter Menschen nicht nur zusammenfassend gewürdigt und einer rechtlichen Gesamtbetrachtung unterzogen, sondern in vielen Facetten und unter Berücksichtigung aller Lebensfelder analysiert und teilweise detailliert beschreiben und bewertet werden. $\mathrm{Zu}$ verdanken ist dieser Erfolg nicht zuletzt der frühen Einbindung behinderter Menschen als „Experten in eigener Sache“ in den Erstellungsprozess des Konventionstextes, an dem führende internationale Behindertenverbände neben den offiziellen Regierungsvertretern beteiligt waren. Dabei wurden die internationalen Dachorganisationen der Behindertenverbände und Selbsthilfegruppen häufig durch Menschen mit Behinderung vertreten.

Die persönliche Autonomie und die Freiheit, eigene Entscheidungen zu treffen, ist ein zentrales Anliegen auch für Menschen mit geistiger oder psychischer Erkrankung. Der Zweck der Konvention besteht nämlich darin, die Menschenwürde sowie die volle und gleichberechtigte Ausübung aller Menschenrechte und Grundfreiheiten durch alle behinderten Menschen zu fördern, zu schützen und zu gewährleisten.

Bereits in der Präambel verankert ist der Satz, dass die Familie die natürliche und grundlegende Gruppeneinheit der Gemeinschaft ist und Menschen mit Behinderung und ihre Angehörigen den Schutz und die Hilfe erhalten sollten, die es Familien ermöglicht, zur uneingeschränkten und gleichberechtigten Anwendbarkeit der Rechte von Menschen mit Behinderung beizutragen.

$\mathrm{Zu}$ den Allgemeinen Grundsätzen des Übereinkommens zählen die Achtung der Menschen Würde, die Nichtdiskriminierung, die volle und wirksame Teilhabe und Einbeziehung in die Gesellschaft

Besondere Erwähnung verdient der Respekt vor der Unterschiedlichkeit und die Akzeptanz von Behinderung als Teil der menschlichen Diversität. Behinderung wird 
demgemäß nicht als Defizit betrachtet, sondern als Quelle möglicher kultureller Bereicherung angesehen und geschätzt.

Dieser „Diversity“-Ansatz findet eine exemplarische Ausprägung in kulturellem Leben, Feizeit und Sport (Artikel 30), wo anerkannt wird, dass gehörlose Menschen nicht nur mit der Gebärdensprache und auf andere Weise kommunizieren können, sondern dass es eine eigene „Kultur der Gehörlosen“ gibt.

$\mathrm{Zu}$ den "Allgemeinen Verpflichtungen" der Vertragsstaaten gehören alle geeigneten Maßnahmen zur Umsetzung der in diesem Übereinkommen anerkannten Rechte zu treffen und entgegenstehende Regelungen, Gepflogenheiten und Praktiken zu beseitigen.

Frauen und Mädchen (Artikel 6) und Kinder mit Behinderung (Artikel 7) werden wegen ihrer besonderen Schutzbedürftigkeit besonders erwähnt.

Der Grundsatz der Barrierefreiheit (Artikel 9) verlangt Zugänglichkeit; Hindernisse und Zugangsbarrieren sind zu beseitigen und für Gebäude, Straßen, Transport-, Informations-, Kommunikations- und andere Dienstleistungen ein Höchstmaß an Barrierefreiheit zu erreichen.

Für Menschen mit geistiger Behinderung und/oder seelischer Erkrankung oder psychischer Beeinträchtigung wird bekräftigt, dass sie gleichberechtigt rechtsfähig in allen Lebensbereichen sind und Anspruch haben auf die Unterstützung, die sie zur Wahrnehmung ihrer Rechtsfähigkeit benötigen. Zugleich ist sicherzustellen durch entsprechende Schutzvorkehrungen, dass ein Missbrauch ihrer besonderen Lebenssituation ausgeschlossen wird (Artikel 12).

Auch ist ihnen der Zugang zur Justiz zu erleichtern. Die dort tätigen Personen sollen durch geeignete Fortbildungsmaßnahmen in die Lage versetzt werden, mit behinderten Menschen zu kommunizieren (Artikel 13).

Freiheitsentziehende Maßnahmen sind nur zulässig im Einklang mit dem Gesetz. Das Vorliegen einer Behinderung allein rechtfertigt in keinem Fall eine Freiheitsentziehung (Artikel 14).

Auch Behinderte dürfen ihren Wohnsitz frei wählen und entscheiden, wo und mit wem sie leben wollen. Sie dürfen insbesondere nicht verpflichtet werden, in besonderen Wohnformen zu leben.

Wirtschaftliche, soziale und kulturelle Menschenrechte sind von dem jeweiligen Unterzeichnerstaat „unter Ausschöpfung seiner verfügbaren Mittel und erforderlichenfalls im Rahmen der internationalen Zusammenarbeit" umzusetzen (Artikel 4 Ziff. 2); diese Formulierung entspricht Art. 3 des Internationalen Paktes über wirtschaftliche, soziale und kulturelle Rechte von 1964 und trägt dem Umstand Rechnung, dass insbesondere die weniger entwickelten Länder sich oft nicht in der Lage sehen, die gleichen wirtschaftlichen, sozialen und kulturellen Rechte zu gewähren, die in Industrienationen üblich sind.

Der Schutz der Privatsphäre verlangt, dass personenbezogene Informationen und Daten vertraulich behandelt werden (Artikel 22).

Auch behinderte Menschen dürfen eine Ehe eingehen, eine Familie gründen, selbst über die Anzahl ihrer Kinder entscheiden u.ä.. Die Sterilisation eines Menschen wegen seiner Behinderung ist unzulässig. 
Behinderte Menschen dürfen nicht auf Grund ihrer Behinderung vom allgemeinen Bildungssystem ausgeschlossen werden. Die Eltern eines geistig behinderten Kindes können deshalb darauf bestehen können, dass ihr Kind eine allgemeine Schule besuchen darf.

Auch die Rechte auf Gesundheit (Artikel 25), Habilitation und Rehabilitation (Artikel 26), auf Arbeit und Beschäftigung (Artikel 27), das Recht auf einen angemessenen Lebensstandard und sozialen Schutz (Artikel 28) werden garantiert. Gleiches gilt für die Teilname am politischen öffentlichen Leben und das aktive und passive Wahlrecht, ggf. auch durch eine Person der Wahl des Behinderten, falls dieser selbst nicht an der Wahl teilnehmen kann (Artikel 29).

Das Vertragswerk ist das erste universelle Rechtsinstrument, das bestehende Menschenrechte, bezogen auf die Lebenssituation behinderter Menschen, konkretisiert. Es würdigt Behinderung als Teil der Vielfalt menschlichen Lebens und überwindet damit das noch in vielen Ländern vorherrschende defizitorientierte Verständnis.

Bereits am 30. März 2007 hatte der Großteil der EU-Mitgliedstaaten das Abkommen unterzeichnet. Daneben hat auch die Europäische Gemeinschaft das Übereinkommen auf der Grundlage eines Beschlusses des Rates der Europäischen Union vom 20. März 2007 unterschrieben und damit erstmalig einen menschenrechtlichen Vertrag unterzeichnet.

Mit Artikel 32 erkennen die Vertragsstaaten die Bedeutung der internationalen Zusammenarbeit und ihrer Förderung zur Unterstützung einzelstaatlicher Anstrengungen zur Umsetzung des Übereinkommens an. Die Vertragsstaaten treffen dafür geeignete und wirksame Maßnahmen, zwischenstaatlich, oder auch in Partnerschaft mit internationalen und regionalen Organisationen und der Zivilgesellschaft, insbesondere Organisationen von Menschen mit Behinderungen.

Artikel 44 Abs. 1 definiert den Begriff „Organisationen der regionalen Integrati-

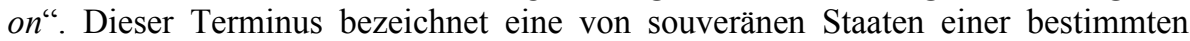
Region geschaffene Organisation, deren Mitgliedstaaten ihr Zuständigkeiten für bestimmte von diesem Übereinkommen erfasste Angelegenheiten übertragen haben. Die Vorschrift ist auf die Europäische Gemeinschaft zugeschnitten, die selbst nicht Vertragsstaat werden kann, da ihr die Staatsqualität fehlt. Sie ist aber eine internationale Regionalorganisation, die für die Umsetzung von Teilen des Artikels 27 des Übereinkommens, nämlich Arbeit und Beschäftigung im Rahmen des Artikels 13 EG i.V. mit der RL 2000/78/EG zur Verwirklichung der Gleichbehandlung in Beschäftigung und Beruf nach Gemeinschaftsrecht zuständig ist, ${ }^{65} \mathrm{da}$ ihr die Mitgliedstaaten der Europäischen Union entsprechende Gesetzgebungskompetenzen übertragen haben.

Insofern spiegelt auch das Übereinkommen der Vereinten Nationen über die Rechte von Menschen mit Behinderungen den für das Behindertenrecht und die 
Behindertenpolitik in den EU-Staaten bedeutsamen Prozess der Europäischen Integration wider.

Die durch die Europäische Gemeinschaft rechtlich wie politisch - im Wege der OMK - angestrebte Angleichung von Politik und Recht der Mitgliedstaaten in bestimmten Rechtsbereichen begegnet zuweilen dem Einwand, sie trage den unterschiedlichen Rechtskulturen der Mitgliedstaaten nicht hinreichend Rechnung, „propfe“ gelegentlich den nationalen Rechtssystemen Regeln auf, die dann aufgrund der unterschiedlichen rechtskulturellen Vorgaben ganz unterschiedliche praktische Wirkungen im Rechtsleben entfalteten. ${ }^{66}$ Dieser Kritik ist entgegenzuhalten, dass die Rechtskultur zwar auch eine nationale Kultur ist, aber jedenfalls heute nicht mehr nur, sondern dass die Technizität des Rechts, die allgemein anerkannte „rule of law“ (Rechtsstaatlichkeit), die Rolle des Rechts bei der Steuerung wirtschaftlicher und gesellschaftlicher Abläufe sowie bei der Lösung von Konflikten, last but not least die Existenz allgemein anerkannter Menschenrechte sowie allgemeiner Rechtsgrundsätze, dann aber auch die Herausbildung sowohl einer - regionalen - europäischen Rechtskultur als auch eine globale Weltrechtskultur befördern.

Faktoren, die diese Entwicklung befördern, sind zum einen die Globalisierung, die u. a. zu einer Einschränkung sowohl rechtlicher Souveränität als auch politischer Autonomie der Staaten führt, sowie die Individualisierung, die u. a. dem einzelnen sowohl gegenüber dem Staat als auch gegenüber internationalen Organisationen eine mit Rechten ausgestattete Position verleiht. (Namentlich die Globalisierung auch der Information durch moderne Kommunikationstechniken wie das Internet trägt dazu bei, diesen Prozess weltweit zu befördern.)

Das Völkerrecht reflektiert diese Entwicklungen: Die Dynamisierung von Rechtsbildungsprozessen in speziellen „Regelungsregimes“ auf regionaler $-\mathrm{z}$. B. Europäischer - wie auf universaler Ebene sind ein neues Element der Verrechtlichung, wobei die Intensivierung der Rechtsfortbildung und Durchsetzung nicht zuletzt auch auf eine Diversifizierung und Multiplizierung der beteiligten Akteure zurückzuführen ist, wobei namentlich die Organisationen der Zivilgesellschaft hervorzuheben sind (und die Internationale Arbeitsorganisation mit der Einbeziehung der Sozialpartner in den Rechtssetzungsprozess durchaus als Vorläuferin angesehen werden kann. ${ }^{67}$ Das Völkerrecht ist eben heute nicht nur eine Rechtsordnung, welche die Beziehungen zwischen Staaten regelt, sondern es begünstigt auch den Einzelnen, indem es die internationale Gemeinschaft verpflichtet, die Menschenrechte zu schützen - letzteres eine zentrale Innovation des universellen Völkerrechts unter der Ägide der Vereinten Nationen, die nach dem Zweiten Weltkrieg eingesetzt hat. ${ }^{68}$

Vgl. zu dieser Kritik Basedow, Rechtskultur - Zwischen nationalem Mythos und europäischen Ideal, in: Zeitschrift für Europäisches Privatrecht (ZEuP) 1996, S. 379 ff.

7 Vgl. in diesem Sinne etwa Bothe, Wandel des Völkerrechts - Herausforderung an die Steuerungsfähigkeit des Rechts im Zeitalter der Globalisierung, in: Kritische Vierteljahresschrift für Gesetzgebung und Rechtswissenschaft (KritV) 2008, S. 233 ff.

Vgl. zu dieser Entwicklung grundlegend Köhler, Sozialpolitische und sozialrechtliche Aktivitäten in den Vereinten Nationen, Baden-Baden 1997 
Das Übereinkommen der Vereinten Nationen über die Rechte von Menschen mit Behinderungen des Jahres 2006, das nach seinem Inkrafttreten - in Deutschland, wie erwähnt Anfang 2009 - auch in einzelnen Mitgliedstaaten der Europäischen Union, und damit eben auch der Europäischen Union selbst seine Wirkung entfaltet, ist ein Ergebnis dieser Entwicklung, denn

,the UN-Convention on the Rights of Persons with Disabilities is about more than making sure that existing human rights are applied to persons with disabilities. It also subtly reformulates and extends existing human rights to take into account the specific rights experience of persons with disability. In fact, the argument can be made that the Convention comes close to creating new rights, or at least very new ways of seeing common rights. This suggests a deeper point about the fragmentation of international human rights law and the increasingly recognized need to take into account the irreducibility of the experience of certain categories of persons." 69

Umsetzung und Durchsetzung der in diesem Rechtsinstrument verankerten Rechte für Menschen mit Behinderungen auf unterschiedlichen Wegen und mit unterschiedlicher Intensität mögen ein Indikator sein für Behindertenpolitik, das Behindertenwesen und das Behindertenrecht in den Staaten Europas und Asiens und auch ein Ansatz für die weitere vergleichende Arbeit. Dabei ist die advocatorische Rolle (,advocacy') der Menschen mit Behinderungen selbst ein Element, dem besondere Aufmerksamkeit geschenkt werden muss. ${ }^{70}$ Ein zweites wichtiges Element, dem verstärkt Rechnung zu tragen ist, ist die Entstaatlichung des Rechts, die sich in vielerlei Hinsicht Bahn bricht. ${ }^{71}$

Diese Elemente gilt es zu berücksichtigen, wenn im Zusammenhang mit den Vorschlägen zur Fortführung und zum Abschluss des Projekts die Vorschläge diskutiert werden, die im Generalbericht von Alexander Graser in diesem Band zur Diskussion gestellt worden sind.

69 Mégret, The Disabilities Convention: Human Rights of Persons with Disabilities or Disability Rights? In: Human Rights Quarterly 2008, S. 494 ff.

70 Vgl. dazu Beiträge in: Bresling/Yee (Hrsg.), Disability Right Law and Policy. International and National Perspectives, Ardsley, NY (USA) 2002

71 Vgl. dazu etwa Zimmermann, Globalisierung und Entstaatlichung des Rechts, 2 Bde, Tübingen 2008 


\title{
Annex 1: UN-Konvention über Rechte von Menschen mit Behinderung (2006) (Auszug)
}

\author{
United Nations General Assembly
}

6 December 2006, Sixty-first session: item 67 (b): Human rights questions including alternative approaches for improving the effective enjoyment of human rights and fundamental freedoms

I. Convention on the Rights of Persons with Disabilities and Optional Protocol to the Convention

Übereinkommen der Vereinten Nationen über die Rechte von Menschen mit Behinderungen $^{72}$

(Auszug)

Die Vertragsstaaten dieses Übereinkommens,

\section{Präambel}

a) unter Hinweis auf die in der Charta der Vereinten Nationen verkündeten Grundsätze, denen zufolge die Anerkennung der Würde und des Wohles, die allen Mitgliedern der Gesellschaft innewohnen, sowie ihrer gleichen und unveräußerlichen Rechte die Grundlage von Freiheit, Gerechtigkeit und Frieden in der Welt bildet,

c) bekräftigend, dass alle Menschenrechte und Grundfreiheiten allgemein gültig und unteilbar sind, einander bedingen und miteinander verknüpft sind und dass Menschen mit Behinderungen der volle Genuss dieser Rechte und Freiheiten ohne Diskriminierung garantiert werden muss,

e) in der Erkenntnis, dass das Verständnis von Behinderung sich ständig weiterentwickelt und dass Behinderung aus der Wechselwirkung zwischen Menschen mit Beeinträchtigungen auf einstellungs- und umweltbedingten Barrieren entsteht, die sie an der vollen, wirksamen und gleichberechtigten Teilhabe an der Gesellschaft hindern,

f) in der Erkenntnis, dass die in dem Weltaktionsprogramm für Behinderte und den Rahmenbestimmungen für die Herstellung der Chancengleichheit für Be-

72 Deutsche Fassung: Bundesrat-Drucksache 760/08 v. 17. Oktober 2008: Entwurf eines Gesetzes zu dem Übereinkommen der Vereinten Nationen vom 13. Dezember 2006 über die Rechte von Menschen mit Behinderungen sowie Denkschrift zu dem Übereinkommen vom 13. Dezember 2006 über die Rechte von Menschen mit Behinderungen 
hinderte enthaltenen Grundsätze und Leitlinien einen wichtigen Einfluss auf die Förderung, Ausarbeitung und Bewertung von politischen Konzepten, Plänen, Programmen und Maßnahmen auf einzelstaatlicher, regionaler und internationaler Ebene zur Verbesserung der Chancengleichheit für Menschen mit Behinderungen haben,

h) ebenso in der Erkenntnis, dass jede Diskriminierung aufgrund von Behinderung eine Verletzung der Würde und des Wertes darstellt, die jedem Menschen innewohnen,

j) in Anerkennung der Notwendigkeit, die Menschenrechte aller Menschen mit Behinderungen, einschließlich derjenigen, die intensivere Unterstützung benötigen, zu fördern und zu schützen,

1) in Anerkennung der Bedeutung der internationalen Zusammenarbeit für die Verbesserung der Lebensbedingungen der Menschen mit Behinderungen in allen Ländern, insbesondere den Entwicklungsländern,

n) in der Erkenntnis, wie wichtig die individuelle Autonomie und Unabhängigkeit für Menschen mit Behinderungen ist, einschließlich der Freiheit, eigene Entscheidungen zu treffen,

o) in der Erwägung, dass Menschen mit Behinderungen die Möglichkeit haben sollen, aktiv an Entscheidungsprozessen über politische Konzepte und Programme mitzuwirken, insbesondere wenn diese sie unmittelbar betreffen,

r) in der Erkenntnis, dass Kinder mit Behinderungen gleichberechtigt mit anderen Kindern alle Menschenrechte und Grundfreiheiten in vollem Umfang genießen sollen, und unter Hinweis auf die zu diesem Zweck von den Vertragsstaaten des Übereinkommens über die Rechte des Kindes eingegangenen Verpflichtungen,

s) nachdrücklich darauf hinweisend, dass es notwendig ist, bei allen Anstrengungen zur Förderung des vollen Genusses der Menschenrechte und Grundfreiheiten durch Menschen mit Behinderungen die Geschlechterperspektive einzubeziehen,

t) unter besonderem Hinweis darauf, dass die Mehrzahl der Menschen mit Behinderungen in einem Zustand der Armut lebt, und diesbezüglich in der Erkenntnis, dass die nachteiligen Auswirkungen der Armut auf Menschen mit Behinderungen dringend angegangen werden müssen,

$\cdots$

v) in der Erkenntnis, wie wichtig es ist, dass Menschen mit Behinderungen vollen Zugang zur physischen, sozialen, wirtschaftlichen und kulturellen Umwelt, zu Gesundheit und Bildung sowie zu Information und Kommunikation haben, damit sie alle Menschenrechte und Grundfreiheiten voll genießen können,

$\mathrm{x}$ ) in der Überzeugung, dass die Familie die natürliche Kernzelle der Gesellschaft ist und Anspruch auf Schutz durch Gesellschaft und Staat hat und dass Menschen mit Behinderungen und ihre Familienangehörigen den erforderlichen Schutz und die notwendige Unterstützung erhalten sollen, um es den Familien $\mathrm{zu}$ ermöglichen, zum vollen und gleichberechtigten Genuss der Rechte der Menschen mit Behinderungen beizutragen, 
y) in der Überzeugung, dass ein umfassendes und in sich geschlossenes internationales Übereinkommen zur Förderung und zum Schutz der Rechte und der Würde von Menschen mit Behinderungen sowohl in den Entwicklungsländern als auch in den entwickelten Ländern ... einen maßgeblichen Beitrag zur Beseitigung der tiefgreifenden sozialen Benachteiligungen von Menschen mit Behinderungen leisten und ihre Teilhabe am bürgerlichen, politischen, wirtschaftlichen, sozialen und kulturellen Leben auf der Grundlage der Chancengleichheit fördern wird,

haben Folgendes vereinbart:

\section{Artikel 1 Zweck}

Zweck dieses Übereinkommens ist es, den vollen und gleichberechtigten Genuss aller Menschenrechte und Grundfreiheiten durch alle Menschen mit Behinderungen zu fördern, zu schützen und zu gewährleisten und die Achtung der ihnen innewohnenden Würde zu fördern.

$\mathrm{Zu}$ den Menschen mit Behinderungen zählen Menschen, die langfristige körperliche, seelische, geistige oder Sinnesbeeinträchtigungen haben, welche sie in Wechselwirkung mit verschiedenen Barrieren an der vollen, wirksamen und gleichberechtigten Teilhabe an der Gesellschaft hindern können.

\section{Im Sinne dieses Übereinkommens}

\section{Artikel 2 Begriffsbestimmungen}

bedeutet „Diskriminierung auf Grund von Behinderung“ jede Unterscheidung, Ausschließung oder Beschränkung aufgrund von Behinderung, die zum Ziel oder zur Folge hat, dass das auf die Gleichberechtigung mit anderen gegründete Anerkennen, Genießen oder Ausüben aller Menschenrechte und Grundfreiheiten im politischen, wirtschaftlichen, sozialen, kulturellen, bürgerlichen oder jedem anderen Bereich beeinträchtigt oder vereitelt wird. Sie umfasst alle Formen der Diskriminierung, einschließlich der der Versagung angemessener Vorkehrungen;

bedeutet „angemessene Vorkehrungen“ notwendige und geeignete Änderungen und Anpassungen, die keine unverhältnismäßige oder unbillige Belastung darstellen und die, wenn sie in einem bestimmten Fall erforderlich sind, vorgenommen werden, um zu gewährleisten, dass Menschen mit Behinderungen gleichberechtigt mit anderen alle Menschenrechte und Grundfreiheiten genießen oder ausüben können;

bedeutet ,universelles Design“ ein Design von Produkten, Umfeldern, Programmen und Dienstleistungen in der Weise, dass sie von allen Menschen möglichst weitgehend ohne eine Anpassung oder ein spezielles Design genutzt werden können. „Universelles Design“ schließt Hilfsmittel für bestimmte Gruppen von Menschen mit Behinderungen, soweit sie benötigt werden, nicht aus. 


\section{Artikel 3 Allgemeine Grundsätze}

Die Grundsätze dieses Übereinkommens sind:

a) die Achtung der dem Menschen innewohnenden Würde, seiner individuellen Autonomie, einschließlich der Freiheit, eigene Entscheidungen zu treffen, sowie seiner Unabhängigkeit;

b) die Nichtdiskriminierung;

c) die volle und wirksame Teilhabe an der Gesellschaft und Einbeziehung in die Gesellschaft;

d) die Achtung vor der Unterschiedlichkeit von Menschen mit Behinderungen und die Akzeptanz dieser Menschen als Teil der menschlichen Vielfalt und der Menschheit;

e) die Chancengleichheit;

f) die Zugänglichkeit;

g) die Gleichberechtigung von Mann und Frau;

h) die Achtung vor den sich entwickelnden Fähigkeiten von Kindern mit Behinderungen und die Achtung ihres Rechts auf Wahrung ihrer Identität.

\section{Artikel 4 Allgemeine Verpflichtungen}

1. Die Vertragsstaaten verpflichten sich, die volle Verwirklichung aller Menschenrechte und Grundfreiheiten für alle Menschen mit Behinderungen ohne jede Diskriminierung aufgrund von Behinderung zu gewährleisten und zu fördern. $\mathrm{Zu}$ diesem Zweck verpflichten sich die Vertragsstaaten,

a) alle geeigneten Gesetzgebungs-, Verwaltungs- und sonstigen Maßnahmen zur Umsetzung der in diesem Übereinkommen anerkannten Rechte zu treffen;

b) alle geeigneten Maßnahmen einschließlich gesetzgeberischer Maßnahmen zur Aufhebung oder Änderung bestehender Gesetze, Verordnungen, Gepflogenheiten und Praktiken zu treffen, die eine Diskriminierung von Menschen mit Behinderungen darstellen;

c) den Schutz und die Förderung der Menschenrechte von Menschen mit Behinderungen in allen politischen Konzepten und allen Programmen zu berücksichtigen;

d) Handlungen oder Praktiken, die mit diesem Übereinkommen unvereinbar sind, zu unterlassen und dafür zu sorgen, dass die staatlichen Behörden und öffentlichen Einrichtungen im Einklang mit diesem Übereinkommen handeln;

e) alle geeigneten Maßnahmen zur Beseitigung der Diskriminierung aufgrund von Behinderung durch Personen, Organisationen oder private Unternehmen zu ergreifen;

f) Forschung und Entwicklung für Güter, Dienstleistungen, Geräte und Einrichtungen in universellem Design, wie in Artikel 2 definiert, die den besonderen Bedürfnissen von Menschen mit Behinderungen mit möglichst geringem Anpassungs- und Kostenaufwand gerecht werden, zu betreiben oder zu fördern, ihre Verfügbarkeit und Nutzung zu fördern und sich bei der Entwicklung von Normen und Richtlinien für universelles Design einzusetzen; 
h) für Menschen mit Behinderungen zugängliche Informationen über Mobilitätshilfen, Geräte und unterstützende Technologien, einschließlich neuer Technologien, sowie andere Formen von Hilfe, Unterstützungsdiensten und Einrichtungen zur Verfügung zu stellen;

i) die Schulung von Fachkräften und anderem mit Menschen mit Behinderungen arbeitendem Personal auf dem Gebiet der in diesem Übereinkommen anerkannten Rechte zu fördern, damit die aufgrund dieser Rechte garantierten Hilfen und Dienste besser geleistet werden können.

2. Hinsichtlich der wirtschaftlichen, sozialen und kulturellen Rechte verpflichtet sich jeder Vertragsstaat, unter Ausschöpfung seiner verfügbaren Mittel und erforderlichenfalls im Rahmen der internationalen Zusammenarbeit Maßnahmen zu treffen, um nach und nach die volle Verwirklichung dieser Rechte zu erreichen, unbeschadet derjenigen Verpflichtungen aus diesem Übereinkommen, die nach dem Völkerrecht sofort anwendbar sind.

3. Bei der Ausarbeitung und Umsetzung von Rechtsvorschriften und politischen Konzepten zur Durchführung dieses Übereinkommens und bei anderen Entscheidungsprozessen in Fragen, die Menschen mit Behinderungen betreffen, führen die Vertragsstaaten mit den Menschen mit Behinderungen einschließlich Kindern mit Behinderungen, über die sie vertretenden Organisationen enge Konsultationen und beziehen sie aktiv ein.

4. Dieses Übereinkommen lässt zur Verwirklichung der Rechte von Menschen mit Behinderungen besser geeignete Bestimmungen, die im Recht eines Vertragsstaats oder in dem für diesen Staat geltenden Völkerrecht enthalten sind, unberührt. Die in einem Vertragsstaat durch Gesetze, Übereinkommen, Verordnungen oder durch Gewohnheitsrecht anerkannten oder bestehenden Menschenrechte und Grundfreiheiten dürfen nicht unter dem Vorwand beschränkt oder außer Kraft gesetzt werden, dass dieses Übereinkommen derartige Rechte oder Freiheiten nicht oder nur einem geringeren Ausmaß anerkenne.

\section{Artikel 5 Gleichberechtigung und Nichtdiskriminierung}

1. Die Vertragsstaaten anerkennen, dass alle Menschen vor dem Gesetz gleich sind, vom Gesetz gleich zu behandeln sind und ohne Diskriminierung Anspruch auf gleichen Schutz durch das Gesetz und gleiche Vorteile durch das Gesetz haben.

2. Die Vertragsstaaten verbieten jede Diskriminierung aufgrund von Behinderung und garantieren Menschen mit Behinderungen gleichen und wirksamen rechtlichen Schutz vor Diskriminierung, gleichviel aus welchen Gründen.

3. Zur Förderung der Gleichberechtigung und zur Beseitigung von Diskriminierung unternehmen die Vertragsstaaten alle geeigneten Schritte, um die Bereitstellung angemessener Vorkehrungen zu gewährleisten.

4. Besondere Maßnahmen, die zur Beschleunigung oder Herbeiführung der tatsächlichen Gleichberechtigung von Menschen mit Behinderungen erforderlich sind, gelten nicht als Diskriminierung im Sinne dieses Übereinkommens. 
Artikel 6 Frauen mit Behinderungen

Artikel 7 Kinder mit Behinderungen

Artikel 8 Bewusstseinsbildung

\section{Artikel 9 Zugänglichkeit}

1. Um Menschen mit Behinderungen eine unabhängige Lebensführung und die volle Teilhabe in allen Lebensberechen zu ermöglichen, treffen die Vertragsstaaten geeignete Maßnahmen, um für Menschen mit Behinderungen den gleichberechtigten Zugang zur physischen Umwelt, zu Transportmitteln, Information und Kommunikation, einschließlich Informations- und Kommunikationstechnologien und -systemen, sowie zu anderen Einrichtungen und Diensten, die der Öffentlichkeit in städtischen und ländlichen Gebieten offenstehen oder für sie bereitgestellt werden, zu gewährleisten. Diese Maßnahmen, welche die Feststellung und Beseitigung von Zugangshindernissen und- barrieren einschließen, gelten unter anderem für

a) Gebäude, Straßen und Transportmittel sowie andere Einrichtungen in Gebäuden und im Freien, einschließlich Schulen, Wohnhäusern, medizinischer Einrichtungen und Arbeitsstätten;

b) Informations-, Kommunikations- und andere Dienste einschließlich elektronischer Dienste und Notdienste,

2. Die Vertragsstaaten treffen außerdem geeignete Maßnahmen,

a) um Mindeststandards und Leitlinien für die Zugänglichkeit von Einrichtungen und Diensten, die der Öffentlichkeit offenstehen oder für sie bereitgestellt werden, auszuarbeiten und zu erlassen und ihre Anwendung zu überwachen.

b) um sicherzustellen, dass private Rechtsträger, die Einrichtungen und Dienste, die der Öffentlichkeit offenstehen oder für sie bereitgestellt werden, anbieten, alle Aspekte der Zugänglichkeit für Menschen mit Behinderungen berücksichtigen;

c) um betroffenen Kreisen Schulungen zu Fragen der Zugänglichkeit für Menschen mit Behinderungen anzubieten;

d) um in Gebäuden und anderen Einrichtungen, die der Öffentlichkeit offenstehen, Beschilderungen in Brailleschrift und in leicht lesbarer und verständlicher Form anzubringen;

e) um menschliche und tierische Hilfe sowie Mittelspersonen, unter anderem Personen zum Führen und Vorlesen sowie professionelle Gebärdensprachdolmetscher und -dolmetscherinnen, zur Verfügung zu stellen mit dem Ziel, den Zugang zu Gebäuden und anderen Einrichtungen, die der Öffentlichkeit offenstehen, zu erleichtern;

f) um andere geeignete Formen der Hilfe und Unterstützung für Menschen mit Behinderungen zu fördern, damit ihr Zugang zu Informationen gewährleistet wird; 
g) um den Zugang von Menschen mit Behinderungen zu den neuen Informationsund Kommunikationstechnologien und -systemen, einschließlich des Internets, zu fördern;

h) um die Gestaltung, die Entwicklung, die Herstellung und den Vertrieb zugänglicher Kommunikationstechnologien und -systeme in einem frühen Stadium zu fördern, sodass deren Zugänglichkeit mit möglichst geringem Kostenaufwand erreicht wird.

\section{Artikel 10 Recht auf Leben}

\section{Artikel 11 Gefahrensituationen und humanitäre Notlagen}

\section{Artikel 12 Gleiche Anerkennung vor dem Recht}

1. Die Vertragsstaaten bekräftigen, dass Menschen mit Behinderungen das Recht haben, überall als rechtsfähige Person anerkannt zu werden.

2. Die Vertragsstaaten anerkennen, dass Menschen mit Behinderungen in allen Lebensbereichen gleichberechtigt mit anderen Rechts- und Handlungsfähigkeit genießen.

3. Die Vertragsstaaten treffen geeignete Maßnahmen, um Menschen mit Behinderungen Zugang zu der Unterstützung zu verschaffen, die sie bei der Ausübung ihrer Rechts- und Handlungsfähigkeit gegebenenfalls benötigen.

4. Die Vertragsstaaten stellen sicher, dass zu allen die Ausübung der Rechts- und Handlungsfähigkeit betreffenden Maßnahmen im Einklang mit den internationalen Menschenrechtsnormen geeignete und wirksame Sicherungen vorgesehen werden, um Missbräuche zu verhindern. Diese Sicherungen müssen gewährleisten, dass bei den Maßnahmen betreffend die Ausübung der Rechts- und Handlungsfähigkeit die Rechte, der Wille und die Präferenzen der betreffenden Person geachtet werden, es nicht zu Interessenkonflikten und missbräuchlicher Einflussnahme kommt, dass die Maßnahmen verhältnismäßig und auf die Umstände der Person zugeschnitten sind, dass sie von möglichst kurzer Dauer sind und einer regelmäßigen Überprüfung durch eine zuständige, unabhängige und unparteiische Behörde oder gerichtliche Stelle unterliegen. Die Sicherungen müssen im Hinblick auf das Ausmaß, in dem diese Maßnahmen die Rechte und Interessen der Person berühren, verhältnismäßig sein.

5. Vorbehaltlich dieses Artikels treffen die Vertragsstaaten alle geeigneten und wirksamen Maßnahmen, um zu gewährleisten, dass Menschen mit Behinderungen das gleiche Recht wie andere haben, Eigentum zu besitzen oder zu erben, ihre finanziellen Angelegenheiten selbst zu regeln und gleichen Zugang zu Bankdarlehen, Hypotheken und anderen Finanzkrediten zu haben, und gewährleisten, dass Menschen mit Behinderungen nicht willkürlich ihr Eigentums entzogen wird. 


\section{Artikel 13 Zugang zur Justiz}

1. Die Vertragsstaaten gewährleisten Menschen mit Behinderungen gleichberechtigt mit anderen wirksamen Zugang zur Justiz, unter anderem durch verfahrensbezogene und altersgemäße Vorkehrungen, um ihre wirksame unmittelbare und mittelbare Teilnahme, einschließlich als Zeugen und Zeuginnen, an allen Gerichtsverfahren, auch in der Ermittlungsphase und in anderen Vorverfahrensphasen, zu erleichtern.

2. Um zur Gewährleistung des wirksamen Zugangs von Menschen mit Behinderungen wirksamen Zugang zur Justiz beizutragen, fördern die Vertragsstaaten geeignete Schulungen für die im Justizwesen tätigen Personen, einschließlich des Personals von Polizei und Strafvollzug.

\section{Artikel 14 Freiheit und Sicherheit der Person}

\section{Artikel 19 Unabhängige Lebensführung und Einbeziehung in die Gemeinschaft}

Die Vertragsstaaten dieses Übereinkommens anerkennen das gleiche Recht aller Menschen mit Behinderungen, mit gleichen Wahlmöglichkeiten wie andere Menschen in der Gemeinschaft zu leben, und treffen wirksame und geeignete Maßnahmen, um Menschen mit Behinderungen den vollen Genuss dieses Rechts und ihre volle Einbeziehung in die Gemeinschaft und Teilhabe an der Gemeinschaft zu erleichtern, indem sie unter anderem gewährleisten, dass

a) Menschen mit Behinderungen gleichberechtigt die Möglichkeit haben, ihren Wohnsitz zu wählen und zu entscheiden, wo und mit wem sie leben, und nicht verpflichtet sind, in besonderen Wohnformen zu leben;

b) Menschen mit Behinderungen Zugang zu einer Reihe von gemeindenahen Unterstützungsdiensten zu Hause und in Einrichtungen sowie zu sonstigen gemeindenahen Unterstützungsdiensten haben, einschließlich der persönlichen Assistenz, die zur Unterstützung des Lebens in der Gemeinschaft und der Einbeziehung in die Gemeinschaft sowie zur Verhinderung von Isolation und Absonderung von der Gemeinschaft notwendig ist;

c) gemeindenahe Dienstleistungen und Einrichtungen für die Allgemeinheit Menschen mit Behinderungen auf der Grundlage der Gleichberechtigung zur Verfügung stehen und ihren Bedürfnissen Rechnung tragen.

\section{Artikel 20 Persönliche Mobilität}

Die Vertragsstaaten treffen wirksame Maßnahmen, um für Menschen mit Behinderungen persönliche Mobilität mit größtmöglicher Unabhängigkeit sicherzustellen, indem sie unter anderem

a) die persönliche Mobilität von Menschen mit Behinderungen in der Art und Weise und zum Zeitpunkt ihrer Wahl und zu erschwinglichen Kosten erleichtern;

b) den Zugang von Menschen mit Behinderungen zu hochwertigen Mobilitätshilfen, Geräten, unterstützenden Technologien und menschlicher und tierischer 
Hilfe sowie Mittelspersonen erleichtern, auch durch deren Bereitstellung zu erschwinglichen Kosten;

c) Menschen mit Behinderungen und Fachkräften, die mit Menschen mit Behinderungen arbeiten, Schulungen in Mobilitätsfertigkeiten anbieten;

d) Hersteller von Mobilitätshilfen, Geräten, unterstützenden Technologien ermutigen, alle Aspekte der Mobilität für Menschen mit Behinderungen zu berücksichtigen.

\section{Artikel 22 Achtung der Privatsphäre}

1. Menschen mit Behinderungen dürfen unabhängig von ihrem Aufenthaltsort oder der Wohnform, in der sie leben, keinen willkürlichen oder rechtswidrigen Eingriffen in ihr Privatleben, ihre Familie, ihre Wohnung oder ihren Schriftverkehr oder andere Arten der Kommunikation oder rechtswidrigen Beeinträchtigungen ihrer Ehre oder ihres Rufes ausgesetzt werden. Menschen mit Behinderungen haben Anspruch auf rechtlichen Schutz gegen solche Eingriffe oder Beeinträchtigungen.

2. Die Vertragsstaaten schützen auf der Grundlage der Gleichberechtigung mit anderen die Vertraulichkeit von Informationen über die Person, die Gesundheit und die Rehabilitation von Menschen mit Behinderungen.

\section{Artikel 23 Achtung der Wohnung und der Familie}

1. Die Vertragsstaaten treffen wirksame und geeignete Maßnahmen zur Beseitigung der Diskriminierung von Menschen mit Behinderungen auf der Grundlage der Gleichberechtigung mit anderen in allen Fragen, die Ehe, Familie und Partnerschaften betreffen, um zu gewährleisten, dass

a) das Recht aller Menschen mit Behinderungen im heiratsfähigen Alter, auf der Grundlage des freien und vollen Einverständnisses der künftigen Ehegatten eine Ehe zu schließen und eine Familie zu gründen, anerkannt wird;

b) das Recht von Menschen mit Behinderungen auf freie und verantwortungsbewusste Entscheidung über die Anzahl ihrer Kinder und die Geburtenabstände sowie auf Zugang zu altersgemäßer Information sowie Aufklärung über Fortpflanzung und Familienplanung anerkannt wird und ihnen die notwendigen Mittel zur Ausübung dieser Rechte zur Verfügung gestellt werden.

2. Die Vertragsstaaten gewährleisten die Rechte und Pflichten von Menschen mit Behinderungen in Fragen der Vormundschaft, Pflegschaft, Personen- und Vermögenssorge, Adoption von Kindern oder ähnlichen Rechtsinstituten, soweit das innerstaatliche Recht solche kennt; in allen Fällen ist das Wohl des Kindes ausschlaggebend. Die Vertragsstaaten unterstützen Menschen mit Behinderungen in angemessener Weise bei der Wahrnehmung ihrer elterlichen Verantwortung. 


\section{Artikel 25 Gesundheit}

Die Vertragsstaaten anerkennen das Recht von Menschen mit Behinderungen auf das erreichbare Höchstma $\beta$ an Gesundheit ohne Diskriminierung auf Grund von Behinderung.

\section{Artikel 26 Habilitation und Rehabilitation}

1. Die Vertragsstaaten treffen wirksame und geeignete Maßnahmen, einschließlich durch die Unterstützung durch andere Menschen mit Behinderungen, um Menschen mit Behinderungen in die Lage zu versetzen, ein Höchstmaß an Unabhängigkeit, umfassende körperliche, geistige soziale und berufliche Fähigkeiten sowie die volle Einbeziehung in alle Aspekte des Lebens und die volle Teilhabe an allen Aspekten des Lebens zu erreichen und zu bewahren. Zu diesem Zweck organisieren, stärken und erweitern die Vertragsstaaten umfassende Habilitations- und Rehabilitationsdienste und -programme, insbesondere auf dem Gebiet der Gesundheit, der Beschäftigung, der Bildung und die Sozialdienste.

\section{Artikel 27 Arbeit und Beschäftigung}

1. Die Vertragsstaaten anerkennen das gleiche Recht von Menschen mit Behinderungen auf Arbeit; dies beinhaltet das Recht auf die Möglichkeit, den Lebensunterhalt durch Arbeit zu verdienen, die in einem offenen, integrativen und für Menschen mit Behinderungen zugänglichen Arbeitsmarkt und Arbeitsumfeld frei gewählt oder angenommen wird. Die Vertragsstaaten sichern und fördern die Verwirklichung des Rechts auf Arbeit, einschließlich für Menschen, die während der Beschäftigung eine Behinderung erwerben, durch geeignete Schritte, einschließlich des Erlasses von Rechtsvorschriften, um unter anderem

a) Diskriminierung aufgrund von Behinderung in allen Angelegenheiten im $\mathrm{Zu}$ sammenhang mit einer Beschäftigung gleich welcher Art, einschließlich der Auswahl-, Einstellungs- und Beschäftigungsbedingungen, der Weiterbeschäftigung, des beruflichen Aufstiegs sowie sicherer und gesunder Arbeitsbedingungen, zu verbieten;

\section{Artikel 28 Angemessener Lebensstandard und sozialer Schutz}

1. Die Vertragsstaaten anerkennen das Recht von Menschen mit Behinderungen auf einen angemessen Lebensstandard für sich selbst und ihre Familien, einschließlich ausreichender Ernährung, Bekleidung und Wohnung, sowie auf eine stetige Verbesserung der Lebensbedingungen und unternehmen geeignete Schritte zum Schutz und zur Förderung die Verwirklichung dieses Rechts ohne Diskriminierung auf Grund einer Behinderung. 


\section{Artikel 32 Internationale Zusammenarbeit}

1. Die Vertragsstaaten anerkennen die Bedeutung der internationalen Zusammenarbeit und deren Förderung zur Unterstützung der einzelstaatlichen Anstrengungen für die Verwirklichung und des Zwecks und der Ziele dieses Übereinkommens und treffen diesbezüglich geeignete und wirksame Maßnahmen, zwischenstaatlich sowie, soweit angebracht in Partnerschaft mit den einschlägigen internationalen und regionalen Organisationen und der Zivilgesellschaft, insbesondere Organisationen von Menschen mit Behinderungen. Unter anderem können sie Maßnahmen ergreifen, um

a) sicherzustellen, dass die internationale Zusammenarbeit, einschließlich internationaler Entwicklungsprogramme, Menschen mit Behinderungen einbezieht und für sie zugänglich ist. $\cdots$

\section{Artikel 33 Nationale Durchführung und Überwachung}

1. Die Vertragsstaaten bestimmen nach Maßgabe ihrer staatlichen Organisation eine oder mehrere staatliche Anlaufstellen für Angelegenheiten im Zusammenhang mit der Durchführung dieses Übereinkommens und prüfen sorgfältig die Schaffung oder Bestimmung eines staatlichen Koordinierungsmechanismus, der die Durchführung der entsprechenden Maßnahmen in verschiedenen Bereichen und auf verschiedenen Ebenen erleichtern soll. ...

3. Die Zivilgesellschaft, insbesondere Menschen mit Behinderungen und die sie vertretenden Organisationen, wird in den Überwachungsprozess einbezogen und nimmt in vollem Umfang daran teil.

Artikel 34 Ausschuss für die Rechte von Menschen mit Behinderungen

\section{Artikel 35 Berichte der Vertragsstaaten}

1. Jeder Vertragsstaat legt dem Ausschuss über den Generalsekretär der Vereinten Nationen innerhalb von zwei Jahren nach Inkrafttreten dieses Übereinkommens für den betreffenden Vertragsstaat einen umfassenden Bericht über die Maßnahmen, die er zur Erfüllung seiner Verpflichtungen aus dem Übereinkommen getroffen hat, und über die dabei erzielten Fortschritte vor

Dieses Übereinkommen liegt für alle Staaten und für Organisationen der regionalen Integration ab dem 30. März 2007 am Sitz der Vereinten Nationen in New York zur Unterzeichnung auf. 


\section{Artikel 45 Inkrafttreten}

1. Dieses Übereinkommen tritt am dreißigsten Tag nach Hinterlegung der zwanzigsten Ratifizierungs- oder Beitrittsurkunde in Kraft.

2. Für jeden Staat und jede Organisation der regionalen Integration, der beziehungsweise die dieses Übereinkommen nach Hinterlegung der zwanzigsten entsprechenden Urkunde ratifiziert, förmlich bestätigt oder ihm beitritt, tritt das Übereinkommen am dreißigsten Tag nach Hinterlegung der eigenen Urkunde in Kraft.

\section{Artikel 50 Verbindliche Wortlaute}

Der arabische, der chinesische, der englische, der französische, der russische und der spanische Wortlaut dieses Übereinkommens sind gleichermaßen verbindlich.

Fakultativprotokoll zum Übereinkommen über die Rechte von Menschen mit Behinderungen

... (vom Abdruck abgesehen) 
Annex 2:Richtlinie 2000/78/EG des Rates vom 27. November 2000 zur Festlegung eines allgemeinen Rahmens für die Verwirklichung der Gleichbehandlung in Beschäftigung und Beruf (Auszug)

Der Rat der Europäischen Union - gestützt auf den Vertrag zur Gründung der Europäischen Gemeinschaft, insbesondere auf Art. 13

in Erwägung nachstehender Gründe:

(1) Nach Artikel 6 Absatz 2 des Vertrags über die Europäische Union beruht die Europäische Union auf Freiheit, Demokratie, der Achtung der Menschenrechte und Grundfreiheiten sowie der Rechtsstaatlichkeit: diese Grundsätze sind allen Mitgliedstaaten gemeinsam. Die Union achtet die Grundrechte, wie sie in der Europäischen Konvention zum Schutze der Menschenrechte und Grundfreiheiten gewährleistet sind und wie sich aus den gemeinsamen Verfassungsüberlieferungen der Mitgliedstaaten als allgemeine Grundsätze des Gemeinschaftsrechts ergeben.

(2) Der Grundsatz der Gleichbehandlung von Männern und Frauen wurde in zahlreichen Rechtsakten der Gemeinschaften verankert, insbesondere in der Richtlinie 76/207/EWG des Rates vom 9. Februar 1976 zur Verwirklichung des Grundsatzes der Gleichbehandlung von Männern und Frauen hinsichtlich des Zugangs zur Beschäftigung, zur Berufsbildung und zum beruflichen Aufstieg sowie in Bezug auf die Arbeitsbedingungen.

(3) $\ldots$

(4) Die Gleichheit aller Menschen vor dem Gesetz und der Schutz vor Diskriminierung ist ein allgemeines Menschenrecht; dieses Recht wurde in der Allgemeinen Erklärung der Menschenrechte, im VN-Übereinkommen zur Beseitigung aller Formen von -Diskriminierung von Frauen, im Internationalen Pakt der VN über bürgerliche und politische Rechte, im Internationalen Pakt der VN über wirtschaftliche, soziale und kulturelle Rechte sowie in der Europäischen Konvention zum Schutz der Menschenrechte und Grundfreiheiten anerkannt, die von allen Mitgliedstaaten unterzeichnet wurden. Das Übereinkommen 111 der Internationalen Arbeitsorganisation untersagt Diskriminierungen in Beschäftigung und Beruf.

(5) $\ldots$

(6) $\ldots$

(7) $\ldots$

(8) $\ldots$

(9) Beschäftigung und Beruf sind Bereiche, die für die Gewährleistung gleicher Chancen für alle und für eine volle Teilhabe der Bürger am wirtschaftlichen, sozialen und kulturellen und sozialen Leben sowie für die individuelle Entfaltung von entscheidender Bedeutung sind.

(10) Der Rat hat am 29. Juni 2000 die Richtlinie 2000/43/EG zur Anwendung des Gleichbehandlungsgrundsatzes ohne Unterschied der Rasse oder der ethnischen 
Herkunft angenommen, die bereits einen Schutz vor solchen Diskriminierungen in Beschäftigung und Beruf gewährleistet.

(11) Diskriminierungen wegen der Religion oder der Weltanschauung, einer Behinderung, des Alters oder sexuellen Ausrichtung können die Verwirklichung der im EG-Vertrag festgelegten Ziele unterminieren, insbesondere die Erreichung eines hohen Beschäftigungsniveaus und eines hohen Maßes an sozialem Schutz, die Hebung des Lebensstandards und die Lebensqualität, den wirtschaftlichen und sozialen Zusammenhalt, die Solidarität sowie der Freizügigkeit.

(12) Daher sollte jede unmittelbare oder mittelbare Diskriminierungen wegen der Religion oder der Weltanschauung, einer Behinderung, des Alters oder sexuellen Ausrichtung in den von der Richtlinie abgedeckten Bereichen gemeinschaftsweit untersagt werden. Dieses Diskriminierungsverbot sollte auch für Staatsangehörigen dritter Länder gelten, betrifft jedoch nicht die Ungleichbehandlungen über die Einreise und den Aufenthalt von Staatsangehörigen dritter Länder und ihren Zugang zu Beschäftigung und Beruf unberührt.

(13)Diese Richtlinie findet weder Anwendung auf die Sozialversicherungs- und Sozialschutzsysteme, deren Leistungen nicht einem Arbeitsentgelt in dem Sinne gleichgestellt werden, der diesem Begriff für die Anwendung des Artikels 141 des EG-Vertrags gegeben wurde, noch auf Vergütungen jeder Art seitens des Staates, die den Zugang zu einer Beschäftigung oder die Aufrechterhaltung eines Beschäftigungsverhältnisses zum Ziel haben.

(14) ...

(15) ...

(16) Maßnahmen, die darauf abstellen, den Bedürfnissen von Menschen mit Behinderung am Arbeitsplatz Rechnung zu tragen, spielen eine wichtige Rolle bei der Bekämpfung von Diskriminierungen wegen einer Behinderung.

(17) Mit dieser Richtlinie wird unbeschadet der Verpflichtung, für Menschen mit Behinderung angemessene Vorkehrungen $\mathrm{zu}$ treffen, nicht die Einstellung, die berufliche Aufstieg, die Wiedereingliederung oder Teilnahme an Aus- und Weiterbildungsmaßnahmen einer Person vorgeschrieben, wenn diese Person für die Erfüllung der wesentlichen Funktionen des Arbeitsplatzes oder zur Absolvierung nicht kompetent, fähig oder verfügbar ist.

(18) $\ldots$

(19) ...

(20) Es sollten geeignete Maßnahmen vorgesehen werden, d. h. wirksame und praktikable Maßnahmen, um den Arbeitsplatz der Behinderung entsprechend einzurichten, z. B. durch eine entsprechende Gestaltung der Räumlichkeiten, oder eine Anpassung des Arbeitsgeräts, des Arbeitsrhythmus, der Aufgabenverteilung oder des Angebots der Ausbildungs- und Einarbeitungsmaßnahmen.

(21) ...

(22) ...

(23) Unter sehr begrenzten Bedingungen kann eine unterschiedliche Behandlung gerechtfertigt sein, wenn es Merkmal das mit Merkmal der Religion oder Weltanschauung, einer Behinderung, des Alters oder der sexuellen Ausrichtung zu- 
sammenhängt, eine wesentliche und entscheidende berufliche Anforderung darstellt, sofern es sich um einen rechtmäßigen Zweck und eine angemessene Forderung handelt.

(24) $\ldots$

(25) $\ldots$

(26) Das Diskriminierungsverbot sollte nicht der Beibehaltung oder dem Erlass von Maßnahmen entgegenstehen, mit denen bezweckt wird, Benachteiligungen von Personen mit einer bestimmten Religion, Religion oder Weltanschauung, einer Behinderung, des Alters oder der sexuellen Ausrichtung verhindern oder auszugleichen, und diese Maßnahmen können die Einrichtung und Beibehaltung von Organisationen von Personen mit einer Bestimmte Religion oder Weltanschauung, einer Behinderung, des Alters oder der sexuellen Ausrichtung zulassen, wenn deren Zweck hauptsächlich darin besteht, diese Bedürfnisse der Personen zu fördern.

(27) ...

(28) In dieser Richtlinie werden Mindestanforderungen festgelegt; es steht den Mitgliedstaaten somit frei, günstigere Vorschriften einzuführen und beizubehalten. $\cdots$

(29) Opfer von Diskriminierungen sollten über einen angemessenen Rechtsschutz verfügen. Um einen effektiveren Schutz zu gewährleisten, sollte auch die Möglichkeit bestehen, dass sich Verbände oder andere juristische Personen unbeschadet der nationalen Verfahrensordnung bezüglich der Vertretung und Verteidigung vor Gericht bei einem entsprechenden Beschluss der Mitgliedstaten im Namen eines Opfers oder zu seiner Unterstützung an einem Verfahren beteiligen.

(30) Die effektive Anwendung des Gleichheitsgrundsatzes erfordert einen angemessenen Schutz vor Viktimisierisung.

(31)Eine Änderung der Regeln für die Beweislast ist geboten, wenn ein glaubhafter Anschein einer Diskriminierung besteht. ...

(32) ...

(33) Die Mitgliedstaaten sollten den Dialog zwischen den Sozialpartnern und im Rahmen der einzelstaatlichen Gepflogenheiten mit Nichtregierungsorganisationen mit dem Ziel fördern, die Diskriminierung am Arbeitsplatz anzugehen und diese zu bekämpfen.

(34) ...

(35)Die Mitgliedstaaten sollten wirksame, verhältnismäßige und abschreckende Sanktionen für den Fall vorsehen, dass gegen die aus diesen Richtlinien erwachsenden Verpflichtungen verstoßen wird.

(36) Die Mitgliedstaaten können den Sozialpartnern auf deren gemeinsamen Antrag die Durchführung der Bestimmungen dieser Richtlinie übertragen, die in den Anwendungsbereich von Tarifverträgen fallen, ...

hat folgende Richtlinie erlassen: 
Kapitel I

Allgemeine Bestimmungen

Artikel 1 Zweck

Zweck dieser Richtlinie ist die Schaffung des allgemeinen Rahmens zur Bekämpfung der Diskriminierung wegen der Religion oder Weltanschauung, einer Behinderung, des Alters oder der sexuellen Ausrichtung in Beschäftigung und Beruf im Hinblick auf die Verwirklichung de Grundsatzes der Gleichbehandlung in den Mitgliedstaaten.

\section{Artikel 2 Der Begriff ,Diskriminierung “}

(1) Im Sinne dieser Richtlinie bedeutet „Gleichbehandlungsgrundsatz“, dass es keine unmittelbare oder mittelbare Diskriminierung wegen eines der in Artikel 1 genannten Gründe geben darf.

(2) Im Sinne des Absatzes 1

a) liegt eine unmittelbare Diskriminierung vor, wenn eine Person wegen eines der in Artikel 1 genannten Gründe in einer vergleichbaren Situation eine weniger günstigere Behandlung erfährt als eine andere Person erfährt, erfahren hat oder erfahren würde

b) liegt eine mittelbare Diskriminierung vor, wenn dem Anschein nach neutrale Vorschriften, Kriterien oder Verfahren der Personen mit einer bestimmten Religion oder Weltanschauung, einer bestimmten Behinderung, eines bestimmten Alters oder bestimmten sexuellen Ausrichtung gegenüber anderen Personen in besonderer Weise benachteiligen können, es sei denn:

(i) diese Vorschriften, Kriterien oder Verfahren sind durch ein rechtmäßiges Ziel sachlich gerechtfertigt, und die Mittel sind zur Erreichung dieses Ziels angemessen und erforderlich, oder

(ii) der Arbeitgeber oder jede Person oder Organisation, auf die diese Richtlinie Anwendung findet, ist im Falle von Personen mit einer bestimmten Behinderung aufgrund der einzelstaatlichen Rechts verpflichtet, geeignete Maßnahmen entsprechend den in Artikel 5 enthaltne Grundsätzen vorzusehen, um die sich durch diese Vorschrift, dieses Kriterium oder Verfahren ergebenden Nachteile zu beseitigen.

(3) Unerwünschte Verhaltensweisen, die mit einem der Gründe nach Artikel 1 in Zusammenhang stehen und bezwecken oder bewirken, dass die Würde der betreffenden Person verletzt und ein von Einschüchterungen , Anfeindungen, Erniedrigungen, Entwürdigungen oder Beleidigungen gekennzeichnetes Umfeld geschaffen wird, sind Belästigungen, die als Diskriminierung im Sinne von Absatz 1 gelten. In diesem Zusammenhang können die Mitgliedstaaten den Begriff Belästigungen im Einklang mit den einzelstaatlichen Rechtsvorschriften und Gepflogenheiten definieren. 


\section{Artikel 3 Geltungsbereich}

(1) Im Rahmen der auf die Gemeinschaft übertragenen Zuständigkeiten gilt diese Richtlinie für alle Personen in öffentlichen und privaten Bereichen, einschließlich öffentlicher Stellen, in Bezug auf

a) Die Bedingungen - einschließlich Auswahlkriterien und Einstellungsbedingungen für den Zugang zu unselbständiger und selbständiger Erwerbstätigkeit, unabhängig von Tätigkeitsfeld und beruflicher Position, einschließlich des beruflichen Aufstiegs

b) den Zugang zu allen Formen und allen Ebenen der Berufsberatung, der Berufsausbildung, der beruflichen Weiterbildung und der Umschulung, einschließlich der praktischen Berufserfahrung;

c) die Beschäftigungs- und Arbeitsbedingungen einschließlich der Entlassungsbediungen und des Arbeitsentgelts;

d) die Mitgliedschaft und Mitwirkung in einer Arbeitnehmer- oder Arbeitgeberorganisation oder einer Organisation, deren Mitglieder einer bestimmten Berufsgruppe angehören, einschließlich der Inanspruchnahme der Leistungen solcher Organisationen;

(2) Diese Richtlinie betrifft nicht unterschiedliche Behandlungen aus Gründen der Staatsangehörigkeit und berührt nicht die Vorschriften und Beschäftigungen für die Einreise von Staatsangehörigen dritter Länder oder staatenlosen Personen in das Hoheitsgebiet der Mitgliedstaaten oder deren Aufenthalt im Hoheitsgebiet sowie eine Behandlung, die sich aus der Rechtstellung von Staatsangehörigen dritter Länder oder staatenloser Personen ergibt.

(3) Diese Richtlinie gilt nicht für Leistungen jeder Art seitens der staatlichen Systeme oder damit gleichgestellten Systeme einschließlich der staatlichen Systeme der sozialen Sicherheit oder des sozialen Schutzes.

(4) Die Mitgliedstaaten können vorsehen, das diese Richtlinie hinsichtlich von Diskriminierungen wegen einer Behinderung und des Alters nicht für die Streitkräfte gilt.

\section{Artikel 4 Berufliche Anforderungen}

(1) Ungeachtet des Artikels 2 Absätze 1 und 2 können die Mitgliedstaaten vorsehen, dass eine Ungleichbehandlung wegen eines Merkmals, das im Zusammenhang mit einem der in Artikel 1 genannten Diskriminierungsgründe steht, keine Diskriminierungen darstellt, wenn das betreffende Merkmal aufgrund der Art einer bestimmten beruflichen Tätigkeit oder Bedingungen ihrer Ausübung eine wesentliche und entscheidende berufliche Anforderung darstellt, sofern es sich um einen rechtmäßigen Zweck und eine angemessene Anforderung handelt.

\section{Artikel 5 Angemessene Vorkehrungen für Menschen mit Behinderung}

Um die Anwendung des Gleichbehandlungsgrundsatzes auf Menschen mit Behinderung zu gewährleisten, sind angemessene Vorkehrungen zu treffen. Das bedeutet dass der Arbeitgeber die geeigneten und im konkreten Fall erforderlichen Maßnah- 
me ergreift, um den Menschen mit Behinderung den Zugang zur Beschäftigung, die Ausübung eines Berufs, des beruflichen Aufstiegs, Teilnahme an Aus- und Weiterbildungsmaßnahmen zu ermöglichen, es sei denn diese Maßnahme würden den Arbeitgeber unverhältnismäßig belasten. Diese Belastung ist nicht unverhältnismäßig, wenn sie durch geltende Maßnahmen im Rahmen der Behindertenpolitik des Mitgliedstaats nicht ausreichend kompensiert wird.

\section{Artikel 6 Gerechtfertigte Ungleichbehandlung wegen des Alters}

\section{Artikel 7 Positive und spezifische Maßnahmen}

(1) Der Gleichbehandlungsgrundsatz hindert die Mitgliedstaaten nicht daran, zur Gewährleistung der völligen Gleichstellung im Berufsleben spezifische Maßnahmen beizubehalten oder einzuführen, mit denen Benachteiligungen wegen eines Diskriminierungsgrundes verhindert oder ausgeglichen werden.

(2) Im Falle von Menschen mit Behinderung steht der Gleichbehandlungsgrundsatz weder dem Recht der Mitgliedstaaten entgegen, Bestimmungen zum Schutz der Gesundheit und der Sicherheit am Arbeitsplatz beizubehalten oder zu erlassen, noch steht er Maßnehmen entgegen, mit denen Bestimmungen der Vorkehrungen eingeführt oder beibehalten werden sollen, die einer Eingliederung von Menschen mit Behinderung in die Arbeitswelt dienen oder dieser Eingliederung fördern.

\section{Artikel 8 Mindestanforderungen}

(1) Die Mitgliedstaaten können Vorschriften einführen, oder beibehalten die im Hinblick auf die Wahrung des Gleichbehandlungsgrundsatzes günstiger als die in dieser Richtlinie vorgesehenen Vorschriften sind.

(2) Die Umsetzung dieser Richtlinie darf keinesfalls als Rechtfertigung für eine Absenkung des von den Mitgliedstaten bereits garantierten allgemeinen Schutzniveaus in Bezug auf Diskriminierungen in den von der Richtlinie abgedeckten Bereichen benutzt werden.

\section{Kapitel II}

\section{Rechtsbehelfe und Rechtsdurchsetzung}

\section{Artikel 9 Rechtsschutz}

(1) Die Mitgliedstaaten stellen sicher, dass alle Personen, die sich durch die Nichtanwendung des Gleichbehandlungsgrundsatzes in ihren Rechten für verletzt halten, ihre Ansprüche aus dieser Richtlinie auf dem Gerichts- oder Verwaltungsweg sowie, wenn diese Mitgliedstaaten es angezeigt hatten, in Schlichtungsverfahren geltend machen können, selbst wenn das Verhältnis, während dessen die Diskriminierung vorgekommen sein soll, bereits beendet ist.

(2) Die Mitgliedstaaten stellen sicher, dass Verbände, Organisationen oder andere juristische Personen, die gemäß den in ihrem einzelstaatlichen Recht festgeleg- 
ten Kriterien ein rechtmäßiges Interesse daran haben, für die Einhaltung der Bestimmungen dieser Richtlinie zu sorgen, sich entweder im Namen der beschwerten Person oder zu deren Unterstützung und mit deren Einwilligung an den in dieser Richtlinie zur Durchsetzung vorgesehenen Gerichts- und/oder Verwaltungsverfahren beteiligen können.

\section{Artikel 10 Beweislast}

Die Mitgliedstaaten ergreifen im Einklang mit ihrem nationalen Gerichtswesen die erforderlichen Maßnahmen, um zu gewährleisten, dass immer dann, wenn Personen, die sich durch die Nichtanwendung des Gleichbehandlungsgrundsatzes für verletzt halten, und bei einem Gericht oder einer anderen zuständigen Stelle glaubhaft machen, die das Vorliegen einer unmittelbaren oder mittelbaren Diskriminierung vermuten lassen, es dem Beklagte obliegt, Tatsachen zu beweisen, dass keine Verletzung des Gleichbehandlungsgrundsatzes vorgelegen hat.

\section{Artikel 11 Viktimisierung}

Die Mitgliedstaaten treffen im Rahmen ihrer nationalen Rechtsordnung die erforderlichen Maßnahmen, um die Arbeitnehmer vor Entlassung oder anderen Benachteiligungen durch den Arbeitgeber zu schützen, die als Reaktion auf eine Beschwerde innerhalb des betreffenden Unternehmens oder auf die Einleitung eines Verfahrens zur Durchsetzung der Gleichbehandlung erfolgen.

\section{Artikel 12 Unterrichtung}

Die Mitgliedstaaten tragen dafür Sorge, dass die gemäß dieser Richtlinie getroffenen Maßnahmen sowie die bereits geltenden einschlägigen Vorschriften allen Betroffenen in geeigneter Form, zum Beispiel am Arbeitsplatz, in ihrem Hoheitsgebiet bekannt gemacht werden.

\section{Artikel 13 Sozialer Dialog}

(1) Die Mitgliedstaaten treffen im Einklang mit den einzelstaatlichen Gepflogenheiten und Verfahren geeignete Maßnahmen zur Förderung des sozialen Dialogs zwischen Arbeitgebern und Arbeitnehmern mit dem Ziel, die Verwirklichung des Gleichbehandlungsgrundsatzes durch Überwachung der betrieblichen Praxis, oder durch einen Austausch von Erfahrungen und bewährten Verfahren, voranzubringen.

(2) Soweit vereinbar mit den einzelstaatlichen Gepflogenheiten und Verfahren, fordern die Mitgliedstaaten Arbeitnehmer und Arbeitgeber ohne Eingriff in deren Autonomie auf, auf geeigneter Ebene Antidiskriminierungsvereinbarungen zu schließen, die die in Artikel 3 genannten Bereiche betreffen, soweit diese in den Anwendungsbereich der Tarifparteien fallen. Die Vereinbarungen müssen den in dieser Richtlinie sowie den in den einschlägigen nationalen Durchführungsbestimmungen festgelegten Mindestanforderungen entsprechen. 
Artikel 14 Dialog mit Nichtregierungsorganisationen

Die Mitgliedstaaten fördern den Dialog mit den jeweiligen Nichtregierungsorganisationen, die gemäß den Rechtsvorschriften und den einzelstaatlichen Gepflogenheiten ein rechtmäßiges Interesse daran haben, sich an der Bekämpfung von Diskriminierung wegen eines der in Artikel 1 genannten Gründe beteiligen, um die Einhaltung des Grundsatzes der Gleichbehandlung zu fördern.

Kapitel III

Besondere Bestimmungen

Artikel 15 Nordirland

Kapitel IV

Schlussbestimmungen

Artikel 16 Einhaltung

Die Mitgliedstaaten treffen die erforderlichen Maßnahmen, um sicherzustellen, dass

a) die Rechts- und Verwaltungsvorschriften, die dem Gleichbehandlungsrundsatz zuwiderlaufen, aufgehoben werden

b) die mit dem Gleichbehandlungsrundsatz nicht zu vereinbarenden Bestimmungen im Arbeits- und Tarifverträgen, Betriebsordnungen und Statuten der freien Berufe und Arbeitgeber- und Arbeitnehmerorganisationen für nichtig erklärt werden oder erklärt werden können oder geändert werden

\section{Artikel 17 Sanktionen}

Die Mitgliedstaaten legen die Sanktionen fest, die bei einem Verstoß gegen die einzelstaatlichen Vorschriften zur Anwendung dieser Richtlinie zu verhängen sind, und treffen alle erforderlichen Maßnahmen, um deren Durchführung zu gewährleisten. Die Sanktionen, die auch Schadensersatzleistungen an die Opfer umfassen können, müssen wirksam, verhältnismäßig und abschreckend sein.

\section{Artikel 18 Umsetzung der Richtlinie}

Die Mitgliedstaaten erlassen die erforderlichen Rechts -und Verwaltungsvorschriften um dieser Richtlinie spätestens zum 2. Dezember 2003 nachzukommen, oder können den Sozialpartnern auf deren gemeinsamen Antrag die Durchführung übertragen, die in den Anwendungsbereich von Tarifverträgen fallen ....

\section{Artikel 19 Bericht}

(1) Bis zum 2. Dezember 2005 und in der Folge alle fünf Jahre übermitteln die Mitgliedstaaten der Kommission sämtliche Informationen, die diese für die Erstellung eines dem Europäischen Parlament und dem Rat vorzulegenden Berichts über die Anwendung dieser Richtlinie benötigt. 
(2) Die Kommission berücksichtigt in ihrem Bericht in angemessener Weise die Standpunkte der Sozialpartner und der einschlägigen Nichtregierungsorganisationen. Im Einklang mit dem Grundsatz der systematischen Berücksichtigung geschlechtsspezifischer Fragen wird ferner in dem Bericht die Auswirkung auf Frauen und Männer bewertet. Unter Berücksichtigung der übermittelten Informationen enthält der Bericht erforderlichenfalls auch Vorschläge zu Änderungen und Aktualisierungen dieser Richtlinie.

Artikel 20 Inkrafttreten

Diese Richtlinie tritt am Tag ihrer Veröffentlichung im Amtsblatt der Europäischen Gemeinschaften in Kraft.

Artikel 21 Adressaten

Diese Richtlinie ist an die Mitgliedstaaten gerichtet.

Geschehen zu Brüssel am 27. November 2000 


\title{
Annex 3: Charta der Grundrechte der Europäischen Union (2002) $\left(\right.$ Auszug) ${ }^{73}$
}

\author{
PRÄAMBEL
}

... erkennt die Union die nachstehend aufgeführten Rechte, Freiheiten und Grundsätze an.

\section{KAPITEL I \\ WÜRDE DES MENSCHEN}

Artikel 1 Würde des Menschen

Die Würde des Menschen ist unantastbar. Sie ist zu achten und zu schützen.

Artikel 2 Recht auf Leben

(1) Jede Person hat das Recht auf Leben.

\section{Artikel 3 Recht auf Unversehrtheit}

(1) Jede Person hat das Recht auf körperliche und geistige Unversehrtheit.

(2) Im Rahmen der Medizin und der Biologie muss insbesondere Folgendes beachtet werden:

die freie Einwilligung der betroffenen Person nach vorheriger Aufklärung entsprechend den gesetzlich festgelegten Modalitäten,

das Verbot eugenischer Praktiken, insbesondere derjenigen, welche die Selektion von Personen zum Ziel haben,

das Verbot, den menschlichen Körper und Teile davon als solche zur Erzielung von Gewinnen zu nutzen, das Verbot des reproduktiven Klonens von Menschen.

\section{Artikel 14 Recht auf Bildung}

(1) Jede Person hat das Recht auf Bildung sowie auf Zugang zur beruflichen Ausbildung und Weiterbildung.

(2) Dieses Recht umfasst die Möglichkeit, unentgeltlich am Pflichtschulunterricht teilzunehmen.

(3) Die Freiheit zur Gründung von Lehranstalten unter Achtung der demokratischen Grundsätze sowie das Recht der Eltern, die Erziehung und den Unterricht ihrer Kinder entsprechend ihren eigenen religiösen, weltanschaulichen und erzieherischen Überzeugungen sicherzustellen, werden nach den einzelstaatlichen Gesetzen geachtet, welche ihre Ausübung regeln. 
Artikel 15 Berufsfreiheit und Recht zu arbeiten

(1) Jede Person hat das Recht, zu arbeiten und einen frei gewählten oder angenommenen Beruf auszuüben.

(2) Alle Unionsbürgerinnen und Unionsbürger haben die Freiheit, in jedem Mitgliedstaat Arbeit zu suchen, zu arbeiten, sich niederzulassen oder Dienstleistungen zu erbringen.

(3) Die Staatsangehörigen dritter Länder, die im Hoheitsgebiet der Mitgliedstaaten arbeiten dürfen, haben Anspruch auf Arbeitsbedingungen, die denen der Unionsbürgerinnen und Unionsbürger entsprechen. $\cdots$

KAPITEL III

GLEICHHEIT

Artikel 20 Gleichheit vor dem Gesetz

Alle Personen sind vor dem Gesetz gleich.

\section{Artikel 21 Nichtdiskriminierung}

(1) Diskriminierungen insbesondere wegen des Geschlechts, der Rasse, der Hautfarbe, der ethnischen oder sozialen Herkunft, der genetischen Merkmale, der Sprache, der Religion oder der Weltanschauung, der politischen oder sonstigen Anschauung, der Zugehörigkeit zu einer nationalen Minderheit, des Vermögens, der Geburt, einer Behinderung, des Alters oder der sexuellen Ausrichtung sind verboten.

(2) Im Anwendungsbereich des Vertrags zur Gründung der Europäischen Gemeinschaft und des Vertrags über die Europäische Union ist unbeschadet der besonderen Bestimmungen dieser Verträge jede Diskriminierung aus Gründen der Staatsangehörigkeit verboten.

\section{Artikel 22 Vielfalt der Kulturen, Religionen und Sprachen}

\section{Artikel 23 Gleichheit von Männern und Frauen}

Die Gleichheit von Männern und Frauen ist in allen Bereichen, einschließlich der Beschäftigung, der Arbeit und des Arbeitsentgelts, sicherzustellen.

Der Grundsatz der Gleichheit steht der Beibehaltung oder der Einführung spezifischer Vergünstigungen für das unterrepräsentierte Geschlecht nicht entgegen.

\section{Artikel 24 Rechte des Kindes}

(1) Kinder haben Anspruch auf den Schutz und die Fürsorge, die für ihr Wohlergehen notwendig sind. Sie können ihre Meinung frei äußern. Ihre Meinung wird in den Angelegenheiten, die sie betreffen, in einer ihrem Alter und ihrem Reifegrad entsprechenden Weise berücksichtigt.

(2) Bei allen Kinder betreffenden Maßnahmen öffentlicher oder privater Einrichtungen muss das Wohl des Kindes eine vorrangige Erwägung sein. 
(3) Jedes Kind hat Anspruch auf regelmäßige persönliche Beziehungen und direkte Kontakte zu beiden Elternteilen, es sei denn, dies steht seinem Wohl entgegen.

\title{
Artikel 25 Rechte älterer Menschen
}

Die Union anerkennt und achtet das Recht älterer Menschen auf ein würdiges und unabhängiges Leben und auf Teilnahme am sozialen und kulturellen Leben.

\section{Artikel 26 Integration von Menschen mit Behinderung}

Die Union anerkennt und achtet den Anspruch von Menschen mit Behinderung auf Maßnahmen zur Gewährleistung ihrer Eigenständigkeit, ihrer sozialen und beruflichen Eingliederung und ihrer Teilnahme am Leben der Gemeinschaft.

\section{KAPITEL IV \\ SOLIDARITÄT}

\begin{abstract}
Artikel 27 Recht auf Unterrichtung und Anhörung der Arbeitnehmerinnen und Arbeitnehmer im Unternehmen
\end{abstract}

Artikel 28 Recht auf Kollektivverhandlungen und Kollektivmaßnahmen

Artikel 29 Recht auf Zugang zu einem Arbeitsvermittlungsdienst

Artikel 30 Schutz bei ungerechtfertigter Entlassung

...

Artikel 31 Gerechte und angemessene Arbeitsbedingungen

$$
\text { ... }
$$

Artikel 32 Verbot der Kinderarbeit und Schutz der Jugendlichen am Arbeitsplatz

\section{Artikel 33 Familien- und Berufsleben}

(1) Der rechtliche, wirtschaftliche und soziale Schutz der Familie wird gewährleistet.

(2) Um Familien- und Berufsleben miteinander in Einklang bringen zu können, hat jede Person das Recht auf Schutz vor Entlassung aus einem mit der Mutterschaft zusammenhängenden Grund sowie den Anspruch auf einen bezahlten Mutterschaftsurlaub und auf einen Elternurlaub nach der Geburt oder Adoption eines Kindes.

\section{Artikel 34 Soziale Sicherheit und soziale Unterstützung}

(1) Die Union anerkennt und achtet das Recht auf Zugang zu den Leistungen der sozialen Sicherheit und zu den sozialen Diensten, die in Fällen wie Mutterschaft, Krankheit, Arbeitsunfall, Pflegebedürftigkeit oder im Alter sowie bei Verlust des Arbeitsplatzes Schutz gewährleisten, nach Maßgabe des Gemeinschaftsrechts und der einzelstaatlichen Rechtsvorschriften und Gepflogenheiten. 
(2) Jede Person, die in der Union ihren rechtmäßigen Wohnsitz hat und ihren Aufenthalt rechtmäßig wechselt, hat Anspruch auf die Leistungen der sozialen Sicherheit und die sozialen Vergünstigungen nach dem Gemeinschaftsrecht und den einzelstaatlichen Rechtsvorschriften und Gepflogenheiten.

(3) Um die soziale Ausgrenzung und die Armut zu bekämpfen, anerkennt und achtet die Union das Recht auf eine soziale Unterstützung und eine Unterstützung für die Wohnung, die allen, die nicht über ausreichende Mittel verfügen, ein menschenwürdiges Dasein sicherstellen sollen, nach Maßgabe des Gemeinschaftsrechts und der einzelstaatlichen Rechtsvorschriften und Gepflogenheiten.

\section{Artikel 35 Gesundheitsschutz}

Jede Person hat das Recht auf Zugang zur Gesundheitsvorsorge und auf ärztliche Versorgung nach Maßgabe der einzelstaatlichen Rechtsvorschriften und Gepflogenheiten. Bei der Festlegung und Durchführung aller Politiken und Maßnahmen der Union wird ein hohes Gesundheitsschutzniveau sichergestellt.

Artikel 36 Zugang zu Dienstleistungen von allgemeinem wirtschaftlichem Interesse Die Union anerkennt und achtet den Zugang zu Dienstleistungen von allgemeinem wirtschaftlichen Interesse, wie er durch die einzelstaatlichen Rechtsvorschriften und Gepflogenheiten im Einklang mit dem Vertrag zur Gründung der Europäischen Gemeinschaft geregelt ist, um den sozialen und territorialen Zusammenhalt der Union zu fördern.

\section{KAPITEL VII \\ ALLGEMEINE BESTIMMUNGEN}

\section{Artikel 51 Anwendungsbereich}

(1) Diese Charta gilt für die Organe und Einrichtungen der Union unter Einhaltung des Subsidiaritätsprinzips und für die Mitgliedstaaten ausschließlich bei der Durchführung des Rechts der Union. Dementsprechend achten sie die Rechte, halten sie sich an die Grundsätze und fördern sie deren Anwendung gemäß ihren jeweiligen Zuständigkeiten.

(2) Diese Charta begründet weder neue Zuständigkeiten noch neue Aufgaben für die Gemeinschaft und für die Union, noch ändert sie die in den Verträgen festgelegten Zuständigkeiten und Aufgaben. 
6. Behindertenrecht und Behindertenpolitik in Asien 


\title{
6.1. Behinderte Menschen in der Volksrepublik China
}

\author{
Sicht einer Betroffenen
}

\author{
Von Ding $\mathrm{Na}$
}

Die Volksrepublik China ist nach wie vor ein Entwicklungsland, allerdings das mit der weltweit größten Bevölkerungszahl. Bei der Gesamtbevölkerung von ca. 1,3 Milliarden wird die Zahl der behinderten Menschen auf 83 Millionen - mehr als 6\% der Gesamtbevölkerung - geschätzt. Eine genaue Angabe über die Zahl der Menschen mit Behinderung in der VR China liegt nicht vor, da von der Regierung bisher nur ein Mal alle zehn Jahre nationale Stichproben in ausgewählten Provinzen und autonomen Regionen durchgeführt worden sind. Diese Schätzungen gelten als höchst unzuverlässig und es wird mittlerweile von einer Dunkelziffer von mindestens 130 Millionen Behinderten gesprochen. Erschwert wird die Feststellung der Anzahl behinderter Menschen in der VR China noch dadurch, dass es keine zentrale Stelle, d.h. kein Ministerium oder ähnliche administrative Einrichtungen gibt, die speziell für das Problem Behinderung zuständig sind. Es gibt auch keine Sozialversicherung für Behinderte. Menschen mit Behinderung genießen grundsätzlich die gleichen Bürgerrechte wie ,normale“ Bürger und der Staat hat lediglich spezielle rechtliche Bestimmungen ausgearbeitet, um die legitimen Interessen der Behinderten zu sichern. So gibt es neben dem Behindertengesetz der Volksrepublik China zahlreiche weitere Rechtsvorschriften, Verordnungen und Sonderbestimmungen zum Schutz Behinderter, z.B. in der Verfassung, im Erziehungsgesetz, im Eherecht etc. In den Erziehungsvorschriften für Behinderte, die seit 23.8.1994 in Kraft sind, wird geregelt, dass für Behinderte eine Chancengleichheit an Universitäten und Hochschulen gewährleistet sein muss. Ferner ist im Behindertengesetz der VR China die Gleichberechtigung der Behinderten in den Bereichen Politik, Wirtschaft, Kultur, Gesellschaft und im Familienleben festgeschrieben. Die Rechte und die Würde Behinderter stehen unter dem Schutz des Staates. Diese Bestimmungen, Verordnungen etc. begründen jedoch in der Praxis oft keinen durchsetzbaren Anspruch für die Betroffenen. Nach dem Motto: „der Himmel ist blau und Peking ist weit" stehen sie im Ermessen der Verwaltungen und scheitern in vielen Fällen an den Hürden der Bürokratie, die sie im Sande verlaufen lässt.

Als ,,behindert“ gelten in der Volksrepublik China Blinde, Taubstumme, körperlich und/oder geistig Behinderte, psychisch Kranke, mehrfach Behinderte u.a. Die Kriterien für die Feststellung einer Behinderung in China sind im Vergleich zu Europa oder Amerika sehr streng.

So gilt z.B. in Amerika schon als behindert, wer nur einen Teil eines Fingers verloren hat, während nach chinesischem Verständnis noch keine Behinderung vorliegt, solange noch Daumen und Zeigefinger vorhanden sind, weil es noch möglich ist, etwas damit zu greifen bzw. festzuhalten. Ebenso gilt ein Mensch, der nur auf einem 
Auge blind ist, oder dessen Sehschärfe an einem Auge mehr als 0,01\% beträgt, nicht als blind.

Der Staat ergreift zahlreiche Maßnahmen zur Unterstützung Behinderter. Vor allem Kriegsinvalide und aufgrund von Dienstunfällen behinderte Personen werden vom Staat bevorzugt behandelt und unterstützt, weil sie ihre Gesundheit im Dienste des Vaterlandes geopfert haben. Die Regierungen auf allen Ebenen werden vom Staat angehalten, dafür Sorge zu tragen, dass Behinderten eine Eingliederung in das Berufsleben ermöglicht werden kann.

Zudem sind in China in den letzten Jahren zahlreiche Maßnahmen ergriffen worden, um eine adäquate medizinische Versorgung behinderter Menschen sicherzustellen. So sind spezielle Krankenhäuser, orthopädische Kliniken und psychiatrische Anstalten erbaut worden, in denen Behinderte medizinisch angemessen versorgt werden mit dem Ziel einer Wiedereingliederung in die Gesellschaft, indem sie selbst für ihren Unterhalt sorgen können.

Das Recht auf Bildung ist für Behinderte im Behindertengesetz der VR China geregelt; dieses bestimmt, dass der Staat, die Schulen und die Familien dafür verantwortlich sind, behinderten Kindern und Jugendlichen den Schulbesuch zu ermöglichen. Der Staat stellt Lernhilfen und Studienbeihilfen zur Verfügung, um finanziell bedürftige behinderte Schüler zu unterstützen. Grund- und Mittelschulen sowie Berufsfachschulen und Universitäten sollen Behinderte aufnehmen, sofern diese in der Lage sind, dem Unterricht zu folgen.

Daneben gibt es in China Sonderschulen, in denen Kinder und Jugendliche eine Ausbildung absolvieren können.

Im Jahre 2007 gab es in China z.B. über 1.600 Sonderschulen und Sonderklassen mit ca. 60.000 Schülern. 77\% der Behinderten im schulpflichtigen Alter ist auf diese Weise ein Schulbesuch ermöglicht worden und ca. 2,5 Mio. Behinderte absolvieren darauf aufbauend eine Berufsausbildung.

Im Behindertengesetz der VR China finden sich auch Vorschriften über die Einstellung von Behinderten in Betrieben, sowie über die Erschließung selbständiger Erwerbs- und Beschäftigungsmöglichkeiten. Zu diesem Zweck sind spezielle betriebliche Einrichtungen für Behinderte errichtet worden und die Regional- und Lokalregierungen sollen bevorzugt Behinderte einstellen und Schutzmaßnahmen ergreifen, damit arbeitsfähige Behinderte in die Gesellschaft eingegliedert werden können. Laut Statistik werden ca. 73,3\% der Behinderten in den Arbeitsmarkt eingegliedert.

Die Einsatzbereiche Behinderter sind sog. Wohlfahrtsfabriken, Genossenschaften (jeweils gemessen an Art und Grad der Behinderung) und als Selbständige (ebenfalls je nach Grad und Art der Behinderung) selbständige Tätigkeit. Als Selbständige werden z.B. Blinde und taube Menschen als Masseure ausgebildet und vorzugsweise Blinde als Klavier- und Instrumentenstimmer. (Blinde haben seit langer Zeit die größte Lobby der Behinderten. In Shanghai existiert ein sog. „Haus der Blinden“, das dem Vernehmen nach von den „Triaden“ geführt wird und dessen Bewohner sich ihren Lebensunterhalt und Unterkunft durch Diebstähle etc. „erarbeiten“ müssen.) 
Seit einiger Zeit gibt es ein sog. Quotensystem, d.h. von den Arbeitnehmern eines Unternehmens sollen ca. 1,5\% behindert sein. Diese Zahl ist aber schon deshalb unrealistisch, weil die Situation der Behinderten von Region zu Region unterschiedlich ist. Vorbildfunktion auf diesem Sektor haben Großstädte wie Peking, Shanghai, Guangdong und Shandong. Es ist gesetzlich geregelt, dass staatliche Organe, Kader und Unternehmen Behinderte zu einem festgelegten Vomhundertsatz einstellen und ihnen einen Arbeitsplatz anbieten müssen. Das gilt sowohl in Städten in Fabriken, wo Behinderte für einfache Arbeiten am Fließband eingesetzt werden können, als auch auf dem Land, wo sie in landwirtschaftlichen Betrieben beim Anbau von Nahrungsmitteln, der Bewässerung der Felder oder sonstigen leichten handwerklichen Tätigkeiten nachkommen können und so in die Gesellschaft integriert werden. Dafür sind im Jahre 2007 vom Staat ca. 150 Mio Yuan RMB (ca. 15 Mio. $€$ ) bereitgestellt worden.

Die Versorgung Behinderter in der VR China in Bezug auf ihre persönlichen Rechte, Rehabilitation, Bildung, Beschäftigung, Existenzsicherung (Unterkunft, Nahrung und Kleidung), Barrierefreiheit sowie der Zugang zu Rechtsvorschriften und Normen wurden verbessert. 2007 sind für 6 Provinzen, 76 Städte und 350 Landkreise behindertenspezifische Rechts- und VerwaltungsvorschriftenDekrete erlassen worden. Der Zugang zu kulturellen Aktivitäten, Sensibilisierung der Öffentlichkeit und Sport (Paralympics) ist gesetzlich gewährleistet.

In der Praxis ergeben sich jedoch bei der Ausführung der Gesetze, Vorschriften und Dekrete erhebliche Probleme. Mit Ausnahme der Kriegsinvaliden, die (wie oben bereits erwähnt) unter dem besonderen Schutz des Staates stehen und der aufgrund von Arbeitsunfällen behinderten Menschen für die besondere Vorschriften einer Berufsunfallversicherung vorgesehen sind, ist der soziale Schutz behinderter Menschen schwer durchsetzbar, weil dem chinesischen Rechtsverständnis immer noch der Gedanke der Erziehung zugrunde liegt, d.h. der Staat übernimmt in erster Linie Verantwortung für die Bürger, die etwas für den Staat leisten oder geleistet haben.

Dies liegt vor allem auch begründet in dem jahrhundertelangen Denken der Menschen, (das bis heute tief verwurzelt ist) dass Behinderung ein Fluch, die Strafe Gottes für eine böse Tat in einem anderen Leben oder in diesem Leben ist. Bringt eine Frau ein behindertes Kind zur Welt, so werden sie und ihre ganze Familie von den Mitmenschen diskriminiert und isoliert und die „Ursache“ für den Schicksalsschlag im Leben der Eheleute bzw. ihrer Vorfahren gesucht.

Gesetzlich festgeschrieben ist in der VR China, dass z.B. lebend geborene Kinder mit Down-Syndrom aufgezogen werden müssen. Die normalerweise greifende „EinKind-Regelung“ gilt nach ärztlich bestätigter Diagnose der Behinderung nicht. Eltern eines Kindes mit Down Syndrom haben das Recht auf ein weiteres Kind. In den Städten werden bis auf ganz wenige Ausnahmen geistig behinderte Kinder nach der Geburt im Krankenhaus zurückgelassen und im Falle des Überlebens in Heimen untergebracht. Erfolgt die Geburt zuhause oder in ländlichen Gebieten, so werden diese Kinder unmittelbar nach der Geburt ausgesetzt oder es wird die Empfehlung befolgt, sie gleich zu töten. 
Körperlich behinderte Kinder werden je nach dem Grad der Behinderung entweder ebenfalls getötet, ausgesetzt oder bis zum Alter von ca. 8-9 Jahren großgezogen und dann als rechtlose Arbeitskräfte an Menschenhändler oder in die Prostitution verkauft.

Vereinzelt gibt es in den Städten Stiftungen, die traditionell von reichen Wohltätern finanziert werden und die Unterkunft, Essen, Kleidung und - wenn möglich auch die Ausbildung behinderter Kinder ermöglichen. Das Überleben behinderter Kinder liegt aber nahezu ausschließlich in privater Hand. Eltern, die finanziell in der Lage sind und sich dazu entschließen, ihr Kind selbst aufzuziehen, sehen sich meist sowohl mit gesamtgesellschaftlichen als auch mit großen innerfamiliären Widerständen konfrontiert. Es gibt keine Frühförderung, Schulbildung wird für Kinder mit Behinderung meist als unnütze Verschwendung angesehen, medizinische Eingriffe (z.B. eine Herzoperation) müssen schwer erkämpft werden, und wenn sie durchgesetzt worden sind, müssen sie von den Eltern komplett selbst finanziert werden

Am 26. Juni 2008 ist vom 11. Nationalen Volkskongress der VR China ein Gesetzentwurf über die Rechte von Menschen mit Behinderung ratifiziert worden. Es ist vorgesehen, dass die Regierung der VR China staatliche Bestimmungen und Maßnahmen ausarbeitet, um mehr für die soziale Absicherung behinderter Menschen zu tun. So sollen z.B. in Zukunft alle behinderten Angestellten und Arbeiter gesetzlich dazu verpflichtet werden, sich zu versichern, damit sie eine soziale Grundabsicherung haben. Diese Maßnahmen sollen auch für die ländlichen Gebiete erarbeitet und den dortigen Verhältnissen angepasst werden.

Seit einigen Jahren werden in der VR China auch immer mehr staatlich geförderte Pflegeheime gebaut, die eine allerdings nur begrenzte Zahl Behinderter aufnehmen können. In diesen Pflegeheimen wird für die alltägliche Versorgung (Wohnen, Essen, Kleidung), die medizinische Betreuung und die Ausbildung der Behinderten gesorgt - allerdings ist es noch dringend notwendig, die Kontrolle über die Verwaltung der sozialen Pflegeheime zu verstärken.

Mittlerweile wird das Vorhandensein und die Problematik der Behinderung in der VR China nicht mehr ignoriert, sondern auch von den Medien aufgegriffen. So gibt es eine berühmte „Botschafterin der Behinderten in der VR China“ Frau Zhang Haidi. Frau Zhang ist Schriftstellerin. Sie wurde 1955 in der Provinz Shandong geboren und sitzt aufgrund einer Krankheit im Rollstuhl. Sie ist Mitglied des Nationalen Komitees der politischen Beratungskonferenz, Mitglied des Nationalen Komitees des chinesischen Schriftstellerverbandes und Vizepräsidentin des chinesischen Behindertenverbandes. Aufgrund ihrer Behinderung konnte sie nie eine Schule besuchen und schloss so im Selbststudium ihre Ausbildung mit einem Master of Arts an der Jilin Universität ab. Frau Zhang wird aufgrund ihrer Leistungen trotz einer schweren Behinderung in China sehr verehrt. In den Klassenzimmern hängen Aufforderungen für die Kinder, es ihr gleich zu tun. Frau Zhang bereist im Namen der Regierung die ganze Welt um auf die Probleme der Behinderten in China aufmerksam zu machen. Ihr Wahlspruch um anderen Betroffenen Mut zu machen lautet: „Auch wenn es links und rechts nicht danach aussieht, muss es irgendwo doch einen Weg geben. Da ich nicht gehenden Fußes nach diesem Weg suchen kann, suche ich 
ihn mit der Feder“. Frau Zhang ist - neben dem Sohn des früheren Ministerpräsidenten Deng Xiao Ping - eine der prominentesten Behinderten in der VR China und gilt als internationales „Aushängeschild“ für Kraft und Selbständigkeit bei der Problembewältigung. Ihre Aktivitäten kommen vielen Behinderten zugute, da sie für ihr Land im Ausland Geld sammelt.

Ein anderer bekannter Schriftsteller, Shi Tieshen, 1951 in Peking geboren, wurde während der Kulturrevolution als Jugendlicher mit Schulbildung aufs Land geschickt. 1972 wurde er infolge einer Erkrankung gelähmt, durfte nach Peking zurückkehren und arbeitete einige Jahre als Behinderter in einer Straßenfabrik. In dieser Zeit schrieb er den Roman „Der Juraprofessor und seine Frau“ und wurde später Mitglied des chinesischen Schriftstellerverbandes.

Natürlich sind diese Beispiele nicht repräsentativ für die Behinderten in der VR China überhaupt. Internationale Veranstaltungen wie die Ausrichtung der Olympischen Spiele und der Paralympics in Peking 2008 sind eine gute Gelegenheit für die Integration Behinderter. So wurden einige neue Möglichkeiten zur Eingliederung Behinderter in den Beschäftigungsprozess erschlossen. Die Regierung hat z.B. Taxilizenzen vorzugsweise an Behinderte vergeben und in vielen Straßenrestaurants und Verkaufsständen sowie bei der Herstellung von Souvenirs werden Behinderte in den Produktions- bzw. Serviceleistungsprozess einbezogen.

Als Schlagworte im Kampf gegen die Vernachlässigung Behinderter gilt: „In Sachen Behindertenhilfe ist Humanismus die Flagge“, oder „Das Verhalten den Behinderten gegenüber symbolisiert die Entwicklungsstufe der jeweiligen Gesellschaft".

Die VR China ist auf einem guten Weg. Ziel der Regierung der VR China ist es, den Aufbau eines Wohlfahrtsstaates bis zum Jahre 2020 zu erreichen. Dabei kann es sich aber nur um eine Grundsicherung handeln, die mit westlichen Systemen der sozialen Sicherheit nicht vergleichbar ist. Das zeigt schon das Beispiel der mittlerweile in Deutschland lebenden behinderten Autorin, deren in Deutschland von der Krankenkasse finanzierten orthopädischen Schuhe in China mindestens ein Jahresgehalt eines Akademikers kosten würden und somit unerschwinglich wären.

Voraussetzungen für eine Grundsicherung sind ein lückenloses Sozialversicherungssystem und ein umfassendes sozialistisches Rechtssystem. Die Verabschiedung des Sozialversicherungsgesetzes ist dazu ein wichtiger erster Schritt. 


\title{
6.2. Politik und Recht der Eingliederung von behinderten Menschen in Indien
}

\author{
Von William Gnanasekaran
}

1. Geschichtlicher Hintergrund 374

1.1. Die koloniale Erfahrung 377

1.2. Nehrus Modell des Wohlfahrtsstaates 377

1.3. Ein Jahrzehnt der Behinderten 378

2. Institute für die Behinderten auf nationaler Ebene 382

3. Kultureller Glaube und Gemeinde basierte Rehabilitierung und die Rolle der

Nicht-Regierungsorganisationen bei der Rehabilitierung der Behinderten 383

3.1. Kultureller Glaube und Gemeinde basierte Rehabilitierung der Behinderten

3.2. Rolle der Nicht-Regierungsorganisationen bei der Rehabilitierung der Behinderten 
Indien ist, wie Sie alle wissen, ein demokratisches Land mit einer gut entwickelten Verfassung. ${ }^{1}$ Die Verfassung garantiert allen Bürgern im 3. Teil (Art.12 bis 35) grundsätzliche Rechte: Sie sichert jedem das Recht auf Gleichheit - auch vor dem Gesetz - zu. Außerdem verbietet sie die Diskriminierung auf Grund der Religionszugehörigkeit, der Rasse, der Kaste, des Geschlechts oder des Geburtsortes und gewährleistet Chanchengleichheit bei der Arbeitssuche. Die Verfassung verbietet auch alle Formen von Zwangsarbeit, sexuellen Mißbrauch und Menschenhandel. Und schließlich garantiert sie auch einen gesetzlichen Weg zur Durchsetzung dieser verfassungsmäßigen Rechte.

Im Gegensatz zu Europa gibt es jedoch in der Praxis kein Sozialversicherungssystem, das für die Gesundheit und sozialen Bedürfnisse der Bürger sorgt. Deshalb lebt noch immer ein großer Teil der Bevölkerung (ein Drittel) unter der Armutsgrenze und 65\% der Bevölkerung verdienen ihren Lebensunterhalt durch Agrarwirtschaft.

\section{Geschichtlicher Hintergrund}

In den meisten südasiatischen Ländern werden seit einigen Jahrzehnten Rehabilitierungsmaßnahmen unternommen. Diese Initiativen waren die Folge nationaler oder hiesiger Interessen. Die Entwicklung in Indien ist ganz verschieden mit großen Unterschieden zwischen den verschiedenen Bundesländern in Bezug auf Gesundheit, Bildung und pro Kopf Einkommen. Die Regierung hat jahrzehntlich eine Umfrage gemacht und danach sind 3 bis $4 \%$ der Bevölkerung behindert und brauchen irgendeine Form von Rehabilitierung. Die Hauptursachen von Behinderung in Indien sind Polio, orthopädische Behinderungen, altersbedingte Krankheiten und angeborene Behinderungen und letztlich kommunikative Behinderungen.

Den größten Anteil an Diensten für Behinderte in Indien gewährt die indische Regierung, aber es mangelt ihr an Koordination und Professionalität. Es gibt nur sehr wenige internationale Nicht-Regierungsorganisationen wie MISEROR, Action Aid, CBM (Christofel Blinden Mission), CORDAID und Save the children Fund UK, die für die Behinderten in der Gemeinschaft sorgen. In den dörflichen Gebieten sorgen vor allem die Familien und Gemeinschaften für die Behinderten.

Im Allgemeinen haben die Inder eine sehr gespaltene Einstellung zu Behinderten. Wenn sie es mit einem Behinderten zu tun haben, dann ist es wie ein Konflikt, ob man helfen soll oder nicht und sie sind sehr gespannt. Der religöse Glaube - besonders des Hinduismus - verstärkt diese Einstellung noch. In allen Religionen wird an die göttliche Vergeltung geglaubt und die Menschen akzeptieren den Behindertenzustand als etwas, was sie verdient hätten. Es wird auch angenommen, dass Gott die Menschen bestraft, um sie auf die Probe zu stellen. 
"Die Heiligen Schriften der Hindus haben viele ausführliche Kommentare gegeben, um die Frage 'warum leiden die Menschen?' zu beantworten. Die Theorie des Karma wird gebraucht, um alle Arten von Leiden zu erklären. Nach dieser Theorie wird ein Mensch für die Fehler, die er im früheren Leben gemacht hat, bestraft und muss die Folgen tragen. Die Behinderung wird als Strafe für die Sünden der letzten Geburt angesehen und man sollte diese Behinderung als Strafe Gottes annehmen. Der Glaube an diese Karma Theorie ermöglicht es die Behinderung anzunehmen, ohne die Lebensbedingungen der Behinderten zu verbessern“"2

Den Glauben an die göttliche Vergeltung gibt es in fast allen Religionen und die Menschen nehmen ihre Behinderung als etwas selbst Verdientes an. Es wird geglaubt, dass die Strafe für sündige Taten vergolten wird und man könnte die Leiden überwinden durch gerechtes moralisches Benehmen. Ein anderer Glaube ist, dass Gott den Menschen Leiden schickt, damit ihre innere Stärke entfaltet wird. Auf jeden Fall muss man sich dem Willen Gottes unterordnen. Die Glücklichen sind durch die religösen Texte ermahnt worden, den Leidenden Erbarmen und Mitleid zu zeigen.

"Manusmriti, das uralte Gesetz sozialen Benehmens riet den Menschen, einen Teil ihrer materiellen Resourcen für ihre hoffnungslosen Mitmenschen zu geben und ihr tägliches Leben zu unterstützen. Die Dharmashastra (auch ein religöser Text) bat die Menschen, die Schwachen und Behinderten zu versorgen und denjenigen, die so taten, wurde ein Platz im Himmel zugesprochen. Die Versorgung sollte ohne irgendwelche Gegenleistung gemacht werden. "3

Der Glaube an das Karma als die Ursache aller Leiden war so fest verankert, dass die Behinderten weder als eine seperate Gruppe gesehen, noch als Ausgestoßene behandelt wurden.

"In den meisten alten Schriften werden die Witwen, Alten, Kranken und Behinderten unter einen Hut gebracht. Nach dem Rig Veda wurden Schutzheime von den Hindu Königen und Adligen für alle gebaut, die kein Heim hatten, egal was für ein Problem sie hätten - sozial, ökonomisch oder körperlich. Dieser Gebrauch wurde im mediävalen und kolonialen Zeitalter weiter praktiziert. Auch nach der Verfassung Indiens wurden sie gemeinsam behandelt. Nach Artikel 41 der indischen Verfassung, die 1950 in Kraft trat, soll der Staat, sofern seine wirtschaftliche Kapazität es erlaubt, die Rechte zur Arbeit, zur Ausbildung gewährleisten und auch in Zeiten der Arbeitslosigkeit und in Alter und Krankheit und Behinderung die Bevölkerung unterstützen“4

Studien haben erwiesen, dass viele Menschen die Schuld an ihrer Krankheit und Behinderung metaphysischen Faktoren, besonders dem Karma, zuschieben. Sogar Patienten, die an Krankheiten wie Herzinfarkt, Tuberkulose, Krebs und orthopädischen Beschwerden litten, fanden den Grund in ihrem Karma.

In Indien wird die Rehabilitierung der Behinderten primär als die Verantwortung der Familie angesehen. Große Familien sorgen für die physische, emotionale und ökonomische Unterstützung der Behinderten.

2 Maheswari Shriram, Voluntary Action in Rural Developmemnt in India, The Indian Journal of Public Adminisration, July -September 1987 No. 3 Vol. 33, New Delhi 1987, S. 554.

3 Prabhu PN, Hindu Social Organisations, Popular Prakashan, Bombay 1963.

4 Maheshwari Shriram, Local Government in India, Lakshmi Narain Agarwal, Agra 2004. 
Familien, besonders die Großfamilien, gaben den Behinderten eine Identität und ein Gefühl der Geborgenheit, trotz ihrer Behinderung. Das Konzept der Rehabilitierung im modernen Sinne existierte nicht in diesen traditionellen Familien. Die Verantwortung wurde von allen Familienmitgliedern geteilt und das gewährte lebenslange soziale und ökonomische Unterstützung. Die Behinderten hatten keine $\mathrm{Zu}-$ kunftsperspektive. Sie lebten genauso wie die anderen Familienmitglieder. Solche Familienbräuche funktionierten gut, solange die indische Gesellschaft agrarwirtschaftsorientiert blieb. Die Agrarwirtschaft war nicht nur Lebensunterstützung, sondern auch Lebensweise. Auch mit der neuen Wirtschaftspolitik bleiben $65 \%$ der Bevölkerung noch fest auf diesem Gebiet verankert. Soziale und berufliche Eingliederung war kaum ein Problem für diejenigen, die in einer Familie wohnten.

Der Begriff Rehabilitierung im modernsten Sinne existierte nicht in den traditionellen Familien. Die Versorgung war meistens der übliche Wartungstyp. Die Verantwortung wurde von allen Familienmitgliedern geteilt und das gewährleistete lebenslange soziale und ökonomische Unterstützung. Eine Behinderung veränderte den Status nicht. Jeder hatte seinen Platz in der Familienhierarchie und war mit anderen durch ihre Rollenbeziehung verbunden. Ein Zusammengehörigkeitsgefühl gab es immer. Solche Maßnahmen wirkten gut, weil die indische Gesellschaft seit langem agrarwirtschaftlich geblieben war. Die Landwirtschaft war nicht nur ihre Erwerbstätigkeit, sondern auch Lebensstil. Weil die Landwirtschaft eine kollektive Tätigkeit war, waren die meisten Familienmitglieder daran beteiligt und auf irgendeine Weise leisteten sie ihren Beitrag zum Familieneinkommen. Es gab keine individuellen Berufe, kein persönliches Einkommen und sie beschäftigten sich an der Frucht ihrer Arbeit. Eine andere Merkwürdigkeit war, dass meistens Arbeitsort und Wohnort derselbe waren. Folglich fühlten sich die Behinderten in einem zusammengesetzten Familienberuf nicht behindert.

"Außerhalb der Familie gab es nur sehr wenige Berufe. Einige Berufe wurden besonders für Behinderte ausgesondert z.B. die Tauben und Stummen wurden als Gehilfen und Diener vorgezogen, denn die Gespräche, die im Palast stattfanden, blieben dann geheim. Dieser Gebrauch war seit langem üblich in Indien. Einige Berufe wurden besonders für Blinde ausgesondert. In Südindien wurde der Beruf des Girlandenmachens besonders für Frauen mit Blindheit reserviert. In Nordindien waren viele Blinde als Musiklehrer tätig und übten instrumentalische Musik in den Tempeln. Unter den indischen Moslems verdienten die Blinden ihren Lebensunterhalt durch das Beibringen und das Rezitieren des Korans. Leute mit orthopädischer Behinderung wurden als normal angesehen und machten viele Jobs ${ }^{\text {“5 }}$

Die drei sozialen Anstalten - die Großfamilie, Kastengruppe und Dorfgemeinden spielten eine aktive Rolle bei der Unterstützung der Ausgestoßenen und Waisenkinder. Die Versorgung der hiesigen Gemeinden in den indischen Dörfern wird deutlich durch die Gründung der vielen sozialen und religösen Institute, um für die Behinderten zu sorgen. Die Dorf Fünfräte dienten, um die Armen, Behinderten, Alten und Ausgestoßenen zu versorgen. Vedische und maurische Quellen berichten von der 1986. 
Zeitperiode 500 vor Christus und sie ermahnen auch für das Wohl der anderen zu arbeiten.

\subsection{Die koloniale Erfahrung}

Eine große Missionierung begann mit der Kolonisierung Indiens durch die Briten. Die missionarischen Institute folgten der gleichen Philosphie und den Taten, die sie aus dem Westen brachten.

"Nach Miles wurden viele Institute für Behinderte gegründet, mit dem missionarischen Eifer, die Botschaft von Jesus Christus zu verbreiten. Sie glaubten an das 'Seife, Suppe und Rettung' Konzept als Vorstufe in der missionarischen Arbeit. Sie vernachlässigten das indigene kulturelle und Glaubenssystem. Die politischen, ökonomischen und Bildungsanstalten der Briten hatten nur das Ziel, ihre Herrschaft zu konsolidieren. Die Behinderung war ihnen kein großes Anliegen. Die Industrialisierung machte auch Fortschritte in Indien. Urbanisierung und industrielles Wachstum brachten eine Änderung im Konzept von Arbeitsort und -weise “6

Das kapitalistische ökonomische Modell legte großen Wert auf das private Individuum, damit es einen produktiven Beitrag zur Marktwirtschaft leiste. Danach wurden die Behinderten als nicht-produktive Mitglieder der Gesellschaft angesehen. Diese Ideologie stand in krassem Gegensatz zum traditionellen Ethos und affektierte den Status der Behinderten. In der langen Geschichte der Kolonisation wurde die indische Elite der westlichen Weltanschauung ausgesetzt und sie folgte den Rehabilitierungsmaßnahmen blind.

\subsection{Nehrus Modell des Wohlfahrtsstaates}

Im Zeitalter nach der Unabhängigkeit Indiens wurde Nehru Indiens erster Premierminister. Nehru hatte die Wohlfahrtsprinzipien und westlichen Demokratien an der Harrow Schule und Oxford Universität gelernt und er führte sein Modell des Wohlfahrtsstaates als leitendes Prinzip aller sozialen Entwicklungsprogramme in Indien ein.

„Immer mehr öffentliche Organisationen wurden gegründet und die Aufgabe, alle zu rehabilitieren, wurde sowohl von der Regierung als auch von den staatlich fundierten Organisationen aufgenommen. Das führte zu einem zentral institutionalisierten Dienst für das Wohl der Behinderten. Die Minister auf Bundes- und Landesebene spielten eine Hauptrolle beim Entwurf der Pläne und Programme und auch bei der Entwicklung der Infrastruktur.

Eine nationale Ratsversammlung für das Wohl der Behinderten wurde gegründet, um Richtlinien für das ganze Land zu formulieren und die Rehabilitierungsprogramme einzuordnen. Wegen der Unterschiede beim Einsatz der Wohlfahrtsprogramme gab es in Ländern wie Bengal, Gujarat und Maharashtra gut entwickelte Programme mit großzügigen Finanzen und auch

6 Miles, Disability Care and Education in 19th Century India: Some Dates, Places and Documents, Action Aid disability News, Bangalore 1994. 
Nicht-Regierungsorganisationen nahmen zu, aber in den anderen Ländern waren die Programme überschaubar und litten unter mangelnden Finanzen"7

Die Bundesregierung und die Landesregierung haben viele Gesetze erlassen, um das Funktionieren der Regierungsorganisationen und Nicht-Regierungsorganisationen zu erfassen. Vier nationale Institute für vier Arten von Behinderung nämlich - Blindheit, orthopädische Behinderung, Gehörbehinderung und Geisteskrankheit wurden in dieser Zeit gegründet.

Alle diese Institute auf nationaler Ebene dienten als höchste Gremien bezüglich der Arbeitskraftentwicklung, sie unternahmen Forschung und dienten auch als Informationszentren. Regionale berufsorientierte Trainingszentren wurden in verschiedenen Teilen des Landes gegründet. Die Landesregierung und freiwillige Gruppen haben viele andere Trainingsorganisationen gegründet. Um einheitliche Standards in den technischen Kursen zu gewährleisten, gründete die indische Regierung The Rehabilitation Council of India (Rehabilitierungsrat Indiens) nach dem Modell des Medical Council of India (Medizinischer Rat Indiens).

Die Behinderung wurde als Staatskrankheit angesehen und die Betonung lag darin, das Problem zu lösen, zu heilen und zu korrigieren, damit die Behinderten so normal wie möglich leben konnten. Diese Perspektive passte sich dem Wohlfahrtsmodell an und war auch anpassunsfähig an internationale Organisationen wie WHO und UNICEF. Die Menschen wurden tatsächlich als passive Empfänger von Wohlfahrtsdiensten behandelt und diese bestanden hauptsächlich aus der gratis Verteilung von medizinischen Mitteln und Geräten für die Armen und Notleidenden. Diese Maßnahmen verstärkten die Abhängigkeitskultur. Wenn man die Größe des Problems und die dürftigen Resourcen und Infrastruktur betrachtet, hatten diese Maßnahmen kaum eine bemerkbare Wirkung auf $2-3 \%$ der indischen Bevölkerung.

\subsection{Ein Jahrzehnt der Behinderten}

Die Zentral- und Landesregierungen führten eine große Zahl von Wohlfahrtsprogrammen durch und Gesetze wurden erlassen, um das Funktionieren der NichtRegierungsorganisationen und Regierungsorganistionen zur Rehabilitierung der Behinderten einzuschätzen. Nach der Verfassung ist die Rehabilitierung der Behinderten eine Sache der Landesregierung, aber die Bundesregierung hat auch eine wichtige Rolle zu spielen.

Die Bevölkerungszahl Indiens ist über eine Milliarde und davon leiden 21,9 Millionen (etwa 2\%) an irgendeiner Form von Behinderung wie Blindheit, TeilBlindheit, orthopädische Behinderung, Gehörbehinderung, Geisteskrankheit oder Sprechbehinderung. Eine Umfrage stellte 2001 fest, dass 4\% der indischen Bevölkerung verspätete Entwicklung des Gehirns hat. Heute werden alle Initiativen im Ge- 
biet des Wohlergehens der Behinderten vom Sozialjustiz- und Bekräftigungsministerium gemacht, früher Sozialwohlfahrtsministerium genannt.

"Das Jahrzehnt der Behinderten begann in den 80er Jahren. Es gab eine Initiative um die Lage der Behinderten in Indien zu analysieren und nach alternativen Modellen zu suchen, um die Behinderten einzugliedern. Während dieser Phase gab es auch ein ideologisches Umdenken von den Wohlfahrtsplänen zur Entwicklung der menschlichen Ressourcen. Nach dieser Idee wurden die Menschen als Ressource angesehen und durch Ausbildung und Training konnte man sie entwickeln. 1981 wurde das Nationale Kommittee zum Jahr der Behinderten unter dem Vorstand Nehrus ins Leben gerufen. Es war eine Folge der Ankündigung der Generalversammlung der Vereinten Nationen zu einem Jahr der Behinderten. Viele Arbeitsgruppen und Aufgabenkräfte wurden von den Bundesministern gegründet, um den Plänen der Behinderten höchste Priorität zu geben. ${ }^{\text {“8 }}$

Das Jahrzehnt der Behinderten (1983-92) kennzeichnete auch das Umdenken in der Debatte über die Ziele der Rehabilitierung. Die Ansicht, dass Rehabilitierungsdienste Gemeinde basiert sein müssen, wurde von vielen angenommen. Es gab die erleuchtende Wahrheit, dass weder Wohlfahrt noch Gratisspenden, noch die Entwicklung von Menschenressourcen in einem Land wie Indien erfolgreich sein konnten. Es gab keinen anderen Weg für die Regierungs- und die Nicht-Regierungsorganisationen, den riesigen Herausforderungen der dörflichen und städtischen Behinderung zu begegnen.

Es wurde notwendig, die hiesigen Ressourcen und Unterstützung zu mobilisieren, um die Behinderungsrehabilitierungsprogramme zu führen. Zweitens gab es eine zunehmende Beteiligung der internationalen Organisationen an den nationalen Programmen für Behinderte. Viele Großprojekte waren von internationalen Organisationen fundiert. Westliche Modelle und Theorien wurden ein Teil der Fundierung. Diese internationalen Organisationen brachten eine Verwandlung in der Kultur, Ideologie und Arbeitsweise der Regierungs- und Nicht-Regierungsorganisationen.

Heutzutage sind die Dienstorganisationen in einer Phase der Umwandlung, weil sie immer mehr mit internationalen Bewegungen verknüpft sind. Ein großer Meilenstein war der Erlass des Gesetzes Persons with Disabilities Act 1995 (Personen mit Behinderung Gesetz 1995) durch das Indische Parlament.

"Dieses allumfassende Gesetz gewährleistet gleiche Möglichkeiten, gleichen Rechtsschutz und volle Teilnahme der Behinderten in allen Bereichen des nationalen Lebens.

Mit diesem Gesetz hat die Regierung das Gratisspendenmodell abgeschafft und das wohlfahrtsbasierte Modell ist eingeführt worden. Dieses Gesetz gewährleistet gleiche Rechte für alle und erlaubt es auch diejenigen, die Behinderte diskriminieren, ins Gefängnis zu sperren. Es ist interessant zu bemerken, dass das Gesetz vom Präsidenten unterzeichnet worden war und am 3. Februar 1996 in Kraft trat, aber die Regierung etwa 3 Jahren brauchte, um die nötige Nacharbeit zu leisten, so dass das Gesetz wirklich anwendbar wird“9

8 Government of India, Persons with Disabilities (Equal Opportunities, Protection of Rights and full Participation) Act 1995, Ministry of Social Justice and Empowerment, New Delhi 1996.

9 Government of India, Persons with Disabilities (Equal Opportunities, Protection of Rights and Full Participation) Act 1995, Ministruy of Social Justice and Empowerment, New Delhi 1996, S. 58. 
Deshalb konzentrierte sich die Rehabilitierungsratsversammlung der Regierung Indiens auf

- Ausbildungsmöglichkeiten und Programme für Behinderte

- Vereinheitlichung der Ausbildung von Behinderten unter Einbeziehung vonFachleuten

- Anerkennung/Würdigung von Institutionen, die Ausbildungen anbieten

- Erstellen eines Adressenverzeichnisses von Fachleuten für Rehabilitationsprogramme

- Förderung der Forschung zur Rehabilitation und von besonderen Ausbildungen

Im Parlament wurde ein Gesetz erlassen um, dieser Ratsversammlung gesetzliche Macht zu verleihen, damit sie die Aufgaben effektiv macht. Dieses Gesetz wurde The Rehabilitation Council of India Act 1992 genannt und trat Juni 1993 in Kraft.

"Dieses Gesetz stellte das frühere Konzept der Wohlfahrt der Behinderten um. Deshalb bietet die Rehabilitierungsratsversammlung mehrere herausfordernde Programme durch Professionelle zur sozialen Eingliederung. Eine Gleichheit in den verschiedenen Trainingsprogrammen der Ratsversammlung wird gewährleistet und ständige Evaluierung und Prüfung der Programme sind möglich. Den Behinderten wurde durch das Gesetz Equal opportunities, protection of rights and full participation Act of 1995 Volljustiz gegeben, das am 7. Feb.1996 in Kraft trat. Es war ein bahnbrechender Schritt, um den Behinderten gleiche Chancen und ihre volle Beteiligung im Aufbau der Nation zu gewähren." ${ }^{\text {"10 }}$

"Das Behinderungsszenario in Indien ändert sich schnell. Die Bemühungen der letzten vielen Jahrzehnte haben eine qualitative Wandlung im Leben der vielen Behinderten gebracht. Es gibt jetzt eine große Menge von Behinderten, die gut ausgebildet, professionell erfolgreich und in führenden Positionen sind.

Viele sind sehr aktiv und machen von ihrem Recht Gebrauch, sich in alle Entscheidungen, die mit ihrem Leben zu tun haben, einzumischen und daran teilzunehmen. Internationaler Austausch und Zusammenarbeit haben ihnen die Probleme und Herausforderungen der Behinderten in anderen Ländern bewußt gemacht. Diese Gruppe spielt eine wichtige Rolle bei dem Fortschritt der Behindertenbewegung in Indien. “11

Die Regierung erlaubt den Behinderten auch, an allen Entwicklungsprogrammen, die ihr Leben betreffen, teilzunehmen. Der Erfolg solcher Partnerarbeit in der letzten Zeit ist ein klarer Beweis für die Änderung der gesellschaftlichen Meinungen und der Meinungen von Regierung und Dienstorganisationen.

Die Schwerpunkte des Disabilities Act sind:

- Vorbeugung und Entdeckung von Behinderungen

- Bildung

- Chancengleichheit bei der Arbeitssuche

10 Kakar S. Shamans, Mystics and Doctors: A Psychological Inquiry in to India and its Healing Traditions Bombay, Oxford University Press 1982.

11 Mellory, Changing Beliefs about Disability in Developing Countries: Historical Factors and Sociocultural Variables, in: Mellory/Nicholls/Charlton/Marfo (Hrsg), Traditional and Changing views of Disability in Developing Societies, University of Hampshire Press 1994. 
- Schutz vor Diskriminierung

- Forschung und Personalentwicklung

- aufbauende Maßnahmen

- $\quad$ soziale Absicherung und Integration

- Problemlösung

Umfragen werden durchgeführt, um die Ursachen von Behinderungen festzustellen und adäquate Schritte zur Versorgung der Mutter und des Kindes vor und nach der Entbindung zu machen.

Nach diesem Gesetz gibt es gesetzliche Rechte:

- Behinderte Kinder haben das Recht, gebührenfreie Erziehung bis 18 Jahren in Regierungsschulen zu bekommen.

- Behinderte Kinder haben das Recht auf entsprechende Verkehrshilfsmittel wie Rollstühle und Reservierung von Sitzplätzen in Bus und Bahn.

- Behinderte Kinder haben das Recht auf Bücher, Uniformen, Stipendien und andere Lernmittel

- Sonderschulen für behinderte Kinder werden auch mit berufsorientierter Trainingsinfrastruktur ausgestattet.

- Nicht-formelle Erziehung für behinderte Kinder wird auch gefördert.

- $3 \%$ der Berufe in der Regierung werden für Behinderte reserviert, wovon $1 \%$ für die Blinden, $1 \%$ für die Gehörbehinderten und $1 \%$ für die physisch Behinderten.

- Erziehungsinstitute und andere Institute, die vom Staat finanziert werden, müssen mindestens 3\% der Studienplätze für Behinderte reservieren.

- Kein Arbeitnehmer im staatlichen Dienst darf wegen einer Behinderung während der Dienstperiode entlassen werden, wenn er in eine andere Stelle mit dem gleichen Gehalt versetzt werden könnte. Keine Beförderung darf wegen einer Behinderung verweigert werden.

- Finanzielle Unterstützug der Nicht-Regierungsorganisationen für die Rehabilitierung der Behinderten.

- Versicherung für die behinderten staatlichen Arbeitnehmer

- Arbeitslosengeld für die arbeitslosen Behinderten, die sich im Arbeitsamt gemeldet haben

Im Januar 1996 wurde ein Sondererlaß durch das Justizministerium erarbeitet und vom Parlament erlassen. Dieses Gesetz erkannte die Probleme der Behinderten auf Basis des Asiatische und Pazifische Jahrzehnt der Behinderten (1993-2003) Vertrag und machte die folgenden Schritte

- Schutz der Rechte und volle Beteiligung der Behinderten

- Einsetzung von Koordinierungskommittees auf Bundes- und Landesebene um die Behinderten zu rehabilitieren.

- Visuelle Hilfsmittel für die Blinden und Teilblinden.

- Versorgung mit Rollstühlen für die Gehbehinderten 
- Versorgung mit Gehörmitteln

- Rehabilitierung der von der Lepra-Krankheit genesenen Personen

- Rehabilitierung der Geisteskranken

Die Regierung Indiens gewährleistet den Behinderten viele Rechte und Privilegien durch Reiseerlassungen wie

- $\quad$ Rechte der Behinderten nach The Disabilities Act 1995

- Rechte der Behinderten nach The National Trust Act 1997

- Rechte der Behinderten nach The Rehabilitation Council of India Act 1992

- Rechte der Behinderten, die geisteskrank sind

Einem Blinden wird 75\% Erlass bei Reisen mit der Bahn oder mit dem Flugzeug gewährleistet, bei einem orthopädisch Behinderten sind es 50\% und bei Geisteskranken ebenfalls $75 \%$. Ein monatlicher Reiseerlass wird auch den orthopädisch Behinderten entsprechend ihrem Beruf und Gehalt gegeben. Steuererlass wird auch den Behinderten gegeben und verschiedene Arten von medizinischer Versorgung werden auch stark subventioniert.

\section{Institute für die Behinderten auf nationaler Ebene}

Das nationale Institut für die orthopädisch Behinderten wurde 1978 gegründet und ist das höchste Gremium des Landes für die Behinderten. Es funktioniert autonom unter dem Sozialjustiz- und Bekräftigungsministerium der Regierung Indiens. Seine Ziele sind die Entwicklung der Humanressourcen und die Entwicklung der Hilfsmittel für orthopädisch Behinderte. Es hat auch Pläne, Trainingsprogramme in verschiedenen Orten Indiens durchzuführen durch die Regierung und Nicht-Regierungsorganistionen. Nach dem Etat der Regierung Indiens werden 2500 Millionen Rupies jährlich für die Trainingsprogramme der Behinderten ausgegeben.

Es gibt vier verschiedene Insitute auf nationaler Ebene für die Behinderten.

- National Institute for the Visually Handicapped - Dehradun, New Delhi

- National Institute for the Orthopaedically Handicapped - Calcutta

- Aliyavar Jung National Institute for the Hearing Handicapped - Mumbai

- National Institute for the Mentally Handicapped - Hyderabad.

Alle diese Institute sind höchste Gremien im Gebiet der Ausbildung, Training, berufsorientierten Beratung, Forschung und Rehabilitierung. Aber es gibt eine Notwendigkeit die Behinderten auf unterster Ebene zu rehabilitieren. Die Behindertenszene wechselt rapide in Indien. Die Bemühungen der letzten vielen Jahrzehnte haben einen qualitativen Wandel im Leben der vielen behinderten Menschen bewirkt. Es gibt jetzt eine große Zahl von Menschen, die ausgebildet, professionell erfolgreich und in Führungspositionen tätig sind. Viele sind sehr aktiv in Einflussrollen und machen von ihren Rechten Gebrauch um im Entscheidungsprozess mit- 
zuwirken und ihr Leben besser zu gestalten. Internationaler Austausch und Unterstützung haben ihnen die Kämpfe der Behinderten in anderen Ländern bewußt gemacht.

\section{Kultureller Glaube und Gemeinde basierte Rehabilitierung und die Rolle der Nicht-Regierungsorganisationen bei der Rehabilitierung der Behinderten}

\subsection{Kultureller Glaube und Gemeinde basierte Rehabilitierung der Behinderten}

In den letzten zwei Jahrzehnten wurde die Gemeinde basierte Rehabilitierung als die wirksamste und praktischste Lösung des enormen Behindertenproblems in Indien gefördert. Sie sieht eine soziale Umgebung vor, wo die Gemeinde eine Empfindsamkeit für spezielle Notwendigkeiten ihrer behinderten Mitglieder zeigt und sich berufen fühlt, die gewünschten Änderungen hervorzubringen. Um diese Idee zu verwirklichen und solche Programme durch die Ressourcen der Gemeinde in Gang zu halten, wurden von der Regierung und Nicht-Regierungsorganisationen viele ambitionierte Pläne in den 80er Jahren begonnen. Viele Gemeinde basierte Rehabilitierungsprojekte wurden großzügig von der Regierung und internationalen Organisationen unterstützt. Alle diese Bemühungen haben in den letzten zwei Jahrzehnten beigetragen, die Philosophie der Gemeinde basierten Rehabilitierung zu popularisieren und die Hoffnung der Behinderten zu erhöhen.

Die Wirksamkeit der Gemeinde basierten Rehabilitierung liegt darin, dass ein starker Gemeinschaftssinn schon immer im Bewußtsein der Inder verankert war. Seit vielen tausend Jahren haben die hiesigen Gemeinschaften für die Behinderten in ihrer Nähe gesorgt. Auf die Dauer entwickelten diese Gemeinschaften ihre eigenen Systeme und ihre eigenen Ressourcen. Religöse Zentren (Tempel, Moscheen und Gebetsstätten) haben als eine Art Zentralstelle gewirkt, um die hiesigen Ressourcen zu mobilisieren und Rehabilitierungsdienste zu organisieren. Dieser Gebrauch ist auch in der jetzigen Zeit üblich, besonders in dörflichen Gebieten.

\footnotetext{
"Aber in seiner jetzigen Lage ist die Gemeinde basierte Rehabilitierung ein Produkt der westlichen wissenschaftlichen Tradition. Von internationalen Instituten ausgedacht und von einigen städtischen Nicht-Regierungsorganisationen übernommen, wurde die Gemeinde basierte Rehabilitierung als ein Bündel von Versprechen in die indischen Dörfer getragen. Eine Hauptkritik an den Gemeinde basierten Rehabilitierungsprogrammen in Indien ist, dass die Programme geplant werden, ohne die Behinderungstraditionen und -bräuche und Lebensweise der hiesigen Gemeinden zur Kenntnis genommen zu haben. Die Gemeinde basierten Rehabilitierungsprogramme bauen kaum auf der kulturellen Stärke der hiesigen Gemeinden auf. In einer Studie über die 7 Haupt Gemeinde basierten Rehabilitierungsprogramme des Landes fanden Dalal, Kumar und Gokhale heraus, dass Gemeinde basierte Rehabilitierungsprogramme ihr Versprechen nicht gehalten haben und nur als Dienstleistungsagenten von der hiesiegen Bevölkerung angesehen werden. Die hiesige Teilnahme an den Programmen wird nur auf Tagun-
} 
gen, Trainingprogramme und Besprechungen beschränkt, die von Nicht-Regierungsorganisationen und vom Staat unterstützt werden “12

Deshalb haben die Gemeinde basierten Rehabilitierungsprogramme keinen markanten Wandel in die dörfliche Szene bringen können. In einem Land wie Indien, wo Religion alle Bereiche des Lebens beherrscht, ist es völlig in Ordnung, wenn die Gemeinde basierte Rehabilitierung ihre Stärke aus religösen Anstalten herausbringt und deren immense Ressourcen nutzt. Wenn die Gemeinde basierte Rehabilitierung mit den sozialen Festen und Ritualen verknüpft ist, dann kommt die Beteiligung der Gemeinde ganz automatisch.

\subsection{Rolle der Nicht-Regierungsorganisationen bei der Rehabilitierung der Behinderten}

Laut Statistik leiden 21,9 Millionen Menschen in Indien an irgendeiner Form von Behinderung. The National Institute of Development Studies, eine NichtRegierungsorganisation in Delhi, berichtet, dass 14\% der Bevölkerung in den Bundesländern Bihar, Orissa und Tamilnadu an irgendeiner Form der Behinderung leiden. Obwohl die Summe 21,9 Millionen bei einer Bevölkerungszahl von einer Milliarde verhältnismäßig klein erscheint, ist sie ein wichtiger Bestandteil der indischen Szene. Deshalb ist die Rolle der Nicht-Regierungsorganistionen bei der Rehabilitierung der Behinderten sehr zu schätzen. Auch diese Nicht-Regierungsorganisationen brauchen die Unterstützung von der Regierung und internationalen Organisationen z.B. ist die Christoffel Blindenmission sehr bekannt in Indien für ihre Unterstützung von Blinden und Teil-Blinden durch ihre Blindenheime für Kinder, die Gestaltung der Brailleschrift Druckereien und die Finanzierung von Augenkrankenhäuser usw., The Spastic Society of India in Chennai hat auch viele Zweigstellen in verschiedenen Orten Indiens und dient zur Rehabilitierung der Behinderten, Geisteskranken und der Alten.

Viele kirchenverwandte katholische, protestantische und evangelische Institute wie Bethesda, Bethania, KNH (Kinder Nothilfe) funktionieren durch ihr Präsidium und erreichen die Menschen auf der untersten Ebene durch das Errichten von Heimen für behinderte Kinder und Erwachsene und Blindenheime für Schulkinder, Universitätsstudenten und Erwachsene. Lilianna Fonds, eine Nicht-Regierungsorganisation in den Niederlanden ansässig, arbeitet auch in Pondicherry und rehabilitiert die Behinderten. Es gibt viele andere Nicht-Regierungsorganisationen, die auf Landesebene und Gemeindeebene den Behinderten dienen. Sie sind

- Mother Theresa's Homes in Calcutta

- Sisters of Charity - gegründet von der Stiftung Mutter Theresa

- Littel sisters of the poor - Chennai 
- Udavumkarangal (Helfende Hände) - Chennai

- Mercy Home - Eine französische Stiftung in Chennai

- Beatitudes Centre - Ein katholisches Institut für die Behinderten in Chennai

- Agape Rehabilitation Centre - in Chennai ansässig und unterstützt vom japanischen Konsulat in Chennai

- SHEHA - Ein freundliches Heim in Chennai

- Balar Kalvi Nilayam - Heim und Schule für geisteskranke Kinder

- Kirche des Südindiens Heime - unterstützt von Kinder Not Hilfe, Deutschland

Diese Nicht-Regierungsorganisationen und viele andere sind beteiligt, um die Behinderten in Indien durch ihre verschiedenen Dienste zu versorgen.

\section{Fazit}

Die Regierung Indiens gründete 2002 eine Stiftung um für die Geisteskranken durch die Nicht-Regierungsorganisationen zu arbeiten. Die Regierung gibt auch viel Geld aus für die Dokumentation und Forschung der Behinderten. Die Regierung soll auch die kleinen Nicht-Regierungsorganisationen auf der untersten Ebene großzügig finanzieren, damit die Rehabilitierungsprogramme die Leute direkt erreichen. Die Regierung sollte auch kleine Nicht-Regierungsorganisationen durch den Transfer von Geldspenden aus dem Ausland erleichtern durch das FCRA (Foreign Currency Regulation Act). Die Korruption sollte auch geprüft werden, damit immer mehr Geldsummen von den internationalen Nicht-Regierungsorganisationen zu NichtRegierungsorganisationen auf der untersten Ebene in Indien fließen. Wenn solche Schritte verwirklicht werden, bin ich sicher, dass Indien die Probleme seiner Behinderten bestimmt lösen wird.

The National Fund for People with Disabilities wurde 1983 gegründet unter dem Gesetz Charitable Endowment Act 1890, um den freiwilligen Sektor zu fördern, damit er Dienste für die Behinderten anbietet. Finanzielle Unterstützung wird von dieser Quelle an die Nicht-Regierungsorganisationen gegeben, um viele Aktivitäten zu machen. Diese soll regelmäßig überprüft werden, um noch mehr Geldsummen aus dem Ausland zu erhalten und Spenden in hiesigen Regionen zu bekommen. Deshalb kann die Regierung zusammen mit den Nicht-Regierungsorganisationen viele Wunder machen im Bereich der Rehabilitierung der Behinderten.

Das Konzept der Rechte, Politik und sozialen Integration der Behinderten hat seit je her in Indien existiert durch religöse Institute und andere traditionelle und soziale Organisationen. Aber der Erfolg solcher Gemeinde basierten Rehabilitierung hängt viel davon ab, wie man die traditionellen Quellen für so einen guten Zweck benutzt. In einem Land wie Indien, wo die Religion alle Lebensbereiche durchströmt, ist es durchaus nicht falsch, wenn Gemeinde basierte Rehabilitierung ihre Stärke von religösen Instituten bezieht und ihre immense Resourcen anwendet. Wenn Gemeinde basierte Rehabilitierung mit den sozialen Zeremonien, Festen und Ritualen der Gemeinschaft verknüpft sind, wird die Teilnahme der Bevölkerung automatisch 
sein. Wenn dies erreicht wird, wird die Gemeinde basierte Rehabilitierung eine Bewegung der einfachen Menschen sein und wird bestimmt erfolgreich sein. 
6.3. Behindertenrecht und Behindertenpolitik in Japan 


\title{
6.3.1. Japan's Welfare System for People with Disabilities
}

\author{
Von Makoto Arai
}

1. The turning point for the welfare system for people with disabilities

1.1. International developments in welfare for the disabled

1.2. The IYDP and system reform

1.3. The shift from disability-specific measures to community-based welfare measures

2. System review and welfare for the disabled in the future

2.1. "Basic Structural Reform of Social Welfare" report proposals

2.2. The shift from sochi-style welfare to contract-style welfare

2.3. Problems related to the principle of the sochi system and a user's right to choose

2.4. Advocacy system and care management 


\section{The turning point for the welfare system for people with disabilities}

\subsection{International developments in welfare for the disabled}

The campaign for the International Year of Disabled Persons (IYDP) in 1981 has invariably been identified as the turning point for Japan's welfare system for people with disabilities in the years since the end of World War II. Back in 1975, which was six years before the IYDP, the United Nations had adopted the Declaration on the Rights of Disabled Persons. This declaration affirmed the fundamental human rights of disabled persons and also called upon countries to guarantee various rights for the disabled, including their civil rights, political rights, right to medical and social rehabilitation, right to education and vocational training, right to enjoy economic security, and right to participate in activities in society.

But the declaration alone was not expected to generate a very significant impact in terms of specific efforts by individual countries. A campaign to motivate them to undertake concrete action in line with the spirit of the declaration was therefore required. This took the form of the IYDP, and an international action plan was adopted. Full participation and equality were buzz words at the time. In 1982, the year after the IYDP, the United Nations adopted the World Programme of Action concerning Disabled Persons and presented model measures for disabled persons that individual countries could take. Furthermore, the period from 1983 through 1992 was designated as the United Nations Decade of Disabled Persons, and countries were asked to embark on concrete action during this ten-year period.

\subsection{The IYDP and system reform}

Japan was one of the countries that were highly receptive to the major campaign launched by the United Nations beginning with the IYDP. The IYDP aims of "full participation and equality" as well as the normalization underlying them gained public recognition through government efforts and the media.

The first action undertaken as system reform based on these IYDP principles was to amend the Law for the Welfare of Physically Disabled Persons. Japan's existing Law for the Welfare of Physically Disabled Persons was to a considerable extent philosophically out of sync with the IYDP slogan "full participation and equality" and its underlying concept of normalization. Consequently, in 1982 the Advisory Council on Welfare of the Physically Handicapped produced a report outlining a comprehensive strategy for promoting the welfare of persons with physical disabilities in the future. Following that report, the Law for the Welfare of Physically Disabled Persons was then amended in 1984.

Two significant points can be cited in this regard. One is that the philosophy of the Law for the Welfare of Physically Disabled Persons was changed in accordance with awareness of the IYDP concepts. The other point is that it became possible for provisions related to the scope of qualifying physical disabilities to be stipulated 
through an ordinance without having to amend the law. The philosophy of the law is noteworthy in that, with its stated aim being to promote the independence of persons with physical disabilities and their participation in socioeconomic activities, it added the concept of all physically disabled persons being presented with the opportunity to take part as individual members of society in activities in every field, including social, economic, and cultural areas. The inclusion in the law's text of wording corresponding to the IYDP slogan "full participation and equality" is notable from the standpoint that international developments exerted an influence on Japan's legal system. Additionally, allowing the scope of qualifying physical disabilities to be stipulated through an ordinance meant that it became possible to respond to the needs of disabled persons who require new measures by adding their conditions to the list of qualifying disabilities.

Japan's welfare for persons with intellectual disabilities underwent a transition without being influenced by the IYDP to the major extent seen in the case of welfare for the physically disabled. Since Japan's policy of developing residential institutions came under criticism in the context of normalization, the trend for several years from 1981 on was to add community-based welfare systems here and there, without carrying out any overall system reform, in order to demonstrate a willingness to place emphasis on measures for in-home welfare. However, a 1989 initiative for community living support programs for the mentally retarded (the Group Home System for Persons with Intellectual Disabilities) was significant in that residential options other than placement in institutions were created.

In the case of welfare for persons with mental disorders, the impact of the Utsunomiya Hospital Incident in 1984 was greater than any IYDP influence. This incident involved two patients at a hospital in the city of Utsunomiya who were beaten to death by nurses. Instances of routine infringement of the rights of patients in the hospital were revealed one after another in the course of the police investigation. The nature of inhumane treatment within the country's psychiatric hospitals became evident to the public in Japan as well as overseas through this incident. Furthermore, it also became apparent that fundamental problems were surfacing at psychiatric hospitals in Japan. One factor causing this is that psychiatric health care is relatively understaffed in Japan compared with general medical treatment. Another is that the country has few national and local-government-operated hospitals providing psychiatric care, and treatment occurs primarily in private medical institutions.

With these concerns coming to light, Japan needed to revamp its policies related to persons with mental disorders. In 1987 the Mental Hygiene Law was revised and became the Mental Health Law. For the first time it was declared that the law's objective was the improvement of welfare for persons with mental disorders, as seen in Article 1, where the stated aim is to enhance the welfare of persons with mental disorders and to improve public mental health. Voluntary hospitalization, advocacy on behalf of patients in hospitals, designated psychiatrists, psychiatric review boards, halfway houses for persons with mental disorders, and other systems were established under this law. It is not an exaggeration to say that Japan's welfare system for persons with mental disorders commenced with this law. To phrase it differ- 
ently, it can be said that the country did not have a welfare system for persons with mental disorders prior to the 1987 Mental Health Law.

\subsection{The shift from disability-specific measures to community-based welfare measures}

The shift from separate measures for persons with different disabilities to an emphasis on community-based welfare measures for all disabled persons occurred with the amendment in 1990 of Japan's eight laws related to social welfare. As part of the revision of those eight laws, two laws in the field of welfare for persons with disabilities were subject to amendment: the Law for the Welfare of Physically Disabled Persons and the Law for the Welfare of People with Mental Retardation (currently the Law for the Welfare of Intellectually Disabled Persons).

Two points in the case of the Law for the Welfare of Physically Disabled Persons are notable in terms of the advancement of community-based welfare. One is that authority for admitting persons with physical disabilities into social welfare and rehabilitation institutions was transferred from prefectures to municipalities. The other is that in-home welfare services were clearly identified as a system. Two similar points in the case of the Law for the Welfare of Intellectually Disabled Persons are also significant. One is that authority over operations, which had been exercised by prefectures, was shifted to designated cities (large cities). The other is that inhome welfare services were clearly identified as a system.

With the 1990 amendment of the Law for the Welfare of Physically Disabled Persons and the Law for the Welfare of People with Mental Retardation, the Japanese Government clarified its policy of developing in-home welfare services, which had had a minor presence compared with institution-based welfare services up to that point. Additionally, plans to move in the direction of transferring authority for administrative measures pertaining to various welfare services to municipalities were mapped out. These plans called for taking a phased approach in each separate area, such as welfare for the physically disabled and welfare for the intellectually disabled.

I would now like to focus on developments in the reform of the welfare system for persons with disabilities from 1990 on.

Persons with mental disorders were positioned as persons with disabilities under the Disabled Persons Fundamental Law in 1993. It is important that a case was made for welfare services for persons with mental disorders. On top of that, though, the fact that the law identified the need for prefectures and municipalities to formulate basic plans for the disabled (and stipulated their obligation to make efforts in this regard) is significant. With the Disabled Persons Fundamental Law making this provision that basic plans for the disabled be created, the Japanese Government announced its proposed guidelines for the formulation of municipal plans for persons with disabilities in 1995. In a further move, the Government Action Plan for Persons with Disabilities: Seven-Year Normalization Strategy was released that 
same year. Efforts to compile plans for the disabled on the basis of the guidelines for the formulation of municipal plans for persons with disabilities progressed in municipalities, too, and local governments (municipalities) demonstrated their move in the direction of assuming responsibility in the field of welfare for the disabled as well by proceeding to develop the required facilities and human resources.

In 2002 Japan compiled and released its new Basic Plan for the Disabled, which replaced its existing plan for persons with disabilities. This new plan covers the years from fiscal 2003 through fiscal 2012. Japan then revised the Disabled Persons Fundamental Law in 2004. This revision made the formulation of prefectural and municipal plans for persons with disabilities mandatory. However, enforcement of this obligatory formulation of municipal plans did not start until fiscal 2007.

\section{System review and welfare for the disabled in the future}

\section{1. "Basic Structural Reform of Social Welfare" report proposals}

The interim version of a report on the ideal future scenario for health and welfare measures for people with disabilities was released in 1997. As in the case of welfare for the elderly and other areas of welfare, the report proposed making a radical change in the existing philosophy of the provision of welfare services for the disabled as well. The points to be incorporated included multifunctional facilities, the principle of user choice, and participation by private-sector service providers.

The Central Social Welfare Council's Subcommittee on the Structural Reform of Social Welfare then compiled a report entitled "Basic Structural Reform of Social Welfare." The subcommittee released an interim version of this report in June 1998 and issued additional opinions related to it in December 1998. The final version of the report came out in January 1999. Along with that, draft revisions of three laws - the Social Welfare Service Law, the Law for the Welfare of Physically Disabled Persons, and the Law for the Welfare of Intellectually Disabled Persons - were issued in August 1999. These three laws were then amended in May 2005. A program for the provision of assistance benefits was launched in April 2003 as a system allowing disabled persons to utilize contract-based services.

The notion of contract-based service provision appeared in an even more powerful and advanced form in the Law for Supporting Independence of Persons with Disabilities, which was enacted in 2005. This law changed the system of services, which had differed depending on the type of disability, to a unified framework. Because of this switch, it became possible to proceed with changing the welfare system for the disabled from the bottom up.

Focusing chiefly on the aforementioned interim version of the "Basic Structural Reform of Social Welfare" report, I would now like to examine present-day challenges in the area of welfare for the disabled in connection with the contents of that report. Those contents consist of three main points: the need for reform, the principles of reform, and the specific contents of reform (the promotion of social welfare 
operations, the assurance of quality and efficiency, and the establishment of community-based welfare).

With regard to the need for reform, the interim report makes observations in terms of the social climate in Japan - the progression of the country's low birthrate and aging population as well as its shift to a slow-growing economy - and refers to the enactment of the Child Welfare Law and the Long-term Care Insurance Law. At the same time, the report emphasizes, social welfare operations, social welfare foundations, and social welfare offices have not undergone any extensive reform in the half-century since the end of World War Two.

The report identifies seven points as principles of reform. Three of them, I believe, are particularly important in that they are associated with the direction of the reform of welfare for disabled persons from 1990 on that I referred to earlier. These three points are (1) the establishment of a relationship of equality between service users and providers, (2) the promotion of the participation of providers offering a diversity of services, and (3) the improvement of service quality and efficiency through market mechanisms.

Looking at the specific contents of reform related to those three points identified as principles, we can see that three changes have been pinpointed as essential action: (1) establish the premise that the system will allow users to choose services themselves and to utilize them based on contracts with providers (make a shift from socalled sochi-style welfare based on administrative measures to contract-style welfare); (2) modify regulations that pertain to the scope of approved management entities so that they are commensurate with the nature of social welfare work (permit the participation of various operating entities, including private not-for-profit organizations); and (3) create conditions that are conducive to competition among providers as a result of user choice and will therefore lead to service quality improvements (disclosure of information about services and service assessments by third-party experts).

The most important focus of fundamental structural reform of social welfare is the question of the revision of the Social Welfare Service Law, a law that affects all entities providing welfare services. The specific points listed in this regard include the diversification of provider entities that are engaged in social welfare work (the revitalization of private-sector groups, the consideration of participation by private not-for-profit organizations, and the stimulation of competition among operators), the problematic nature of the sochi system (this system is a mechanism under which services are provided in accordance with administrative judgments with regard to the persons targeted for services), and the establishment of service provision methodologies through care management techniques. The list also includes the need to improve the efficiency of the provision of services in order to allocate limited resources, the need to establish community-based welfare and convert the function of social work offices so that they serve as general consultation centers, and other points. 


\subsection{The shift from sochi-style welfare to contract-style welfare}

Sochi-style welfare involves three parties: persons who are covered (disabled persons and so on), holders of sochi authority (administrative authorities and so on), and commissioned organizations (service providers). To utilize welfare services, a person who is covered first files an application with the holder of sochi authority. That holder of authority then conducts an investigation into eligibility for services based on the application. This eligibility screening includes checking such points as whether or not the applicant has a disability certificate, the degree of the disability, and the applicant's age (in the case of welfare services for the elderly). In the event that the outcome is a judgment that service utilization is possible, the holder of soch $i$ authority directs a commissioned organization to provide service and pays sochi system funds to that organization. The distinguishing feature here is that a person who is covered by the system does not have the right to choose the commissioned organization providing service since there is not any direct contractual relationship between that person and the service provider.

By contrast, the three parties involved in contract-style welfare (an assistance benefits system) are users, administrative authorities, and approved organizations (service providers). To utilize welfare services, a user first registers with an approved organization. Service provision comes at the stage when those two parties have entered into a contract. In this case the job of administrative authorities is to check whether approved organizations are entities that are qualified to provide welfare services and to identify appropriate entities as approved organizations. Additionally, in the case of welfare services for the disabled, the system is such that administrative authorities assume the role of paying subsidies to approved organizations for the cost of service utilization after the conclusion of contracts between users and those approved organizations. The distinguishing feature here is the existence of the user's right to choose an approved organization since the user and the approved organization enter into a direct contract.

From a practical standpoint, however, multiple problems are also anticipated. At this point I would like to touch upon issues associated with the principles of the sochi system and the user's right to choose as well as the advocacy system and care management.

\subsection{Problems related to the principle of the sochi system and a user's right to choose}

The premise of Japan's basic structural reform of social welfare is that users of services under the sochi system are subject to administrative procedures and the rights and obligations associated with relationships between users and providers are legally ambiguous. This is significant as an indication of the notion of user-centeredness, a concept that has been lacking in welfare services up to now. Thinking about this from the standpoint of social welfare principles, though, this premise is probably problematic in terms of Article 25 of the Japanese Constitution, for this article stipu- 
lates that all people of Japan the right to receive social welfare services. Even the sochi system incorporates the principle of protecting the users of services, since an advocacy function is fundamentally built into the judgments of welfare administrative authorities.

I did some checking in connection with the point that users would be given a choice with the introduction of contract-style welfare. In doing this I drew on the following example from the current welfare system.

The Law Concerning Mental Health and Mental Health Welfare (the Mental Health and Welfare Law) provides for admission into halfway houses, with such admission being based on a contract between a user and a service provider. Japan's 1999 patient census indicates that the total number of persons with mental disorders was 2,04 million. Meanwhile, the patient capacity of facilities as of 2001 was such that the country was equipped to accommodate 4.775 persons in life skills training facilities (232 facilities), 1.379 persons in welfare homes (127 homes), 4.941 persons in vocational training centers (208 centers), and 348 persons in welfare factories (12 factories). In actuality, user choice is not possible given this volume of service provision. Consequently, the simple reasoning that a choice of services would exist for users as a result of having contract-style welfare does not work. Instead, we must understand that in reality the potential for user choice would not exist even with contract-style welfare in instances when the volume of services provided is extremely limited, as can be seen in the case of halfway houses for persons with mental disorders.

\subsection{Advocacy system and care management}

With regard to advocacy, proposals for Japan's basic structural reform of social welfare encompass not only the adult guardianship system but also the introduction and reinforcement of a system to assist with appropriate utilization of different types of services in the field of social welfare, including daily living support. In connection with this, efforts to look into the development of community welfare advocacy systems are proceeding within social welfare councils and elsewhere around the country. The intent is to provide the users of services with daily living support staff (specific tasks being contemplated include the provision of information on welfare services, advice on service utilization, assistance with service utilization procedures, and daily money management) and to establish specialized third-party organizations that would handle complaints and the filing of claims.

This system would be extremely valuable in terms of checking how well the rights of users were being protected. The problem, though, is whether circumstances have progressed to the point that the system could work effectively. Two concerns in particular are the quantity of neutral daily living support staff that would be available from service providers in communities and the extent to which local people would participate on the side of users in specialized third-party organizations. 
It is said that there are two sharply divided philosophies concerning care management. One philosophy advocates ascertaining the overall needs of users, conducting comprehensive assessments, developing the necessary social resources, protecting users' rights, evaluating service quality, and so on. This way of thinking is the most important philosophy in terms of the practice of care management for disabled persons. For the other philosophy, care managers have budgetary authority, and the packaging of services (providing a combination of services) produces the effect of controlling service-related costs.

As Japan proceeds with the basic structural reform of social welfare that is presently in progress, the country should move forward with the care management philosophy. If social work offices are institutions that specialize in the practice of social work, then of course they ought to carry out care management that is centered on users' needs. Today's social work offices, however, are a long ways from being institutions that specialize in social work centered on users (disabled persons), and tasks are vertically segmented into separate social welfare legal systems. Considering this situation, the context that brought about this kind of basic structural reform of social welfare can be perceived as a fundamental problem of the present welfare administration, which has not been centered on service users. 


\title{
6.3.2. Behinderung und Familie in Japan
}

\author{
Von Miyoko Motozawa
}

1. Gegenwärtige Situation der Menschen mit Behinderung in Japan 400

2. Historischer Hintergrund des Gedankens über Familie in Japan $\quad 400$

3. Reform des Familienrechtes nach dem Zweiten Weltkrieg in Japan 402

3.1. Familienunterhalt 402

3.2. Persönliche Betreuung 403

4. Gesetzgebung für die Menschen mit Behinderung in Japan 404

4.1. Entstehung und Entwicklung der Sozialwohlfahrtsgesetze 404

4.2. Wohlfahrtsgesetze und Familienpflege 405

4.3. Pflegeversicherung und Familie 406

4.4. Gesetze für die Menschen mit Behinderung $\quad 408$

5. Schlußbemerkung $\quad 410$ 


\section{Gegenwärtige Situation der Menschen mit Behinderung in Japan}

Nach Art. 2 des Grundgesetzes über Behinderte, das im Jahre 2004 gründlich geändert wurde, sind „Behinderte“ definiert als Menschen, die bei der Bewältigung ihres alltäglichen oder gesellschaftlichen Leben wegen einer körperlichen, geistigen oder psychischen Behinderung langfristig eingeschränkt sind.

Nach Schätzung des Ministeriums für Gesundheit, Arbeit und Wohlfahrt $\left(\right.$ MGAW) ${ }^{1}$ betrug im Jahre 2006 die Zahl der Menschen mit Behinderung (inkl. Kinder und Jugendliche) in ganz Japan ca. 7.100.000; davon mit einer körperlichen Behinderung ca. 3.520.000, mit einer geistigen Behinderung ca. 550.000 und mit einer psychischen Behinderung ca. 3.030.000 Personen. In einer stationären Einrichtung lebten nur ca. 670.000 Menschen mit Behinderung; davon mit körperlicher Behinderung ca. 190.000, mit geistiger Behinderung ca. 130.000 und mit psychischer Behinderung ca. 350.000 Personen. Also lebten mehr als 90\% aller Menschen mit Behinderung nicht in stationären Einrichtungen, sondern in einer Wohnung mit oder ohne Familie.

Nach einer Umfrage des MGAW aus dem Jahr 2005 über die Situation von Kindern und Jugendlichen mit geistiger Behinderung ${ }^{2}$ betrug der Anteil der zu Hause lebenden Personen 96,2\% aller antwortenden Befragten (nur 581 Personen). Auch in Zukunft möchte mehr als die Hälfte aller befragten Kinder und Jugendlichen mit ihren Familien zusammenleben: 36,0\% mit ihren Eltern, 16,7\% mit dem (künftigen) Ehegatten und 1,2\% mit ihren Geschwistern. Dagegen wollen nur 13,1\% von ihnen in einer betreuten Wohngruppe und 4,3\% in einer stationären Einrichtung wohnen. Lediglich 4,3\% der Befragten wünschen sich, ganz alleine zu leben bzw. 1,2\% zusammen mit Freunden oder Freundinnen. Diese Ergebnisse zeigen, dass behinderte Menschen grundsätzlich relativ abhängig sind von ihren Familien, im Speziellen von ihren Eltern. Dieselbe Tendenz lässt sich auch in anderen Antworten feststellen: $72,3 \%$ der befragten Kinder und Jugendlichen gaben als Ratgeber ihre Eltern und Großeltern an, während nur 43,2\% ihre Lehrer in der Schule oder jemanden am Arbeitsplatz nannten. Diese Abhängigkeit von ihren Eltern ist Ausdruck einer grundsätzlichen Zukunftsangst, vor allem vor dem Leben im Alter, obwohl die Befragten noch nicht 18 Jahre alt waren. Darüber hinaus nannten sie Sorgen hinsichtlich Arbeitsplatz und Alltagsleben in der Zukunft: Arbeitsplätze und Werkstätten $66,3 \%$, Leben im Alter 36,5\%, Tagesstätte 35,1\%.

\section{Historischer Hintergrund des Gedankens über Familie in Japan}

In Japan hatten religiöse Anschauungen aus dem Buddhismus keinen Einfluß auf das Denken über Familie, ganz im Gegenteil zum Familienbild in europäischen

1 MGAW, Weißbuch über die Behinderten 2007 (auf Japanisch), S. $4 \mathrm{f}$.

2 MGAW, Grunduntersuchung über Kinder und Menschen mit geistiger Behinderung (ChitekiShogaiji(sha) Kiso-Chosa) von 2005. 
Ländern, das stark vom Christentum geprägt ist ${ }^{3}$. Eine größere Übereinstimmung mit den japanischen Sitten und Gewohnheiten wies dagegen der Konfuzianismus auf.

Das traditionelle japanische Konzept des familiären Lebens war das Ie-System. Der Begriff des „Ie“ wurde bereits in der Heian-Zeit (794-1183) als eine Nachfolgeeinheit des Gewerbes und Berufs zuerst bei der Familie des Hofadels (Kuge) und dann bei der des Kriegers (Buke) eingeführt. In der Kamakura-Zeit (1185-1333) gab es noch mehrere Vorschriften über die Familienangelegenheiten in der Grundregel der Krieger „Goseibai-Shikimoku“ von 1232. In der Muromachi-Zeit (1392-1573) wurden die Familienangelegenheiten dann nicht mehr offiziell geregelt, sondern ausschließlich dem privaten Bereich überlassen. Dieser wurde in der Edo-Zeit (1603-1867) durch den Grundgedanken des Kriegers (Bushi-doh) nach dem Konfuzianismus geprägt: Danach waren die Treue des Kriegers zum Herrn (Chuhgi) und die Elternliebe der Kinder (Kohkoh) am wichtigsten ${ }^{4}$.

In der Meiji-Zeit (1868-1912) wollte die Meiji-Regierung das Land so schnell wie möglich modernisieren und industrialisieren, um das Kaiserreich vom Tenno zu stabilisieren und führte das Ie-System - ein pyramidenförmig aufgebautes, sich auf die Großfamilie stützendes System gesellschaftlicher Organisation - als Grundlage des Kaisertums ein. Unter dem Ie-System war das Fortbestehen des Ie am wichtigsten. Dafür mußte man die Ehe schließen und Kinder bekommen. Die Stelle des Hausherren $(K o s h u)$ und das gesamte Vermögen des Ie wurden dem ersten Sohn vererbt (Meiji-ZGB §986). Der Hausherr besaß alle Befugnisse über die vermögensrechtlichen und persönlichen Entscheidungen aller Ie-Mitglieder. Zugleich war er aber auch verpflichtet, alle Ie-Mitglieder zu unterhalten. Jeder Japaner gehörte einem Ie an, das seinen Unterhalt sicherte.

Ohne diese Ie-Zugehörigkeit konnte man keine richtige Arbeit finden und keine richtige Ehe schließen. Die richtige Ehe bedeutete, dass eine Frau von guter Herkunft sein und in den Rang und die Tradition des Ie ihres Mannes passen musste. Wenn sie diese Bedingungen erfüllte, wurde sie als seine Ehefrau (Yome) allerdings nicht automatisch ein richtiges Ie-Mitglied ihres Mannes. Dazu musste sie unbedingt ein Kind bekommen. Die Unfruchtbarkeit einer Frau war ein gesetzlicher Scheidungsgrund; genauso wie eine mangelhafte Sache durfte man die unfruchtbare Frau zu ihrem Ie zurückschicken. Auch die gebärende Ehefrau wurde jedoch wegen ihrer Abstammung immer weiter als fremd angesehen. Sie konnte ihre Existenz im Ie ihres Mannes nur stabilisieren, indem sie einen gesunden Sohn als Nachfolger des Ie bekam. Diese gesetzliche, gesellschaftliche Situation der Frauen machte die Beziehung zwischen Mutter und Kindern nicht nur emotional, sondern auch gesellschaftlich ganz nah und eng.

Falls eine Frau einen Bruder oder eine Schwester mit Behinderung hatte, hatte sie keine Chance auf eine richtige Ehe, weil man glaubte, dass Behinderungen vererbt 
würden. Deshalb wurde ein neugeborenes Kind normalerweise heimlich umgebracht oder verlassen, wenn es irgendeine schwere Behinderung hatte. Wenn ein Kind mit Behinderung in einer reichen Familie geboren wurde, konnte das Kind glücklicherweise mit Finanzierung der Eltern heimlich von jemandem auf dem Land oder im Zimmergefängnis zu Hause gepflegt werden. Die Geburt eines behinderten Kindes galt allein als Schuld der Mutter - auch dann, wenn der Grund der Behinderung des Kindes auf Seiten ihres Mannes lag, genauso wie bei der oben erwähnten Ehescheidung wegen Unfruchtbarkeit.

\section{Reform des Familienrechtes nach dem Zweiten Weltkrieg in Japan}

\subsection{Familienunterhalt}

Nach der Niederlage im Zweiten Weltkrieg 1945 sollte Japan unter der Herrschaft der amerikanischen Besatzungstruppen einen demokratischen Staat aufbauen. Das Ie-System mußte abgeschafft werden, weil es die politische und gesellschaftliche Grundlage des Tenno-Systems bildete und weil es gegen die Würde des Individuums und gegen die Gleichberechtigung von Mann und Frau verstieß. Im neuen Familien- und Erbrecht ist die Kernfamilie das Vorbild der modernen Familie. Der Personenkreis und der Umfang des Familienunterhalts sind beschränkt. Alle Kinder sind gleichberchtigte Erben der Eltern.

Die Ehegatten sind zur gegenseitigen Unterstützung verpflichtet (ZGB§752) und teilen sich die Kosten des ehelichen Lebens nach Maßgabe ihres Vermögens, ihres Einkommens und aller sonstigen Umständen (ZGB§760). Es ist durch diese Vorschrift nicht klar, was in diesen Kosten des ehelichen Lebens enthalten ist. Nach der herrschenden Meinung sind die Kosten der Kinderbetreuung und -erziehung sowie der medizinischen Behandlung der Familienangehörigen usw. enthalten.

Die Blutsverwandten in gerader Linie und auch Geschwister sind verpflichtet, einander Unterhalt zu gewähren (ZGB§877 I). Mit dieser Vorschrift wird die allgemeine Unterhaltspflicht der Eltern für ihr minderjähriges unverheiratetes Kind und ihr erwachsenes Kind, sowie die der erwachsenen Kinder für ihre Eltern begründet. Die Ehefrau, die nach dem alten Familienrecht gegenüber ihren Schwiegereltern eine Unterhaltspflicht hatte, ist jetzt nur noch dann verpflichtet, wenn das Familiengericht ihr die Unterhaltspflicht unter besonderen Umständen auferlegt (ZGB §877II).

Die Reihenfolge der Unterhaltspflichtigen und -berechtigten wird durch die Vorschriften des Familienrechtes (ZGB§878) nicht klar. In erster Linie wird sie durch Vereinbarung der Beteiligten und dann durch die Entscheidung des Familiengerichts festgesetzt. Nach der herrschenden Meinung hat das Unterhaltsrecht der erwachsenen Kinder gegenüber den Eltern den Vorrang vor dem gegenüber den Geschwistern und ist nachrangig gegenüber dem Unterhaltsrecht des Ehegatten und der minderjährigen, unverheirateten Kinder. In der Gesellschaft wird diese Auffassung inzwischen 
auch vertreten; es gibt aber nach wie vor noch viele Menschen, die meinen, dass der erste Sohn seine Eltern unterhalten und seine Ehefrau diese Pflicht mittragen muß.

Über Art und Weise und die Höhe des Unterhalts entscheidet das Familiengericht unter Berücksichtigung des Bedarfs des Unterhaltsberechtigten, der finanziellen Möglichkeiten des Unterhaltsverpflichteten und der sonstigen Umstände, wenn die Parteien sich darüber nicht untereinander einigen können (ZGB§879). Es gibt drei verschiedene Formen von Unterhalt: Entrichtung einer Geldrente, Gewährung der Leistung in natura und Aufnahme in den eigenen Haushalt. Die finanzielle Unterstützung mit barem Geld ist dabei die grundsätzliche Art des Unterhalts. Der Begriff „Unterhalt“ bedeutet normalerweise nur die finanzielle Unterstützung und keine persönliche Betreuung - nicht nur beim Unterhalt der alten Menschen und der erwachsenen Menschen mit Behinderung, sondern auch beim Unterhalt der minderjährigen, unverheirateten Kinder.

\subsection{Persönliche Betreuung}

Die persöhnliche Betreuung und Erziehung der minderjährigen, unverheirateten Kinder ist die Aufgabe beider Eltern während der Ehe (ZGB§§818, 820). Diese Aufgabe der Eltern wird in Japan immer noch als elterliche Gewalt (Shinken) bezeichnet. Die elterliche Gewalt betont man seit der Niederlage im Zweiten Weltkrieg im Zusammenhang mit der staatlichen Gewalt, um einen Eingriff des Staates in das private Familienleben durch das Ie- und Schulsystem, wie man ihn in der Kriegzeit erfahren hat, zu beseitigen. Nach der herrschenden Meinung haben die Eltern mit der elterlichen Gewalt bei der Kindererziehung immer Vorrang vor dem Staat und der Gemeinde - nicht nur nach dem Familienrecht, sondern auch nach dem Kindeswohlfahrtsgesetz. Diese Auslegung ist dann problematisch, wenn die Eltern ihre Kinder nicht richtig betreuen und erziehen können oder wollen. Deshalb wurde im Jahre 2000 ein neues Gesetz zum Schutz der Kinder vor Misshandlungen durch die Eltern verkündet ${ }^{5}$. Ein ähnliches Gesetz für misshandelte alte Menschen wurde im Jahre 2005 verabschiedet und ist am 1. April 2006 in Kraft getreten, aber ein solches Sondergesetz für Menschen mit Behinderung wird noch nicht diskutiert.

Im Zusammenhang mit der persönlichen Betreuung der Familie muss man die Reform des Familienrechts im Jahre 2000 ansprechen. Bei der Einführung der später erwähnten Pflegeversicherung für alte Menschen wurden die Vorschriften über die teilweise und ganze Beschränkung der Geschäftsfähigkeit sowie Vormundschaft geändert, um Verträge mit dem Anbieter über Versicherungsleistungen auch dann und Führung der Gemeinde erleichtert und damit die Kinder vor Misshandlung geschützt werden, und dass das Kindesamt im Notfall die misshandelten Kinder zeitweilig von ihren Eltern entfernt und entscheidet, ob sie bei ihrer Familie weiter betreut werden können oder sie in einer stationären Einrichtung betreut werden müssen. Das Gesetz wurde im Jahre 2007 reformiert, um den Eingriff des Kindesamtes zum Schutz der Kinder mit Hilfe der Polizei zu verstärken. 
rechtswirksam abschließen zu können, wenn die pflegebedürftigen Versicherten keine ausreichende Geschäftsfähigkeit haben. Der vom Familiengericht berufene Vormund hat das Verwaltungsrecht und die Vollmacht hinsichtlich des Vermögens des Mündels (ZGB§859). Bei der Geschäftsführung hinsichtlich des Lebens, der Betreung und der Vermögensverwaltung muss er den Willen des Mündels jedoch berücksichtigen und sich um den körperlichen, geistigen Zustand und die Lebenslage des Mündels sorgen (ZGB§858).

Im Rechnungsjahr 2006 (vom 1. April 2006 bis zum 31. März 2007) betrug die Zahl der Anträge auf Beginn einer Vormundschaft 29.380, während sie noch im Rechnungsjahr 2000 nur 7.451 betrug $^{6}$. Der Anteil aller Antragssteller betrug bei Eltern ca. 31\%, bei Kindern ca. 26\%, bei Geschwistern ca. 19\%, bei Ehegatten und sonstigen Verwandten insgesamt ca. $18 \%$. Der Anteil der männlichen Betroffenen betrug ca. $47 \%$ und davon ca. $18 \% 30$ Jahre alt und ca. $17 \% 40$ Jahre alt. Bei den Männern betrug der Anteil der über 65-Jährigen ca. 34\%, während der Anteil bei den Frauen ca. 55\% betrug ${ }^{7}$. Dies lässt darauf schließen, dass die relativ jüngeren Männer vor allem wegen einer geistigen, psychischen Behinderung ihre Vormunde brauchen, während die älteren Frauen wegen der Demenz ihre Vormunde brauchen.

\section{Gesetzgebung für die Menschen mit Behinderung in Japan}

\subsection{Entstehung und Entwicklung der Sozialwohlfahrtsgesetze}

Nach der Niederlage im Zweiten Weltkrieg konnten die meisten Japaner durch das offizielle Verteilungssystem nicht ausreichende Lebensmittel erhalten. Deshalb mussten sie ihre lebensnotwendige Nahrung am Schwarzmarkt in der Stadt kaufen oder sich selbst mit Tauschhandel auf dem Land beschaffen. Inzwischen waren viele verletzte und behinderte Soldaten von der Front im Ausland nach Japan zurückgekommen. Aber sie konnten nicht alle wieder mit ihrer Familie zusammen leben, weil ihre Familie während des Krieges gestorben war oder das Familienleben ohne sie bereits neu gestaltet wurde, da ihre Familie geglaubt hat, dass sie - wegen des falschen amtlichen Berichtes über die Gefallenen im Krieg - gestorben wären. Sie konnten deshalb keinen Familienunterhalt erwarten, obwohl sie wegen ihrer Behinderung keine angemessene Arbeit finden und sich selbst nicht unterhalten konnten. Auch hatten viele Kinder ihre Eltern während des Krieges verloren und waren obdachlos geworden.

Unter diesen Umständen wurde zum Schutz vor dem Hungertod gemäß der Anweisung der Besatzungstruppe das alte Fürsorgegesetz von 1946 erlassen. Nach dem 
Inkrafttreten der Verfassung 1947 wurde das Fürsorgegesetz ${ }^{8}$ gemäß deren Art. 25, der ein Existenzminimum der Bevölkerung garantiert, grundlegend reformiert. Ferner wurde das Kindeswohlfahrtsgesetz zum Schutz aller pflege- und hilfebedürftigen Kinder und Jugendlichen (inkl. behinderte Kinder und Jugendliche) im Jahre 1947 verkündet. Auch das Wohlfahrtsgesetz für Menschen mit körperlicher Behinderung wurde im Jahre 1949 verkündet.

Seit der Niederlage im Zweiten Weltkrieg zielte Japan in erster Linie auf eine gute wirtschaftliche Entwicklung ab. Erst das hohe Wirtschaftswachstum in den 60er Jahren führte dann zum Erlaß einer Reihe von Gesetzen für Menschen mit Behinderung: Z.B. das Wohlfahrtsgesetz für Geistesschwache von 1960, das Gesetz über das Gesundheitswesen der werdenden Mütter und Neugeborenen von $1965^{9}$, das Unterhaltsbeihilfegesetz für die Unterhaltspflicht der geistig schwerbehinderten Kinder von 1964 und das Kindergeldgesetz für arme Unterhaltspflichtige der kleinen Kinder von 1971. Im Jahre 1970 wurde das Grundgesetz für Menschen mit körperlicher und geistiger Behinderung verkündet, aber in diesem Gesetz blieb die psychische Behinderung außer Betracht.

\subsection{Wohlfahrtsgesetze und Familienpflege}

Die Stagnation der Wirtschaft in den 80er Jahren, die mit der ersten Ölkrise begann, wirkte sich deutlich negativ im Bereich der Sozialwohlfahrt aus. Wegen der Verschlechterung der Staatsfinanzen wollte die Regierung eine Reihe von Gesetzesreformen durchführen, um die Finanzlast des Staates zu verringern. Die konservative Regierung zielte deshalb auf die traditionelle Form der Pflege und Betreuung auf der Grundlage einer stabilen Familie ab, in der die drei Generationen unter einem Dach harmonisch zusammenleben. Tatsächlich hatten die Familien aber nach und nach ihre Pflegefähigkeit verloren, weil sie durch die Industrialisierung und Urbanisierung innerhalb einer kurzen Zeitspanne verkleinert worden waren. Die Regierungspartei LDP sagte in der Öffentlichkeit zwar immer noch, dass dies das eigene japanische Modell des Wohlfahrtsstaates war und Japan auf einem anderen Weg als europäische Länder erfolgreich sein würde. Aber es war nur ihr alter Traum; in der Tat betrug der Anteil der sog. Drei-Generationen-Familie an allen Haushalten schon damals nur noch $16 \%$.

Ende der 80er Jahre war in Japan durch die Grundstücksinvestitionen eine Zeit der Hochkonjunktur, aber nach kurzer Zeit platzte die Seifenblase der „bubbleeconomy“. Die folgende große Wirtschaftskrise zwang erneut zu einer Änderung der sorgerechts; in Leipold (Hrsg.), Selbstbestimmung in der modernen Gesellschaft aus deutscher und japanischer Sicht, C. F. Müller in Heidelberg, 1997, S. 223 ff..

9 Das Gesetz zielt nicht nur auf die Anpassung des Gesundheitszustandes der werdenden Mütter durch regelmäßige Untersuchung, sondern auch auf die Frühdiagnose der Behinderung der Neugeborenen und die angemessene Behandlung der Kinder mit Behinderung. 
japanischen Wirtschafts- und Beschäftigungsstruktur. Gleichzeitig kam es zu einem Geburtenrückgang und zu einem Anstieg der Anzahl älterer Menschen. Daher kam es auch zu einer Änderung im System der sozialen Sicherung, weil die früheren Wohlfahrtsgesetze darauf zielten, die armen und alleinstehenden Menschen mit Behinderung oder im Alter in angemessenen Einrichtungen unterzubringen. Im Jahre 1990 gab es eine große Reform aller acht Wohlfahrtsgesetze, um häusliche und ambulante Pflege zu fördern ${ }^{10}$. Zu diesem Zweck wurde die Zuständigkeit der Sozialwohlfahrt auf die Gemeinden konzentriert und die Einbeziehung privater Träger in die Geschäftsführung ermöglicht.

Aber in der Gesellschaft war es immer noch eine große Schande für eine Familie, öffentliche Hilfeleistungen zu erhalten, weil sie durch Steuermittel finanziert wurden und ursprünglich nur von den armen und alleinstehenden Menschen in Anspruch genommen werden konnten ${ }^{11}$. Zur Änderung dieses Vorurteils und zur Erleichterung der körperlichen und seelischen Belastung der Pflegeperson in der Familie wurde am 17. Dezember 1997 das Gesetz über die Pflegeversicherung erlassen, das am 1. April 2000 in Kraft trat. Damit erhöhte sich die Zahl der Benutzer der ambulanten Pflege und der Leistungserbringer in kurzer Zeit sprunghaft. In der Gesellschaft hat sich die Pflegeversicherung zusätzlich einen großen Verdienst dadurch erworben, dass über die Altenpflege als ein soziales Problem nun in der Öffentlichkeit berichtet und diskutiert wird.

\subsection{Pflegeversicherung und Familie}

Das Pflegeversicherungsgesetz zielt darauf ab, dass Menschen, die wegen des Alters körperlich oder geistig pflegebedürftig sind, soweit wie möglich ein selbständiges Leben führen können ( $§ 1 \mathrm{PVG})$. Der in der Pflegeversicherung versicherte Personenkreis umfasst zum einen Menschen ab 65 Jahren mit Wohnsitz in Japan, unabhängig von Einkommen und Staatszugehörigkeit (§ 9 Nr.1 PVG). Die zweite Gruppe umfasst alle gesetzlich Krankenversicherten sowie deren Mitversicherte zwischen 40 und 64 Jahren ( 99 Nr.2 PVG). Die Versicherten der ersten Gruppe ${ }^{12}$ erhalten Leistungen bei Hilfe- und Pflegebedürftigkeit ${ }^{13}$ ohne Wartezeit. Bei den Versicherten der zweiten Gruppe wird vorausgesetzt, dass ihre Hilfe- oder Pflegebedürftigkeit durch eine altersbedingte Krankheit ${ }^{14}$ verursacht wurde ( $\$ 7$ PVG). Dies bedeutet,

10 Vgl. Motozawa, Familiäre und sonstige Pflege der alten Menschen im japanischen Sozialrecht, ZfSH/SGB April 1995, S. 176 f.

11 Dies zeigt ihre Unfähigkeit und ihr kaltes Herz. Vgl. Motozawa (1995), a.a.O., S. 169 f.

12 Anfang 2005 versicherte die japanische Pflegeversicherung rund 25 Millionen über 65-Jährige. Rund vier Millionen Japaner wurden als hilfe- oder pflegebedürftig eingestuft, darunter mehr als doppelt so viele Frauen wie Männer.

13 Pflegebedürftig sind Menschen, die wegen einer körperlichen oder geistigen Behinderung für die Verrichtungen des täglichen Lebens ständig Hilfe benötigen. Hilfebedürftig sind Menschen, bei denen die Gefahr besteht, dass sie pflegebedürftig werden.

14 Welche Krankheiten dazu gehören, ist in einem Ministerialerlass aufgeführt. 
dass die Versicherten der zweiten Gruppe auch dann normalerweise keine Leistung aus der Pflegeversicherung erhalten können, wenn sie wegen einer Behinderung hilfe- oder pflegebedürftig werden, sondern dass sie nur bestimmte unten erwähnte Hilfe- und Pflegeleistungen für Menschen mit Behinderung verlangen dürfen. Dagegen können die Versicherten der ersten Gruppe beide Leistungen erhalten, wenn sie wegen einer Behinderung hilfe- oder pflegebedürftig sind.

Die Leistungen aus der Pflegeversicherung decken $90 \%$ aller häuslichen und stationären Pflegekosten mit der gemäß der Hilfe- und Pflegestufe ${ }^{15}$ bestimmten Obergrenze. Die Versicherten müssen einen Eigenanteil von 10\% der Kosten tragen. $\mathrm{Zu}$ häuslichen Pflegekosten zählen die der ambulanten Pflege und hauswirtschaftlichen Versorgung, die der ambulanten Krankenpflege und ambulanten Rehabilitation sowie die der Tages- und Kurzzeitpflege. Auch die Versorgung in Wohngemeinschaften für Demenz-Patienten gehört dazu. Bei der Inanspruchnahme der häuslichen Pflegeleistungen können die Versicherten ohne Eigenanteil einen Vertrag mit einer Einrichtung für Care-Management schließen. Diese stellt ihnen einen CareManager zur Seite, der sie hinsichtlich der Zusammenstellung der Leistungen berät, einen Care-Plan aufstellt und dessen Einhaltung kontrolliert. Diese Einrichtung wickelt auch die Vergütung der Leistungserbringer ab.

Im Januar 2005 erhielten rund 3,2 Millionen Versicherte Leistungen aus der Pflegeversicherung $^{16}$, während die Zahl der Hilfe- und Pflegebedürftigen bei rund 4 Millionen lag. Diese Differenz lässt vermuten, dass ca. 0,8 Millionen Pflegebedürftige zu Hause von ihren Familieangehörigen gepflegt werden. Bei der Einführung der Pflegeversicherung gab es im Zusammenhang mit der Familienpflege eine große Diskussion über hierfür zu gewährende Geldleistungen. Eine große Frauengruppe opponierte dagegen, weil sie fürchtete, dass die Geldleistungen den traditionellen gesellschaftlichen Druck auf Frauen, insbesondere Schwiegertöchter (Yome), die nicht berufstätig oder nur teilzeitbeschäftigt sind, verstärken würden, die Aufgabe der Altenpflege zu übernehmen. Die vollzeitbeschäftigten Frauen der Gewerkschaften votierten dafür, weil sie durch die Geldleistungen auch dann ein eigenes Einkommen teilweise weiterbehalten konnten, wenn sie ihre Arbeitsstelle aufgeben sollten oder wollten, um ihre eigenen Eltern oder Schwiegereltern selbst zu betreuen. Die beschäftigten Männer enthielten sich im Allgemeinen einer Meinung.

Das MGAW fürchtete, dass die Geldleistungen zur Erweiterung des Marktes der Pflegedienste nicht wirksam beitragen könnten und nur große finanzielle Belastungen bedeuten würden. Die Gemeinden, insbesondere die auf dem Land gelegenen, wünschten dagegen die Geldleistungen, weil sie nicht ausreichend Personalkräfte zur Gewährung der häuslichen Pflegeleistungen finden konnten. Einige Gemeinden versuchten deshalb, außerhalb des Pflegeversicherungsgesetzes, eine kleine Beloh-

15 Vgl. Motozawa, Problem der japanischen Pflegeversicherung, ZIAS 1/2003, S. 84 f. Die Hilfebedürftigen werden nach dem Änderungsgesetz der Pflegeversicherung 2005 zwei Stufen zugeordnet und können Leistungen zur Verbeugung der Pflegebedürftigkeit, nicht nur hauswirtschaftliche Hilfen, erhalten. Zur Hilfe-Stufe II gehören viele Pflegebedürftige der bisherigen Pflegestufe I.

16 Darunter erhielten 2,46 Millionen Versicherte ambulante Leistungen und 0,77 Millionen Versicherte stationäre Leistungen. 
nung für die Familien, die Hilfe- und Pflegebedürftige ohne Leistungen der Pflegeversicherung selbst betreuen wollten, aus eigener Tasche zu finanzieren. Unter dem starken politischen Druck, insbesonders eines LDP-Politikers musste das Ministerium am Ende den Familien zumindest zusichern, die jährlichen Kosten für Verbandsmaterial zu gewähren, wenn die Familie einen Pflegebedürftigen in Stufe IV oder V ohne Leistungen der Pflegeversicherung zu Hause selbst betreut.

\subsection{Gesetze für Menschen mit Behinderung}

Bei der Einführung der Pflegeversicherung wurde ebenfalls diskutiert, ob hierin auch Menschen mit Behinderung versichert werden sollten. Ohne gemeinsame Vereinbarung hat das MGAW sich entschieden, ab April 2003 ein neues, anderes System zur Deckung der häuslichen Pflegekosten für Menschen mit körperlicher und geistiger Behinderung (Shienpi-Seido) einzuführen. Danach konnte jeder selbst frei entscheiden, welche Art der häuslichen Pflegeleistungen beansprucht und mit welchem Leistungserbringer ein Pflegevertrag abgeschlossen werden sollte. Dabei musste er - entsprechend der Pflegeversicherung - einen Eigenanteil von 10\% aller Kosten selbst tragen. Allerdings wurde der Rest von 90\% nach wie vor durch Steuergelder finanziert. Leider hat dies nicht gut funktioniert, weil viele Menschen mit Behinderung in kurzer Zeit mehr Leistungen erhielten, als das MGAW kalkuliert hatte, und weil die Leistungen ohne systematisches Care-Management zum Teil auch missbraucht wurden. Deshalb musste man das System schnell verbessern. Ferner wurde im Jahre 2004 das Reformgesetz des Grundgesetzes für Menschen mit Behinderung verkündet, das vor allem auf die Förderung der Selbstständigkeit behinderter Menschen zielte. Um dieses Ziel zu erreichen, musste man alle Leistungen für Menschen mit Behinderung gut miteinander verbinden.

Im Jahre 2005 wurde das Gesetz zur Hilfe der Selbstständigkeit der Menschen mit Behinderung (Shogaisha-Jiritsu-Shien-ho) erlassen und am 1. April 2006 in Kraft gesetzt, gleich wie das Änderungsgesetz über die Pflegeversicherung. Nach dem Änderungsgesetz über die Pflegeversicherung muss jede Gemeinde als Versicherungsträger für je 20.000 - 30.000 Einwohner ein neues Regionalzentrum für die Totalhilfe gründen. Die Aufgabe dieses Regionalzentrums entspricht weitestgehend dem früheren Hilfezentrum der Gemeinden für ambulante Pflege alter Menschen, das nach dem Wohlfahrtsgesetz für die Alten gegründet wurde und nur mit Zuschüssen des Staates aus Steuermitteln finanziert wurde. Das Regionalzentrum hat demgegenüber aber auch einige wichtige neue Aufgaben: Z.B. die Care-Manager in schwierigen Fällen zu beraten, die Dementen und ihre Familienangehörigen zu beraten und ihnen zu helfen. Daneben erstellt es auch einen Pflegeplan der häuslichen Pflegeleistungen für jeden Menschen mit Behinderung. Wegen solcher fachlicher 
Aufgaben wird die anzuordnende Zahl der qualifizierten Personen ${ }^{17}$ nach der Richtlinie des MGAW angefordert.

Die Leistungen zur Hilfe der Selbständigkeit können alle Menschen mit körperlicher, geistiger und psychischer Behinderung ohne Altersgrenze erhalten. Die Leistungen werden in drei Gruppen geteilt: Kostendeckung der häuslichen und stationären Pflegeleistungen, Kostendeckung der Berufsausbildung und -förderung, sowie Kostendeckung der medizinischen Behandlung. Leistungen für Familienangehörige als Pflegepersonen gibt es nicht, genauso wie bei der Pflegeversicherung.

Für den Erhalt der Pflegeleistungen muss ein Mensch mit Behinderung bei der zuständigen Gemeinde einen Antrag auf Feststellung der Behinderung stellen, genauso wie bei der Pflegeversicherung. Die Einordnung der Behinderung begründen die Gemeinden auf einer zweistufigen Begutachtung. Auf der ersten Stufe besucht ein Angestellter der Gemeinde jeden Antragsteller, um seinen körperlichen, seelischen Zustand und seine Umgebung zu prüfen. Der Prüfer füllt einen Fragebogen, der 106 Fragen umfasst und den das MGAW abgefasst hat, aus und trägt besondere Bemerkungen im Formular ein, wenn er es für nötig erachtet. Die Daten des Fragebogens gibt er dann in einen Computer ein und dieses Ergebnis ist die erste Begutachtung. Die Gemeinde schickt diese erste Begutachtung, ein Gutachten des Hausarztes und gegebenenfalls die besondere Bemerkung an jedes Mitglied der Fachsitzung zur Festsetzung der Behinderung. Diese zweite Begutachtung der Fachsitzung wird an die Gemeinde weitergeleitet. Daraufhin stellt die Gemeinde fest, ob der Antragsteller hilfebedürftig, pflegebedürftig entsprechend Stufe 1-6 ist. Nach dieser Feststellung schickt die Gemeinde dem Antragsteller einen Berechtigungsschein.

Für eine Berufsausbildung und -förderung kann der Antragsteller bei der zuständigen Gemeinde auch nur einen Antrag auf diese konkrete Leistung stellen, die er dann nach seinem Wunsch ohne die oben erwähnte Feststellung der Behinderung auf Probe erhalten kann. Nach Beratung der Fachsitzung trifft die Gemeinde eine Entscheidung über die Gewährung der Leistung und schickt dem Antragsteller einen Berechtigungsschein.

Auch im Rahmen dieser beiden Leistungen für Menschen mit Behinderung besteht - ähnlich dem Care-Management der Pflegeversicherung - die Möglichkeit, ohne Eigenanteil einen Vertrag mit einer Einrichtung zur Beratung und Hilfe abzuschließen. Sie berät hinsichtlich der Zusammenstellung der Leistungen und stellt einen Plan für den Erhalt der Leistungen auf. Nach diesem Plan kann der Behinderte dann einen Vertrag mit einzelnen Leistungserbringern abschließen und konkrete Leistungen erhalten.

Zum Erhalt von medizinischer Behandlung muss er bei der zuständigen Gemeinde oder Präfektur nach Art der Behinderung einen Antrag auf diese Leistung stellen. Dann kann seine Berechtigung durch bestimmte medizinische Einrichtungen festgestellt werden und der Antragsteller einen Berechtigungsschein bekommen. 
Für den Bezug aller drei Leistungen muss jeder grundsätzlich einen Eigenteil von $10 \%$ aller Leistungskosten tragen. Die finanzielle Belastung des Eigenteils kann aber abhängig vom Jahreseinkommen seines Haushaltes erleichtert werden.

\section{Schlussbemerkung}

Die Familie ist die natürliche Kernzelle jeder Gesellschaft, aber speziell in Japan hatte sie im Zusammenhang mit dem Ie-System eine besondere Bedeutung, die sich teilweise auch in der heutigen Gesellschaft noch wiederfindet. Deshalb gibt es z.B. keinen Begriff der Familienpolitik in Japan. Im Gesetz kann man keine Definition von Familie finden. Was ist Familie? So muss man in Japan anfangen, wenn man sich mit dem Thema „Behinderung und Familie“ beschäftigen möchte.

Im Bereich der Sozialwohlfahrt wird das Thema im Folgenden erklärt. Einerseits hat die Familie eine wichtige Hilfe- und Schutzfunktion für Menschen mit Behinderung als Berater, Betreuer, Pfleger usw. Anderseits hat die Familie selbst eine Lebensfunktion für jedes Familienmitglied: Ein Familienmitglied mit Behinderung bringt für das Leben der anderen Familienmitglieder eine Schwierigkeit bei der alltäglichen Erwerbstätigkeit und Haushaltsführung, eine Beschränkung der sozialen, kulturellen Aktivität und der Gestaltung der persönlichen, gesellschaftlichen Beziehungen, eine Angst vor dem künftigen Leben und ggf. eine große geistige und körperliche Belastung auf Grund der schweren Behinderung mit sich ${ }^{18}$. Diese zwei Funktionen der Familie lassen sich manchmal nicht gut miteinander vereinbaren und führen zu Konflikten in der Familie.

Menschen mit Behinderung wünschen sich, wie ich am Anfang dieses Beitrags erwähnte, einerseits weiter mit ihrer Familie zusammenzuleben, aber andererseits besteht auch der Wunsch, sich von der Familie zu befreien und selbstständig zu leben. Diese beiden Wünsche sind nicht widersprüchlich, sondern passen zu den oben genannten zwei Funktionen der Familie. Wir müssen deshalb versuchen, die geistige, körperliche und finanzielle Belastung der Familien, deren Mitglieder eine Behinderung haben, zu erleichtern und jedem Familienmitglied die Möglichkeit zu geben, sein eigenes Leben zu leben. Damit können die Menschen mit Behinderung selbstständig leben und eine gute geistige, emotionale Beziehung zu ihrer Familie haben. 


\title{
6.3.3. Die Rechtstellung behinderter Menschen in Japan
}

Unterschiede zwischen Deutschland und Japan

\author{
Von Hitohiro Takizawa
}

1. Vorbemerkung

2. Kurze Charakterisierung des Rechtssystems der sozialen Sicherheit für behinderte Menschen in Japan

2.1. Das Krankenversicherungssystem

2.1.1. Allgemeines

2.1.2. Behandlungskosten $\quad 415$

2.2. Alterssicherungssystem 416

2.2.1 Allgemeines 416

2.2.2. Beschädigte, die Grundlagenrente genießen 418

2.2.3. Beschädigte, die Beschädigtenrente, Beschädigtengeld, Beschädigtenunterstützungsrente oder Beschädigtenunterstützungsgeld genießen

2.2.4. Das System der Geldleistungen für besonders Beschädigte

2.2.5. Gesetze für behinderte Menschen

2.2.5.1. Rente für verwundete und erkrankte Soldaten

2.2.5.2. Allgemeine Rente, zusätzliche Rente und Rente für Verwundete und Erkrankte

2.2.6. Die wichtigsten Gesetze für behinderte Menschen

3. Einige Gründe für die Ungleichheit der Rechtsstellung der behinderten

Menschen

3.1. Die Initiativen und die Bewegungen der behinderten Menschen

3.1.1. Die Bewegung der behinderten Menschen

3.1.2. Die Berufsgruppe der behinderten Menschen

3.2. Probleme des Gerichtswesens

3.3. Probleme der Gesetzgebung

4. Schlussbemerkung zu den Unterschieden der Rechtsstellungen zwischen

Behinderten Menschen in Deutschland und in Japan 


\section{Vorbemerkung}

Ich möchte im Folgenden über die Rechte behinderter Menschen in Japan referieren:

In Japan gibt es kein Sozialgesetzbuch wie in Deutschland, welches die Gesamtheit der Normen zusammenfasst, mit allgemeinen Regeln beginnt und durch einen besonderen Teil ergänzt wird. Stattdessen herrscht Vielfalt. Das Recht der sozialen Sicherheit der behinderten Menschen umfast mehr als 16 Gesetze in fast 80 Rechtsverordnungen der Regierung. Diese Texte sind in sehr unterschiedlichem geschichtlichen Zusammenhang entstanden und deshalb nicht nach denselben Grundsätzen verfasst. Insofern ist das japanische Sozialrechtssystem anders als das deutsche.

Das Existenzrecht wird im japanischen Verfassungsrecht vom 3.11.1946 auf folgende Weise festgesetzt:

Artikel 25 lautet:

1) Alle Menschen sollen das Recht haben den Minimumstandard eines gesunden und kultivierten Lebens aufrechtzuerhalten.

2) In allen Bereichen des Lebens sollte der Staat seine Kräfte für die Förderung, den Ausbau und die Erweiterung der Sozialfürsorge, der sozialen Sicherheit und der öffentlichen Gesundheit benutzen.

Bald nach dem Zweiten Weltkrieg im Oktober 1950 wurde durch den Beratenden Ausschuss für Soziale Sicherheit (BsS), der ein beratendes Organ des Premierministers war, ein Bericht über die soziale Sicherheit veröffentlicht. Darin wurden Sozialversicherung, Sozialfürsorge, Sozialhilfe sowie öffentliche Hygiene und ärztliche Behandlung als vier Bereiche der sozialen Sicherheit genannt. Später wurden noch Kindergeld und Wohlfahrt der Alten hinzugefügt.

Die Sozialversicherung ruht auf vier Säulen: Krankenversicherung, Rentenversicherung, Arbeitsunfallversicherung und Arbeitslosenversicherung. Wohngeld fällt in Japan, anders als in Deutschland, nicht in den Bereich der sozialen Sicherheit. Ein Staatsbürger im Sinne der Verfassung ist nur, wer die japanische Staatsangehörigkeit hat. Nach herrschender Meinung ist das Sozialhilfegesetz auf einen Ausländer weder unmittelbar noch entsprechend anzuwenden, so dass Ausländer einen Anspruch auf Sozialhilfe vor Gericht nicht geltend machen können.

Wie einleitend festgestellt, gibt es in Japan keine Kodifikation im Sozialrecht. Das ist eine Besonderheit des Systems der japanischen sozialen Sicherheit. Auch der Begriff „Versicherung“ wird in Japan anders verwandt als in Deutschland. In Deutschland wird z. B. die Höhe der Beiträge normalerweise nach den für Leistungsfälle erforderlichen Kosten festgesetzt und der individuelle Beitrag wird auch danach bemessen. In Japan gilt dieser Grundsatz nicht, weil auch Steuern für die Deckung der Sozialversicherungskosten aufgewendet werden, wenn Beiträge der Bürger aus irgendeinem politischen Grund niedriger liegen sollten. Z. B. werden die 
Hälfte der Kosten ${ }^{1}$ für die Pflegeversicherung, 32-55\% der Kosten für die nationale Krankenkassen ${ }^{2}$ und $33 \%$ Kosten für die Volksrente ${ }^{3}$ über Steuern finanziert. In Japan gibt es somit gleichlautende Systeme der sozialen Sicherheit wie in Deutschland, aber ihr Inhalt ist häufig anders.

\section{Kurze Charakterisierung des Rechtssystems der sozialen Sicherheit für behinderte Menschen in Japan}

„Krankheit“ und „Behinderung“ werden in Japan rechtlich unterschiedlich behandelt. Es gibt einen großen Unterschied zwischen dem Begriff „Behinderung“ in Japan und in Deutschland.

Wenn man in Japan erkrankt, wird man normalerweise nicht als behinderter Mensch behandelt, auch wenn man Bewegungseinschränkungen im Leben davonträgt. Wenn man krank wird oder bei einem Unfall schwer verletzt wird, wird man wahrscheinlich oder vielleicht gesund. Dann zahlt die Krankenversicherung die Behandlung. Wenn man nicht mehr gesund wird, oder verletzt bleibt, wird man erstmals als „,behinderter Mensch“ nach den verschiedenen Gesetzen anerkannt. In Japan werden normalerweise fast alle chronisch kranken Menschen nicht als behinderte Menschen angesehen, während man in Deutschland als ein Behinderter behandelt wird, wenn man chronisch krank ist. Wenn man eine ,nicht heilbare, sich nicht mehr verändernde fortdauernde“ Gesundheitsschädigung hat, wird man in Japan als „behinderter Mensch“" angesehen.

\subsection{Das Krankenversicherungssystem}

\subsubsection{Allgemeines}

Das System der Sicherung im Krankheitsfall in Japan wird getragen durch

- die gesetzliche Krankenversicherung

- sonstige Sicherungssysteme (z. B. Arbeitsunfallversicherung, Sozialhilfe, Kriegsopferversorgung für Soldaten und Zivilbeamte im Militärdienst etc.)

Der größte Teil der Bevölkerung, ca. 127.100.000 Menschen, gehört der gesetzlichen Krankenversicherung an. Das System ist einfacher strukturiert als das deutsche System.

1 Vgl. Herausgegeben von Kouseitoukeukyoukai (Institut für soziale Fürsorge und Statistik) „Hoken to Nenkin no Doukou (Tenden der Versicherung und Renten)“ Koseitoukeikyoukai, 2005, S. 128. Folgende Zahlen der Versicherten sind im oben genannten Buch beschrieben 
Die gesetzliche Krankenversicherung ruht auf zwei großen Säulen:

1) Arbeitnehmerkrankenversicherung

- Arbeitnehmerkrankenkasse

Arbeitnehmerkrankenkasse für Arbeitnehmer und Angestellte, in denen 33.735.000 Versicherte und deren 31.900.000 Familienmitglieder versichert sind (Fast alle Zahlen sind vom 31.3.2005). ${ }^{4}$

- Seeleuteversicherung

Seeleuteversicherung, in der 66.000 Versicherte und deren 109.000 Familienmitglieder versichert sind.

- Genossenschaftswerke

Genossenschaftswerke für alle staatlichen Angestellten (die höchste Stufe nennt man in Japan Beamte), Genossenschaftswerke für gemeindliche Angestellte und Genossenschaftswerke für Angestellte, Lehrer und Professoren in Privatschulen, in denen 4.449.000 Versicherte und deren 5.262.000 Familienmitglieder versichert sind.

\section{2) Ortskrankenversicherung}

Nationale Krankenkassen, in denen versicherte Bauern und alle Selbständigen sowie Abschiednehmende (die Bezeichnung Rentner wäre nicht exakt, weil Abschiednehmende in Japan zwar mit ca. 60 Jahren entlassen werden, die Vollrente jedoch erst mit 65 Jahren beziehen können) und Pensionierte versichert sind. Die Anzahl beträgt 51.579.000.

Alle Personen gehören kraft Gesetzes der gesetzlichen Krankenversicherung als Pflichtversicherte oder als Familienversicherte an. Die Voraussetzungen für diese Versicherungsformen sind im Gesetz festgelegt. Dieser Umstand ist etwas anders als im deutschen Krankenversicherungssystem.

Die Arbeitnehmerkrankenversicherung gewährt medizinische Versorgung und Geldleistung bei ausserbetrieblich entstandener Krankheit, Verletzung, Entbindung, Tod des Mitglieds sowie bei Krankheit, Verletzung, Entbindung, Tod seiner Familienangehörigen. Die Krankenversicherung wird durch die Regierung und die einzelnen Krankenkassen (regierungsverwaltete Krankenkassen und genossenschaftlich verwaltete Krankenkassen) verwaltet. Dem Gesetz nach darf ein Betrieb mit über 300 Beschäftigten eine Krankenkasse gründen. Um die Kapazität der Risikoverwaltung zu erhöhen, wird es aber in Wirklichkeit nur dann zugelassen, wenn die Krankenkasse als einzelner Betrieb mehr als 3.000 Beschäftigte zählt. Für die kleineren Betriebe, wo keine Krankenkasse organisiert ist, übernimmt die Regierung (Sozialversicherungsamt) das Geschäft der Krankenversicherung. In Wirklichkeit nehmen aber ca. 300 landesweit errichtete Sozialversicherungsstellen das Geschäft wahr. 
Die genossenschaftlich verwalteten Krankenkassen dürfen den Beitragssatz sowie den Beitragsanteil zwischen den Arbeitgebern und -nehmern selbstständig festlegen und darüber hinaus, je nach der Finanzlage der einzelnen Genossenschaften, neben den gesetzlichen Leistungen zusätzliche Leistungen freiwillig erbringen. Insofern unterscheiden sie sich von regierungsverwalteten Krankenkassen. ${ }^{5}$

\subsubsection{Behandlungskosten}

Die Leistung für ärztliche Behandlung wird in Form von Sachleistungen erbracht. Der Leistungssatz beträgt bei Patienten bis zur Vollendung des 70. Lebensjahrs 70\% der bei ärztlichen Behandlungen entstandenen Kosten und bei älteren Patienten 90\% der Kosten (jedoch 80\% bei Besserverdienenden). Die Bezugsdauer der Leistungen ist nicht beschränkt. Das rechnerische Verfahren für die bei ärztlichen Behandlungen entstandenen Kosten ist vom Minister für Arbeit, Gesundheit und Wohlfahrt bestimmt. Die Versicherten zahlen beim Kassenarzt oder -apotheker den Betrag, der über den Leistungssatz hinausgeht. Leistungen für ärztliche Behandlung bei gewöhnlicher Schwangerschaft (Entbindung) und Organtransplantation (mit einigen Ausnahmen) werden jedoch nicht erbracht. ${ }^{6}$

Die Krankenkasse stellt den Versicherten Krankenscheine zur Verfügung. Diese können nach Bedarf von den Versicherten in eigener Verantwortung verwendet werden.

Der gesetzlichen Krankenversicherung liegt das Sachleistungssystem zugrunde. In der Regel haben Versicherte Anspruch auf Übernahme der Kosten durch die Krankenversicherung. Die Versicherten hatten früher einen Selbstkostenanteil in unterschiedlicher Höhe zu tragen. In allen Gruppen müssen die Versicherten 30\% der Kosten selbst tragen. Wenn die Summe zu teuer ist, zahlt man folgende Kosten:

- Wenn man ein höheres Einkommen (über Yen 530.000 im Monat durchschnittlich bei Arbeitnehmer) bekommt, muss man Kosten in Höhe von Yen 150.000 (medizinische Kosten -500.000) x $1 \%$ pro Monat bezahlen. ${ }^{7}$

- Wenn man ein normales Einkommen bekommt, muss man Yen 80.100 + (medizinische Kosten - 267.000) x $1 \%$ pro Monat bezahlen.

- Auch wenn man als einkommensschwach anerkannt ist, muss man noch Yen 35.400 pro Monat bezahlen.

Der Arzt stellt dem Versicherten keine Rechnung aus, sondern erhält sein Honorar von der Krankenkasse über die kassenärztliche Vereinigung. Durch die Aushändigung des Krankenscheins an den Arzt macht der Versicherte seinen Anspruch auf

5 Kenporen (Zentralverband der Krankenversicherungsträger) „Krankenversicherung, Pflegeversicherung und Krankenversicherungsträger in Japan 2006“, 2006, S. 6 a.a.O. S. 11 .

7 Vgl. Herausgegeben von Kouseitoukeukyoukai (Institut für soziale Fürsorge und Statistik) „Hoken to Nenkin no Doukou (Tenden der Versicherung und Renten)“"Koseitoukeikyoukai, 2005, S. 47. 
ärztliche Behandlung als Sachleistung geltend. Man spricht daher auch in Japan vom „Sachleistungsprinzip“.

Der Aufgabenschwerpunkt der Krankenkassen liegt in der Übernahme der Kosten für Krankenbehandlungen. Obwohl dabei davon ausgegangen wird, dass der Versicherte durch seinen eigenen Kostenanteil zu einer kostenbewussteren und verantwortungsvolleren Inanspruchnahme von Leistungen angeregt wird, erhöhen sich von Jahr zu Jahr die Kosten.

Die älteren Versicherten, die das 70. Lebensjahr noch nicht vollendet haben und in der Volkskrankenversicherung versichert sind, und deren Ehegatten müssen Beiträge gemäß ihres früheren Jahreseinkommens bezahlen und bei Krankheit $30 \%$ der Kosten der ärztlichen Behandlung selbst tragen. Dies bedeutet manchmal eine große finanzielle Belastung für alte Menschen und ihre Familien. Viele alte Menschen können ihre Beiträge nicht mehr bezahlen und erhalten deshalb keine Krankenscheine und keine Leistungen mehr.

\subsection{Alterssicherungssystem}

\subsubsection{Allgemeines}

In Japan gibt es kein Sozialgesetzbuch, das die Gesamtheit der Normen zusammenfassen würde, beginnend mit den allgemeinen Regeln und gefolgt von Besonderheiten der Systeme. Im Gegenteil: es herrscht Vielfalt. Das Recht im Bereich der sozialen Sicherheit der Behinderten umfasst über 130 Gesetze und viele Rechtsverordnungen der Regierung. Diese Texte stammen aus verschiedenen Zeiträumen und sind selbstverständlich nicht nach derselben Systematik verfasst. Deshalb ist das japanische Sozialrechtssystem ganz anders als das deutsche.

Die gesetzliche Rentenversicherung ist das größte Sicherungssystem in Japan. Sie ist die wesentliche, für einen großen Teil - vor allem für Bezieher niedriger und mittlerer Einkommen - nach wie vor einzige Sicherung gegen das Risiko der Verarmung im Alter und bei Invalidität, sowie der Verarmung Hinterbliebener im Todesfall.

Durch die Rentenreform 1985 in Japan wurde das System der Grundlagenrenten, das alle Staatsbürger betrifft, eingeführt. In der davor bestehenden Volksrentenversicherung wurden nur Bauern und Selbständige versichert, seit 1985 sind alle Menschen zusätzlich in der Volksrentenversicherung gesetzlich versichert. Darüber hinaus blieb es dabei: In Arbeitnehmerrentenversicherungen werden Arbeiter und Angestellte in privaten Gesellschaften versichert. In den Unterstützungskassen für Angestellte, Beamte etc. werden nur diese versichert. In den Unterstützungskassen für Angestellte, Beamte und gemeindliche Beamte etc. werden nur gemeindliche Beamte versichert. In den Unterstützungskassen für Angestellte, Lehrer, Professoren etc. werden nur Angestellte, Lehrer, Professoren in Privatschulen versichert. In den Unterstützungskassen für Angestellte der Landwirtschaft, Forstwirtschaft und Fischerei werden nur solche Angestellten versichert. 
Die Leistungen der Rentenversicherung finanzieren sich im Umlageverfahren, d.h. die Aufwendungen zur gesetzlichen Rentenversicherung werden durch die aktuellen Einnahmen gedeckt. Die frühere Volksrentenversicherung erlitt und erleidet stets einen finanziellen Verlust. Seit dem 1. April 1986 wurde das neue System eingeführt. Zur Grundlagenrente, die die frühere Volksrentenversicherung war, wird ein Teil der früheren Arbeitnehmerversicherungen im Verhältnis zum Mehrverdienst der Arbeitnehmer addiert und darin liegt die Bedeutung der Unterstützungskassen. Deshalb nennt man ein solches System zweistufig.

In Japan gibt es folgende Rentenversicherungssysteme:

1) Grundlagenrente, in der drei Arten von Versicherten zusammengefasst sind.

- Selbständige, die älter als 20, aber jünger als 60 sind und deren Wohnsitz in Japan ist. Der Versicherte Nummer eins. Der Name stammt aus dem ersten Satz eines Artikels des Volksrentenversicherungsgesetzes. Die Anzahl ist 33.494.000 (alle Zahlen sind vom 31.3.2004). Der Teil der Selbständigen, der den Versicherungsbetrag nicht bezahlen kann und deshalb nicht versichert ist, hat sich vermehrt. Es wird vermutet, dass die Anzahl der Nichtversicherten etwa fünf Millionen beträgt. Diese Zahl wird aber nicht veröffentlicht, da man befürchtet, dass diese dann zum schlechten Beispiel für die anderen würden. Ein Vorgehen des Staates gegen die Nichtzahler ist nicht möglich, weil die Veröffentlichung gefürchtet wird.

- Versicherte der Arbeitnehmerversicherungen: Der Versicherte Nummer zwei. Die Anzahl beträgt 32.121.000.

- Der unterhaltsbedürftige Ehegatten, die älter als 20, aber jünger als 60 sind. Der Versicherte Nummer drei. Die Anzahl beträgt 11.236 (31.3.2002).

2) In den Arbeitnehmerversicherungssystemen sind fünf verschiedene Gruppen versichert:

- Rentenversicherungen für Arbeitnehmer und Angestellte inklusive Seeleute mit 32.120.000 Versicherten

- Unterstützungskassen für staatliche Angestellte, Beamte etc. mit 1.091 .000 Versicherten

- Unterstützungskassen für gemeindliche Angestellte, Beamte etc. mit 3.151.000 Versicherten.

- Unterstützungskassen für Angestellte, Lehrer, Professoren etc. in Privatschulen mit 431.000 Versicherten

- Unterstützungskassen für Angestellte in Körperschaften der Landwirtschaft, Forstwirtschaft und der Fischerei mit 440.000 Versicherten

Die Rente, die Personen, die nicht Bauern oder Selbständige sind, erhalten, vermindert sich aber, weil die Beiträge der Arbeitnehmer für die Rente der Bauern und Selbständigen benutzt wird.

Die Höhe der laufenden Altersrenten aus der Grundlagenrente und fünf Arbeitnehmerversicherungen ist ganz unterschiedlich. Denn die meisten Rentner bekom- 
men ihre Altersrenten zur Zeit nach dem alten Rentenversicherungssystem, das 1986 grundlegend reformiert wurde und noch jetzt als Übergangsregelung gültig ist. Selbständige erhielten nur Grundlagenrente, im Durchschnitt ungefähr im Jahr 2006 nur 66.000 Yen (440 EUR) pro Monat (1 EUR = ungefähr 150 Yen). Die Höhe der laufenden Altersrenten aus den Arbeitnehmerversicherungen betrug im Rechnungsjahr 2005 monatlich 133.374 Yen (889.16 EUR).

Die Höhe der Renten aus Grundlagenrente ist niedrig, weil die Beiträge erst ab dem 1. April 1961 eingezahlt wurden und die Höhe der Beiträge, die die Selbständigen anders als andere Versicherte allein bezahlen mussten, aus einem sozialpolitischen Grund auf ein niedriges Niveau begrenzt wurde.

Jetzt beschäftige ich mich mit dem Rentensystem für Beschädigte, die durch Unfälle im privaten Bereich beschädigt wurden. Von Arbeitnehmern, die beim Arbeitsunfall verletzt wurden, und Soldaten und Zivilbeamten im Militärdienst, die während des Krieges verwundet wurden, ist jetzt nicht mehr die Rede.

Die Rente für Beschädigte ist nach der Gruppe, zu der diese gehören, verschieden. Oberflächlich betrachtet, gibt es zwei Gruppen.

\subsubsection{Beschädigte, die Grundlagenrente genießen}

\section{$\mathrm{Zu}$ dieser Gruppe gehören}

- die in der Grundlagenrente Versicherten (s.o.)

- die ehemals Versicherten, die älter als 60 aber jünger als 65 Jahre sind und deren Wohnsitz in Japan ist.

Diese Beschädigten genießen nur die Grundbeschädigtenrente nach dem Beschäftigungsgrad (Grad eins oder zwei), der durch die Rechtsordnung bestimmt wird. Nur diese Grundbeschädigtenrente bekommen Selbständige, Studenten, unterhaltsbedürftige Ehegatten(-gattinnen) etc. Ein Beschädigter ersten Grades bekommt pro Jahr 990.100 Yen (6.600 EUR) und des zweiten 792.100 Yen (5.280 EUR).

\subsubsection{Beschädigte, die Beschädigtenrente, Beschädigtengeld, Beschädigtenunter- stützungsrente oder Beschädigtenunterstützungsgeld genießen}

Beschädigte Arbeitnehmer genießen Beschädigtenrente (Grad eins, zwei oder drei) oder Beschädigtengeld und beschädigte Beamte oder Versicherte in der Unterstützungskasse für Angestellte, Lehrer, Professoren etc. in Privatschulen nach dem Beschäftigungsgrad, der durch die Rechtsordnung bestimmt wird, genießen Beschädigtenunterstützungsrente (Grad eins, zwei oder drei) oder Beschädigtenunterstützungsgeld. Die Beschädigten des ersten und zweiten Grades bekommen auch ihre Grundbeschädigtenrente.

- Die Summe der Beschädigtenrente oder Beschädigtenunterstützungsrente ersten Grades entspricht dem Betrag, den ein Arbeitnehmer im Verhältnis mehr ver- 
dient, plus ein Viertel dieses Betrags plus den Betrag, der für die Ehegatten (-gattinnen) addiert wird.

- Die Summe der Beschädigtenrente oder Beschädigtenunterstützungsrente zweiten Grades entspricht dem Betrag, den ein Arbeitnehmer im Verhältnis mehr verdient, plus den Betrag, der für die Ehegatten(-gattinnen) addiert wird.

- Die Summe der Beschädigtenrente oder Beschädigtenunterstützungsrente dritten Grades entsprich dem Betrag, den ein Arbeitnehmer im Verhältnis mehr verdient. Mindestens 597.800 Yen (=3.985 EUR) pro Jahr.

- Ein beschädigter Arbeitnehmer bekommt Beschädigtengeld oder Beschädigtenunterstützungsgeld. Das Geld wird ihm nur einmal gezahlt, wenn seine Beschädigung den dritten Grad nicht erreicht. Die Summe entspricht zweimal der Summe der Beschädigtenrente oder Beschädigtenunterstützungsrente der Beschädigten dritten Grades.

\subsubsection{Das System der Geldleistungen für besonders Beschädigte}

Nach dem Gesetz über die Leistung des Kinderfürsorgegeldes für Besondersbeschädigte etc. vom 2.7.1964 ist der Besondersbeschädigte jemand, der älter als 20 ist und wegen der Schwerbehinderung i.S. des Gesetzes im täglichen Leben immer Pflege braucht. Nach der Gesetzesausführungsverordnung über die Leistung des Kinderfürsorgegeldes für Besondersbeschädigte etc. vom 4.7.1975 ist der Besondersbeschädigte grundsätzlich jemand, der eine doppelte Behinderung ersten Grades nach dem Volksrentengesetz hat oder folgendermaßen beschrieben werden kann:

- jemand, der mehrere Behinderungen und darüber hinaus eine körperliche Behinderung oder eine Krankheit, beziehungsweise eine geistige Behinderung von eins bis sieben in der folgende Tabelle hat.

- jemand, der neben einer Behinderung, die eine körperliche Behinderung oder eine Krankheit, beziehungsweise eine geistige Behinderung von eins bis sieben in der folgende Tabelle sein kann und zwei Behinderungen zweiten Grades nach dem Volksrentengesetz, also drei Behinderungen hat.

- jemand, der neben einer Behinderung, die eine körperliche Behinderung von drei bis fünf in folgender Tabelle ist, hat, die besonders schwer ist, so dass seine Bewegungsfähigkeit im täglichen Leben als gering eingestuft wird.

- jemand, der neben einer Behinderung, die eine körperliche Behinderung von sechs oder sieben in folgender Tabelle ist, und sein Krankheitszustand strenge Bettruhe erfordert, oder ein Mensch, der eine Behinderung, die eine geistige Behinderung von sechs oder sieben in folgender Tabelle ist, hat die besonders schwer ist, so das seine Teilnahmefähigkeit am täglichen Leben als sehr gering eingeschätzt wird. 
Tabelle der Gesetzausführungsverordnung über die Leistung des Kinderfürsorgegeldes für Besondersbeschädigte etc.

1) jemand, bei dem die Summe der Werte, die sich bei der Messung des Sehvermögens beider Augen ergeben, weniger als 0,04 beträgt

2) jemand, dessen Hörvermögen beider Ohren nicht erlaubt Töne über 100 Dezibel zu hören

3) jemand, der sehr schwere Behinderung in der Bewegungsfähigkeit beider Arme hat, keine Finger hat, oder bei dem Bewegungsfähigkeit aller Finger sehr stark eingeschränkt ist

4) jemand, bei dem die Bewegungsfähigkeit beider Unterglieder stark eingeschränkt ist, oder der mindestens den Verlust von zwei Fußgelenken zu beklagen hat,

5) jemand, der eine Behinderung seines Körpers hat, aufgrund dessen er nicht sitzen oder stehen kann.

6) jemand, der eine Behinderung oben von eins bis fünf hat und bei dem anerkannt wird, dass er wegen seiner körperlichen Funktionsbeeinträchtigung oder seiner Krankheit in gleichem Grad und darüber hinaus, wie oben von eins bis fünf genannt wird, eingeschränkt wird, und lange Bettruhe braucht, so dass er am täglichen Leben nicht teilnehmen kann.

7) jemand, der eine geistige Behinderung von gleichem Grad und darüber, wie oben von eins bis sechs bestimmt wird, hat und dem es anerkannt wird.

(Bemerkung) Bei der Messung des Sehvermögens muss man Sehvermögenstabelle für alle Länder benutzen, und man muss verbessertes Sehvermögen messen, wenn man Reflektionsanomalie hat.

Die Summe des Besondersbeschädigtengeld beträgt 26.440 Yen (176 EUR) pro Monat im Jahr von 2006. Sie wird einem schwerbehinderten Menschen gezahlt, der nach einem geltenden Gesetz als behinderter Mensch ersten Grades eingestuft wird. Wenn er Pflegegeld wegen einer Behinderung durch die Atombombe bekommt, darf er es nicht bekommen (nach dem Artikel 26-4 des Gesetzes und Art. 10 der Verordnung). Wenn er ein nach der Verordnung bestimmtes Einkommen erhält, darf er es auch nicht bekommen (nach dem Artikel 26-5 des Gesetzes und Art. 11 der Verordnung).

Wie oben beschrieben, bekommen nur die schwerbehinderten Menschen, die mehrere bestimmte Behinderungen haben, das Besondersbeschädigtengeld. Aber das Besondersbeschädigtengeld beträgt nur 26.440 Yen (ca. 176 EUR) pro Monat (Werte aus dem Jahr 2006). Nach dem Gesetz über die Leistung des Besondersbeschädigtenkinderfürsorgegeldes etc. soll das Geld für die Pflege von schwerbehinderten Menschen aufgewendet werden. Die Grundbeschädigtenrente beträgt 792.100 Yen (ca. 5.280 EUR) nach dem Absatz 1 des Artikels 33 des Volksrentengesetzes pro Jahr. Nach dem Absatz 2 dieses Artikels entspricht die Summe der Grundbeschädigtenrente ersten Grades der Summe der Grundbeschädigtenrente plus ein Viertel 
dieser Summe (zusammen 990.100 Yen pro Jahr). Deshalb bekommt ein schwerbehinderter Mensch ersten Grades normalerweise 82.500 Yen (ca. 550 EUR) pro Monat. Auch wenn er Besondersbeschädigtengeld bekommt, entspricht die Summe nur 108.900 Yen (ca. 725 EUR). Wenn er z. B. 30 Jahre alt ist und allein in einer großen Stadt ohne Einkommen und Vermögen lebt, bekommt er die folgende Summe als Sozialhilfe: Hilfe zum Lebensunterhalt entspricht 83.700 Yen (2006 Sommer), behinderte Menschen erhalten zusätzlich 26.850 Yen und schwerbehinderte Menschen 14.430 Yen. Die Summe entspricht 124.980 Yen (ca. 833 EUR) pro Monat. Natürlich bekommt er die Lebenshilfe und das Besondersbeschädigtengeld nicht zusammen. Wenn ein schwerbehinderter Mensch in einem großen Haus lebt, das er nach dem japanischen Sozialhilfegesetz nicht besitzen darf, kann er keine Sozialhilfe erhalten. Im Vergleich mit der Summe 1.432 EUR (Sachleistung) in Pflegestufe III der Pflegeversicherung in der BRD ist es klar, dass ein japanischer Schwerbehinderter noch weniger Beihilfe als ein deutscher bekommt.

\subsubsection{Gesetze für behinderte Menschen}

In Japan gibt es verschiedene Gesetze, die die Rechtstellung behinderter Menschen regeln. Jedoch haben nicht alle behinderten Menschen das gleiche Recht auf Fürsorge und Rente.

\subsubsection{Rente für verwundete und erkrankte Soldaten}

Wenn Soldaten und Zivilbeamte im Militärdienst während des Zweiten Weltkriegs während des Dienstes, aber nicht bei Kampfbehandlungen verwundet worden oder erkrankt sind, bekommen sie eine besondere Rente für verwundete und erkrankte Soldaten. Die oben genannte Zeit ist die Zeitdauer vom 8.12.1941 als Japan den USA, Großbritannien, Frankreich und Niederlanden den Krieg erklärte, bis zum 30.11.1945 oder für den Fall, dass ein Soldat nach dem 2.9.45 im Ausland war und danach demobilisiert wurde, bis zum Demobilisierungstag. Diese Gruppe der Soldaten und Zivilbeamten wird in zwölf Klassen eingeteilt. Nach Artikel 13 der Ergänzung zum Pensionsgesetz beträgt die Höhe der Rente wie folgt (Werte aus dem Jahr 2007. 150 Yen = ungefähr 1 EUR):

Gruppe A: Schwerbehinderte Menschen (sieben Klassen) Besonderer Grad: Die Summe beträgt höchstens 1,7mal die Höhe des ersten Grades, deshalb 9.719.150 Yen (ca. 64.861 EUR) pro Jahr. Erster Grad: 5.723.000 Yen (ca. 38.153 EUR). Zweiter Grad: 4.769.000 Yen (ca. 31.793 EUR). Dritter Grad: 3.927.000 Yen (ca. 26.180 EUR9: Vierter Grad: 3.108.000 Yen (ca. 20.720 EUR). Fünfter Grad: 2.514.000 Yen (ca. 16.760.000 EUR). Sechster Grad: 2.033.000 Yen (ca. 13.533 EUR). 
Gruppe B: Behinderte Menschen (fünf Klassen)

Erster Grad: 1.853.000 Yen (ca. 12.353 EUR). Zweiter Grad: 1.686.000 Yen (ca. 11.240 EUR). Dritter Grad: 1.353 .000 Yen (ca. 9.020 EUR). Vierter Grad: 1.089.000 Yen (ca. 7.260 EUR). Fünfter Grad: 961.000 Yen (ca. 6.406 EUR).

\subsubsection{Allgemeine Rente, zusätzliche Rente und Rente für Verwundete und Erkrankte}

Wenn Soldaten und Zivilbeamte im Militärdienst während des Zweiten Weltkriegs im Dienst bei Kampfbehandlungen verwundet worden oder erkrankt sind, bekommen sie eine allgemeine Rente und eine zusätzliche Rente für verwundete und Erkrankte. Es gibt in dieser Gruppe ebenfalls zwölf Klassen. Nach Artikel 46 und 65 des Pensionsgesetzes sowie Artikel 22 der Ergänzung zum Pensionsgesetz beträgt die Höhe der Rente wie folgt (Werte aus dem Jahr 2003):

Gruppe A: Schwerbehinderte Menschen (acht Klassen) Besonderer Grad: Die Summe beträgt höchstens 1,7 mal die Höhe des ersten Grades, deshalb 9.729.100 Yen (ca. 64.861 EUR) pro Jahr. Erster Grad: 5.723.000 Yen (ca. 38.153 EUR). Zweiter Grad: 4.769.000 Yen (ca. 31.793 EUR). Dritter Grad: 3.927.000 Yen (ca. 26.180 EUR). Vierter Grad: 3.108.000 Yen (ca. 20.720 EUR). Fünfter Grad: 2.514.000 Yen (ca. 16.760 EUR). Sechster Grad: 2.033.000 Yen (ca. 13.533 EUR). Siebter Grad: 1.853.000 Yen (ca. 12.353 EUR).

Gruppe B: Behinderte Menschen (vier Klassen)

Erster Grad: 1.686.000 Yen (ca. 11.240 EUR). Zweiter Grad: 1.353.000 Yen (ca. 9.020 EUR). Dritter Grad: 1.089.000 Yen (ca. 7.620 EUR). Vierter Grad: 961.000 Yen (ca. 6.406 EUR).

Den oben genannten Gruppen entsprechen die Definitionen der einzelnen Grade. Ehemalige Soldaten und Zivilbeamte im Militärdienst, die während des Zweiten Weltkriegs während des Dienstes verwundet wurden oder erkrankten, genießen verschiedene staatliche Leistungen. Nach dem Artikel neun des besonderen Kriegsopferbeschädigtenfürsorgegesetzes sind die staatlichen Leistungen dafür, wie folgt:

- ärztliche Behandlung

- Krankengeld

- Begräbnisfeiergeld

- ärztliche Behandlung für die Rehabilitation

- Hilfsmittel, Prothesen und ihre Reparatur

- Kuraufenthalt in einem staatlichen Erholungsheim

- Freie Fahrt mit der Bahn

Ein solches Gesetz über besondere Sozialfürsorge gilt aber selbst für die im Krieg schwer geschädigten Krankenpflegerinnen, Bürger und in Japan lebende Ausländer 
nicht. Soldaten und Zivilbeamte im Militärdienst, die während des Zweiten Weltkriegs während des Dienstes verwundet wurden oder erkrankten, genießen natürlich die Vorteile der Rente. Von Geburt an behinderte Menschen und behinderte Menschen, die nicht Soldaten und Zivilbeamte im Militärdienst sind, haben zwar ein Recht auf Sozialhilfe - wie auch andere Mittellose -, sie haben jedoch selbst dann keinen Anspruch auf Kriegsoperbeschädigtenfürsorge wenn sie auch im Krieg schwer verwundet wurden.

Soldaten und Zivilbeamte im Militärdienst, die die oben genannte Rente bekommen, genießen die oben genannten staatlichen Leistungen, ausgenommen den Kuraufenthalt in einem staatlichen Erholungsheim für Schwerverletzte. Das Gesetz bietet jedoch Soldaten und Zivilbeamte Vorteile, so dass behinderte Menschen untereinander ungleich behandelt werden.

Tabelle 1-2 des Pensionsgesetzes bringt Beispiele für Behinderungen wie folgt:

\section{Gruppe A: Schwerbehinderte Menschen (sieben Klassen)}

Besonderer Grad:

1) Ein Mensch, der wegen seiner körperlichen oder seelischen Behinderung am täglichen Leben nicht teilnehmen kann und deshalb immer intensiver Pflege bedarf. [A-BG-1]

2) Ein Mensch, der kein Licht erkennen kann. [A-BG-2]

3) Ein Mensch, der entweder keine Arme oder keine Beine hat. [A-BG-3]

4) Ein Mensch, der eine Behinderung des ersten Grades und noch eine Behinderung des ersten Grades beziehungsweise des sechsten Grades hat. [A-BG-4]

Erster Grad:

1) Ein Mensch, der wegen seiner körperlichen oder seelischen Behinderung nur sehr eingeschränkt am täglichen Leben teilnehmen kann und deshalb immer Pflege bedarf. [A-1-1]

2) Ein Mensch, der nicht kauen und nicht sprechen kann. [A-1-2]

3) Ein Mensch, dessen Sehvermögen unter 0,01 liegt. [A-1-3]

4) Ein Mensch, der ein großes Loch in seiner Lunge hat und an offener Tuberkulose erkrankt ist und sehr ansteckend ist, so dass er am täglichen Leben nicht teilnehmen kann. [A-1-4]

5) Ein Mensch, der so schwer atmet, dass eine Untersuchung der Lungen nicht möglich ist. [A-1-5]

6) Ein Mensch, der mindestens den Verlust von beiden Ellbogengelenken zu beklagen hat. [A-1-6]

7) Ein Mensch, der mindestens den Verlust von beiden Kniegelenken zu beklagen hat. [A-1-7] 
Zweiter Grad:

1) Ein Mensch, der nicht kauen oder nicht sprechen kann. [A-2-1]

2) Ein Mensch, dessen Sehvermögen unter 0,02 liegt. [A-2-2]

3) Ein Mensch, der nicht hören kann. [A-2-3]

4) Ein Mensch, der eine große Schlagadergeschwulst, eine Schlagadergeschwulst unter seinem Schlüsselbein, ein Halsarterieschlagadergeschwulst, eine Schlagadergeschwulst oder eine Darmbeinschlagadergeschwulst hat. [A-2-4]

5) Ein Mensch, der mindestens den Verlust von beiden Handgelenken zu beklagen hat. [A-2-5]

6) Ein Mensch, dem ein Arm oder ein Bein fehlt. [A-2-6]

7) Ein Mensch, der mindestens den Verlust von beiden Fußgelenken zu beklagen hat. [A-2-7]

Dritter Grad:

1) Ein Mensch, der wegen seiner körperlichen oder seelischen Behinderung sehr schwer am täglichen Leben teilnehmen kann. [A-3-1 ]

2) Ein Mensch, dessen Sehvermögen unter 0,03 liegt. [A-3-2]

3) Ein Mensch, der kein großes Loch in seiner Lunge hat, an Tuberkulose erkrankt, ansteckend ist und relativ viel Bettruhe braucht. [A-3-3]

4) Ein Mensch, der schwerwiegende Atembeschwerden hat. [A-3-4]

5) Ein Mensch, der an sehr schweren Herzbeschwerden (Herzinsuffizienz oder Herzkranzgefäßverengung) leidet. [A-3-5]

6) Ein Mensch, bei dem die Nieren-, Leberfunktion oder Blutbildung sehr eingeschränkt ist. [A-3-6]

7) Ein Mensch, der mindestens den Verlust von einem Ellbogengelenk zu beklagen hat. [A-3-7]

8) Ein Mensch, der mindestens den Verlust von einem Kniegelenk zu beklagen hat. $[\mathrm{A}-3-8]$

Vierter Grad:

1) Ein Mensch, der sehr schwer kauen oder sprechen kann. [A-4-1 ]

2) Ein Mensch, der auf beiden Augen nur noch eine Sehschärfe von weniger als 0,04 hat. [A-4-2]

3) Ein Mensch, der laute Stimmen von keiner größeren Entfernung als 0,05 m von beiden Ohren entfernt verstehen kann. [A-4-3]

4) Ein Mensch, der beide Hoden verloren hat, und diese Sachlage jedoch keine bemerkenswerten negativen Symptome nach sich zieht. [A-4-4]

5) Ein Mensch, der mindestens den Verlust eines Armgelenkes zu beklagen hat. [A-4-5]

6) Ein Mensch, der mindestens den Verlust eines Fußgelenkes zu beklagen hat. [A-4-6] 
Fünfter Grad:

1) Ein Mensch, der wegen seiner körperlichen oder seelischen Behinderung sehr schwer am täglichen Leben in der Gesellschaft teilnehmen kann. [A-5-1]

2) Ein Mensch, der eine große unschöne Narbe am Kopf oder im Gesicht hat. [A-5-2]

3) Ein Mensch, der auf einem Auge nur noch eine Sehschärfe von weniger als 0,01 hat. [A-5-3]

4) Ein Mensch, der kein durch Röntgenaufnahmen nachweisbares Loch in seiner Lunge hat, an Tuberkulose erkrankt ist, lebendige Krankheitskeime in seiner Lunge hat und immer relativ wenig Bettruhe braucht. [A-5-4]

5) Ein Mensch. der an Atembeschwerden mittleren Grades leidet. [A-5-5]

6) Ein Mensch, der an Herzbeschwerden mittleren Grades (Herzinsuffizienz oder Herzkrampf) leidet.

7) Ein Mensch. bei dem die Nieren, die Leber oder die Blutbildung nur eingeschränkt funktionsfähig ist. [A-5-7]

8) Ein Mensch. der alle Finger einer Hand verloren hat. [A-5-8]

Sechster Grad:

1) Ein Mensch, der schwerwiegende Bewegungsbeschwerden im Bereich des Halses oder Rumpfes hat. [A-6-1]

2) Ein Mensch, der auf einem Auge nur noch eine Sehschärfe von weniger als unter 0,02 hat. [A-6-2]

3) Ein Mensch, der seine Milz verloren hat. [A-6-3]

4) Ein Mensch, der einen Daumen und einen Zeigefinger einer Hand verloren hat. [A-6-4]

5) Ein Mensch, der die Bewegungsfähigkeit aller Finger einer Hand verloren hat. [A-6-5]

(Bemerkung) Behinderungen, die nicht oben genannt werden, werden entsprechend ähnlicher Behinderungen anerkannt.

Die Röntgenbilder von Tuberkulosepatienten werden nach der Nihon Kekkakubyou Gakkai Byoukei Bunrui (Klassifikation der Krankheiten durch die japanische wissenschaftliche Vereinigung gegen Tuberkulose) eingestuft.

Bei der Messung des Sehvermögens muss man die Sehvermögenstabelle für alle Länder benutzen, und man muss verbessertes Sehvermögen messen, wenn man Reflektionsanomalie hat. 
Tabelle 1-3 des Pensionsgesetzes bringt Beispiele für Behinderungen wie folgt:

\section{Gruppe B: Behinderte Menschen (fünf Untergruppen)}

Erster Grad:

1) Ein Mensch, der auf einem Auge nur noch eine Sehschärfe von weniger als 0,04 hat. [B-1-1]

2) Ein Mensch, der auf einem Ohr taub ist und normale Stimmen von keiner gröBeren Entfernung als 1,5 m von einem Ohr entfernt verstehen kann. [B-1-2]

3) Ein Mensch, der eine Niere verloren hat. [B-1-3]

4) Ein Mensch, der einen Daumen an einer Hand verloren hat. [B-1-4]

5) Ein Mensch, der den Zeige-, den Mittel-, den Ring- und den kleinen Finger an einer Hand verloren hat. [B-1-5]

6) Ein Mensch, dessen Fußgelenk abgewinkelt und steif ist. [B-1-6]

7) Ein Mensch, der alle Zehen eines Fußes verloren hat. [B-1-7]

Zweiter Grad:

1) Ein Mensch, der auf einem Auge nur noch eine Sehschärfe von weniger als 0,05 hat. [B-2-1]

2) Ein Mensch, der auf einem Ohr taub ist. [B-2-2]

3) Ein Mensch, dessen Daumen an einer Hand bewegungsunfähig ist. [B-2-3]

4) Ein Mensch, dessen Zeige-, Mittel-, Ring- und kleiner Finger an einer Hand bewegungsunfähig ist. [B-2-4]

5) Ein Mensch, dessen Zehen eines Fußes bewegungsunfähig sind. [B-2-5]

Dritter Grad:

1) Ein Mensch, der wegen seiner körperlichen oder seelischen Behinderung nur begrenzt am täglichen Leben in der Gesellschaft teilnehmen kann. [B-3-1]

2) Ein Mensch, der auf einem Auge nur noch eine Sehschärfe von weniger als 0,07 hat. [B-3-2]

3) Ein Mensch, der laute Stimmen von keiner größeren Entfernung als $0,05 \mathrm{~m}$ von seinem Ohr entfernt verstehen kann. [B-3-3]

4) Ein Mensch, der zwar an Tuberkulose erkrankt ist, jedoch weder ein durch Röntgenaufnahmen nachweisbares Loch noch lebende Krankheitskeime in seiner Lunge hat, bei dem aber wegen der Gefahr des Wiederausbrechens der Krankheit eine Verlaufsuntersuchung durchgeführt werden muss. [B-3-4]

5) Ein Mensch, der an leichten Atembeschwerden leidet. [B-3-5]

6) Ein Mensch, der einen Hoden verloren hat. [B-3-6]

7) Ein Mensch, der den Zeigefinger an einer Hand verloren hat. [B-3-7]

8) Ein Mensch, der die große Zehe eines Fußes verloren hat. [B-3-8] 
Vierter Grad:

1) Ein Mensch, dessen Zeigefinger an einer Hand bewegungsunfähig ist. [B-4-1]

2) Ein Mensch, der den Mittelfinger an einer Hand verloren hat. [B-4-2]

3) Ein Mensch, dessen großer Zehe eines Fußes bewegungsunfähig ist. [B-4-3]

4) Ein Mensch, der die zweite Zehe eines Fußes verloren hat. [B-4-4]

Fünfter Grad:

1) Ein Mensch, der auf einem Auge nur noch eine Sehschärfe von weniger als 0,01 hat. [B-5-1]

2) Ein Mensch, der normale Stimmen von keiner größeren Entfernung als $0,5 \mathrm{~m}$ von einem Ohr entfernt verstehen kann. [B-5-2]

3) Ein Mensch. dessen Mittelfinger an einer Hand bewegungsunfähig ist. [B-5-3]

4) Ein Mensch. der den Ringfinger einer Hand verloren hat. [B-5-4]

5) Ein Mensch. dessen zweite Zehe eines Fußes bewegungsunfähig ist. [B-5-5]

6) Ein Mensch. der zwei Zehen von der zweiten bis fünften Zehe eines Fußes verloren hat. [B-5-6]

(Bemerkung) Behinderungen, die nicht oben genannt werden, werden entsprechend ähnlicher Behinderungen anerkannt.

Die Röntgenbilder von Tuberkulosepatienten werden nach der Nihon Kekkakubyou Gakkai Byoukei Bunrui (Klassifikation der Krankheiten durch die japanische wissenschaftliche Vereinigung gegen Tuberkulose) eingestuft.

Bei der Messung des Sehvermögens muss man die Sehvermögenstabelle für alle Länder benutzen, und man muss verbessertes Sehvermögen messen, wenn man Reflektionsanomalie hat.

Das Pensionsgesetz wurde zum erstenmal im Jahre 1923 erlassen, so dass es einige Ausdrücke (z. B. [A-4-3], [B-1-2], [B-3-3], [B-5-2] etc.), welche aus heutiger Sicht fremd und veraltet sind, in seinen Tabellen hat. Der Wortlaut des oben genannten [A-4-3] (Pensionsgesetz) entspricht fast dem Wortlaut des dritten Grades der fünften Tabelle der Gesetzesausführungsverordnung des Sozialfürsorgegesetz für körperlich behinderte Menschen vom 6.4.1950 (Dieser lautet: Ein Mensch, dessen Gehör zur Wahrnehmung von Stimmen und Geräusche unter 90 Dezibel nicht in der Lage ist. - Ein Mensch, der laute Stimmen von keiner größeren Entfernung mit beiden Ohren verstehen kann.) Aber es gibt keine Entsprechung mit [B-1-2], [B-3-3], [B-5-2], obwohl das Pensionsgesetz fast jedes Jahr geändert wird.

\subsubsection{Die wichtigsten Gesetze für behinderte Menschen}

Die wichtigsten Gesetze für behinderte Menschen sind folgende:

1. Grundgesetz für behinderte Menschen vom 21.5.1970

2. Sozialfürsorgegesetz für körperlich behinderte Menschen vom 26.12.1949

3. Sozialfürsorgegesetz für geistig behinderte Menschen vom 31.3.1960

4. Sozialfürsorgegesetz für Alte vom 11.7.1963 
5. Gesetz zur medizinischen Hilfe und Sozialfürsorge für seelisch behinderte Menschen vom 1.5.1950

6. Volksrentengesetz vom 16.4.1959

7. Rentenversicherungsgesetz vom 19.5.1954

8. Gesetz für Unterstützungskassen für staatliche Angestellte, Beamte etc. vom 1.5.1958

9. Gesetz für Unterstützungskassen für gemeindliche Beamte etc. vom 8.9.1962

10. Gesetz für Unterstützungskassen für Angestellte, Lehrer, Professoren etc. in Privatschulen vom 21.8.1953

11. Pensionsgesetz vom 14.4.1923

12. Arbeitsunfallversicherungsgesetz vom 7.4 .1947

13. Arbeitsförderungsgesetz für behinderte Menschen vom 25.7.1960

14. Besondersfürsorgegesetz für Kriegsbeschädigte vom 3.8.1963

15. Pflegeversicherungsgesetz vom 17.12 .1997

16. Gesetz zur Unterstützung der Selbständigkeit behinderte Menschen vom 7.11.2005

Merkwürdigerweise hat weder das Sozialfürsorgegesetz für geistig behinderte Menschen vom 31.3.1960, noch die Gesetzesausführungsverordnung des Sozialfürsorgegesetz für geistig behinderte Menschen vom 18.4.1960 den Begriff des geistig behinderten Menschen definiert, weil Gruppen geistig behinderter Menschen wegen des Schutzes des Privatlebens einzelner einer solchen Einstufung widersprachen. Der Begriff wird in Bekanntmachungen des Ministeriums für Gesundheit und Wohlfahrt über den Nachweis für geistig behinderte Menschen vom 27.9.1973 festgesetzt: Als geistig behinderter Mensch gilt jemand, der vom Jugendamt oder vom Rehabilitationsberatungsamt als solcher anerkannt wird. Der Satz ist unverständlich, weil in der Bekanntmachung der Begriff behinderter Mensch nicht definiert wird. In den Bekanntmachungen des Ministeriums für Gesundheit und Wohlfahrt über Aufnahmeeinrichtungen und Verwaltung für geistig schwerbehinderte Menschen vom 27.9.1973 wird aber ein geistig schwerbehinderter Mensch als jemand beschrieben, der einen IQ von weniger als 35, oder neben einer körperlicher Behinderung weniger als 50 hat. $^{8}$

Die Gesetze, die selbst oder in ihren Gesetzesausführungsverordnungen konkrete Behinderungskataloge enthalten, sind die 2, 6-13. Die Behinderungskataloge von 7-10 sind gleich. Der erste und zweite Behinderungsgrad des Behinderungskataloges des Volksrentengesetzes entspricht den Graden der Gesetze von 7-10. Das Volksrentengesetz hat jedoch nur zwei Grade, obwohl die Gesetze von 7-10 drei Grade enthalten. Behinderte Arbeitnehmer genießen deshalb Behindertenrente (Grad eins, zwei oder drei) oder Behindertengeld. Behinderte Beamte oder Versicherte in der 
Unterstützungskasse für Angestellte, Lehrer, Professoren etc. in Privatschulen genießen abhängig vom Behinderungsgrad Behindertenunterstützungsrente (Grad eins, zwei oder drei) oder Behindertenunterstützungsgeld. Zudem bekommen behinderte Menschen des ersten und zweiten Grades auch noch ihre Grundbehindertenrenten. Das Pensionsgesetz hat einen ganz anderen Behinderungskatalog als sonstige Gesetze. ${ }^{9}$

\section{Einige Gründe für die Ungleichheit der Rechtsstellung der behinderten Menschen}

\subsection{Die Initiativen und die Bewegungen der behinderten Menschen}

\subsubsection{Die Bewegung der behinderten Menschen}

Streng genommen, gab und gibt es einige Bewegungen, mit denen behinderte Menschen für die Verbesserung ihrer Rechtsstellung gekämpft haben. Jedoch waren viele von den Bewegungen fast ohne Erfolg, ausgenommen die Bewegung der Soldaten und der Zivilbeamten im Militärdienst, die während des zweiten Weltkrieges verwundet wurden.

Diese Bewegung war von Erfolg gekrönt, denn es gab und gibt Hinterbliebene von Soldaten und Zivilbeamten im Militärdienst. Sie wählen Abgeordnete der Japanischen Freien Demokratischen Partei (JFDP), die die größte Partei und etwa 60 Jahre lang nach dem zweiten Weltkrieg die einzige herrschende Partei in Japan war, und unterstützten deren Wahlkampf. Nippon Izokukai (Japanische Gesellschaft für die Hinterbliebenen) war und ist auch jetzt eine von den einflussreichsten Verhandlungsgruppen. Ihr Präsident war der frühere Premierminister Ryuutarou Hashimoto. Die Hinterbliebenen der geschädigten Soldaten und Zivilbeamten im Militärdienst haben normalerweise viele Familienmitglieder und Verwandte. Deshalb kann Nippon Izokukai eine starke einflussreiche Verhandlungsgruppe sein.

Andererseits ist die Anzahl anderer Kriegsbeschädigter, z.B. die der im Krieg schwer geschädigten Krankenpflegerinnen und Bürger kleiner als die der Hinterbliebenen sowie der kriegsbeschädigten Soldaten und Zivilbeamten im Militärdienst und sie können in der Wahl kaum eine große Rolle spielen. Die in Japan lebenden Ausländer dürfen überhaupt nicht wählen, obwohl sie seit vor dem zweiten Weltkrieg in Japan leben.

In Japan genießt leider nur der ein Recht, der mit der staatlichen Macht verknüpft ist. Macht schafft Recht. 


\subsubsection{Die Berufsgruppe der behinderten Menschen}

Japanische behinderte Menschen werden ungefähr in vier politische Gruppen aufgeteilt: es sind eine konservative, eine kommunistische, eine radikale und eine unabhängige Gruppe. Die Letzte ist groß in Japan, weil viele behinderte Menschen sehr verschieden klassifiziert und schwer organisiert werden. Man kann nicht alle Gruppen aufzählen, weil es zu viele behinderte Menschengruppen in Japan gibt.

Ich möchte ein typisches Beispiel für eine Gruppe der Blinden anführen. Denn die Behinderung der Blinden ist eine der schwersten Behinderungen, und die Blinden organisieren sich relativ einfach, weil sie nur unter Schwierigkeiten allein gehen, fahren, arbeiten können und stets dringende gemeinsame Bedürfnisse und Ansprüche haben.

Die Blinden werden im Allgemeinen in drei politische Gruppen aufgeteilt. Die Blinden organisieren sich grundsätzlich ordentlicher als andere behinderte Menschen. Die Anzahl der unabhängigen und neutralen Blinden ist deshalb klein in Japan, weil sie normalerweise als Masseure und Akupunkteure (der Akupunkteur behandelt auch mit einem Weihrauchstückchen, deshalb wird diese Arbeit Sanryougyou d.h. dreiartige Therapie in Japan genannt.) arbeiten und diese Berufsgruppe von alters her organisiert wurde.

Die konservative Gruppe der Blinden ist mit der herrschenden Partei, d.h. der Japanischen Freien Demokratischen Partei (JFDP) verknüpft. Sie heißt Nippon Moujin-kai-Rengou (Die Abkürzung ist Nichimouren.), d.h. Japanischer Gesellschaftsbund der Blinden.

Die kommunistische Gruppe ist mit Nihon-kyousan-tou d.h. der Japanischen Kommunis-tischen Partei (NKP) verknüpft. Sie heißt Zennihon-shiryokushougaisha-kyougikai (Die Abkürzung ist Zenshikyou.), d.h. Allgemeine Japanische Gesellschaft der sehbehinderten Menschen.

Die radikale Gruppe ist sonderbarerweise mit Shakai-minshu-tou, d.h. der Japanischen Sozialdemokratischen Partei (JSDP), verknüpft. Sie heißt Zenkoku-shiryokushougaisha-kaihou-undou-renraku-kaigi (Die Abkürzung ist Zenshikairen oder Zenshouren.), d.h. Allgemeine Japanische Verbindungskonferenz der unabhängigen gleichberechtigen Gruppen augenbehinderter Menschen.

Bevor ich diese Gruppen beschreibe, muss ich die Geschichte der Beschäftigung der Blinden seit der Edo-Zeit (von 1603 bis 1868) erklären. Denn man kann ohne eine solche Erklärung nicht verstehen, warum viele Blinde in Japan als Masseuere und Akupunkteure arbeiten und ihre Berufsgruppe von alters her organisiert wurde.

In der Edo-Zeit gab es den Feudalismus in Japan wie in Europa. Man musste unter der ständischen Ordnung des Mittelalters leben, in der der Besitz von Ländereien das wichtigste war. Es ist in Japan bekannt, dass es vier Stände d.h. Shi-Nou-KouShou gab. Shi, Nou, Kou und Shou bedeutet Ritter, Bauer, Handwerker und Verkäufer. Die Ritter hatten einen hohen Rang und Verkäufer einen niedrigen. Die Bauern bauten Reis an, mit dem die damalige japanische Wirtschaft funktionierte. Deshalb war der Bauer vom zweiten Rang, obwohl sein Leben am schwersten unter den vier Ständen war. Verkäufer wurden verachtet, weil sie nicht wie Bauern und Handwer- 
ker etwas produzierten, sondern nur aus Waren Gewinn zogen, obwohl sie die Wirtschaft bewegten und entwickelten und am reichsten waren.

$\mathrm{Zu}$ welchem Stand gehörten dann aber der Kaiser, der Adel und der Priester? Der Kaiser und der Adel (abgesehen von dem Ritteradel) waren von besonders hohem Rang, obwohl sie wenig politische Macht hatten. Das japanische Kaisertum im Mittelalter war ganz anders als das Kaisertum im Ausland. Die Priester waren von besonderem Rang. Sie standen außerhalb der Stände, weil sie als Tote angesehen wurden. Sie hatten einen besonderen Namen und ließen sich eine Glatze schneiden. Sie waren Tote, so dass sie mit Menschen aus fast allen Ständen direkt sprechen konnten.

Zu welchem Stand gehörte der Blinde?

Er war formell Priester, obwohl er natürlich kein Sutra vortragen konnte und sehr verachtet wurde. Viele Blinde mussten sich deshalb eine Glatze schneiden lassen.

Warum musste er formell als Priester behandelt werden?

Die Anzahl der Beschäftigungsmöglichkeiten der Blinden war und ist leider gering. Blinde mussten ganz überwiegend als Masseure und Akupunkteure. Musiklehrer, Musiker (viele waren wandernde Bettler, um die Wahrheit zu sagen), oder als Leihgeber zu Wucherzinsen arbeiten (die Blinden hatten das von der Regierung gegebene Vorrecht als einzige Geld zu verleihen, das ihnen mit dem Ende der Edo-Zeit von der neuen Regierung weggenommen wurde). Insbesondere waren Masseuer und Leihgeber zu Wucherzinsen die berühmtesten Beschäftigungen der Blinden in Japan (Masseur heisst in Japanisch Anma oder Anma-san, deshalb nennt man Blinde jetzt noch oft Anma-san).

Man durfte den Körper des anderen nicht direkt berühren oder mit anderen in der Edo-Zeit nicht direkt sprechen, wenn man nicht dem gleichen Stand angehörte. Deshalb musste der Blinde formell ein Priester sein. ${ }^{10}$

\subsection{Probleme des Gerichtswesens}

Die japanische Gerichtsordnung und das Sozialrecht sind ganz anderes als in Deutschland. In Japan gibt es nur einige Prozesse auf dem Gebiet des Sozialrechts pro Jahr, während es über 200.000 Sozialprozesse in der BRD gibt. Hieraus ergeben sich viele Schwierigkeiten. Das basiert auf vielen Ursachen. Ich kann sie deshalb nicht alle erklären, möchte aber einige von ihnen nennen. Erstens nenne ich ein Verbot des Sondergerichts. Nach dem japanischen Verfassungsrecht $\S 76$ sind keine Sondergerichte vorgesehen. Deshalb muss man vor das Zivilgericht gehen, wenn man mit der Regierung über die Auslegung (Anwendung) des Sozialrechts streiten möchte.

Zweitens nenne ich hohe Prozesskosten in Japan. Die Prozesskosten sind armen Menschen zu hoch. Wenn man eine Sprechstunde mit einem Rechtsanwalt haben 
möchte, muss man nach der Anordnung des Bundes der Rechtsanwaltsgesellschaften halbstündlich mehr als 35 EURO zahlen. Obwohl es jetzt keine solche Anordnung mehr gibt, bezahlt man auch das Gleiche wie früher.

Wenn man eine Klage erheben möchte, muss man normalerweise einem Rechtsanwalt mehr als 667 EURO als Anzahlung leisten, die nicht zurückgegeben wird, auch wenn man den Prozess verliert.

\begin{tabular}{|lll|}
\hline Wirtschaftliche Dimension & Anzahlung & Honorar \\
bei einem Wert von : & & \\
-bis zu 20.000 EURO (drei Millionen Yen) & $8 \%$ & $16 \%$ \\
-mehr als 20.000 EURO bis zu 200.000 EURO & $5 \%+90.000$ Yen & $10 \%+180.000$ Yen \\
(dreißig Millionen Yen) & & \\
-mehr als 200.000 EURO bis zu 2.000.000 EURO & $3 \%+690.000$ Yen & $6 \%+1.380 .000$ Yen \\
(dreihundert Millionen Yen) & $2 \%+3.690 .000$ Yen & $4 \%+7.380 .000$ Yen \\
-mehr als 2.000.000 EURO & & \\
& & \\
Abhängig von dem Inhalt des Falles kann sich die berechnete Summe um 30 $\%$ erhöhen bzw. vermindern. \\
Mindestanzahlung sind 667 EURO (100.000 Yen). Wenn der Wert eines Prozesses unter 8.333 EURO \\
(1.250.000 Yen) liegt, kann die Anzahlung unter Umständen auch weniger als 667 EURO betragen.
\end{tabular}

Selbst wenn ein Kläger seinen Prozess gewinnen kann, werden nur Minimalprozesskosten für seinen Rechtsanwalt vom Gericht anerkannt. Schließlich muss er viel von den Prozesskosten selbst zahlen. Es gibt natürlich auch in Japan ein System der Prozesskostenhilfe. Die Voraussetzungen sind jedoch zu streng. Nur wenn ein Kläger sehr arm ist und gewiss seinen Prozess gewinnen kann, kann er sich von einer Gesellschaft für Prozesskostenhilfe seine Gerichtsgebühren und die Minimalprozesskosten für seinen Rechtsanwalt leihen. Wenn er seine Klage gegen die Regierung erhebt, ist es kaum möglich, dass er seinen Prozess gewinnen kann. Von Anfang an rechnet er deshalb nicht mit Prozesskostenhilfe. Die Prozesskosten werden von einer Gruppe gezahlt, die ihn unterstützen will. Wenn er arm ist und keine solche Gruppe kennt, kann er tatsächlich keine Klage erheben.

Drittens nenne ich lange Prozesszeiten. Wenn man vor Gericht geht, muss man auf eine lange Prozessdauer vorbereitet sein. In Japan gibt es ungefähr 22.000 Rechtsanwälte, ${ }^{11}$ obwohl es etwa einhundertzwanzig Millionen Bewohner gibt. Ein Rechtsanwalt hat normalerweise ungefähr zweihundert Prozesse zur selben Zeit. Deshalb kann er nicht sofort Termine mit dem Rechtsanwalt der anderen Partei ausmachen. Im besten Fall kann ein Kläger seinen Fall in einer Gerichtsverhandlung innerhalb eines Monats erörtern.

Viertens nenne ich zu wenige Normen zum Sozialrecht. Ein einzeler Mensch hat nur wenige Rechte aus dem Sozialrecht. Z.B.: Wenn ein Arbeiter bei einem Arbeits- 
unfall verletzt wird, wird er durch die Arbeitsunfallversicherung besser entschädigt als ein von Geburt an behinderter Mensch, der durch die Sozialversicherung nur unvollständig abgesichert ist. Nach Meinung der japanischen Regierung haben von Geburt an behinderte Menschen zwar ein Recht auf Sozialhilfe, jedoch kein Recht auf Sozialfürsorge. Gegen diese Auffassung gerichtete Klagen vor Gericht haben deshalb keine Aussicht auf Erfolg.

Fünftens nenne ich eine Tendenz der Japaner Streitigkeiten zu vermeiden. Meiner Meinung nach will und möchte kaum jemand gegen seine Feinde kämpfen. Ein armer oder von Geburt an behinderter Mensch kann von seinen Kollegen oder aus seiner Gemeinschaft ausgestoßen werden, wenn er um sein Recht vor Gericht kämpft. Ich kenne eine Mutter und ihre Tochter, die von ihrer Gemeinschaft ausgestoßen wurden, als sie wegen ihres Rechts auf Sozialhilfe vor Gericht gingen. In Japan nennt man das Mura-hachibu. „Mura" bedeutet Gemeinschaft oder Dorf und „hachibu" bedeutet 8/10. Dieser Ausdruck besagt, dass die Einwohner einer Gemeinde 8/10 Verrichtungen nicht mehr bereit sind mit dem Bewohner zu teilen, der gemeinschaftsregelswidrig lebt, d.h. sie helfen diesem nur noch bei zwei Verrichtungen, nämlich bei seiner Begräbnisfeier und bei einem eventuellen Hausbrand (d.h. zwei zehntel. Natürlich ist dies eine Metapher.). Mura-hachibu bedeutet deshalb aus der Gemeinschaft ausgeschlossen zu werden. Man muss sich normalerweise in Japan schämen, wenn man Sozialhilfe erhält. Wenn ein Mensch einen Antrag auf Sozialhilfe an das Sozialamt stellt, werden sein Eigentum und auch alles Eigentum seiner Verwandtschaft untersucht.

Sechstens das „Zirkelsystem“ der Richter und der Staatsanwälte: Wenn man mit der Regierung über Institute des Sozialrechts streitet, arbeiten Staatsanwälte oft als Vertreter der Regierung, weil Staatsanwälte Mitglieder des Justizministeriums sind. Staatsanwälte werden oft Richter. Deshalb kann es vorkommen, dass ein Richter in demselben Fall entscheidet, in dem er als Vertreter der Regierung arbeitete. Dieser Richter wird dabei unglaublicherweise in Japan nicht abgelehnt. Es gibt natürlich ein System, mit dem Rechtsanwälte Richter werden können. Rechtsanwälte möchten jedoch kaum Richter werden. Denn diese verdienen normalerweise mehr als 20.000.000 Yen ${ }^{12}$ pro Jahr, während Richter im Allgemeinen nur 10.000.000 Yen ${ }^{13}$ pro Jahr verdienen und oft versetzt werden.

\subsection{Probleme der Gesetzgebung}

Das japanische Gesetzgebungsverfahren ist etwas anders als in Deutschland. In Japan wird normalerweise ein konkreter Gesetzesentwurf erst bekannt gemacht, wenn über ihn im Reichstag diskutiert wird, obwohl sein Wortlaut vorher in von einem die Gesetzgebung übernehmenden Ministerium fast vollständig bestimmt

13 Vgl. http://www.tuutenkaku.com/naiyou.houritu/shihousiken.html 
wurde. Meiner Meinung nach stimmt das mit dem Grundgesetz nicht überein. Z. B. den Gesetzesentwurf für die Pflegeversicherung in Japan; Obwohl das Gesetz Ende Juni 1996 fertiggestellt wurde, konnte man auch am 20.6.1996 noch überhaupt keine Informationen über einen konkreten Gesetzesentwurf erhalten.

Nach dem Artikel 59 des japanischen Verfassungsrechtes wird ein Gesetzesentwurf in folgendem Falle zu einem Gesetz:

Artikel 59

1) Ein Gesetzesentwurf wird ein Gesetz, wenn es im Abgeordnetenhaus und im Oberhaus angenommen wird, wenn nicht diese Verfassung etwas anderes vorsieht.

2) Ein Gesetzesentwurf, der im Oberhaus angenommen wird und im Abgeordnetenhaus abgelehnt wird, wird ein Gesetz, wenn der Gesetzesentwurf durch mehr als zwei Drittel der anwesenden Abgeordneten im Abgeordnetenhaus bei einer zweiten Abstimmung angenommen wird.

3) Durch die Regelung des Absatzes 2 wird nicht ausgeschlossen, dass das Abgeordnetenhaus den Vermittlungsausschuss wie nach diesem Gesetz vorgesehen anruft.

4) Wenn das Oberhaus über einen vom Abgeordnetenhaus angenommenen Gesetzesentwurf nicht innerhalb von 60 Tagen nach Empfang entscheidet, die Zeit der Reichstagsferien nicht mitgerechnet, kann das Abgeordnetenhaus den Gesetzesentwurf als abgelehnt ansehen.

Nach dem Artikel 41 ist der Reichstag das höchste Organ der Staatsgewalt und das einzige gesetzgebende Organ. Die Realität sieht jedoch anders aus. Über $90 \%$ der Gesetzesvorlagen werden nicht von Abgeordneten des Reichstages, sondern von den Beamten entworfen, weil das gegenwärtige Rechtssystem sehr kompliziert ist, Abgeordnete dazu zeitlich nicht in der Lage sind und einen Entwurf kaum selbst anfertigen können. Streng genommen, hat ein Abgeordneter kaum die Fähigkeit Gesetze zu entwerfen, weil er nicht bereit ist, Einzelheiten des Entwurfes zu studieren, hierzu auch keine Zeit und nicht genügend Helfer hat und nur seinen Wahlsieg anstrebt und nur auf seine Wähler achtet. In Japan gibt es den folgenden Ausdruck: Auch wenn ein Affe vom Baum fällt, ist er immer noch ein Affe, aber ein Abgeordneter, der in den Wahlen durchfällt, ist nur noch ein einfacher Mensch. Wenn er bei der Wahl eine Niederlage erleidet, verliert er alle Befugnisse, die er als Abgeordneter genoß und viel Geld.

Keine Probleme ergeben sich, wenn die Entwürfe einfach und nicht umstritten sind. Wenn sie jedoch komplizierter und streitiger sind, beruft man einen Beratungsausschuss ein. Ausschuss heißt auf japanish „Shingikai". Er wird auch gesetzlich gebildet. Dabei wird das Ernennungsverfahren der Mitglieder durch ein Gesetz oder eine Verordnung vorgeschrieben. Viele von solchen Ausschüssen werden aber von Ausschussmitgliedern gebildet, die durch einen die Gesetzgebung übernehmenden Minister privat (aber öffentlich finanziert) ernannt werden. Deshalb nennt man den Ausschuss: ein privates beratendes Organ. Um die Wahrheit zu sagen, ist es aber eine Tarnkappe für die das Gesetz Entwerfenden.

Ein bei der Gesetzgebung mitwirkender Minister könnte die Mitglieder des Ausschusses selbst ernennen, weil der Ausschuß nur privat ist; praktisch ist er aber dazu nicht in der Lage, denn seine Amtszeit reicht dazu nicht aus: Er wird spätestens nach 
zwei Jahren abgelöst. In Japan gab es in den 17 Jahren von 1990 bis 2007 zehn verschiedene Premierminister. Um überhaupt Minister werden zu können, muss er zuvor aber drei bis viermal als Abgeordneter gewählt worden sein. Da ist kaum Zeit für einen Minister, um sich als Minister Fachkenntnisse zu erwerben. Es ist für die Beamten viel besser, dass der Minister keine Fachkenntnisse hat. Wäre er darin bewandert, würde er die Verwaltung nur in Verwirrung bringen. Es ist durchaus keine Übertreibung, dass je dümmer der Minister ist, desto besser für die Beamten.

Wird in der Verwaltung des Ministeriums etwas rechtlich zweifelhaftes getan, z.B. ein Gesetz nicht beachtet, muss der Minister selbst die politische Verantwortung für eine Fehlentscheidung tragen und seine Kündigung einreichen, während ein Beamter, der einen Irrtum begeht, kaum entlassen wird. Selbst die Ermittlung des Urhebers des Fehlers ist schwierig, weil der Beamte entweder durch den Minister oder durch den Beratungsausschuss gedeckt ist. In Japan wäre es besser, wenn jeder die Verantwortung selbst tragen würde. Deshalb können Beamte die Verwaltung sorglos beherrschen, wie sie wollen. Sie haben immer Tarnkappen.

Auch wenn ein höherer Beamter entlassen wird, wird er nach einiger Zeit oft als Leiter einer besonderen gemeinnützigen juristischen Person beschäftigt, die aufgrund besonderer Gesetze gegründet und vom Staat finanziell unterstützt wird. Wenn er bei keiner solchen juristischen Person beschäftigt wird, wird er sofort in einer Gesellschaft angestellt, die in Beziehung mit seinem Ministerium steht. Wenn er in einer Beförderungskonkurrenz mit seinem Kollegen besiegt wird, will er auch in ähnlicher Weise einen Dienst antreten. In Japan darf er eigentlich frei in einer Gesellschaft angestellt werden, die in Beziehung mit seinem Ministerium steht. Er kann sogar noch mehr Gehalt als früher beziehen. Er wird selbstverständlich in der neuen Organisation herzlich empfangen, weil er viele Informationen und Vorteile mitbringt. Das nennt man in Japan „Amakudari", d.h. vom Himmel herabzukommen.

Ich nenne ein Beispiel dafür, wie ein Beamter einen Beratungsausschuss und Staatsbürger behandelt.

In Japan diskutierte man vor zehn Jahren über die Pflegeversicherung. Es gab einen Beratungsausschuss für die Gesundheit und die Wohlfahrt der Alten (RoujinHoken-Fukshi-Shingikai auf Japanisch) als ein privates beratendes Organ des Gesundheitsministers. Der Ausschuss wurde aus Mitgliedern gebildet, die Gelehrte, Direktoren der großen Gesellschaften, Vorsitzende der großen Gewerkschaften, Vorsitzende der Pflegeeinrichtungen (Altenheimen) und populäre Fernsehdarsteller sind. Im Ausschuss gab es natürlich wenige, die gegen die Regierung in Oposition gehen. Es gab dort auch weder gepflegte alte Menschen, noch pflegende Familienmitglieder, noch Pflege brauchende schwerbehinderte Menschen. Beiläufig gesagt, gab es drei Beratungsausschüsse nach den Gesetzen, d.h. den Beratungsausschuss für die Wohlfahrt der körperlich behinderten Menschen, den zentralen Wohlfahrtsberatungsausschuss, der auch die Wohlfahrt der alten Menschen beriet und den Jugendwohlfahrtsberatungsausschuss, der auch die Wohlfahrt der geistig behinderten Menschen beriet. Nur im Beratungsausschuss für die Wohlfahrt der körperlich be- 
hinderten Menschen wird ein Teil von ihren Mitgliedern von Vertretern der behinderten Menschen organisiert.

Im Beratungsausschuss für die Gesundheit und die Wohlfahrt der alten Menschen wurde die Gründung einer Pflegeversicherung drei Jahre lang diskutiert. Zwei vorläufige Berichte wurden veröffentlicht und von vielen Leuten kritisiert. Am 22. April 1996 wurde der letzte Bericht bekannt gegeben. Sie enthalten verschiedene Meinungen, weil sich eine einheitliche Meinung nicht bilden konnte. Nach deutschem Verständnis könnte man das wohl keinen Abschluss nennen. In ihm gab es folgende verschiedene Meinungen: (1) das Geld der Pflegeversicherung wird direkt an den Pfleger geleistet, und weder an den gepflegten alten Menschen noch an die pflegenden Familienmitglieder. (2) Die Versicherungsleistung wird grundsätzlich nur an alte Menschen über 65 Jahre geleistet. (3) Über 20 jährige Leute müssen ihren Versicherungsbeitrag zahlen. (4) Wenn ein Mensch mit seinem Versicherungsbeitrag in Rückstand ist, wird die Versicherungsleistung nicht erbracht.

Am Anfang des Berichts wird folgendes geschrieben: Dieser Beratungsausschuss hat sich bemüht, in- und ausländische verschiedene Meinungen zu hören, um eine öffentliche Anhörung zu gewährleisten, um verschiedene Meinungen der Staatsbürger direkt in der Beratung widerzuspiegeln und um mit verantwortlichen Personen der deutschen Regierung, die die Pflegeversicherung vor Japan eingeführt haben, Meinungen auszutauschen. Der Gesetzentwurf des Berichts ist ganz anderes als die Pflegeversicherung in der BRD. Es ist ziemlich sicher, dass der Bericht von Beamten geschrieben wurde.

Am 22. Mai 1996 wurde unerwartet versuchsweise ein neuer Vorschlag durch das japanische Wohlfahrtsministerium veröffentlicht. Er ist ganz anderes als der letzte Bericht des Beratungsausschusses über die Gesundheit und die Wohlfahrt der alten Menschen (BGWA). Ich kann drei Unterschiede zwischen dem Bericht des BG A,A und dem Vorschlag nennen. (1) Alle Menschen, die älter als 40 Jahre sind, müssen einen Versicherungsbeitrag zahlen. (2) Die Versicherungsleistung wird auch an Menschen über 40 Jahren geleistet. (3) Einen Anspruch auf die Versicherungsleistung hat man ab 40, wenn die Pflegebedürftigkeit auf verfrühter altersbedingter Gebrechlichkeit beruht, sofern diese Folge einer von 15 in einem Katalog genannten Krankheiten ist.

Dieser Vorschlag wurde formell vom Wohlfahrtsministerium veröffentlicht. In der Tat wurde er aber auf Druck der drei herrschenden Parteien, der Jiyuu-minshutou d.h. die Japariiche Freie Demokratische Partei (JFDP), der Shakai-minshu-tou d.h. die Japanische Sozialdemokratische' Partei (JSP) und der Sintou-sakigake d.h. die neue Bahnbrecher-Partei veröffentlicht. Das Abgeordnetenhaus wurde vielleicht in diesem Jahr aufgelöst. Abgeordnete wurden spätestens bis Juli 1997 aus ihren Ämtern entlassen, weil ihre Amtszeit vier Jahre dauerte. Die drei herrschenden Parteien fürchteten, dass sie bei der nächsten Wahl eine Niederlage erleiden würden. Nach dem letzten Bericht des BGWA wird grundsätzlich die Pflegeversicherungsleistung in Japan nur an alte Menschen über 65 Jahre geleistet, obwohl man mit 20 Jahren bereits den Versicherungsbeitrag zahlen musste. Wenn ein solches Gesetz 
erlaubt würde, würden die drei herrschenden Parteien in den nächsten Wahlen mit Sicherheit verlieren.

\section{Schlussbemerkung zu den Unterschieden der Rechtsstellungen zwischen Behinderten Menschen in Deutschland und in Japan}

Die Rechtssysteme für schwerbehinderte Menschen in Deutschland und Japan, z.B. das Rechtssystem für die Sozialhilfe, das Rentensystem, das Krankenversicherungssystem, das Unfallversicherungssystem, das Pflegeversicherungssystem, das Beschäftigungspflichtsystem der schwerbehinderten Menschen u.a. sehen sich ein bisschen ähnlich. Um es offen zu sagen, stammen viele japanische soziale Sicherheitssysteme aus den Systemen in Deutschland. Obwohl Japan von der Amerikanischen Armee besetzt und „demokratisiert“ wurde, bleibt das Rechtssystem ähnlich wie in Deutschland vor dem Zweiten Weltkrieg. Alle Staatsbürger werden von rechtlichen Krankenversicherungssystemen gesichert. Das Versicherungssystem von Bismarck ist echt in Japan übernommen worden.

Aber die Rechtsstellung der Staatsbürger, insbesondere der schwerbehinderten Menschen in Deutschland und in Japan sieht ganz anders aus. Ich habe das Rechtssystem der schwerbehinderten Menschen in Deutschland erforscht. Ich möchte kurz nur wenige folgende Unterschiede nennen:

1.) Fehlen konkreter Normen zum Sozialrecht für schwerbehinderte Menschen in Japan

Die Wörter „Recht auf ...“ werden oft in Sozialgesetzbüchern in Deutschland verwendet. Es gibt leider keinen solcher Ausdrücke in japanischen Gesetzen für schwerbehinderte Menschen.

2) Fehlen einer Kodifikation in Japan

In Japan gibt es kein Sozialgesetzbuch, deshalb können Staatsbürger nur sehr schwer ihre Rechtsetzung hinsichtlich der sozialen Sicherheit kennen. Wenn sie Einzelheiten über ihre Rechtstellung wissen möchten, müssen sie Beamte in Gemeinden darüber fragen. Auch Beamte wissen vielleicht wenige Einzelheiten. Nach der Meinung der japanischen Regierung haben Beamte keine Aufklärungspflicht gegenüber Staatsbürgern. $^{14}$

3) Kein Finalprinzip in japanischen Gesetzen über die soziale Sicherheit

Es gibt natürlich formal ein „Finalitätsprinzip“ im japanischen Sozialfürsorgegesetz. Aber Staatsbürger können nicht einfach Sozialfürsorge genießen, weil ein Gesetz auf Sozialfürsorge schwer von Beamten in Gemeinden bewilligt wird. Auch jetzt stirbt man in Japan vor Hunger, wenn man keine Sozialfürsorge erhalten kann. 
4) Unterschiede der Gerichtssysteme

In Japan gibt es ganz wenige Rechtsstreitigkeiten für soziale Sicherheit. Es gibt zu wenige konkrete Normen zum Sozialrecht. Ein einzelner Mensch hat nur wenige Rechte aus dem Sozialrecht. Es gibt weniger Rechtsanwälte in Japan als in Deutschland. Die Prozesskosten sind im Allgemeinen behinderten Menschen zu hoch.

5) Politische Streitigkeiten zwischen Behindertengruppen

Japanische behinderte Menschen werden in verschiedene politische Gruppen unterteilt. Viele behinderte Menschen werden auch jetzt sehr unterschiedlich klassifiziert und lassen sich nur schwer organisieren. 
6.4. Behindertenrecht und Behindertenpolitik in Korea 


\title{
6.4.1. Leistungen für behinderte Menschen in der Republik Korea
}

\author{
Von Kwang Seok Cheon
}

1. Geschichte

1.1. Wirklichkeit

1.2. Norm

2. Gesetzeslage im Allgemeinen

3. Behinderung-Begriff und Bestand

4. Leistungen

4.1. Einleitung

4.2. Prävention

4.3. Medizinische Leistungen

4.4. Ergänzende Geldleistung, Unterstützung der Ausbildung

4.5. Rehabilitation im alltäglichen und sozialen Lebensbereich

4.6. Förderung der Erwerbstätigkeit

4.6.2. Förderung der Unternehmenstätigkeit

4.7. Bildungsförderung

4.8. Maßnahmen zur Gleichstellung der Behinderten nach dem Gesetz über die Antidiskriminierung wegen Behinderung 


\section{Geschichte}

\subsection{Wirklichkeit}

Wie in anderen Ländern und Kulturen auch wurde den Behinderten in Korea bis vor etwa 30 Jahren ein Stigma angehaftet, was seine Spuren z.B. in einer Gesetzesvorschrift hinterließ, die den Schwangerschaftsabbruch aus solchem genetischen Grunde erlaubte ${ }^{1}$. Vor diesem Hintergrund standen Behinderte lange Zeit eher am Rande des gesellschaftlichen sowie politischen Arrangements. Dies nahm auch Einfluss auf das Verständnis von Behinderung. Nicht nur der körperliche oder geistige Zustand, sondern auch soziales Stigma zählte Anfang der 60er Jahre zu den Merkmalen der Behinderung wie etwa ein nicht-koreanisches Elternteil ${ }^{2}$.

Die Kriegsbeschädigten während des Koreakrieges, denen medizinische sowie berufliche Maßnahmen rehabilitativen Charakters gewährt wurden, waren dagegen von Anfang an vom Staat als schutzwürdig angesehen ${ }^{3}$. Davon abgesehen gab es nur wenige rechtliche Regelungen, die die Situation behinderter Menschen berücksichtigten. Ein Beispiel, das sich zwar nicht spezifisch, aber partiell der Behinderung zuwandte, war das Gesetz über die Unfallversicherung von 1963.

Das im obigen Sinne herrschende gesellschaftliche Verständnis von Behinderung und der demzufolgende Rückstand in der Behindertenpoltik sowie -gesetzgebung erfuhr aber im Laufe der Zeit, vor allem seit den 80er Jahren, einen Gesinnungswandel, der auf verschiedene Aspekte zurückzuführen war.

Erstens wandelte sich das gesellschaftliche Bewusstsein. Es wurde anerkannt, dass Behinderte nichts anderes als "differently abled" sind. Dieser Wandel vollzog sich einerseits durch einen neuen Solidaritätsgedanken und die weiter verbreitete Sensibilität für Menschenrechte, andererseits durch den größer werdenden Einfluss von Tätigkeiten der Behinderten und deren Organisationen.

Zweitens war Folgendes zu beobachten: Ein Anwachsen des Selbstbewusstseins der Behinderten selbst, sowie ihrer Familienmitglieder und der Behindertenbewegung in Gruppen. Die Demokratisierung von 1987 in Korea forderte zur Gleichheit der Behinderten im Staat sowie in der Gesellschaft auf, was die Gründung und Betätigung verschiedener Behindertengruppen zur Folge hatte. Die Demokratisierung war auch ein Signal dafür, dass inzwischen dem Wirtschaftswachtum geopferte Werte wieder hervorgehoben sowie Versäumtes kompensiert werden sollte. Zudem ergab die Beschäftigung mit den Sozialpolitiken anderer Länder die Beurteilung, dass Korea allgemein für seine wirtschaftliche Kapazität in der Sozialpolitik zurück

1 Zum Bericht über China wo ähnliches zu beobachten ist. Vgl. Darimont, Teilhabe behinderter Menschen an der Bürgergesellschaft in VR China (Eingliederung im sozial- und Rechtsvergleich), in: v. Maydell/Pitschas/Schulte (Hrsg.), Teilhabe behinderter Menschen an der Bürgergesellschaft in Asien und Europa - Eingliederung im Sozial- und Rechtsvergleich, Speyer 2002, S. 172 ff. Vgl. Bericht über behinderte Kinder 1961.

3 Zur Entwicklung der Behindertenpolitik in Korea, siehe Jeong Keun Sik, Neues Verständnis der Behinderung, in: Kim Chang Yop et. al., Ich werde ,bad guy“, Samin 2003, S. 23 ff. 
lag. Mit zeitlichem Abstand erkannte man diese Tatsache auch zutreffend für die Politik für sozial Schwächere, repäsentativ waren Behinderte. Realpolitisch gesehen war nun der Bevölkerung erlaubt politisch aktiv zu sein. Behinderte und ihre Verbände engagierten sich politisch und erhielten politisches Gewicht.

Drittens wurde die Behindertenpolitik von außen beeinflusst. Vor allem die Tätigkeiten der UNO im Bereich der Behindertenpoltik, wie z.B. zwei Erklärungen von 1971 (Declaration on the rights of mentally retarded persons) und 1975 (Declaration on the rights of disabled persons), sowie das Jahr für die Behinderten 1981 veranlassten neue Denkweisen hinsichtlich der Behinderung und Behindertenpoltik.

Nach der Jahrhundertwende wurde eine Diskussion über einen neuen Ansatz in der Behindertenpolitik entfacht, nämlich über die Ergänzung um Antidiskriminierungsvorschriften wegen Behinderung. Diese wurde stark von ausländischen Erfahrungen beeinflusst. Vor allem wurden der ADA (Americans with Disabilities Act) der USA, aber auch Gesetzgebungen von Großbritannien, Australien, Hongkong etc. herangezogen. Bemerkenswert war dabei, dass diesbezügliche Studien und Aktivitäten hauptsächlich von verschiedenen Behindertenorganisationen initiert wurden.

$\mathrm{Zu}$ dieser Zeit wurde zumindest auf gedanklicher Ebene allgemein anerkannt, dass Behinderte nicht mehr nur fürsorgerische Objekte sind, sondern den Behinderten die Subjektstellung eingeräumt werden sollte.

\subsection{Norm}

Wie oben erwähnt, wurde in Korea die erste Gesetzgebung für Behinderte 1981 erlassen: Das Gesetz über die Wohlfahrt der körperlich und geistig Behinderten. Dieses Gesetz konnte hinsichtlich der Ideen, sowie der Leistungsart und des Leistungsumfangs der sich im Wandel befindlichen Vorstellung von Behinderung jedoch nicht gerecht werden. Es hatte eher symbolischen Charakter. So blieb es bis zur zweiten Hälfte der 80er Jahre.

Die Behindertenpolitik bekam dann eine Grundlage auf Ebene der Verfassung. Die Verfassung von 1987 sah in Art. 34 V zum ersten Mal den Schutz der Behinderten vor. Dieser war jedoch unsystematisch und nicht realitätsnah formuliert. Erstens wurde der Behindertenschutz auf die Hilfe für die Bedürftigen beschränkt. Dass die Behinderung an sich des staatlichen Schutzes bedürftig ist, war noch nicht in Sicht. Zweitens gehörten zum geschützten Personenkreis explizit nur die körperlich Behinderten. Der Verfassungsgeber sah nur sichtbaren Behinderungen ins Auge. Sollte dies aus Versehen geschehen sein, würde das eine Leichtsinnigkeit des Verfassungsgebers im Verständnis von Behinderung aufzeigen.

Trotzdem trug die verfassungsrechtliche Forderung dazu bei, den Blickwinkel auf dem Gebiet der Behindertenpolitik sowie -gesetzgebung zu erweitern. Oder anders formuliert: Die weiteren Bemühungen in der Behindertenpolitik und -gesetzgebung konnten nun auf die verfassungsrechtliche Forderung gestützt werden. Dies wirkte sich in zweierlei Hinsicht aus. Erstens zeigte sich die Tendenz, die Merkmale von Behinderung weiter auszubauen und entsprechend zusätzliche anzuerkennende Ka- 
tegorien in den Behindertenschutz einzubeziehen. Zweitens folgte der absoluten Ebene des Schutzes dann die relative Ebene: als verfassungsrechtlich verankert wurde anerkannt, dass die Ungleichbehandlung wegen des Merkmals „Behinderung" verboten ist, obwohl es im Verfassungsrecht nicht explizit vorgeschrieben war".

Ende der 80er Jahre begann sich die Rehabilitation in der Behindertenpolitik einen eigenen Platz zu behaupten. Damit wurde der Schwerpunkt darauf gesetzt, Voraussetzungen für die Teilhabe in alltäglichen, sozialen und beruflichen Bereichen zu schaffen. Das oben erwähnte Gesetz von 1981 wurde 1989 umbenannt in Gesetz über die Behindertenwohlfahrt. Seine Aufgaben wurden um Prävention und Rehabilitation erweitert (BWG $\S 1$ ).

Aktivere Ansätze in der Behindertenpolitik wurden 1990 im Gesetz über die Förderung der Beschäftigung von Behinderten anerkannt. Es zielte darauf ab, die Behinderten durch Teilhabe am beruflichen Leben zu integrieren. 1999 wurde dieses Gesetz in Gesetz für die Beschäftigung der Behinderten sowie berufliche Rehabilitation umbenannt. Diese Änderung ist inhaltlich durch die Ergänzung um die berufliche Rehabilitation und die Bevorzugung von Schwerbehinderten gekennzeichnet ${ }^{5}$.

Um die Jahrhundertwende stand eine Lücke in der Behindertenpolitik zur Diskussion. Die bisherigen Gesetze hatten allein die Behinderten als Regelungsgegenstand. Nun sollte auch deren Umwelt ins Zentrum des Denkens und Tuns gerückt werden. Motto war: Durch eine behindertengerechte Umstrukturierung des Umfeldes sollten die Behinderten ein „normales“ Leben führen können. Erst dann sei es ihnen möglich, über sich selbst zu entscheiden (Selbstbestimmungsansatz).

Ansatzweise gab es bereits einige Gesetze, die zu diesem Zweck erlassen wurden: das Gesetz über die Förderung der Sonderschule von 1977, das Gesetz über die Förderung des Alltagslebens für Behinderte, Alte sowie Schwangere von 1997 und das Gesetz über die Barrierefreiheit im Verkehr für die Schwachen von 2005. Diese Gesetze waren jedoch in ihrer Wirkung begrenzt. Vor allem fehlte es an Durchsetzungsmöglichkeiten. Und die beiden letztgenannten richteten sich nicht nur an Behinderte, so dass sie der besonderen Lage der Behinderten nicht voll Rechnung tragen konnten.

Das Unbehagen darüber führte zur Forderung nach Erlass eines Antidiskriminierungsgesetzes wegen Behinderung ${ }^{6}$. Die Diskussion um die Gleichbehandlung von Behinderten in den letzten Jahren war eine gute Gelegenheit, den wissenschaftlichen gerichtshofs. Siehe Entscheidung des Verfassungsgerichtshofs 1999.12.23, 98 hon ma 363, 11-2, S.770 ff.

5 Zur Entwicklung der Beschäftigungspolitik für die Behinderten, siehe Choi Mu Hyun, Über die Evolution der Beschäftigungspolitik für die Behinderten im Bereich des Gesetzes für die Beschäftigung der Behinderten sowie berufliche Rehabilitation in Korea, Korean Public Administration Journal 2003, S. 33 ff.; Lee Sung Kyu, Soziale Integration und Wohlfahrtspolitik für die Behinderten, Nanam 2000, S. 134 ff.

6 Zum Erlass dieses Gesetzes siehe Park Jong Un, Aufgabe und Entwicklungsaussicht des Gesetzes über die Antidiskriminierung wegen der Behinderung, International Human Rights Journal 2004, S. $51 \mathrm{ff}$. 
Horizont der Behindertenforschung zu erweitern. Es musste etwa festgestellt werden, ob ein solches Gesetz neben den bisherigen Gesetzen wirklich notwendig war. Stichwort ist die Wechselwirkung von Wohlfahrt und Gleichstellung ${ }^{7}$. Dies hatte auch zur Folge, dass die bestehenden, die Behindertenpolitik betreffenden Gesetze in Bezug auf ihre Zielgerechtigkeit und Effizienz zur Prüfung standen. Die Verabschiedung des Gesetzes über die Antidiskriminierung wegen Behinderung folgte. Das Gesetz über die Sonderschule von 1977 wurde 2007 in Gesetz für Sonderausbildung der Behinderten umbenannt. Das Gesetz über die Förderung der Unternehmenstätigkeit der Behinderten wurde ebensfalls 2007 verabschiedet. Alle drei Gesetze treten 2008 in Kraft.

\section{Gesetzeslage im Allgemeinen}

Das Grundgesetz für die Behindertenpolitik in der Republik Korea ist das Gesetz über die Behindertenwohlfahrt (BWG). Verschiedene Maßnahmen sollen bereitgestellt werden, die darauf zielen, Behinderte in die Gesellschaft zu integrieren und ihnen damit die Lebensführung wie Nicht-Behinderten zu ermöglichen. Dieses Ziel soll dadurch verwirklicht werden, ihre persönliche Entfaltung zu fördern sowie Diskriminierungen wegen der Behinderung zu verbieten. Damit erkennt der Gesetzgeber den Weg zur Verwirklichung der Behindertenpolitik an: Wohlfahrt und Gleichstellung.

Das BWG sieht selbst verschiedene medizinische, soziale und berufliche Maßnahmen vor. Sie betreffen die Prävention sowie Rehabilitation, meist in Form von Sach- und Dienstleistungen. Demgegenüber sind die Leistungen für die Einkommenssicherung oder -ergänzung in Form von Geldleistungen minimal entwickelt ${ }^{8}$. Selbst Sach- sowie Dienstleistungen sind, von einigen Vorschriften abgesehen, plakativ formuliert, so dass daraus kein gerichtlich durchsetzbarer Rechtsanspruch hergeleitet werden kann.

Um das Gesetzesziel des BWGs zu verwirklichen, wurden einige zusätzliche Gesetze verabschiedet, die den Behinderten in wichtigen Lebensbereichen zugute kommen. Um die Behindertenpolitik in Korea zu überblicken, sind dieser gesamte Normenkomplex und seine Zusammen- und Wechselwirkung in Aufgaben und Funktionen zu betrachten ${ }^{9}$. Es sind das Gesetz für die Beschäftigungsförderung und berufliche Rehabilitation der Behinderten, das Gesetz über die Förderung der Sonderausbildung der Behinderten, das Gesetz über die Förderung der Unternehmens-

7 Zur dieser Problematik siehe etwa Cheon Kwang Seok, Zum Erlass des Gesetzes über die Antidiskriminierung wegen der Behinderung, Disability and Employment 2004, S. 5 ff.

8 Zum Aufbau der Einkommenssicherung für die Behinderten, siehe Byun Yong Chan et. al., Eine Studie über die Einführung der Renten für die Behinderten (Korea Institute for Health and Social Affairs, 2004).

9 Hierzu vgl. Zacher, Der Behinderte als Aufgabe der Rechtsordnung, in: Zacher, Abhandlungen zum Sozialrecht, Heidelberg 1993, S. 533 ff. 
tätigkeit der Behinderten und nicht zuletzt das Gesetz über die Antidiskriminierung wegen der Behinderung und Rechtsschutzvorschriften.

Wie unten erwähnt wird, sind alle Sozialgesetze wie Sozialversicherungsgesetze und Sozialhilfegesetze im Großen und Ganzen relevant für die Behindertenpolitik. Hierbei erwähnenswert ist das Gesetz über die Pflegeversicherung, das ebenfalls 2007 verabschiedet wurde und 2008 in Kraft tritt. Die dadurch eintretende Überlappung beim Schutz der Behinderten im Alter bedarf noch genauerer Analyse ${ }^{10}$.

\section{Behinderung-Begriff und Bestand}

Der Begriff „Behinderte“ ist gesetzlich definiert. Man versteht darunter diejenigen, die wegen körperlichen und geistigen Behinderungen an der alltäglichen sowie gesellschaftlichen Lebensführung erheblich gehindert sind (§ 2 BWG). Das BWG listet 15 Arten und verschiedene Grade, je nach Schwere der Behinderung, tabellerisch auf ${ }^{11}$. Diese Regelungstechnik verschafft den Betroffenen Rechtssicherheit; es scheint jedoch an Flexibilität zu fehlen.

2005 existierten schätzungsweise etwa 2,14 Mio. Behinderte in Korea. Davon waren 2006 1.967.326 als Behinderte registriert (etwa 4,3\% der gesamten Bevölkerung von 47 Mio.). 2007 waren insgesamt 1.559 Einrichtungen für Behinderte in Betrieb, wo 27.352 Behinderte betreut wurden ${ }^{12}$. Diese Daten zeigen zweierlei: Eine Differenz zwischen tatsächlich vorhandener und registrierter Zahl der Behinderten sowie eine Differenz zwischen der Zahl der Behinderten und der Aufnahmefähigkeit der Einrichtungen. Wie problematisch dieses ungleiche Verhältnis einzuschätzen ist, bedarf noch vertiefter Untersuchung ${ }^{13}$. Dies ändert aber nichts an einer grundsätzlichen Knappheit an Betreuungseinrichtungen in Korea.

\section{Leistungen}

\subsection{Einleitung}

Mit Leistungen sind alle Maßnahmen gemeint, die dem oben genannten Gesetzesziel dienen. Es umfasst individuelle sowie allgemeine Leistungen, direkte Leistungen sowie persönliche Betreuung, gewährende Leistungen sowie Förderung der Infrastruktur. Persönliche Betreuung und Förderung der Infrastruktur sind in diesem beits- und Sozialrecht 2003, S. 332 ff.

11 Vgl. Tabelle 1 BWG-Ausführungsverordnung.

12 Vgl. Weisses Buch für Gesundheit und Soziale Angelegenheit 2007, S. 245, 247 ff.

13 Es kann theoretisch so sein, dass politisch betrieben ist, Behinderte möglichst in ihren oder anderen Familien zu unterstützen. Dies ist aber in der Tat in Korea sicherlich nicht der Fall. 
Bereich deswegen von Bedeutung, weil die Leistungen für die Behinderten erst durch Vorverfahren der Beratung sowie Auskunft und persönliche Intervention, gegebenenfalls in den Einrichtungen ihre Wirkungen entfalten können ${ }^{14}$. Dieser Aspekt wird noch zum Schluss erwähnt. Wenn man den Blick über die speziell der Behindertenpolitik dienlichen Maßnahmen hinaus richtet, zählen die steuerlichen Begünstigungen auch dazu ${ }^{15}$.

\subsection{Prävention}

Das BWG erklärt die präventive Tätigkeit zu einer wichtigen Aufgabe der Behindertenpolitik. Es ist plakativ so formuliert: Staat und kommunale Verwaltungen sollen sich um die Vorbeugung von Behinderung bemühen. $\mathrm{Zu}$ diesem Ziel sollen sie Ursachen von Behinderungen untersuchen, und sich bemühen, gesundheitliche Anomalien früh zu erkennen und zu heilen ( 99 BWG). Die Durchführung dieser Aufgabe wurde hauptsächlich institutionell ausgestaltet.

Die Unfallversicherung ist ein entscheidender Zweig in Bezug auf die präventive Tätigkeit. Einerseits regelt sie selbst einige Maßnahmen für die Prävention ( $\S 78 \mathrm{ff}$ Gesetz über die Unfallversicherung), andererseits veranlasst sie die Unternehmen, diesbezügliche Maßnahmen zu ergreifen. Dies erfolgt dadurch, dass ein Teil des Beitragssatzes in der Unfallversicherung nach oben oder unten variiert, abhängig von der Höhe der vom jeweiligen Unternehmen in Anspruch genommenen Leistungen $^{16}$.

Die allgemeine Aufgabe der Vorbeugung von Behinderung soll hauptsächlich der Krankenversicherung zugeschrieben werden. In dieser sind aber bisher die präventiven Leistungen nur minimal vorgesehen. 1995 wurde die gesundheitliche Vorsorgeuntersuchung als Leistung in die Krankenversicherung einbezogen (§ 47 I Gesetz über die Volkskrankenversicherung). Der Personenkreis ist aber auf Versicherte und Mitversicherte über 40 Jahre beschränkt ( $\$ 26$ Gesetz über die Volkskrankenversicherung-Ausführungsverordnung). Wünschenwert ist eine Ergänzung um die allgemeinen Früherkennungsmaßnahmen und regelmäßige Gesundheitsuntersuchungen.

Die Vorbeugung vor Behinderungen der Versicherten sollte schon per se auch Aufgabe der Rentenversicherung sein. Die Übernahme präventiver Maßnahmen würde sich für diese auch aus Eigeninteresse anbieten, da sich so ein früherer Eintritt des Rentenanspruchs in vielen Fällen vermeiden ließe, was sich finanziell entlastend auswirken würde. Dieser Aspekt findet aber in der koreanischen Rentenversicherung keine Anerkennung.

14 Allgemein zu diesem Thema siehe Pitschas, Die Infrastruktur sozialer Dienste als Wirkungsbedingungen der Sozialrechtsentwicklung, Vierteljahresschrift für Sozialrecht 1990, S. 3 ff.

15 Allgemein zum steuerrechtlichen Beitrag zum Sozialrecht, siehe Cheon Kwang Seok, Wechselwirkung von Steuer- und Sozialrecht, Korean Journal of Public Law 2003, 32-1, S. 207 ff.

16 Vgl. § 15 II Gesetz über die Beitragserhebung in der Unfallversicherung und Arbeitslosenversicherung i.V.m. $§ 18$ und Tabelle 1 Ausführungsverordnung. 


\subsection{Medizinische Leistungen}

In Korea sind etwa 97,5\% der Bevölkerung durch die Krankenversicherung vor Kosten aufgrund Krankheiten geschützt. Für die übrigen gilt die Krankenhilfe. Beide enthalten aber kaum Vorschriften, die die besonderen Belange der Behinderten betreffen. Vorgesehen ist nur, dass die Krankenversicherung den Behinderten Prothesen gewähren kann. Diese ist eine Ermessensleistung ( $§ 46$ Gesetz über die Volkskrankenversicherung).

Grundsätzlich ist gesetzlich vorgeschrieben, dass sowohl die Versicherten in der Krankenversicherung, als auch die Berechtigten in der Krankenhilfe einen Eigenanteil an den Krankenbehandlungskosten zu tragen haben. Menschen mit Behinderung können nach dem BWG von diesem Eigenanteil befreit werden, allerdings ist die Leistung auf Behinderte mit geringem Einkommen beschränkt. Der Berechtigtenkreis umfasst nämlich nur diejenigen, die zur Sozialhilfe berechtigt sind und sich in einer ähnlichen Lage befinden wie Sozialhilfeberechtigte ( $§ 18$ BWG-Ausführungsrichtlinie).

Außerdem können den Behinderten verschiedene Hilfsmittel sowie Geräte als Sachleistung gewährt werden, falls sie sich diese nicht aus eigenem Vermögen beschaffen können ( $\$ 57$ BWG).

Dieser Regelungszustand gibt Anlass zur Verbesserung der Lage der Behinderten. Erstens deckt die Krankenversicherung in Korea nicht alle medizinischen Behandlungen. Die Kosten für medizinische Behandlungen außerhalb des Leistungskatalogs sind von den Versicherten selbst zu tragen. Dem liegt zugrunde, dass die Versicherten meist erwerbstätig sind oder ihnen zumindest eine Erwerbstätigkeit zugemutet werden kann, so dass sie grundsätzlich zahlungsfähig sind. Dies ist jedoch im Großen und Ganzen bei Behinderten nicht der Fall. Zweitens bedürfen die Behinderten typischerweise einer ständigen und intensiven medizinischen Behandlung. Dieser Besonderheit bei der Behandlung von Behinderten muss Rechnung getragen werden. Wie unterschiedlich und spezifisch im Vergleich mit Nichtbehinderten die Behandlung der Behinderten tatsächlich aussieht und sein soll, ist noch eine offene Frage. Drittens unterliegt die Gewährung der Leistungen meistens dem Ermessen der Verwaltung, was eine gewisse Unsicherheit mit sich bringt. Wünschenswert wäre die Anerkennung eines Rechtsanspruchs zumindest hinsichtlich des Leistungsgrundes.

\subsection{Ergänzende Geldleistung, Unterstützung der Ausbildung}

Den Behinderten können Zuschläge zur Hilfe bei der Lebensführung gewährt werden. Den Anspruch darauf haben jedoch nur sozialhilfeberechtigte Behinderte, bei denen die Leistungen der Sozialhilfe den Sonderbedarf wegen der Behinderung nicht decken können ( $§ 44$ BWG). Der Zuschlag betrug 2007 monatlich 30.000 
Won, 120.000 Won und 130.000 Won (etwa 20 Euro, 80 Euro und 87 Euro) je nach dem Grad der Behinderung ${ }^{17}$.

Die Eltern von Behinderten erhalten einen Zuschlag, der dazu dienen soll, die Betreuung dieser Kinder zu unterstützen. Als Empfänger dieser Leistungen kommen ebenfalls nur die Eltern mit behinderten Kindern in Betracht, die sozialhilfeberechtigt sind und aus eigener Kraft ein Alltagsleben nicht führen können ( $\$ 45 \mathrm{BWG}$ ). Die Altersgrenze ist bis zum 18. Lebensjahr gesetzt. Für Schwerbehinderte gilt die Altersgrenze nicht. 2005 betrug der Zuschlag 100.000 Won, 150.000 Won und 200.000 Won (etwa 67 Euro, 100 Euro und 130 Euro) je nach dem Grad der Behinderung ${ }^{18}$.

Reformbedürftig ist zweierlei. Erstens sind die Geldleistungen nur im Zusammenhang mit der Sozialhilfe vorgesehen. Es ist nicht berücksichtigt, dass die Behinderung individuell sowie in der Familie zusätzliche Kosten verursacht. Entweder sollte anerkannt werden, dass sich eine Behinderung grundsätzlich einkommensmindernd auswirkt und zusätzliche Lebenskosten verursacht. Oder zumindest muss wegen der zusätzlichen Kosten die Einkommensgrenze für den Bezug von Sozialhilfe bei Behinderten höher gesetzt werden. Zweitens ist die Höhe der Leistung realitätsfern niedrig. Es fehlt an Anpassungsregeln.

Den Behinderte erziehenden Eltern werden die Schulgebühren in der Middle- sowie Highschool erlassen (§ $34 \mathrm{BWG})$.

\subsection{Rehabilitation im alltäglichen und sozialen Lebensbereich}

Die beruflichen Rehabilitationen sind spezifisch im diesbezüglichen Gesetz, nämlich im Gesetz über die Arbeitsförderung und berufliche Rehabilitation der Behinderten näher geregelt ( $\$ 30 \mathrm{BWG})$. Dies wird im nächsten Abschnitt behandelt. Das Gesetz über die Antidiskriminierung wegen Behinderung ergänzt es in der Beschäftigung und Arbeitsführung aus den Perspektiven der Gleichbehandlung.

Für die Behinderten sind verschiedene Rehabilitationsmaßnahmen für die alltägliche Lebensführung vorgesehen. Sie erhalten hierfür Beratung in den kommunalen Verwaltungen ( $\$ 30$ BWG). In erforderlichen Fällen werden Behinderte an die medizinischen Versorgungsstellen oder Wohlfahrtseinrichtungen verwiesen, um dort ambulant oder stationär geeignete rehabilitative Maßnahmen zu erhalten (§31 BWG). Intervenieren können auch die Beratungsstellen der kommunalen Verwaltungen.

Was die alltägliche Lebensführung der Älteren anbelangt, wird erwartet, dass es die 2008 in Kraft tretende Pflegeversicherung übernimmt, diesen Bedarf zu decken. Es ist noch eine offene Frage, wie dann das BWG und das Pflegeversicherungsgesetz koordiniert werden sollen.

Vgl. Weisses Buch für Gesundheit und Soziale Angelegenheit 2007, S. 247. 
Für die soziale wie kulturelle Rehabilitation im Sinne von Teilnahme sieht das BWG ebenfalls einige Maßnahmen vor. Allgemein wird ein Training zur Anpassung an das gesellschaftliche Leben durchgeführt ( $\S \S 17$ ff. BWG). Noch konkreter wird die Benutzung der Sport- und kulturellen Einrichtungen sowie der Verkehrsmittel erleichtert ( $\$ 25$ BWG). Der Zugang zu Informationsquellen soll ermöglicht werden ( $\$ 22$ BWG). Es wird erwartet, dass das Antidiskriminierungsgesetz etwa im Bereich der Benutzung von Boden und verschiedenen Diensten wie Verkehr, Information sowie Informationsmitteln, Kultur und Sport noch weiter zur sozialen und kulturellen Teilnahme der Behinderten beiträgt.

\subsection{Förderung der Erwerbstätigkeit}

Diesem Ziel dienen zwei Gesetze: das Gesetz über die Arbeitsförderung und berufliche Rehabilitation der Behinderten (AFRG) und das Gesetz über die Förderung der Unternehmenstätigkeit der Behinderten (UFG).

\subsubsection{Arbeitsförderung und Rehabilitation}

Es wird versucht, Behinderten den Zugang zu Arbeit sowie Berufsausbildung durch verschiedene Maßnahmen zu erleichern: Zunächst verschaffen Berufsberatungen, Untersuchungen zur Berufsfähigkeit und Berufsausbildungen den Behinderten grundsätzliche Voraussetzungen zur Beschäftigung. Sofern es erforderlich ist, kann ihnen eine Berufsausbildung in speziellen Berufsrehabilitationseinrichtungen gewährt werden ( $\S \S 15 f f$. AFRG). Während dieser Maßnahmen werden den Behinderten Trainingsgeld und ein Zuschlag für die Beschäftigungsförderung gewährt (§ 37 AFRG).

Neben der Ausbildungsförderung ist die Vermittlung von Behinderten in Beschäftigung von entscheidender Bedeutung. Es wird zum einen unterstützt, Eigenbetriebe zu gründen. Für diese Unterstützung wurde ein eigenes Gesetz erlassen (siehe unten). Zum anderen werden Arbeitgebern erforderliche Informationen für die Beschäftigung von Behinderten bereitgestellt und finanzielle Unterstützung gewährt. Letztere soll sachliche sowie personelle Bedürfnisse decken, die die Beschäftigung und Arbeitsführung der Behinderten zusätzlich erfordern.

Behindertenwerkstätten erhalten ähnliche Unterstützungen. Staat und kommunale Verwaltungen sind außerdem angehalten, vorrangig Produkte von diesen Werkstätten zu kaufen ( $\$ 40$ BWG-Ausführungsverordnung).

Das AFRG erlegt dem Staat und den Kommunalverwaltungen, sowie den privaten Unternehmen die Pflicht auf, zu einem gesetzlich festgeschriebenen Pflichtsatz Behinderte zu beschäftigen. Der Staat und die kommunalen Verwaltungen sind verpflichtet, mindestens 2\% aller Arbeitsplätze mit behinderten Arbeitnehmern zu besetzen. Einige Tätigkeitsbereiche sind davon ausgenommen: Z.B. Tätigkeiten von Staatsanwaltschaft, Feuerwehr, Polizei, Soldaten etc ( $\$ 23$ AFRG). Private Unter- 
nehmen mit über 50 Beschäftigten sind verpflichtet zwischen 2 und 5\% der gesamten Arbeitnehmer Behinderte zu beschäftigen. Dieser Prozentsatz kann in bestimmten Berufssparten durch Verordnung höher gesetzt werden (§ 24 AFRG).

Arbeitgebern, die diese Pflicht zur Beschäftigung von Behinderten nicht erfüllen, werden Arbeitsförderungsabgaben auferlegt ${ }^{19}$. Zum Schutz von Kleinunternehmern gilt dies nicht für Unternehmen mit weniger als 100 Beschäftigten. Arbeitgeber müssen für jede unbesetzte Pflichtstelle einen Grundbetrag zahlen. Schwerbehinderte werden dabei zweifach gezählt. Der Grundbetrag entspricht dem Betrag, der sonst für die Beschäftigung und Arbeitsführung der Behinderten zusätzlich aufgewendet werden müsste. Er soll $60 \%$ des gesetzlich festgelegten Mindestlohns nicht unterschreiten (§ 27 AFRG).

Die bisherige Erfahrung zeigt aber, dass die privaten Unternehmen eher Sonderabgaben zahlen als eine Pflichtanzahl von Behinderten zu beschäftigen. Wenn sie es zahlen, sind sie von der Beschäftigungspflicht komplett freigestellt. Dass Arbeitgeber in der Regel eine Geldzahlung bevorzugen, liegt einerseits am noch vorhandenen Vorurteil der Unternehmen gegenüber der Arbeitsfähigkeit von Behinderten, andererseits aber auch daran, dass Behinderte zum Teil tatsächlich nicht über die erforderlichen Kenntnisse für eine Beschäftigung verfügen.

Arbeitgeber, die dagegen mehr Behinderte als verpflichtet beschäftigen, erhalten ein Beschäftigungsförderungsgeld. Das gilt auch für die Arbeitgeber, die überhaupt nicht der Pflicht zur Beschäftigung von Behinderten unterliegen. Diese Leistung entspricht dem fiktiven Lohn für jeden zusätzlich beschäftigten Behinderten. Sie soll im Rahmen des Mindestlohns unter Berücksichtigung von Grundbetrag und tatsächlichem Lohn der Beschäftigten im jeweiligen Unternehmen festgesetzt werden (§ $26 \mathrm{AFRG}$ ).

\subsubsection{Förderung der Unternehmenstätigkeit}

Für diese Aufgabe wurde ein eigenes Gesetz erlassen. Dieses Gesetz unterstützt die Behinderten, die zur selbstständigen Unternehmensführung relevanter Berufskenntnisse bedürfen. Außerdem kommen die Unternehmen von Behinderten bevorzugt in den Genuss von Förderungsmaßnahmen für Klein- sowie Mittelunternehmen. Dies erfolgt in Form von Darlehen und steuerlichen Begünstigungen (§ 37 AFRG). Diese sollen für das Betreiben der Geschäfte, für die Beschaffung eines Autos und sonstiger erforderlicher Geräte speziell für Behinderte und für das Training zur Erhaltung der Betriebskenntnisse verwendet werden (§ 19 AFRG-Ausführungsverordnung).

Behinderten ist es bevorzugt erlaubt, Geschäfte für Postsachen und Zigaretten, sowie Ginsengprodukte zu betreiben ( $§ 38$ AFRG). Staat und kommunale Verwal-

19 Zur verfassungsrechtlichen Beurteilung dieser Vorschrift: Entscheidung des Verfassungsgerichtshofs 2003.7.24, 2001 hon ba 96, 15-2 (1.Bd), S. $58 \mathrm{ff}$. 
tungen sollen vorrangig Produkte aus den Behindertenwohlfahrtseinrichtungen erwerben.

\subsection{Bildungsförderung}

Um den besonderen Bildungsbedürfnissen der Behinderten gerecht zu werden, wurde das Gesetz über die Sonderausbildung der Behinderten (BSAG) verabschiedet, das 2008 in Kraft tritt.

Der Gesetzgeber erkannte, dass Behinderte zur selbstständigen Lebensführung, sowie Teilnahme an der Gesellschaft „frühzeitig, lange und dauernd“ gebildet werden sollten. Für Behinderte beginnt die Schulpflicht bereits im Kindergarten und gilt bis zur High school ( $§ 2 \mathrm{Nr}$.2 BSAG). Sie dauert damit länger als die allgemeine Schulpflicht, die nur von der Volksschule bis zur Middleschool besteht (§ 8 I Grundgesetz über die Bildung). Hervorgehoben wird außerdem die Durchführung der lebenslangen Bildung für Behinderte. Sie soll den Behinderten ermöglichen, sich den ständig und dynamisch wandelnden Umständen in der modernen Gesellschaft anzupassen. Diese Bildung ist prinizipiell ohne Schulgebühren.

Die erforderlichen Kenntnisse für die weitere Lebens- und Berufsführung werden hauptsächlich durch die Schulbildung vermittelt. Hier wird auch gefördert, Persönlichkeit und Integrationsbereitschaft sowie -fähigkeit im sozialen Gefüge zu entfalten. Dementsprechend hebt das Gesetz das Prinzip auf, Behinderte in die allgemeinen Schulen zu integrieren und dort zu bilden ( $§ 15$ BSAG). Um den besonderen Bedürfnissen der Behinderten gerecht zu werden, sollen Sonderpädagogen eingesetzt und Sondermaßnahmen planmässig durchgeführt werden (§§ 16ff. BSAG).

\subsection{Maßnahmen zur Gleichstellung der Behinderten nach dem Gesetz über die Antidiskriminierung wegen Behinderung}

Das BWG enthält einige Vorschriften, die unter Berücksichtigung der Bedürfnisse von Behinderten eine behindertenfreundliche Umwelt im Alltagsleben bereitzustellen versuchen (§ 8 BWG). Diese sind meistens im Gesetz über Antidiskriminierung wegen der Behinderung (ADBG) absorbiert.

Die Lebensbereiche, für die dieses Gesetz angewendet wird, sind Folgende: Beschäftigung, Ausbildung, Benutzung verschiedener Sachen sowie Dienste (Boden und Häuser, Verkehr, Information, Kulturgüter, Sport), Justiz sowie Verwaltungsverfahren, politische Beteiligung, Geschlecht, Familie, Wohlfahrtsdienst sowie Gesundheit (Kapitel 2 ADBG).

Dieses Gesetz ist nicht nur an den Staat und die öffentliche Verwaltung adressiert, sondern auch an Privatpersonen. Diskriminierende Handlungen werden grundsätzlich nicht geduldet, außer wenn rechtfertigende Gründe vorliegen (§ 4 III ADBG). Dazu zählen: (1) wenn gleiche Behandlung übermäßige Aufwendungen erfordert oder erhebliche Schwierigkeit bereitet oder (2) wenn ungleiche Behandlung wegen 
besonderer Anforderungen der in dem Bereich ausgeübten Tätigkeiten unvermeidlich ist. Es ist vorauszusehen, dass es viele Streitigkeiten darüber geben wird, ob einer der oben genannten, Diskriminierung rechtfertigenden Gründe gegeben ist oder nicht. Es liegt dann an der Menschenrechtskommission und dem Gericht, ob und wie das Gesetzesziel verwirklicht werden kann.

Für die Verletzung des Ge- und Verbotes sind primäre sowie sekundäre Rechtsschutzverfahren installiert:

Als primärer Rechtsschutz können Behinderte eine Beschwerde bei der Menschenrechtskommission einlegen, wenn die Gleichsstellungsgebote verletzt sind. Dafür ist eine Unterkammer zuständig. Die Kommission erteilt Empfehlungen für die Berichtigung der nachteiligen Maßnahmen. Wenn der Empfehlung ohne rechtfertigende Gründe nicht gefolgt wird, kann der Minister für Justiz einen Berichtigungsbefehl erteilen. Dieser umfasst das Ruhen der diskriminierenden Behandlung, Wiedergutmachung und Maßnahmen zur Verhütung wiederholter Diskriminierung. Gegen diesen Befehl steht dem Betroffenen der Rechtsweg zum Verwaltungsgericht offen.

Als sekundärer Rechtsschutz sind diejenigen, die gegen dieses Gesetz anderen Personen Schaden zufügen, zum Schadensersatz verpflichtet. Die Beweislast für die Tatsachen der Diskriminierung trägt der Beschwerende. Die Beweislast bezüglich der Schuld wird demgegenüber vom Beschwerten getragen.

\section{Abschliessende Bemerkung}

Kulturelle Vorstellungen über die Behindertenpolitik in Korea haben sich seit den 80er Jahren des letzten Jahrhunderts gründlich und rasant geändert. Hintergründe sind folgende Faktoren: Erstens kompensatorische sowie gleichheitliche Ansprüche der Behinderten, zweitens allgemeine Sensibilität der Gesellschaft gegenüber Menschenrechten und drittens aktive politische Teilnahme der Behinderten. Diese Grundlage fordert den Gesetzgeber seit den 80er Jahren. Dieser Bestrebung in der Wohlfahrtsgesetzgebung folgt dann der Gesichtspunkt der Antidiskriminierung.

$\mathrm{Ob}$ die so ausgebaute Gesetzgebung tatsächlich in Wirklichkeit umgesetzt werden kann, ist eine andere Frage. Zweierlei ist erwähnenswert: Erstens vernachlässigt der Elan zur Gesetzgebung die Koordinierung mit anderen Gesetzeszweigen sowie der Behindertengesetze untereinander. Ein Beispiel ist die Frage der Koordinierung und Wechselwirkung zwischen Behindertengesetzen und Pflegeversicherungsgesetz. Anderes Beispiel ist die Frage der Koordinierung und Wechselwirkung zwischen Behindertenwohlfahrtsgesetzen und Antidiskriminierungsgesetz. Zweitens geht es um die Umsetzungsprobleme. Diese sind zwar mehr oder weniger in allen Rechtsbereichen zu beobachten, stechen aber besonders in den Behindertengesetzen hervor. Denn die Gesetze sind zwar an Behinderte adressiert, aber meistens vermittelt durch Organisationen, Einrichtungen sowie Verfahren. All dies erfordert unendliches Gespräch auf normativer sowie tatsächlicher Ebene. 
Die Behinderung ist in Ursachen, Phänomenen sowie Auswirkungen durch Vielfältigkeit ausgezeichnet. Dementsprechend wird vom Gesetz eine Steuerungsfunktion erwartet. Unter diesem Gesichtspunkt sind die Gesetzgebung sowie -anwendung zu überprüfen. Dies wird nicht zuletzt durch den demokratischen Diskurs ermöglicht, wobei die verschiedenen Positionen von Staat (Gesetzgeber, Verwaltung und Justiz), Gesellschaft, Unternehmen und Behinderten selbst abgewogen werden sollten. Dabei sind die ausländischen Erfahrungen in diesem ganzen Fragenkomplex eine Hilfe für den weiteren ausbalancierten Diskurs ${ }^{20}$.

20 In dieser Hinsicht sind erwähnenswert v. Maydell/Pitschas./Schulte (Hrsg.), Teilhabe behinderter Menschen an der Bürgergesellschaft in Asien und Europa-Eingliederung im Sozial- und Rechtsvergleich, Speyer 2002; v. Maydell/Pitschas/Schulte (Hrsg.), Behinderung in Asien und Europa im Politik- und Rechtsvergleich, Baden-Baden 2003. 


\title{
6.4.2. Bildungspolitik für junge behinderte Menschen in Korea und die Entwicklung von Gesetzgebung und Rechtsprechung
}

\author{
Von Soh-Yeon Won
}

1. Einführung

2. Rahmenbedingungen für die sonderpädagogische Förderung 456

2.1 Begriff des sonderpädagogischen Förderbedarfs 456

2.2 Instrumente der sonderpädagogischen Förderung 457

2.3 Gegenwärtiger Zustand der sonderpädagogischen Förderung 458

2.3.1. Zahl der Menschen mit sonderpädagogischer Förderung $\quad 458$

2.3.2. Sonstige infrastrukturelle Bedingungen zur sonderpädagogischen Förderung

3. Rechtliche Grundlage und Rechtsprechung

3.1. Das Recht auf Bildung für behinderte Menschen im Verfassungsrecht 461

3.2. In einfachen Gesetzen unter Berücksichtigung des Gesetzes für die sonderpädagogische Förderung

3.2.1. Aufbau des Sonderschulwesens

3.2.2. Leistungen im Gesetz für die sonderpädagogische

Förderung (GSF)

3.3. Entwicklung der Rechtsprechungen 463

4. Bildungspolitik für Menschen mit sonderpädagogischem Förderbedarf $\quad 464$

4.1. Fünfjahres-Plan für die sonderpädaogische Entwicklung 464

4.2. Sonderpädagogische Entwicklung durch die Fünfjahres-Pläne 465

5. Gewährleistungsmängel

5.1. Probleme mit der rechtlichen Konkretisierung des geschützten

Personenkreises

5.2. Unzulänglichkeit der Aufsichtsmechanismen 466

5.3. Mängel in der Infrastruktur 467

6. Änderung des Konzepts für die sonderpädagogische Förderung: Geplantes

Gesetz für die sonderpädagogische Förderung behinderter Menschen $\quad 468$

6.1. Erweiterung des berechtigen Personenkreis 469

6.2. Inhaltliche Erweiterung für die sonderpädagogische Förderung $\quad 469$

6.3. Verbot der behinderungsbedingten Diskriminierung 469

7. Fazit - Perspektive 


\section{Einführung}

In den letzten Jahrzehnten gewinnt das Thema der Teilhaberechte und Rehabilitation behinderter Menschen weltweit immer mehr an Bedeutung. Mit Bezug auf die internationale Entwicklung, nämlich dass die Weltgesundheitorganisation das Jahr 1981 zum „Jahr für behinderte Menschen“ und die UNO das Jahrzehnt von 1983 bis 1992 zum „Jahrzehnt für behinderte Menschen“ erklärt hat, wurde in Korea das staatliche Bestreben zur Teilhabesicherung behinderter Menschen erst seit der zweiten Hälfte der 80er Jahren in dem letzten Jahrtausend systematisch angefangen. Danach hat die Behindertenpolitik in Korea durch zwei Gesetzgebungen für die Teilhabesicherung am Arbeitsleben und für die Barrierefreiheit in der Gesellschaft einen großen Fortschritt gemacht.

Obwohl sich die Behindertenpolitik in Korea bisher stets weiter entwickelt hat, müssen behinderte Menschen in Korea teils immer noch unter Gewährleistungsmängeln bei der Teilhabesicherung leiden und daher ist in den letzten Jahren ein Modernisierungsbedarf vorgelegt worden. Eine wichtige Forderung hierbei ist mit der Ungleichheit zwischen Behinderten und Nicht-Behinderten bei Bildungschancen verbunden. Die Bildung spielt eine grundlegende Rolle, um die von Anfang an im menschlichen Sein angelegten potentiellen Fähigkeiten weiter zu entwickeln und damit die Grundlage für ein menschenwürdiges Arbeits- und Gesellschaftsleben herzustellen. Zur individuellen Selbstbestimmung ist deshalb die Förderung der individuellen Fähigkeiten im Bildungs- und Erziehungsprozess notwendig. In der heutigen hoch differenzierten und entwickelten Gesellschaft gewinnt Bildung immer mehr an Bedeutung. ${ }^{1}$ Dennoch ist das Recht auf Bildung für behinderte Menschen in Korea noch nicht völlig gewährleistet.

Insofern ist es bedeutsam, die rechtliche und politische Grundlage im Bereich des Bildungssystems für behinderte Menschen in Korea zu untersuchen und damit die Problemlage herauszufinden. Dies leistet letztendlich zur Integration behinderter Menschen in die koreanische Gesellschaft einen Beitrag.

\section{Rahmenbedingungen für die sonderpädagogische Förderung}

\subsection{Begriff des sonderpädagogischen Förderbedarfs}

Um das Recht auf Bildung für behinderte Menschen zu besprechen, soll der Begriff des sonderpädagogischen Förderbedarfs klargestellt werden. Sonderpädagogischer

1 Entscheidung des koreanischen Verfassungsgerichts am 27.02.2003, Case-Code 2000Hunba26; Hu, Young, Koreanisches Grundgesetz, 28. Aufl. 2005, S.427; Kim, Chul-Su, Koreanisches Grundgesetz, 18. Aufl. 2006, S. 823-824; Ramm, Bildung, Erziehung und Ausbildung als Gegenstand von Grundrechten, in: Festschrift für Erwin Stein, Avenarius/Engelhardt/Heussner/v. Zezschwitz (Hrsg.), 1983, S. 246. 
Förderbedarf ist eine Definition, die sich nicht auf gesundheitliche Zustände, sondern auf den umstandbezogenen „Interventionsbedarf " bezieht. ${ }^{2}$

Zur Garantie des Bildungsrechts für behinderte Menchen sind besondere Förderungen erforderlich, weil die Bildungschancen behinderter Menschen wegen ihres körperlichen, geistigen und seelischen Schadens im allgemeinen Bildungssystem nicht hinreichend gewährleistet werden. Daher entsteht ein besonderer Förderungsbedarf für sie. Im Hinblick auf das Recht auf Bildung überlappen sich die berechtigten Personenkreise beider Kategorien, „Menschen mit sonderpädagogischem Förderbedarf“ und „Menschen mit Behinderung“, sind aber nicht völlig identisch. Obwohl jemand behindert sein kann, kann er möglicherweise keinen sonderpädagogischen Förderbedarf haben. Und umgekehrt ist jemand nicht behindert, kann er trotzdem einen sonderpädagogischen Förderbedarf haben. Ein Beispiel für Letzteres sind diejenige Schüler und Schülerinnen mit Förderbedarf auf Lernen. Nach dem jährlichen Bericht über den sonderpädagogischen Zustand 2006 vom Ministerium für Bildung machen Schüler und Schülerinnen in sonderpädagogischen Einrichtungen mit Förderschwerpunkt auf Lernen in Korea etwa 10,5\% aus. ${ }^{3}$ In der Tat orientiert sich die koreanische Bildungspolitik für Menschen mit sonderpädagogischem Förderbedarf auch zumeist daran, das Recht auf Bildung für Menschen mit Behinderung zu garantieren. ${ }^{4}$ Insofern wird es in Korea im Allgemeinen verstanden, dass der berechtigte Personenkreis der Sonderpädagogik zumeist Menschen mit Behinderung ist. Auch wäre der Unterschied der beiden berechtigten Personenkreise in Korea relativ klein. ${ }^{5}$

\subsection{Instrumente der sonderpädagogischen Förderung}

Die Schülerinnen/Schüler mit sonderpädagogischem Förderbedarf in Korea werden gemäß dem Behinderungsgrad und den Umständen der Betroffenen sowohl in Schulen, als auch in Krankenhäusern, zu Hause und in sonstigen Einrichtungen gefördert. ${ }^{6}$ Der Bildungsprozess, nicht in Schulen, sondern in Krankenhäusern und zu Hause sowie in öffentlichen Heimen, wird aber grundsätzlich für diejenigen, die wegen der Schwerbehinderung nicht in der Lage sind, die Schule zu besuchen, vorübergehend durchgeführt. ${ }^{7}$

2 Welti, Behinderung und Rehabilitation im sozialen Rechtsstaat, 2005, S. 48.

3 Nch diesem Bericht es gibt aber keine Förderschule für Schülerinnen und Schüler mit Förderschwerpunkt auf Lernen.

4 Zur Bildungspolitik für Menschen mit sonderpädagogischem Förderbedarf; Der zweite FünfjahresPlan zur sonderpädagogischen Entwicklung von 2003-1007.

5 In Deutschland waren im Jahr 2003 etwa 53\% der Schülerinnen und Schüler mit sonderpädagogischer Förderung dem Schwerpunkt auf Lernen zugeordnet ; statistische Veröffentlichungen der Kultusministerkonferenz - Dokumentation Nr.177, Sekretariat der ständigen Konferenz der Kultusminister der Länder in der Bundesrepublik Deutschland (Hrsg.), November 2005.

$6 \quad \S 14-15$ im Gesetz für die sonderpädagogische Förderung am 24.03.2005, Gesetz-Nr.7395.

7 Woo, Ju-Hyung, Das Recht auf Bildung für behinderte Menschen und die rechtliche Grundlage, in: Journal of the Korea Rehabilitation Fund, Vol.10, 2001, S. 97-157, hier S. 110. 
Die sonderpädagogische Förderung wird in der Regel durch das Schulwesen gewährleistet. Das öffentliche Schulwesen in Korea gliedert sich in der Regel in den Elementarbereich (Kindergarten), den Primarbereich (Grundschule), den Sekundarbereich I (Mittelschule) und II (Oberschule). ${ }^{8}$ Für Schülerinnen/Schüler mit sonderpädagogischem Förderbedarf, deren Förderung in allgemeinen Schulen nicht hinreichend gewährleistet werden kann, gibt es in jedem Bereich entsprechend den Behinderungsarten unterschiedliche Typen von Sonderschulen. ${ }^{9}$ Daneben werden sie auch in Sonderklassen in allgemeinen Schulen sonderpädagogisch gefördert. Die allgemeine Schulpflicht in Korea beginnt für alle Kinder mit dem Primarbereich und endet mit dem Abschluss des Sekundarbereichs I. ${ }^{10}$

\subsection{Gegenwärtiger Zustand der sonderpädagogischen Förderung}

\subsubsection{Zahl der Menschen mit sonderpädagogischer Förderung}

Die gesamte Zahl an jungen Menschen im Alter von drei bis siebzehn Jahren im Jahr 2006 betrug 9.751.259. Nach der Veröffentlichung über den sonderpädagogischen Zustand 2006 wurden davon 65.940 Schüler und Schülerinnen mit sonderpädagogischem Förderbedarf in Sonderschulen und auch in allgemeinen Schulen unterrichtet. Im Hinblick auf die Förderschwerpunkte entfielen von den Beteiligten etwa 55\% (36.041) auf den Förderschwerpunkt geistige Entwicklung und 45\% (29.899) auf die sonstigen Förderschwerpunkte.

9 Es gibt in Korea im Jahr 2007 fünf Typen von Sonderschulen, nämlich mit Förderschwerpunkt auf Sehen, Hören, geistige Entwicklung, motorische Entwicklung und emotionale Entwicklung, Jährlicher Bericht über Sonderpädagogik zur Parlament 2007 vom Ministerium für die Bildung, S. 24. $\S 8$ Abs.1, Gesetz der Bildung. 
Tabelle 1: Schüler und Schülerinnen in sonderpädagogischer Förderung nach Förderschwerpunkten

\begin{tabular}{|l|c|c|c|c|}
\hline & \multicolumn{2}{|c|}{ Korea (2007) } & \multicolumn{2}{c|}{ Deutschland (2003)*** } \\
\hline Förderschwerpunkt & $\begin{array}{l}\text { Schüler* } \\
\text {-Anzahl- }\end{array}$ & $\begin{array}{l}\text { Förderquote** } \\
\text {-In Prozent- }\end{array}$ & $\begin{array}{l}\text { Schüler } \\
\text {-Anzahl- }\end{array}$ & $\begin{array}{l}\text { Förderquote } \\
\text {-In Prozent- }\end{array}$ \\
\hline Lernen & 6.982 & 0,07 & 258.618 & 2,9 \\
\hline Sehen & 2.292 & 0,02 & 6.167 & 0,1 \\
\hline Hören & 2.864 & 0,03 & 13.717 & 0,2 \\
\hline Sprache & 1.185 & 0,01 & 45.837 & 0,5 \\
\hline $\begin{array}{l}\text { Körperliche, motori- } \\
\text { sche Entwicklung }\end{array}$ & 7.739 & 0,08 & 27.324 & 0,3 \\
\hline Geistige Enwicklung & 36.041 & 0,37 & 72.277 & 0,8 \\
\hline $\begin{array}{l}\text { Emotionale und } \\
\text { soziale Entwicklung }\end{array}$ & 7.695 & 0,08 & 42.627 & 0,5 \\
\hline Kranke & 1.142 & 0,01 & 9.844 & 0,2 \\
\hline $\begin{array}{l}\text { Föerderschwerpunkt } \\
\text { übergreifend bzw. } \\
\text { ohne Zuordnung }\end{array}$ & - & - & 16.310 & 0,1 \\
\hline Insgesamt & 65.940 & 0,67 & 492.721 & 5,6 \\
\hline
\end{tabular}

Quelle:

\footnotetext{
* Bericht für das Parlament 2007, Ministerium für Bildung und Humankapital.

** als Förderquote wird der Anteil der Schüler $\left.{ }^{*}\right)$ an der Gesamtzahl der Schüler im schulpflichtigen Alter in Korea (im Alter 3 bis 17) bezeichnet.

***Statistische Veröffentlichungen der Kultusministerkonferenz, Dokumentation Nr.177- November 2005, S.XI.
}

Von den Schülern und Schülerinnen mit sonderpädagogischem Förderbedarf in Korea besuchten 32.752 (49,6\%) die Grundschule, 15.267 (23,3\%) die Mittelschule, $14.796(22,4 \%)$ die Oberschule und 3.125 (4,7\%) den Kindergarten ${ }^{11}$.

Entsprechend der Tendenz in Korea, dass Schüler und Schülerinnen mit sonderpädagogischem Förderbedarf immer weiter in allgemeine Schulen integriert werden sollen, werden 65,2 \% (42.977) in allgemeinen Schulen und etwa 34,8 \% (22.963) in Sonderschulen unterrichtet.

\subsubsection{Sonstige infrastrukturelle Bedingungen zur sonderpädagogischen Förderung}

Als sonderpädagogische Einrichtungen gab es in Korea im Jahr 2006 insgesamt 3.278 Klassen in 143 Sonderschulen und 5.753 Sonderklassen in allgemeinen Schulen. Nach der Veröffentlichung des Ministeriums für Bildung wurden etwa 7.637

11 In Korea wird die Schulstufe in Sonderschulen wie in allgemeinen Schule auf die Grundschule, Mittelschule, Oberschule und Kindergarten aufgeteilt. 
Schüler und Schülerinnen in allgemeinen Klassen in allgemeinen Schulen mit nichtbehinderten Schülern zusammen unterrichtet.

Die Zahl der Lehrer und Lehrerinnen in sonderpädagogischen Einrichtungen beträgt insgesamt 12.249. Davon unterrichten etwa die Hälfte (6.141) in Sonderschulen und die andere Hälfte $(6.108)$ in allgemeinen Schulen. Die durchschnittliche Schülern-Lehrer Relation ist 5,6.

Tabelle 2: Lehrer, Schüler je Lehrer in Korea

\begin{tabular}{|l|c|c|c|}
\hline & Sonderschule & Allgemeine Schule & Insgesamt \\
\hline Lehrer insgesamt & 6.141 & 6.108 & 12.249 \\
\hline Schüler je Lehrer & \multicolumn{3}{|c|}{5,6} \\
\hline
\end{tabular}

Quelle: Ministerium für Bildung und Humankapital, Untersuchung des Standes der sonderpädagogischen Förderung 2007

Zur Sicherung des Bildungsrechts für Menschen mit Behinderung sind auch behindertengerechte Ausstattungen in Schulen erforderlich. Diesbezügliche Bestimmungen in Korea sind im Vergleich zu anderen Bedingungen relativ unterentwickelt. In der Verwaltungsverordnung des Gesetzes zur Barrierefreiheit für Alte, Schwangere und Behinderte werden als behindertengerechte Ausstattungen in Schulen die Türen, die Toiletten, der Aufzug bzw. Rollstuhlweg und der Parkplatz kodifiziert. $^{12}$

Die durchschnittliche Quote behindertengerechter Einrichtung in allgemeinen Schulen, die Sonderklassen für Menschen mit sonderpädagogischem Förderbedarf haben, erreicht etwa $50 \%{ }^{13}$

Tabelle 3: Durchschnittliche Quote der behindertengerechten Ausstattungen in allgemeinen Schulen

\begin{tabular}{|c|c|c|c|c|c|}
\hline & Türen & $\begin{array}{c}\text { Aufzug/ } \\
\text { Rollstuhlweg }\end{array}$ & Toilette & Parkplatz & $\begin{array}{l}\text { Insgesamt } \\
\text { (Durchschnitt) }\end{array}$ \\
\hline $\begin{array}{c}\text { Einrichtungsquote } \\
\text { (\%) }\end{array}$ & 57,2 & 29,6 & 65,4 & 50,6 & 51,2 \\
\hline
\end{tabular}

Quelle: der überarbeitete zweite Fünfjahres-Plan für sonderpädagogische Entwicklung, Ministerium für Bildung 2004.

$12 \S 8$, Gesetz zur Barrierenfreiheit für Alte, Schwangere und Behinderte vom 31.03.2005, GesetzNr.7476; §4, Verwaltungsverordnung desselben Gesetzes vom 15.10.2007, Verordnung-Nr.20323.

Der überarbeitete zweite Fünfjahres-Plan zur sonderpädagogischen Entwicklung 2004. 


\section{Rechtliche Grundlage und Rechtsprechung}

\subsection{Das Recht auf Bildung für behinderte Menschen im Verfassungsrecht}

Das Recht auf Bildung für behinderte Menschen ist seit dem ersten Verfassungsrecht 1948 in Korea dadurch mittelbar anerkannt worden, dass alle Menschen gemäß ihren Fähigkeiten ein gleiches Recht auf Bildungserwerb haben. ${ }^{14}$ Außer dieser expliziten Kodifikation steht der grundrechtlich garantierte Ansatz für das Recht auf Bildung mit dem Recht auf die freie Wahl des Berufs in Art.15 und auf die zu achtende Menschenwürde, sowie auf die Verfolgung des persönlichen Wohls ${ }^{15}$ in Art.10 in engem Zusammenhang. Obwohl das Verfassungsrecht keine unmittelbare Garantie für das Recht auf Bildung behinderter Menschen enthält, ist es unbestritten, dass niemand wegen seiner Behinderung beim Bildungserwerb benachteiligt werden darf. ${ }^{16}$

Darüber hinaus legt diese verfassungsrechtliche Garantie in Verbindung mit dem allgemeinen Gleichheitssatz in Art.11 und dem Sozialstaatsprinzip ${ }^{17}$ dem Staat eine besondere Aufgabe auf, angemessene Voraussetzungen wie Gesetzgebung, Institutionen und soziale Infrastruktur mit dem Ziel zu schaffen, die Chancengleichheit behinderter Menschen beim Bildungserwerb herzustellen. ${ }^{18}$ Aber es ist immer noch umstritten, wieweit das Bildungsrecht für behinderte Menschen garantiert werden und wieweit der Staat die Veranwortung übernehmen soll.

\subsection{In einfachen Gesetzen unter Berücksichtigung des Gesetzes für die sonder-} pädagogische Förderung

Um die grundrechtliche Garantie für das Recht auf Bildung von behinderten Menschen umzusetzen, wurde im Gesetz für Bildung 1949 die Errichtung der Sonderschulen bzw. Sonderklassen für behinderte Menschen als staatliche Verantwortung kodifiziert. Danach ist der verfassungsrechtliche Schutz auf Bildung für behinderte Menschen in Korea in Bildungsgesetzen und Behindertengesetzen weiter ausgestaltet worden. Damit gewinnt das Gesetz für die sonderpädagogische Förderung $(G S F)$, das umfangreiche Leistungen und Maßnahmen für die sonderpädagogische Förderung bzw. Entwicklung umfasst, seit der ersten Fassung von 1977 als ein kom-

14 Art.16, Koreanisches Verfassungsrecht vom 17.07.1948.

15 Diese Vorschrift ist funtionell vergleichbar mit dem Recht auf freie Entfaltung in Art.2 GG Deutschland

16 Zur verfassungsrechtlichen Bedeutung des Rechts auf Bildung, Entscheidung des koreanischen Verfassungsgerichts vom 24.02.1994, Case-Code Hunma 192, 173 (177).

17 Das koreanische Verfassungsrecht nimmt das Sozialstaatsprinzip nicht durch eine explizite Kodifikation wie im GG Deutschland, sondern dadurch an, zahlreiche soziale Grundrechte zu garantieren.

18 Entscheidung des koreanischen Verfassungsgerichts vom 27.4.2000, Case-Code 98Hunga16, KCCR 12-1, S. 427 (428). 
plexes Gesetz zur Teilhabesicherung am Bildungwesen für behinderte Menschen an Bedeutung.

\subsubsection{Aufbau des Sonderschulwesens}

Die Rechtsgrundlage des Sonderschulwesens für behinderte Menschen findet sich unmittelbar in drei Bildungsgesetzen ${ }^{19}$ und drei Behindertengesetzen ${ }^{20}$. Im Hinblick auf das Bildungswesen sind in Korea nach der Schulstufe fünf Gesetze vorhanden, nämlich Gesetz der Bildung (,BildungG“), Gesetz der Bildung für Vorschulkinder (,VorschulG“), Gesetz der Bildung für den Primar- und Sekundarbereich (,,SchulG“), Gesetz der Hochschulbildung (,HochschulG“) und Gesetz für die Förderung des lebenslangen Lernens (,WeiterbildungG“"). Dadurch ist die sonderpädagogische Förderung im BildungG, VorschulG und SchulG unmittelbar niedergelegt. Der verfassungsrechtliche Schutz auf Bildung für behinderte Menschen bezieht sich aber nicht nur auf die Schulbildung, sondern umfasst auch die berufliche Ausbildung und das lebenslange Lernen. Aber eine Garantie auf Hochschulbildung und das lebenslange Lernen behinderter Menschen findet sich nicht unmittelbar im Gesetz. ${ }^{21}$ Aufgrund dieser Abwesenheit des gesetzlichen Schutzes ist das Bildungsrecht für behinderte Menschen im Bereich der Hochschulbildung und des lebenslangen Lernens tatsächlich eingeschränkt. Das Bildungsrecht für behinderte Menschen in Korea ist deshalb zumeist mit Schwerpunkt auf die Schulbildung ausgestaltet.

\subsubsection{Leistungen im Gesetz für die sonderpädagogische Förderung (GSF)}

GSF gewinnt als das erste Gesetz im Bereich der Teilhaberechte und Rehabilitation für behinderte Menschen an Bedeutung. In dem Gesetz wurden zunächst als berechtiger Personenkreis für die sonderpädagogische Förderung Sehbehinderte, Gehörlose, Körperbehinderte, geistig Behinderte, Sprachbehinderte und Menschen mit Behinderung in der emotionalen Entwicklung anerkannt (§ 3). Durch das Verbot der behinderungsbedingten Diskriminierung im $\S 10$ versuchte das Gesetz auch, die Chancengleichheit beim Bildungserwerb umzusetzen. Außerdem ist der Staat bzw. die Lokalregierung verpflichtet, angemessene Maßnahmen zur sonderpädagogischen

19 Gesetz der Bildung (Framework Act on Education), Gesetz der Bildung für Vorschulkinder (Early Childhood Education Promotion Act), Gesetz der Schulbildung im Primar- und Sekundarbereich (Elementary and Secondary Education Act).

20 Gesetz für die sonderpädagogische Förderung (Act on the Promotion of Education for the Handicapped), Gesetz zur Förderung der Einstellung und beruflichen Rehabilitation für behinderte Menschen (Employment promotion and Vocational Rehabilitation of Disabled Persons Act), Gesetz der Wohlfahrt für behinderte Menschen (Welfare of Disabled Persons Act).

21 Das Gesetz für die Hochschulbildung und das Gesetz für das lebenslange Lernen umfassen dafür keine unmittelbare Vorschrift, das Bildungsrecht für behinderte Menschen in diesen Bereichen umzusetzen. 
Entwicklung einzuleiten (§ 4). Für die finanzielle Förderung wurde auch kodifiziert, dass die Kosten für die sonderpädagogischen Leistungen vom Fiskus unterstützt werden können. Aber das Gesetz umfasste keine Sanktionen gegen die behinderungsbedingte Diskriminierung und gegen die Nicht-Erfüllung der Verantwortung. Außerdem waren die Vorschriften in diesem Gesetz zumeist Ermessensbestimmungen. Deshalb war das Gesetz damals schwer einzuklagen.

Dieses Gesetz wurde im Jahr 1994 völlig neu gefasst. Dadurch wurde zum ersten Mal die Lernbehinderung in den Kreis der sonderpädagogischen Förderung aufgenommen ( $\$ 10$ Abs.1). Der Inhalt der sonderpädagogischen Förderung wurde auch erweitert. Früher konnten nur die sonderpädagogischen Leistungen in Sonderschulen bzw. in Sonderklassen vom Staat gefördert werden. Daher wurden Schüler und Schülerinnen, die wegen einer Schwerbehinderung oder chronischen Krankheit nicht in Schulen unterrichtet werden konnten, vom berechtigen Personenkreis ausgeschlossen. Bei der Änderung 1994 wurden diejenigen Kinder, die zu Hause oder im Krankenhaus sind, auch dadurch sonderpädagogisch gefördert, dass Lehrer und Lehrerinnen persönlich Häuser und Krankenhaus besuchten und dort unterrichteten. Eine andere wichtige Änderung war, dass die Bildung in Grundschulen bzw. in Mittelschulen als Schulpflicht und im Kindergarten bzw. in Oberschulen als unentgeltliche Schulbildung zuerkannt wurde ( $§ 5$ Abs.1). Außerdem wurde im $\S 28$ eine Sanktion gegen die Ablehnung der Einschulung behinderter Menschen neu aufgenommen. Aber das Gesetz enthielt immer noch keine konkrete Aussage, was als behinderungsbedingte Diskriminierung beim Bildungserwerb anzusehen ist.

Außer diesen Bildungsgesetzen enthält das Gesetz der Wohlfahrt für behinderte Menschen („WohlfahrtG“) auch die Teilhabesicherung im Bildungsbereich (§ 18). ${ }^{22}$ Mit Bezug auf die berufliche Tätigkeit sind die berufliche Ausbildung und Anpassungshilfe zur beruflichen Tätigkeit (§ 10-12) im Gesetz zur Förderung der Einstellung und beruflichen Rehabilitation für behinderte Menschen („BerufRehaG“) niedergelegt. ${ }^{23}$

\subsection{Entwicklung der Rechtsprechungen}

Das Verfassungsgericht hat in Korea für einen materiellen Rechtsstaat eine entscheidende Rolle gespielt. Zur Garantie des Bildungsrechts im Art. 31 der koreanischen Verfassung leistete auch die Rechtsprechung des Verfassungsgerichts einen Beitrag. Die meisten Entscheidungen sind allerdings mit dem generellen Bildungsrecht verbunden. In der Tat gibt es in Korea nur sehr wenige Rechtsprechungen für die Teilhabesicherung der behinderten Menschen. In Bezug auf das Bildungsrecht für behinderte Menschen gibt es zwei gerichtliche Entscheidungen gegen die Verlet- 
zung des Rechts auf Hochschulbildung von behinderten Menschen. ${ }^{24}$ Die Entscheidungen gewinnen insofern an Bedeutung, als dass das Recht auf Hochschulbildung für behinderte Menschen vorgeschrieben wird. Dennoch ist in der Realität die Hochschulbildung bei behinderten Menschen schwer zu erreichen. Außerdem lassen sich keine Entscheidungen für das Bildungsrecht von Schulkindern finden. Dies kann damit erklärt werden, dass der Rechtschutz durch das Gericht in Korea nicht üblich ist. Zu den Gründen dafür gehören sowohl die hohen Gerichtskosten, als auch die Unzulänglichkeit der gerichtlichen Kontrolle bei der Verletzung des Bildungsrechts für behinderte Menschen.

\section{Bildungspolitik für Menschen mit sonderpädagogischem Förder- bedarf}

Eine staatliche Bildungspolitik für behinderte Menschen in Korea wurde bereits in den sechziger Jahren beobachtet. Damals hatte die koreanische Regierung einen ersten Fünfjahres-Plan für die Wirtschaftsentwicklung eingeleitet und seit dem zweiten Plan war auch die sonderpädagogische Förderung berücksichtigt; dieser Bereich wurde aber auf Grund der Wirtschaftsentwicklung nicht verwirklicht. 1998 ist dann endgültig ein Fünfjahres-Plan für die sonderpädagogische Entwicklung unter der Verantwortung des Ministeriums für Bildung begonnen worden und seit dem Jahr 2003 ist der zweite Plan in Gange.

\subsection{Fünfjahres-Plan für die sonderpädagogische Entwicklung}

Die erste komplexe Planung für die sonderpädagogische Förderung wurde 1998 mit dem Fünfjahres-Plan unter dem Motto „eine sonderpädagogische Entwicklung zur Erreichung des Sozialstaates durch die Integration“ eingeleitet. Das Ziel des ersten Plans war, entprechend der Behinderungsarten und des -grades sowie der individuellen Anforderungen, eine angemessene Bildung anzubieten. Dazu wurde die Bildungspolitik für Menschen mit sonderpädagogischem Förderbedarf gemäß dem Behinderungsgrad durch zwei unterschiedliche Maßnahmen, jeweils für Kinder mit Schwerbehinderung und Leichtbehinderung, durchgeführt. Um bessere Bildungschancen für behinderte Kinder zu sichern wurde auch ein flexibles Schul- und Unterrichtssystem eingeleitet. Daneben wurde das Ausbildungssystem für sonderpädagogische Lehrer und Lehrinnen reformiert. ${ }^{25}$ Dieser Plan hat damit einen Beitrag

24 Vom Verband für das Recht auf Bildung von behinderten Menschen werden zwei Entscheidungen berichtet. Aber nur eine Entscheidung davon ist bekannt; Entscheidung des Stadtsgerichts Seoul vom 06.12.2001, Case-Code 2001Na25719.

25 Das Ministerium für Bildung, Der erste Fünfjahres Plan zur sonderpädagogischen Entwicklung, 1996. 
dazu geleistet, durch die Erweiterung der Bildungschancen ein sonderpädagogisches Fundament in Korea aufzubauen.

Demgegenüber hat der zweite Plan sowohl die quantitative Erweiterung, als auch die Erhöhung der Bildungsqualität ins Auge gefasst, mit dem Ziel, den Bildungseffekt der Schüler und Schülerinnen mit sonderpädagogischem Förderbedarf zu maximieren. Zur Zielverwirklichung hat der zweite Plan etwa vier mittlere Aufgaben festgestellt, d.h. Garantie der Bildungschancen durch die Integration in die allgemeinen Schulen, Erhöhung der Bildungsqualität durch die Verbesserung bzw. Pluralisierung der Bildungsmethoden, Verstärkung der Qualität bzw. Verantwortung von Lehrern, Modernisierung des sonderpädagogischen Förderungssystems.

\subsection{Sonderpädagogische Entwicklung durch die Fünfjahres-Pläne}

In den letzten Jahren haben die beiden Fünfjahres-Pläne zur sonderpädagogischen Entwicklung vor allem zur quantitativen Erweiterung einen Beitrag geleistet. Die Entwicklung wird durch den geschichtlichen Überblick deutlich gemacht.

Tabelle 4: Geschichtlicher Überblick der sonderpädagogischen Entwicklung

\begin{tabular}{|l|l|l|l|l|}
\hline Jahre & Sonderschule & $\begin{array}{l}\text { Sonderklasse } \\
\text { in allgemeinen Schule }\end{array}$ & Schüler & Lehrer \\
\hline 1962 & 10 & - & 1.343 & 120 \\
\hline 1972 & 38 & $1(1971)$ & 5.188 & 505 \\
\hline 1980 & 56 & 355 & 8.094 & 904 \\
\hline 1990 & 102 & 3.181 & 19.947 & 1.746 \\
\hline 1995 & 108 & 3.440 & 21.569 & 3.422 \\
\hline 2000 & 129 & 3.802 & 24.196 & 8.376 \\
\hline 2003 & 137 & 4.102 & 53.404 & 9.175 \\
\hline 2005 & 142 & 4.697 & 58.362 & 10.429 \\
\hline 2007 & 144 & 5.753 & 65.940 & 12.249 \\
\hline
\end{tabular}

Quelle: Bericht über die sonderpädaogische Förderung zum Parlament 2007, Ministerium für Bildung

\section{Gewährleistungsmängel}

Obwohl sich die sonderpädagogische Förderung bisher quantitativ und auch qualitativ stark entwickelt hat, gibt es noch Gewährleistungsmängel bei der Sicherung des Rechts auf Bildung für Menschen mit sonderpädagogischem Förderbedarf und daher ist ständig ein Modernisierungsbedarf angemahnt worden.

Die Gewährleistungsmängel in Korea stehen zunächst mit der negativen allgemeinen Einstellung gegenüber behinderten Menschen in enger Verbindung. Nach Umfragen von behinderten Menschen wird stets berichtet, dass sie wegen der Behinderung im Bereich der Bildung, der Arbeit und im alltäglichen Leben nicht nur 
von privaten Personen, sondern auch von öffentlichen Institutionen benachteiligt werden. ${ }^{26}$ Dem staatlichen Komittee für Menschenrechte in Korea werden auch fortlaufend behinderungsbedingte Diskrimierungsfälle gemeldet. ${ }^{27}$ Allerdings ist es schwer zu belegen, welche Faktoren diese negative Einstellung verursachen und wieweit sie bezüglich des Bildungsrechts negativ beeinflusst werden. Dennoch ist die negative Einstellung gegenüber behinderten Menschen in Korea immer noch ein grundsätzliches Hindernis bei der Teilhabesicherung behinderter Menschen in allen Bereichen. Nach Untersuchungen haben 75\% der Eltern von Kindern mit potentiellem Förderbedarf angegeben, dass sie ihre Kinder nicht als berechtigten Personenkreis für sonderpädagogische Leistungen anerkennen lassen wollen. Dies kann bedeuten, dass sie - solange es nicht unbedingt notwendig ist - von dem behinderten Personenkreis ausgeschloßen werden wollen.

\subsection{Probleme mit der rechtlichen Konkretisierung des geschützten Personenkreises}

Im Vergleich mit Deutschland ist der geschützte Personenkreis mit sonderpädagogischer Förderung in Korea relativ eng. Bezüglich des Umfangs der Schüler und Schülerinnen mit sonderpädagogischem Förderbedarf gibt es momentan in Korea keine offiziell einheitlichen Daten. Stattdessen werden vom Ministerium für Bildung die statistischen Untersuchungen über Schüler und Schülerinnen mit sonderpädagogischer Förderung jedes Jahr veröffentlicht. Mit Bezug auf den Untersuchungsbedarf über den berechtigen Personenkreis mit sonderpädagogischem Förderbedarf gibt es in Korea ein interessantes Ergebnis des Ministeriums für Gesundheit und Wohlfahrt. Das Ministerium führt alle fünf Jahre eine Untersuchung über den Zustand behinderter Menschen in Korea durch. Nach den Ergebnissen von 2005 wurde berichtet, dass 45\% der Beantworter der Umfrage keine Mittelschule besucht haben bzw. besuchen und $62 \%$ keine Oberschule sowie 15,4\% keine Grundschule. Als ersten Grund, dass sie die Schule nicht besucht haben, nannten davon $71 \%$ finanzielle Schwierigkeiten. Zur weiteren sonderpädagogischen Entwicklung ist es deshalb zunächst erforderlich, den Gegenstand genau zu definieren.

\subsection{Unzulänglichkeit der Aufsichtsmechanismen}

Abgesehen davon, ob alle Menschen mit sonderpädagogischem Förderbedarf gerechte Bildungschance haben, ist der Schutzmechanismus für Menschen in sonderpädagogischer Förderung zu kritisieren. 2006-04, Ministerium für Wohlfahrt, 2006, S. 278-312.

27 Nach der Statistik des Komittees werden Diskriminierungsfälle aufgrund des sozialen Status am häufigsten angemeldet, gefolgt von behinderungsbedingter Diskriminierung auf dem zweiten Platz. 
Der zweite Problemkreis bei der Sicherung des Bildungsrechts für behinderte Menschen ist mit der behinderungsbedingten Diskriminierung verbunden. Wegen der Behinderung werden Schulkinder nicht selten bei der Einschulung abgelehnt und zur Umschulung in andere Schulen gezwungen. Wenn sie denn zugelassen werden, werden sie nicht selten von verschiedenen Schultätigkeiten ausgeschloßen, beispielsweise Schulausflug oder Schulsportprogramm usw.

Dass diese diskriminierenden Behandlungen häufig passieren, ist in erster Linie auf die Grenzen des Gesetzes selbst zurückzuführen, zum einen weil die Leistungen für die Sicherung des Bildungsrechts im Gesetz meistens nur als Kann-Bestimmungen formuliert sind und zum anderen weil gesetzliche Regelungen für die Förderung der integrierten Bildung in allgemeinen Schulen fast vollständig fehlen. Daneben ist die Unzulänglichkeit der Aufsichtsmechanismen ein anderer Grund dafür. In Korea werden diese Diskriminierungsfälle entweder durch den Eingriff vom städtischen Bildungsamt oder vom staatlichen Komittee für Menschenrechte behandelt.

Aber die sachliche Zuständigkeit des städtischen Bildungsamts ist auf solche Schulen beschränkt, die vom Staat bzw. von der Lokalregierung getragen werden. Gegen Direktoren in Schulen von privaten Akteuren kann das Bildungsamt bei Verstoß gegen das Diskriminierungsverbot keine disziplinarischen Maßnahmen verhängen.

Das staatliche Komittee für Menschenrechte ist für den Schutz vor Verletzungen des Bildungsrechts auch unzulänglich, weil seine Entscheidungen keine gerichtliche Befugnis haben.

Insofern ist das gegenwärtige System nicht ausreichend, die Diskriminierung zu beseitigen und das Bildungsrecht für behinderte Menschen zu sichern. Im Hinblick auf die koreanische Konstellation, dass $65 \%$ des berechtigten Personenkreises für die sonderpädagogische Förderung in allgemeinen Schulen unterrichtet werden und etwa $60 \%$ dieser Schulen von privaten Akteuren getragen werden, sind effektive Aufsichtsmechanismen in besonderem Maße erforderlich. Allerdings kann das Bildungsrecht nicht allein durch den äußeren Druck von Aufsicht oder gerichtlicher Kontrolle effektiv geschützt werden. Vielmehr sollten die Leistungsträger durch angemessene Förderungsmaßnahmen zu eigenem Engagement animiert werden.

\subsection{Mängel in der Infrastruktur}

Für die Sicherung des Bildungsrechts für behinderte Menschen sind auch infrastrukturelle Voraussetzungen erforderlich. Diesbezüglich sind die Bedingungen in Korea trotz der Erweiterung in den letzten Jahren nicht ausreichend, alle Berechtigten mit sonderpädagogischem Förderbedarf zu versorgen. Vor allem ist die Verteilung der Einrichtungen problematisch. Beispielsweise erreichen die Sonderklassen in allgemeinen Schulen eine Gesamtzahl von 3.892 (22.498 Schüler) in Grundschulen, diese sinkt aber in Mittelschulen bis auf 1.125 (7.500) Sonderklassen und in Oberschulen 
bis auf 562 (4.743). Daneben beträgt die Zahl der Einrichtungen für Vorschulkinder nur 174 (599). ${ }^{28}$ Die asymmetrische Verteilung der Sonderklassen wird nicht nur bei der Schulstufe, sondern auch bei der örtlichen Verteilung beobachtet. Insofern sind sowohl die quantitative Erweiterung der Infrastruktur, als auch eine harmonisierende Entwicklung erforderlich.

Daneben ist die Knappheit behindertengerechter Ausstattungen in Schulen ein anderes großes Hindernis. Immer mehr Kinder mit sonderpädagogischem Förderbedarf werden in allgemeinen Schulen integriert unterrichtet. In der Tat werden etwa $65 \%$ der Schülerinnen und Schüler mit sonderpädagogischem Förderbedarf in Korea in allgemeinen Schulen unterrichtet. Dennoch erreicht die Quote der Einrichtung behindertengerechter Ausstattungen z.B. der Türen, des Aufzug, der Toiletten durchschnittlich nur knapp 50\% in Schulen, die Sonderklassen für Schülerinnen und Schüler mit sonderpädagogischem Förderbedarf haben. Die Quote in allgemeinen Schulen ohne Sonderklasse ist noch niedriger und erreicht nur etwa knapp 30\%. ${ }^{29}$

Dies führt dazu, dass die Schulen wegen der Knappheit bzw. Abwesenheit von behindertengerechten Ausstattungen die Einschulung der Kinder mit sonderpädagogischem Förderbedarf weiterhin ablehnen. Selbst wenn die Kinder Chancen haben, in den Schulen unterrichtet zu werden, ist es unklar, ob ihr Bildungsprozess in den Schulen effektiv vollzogen werden kann. ${ }^{30}$

\section{6. Änderung des Konzepts für die sonderpädagogische Förderung: Geplantes Gesetz für die sonderpädagogische Förderung behinderter Menschen}

Aufgrund dieser Probleme ist ein Modernisierungsbedarf bei der sonderpädagogischen Förderung seit Anfang diesen neuen Jahrtausends vorgelegt worden. Daraufhin wurde im Mai 2007 das Gesetz der Sonderpädagogik für behinderte Menschen verabschiedet. In diesem Gesetz spiegeln sich die Forderungen der sonderpädagogisch Berechtigten wider. Deshalb wird es sehr positiv bewertet. Die Änderungen bei dem Gesetz sind etwa in drei Kategorien aufgeteilt.

S. 7.

29 Der überarbeitete zweite Fünfjahres-Plan 2004.

30 Nach der Pressekonferenz des Verbands des Rechts auf Bildung für behinderte Menschen gegen die behinderungsbedingte Diskriminierung beim Bildungserwerb sind die meisten Diskriminierungsfälle mit der Knappheit bzw. Abwesenheit der behindertengerechten Ausstattungen in Schulen verbunden. Mit solcher Ausrede haben manche Schulen abgelehnt, behinderte Kinder aufzunehmen. In der Tat werden solche Ausflüchte der Schulen bei der Anordnung der schulpflichtigen Kinder nicht selten akzeptiert. Dann müssen die Kinder andere Schule besuchen, wo Sonderklassen und behindertengerechte Ausstattungen eingerichtet sind, obwohl es eine Schule in der Nähe ihres Wohnortes gibt. 


\subsection{Erweiterung des berechtigen Personenkreis}

Erstens wird der berechtigte Personenkreis erweitert. Während das alte Gesetz keine konkreten Vorschriften über die Bildung der Vorschulkinder und die Ausbildung der Erwachsenen mit Behinderung enthielt, beschränkt sich die sonderpädagogische Förderung im neuen Gesetz nicht auf die Schulbildung. Vielmehr wird der sonderpädagogische Umfang um die Förderung für Vorschulkinder und für Erwachsene erweitert. Daneben war der Maßstab für den berechtigten Personenkreis im alten Gesetz sehr streng und wurde nur anerkannt, wenn der Betroffene die im $\S 10$ kodifizierten Behinderungen in acht Kategorien und zugleich einen sonderpädagogischen Förderbedarf aufwies. Demgegenüber werden die Behinderungskategorien im neuen Gesetz bis auf zehn Kategorien erweitert. Der Personenkreis wird nicht nur auf Grund der Behinderung zugeordnet. Vor allem können durch die Aufnahme der neuen Kategorie „Behinderung bei der körperlichen bzw. geistigen Entwicklung (developmental disability)“ Menschen ohne deutliches Behinderungsymptom als berechtigte Personen für die sonderpädagogische Förderung anerkannt werden.

\subsection{Inhaltliche Erweiterung für die sonderpädagogische Förderung}

Zweitens wird auch der Inhalt der sonderpädagogischen Förderung erweitert. Im alten Gesetz hatte sich die sonderpädagogische Förderung auf den Bildungserwerb in Schulen konzentriert. Daher wurden Schüler und Schülerinnen, die die „Sonderpädagogik" nicht benötigten, aber eine andere Förderung (wie die Förderung von behindertengerechten Ausstattungen, medizinische Rehabilitation, Hilfe für den Schulbesuch) von der gesetzlichen Förderung ausgeschlossen. Demgegenüber werden im $\S 28$ des neuen Gesetzes Dienstleistungen der medizinischen Förderung, des Beihilfepersonals beim Bildungserwerb und der Verkehrshilfe zum Schulbesuch neu aufgenommen. Dadurch beschränkt sich die sonderpädagogische Förderung nicht auf die lernmethodische „Sonderpädagogik“. Vielmehr umfasst sie komplexe Maßnahmen zur Sicherung des Bildungsrechts von Menschen mit Förderbedarf beim Bildungserwerb.

\subsection{Verbot der behinderungsbedingten Diskriminierung}

Drittens verbietet das neue Gesetz ausdrücklich die behinderungsbedingte Diskriminierung beim Bildungserwerb. Im alten Gesetz wurde nur das Diskriminierungsverbot kodifiziert. Aber es gab keine konkreten Aussagen zur Ausgestaltung dieses Verbotes. Demgegenüber wird im neuen Gesetz im $\S 4$ die behinderungsbedingte Diskriminierung beim Bildungserwerb konkret kodifziert. Demnach werden folgende Behandlungen als Diskriminierung anerkannt: Wenn behinderte Menschen von den in $\S 28$ verankerten Dienstleistungen ausgeschlossen werden, wenn sie von bestimmtem Unterricht oder Tätigkeiten ausgeschlossen werden, wenn die Eltern 
von verschiedenen Teilnahmeprogrammen ausgeschlossen werden, wenn von behinderten Menschen bei der Immatrikulation an der Hochschule zusätzliche körperliche bzw. mündliche Untersuchungen verlangt werden.

\section{Fazit-Perspektive}

Das Gesetz der Sonderpädagogik für behinderte Menschen kann zur Sicherung des Bildungsrechts für behinderte Menschen ein neuer Wendepunkt sein. Während das alte Gesetz sich darauf beschränkte, im Rahmen der vorhandenen sonderpädagogischen Einrichtungen die Bildungsqualität zu erhöhen, versucht das Gesetz seitens der Empfänger das Bildungsrecht zu sichern. Die sonderpädagogische Förderung im neuen Gesetz wird nicht nur im Sinne der lernmethodischen Leistungen, sondern auch der komplexen Maßnahmen für die Sicherung des Bildungsrechts von behinderten Menschen verstanden.

Allerdings kann das Bildungsrecht der behinderten Menschen nicht allein mit der Gesetzgebung verwirklicht werden. Um das Vollzugsdefizit zu decken, muss der Staat durch eine Aufgabenverteilung den Leistungsträgern eigene Verantwortung zuweisen und gleichzeitig durch effektive Aufsichtsmechanismen seine Gewährleistungsfunktion erfüllen. 


\title{
6.5. The Foundation of Disabled Welfare Policy in Asia: Focusing on China
}

\author{
Von Ming-Cheng Kuo
}

1. Foreword

2. Comparison of Disabled Welfare Policy in Taiwan and China

2.1. The Basic Social Provision for Public Health Care, House and Food etc.

2.2. Social Insurance

2.3. Welfare Services and Allowances

2.4. Enterprises Welfare

2.5. The Third Sector

2.6. Preliminary Conclusion

3. Foundation of Disabled Welfare Policy in China and Taiwan

3.1. Introduction

3.2. Cultural foundation

3.3. The International foundation

3.4. Academic Foundation

483

4. Concluding Remarks 


\section{Foreword}

There are significant differences between Asian countries in terms of culture, economic development, and political systems. Culturally speaking, there exist huge differences among Asian countries, particularly between West Asia, East Asia and South Asia. The economies of the different Asian countries are also at various stages of development. In East Asia, for example, there is an advanced industrialized country like Japan, newly-industrialized countries like Korea and Taiwan, as well as developing countries like China and Vietnam. In terms of political systems, China and Vietnam are no longer the socialist countries they were in the past due to various reforms. China now proclaims itself as having a socialist market economy in which it still retains aspects of a socialist political and economic system. China's socialist market economy differs greatly from the market economy in Japan, Korea and Taiwan.

The huge differences between Asian countries make it a highly difficult task to discuss the foundation of disabled welfare in all of the Asian countries. Therefore, in this paper, the focus is on East Asia, particularly China and Taiwan. It seems undeniable that Taiwan falls within the realm of Chinese culture although the type of political relationship that should exist between Taiwan and China has been a very controversial issue. Among the roughly 23 millions inhabitants in Taiwan, some 97\% are descendents of immigrants from China, $2 \%$ are indigenous people, and $1 \%$ are migratory workers. The languages commonly used in Taiwan are the same as in China, with Mandarin being the most prominent. Most of the people in Taiwan believe in Taoism or Buddhism in the forms originating from China. The Nationalist Party (Kuomintang, hereafter referred to as the KMT), which ruled Taiwan from 1949 to 2000, is the remnant of the same party that previously ruled in China and the KMT regards itself as a defender of Chinese culture. It could thus be said there is much common ground between Taiwan and China, especially in culture. They are also very diverse societies in many ways.

First of all, Taiwan is an immigrant society formed by immigrants from China over 400 years ago who took with them the cultural artifacts of Chinese society. However, the current cultural traits of China differ from traditional Chinese culture as a result of the revolution led by the Chinese Communist party with their MarxistLeninist and Maoist ideologies.

Secondly, the differences between Taiwan and China have become larger and larger due to the fact that they have had different political systems and rulers. Taiwan was ruled by Japan from 1895 to 1945 . Both Taiwan and China were ruled by the KMT after 1945. The Chinese Communist party then took power in China while the KMT continued in power in Taiwan. Political relations have frequently been hostile between China and Taiwan over the intervening years. At the same time, the economic system and economic development in Taiwan and China have followed different paths.

Despite the large differences between Taiwan and China; nevertheless, there is still much common ground that can be found. For one thing, both sides had authori- 
tarian regimes ruled by a strongman until the 1970s and 80s. Discernible reforms appeared on both sides after the death of Chiang Kai-shek and Mao Zedong though the focus of reforms for both sides was different. While Taiwan's reforms focused on politics, China's reforms focused mainly on the economy. It should be noted that the level of economic development of both sides has become closer and closer. There has been extensive investment from Taiwan into China and there are increasing amounts of Taiwanese working there. Consequently, the level of interaction between Taiwan and China has dramatically increased and the differences between the both sides have quickly decreased.

Having so much common ground, but also having lots of diversity, Taiwan and China provide an excellent sample for cultural, economic and political research. It would certainly be worthwhile to extend the sample to Hong Kong and Singapore and even to include a comparison of Korea, Japan, and Southeast Asian countries at the same time. However, due to time constraints, this report will focus only on Taiwan and China.

In order to finish this report, I not only referred to the materials used in the conferences held in Speyer and Berlin ${ }^{1}$ and the Workshop on Social Security on Social Security in the PRC held by Das Instititut für Asienkunde in Hamburg ${ }^{2}$, but I also headed for Beijing to pay a research visit before attending this conference. During my stay in Beijing, I had several meetings with Professor Zheng Gongcheng, Professor Lin Jia, Professor Yang Tuan and Professor Liu Chueixiao from which I benefited greatly. I hereby express my gratitude for their assistance.

\section{Comparison of Disabled Welfare Policy in Taiwan and China}

Disabled welfare, as defined here, is confined to rehabilitation, education, employment, nursing, living assistance, provision of a non-handicapping environment, and social and cultural participation. These benefits and measures as defined here match the main content of Taiwan's Disabled Welfare Act of 1980 which later was amended as the Physically and Mentally Disabled Protection Act of 1997 and later the Protection of Rights and Interests of the Physically and Mentally Disabled Act of 2007. China enacted The Law on the Protection of Disabled Persons in 1991, which is currently under revision, and which has similar provisions to the Taiwan acts. The measures stipulated in the acts in Taiwan and China are mainly public benefits provided by the government, particularly welfare services like social work, with the

1 Ming-Cheng Kuo, „Taiwan“, in: v. Maydell/Pitschas/Schulte (Hrsg.), Teilhabe behinderter Menschen an der Bürgergesellschaft in Asien und Europa - Eingliederung im Sozial- und Rechtsvergleich, 2002, 185-191; ders., Status quo and Perspectiven des Rechts und der Politik für Menschen mit Behinderung: Taiwan, in: v. Maydell/Pitschas/Schulte (Hrsg), Behinderung in Asian und Europa im Politik- und Rechtsvergleich, 2003, 277-293.

2 Social Change and Social Security in Taiwan: Lessons for the PRC, in: Krieg/Schädler (Hrsg.), Social Security in the People's Republic of China, 1994, 340-365. 
financial resources coming from the general revenue of the state or local government. In addition the acts include the state's administrative intervention measure regarding a system of compulsory employment of handicapped persons by enterprises.

The protection of disabled persons is definitely not restricted only to these welfare measures but should also include public health care, house and food provisions, and a social insurance system, particularly health insurance, pension insurance, occupational accident insurance and even long-term care insurance. Whether such a kind of system is established and whether it operates effectively should be the precondition for any discussion of welfare policy such as welfare services. In other words, the need for welfare services would be alleviated when such a social insurance system provides a protective function. If not, the expectations put on the welfare service are naturally increased. Besides public welfare services, the welfare service system in the private sector and the third sector including enterprises, welfare institutions and volunteer groups and volunteer services etc. is also an essential issue in disabled welfare services, and perhaps is even the area for the greatest potential development in disabled welfare policy, and is thus a worthwhile area to explore.

\subsection{The Basic Social Provision for Public Health Care, House and Food etc.}

Before China began its economic reform, the communist economic system, as implemented by China's government, made their citizen's life and their work units closely integrated and such units bore the responsibility for providing living resources and risk protection. In addition, people could receive, to a certain extent, medical protection provided by the so-called "barefoot doctors", a medical cooperative system, though the medical resources provided could be very limited.

This economic system in China underwent huge changes after economic reforms. Nowadays, as public enterprises have been marketized, the relationship between workers and the public enterprises they belong to is close to the labor relationship found in industrialized countries. Furthermore, the medical cooperative system with its "barefoot doctors" has also collapsed and been replaced by a medical market economy. To date, the medical system in China has become highly marketized due to the limited development of health insurance.

In Taiwan, the living resources provided by the government has traditionally been relatively limited. Taking medical resources for example, the government set up local public health centers or hospitals, but such public medical institutions were basically public enterprises where the patients have to pay for services except for some public health measures such as preventive injections. Before the implementation of the National Health Insurance in 1995, roughly half of the people in Taiwan were still uncovered by health insurance in which case they needed to pay for any medical services provided. 


\subsection{Social Insurance}

With the establishment of the People's Republic of China in 1949, China implemented a labor insurance system. However, such insurance is better classified as employer's liability rather than social insurance. Namely, it is a kind of enterprise benefit provided by law which obliges every employer to be responsible for taking care of its unit members. In recent years, social insurance schemes have been continuously established following economic reforms, in particular, unemployment insurance and accident insurance. The coverage of the insured persons under such unemployment insurance or accident insurance is still limited and thus it cannot protect all workers but the measures it provides are somewhat similar to social insurance in industrialized countries. However, there is a vast difference in the pension insurance and medical insurance in China compared to industrialized countries.

The pension insurance and medical insurance schemes implemented in China up to the present day are still at an experimental stage and lack clear content. As regards these two kinds of social insurance, such systems are still based on administrative guidelines set out by the central government in Beijing. Only urban workers are obliged to join these insurance schemes while rural areas have the discretion to decide whether to implement them or not. As to the content, besides the basic principles which should be followed, there is still large room for every local government to formulate it. The health insurance not only limits the benefits but establishes individual medical account, which is different from health insurance in industrialized countries.

Its main characteristics consist of:

- $\quad$ very limited benefits with comparatively limited contributions. In rural areas the contribution rate of experimental health insurance costs only $10 \mathrm{RMB}$ every year, plus a subsidy of $10 \mathrm{RMB}$ from the government, which makes for a total of only 20 RMB every year.

- a so-called individual medical account system in addition to an ordinary social insurance system

Similarly, the current old-aged security system in China is called old age insurance, but it is still not pure social insurance as found in most industrialized countries. Such a system remains experimental just like the medical insurance, lacking clear content and having the coverage limited to specific regions or workers e.g. socalled farmer workers and industrial workers from agricultural regions are excluded. The most important thing to note is that such a system is not pure social insurance but a mixture of social insurance and individual accounts. By current regulation, the contribution rate of social insurance is $20 \%$ of basic salary while the rate of individual accounts is $8 \%$ of basic salary.

In Taiwan, the Labor Insurance implemented in 1950 and enacted in 1958, the Soldier Insurance enacted in 1953, and Government Employees and Educators Insurance enacted in 1958, demonstrates the importance attached by Chiang Kai Shek's regime to social insurance. Such a social insurance system was essentially 
similar to social insurance in industrialized countries but the medical benefit did not cover family members and the old-age, disability and survivors' benefits were a lump-sum payment.

There was continuous expansion of the insured persons under the Labor Insurance throughout the 1960s, 70s and 80s. A significant growth of social insurance in Taiwan appeared with the implementation of the National Health Insurance in 1995 and the Unemployment Insurance in 1999. However, the payment of the abovementioned benefits failed to be changed into pension benefits. On the contrary, the Labor Retirement Benefit system implemented in 2004 set up individual retirement accounts. By law, every employer shall contribute at least $6 \%$ of salary monthly for their workers to each account. Such a development has fully demonstrated the skepticism towards social insurance and the preference for individual accounts shown by Taiwan society.

In 2007, the Congress in Taiwan passed the National Pension Act which is a social insurance scheme covering non-workers and non-government employees. Furthermore, the reform of changing the lump-sum payment of old-age, disability and survivor's benefits into regular pension payment under the Labor Insurance has been discussed in the Congress but the reform bill had not been passed at the time this paper was finished.

\subsection{Welfare Services and Allowances}

Non-insurance disabled welfare is perhaps of much higher importance than that of social insurance in China. We can refer to the report presented by Darimond in the Berlin conference in this respect ${ }^{3}$. The China Disabled Persons' Federation is basically a kind of public institution for disability protection. This institution has attracted special attention because of its leader, Deng Pufang, who is the son of Deng Xiaoping. The Beijing government has attached importance to various welfare measures for disabled persons by announcing in its 11th five-year plan for the National Economy and Social Development in 2006 of its Implementation Program of Development Guidelines and Corresponding Measures for the Disabled in China. Nevertheless, we can learn from the Communiqué on Major Statistics of the Second China National Sample Survey on Disability (2006-2007) that:

- In 2005, the yearly household income of a family with disabled persons was on average, $4864 \mathrm{RMB}$ in urban areas and $2260 \mathrm{RMB}$ in rural areas. However, $12.95 \%$ of yearly household income of family with disabled persons in rural areas was below 683 RMB and 7.96\% was between 684 RMB and 944 RMB.

- Among the disabled, the rate of people with medical and assistance needs was $72.78 \%$ but in reality only $35.61 \%$ received medical service and assistance.

3 Darimont, Status quo and Perspectiven des Rechts und der Politik für Menschen mit Behinderung: VR China, in: v. Maydell/Pitschas/Schulte (Hrsg), Behinderung in Asian und Europa im Politikund Rechtsvergleich, 2003, 255-275. 
- Among the disabled persons in urban areas, roughly $13.28 \%$ of them enjoyed locally provided minimum living protection, while $0.75 \%$ of them received regular or irregular assistance. In the case of rural areas, the percentage of disabled persons who enjoyed locally provided minimum living protection was $5.12 \%$ of the total number of disabled persons. $11.68 \%$ of these rural disabled received regular or irregular assistance.

In Taiwan, Public Welfare Services and Allowances, the main provision in the Handicapped Welfare Act, is for the most part the core issue that social movements and welfare groups are most concerned about. In this regard, the government always responds to their needs to some extent with a view to stabilizing society and legitimatizing its rule. Nowadays various kind of allowances have played quite an important role in providing income replacement due to the fact that only public servants, military servicemen and educators can enjoy pensions and the Labor Insurance program still only provides old-age, disability and survivor benefits in the form of a lump-sum payment. Currently the allowances in Taiwan mainly include an Old-Age Citizens' Allowance of a monthly amount of NT\$ 3,000 under the Provisional Act of the Old-Age Citizens' Welfare Living Allowance, an Old-Age Farmers' Allowance of a monthly amount of NT\$ 6,000 under Article 4 of the Provisional Act of the Old-Age Farmers' Allowance and a monthly amount of NT\$3,000 or 6,000 of Living Allowance for Mid or Low-income Senior Citizens under the Senior Citizen Welfare Act and the Regulations on Living Allowance For Mid or Low-income Senior Citizens ${ }^{4}$.

As to the allowance targeted at disabled persons, the decision as to how much to pay out is in the hands of a small number of local governments. The level of benefits under this program is between NT\$ 1,000 and NT\$ 6,000 monthly. In addition, the welfare provision for disabled persons includes schooling, nursing, and economic support programs etc. Notwithstanding, the development of social assistance in Taiwan is still quite underdeveloped. Till now, the beneficiaries of the social assistance system in Taiwan is less than $1 \%$ of the population. In other words, it is still the case that the disabled have difficulty in obtaining protection through social assistance in Taiwan. amount is less than 1.5 times of the average individual monthly living expense and also less than 1.5 times the average individual monthly living cost in Taiwan. 2.an allowance shall be limited to NT $\$ 3,000$ dollars if the average individual monthly living expense exceeds 1.5 times but is less than 2.5 times the average individual monthly living expense, and is also less than 1.5 times the average individual monthly living cost in Taiwan. 


\subsection{Enterprises Welfare}

To impose obligations upon enterprises to pay benefits by way of policy or legislation could be said to be a very easy approach. The typical example includes the Labor Insurance implemented in China after 1949, and the Labor Standards Act enacted in 1984 in Taiwan, both of which require employers to take responsibilities to pay retirement benefits and severance pay to their workers. These approaches are rich in patriarchal thought and clearly reject modern social insurance systems. To oblige employers to hire disabled persons and intensify enterprises' social responsibilities is atypical of modern approaches to social insurance. Both Taiwan and China have similar measures in this respect.

\subsection{The Third Sector}

In Taiwan and China, the welfare institutions for the disabled play an important role. In China, the aforementioned national and local protection institutions of the disabled are of great importance. Such institutions are more a part of the governmental administration than of non-governmental welfare institutions and have a clear bureaucratic character. Comparatively, the non-governmental welfare institutions in China obviously play a very limited role. The development of the private or nongovernmental welfare institutions is closely related to the openness of society and political democracy.

The welfare institutions of Taiwan tend to be private and primarily from the third sector. In the past, the welfare institutions from abroad, religious organizations in particular, played an important role. These welfare institutions or organizations achieved their mission at the time. The Bethesda Protectory, a German-established institution in Hualien, Taiwan, can be a significant case. The institution founded in 1955, has now been taken over by Taiwanese and funded by local donations and governmental subsidies. In addition, the Buddhist Compassion Relief Tzu Chi Foundation based in Hualien, Taiwan, a Buddhist charity organization established in 1966, was founded and is run by local Taiwanese. It has more than two hundred branches distributed throughout more than thirty countries and enjoys a prestigious reputation as a welfare organization.

Increasingly, the welfare institutions for the disabled in Taiwan have been conspicuous for their strong growth, including the Eden Social Welfare Foundation and the Parents' Association for Persons with Intellectual Disability. These welfare institutions for the disabled are well-organized and provide an effective service. They are part of the National Association of Disabled Welfare Organizations. A former general secretary Wang of this National Association who was also a legislator from 2005 to 2008, is a key proponent moving forward the process of the revision of the Protection of Rights and Interests of the Physically and Mentally Disabled Act. In recent years, with the increase of the governmental subsidy to the welfare of the 
disabled, the funds and the quality of service of such organizations have been greatly improved.

\subsection{Preliminary Conclusion}

From the comparison between Taiwan and China, it can be concluded that there is no provision of sufficient pension insurance for both sides and both prefer an individual account system when it comes to pension reform.

The labor insurance system in Taiwan has almost 60 years of history but the payment of old-age/disability/survivor benefits still remains a lump-sum payment rather than regular pension payments as in other industrialized countries. Even if in the near future it could be changed into a regular pension payment, the question is why such reform has experienced difficulties for decades and even has resulted in the implementation of an individual account system in Taiwan. In China, where pension systems do exist, the question is why they have chosen to adopt an individual account system even when the development of the pension systems is still very limited.

As far as medical care is concerned, the implementation of the National Health Insurance in Taiwan was definitely a very important and significant breakthrough. The overall implementation of health insurance in Taiwan and South Korea demonstrates the implementation possibility of health insurance in other Asian countries, outside of Japan. Such a form of health insurance, though garnering wide public support, still attracts lots of criticism, mainly from economists who go so far as to propose a individual medical account system instead. In comparison, the implementation of medical insurance in China is very limited and the introduction of individual medical accounts is an example of another system that is obviously different from that of industrialized countries.

As regards social assistance, its development is very limited no matter if you are referring to China or Taiwan. The result is that the disabled can hardly expect to enjoy even basic subsistence protection.

\section{Foundation of Disabled Welfare Policy in China and Taiwan}

\subsection{Introduction}

Disabled welfare policy includes various welfare measures like education, employment, medical care, pension, long term care, social assistance and so on. However, just as Grasser said: Wie soll ein Menschen mit Behinderung mit gut \$ 600 im Monat 
durchkommen $?^{5}$ (How can a disabled person live on US\$ 600 dollar per month?) If a disabled person cannot expect to receive a reasonable amount of pension, perhaps a discussion of other measures makes no sense. Besides a pension, medical care and social assistance for basic living protection are even more important and urgent to the disabled. Similarly, it must be asked how the disabled survive if basic medical care, a disability pension and social assistance become impossibilities?

In the existing literature, there have already been many papers discussing the factors affecting social welfare development in Asian countries. Industrialization and democratization are two of the most crucial of the various factors. In this regard, Kuo's papers including; "Social Change and Social Security in Taiwan: Lessons for the PRC " " presented at Hamburg," Volkskrankenversicherung in Taiwan" presented in 1997, "50 years of Social Insurance in Taiwan"" presented in 2000 and "Development and Reform of Social Insurance in Taiwan" ${ }^{8}$, in 2002, have elaborated on this issue. Recently, a paper "Comparative Analysis on the History of Medical Insurance in South Korea and Taiwan: Industrialization, Democratization and Social Policy in Latecomer Societies" presented in China made a similar analysis".

Similarly, Japan's social policy has a very close relationship to its industrialization and democratization. It can thus be concluded that industrialization and democratization are very important factors in constructing a modern social security system. Without these two factors, the initial development of a social security system still occurred in a country like Taiwan in the 1950s ; however, it could hardly be expected to be an overall and complete development, in particular a social security system covering the disabled.

On the other hand, the question arises, why have Asian countries, particularly Taiwan and China, been unable to establish a complete modern social security system following their social development? As mentioned earlier, industrialization and democratization are very important factors. Accordingly, the lack of industrialization and democratization are the main factors making it difficult for a social security system to develop soundly. In addition to these crucial factors, what other things could explain the inadequate development of a modern social security system. Among other things, cultural and international factors are often mentioned as important explanations for this inadequate development. For example, Leisering's paper

5 Graser, Staus quo und Perspectiven des Rechts und der Poloitik für Menschen mit Behinderung: USA, in: v. Maydell/Pitschas/Schulte (Hrsg.), Behinderung in Asien und Europa im Politik- und Rechtsvergleich, 2003, 233-253 (253)

6 In: Krieg/Schädler (Hrsg.), Social Security in the Republic of China, 1994, 340-365

7 Ming-Cheng Kuo, Fifty Years of Social Insurance in Taiwan, in: Boecken/Ruland/Steinmeier (Hrsg.), Sozialpolitik und Sozialrecht in Deutschaland und Europa, 2002, 421-433.

8 Ming-Cheng Kuo, Development, Reform and Perspectives on Social Insurance in Taiwan, in: Kuo/Zacher/Chan (eds.), Reform and Perspectives on Social Insurance: Lessons from the East and West, 2002, 121-144.

9 Lianhua Li, in: Social Security Studies, 2/2007, 81-104. 
presented in the Berlin conference made a relevant analysis of this ${ }^{10}$. However, although Leisering mentioned the influence of Confucianism and international organizations, his paper did not make an in-depth analysis of these factors which left some serious unanswered questions. The next section of this paper will begin with this point. Besides the role of Confucianism and international organizations, the influence of the academic factor will be particularly looked at.

\subsection{Cultural foundation}

It is highly farfetched or even nonsensical to attribute the inadequate development of a social welfare system, particularly in Asia, to reasons of traditional culture. All Asian countries, including India, Indonesia and Vietnam, have been reported to be traditionally unfriendly and severely discriminatory towards disabled people ${ }^{11}$.

In regards to China, and even in countries like Taiwan, Korea and Japan which have been under the influence of Confucianism, the Confucianism factor should not be ignored. Related to this, there has been lots in the literature mentioning the importance of family within Confucianism influencing the development of social security ${ }^{12}$. However, such a kind of argument doesn't make a lot of sense because the importance of family is absolutely not a strictly Confucian characteristic. It can be found that in Europe, under Christian culture, the importance of family is not second to that in China. The importance of family in China is more attributable to the agricultural economic system, especially the production mode based on the family unit, rather than Confucianism.

Families served the function of protection in agricultural times, as was true in Western countries as well. Therefore, it is not solely a characteristic of Asian countries. To resort to the traditional family unit in Asian industrialized countries or areas does not only hinder the development of social security but also deliberately prevents the development of welfare for the disabled.

With Confucianism, its social ideas should be mentioned first. The most famous Confucian literature is the Analects of Confucius which includes the CHAPTER OF GREAT HARMONY (TA TUNG ). This states that

"Provision is secured for the aged till death, employment for the able-bodied, and the means of growing up for the young. Helpless widows and widowers, orphans and the lonely, as well as the sick and the disable, are to be well cared for."

10 Leisering, Ssozuakpolitische und rechtliche Gestaltung der Behindertenpolitik in Asien, in: v. Maydell/Pitschas/Schulte (Hrsg.), Behinderung in Asien und Europa im Politik- und Rechtsvergleich, 2003, 425-439.

11 Please refer to the reports by Ninh Do Thi Hai, Kruse/Schmidt, Prasojo and Gnanasekaran, all in the Books "Teilhabe behinderter Menschen an der Bürgergesellschaft in Asien und Europa" and "Behinderung in Asien und Europa im Politik- und Rechtsvergleich", both edited by von Maydell, Pitschas and Schulte.

12 Leisering, Fn. 10, 429. 
Both Taiwan and China have a similar conservative attitude towards a social security system, in particular their passive attitude toward social assistance, which could be derived from the traditional Confucian attitude towards the aged, sick and disabled. It is a very interesting question as to why there exists such a similarity in attitudes between Taiwan and China when the economic and political developments for both sides have been quite divergent. It could be argued that Confucian social thought has had only a very limited influence in practice. However, in reality, Confucianism has had a lot of influence on social security attitudes in both Taiwan and China.

It is quite clear that Taiwan and China provide special protection for public servants, including military servicemen, civil servants and educators. The extent of the protection they enjoy is not only more comprehensive than for other citizens but far exceeds that which is available to public servants in Europe and the U.S.

Such a system is closely connected to the Confucian idea of class where society is divided into a ruling class with knowledge and a ruled class without knowledge. Government officials traditionally enjoyed many privileges. Nowadays, social security has been turned into privileges for government officials. Such a misunderstood and abused social security system is most likely far from what the founders of social security expected it to be.

\subsection{The International foundation}

As mentioned above, the social policy of the PRC before the 1970s was deeply influenced by communists, that is to say, the Communist International.

Similarly, the origin of Taiwan social insurance also has a close relationship with international society and international organizations. In addition to the advocating of scholars who studied in Europe or America, the influence of the implementation of Japan's social insurance system, the influence of America during WWII and the participation by Taiwan in international organizations, the UN and the ILO in particular, all have contributed greatly to the implementation of Taiwan's social insurance system.

The role and influence of international organizations play an essential role. Taiwan was a member of the ILO and the UN from the 1950s into the 1970s when the establishment of social insurance was closely connected to the goals of the ILO and the U.N. Afterwards, Taiwan's social policy was predisposed towards a high level of privatization, characterized by the unique development of compulsory employer's retirement benefits and severance pay in 1984, when Taiwan was withdrawing from international organizations one after another.

Such a development was very different from other countries at that time and concurrently it was hard for Taiwan to seek for international support of this policy. Therefore, the privatization process did not move further until the 1990s. At this period of time there came huge changes. 
Under the situation, the pension reform proposal of the World Bank-the replacement of social insurance by individual retirement accounts, facilitated all the anti-welfare proponents in gaining full support and becoming more arrogant. The pension reform proposal of the World Bank is the most important factor in the implementation of individual retirement accounts in Taiwan. The governments in Taiwan, China, and in several other Asian countries as well as countries hostile to social welfare all strongly endorsed the World Bank proposal. Without the World Bank, China and Taiwan would not have necessarily introduced individual retirement accounts.

Presently, even Stiglitz and Modigliani sternly criticize individual retirement accounts. They think that this view of individual accounts is wrong and is based on a series of myths which only serve the interests of asset managers ${ }^{13}$. Such accusations, if confirmed, will prove the World Bank to be a misguided or perhaps even an evil institution which brings disasters, rather than blessings, to human beings. Labor, especially the disabled labor in question, who cannot get social protection and even suffer loss, could possibly file lawsuits and claim damages against the World Bank. The question is whether the World Bank can afford it.

It is regrettable when the World Bank has promoted its policy of individual accounts which has become the accepted system in many countries. In the meantime, what is the ILO doing? The ILO not only is passive, but in fact is doing nothing. The passiveness of the ILO is a major cause of this disaster of the widespread introduction of an individual account system.

\subsection{Academic Foundation}

The above described privatization has not just impeded development of social security systems but also made it hard to expect much protection for the disabled. Such a development is attributable to domestic cultural factors and political factors, particularly politicians' ignorance of social security under limited democracy. As mentioned earlier, the politicians' can justify their choice of individual retirement accounts as opposed to social insurance by the support given to individual retirement accounts by the World Bank.

Besides the international support for individual retirement accounts, who are the other supporters of individual retirement accounts in the home countries besides the politicians? The answer is academics, particularly economists. Economists in Taiwan, most of them studied in the US, are mostly against a social security system. They favor the market mechanism of privatization, especially individual accounts. This is why the labor insurance of Taiwan up till now has not adopted a pension system and the key reason for why Taiwan practices an obligatory individual ac- 
count system. In Taiwan, a lot of economists refer to the economist and political philosopher, Friedrich Hayek, to justify their opposition to social security. However, the economists in Taiwan confuse the situation by seemingly not being aware or letting it be known that Hayek, who strongly defended a free economy and classical liberalism, was not against welfare states as long as the social welfare was not monopolized by the government. In addition, the economists in Taiwan ignore the criticisms of the World Bank's welfare policies by Stiglitz and Modigliani. Thus, we can see that the opposition of the economists is not based on their academic knowledge but on their own personal or political preferences and interest groups. Such economists work for the government for the interest of themselves, specific groups, or for the governing class.

Various academic fields in Taiwan are guilty of opposing or not supporting social welfare systems. The academic fields of political science and public administration have long ignored social welfare. The field of law has been no better. In Taiwan, some Grand justices who have been working as jurists, for example, Yu Shei-ming, have strongly advocated an individual account system in their opinions given on a Constitutional interpretation. Even many scholars and professors of sociology, social work, social policy, and social welfare prefer privatization. Some of them have shown high hopes for welfare states, but they do not necessarily identify with the social insurance systems implemented in industrialized countries such as Germany. Basically, such scholars are mostly educated in the US or the UK. Some of these scholars and professor have gone so far as to indicate that they regard social insurance as a low-level social protection system, a means of social control, and a system which is only limited to the socially advantaged workers ${ }^{14}$. They always cite EspingAnderson and refer to Germany's social welfare as a conservative welfare state, or as second-rate welfare state. What sociologists prefer is definitely not social insurance but a public medical system and more social service of the kind provided by the National Health Service. Furthermore, a lot of sociologists also prefer a private individual account system. For example, Professor Ho-Sheng Chan, the former minister of the Council of Labor Affairs, is an advocate and promoter of individual accounts.

Such a phenomenon of favoring privatization is also seen in China. Li Tieying, the former President of the Chinese Academy of Social Sciences once stated: "As far as I know, European and American states are also reforming their social security systems, mainly to resolve the problem of "welfare illness" formed as a result of excessively good welfare treatment. Singapore, Chile and other countries are applying the model of compulsory savings social security ${ }^{15}$."

$14 \mathrm{Li}$-Yeh Fu, The Social Control Essence of the social Insurance System in Taiwan (in Chinese), A Radical Quarterly in Social Studies, No. 15 (1993), 165-190.

15 Tieying $\mathrm{Li}$, Reform of China's Social Security System Faces Arduous Tasks and Long Way to Go General Preamble to SSSS - Series of Social Securities, in: Dongjin Wang (ed.), The Reform and Development of Social Security System in China, 2001, 1-19. (17). 
It is quite obvious that in China, particularly in academia, a social security system in which social insurance constitutes a major part is highly questioned. On the contrary, the Chilean model, where you have individual accounts of compulsory savings is highly welcome, and even is regarded as the best solution to solve the welfare state issues. From what we have written, it can now be more easily understood why China would adopt the path of individual accounts. However, through such a selection of individual accounts, how can the disabled expect to obtain effective protection?

Such a loss of confidence in social insurance as seen in welfare states does not come just from academia, in particular economists, and other sources in Taiwan or China. As a matter of fact, even some European sociologists also strongly criticize social insurance. When Leisering cited Esping-Andersen to classify Continental countries like Germany as a Conservative welfare state which particularly attaches importance to security while relatively ignoring freedom and equality ${ }^{16}$, this also explains why the social security system in Germany or other Continental countries, particularly social insurance, is considered by some to be an inferior second-rate system. This argument that social insurance is absolutely not the best choice appears to have become dominant among sociologists.

\section{Concluding Remarks}

From what was mentioned above, we can see that the influence of academic factors is far greater than that of political or cultural factors on disabled welfare policy in China and Taiwan. Academe has played a vital role in both social policy and disabled welfare policy. The behavior of a lot of political figures is a sort of conditioned behavior dominated by academic culture.

We can also see that international factors, most of the time, outweigh domestic factors. The establishment and development of social security can be attributed to international influence and so can its stagnation and obsoleteness. As a result, the impact of the international foundation for disabled policy cannot be ignored and obviously has a greater influence than domestic culture.

If academic obstacles and international obstacles cannot be removed, the development of social security is doomed. Without a complete health insurance, how can a disabled person gain sufficient medical care and rehabilitation? This is a key issue in China. If we keep being frenzied with an individual retirement account system, how can the disabled, especially the economic security of disabled labor, be fully protected? The same problem exists in many Asian countries such as Taiwan, China, Hong Kong, and Singapore. Taking into consideration this problem, Japan implemented long term care insurance in 2000, which has been a tremendous exception to welfare policies in other Asian countries. The experience of Japan also explains that 
it is not impossible to practice a social security system in Asia which incorporates the key functions of social insurance. The National Health Insurance implemented in Taiwan also demonstrates the possibility of practicing a beneficial form of health insurance in an area dominated by Chinese culture.

If the Word Bank, and in addition, Esping-Andersen still play a dominant role in the formation of social welfare policy, a sound social security system in Asia will always be an impossibility. With the influence of the World Bank and EspingAndersen, a competent disabled welfare policy in Asia will always be difficult to achieve.

To sum up, the underdevelopment of disabled welfare in Asia can be partially attributable to the traditional culture, religion and the political development in the area. However, this underdevelopment is also attributable to international factors, especially the anti-welfare stance of the Word Bank and academic factors, especially the anti-welfare viewpoint of many international and domestic economists. What can not be overlooked though is that without the passive reaction of the ILO and the sociologists, the anti-welfare policies that have been put in place in China and Taiwan could definitely not have been implemented. 


\title{
7. Generalbericht
}

\author{
Von Alexander Graser
}

1. Die Zielsetzung des vorliegenden Beitrags 488

2. Das Potential des Projekts 488

2.1. Der bisherige Projektverlauf 488

2.2. Die Attraktivität des Projekts und ihre Gründe 489

2.2.1. Verschiedenheit der betrachteten Regionen 489

2.2.2. Behindertenrecht also Lackmustest der Sozialstaatlichkeit $\quad 490$

2.3. Zur Ambitioniertheit des Projekts 491

2.3.1. Vielzahl der einbezogenen Länder 491

2.3.2. Verschiedenheit der betrachteten Regionen 492

2.3.3. Breite des Themas 492

2.3.4. Komplexität des Themas 492

3. Der Workshop im Kontext der vorangegangenen Tagungen 493

3.1. Zielsetzungen des Workshops im Lichte der vorangegangenen Projektschritte

3.2. Ausleuchten des „kulturwissenschaftlichen Hintergrundes“ 494

3.3. Brückenbau ausgehend vom Boden der Rechts- und Verwaltungswissenschaft 495

3.3.1. Vom materiellen Recht ausgehende Erwägungen 495

3.3.2. Vom prozeduralen Recht ausgehende Erwägungen 496

3.3.3. Verwaltungsstrukturen und Zivilgesellschaft 497

3.4. Demographischer Wandel als Querschnittsthema 498

3.4.1. Zunahme von Behinderungen aufgrund steigender Lebenserwartung

498

3.4.2. Steigerung der Lebenserwartung für Menschen mit Behinderung

4. Überlegungen zur Fortsetzung des Projekts 499

4.1. Bisherige Erträge $\quad 499$

4.2. Ausrichtung weiterer Schritte $\quad 500$

4.2.1. Ausgleich bestehender Asymmetrien 501

4.2.2. Exemplarische Fokussierung 501 


\section{Die Zielsetzung des vorliegenden Beitrags}

Der vorliegende Generalbericht versucht ein Resümee des Workshops, stellt ihn in Zusammenhang mit den vorangegangenen Tagungen und erörtert die Perspektiven einer Fortsetzung des Projekts. Eine Zusammenfassung der einzelnen Beiträge ist dagegen nicht Ziel dieses Berichts. Eine vorläufige und knappere Fassung der hier entfalteten Gedanken wurde bereits während der Tagung vorgestellt, und zwar zu Beginn der Schlussdiskussion, deren Inhalt im Folgenden so weit wie möglich berücksichtigt wurde.

\section{Das Potential des Projekts}

Behindertenpolitik und -recht in Asien, Europa und darüber hinaus vielleicht auch noch in weiteren Regionen der Welt zu vermessen, ist ein faszinierendes Vorhaben, und zugleich auch ein höchst ambitioniertes. In diesem einleitenden Abschnitt wird zunächst der bisherige Projektverlauf knapp skizziert. Sodann soll substantiiert werden, was die besondere Attraktivität und Ambitioniertheit des Projekts ausmacht.

\subsection{Der bisherige Projektverlauf}

Die Faszination, die das Projekt ausübt, lässt sich bereits daran ablesen, dass es eine wachsende Gruppe von Projektpartnern und darüber hinaus eine Vielzahl weiterer Tagungsteilnehmer über einen inzwischen mehrjährigen Zeitraum beschäftigt hat. Erwähnung verdienen insoweit zunächst die in den Jahren 2001 und 2002 durchgeführten Konferenzen, deren Ergebnisse in jeweils einem Sammelband unter den Titeln „Teilhabe behinderter Menschen an der Bürgergesellschaft in Asien und Europa - Eingliederung im Sozial- und Rechtvergleich“ sowie „Behinderung in Asien und Europa im Politik- und Rechtsvergleich (mit einem Beitrag zu den USA)“ in den Jahren 2002 und 2003 veröffentlicht worden sind. Als Herausgeber fungierten die Herren von Maydell, Pitschas und Schulte, die dieses Projekt konzipiert und seither in enger Kooperation verfolgt haben. Auch in der Phase zwischen dem zweiten Band und dem nunmehr durchgeführten Workshop sind sie in Kontakt und dem Thema verbunden geblieben, wovon eine Reihe weiteren Arbeiten zeugen, die in engem Bezug hierzu stehen. ${ }^{1}$ Für den jüngsten Workshop sind zu den drei bisherigen

1 So hat etwa Schulte in der Zwischenzeit vergleichend zum eng mit dem Behindertenrecht verbundenen Betreuungsrecht gearbeitet, und zwar insbesondere auch unter Einbezug asiatischer Perspektiven, vgl. dazu Schulte, Betreuungsrecht und soziale Grundsicherung, BtPrax 2006, 210 ff., ders., Betreuung: Rechtsfürsorge im Sozialstaat aus sozialrechtlicher Perspektive, in: Zander (Hrsg.), Rechtsfürsorge im Sozialstaat: Was ist Aufgabe der Betreuung? Ergebnisse des 9. Vormundschaftsgerichtstags, Bochum 2005, S. 29 ff.; sowie schließlich den auf Japanisch publizierten Beitrag „Theoretische Aufgaben des deutschen Betreuungsrechts“, in: Betreuungsrecht (Japan), 
Organisatoren noch die Herren Pörtner und Zachmann hinzugekommen, um die kulturwissenschaftliche Perspektive in der Konzeption stärker zu betonen.

\subsection{Die Attraktivität des Projekts und ihre Gründe}

Die augenfällige Faszination, die von diesem Projekt ausgeht, erscheint bei näherem Hinsehen durchaus erklärlich. Vor allem zwei Gründe kommen hierfür in Betracht.

\subsubsection{Verschiedenheit der betrachteten Regionen}

Der erste Grund hierfür dürfte zunächst darin liegen, dass es derart unterschiedliche Regionen sind, die einander gegenübergestellt werden. Auf der einen Seite wird Europa häufig und wohl auch mit einiger Berechtigung als Wiege des modernen Sozialstaats apostrophiert - bei allen Problemen, die dessen Erhalt und Anpassung seit langem aufwerfen. ${ }^{2}$ In Asien auf der anderen Seite geht es demgegenüber um eine deutlich jüngere Entwicklung, die sich mitunter in Anlehnung oder Abgrenzung zu den europäischen Erfahrungen vollzogen, dabei aber durchaus neue Formen hervorgebracht hat - oder diese angesichts der erst allmählich durchgreifenden ökonomischen Entwicklung in manchen der in das Projekt einbezogenen Staaten auch erst hervorzubringen begonnen hat.

Diese Konstellation verspricht Erkenntnisgewinne gleich in mehrfacher Hinsicht. ${ }^{3}$ Nicht nur besteht die Aussicht, dass der Vergleich prospektiv eine - ggf. adaptierte Rezeption übertragbarer Politiken vorbereiten könnte. ${ }^{4}$ Vielmehr verheißt retrospek-

4/2003, S. 352 ff.; Erwähnung verdient des Weiteren die von Pitschas betreute vergleichende Dissertation von Won über die bildungs- und insbesondere schulpolitische Behandlung junger behinderter Menschen; vgl. schließlich ferner noch den im Max-Planck-Institut für ausländisches und internationales Sozialrecht entstandenen Band über Perspektiven der schulischen Integration von Kindern mit Behinderung - Vergleichende und interdisziplinäre Betrachtungen, Baden-Baden 2004 (herausgegeben von Becker/Graser).

2 Die verschiedenen Herausforderungen, denen sich der Wohlfahrtsstaat gegenübersieht, werden in unzähligen Publikationen thematisiert; vgl. statt vieler beispielsweise zu den Folgen immer durchlässiger werdender Grenzen, Leibfried/Pierson (Hrsg.), Standort Europa. Sozialpolitik zwischen Nationalstaat und Europäischer Integration, Frankfurt am Main 1995, zur gesellschaftlichen Alterung Reinhard (Hrsg.), Demographischer Wandel und Alterssicherung, Rentenpolitik in neun europäischen Ländern und den USA im Vergleich, Baden-Baden 2001; mit einem vergleichenden Überblick zum Kostenanstieg im Gesundheitswesen vgl. Henke/Schreyögg, Towards sustainable health care systems, Berlin 2004, S. 27 ff.; zum Wandel der Arbeitswelt schließlich Zacher, Der Wandel der Arbeit und der sozialen Sicherheit im internationalen Vergleich, ZIAS 1999, S. 1 ff.

3 Für eine ausführlichere Auseinandersetzung mit den Erkenntnismöglichkeiten der Rechtsvergleichung, auf der die vorliegende Darstellung aufbaut, vgl. Graser, Dezentrale Wohlfahrtsstaatlichkeit im föderalen Binnenmarkt?, Berlin 2001, S. $110 \mathrm{ff}$.

4 Als klassisch gilt insofern die Formulierung, dass der Rechtsvergleich der Anreicherung des ,Vorrates an Lösungen“; vgl. dazu etwa Zweigert/Kötz, Einführung in die Rechtsvergleichung, 3. Aufla- 
tiv die Betrachtung vergangener Transfers solcher Politiken, der dabei erfolgten Anpassungen sowie der tatsächlich erzielten Wirkungen vielfältige Aufschlüsse über die Interaktion politischer Steuerung und sozialer Wirklichkeit. ${ }^{5}$ Und nicht zuletzt verspricht ferner die Gegenüberstellung der unterschiedlichen regionalen Entwicklungen in einer breiteren Makro ${ }^{6}$-Perspektive ein klareres Bild von den jeweiligen regionalen Charakteristika in diesem Politikfeld, das - bei aller Vorsicht - Rückschlüsse auf die institutionalisierten Werthaltungen und deren Kontrast erlaubt. ${ }^{7}$

\subsubsection{Behindertenrecht also Lackmustest der Sozialstaatlichkeit}

Ein weiterer Grund für die Faszination dürfte sein, dass mit der Behindertenpolitik ein Politikfeld gewählt wurde, in dem die humanitäre, menschenrechtliche Dimension noch stärker im Vordergrund steht als in den meisten anderen Bereichen der Sozialpolitik. Gewiss, auch in diesem Bereich können sich sozial- und wirtschaftspolitische Interessen einmal decken, kann Sozialpolitik, um es unverblümt zu fassen, sich „rechnen“, so wie es etwa von Studien- und Arbeitsförderung, ja in mancher Hinsicht auch von Gesundheits- und Rentenpolitik gesagt werden könnte. Man sollte diese Dimension schon deswegen nicht aus den Augen lassen, weil sie stets und auch im der Fall der Behindertenpolitik Möglichkeiten eröffnen könnte, die sonst nicht erreichbar wären. Doch bei allen Fortschritten, die ein in dieser Hinsicht geschärftes Bewusstsein der Behindertenpolitik bereits beschert haben mag oder noch zu bescheren verheißt, so wäre doch die Hoffnung, eine weitgehende Deckung ökonomischer und sozialpolitischer Interessen zu erreichen, in diesem Bereich wohl illusorischer noch als in den meisten anderen Feldern der Sozialpolitik.

Ähnliches gilt für die Möglichkeit, Regelungen in diesem Politikfeld als bloßes Produkt einer erfolgreichen Interessendurchsetzung durch die Betroffenen zu deuten. Wieder gilt, dass dies auch im Bereich der Behindertenpolitik keineswegs ausgeschlossen ist. So verzeichnet man vielerorts eine zunehmende Organisiertheit der Betroffenen $^{8}$ und hat es auch in diesem Politikfeld mit zuweilen schlagkräftigen Organisationen zu tun. Aber bei allem Respekt für diese Entwicklung wäre es meist doch irreführend, die entsprechenden Verbände mit Blick auf ihren Einfluss auch

ge Tübingen 1996, S. 14, sowie Rheinstein, Einführung in die Rechtsvergleichung, (herausgegeben von v. Borries), 2. Auflage München 1987, S. 26.

$5 \mathrm{Zu}$ diesem methodologischen Verständnis solcher Transferstudien - oder mit einem anderen Wort: der Rezeptionsforschung - als einer invertierten Form des Rechtsvergleichs siehe Hänlein, Sozialrechtsvergleichung als Rezeptionsforschung?, ZIAS 1998, S. $104 \mathrm{ff}$.

6 Eingehend zum Begriff des „Makrovergleichs“ Bartels, Methode und Gegenstand intersystemarer Rechtsvergleichung, Tübingen 1982, S. 127 f.

7 Prägend zu diesem Verständnis des Rechtsvergleichs als Kulturvergleich Häberle, Rechtsvergleichung im Kraftfeld des Verfassungsstaates, Berlin 1992; besonders deutlich beispielsweise auf S. 40 .

8 Insbesondere für China hat Ding $\mathrm{Na}$ diesen Befund hervorgehoben; mit einer entsprechenden Beobachtung auch bereits Darimont, Landesbericht China, in: v.Maydell/Pitschas/Schulte (Hrsg.), Behinderung in Asien und Europa im Politik- und Rechtsvergleich, S. 272 f. 
nur in die Nähe der großen Interessenorganisation im Bereich der Sozialpolitik zu rücken. Allein die quantitative Dimension dürfte, gerade wenn es um Menschen mit schweren Behinderungen geht, jeder solchen Analogie entgegenstehen. Umso mehr freilich sind vorgefundene Regulierungen eher humanitär denn interessenpolitisch zu deuten.

In diesem zweifachen Sinne also kann das Behindertenrecht als Lackmustest für die Existenz und Umsetzung sozialpolitischer Steuerungsambitionen - oder kurz: für Sozialstaatlichkeit ${ }^{9}$ - gelten. Es eignet sich mithin ideal für einen Vergleich, der nicht allein Regulierungstechniken, sondern auch die ihrer Wahl zugrunde liegenden Werthaltungen in den Blick nimmt.

\subsection{Zur Ambitioniertheit des Projekts}

So faszinierend das Projekt sein mag, und so groß seine Erkenntnispotenziale, so ambitioniert ist es auch. Wieder lässt sich dies an seinem bisherigen Verlauf ablesen: Die drei Tagungen wurden mit großem Aufwand vorbereitet, konzentriert durchgeführt und gründlich nachbereitet. Sie haben, davon zeugen die jeweiligen Publikationen, deutliche Fortschritte gebracht. Dennoch ist das Erkenntnispotenzial des Projekts noch nicht ausgeschöpft und könnte es in diesem Stadium auch noch nicht sein. Die Ursachen hierfür liegen jedenfalls im Rückblick auf der Hand: Verantwortlich sind die Vielzahl der einbezogenen Länder, die Verschiedenheit der Regionen, die Breite des Themas sowie schließlich auch dessen Komplexität.

\subsubsection{Vielzahl der einbezogenen Länder}

Was die Vielzahl angeht, so bedarf diese Feststellung kaum der weiteren Erläuterung. Zwar sind Mehrländervergleiche in den Sozialwissenschaften und insbesondere in den sozialwissenschaftlichen Politikanalysen keine Seltenheit. ${ }^{10}$ Etwas anderes gilt aber, wenn diese Vergleiche mit einer Tiefenschärfe angestellt werden, wie sie bei rechtsvergleichenden Ansätzen anzutreffen und insbesondere auch im vorliegenden Projekt angestrebt ist. Gemessen daran handelt es sich bereits aufgrund der schieren Zahl einbezogener Länder um ein breit angelegtes Unterfangen.

9 Umfassend zum Begriff bereits Zacher, Das soziale Staatsziel, § 25 in: Isensee/Kirchhof (Hrsg.), Handbuch des Staatsrechts, Heidelberg 1987.

10 Kritisch über die Erträge vieler solcher vergleichender Studien hat sich in jüngerer Zeit u.a. Marmor geäußert. Er attestiert dem (seinem!) Feld der "comparative policy studies...a considerable gap between promise and performance", und konstatiert darüber hinaus "an extraordinary imbalance between the magnitude and speed of the information flows and the capacity to learn useful lessons from them"; vgl. Marmor/Freeman/Okma, Comparative Perspectives and Policy Learning in the World of Health Care, Journal of Comparative Politics Vol. 7 (2005), S. 331. 
Mehr noch als die Anzahl schlägt freilich die Verschiedenheit der betrachteten Systeme zu Buche. Zwischen Japan, Südkorea, Indien und China liegen buchstäblich Welten, im Hinblick auf die ökonomische Entwicklung, Rechts- und Verwaltungstraditionen sowie geistesgeschichtlich-kulturelle Wurzeln. Die auf europäischer Seite betrachteten Systeme mögen demgegenüber zwar relativ homogen erscheinen. Aber auch hier sind die Unterschiede erheblich, gerade wenn man bedenkt, dass sich der Kreis der bisher einbezogenen Systeme keineswegs auf die Kernstaaten des vereinten Europas beschränkt.

\subsubsection{Breite des Themas}

Ein weiterer, nicht minder bedeutsamer Aspekt ist in diesem Zusammenhang die Breite des betrachteten Politikfeldes. Mit dem Begriff der Behinderung ist ein Phänomen umschrieben, dessen Ursachen und Erscheinungsformen ebenso vielfältig sind wie die Handlungen oder Handlungsabsichten, die es auf Seiten der Akteure kollektiver (Selbst-)Steuerung auslöst. Das Spektrum möglicher Maßnahmen reicht von Geldleistungen zur Deckung der Grundbedarfe bis hin zum gesetzlich festgeschriebenen Erfordernis rollstuhlgerechter Zugänge für Neubauten, von der Organisation und Finanzierung ambulanter heilpädagogischer Dienste zur Förderung entwicklungsverzögerter Kinder bis hin zur Definition steuerlich absetzbarer Sonderbelastungen infolge einer Behinderung.

\subsubsection{Komplexität des Themas}

Man hat es also mit einer Vielfalt sozialer Problemlagen, möglicher Politikziele und Umsetzungsinstrumente zu tun, und entsprechend komplex ist die Materie, die im Projekttitel so scheinbar schlicht mit „Behindertenrecht und -politik“ bezeichnet ist. Pflege-, Kranken-, Mindestsicherungssysteme sind angesprochen. Regulierungen vom Arbeits-, über das Steuer-, bis hin zum Baurecht sind einschlägig. Man muss die Organisation von Kindergärten, Schulen, Werkstätten, Heimen, die Interaktion staatlicher und privater Akteure einschließlich der Zwischenformen studieren, und dazu typischer Weise auch das Zusammenspiel verschiedener politischer Ebenen einbeziehen, von der Gemeinde bis - gerade in Europa - hinein in den transnationalen Raum. 


\section{Der Workshop im Kontext der vorangegangenen Tagungen}

Sind das Potenzial des Projekts, sein Reiz und seine Herausforderungen damit immerhin grob nachgezeichnet, so soll im Folgenden eine Zwischenbilanz gezogen werden. Oder - direkt gefragt: Wie ist der Stand nach der dritten Tagung?

\subsection{Zielsetzungen des Workshops im Lichte der vorangegangenen Projektschritte}

Wie von Maydell in seinem Einführungsreferat zum Workshop ausführte, war es ein Verdienst bereits der ersten beiden Tagungen innerhalb des Projekts gewesen, die Vielfalt und Komplexität des Themas, wie sie im Vorangegangenen nur angedeutet werden konnte, zu erfassen und zu konturieren. Zunächst und vor allem war dies aus rechts- und verwaltungswissenschaftlicher Perspektive geschehen, wie es auch der disziplinären Ausrichtung der in diesem Stadium am Projekt beteiligten Personen entspricht. Zugleich waren mit der zunehmenden Differenziertheit dieser rechts- und verwaltungswissenschaftlichen Analysen aber auch deren zahlreiche Bezüge zu jenen Rahmenbedingungen zunehmend deutlich geworden, die dem juristisch oder verwaltungswissenschaftlich geschulten Blick nicht unmittelbar zugänglich sind.

Folgerichtig wurde die nächste Tagung - der Workshop, der im Zentrum der vorliegenden Betrachtungen steht - ganz dem Ziel gewidmet, diesen Bereich besser auszuleuchten. Es galt also, vom Boden der rechts- und verwaltungswissenschaftlichen Bestandsaufnahmen ausgehend, die soziale Ambiance ${ }^{11}$ der rechtlichinstitutionellen Strukturen näher zu erkunden. Man kann insofern von einem im weiten Sinne rechts- und verwaltungssoziologischen Erkenntnisinteresse sprechen und dem Projekt in dieser Hinsicht ein beachtliches Innovationspotenzial attestieren. Denn bei allem Zuspruch, den die Interdisziplinarität seit einiger Zeit erfährt, werden derartige Fragestellungen gegenwärtig dennoch allzu selten verfolgt. ${ }^{12}$

$11 \mathrm{Zu}$ diesem Begriff vgl. Häberle, Die Wesensgehaltsgarantie des Art. 19 Abs. 2 Grundgesetz, 3. Aufl. 1983, S. 415; auch schon Zacher, Vorfragen zu den Methoden der Sozialrechtsvergleichung, S. 21 ff., in: ders. (Hrsg.), Methodische Probleme des Sozialrechtsvergleiches, Colloquium der Projektgruppe für Internationales und Vergleichendes Sozialrecht der Max-Planck-Gesellschaft, Berlin 1977, der auf S. 61-66 zahlreiche Facetten dieser „Ambiance“ differenziert.

12 Eine Renaissance der Rechtssoziologie (und -anthropologie) im Gefolge der zunehmenden Pluralisierung positiver Rechtsordnungen verzeichnet in jüngerer Zeit Günther, Rechtspluralismus und universaler Code der Legalität: Globalisierung als rechtstheoretisches Problem, in: ders./Wingert (Hrsg.), Die Öffentlichkeit der Vernunft und die Vernunft der Öffentlichkeit, Festschrift für Jürgen Habermas, Frankfurt am Main 2001, S. 539 ff. Zugleich attestiert er dieser Disziplin bei dieser Gelegenheit zu Recht, seit langem ein Schattendasein geführt zu haben (S. 552). 


\subsection{Ausleuchten des „kulturwissenschaftlichen Hintergrundes“}

Der explorativen Zielsetzung des Workshops entsprechend, wurde weit ausgegriffen. Um eine „kulturwissenschaftliche Grundlegung und (um) Erklärungshypothesen" sollte es gehen. Dementsprechend reichte das Spektrum von Aspekten der Religions- und Geistesgeschichte ${ }^{13}$ über mikrosoziologische Betrachtungen der Bedingungen in Familie, ${ }^{14}$ Schule $^{15}$ und am Arbeitsplatz ${ }^{16}$ bis hin zu Analysen der politischen Interessen- und Entscheidungskonstellationen ${ }^{17}$ im Bereich der Behindertenpolitik sowie deren ökonomischer Voraussetzungen. ${ }^{18}$

Bereits diese Aufzählung macht deutlich, dass es nicht um eine umfassende Würdigung all dieser Umstände und ihrer Relevanz für die rechts- und verwaltungswissenschaftlich erfassten Strukturen gehen konnte. Im Vordergrund stand stattdessen das Bestreben, anhand einzelner Betrachtungen exemplarisch zu plausibilisieren, welche Bedeutung diesen Aspekten im Kontext des Projekts zukommen kann. Diese Plausibilisierung ist ohne Frage gelungen, und zugleich wurde dabei eine Vielzahl von Impulsen für künftige Projektschritte gegeben.

So wurde beispielsweise in mehreren Beiträgen ${ }^{19}$ zur Situation in unterschiedlichen Regionen Asiens thematisiert, dass von Geburt an behinderte Menschen als Strafe für Eltern und Familie wahrgenommen würden. Dies ist ein Umstand, der die Rahmenbedingungen behindertenpolitischer Interventionen wesentlich beeinflusst und von den in Europa vorgefundenen absetzt.

Einen deutlichen Kontrast insbesondere mit Blick auf die politischen Zielsetzungen hat ferner der Bericht über die Förderschulentwicklung in Südkorea zu Tage gefördert. ${ }^{20}$ Während man in Europa gegenwärtig einen deutlichen Akzent auf integrative Beschulungsansätze legt, dominiert in der koreanischen Entwicklung klar die Idee einer institutionellen Separation. Auch wenn derlei Leitbilder gerade in einer historischen Perspektive keineswegs unveränderlich erscheinen - gerade in Deutschland liegt der Paradigmenwechsel in diesem Bereich noch nicht lange zurück,$-{ }^{21}$ so müssen die hier hervortretenden Unterschiede doch berücksichtigt werden, wenn es um einen Vergleich der aktuellen Lage geht.

Außer im Referat von Pörtner und in den Diskussionsbeiträgen von Zachmann wurden diese Aspekte insbesondere auch in den Beiträgen von Gnanasekaran und Won thematisiert. Vgl. dazu vor allem den Beitrag von Motozawa.

15 Vgl. dazu den Beitrag von Won.

16 Vor allem im Zusammenhang mit den Beiträgen über Korea und Japan wurden diese Aspekte diskutiert.

17 Eine besondere Betonung hat dieser Aspekt im Referat von van Langendonck erfahren.

18 Dieser Aspekt wurde insbesondere in Pitschas' Analyse der Interaktion mehrerer Verwaltungsebenen in Deutschland eingehend behandelt.

19 Das gilt namentlich für die Beiträge von Gnanasekaran, Won und $\mathrm{Na}$.

20 Von Won zum Thema „Bildungspolitik für junge Menschen und die Entwicklung von Gesetzgebung und Rechtsprechung“.

21 Vgl. dazu jüngst Powell, Behinderung in der Schule, behindert durch Schule? Wie ,schulische Behinderung" institutionalisiert wurde, in: Waldschmidt/Schneider (Hrsg.), Disability Studies, Kultursoziologie und Soziologie der Behinderung, Bielefeld 2007, S. $321 \mathrm{ff}$. 
Ebenfalls die politischen Zielsetzungen und Problemdefinitionen betreffend, hat Pörtner in seinem einleitenden Beitrag vorgeführt, dass nicht einmal die grundlegende Prämisse des Projektes unhinterfragt bleiben könne. Gemeint ist die implizite Annahme, dass die Verbesserung der Situation behinderter Menschen Gegenstand und Auftrag kollektiver Selbststeuerung - „der Politik“ mit anderen Worten - zu sein habe. Offensichtlich ist dieser Aspekt für das gesamte Projekt von fundamentaler Bedeutung. Zwar haben die neueren internationalrechtlichen Vorgaben, ${ }^{22}$ wie sie insbesondere in der Referaten von Trenk-Hinterberger und Schulte erläutert wurden, eine gewisse Universalisierung dieser womöglich westlich-europäischen Prämisse gebracht, auf deren Grundlage die ,globalere“ Ausrichtung des vorliegenden Projektes jedenfalls berechtigt erscheint. Zumal aber Konkretion und Bindungsgrad solcher internationalrechtlicher Zielvorgaben bekanntlich gering und damit die Räume für unterschiedliche nationale Ausprägungen groß sind, bleibt der Hinweis von Pörtner dennoch gültig und sollte die weiteren Schritte des Projekts im Sinne eines, wie er es selbst formuliert hat, ,mitlaufenden Zweifels“ begleiten.

\subsection{Brückenbau ausgehend vom Boden der Rechts- und Verwaltungswissenschaft}

Parallel und gleichsam als Gegenstück zu den primär gesellschafts- oder kulturwissenschaftlich orientierten Beobachtungen gab es eine Reihe von Beiträgen, die ihren Ausgangspunkt erneut in den Rechts- oder Verwaltungswissenschaften genommen haben, um aber von dort dann mit dem Bau von Brücken zu jenen Umständen außerhalb ihrer genuinen disziplinären Zuständigkeit zu beginnen. Wieder war das Spektrum breit.

\subsubsection{Vom materiellen Recht ausgehende Erwägungen}

Ausgehend von den Darstellungen zum materiellen Recht wurde beispielsweise wiederholt erörtert, welche Leitbilder ${ }^{23}$ den jeweils betrachteten behindertenpolitischen Maßnahmen zugrunde lägen - von Fürsorge über Gleichstellung bis hin zu Inklusion - und inwiefern sie unter den jeweiligen Bedingungen adäquat und für die verschiedenen Gruppen von Betroffenen interessengerecht seien. Dabei wurde nicht nur ein breites Arsenal rechtlicher Instrumente sichtbar, das der gesetzgeberischen Inspiration und so einem klassischen Ziel der Rechtsvergleichung dienen könnte. ${ }^{24}$ Vielmehr wurde auch die Kontextgebundenheit dieser Instrumente deutlich, deretwegen simple Übertragungsversuche von vornherein ausscheiden. Besonders einge-

22 Namentlich handelt es sich um die UN Convention on the Rights of Persons with Disabilities aus dem Jahr 2006; für nähere Angaben vgl. insbesondere das Referat von Schulte.

23 Besonders prononciert hat solche Leitbilder insbesondere Trenk-Hinterberger in seinem Beitrag über „Leistungen für behinderte Menschen in Europa“ herausgearbeitet.

Vgl. dazu oben Fn. 3-7 sowie den zugehörigen Text. 
hend behandelt wurde dies in Cheons Bericht über die Entwicklung des südkoreanischen Behindertenrechts, die gekennzeichnet ist von einer raschen, aber doch kontextsensiblen und mithin adaptierenden Rezeption westlicher Regelungsansätze.

Ebenfalls aus den Erörterungen des materiellen Rechts ergaben sich wiederholt aufschlussreiche Perspektiven auf die Interdependenz juristischer Kategorisierungen und politischer Interessenformationen. ${ }^{25}$ Auffällig ist dies beispielsweise im Hinblick auf den Behindertenbegriff. Ein breiter und umfassender Zuschnitt der entsprechenden gesetzlichen Begriffe könnte insofern zur Formation großer und zunächst ähnlich interessierter Gruppen führen und diesen mehr Durchsetzungskraft verleihen. Als ein einschlägiges Beispiel wurden in der Diskussion die USA erwähnt. ${ }^{26}$ Dagegen trifft man nicht selten auf eher enge Definitionen. Beispielsweise lässt sich wohl nur so erklären, warum man in Indien von einer insgesamt soviel geringen Zahl behinderter Menschen ausgeht. Hinzu kommt, dass die Grenzziehungen, wie sie gerade in stark ausdifferenzierten Systemen des sozialen Schutzes unvermeidbar sind, mitunter diffizil sind und ähnliche Interessenlagen künstlich auseinanderdividieren könnten. Als Beispiel mag in Deutschland etwa die Abgrenzung zwischen Rehabilitation und Pflege dienen.

\subsubsection{Vom prozeduralen Recht ausgehende Erwägungen}

Viel Aufmerksamkeit wurde ferner den sogenannten „enforcement“-Fragen zuteil, also dem Aspekt der effektiven Durchsetzung von (hier: behinderten-) politischen Zielen und Regelungen. Dabei kann es sich um Durchsetzung mittels sowohl exekutiver als auch judikativer Mechanismen handeln, und wie zuvor wurde auch hier das Wechselspiel dieser institutionellen Strukturen mit den im weiten Sinne sozialen Rahmenbedingungen augenfällig.

Im Einzelnen ging es auch in diesem Themenfeld zunächst erneut darum, aus der vergleichenden Betrachtung ein Arsenal unterschiedlicher Instrumente einschließlich der Erfahrungen mit ihrem praktischen Einsatz zusammenzutragen. Eingehend wurden erstens die verschiedenen Wege administrativer Rechtsdurchsetzung erörtert, die von kommunalen oder betrieblichen Behindertenbeauftragen über weniger dezentral angesiedelte Ombudspersonen bis hin zu Behörden reichen, die eigens mit der Überwachung oder gar Durchsetzung der entsprechenden Normen befasst sind. Insbesondere Köhlers Analyse der entsprechenden Strukturen in Schweden hat diese Perspektive in den Vordergrund gerückt. these zum Zusammenhang zwischen dem Geltungsbereich eines sozialrechtlichen Regelwerks und seinem materiellen Schutzgehalt, ZIAS 2003, S. 319 ff.

26 Näher zum breiten Behindertenbegriff in den USA und zum entsprechend hohen Anteil der Behinderten an der dortigen Bevölkerung, Graser, ebda., S. 320 f. sowie ders., Landesbericht USA, in: v.Maydell/Pitschas/Schulte (Hrsg.), Behinderung in Asien und Europa im Politik- und Rechtsvergleich, S. 234. 
Im Hinblick auf die Formen der klassischen, also judikativen (Rechts-)Durchsetzung wurde zweitens vor allem über die Beschränkungen gesprochen, wie sie mit der typischer Weise retrospektiven und auf den Einzelfall beschränkten Ausrichtung dieser Verfahren einhergehen. Als alternative Optionen stehen class actions oder Verbandsklagen zur Diskussion. Freilich handelt es sich auch dabei um Instrumente, die nicht unbesehen in einen neuen Kontext importiert werden sollten.

Mit dem Verweis auf die Kontextabhängigkeit dieser Instrumente ist drittens ein weiterer Ertrag angesprochen, den die Betrachtungen der enforcement-Fragen gebracht haben. Denn auch in diesem Zusammenhang wurde deutlich, wie sehr die Effektivität der rechtlichen und Verwaltungsstrukturen anhängt von in weiterem Sinne sozialen Parametern. So läuft etwa das Instrument der Verbandsklage offensichtlich leer, solange es an einer hinreichend organisierten Verbandsstruktur fehlt. Grundlegender noch wurde mit Blick auf alle Instrumente, die einen subjektivrechtliche Komponente enthalten, wiederholt die These aufgestellt, dass deren gerichtliche Rechtsverfolgung in manchen asiatischen Ländern deutlich weniger üblich sei als in den westlichen und insbesondere auch den europäischen Kulturen. Gewiss bedürfte diese Behauptung noch einer empirischen Fundierung speziell für den gegebenen Kontext des Behindertenrechts. Fest steht aber, dass dieser Unterschied für einen eventuellen Transfer von Regelungsinstrumenten aus oder nach Europa offensichtlich von erheblicher Bedeutung wäre.

\subsubsection{Verwaltungsstrukturen und Zivilgesellschaft}

Die soeben erwähnten Verbandsstrukturen sind freilich nicht nur aus prozessrechtlicher, sondern auch aus verwaltungswissenschaftlicher Perspektive von zentraler Bedeutung. Diese - oft mit dem schillernden Begriff der Zivilgesellschaft umschriebenen - Strukturen „Zwischen Staat und Markt“, wie sie im (leider entfallenen) Referat von Lachwitz bezeichnet sind, sind eine entscheidende Determinante für die konkrete Gestalt der behindertenpolitischen Verwaltungsorganisation - und darüber hinaus auch für die Möglichkeiten der Behindertenpolitik insgesamt. Ein Blick auf die Rolle, welche die freien Träger in Deutschland für die Erbringung behindertenpolitischer Leistungen spielen, verdeutlicht dies eindrucksvoll. ${ }^{27}$

Wenn es darum geht, Behindertenrecht und -politik zu vergleichen und insbesondere mit Blick auf ihre Gestaltungsmöglichkeiten zu erfassen, kommt man nicht umhin, diese zivilgesellschaftlichen Strukturen und ihre vielfältigen Verschränkungen mit den unterschiedlichen Ebenen staatlicher Verwaltung einzubeziehen. Dieser Blickwinkel der - mit einem Modewort - ,governance“-Forschung ist für die Behin-

27 Für eine systematische Auseindersetzung mit diesen „privaten“ Erscheinungsformen unter dem Blickwinkel ihrer Beziehung zum Staat siehe Geis, Die öffentliche Förderung sozialer Selbsthilfe Verfassungsrechtliche Grundlagen und verwaltungsrechtliche Ausgestaltung, Baden-Baden 1997. 
dertenpolitik unumgänglich. Insbesondere Pitschas hat diese Aspekte in seinem Referat $^{28}$ wie auch im Rahmen der Diskussionen wiederholt eingebracht.

\subsection{Demographischer Wandel als Querschnittsthema}

Besondere Berücksichtigung erfuhr im Rahmen des Workshops ferner der Aspekt des demographischen Wandels - teils in Form eines Querschnittsthemas, das sämtliche Referate berührte und dementsprechend in unterschiedlichen Zusammenhängen immer wieder zur Sprache kam; teils aber auch im Rahmen des eigens hierauf fokussierenden Beitrags von Kruse. Danach ist der demographische Wandel ist für die Behindertenpolitik vor allem in zweierlei Hinsicht relevant.

\subsubsection{Zunahme von Behinderungen aufgrund steigender Lebenserwartung}

Zum einen ist im Zuge einer steigenden Lebenserwartung damit zu rechnen, dass das Phänomen altersbedingter Behinderungen zunehmen wird. Das kann jedenfalls dann gelten, wenn dieser Zuwachs auf einer Verlängerung einer dem Lebensende vorangehenden Phase wahrscheinlicher Gebrechlichkeit beruht. Für diese Annahme spricht der Umstand, dass die Lebenserwartung gerade in den Industriestaaten unter anderem aufgrund einer Ausdehnung der Phase des hohen Alters steigt. ${ }^{29}$ Allerdings bedürfte diese Annahme weiterer empirischer Erhärtung. Dabei kommt dem rechtlichen Begriff der Behinderung und den damit einhergehenden Abgrenzungsproblemen offensichtlich gesteigerte Bedeutung zu. Hier eröffnet sich eine Perspektive für künftige Forschung an der Schnittestelle von empirischer Soziologie und insbesondere demographischer Forschung auf der einen Seite und Rechtswissenschaft auf der anderen.

Ein Konnex zwischen steigender Lebenserwartung und der relativen Zunahme von Behinderungen kann ferner auch noch auf andere Entwicklungen zurückzuführen sein, die nicht allein die Phase des hohen Alters betreffen. So könnten medizinische Fortschritte etwa im Bereich der Versorgung Frühgeborener nicht nur die Lebenserwartung, sondern auch die relative Häufigkeit von Behinderungen steigern. Ähnliches könnte - mutatis mutandis - auch für Behandlungsmöglichkeiten in anderen Lebensphasen gelten. Abermals kann zu diesem Aspekt nur auf die Notwendigkeit einer weiteren Erforschung dieses relativ wenig behandelten Gebiets verwiesen werden, und insbesondere auf die Notwendigkeit (einer Rezeption) von demographi-

29 Einen weiteren Rückgang der Sterblichkeit in hohem Alter verzeichnen in jüngerer Zeit Rau/Soroko/Jasilionis/Vaupel: 10 years after Kannisto: further evidence for mortality decline at advanced ages in developed countries. MPIDR working paper WP-2006-033, Rostock 2006, im Internet verfügbar unter: http://www.demogr.mpg.de/papers/working/wp-2006-033.pdf 
schen Studien speziell zu den in diesem Projekt betrachteten Regionen und Fragestellungen.

\subsubsection{Steigerung der Lebenserwartung für Menschen mit Behinderung}

Noch ein weiterer, seltener thematisierter Konnex zwischen demographischer Entwicklung und Behinderung wurde in dem Referat von Kruse mit großer Eindringlichkeit unterstrichen. Kruse bezog sich auf neuere Studien, welche die Vermutung nahelegen, dass die Lebenserwartung von Menschen mit Behinderung wesentlich gesteigert werden könne, wenn sie auch nach der Phase der Berufsqualifikation und mithin in höherem Alter noch systematisch gefördert werden.

In einer humanitären Perspektive erfordern diese Befunde dringend ein Umdenken in der Gestaltung speziell von Heimumgebungen, aber auch des breiteren Angebotsspektrums der einschlägigen Sozialleistungssysteme. Diese Erkenntnisse betreffen, wie Kruse betonte, zunächst einmal Deutschland, dürften aber generalisierbar sein und könnten mithin maßgebend für eine entsprechende Rechtsentwicklung auch in anderen Regionen werden. Es wäre lohnende Aufgabe weiterer Forschung in diesem Bereich, die konkreten Erfordernisse und Möglichkeiten solcher eventuell gebotenen Anpassungen der Rechts- und Verwaltungsstrukturen in den verschiedenen Betrachtungslängern näher zu eruieren.

\section{4. Überlegungen zur Fortsetzung des Projekts}

Die vorangegangenen Erörterungen mögen jenen Befund untermauert haben, der vorab bereits angedeutet worden war: So ertragreich die bisherigen Tagungen einschließlich des hier referierten Workshops auch gewesen sein mögen, die Erkenntnispotentiale des Projekts sind noch nicht ausgeschöpft. Zuvor wurden mehrfach einzelne Forschungsfragen benannt, die sich aus den bisherigen Arbeiten ergeben haben und künftig weiter verfolgt werden könnten. In diesem letzten Abschnitt soll nun eine breitere Perspektive eingenommen und gefragt werden, wie das Projekt insgesamt auf Grundlage der bisherigen Erkenntnisse fortgeführt werden könnte.

\subsection{Bisherige Erträge}

Ohne die bisherigen Erfolge im Geringsten zu schmälern, lässt sich festhalten, dass die beiden großen Hoffnungen, die diesem Ansatz zu Anfang des vorliegenden Resümees zugeschrieben wurden, noch nicht vollends eingelöst sind. Denn weder lassen sich auf Ebene einer Makrobetrachtung der verglichenen Regionen gesicherte Aussagen treffen über divergierende Werthaltungen und deren Manifestationen im Recht der Menschen mit Behinderung. Noch könnten in einer Mikroperspektive auf 
Grundlage der bisherigen Erkenntnisse fundierte Empfehlungen zur Übertragung einzelner Maßnahmen von einer Region in die andere getroffen werden.

Gewiss wurden in beiderlei Hinsicht erhebliche Fortschritte erzielt. So konnte erstens das Arsenal denkbarer Regelungsinstrumente in sowohl materieller als auch prozeduraler als auch institutioneller Hinsicht angereichert werden, und zwar in einem Ausmaß, wie es wohl nur in einem derart ausgreifenden Vergleich möglich ist. Zweitens sind auch die Hypothesen über das Bestehen und die Relevanz erheblicher - im weiten Sinne: - „kultureller“ Unterschiede zwischen den betrachteten Regionen konkretisiert und erhärtet worden. Dem insbesondere sollte der Workshop ja dienen.

Und noch in einer dritten Hinsicht, die gleichsam dazwischen liegt, wurden wesentliche Fortschritte erzielt, nämlich beim Bemühen um einen Brückenbau zwischen diesen beiden Perspektiven, bei der Identifikation der konkreten Rahmenbedingungen also, die für das Funktionieren von Recht und Verwaltung maßgeblich sind. Vielleicht sind dies die wichtigsten Erträge. Jedenfalls wäre dies nicht untypisch für rechts- und wohl auch verwaltungsvergleichende Unternehmungen, wie das vorliegende Projekt eine ist. Diesseits von weitreichenden kulturwissenschaftlichen Erklärungsansätzen und praktisch verwertbarem Lösungsimport schärft sich im Wege eines solchen Vergleichs das Verständnis der mannigfaltigen Wechselbezüge von Rechts- und Verwaltungsstrukturen einerseits und ihrer sozialen Umgebung andererseits. In diesem Sinne war eingangs von einem rechts- und verwaltungssoziologischen Erkenntnisinteresse die Rede.

\subsection{Ausrichtung weiterer Schritte}

Freilich sollten die beachtlichen Erträge, die das Projekt bislang erbracht hat, nicht als Grund dafür dienen, weitere Schritte zu unterlassen. Dafür haben sich allzu viele fruchtbare Perspektiven eröffnet während der bisherigen, nicht umsonst durchwegs als Explorationen konzipierten Tagungen. Und auch eine mögliche Unerreichbarkeit der zuvor als „große Hoffnungen“ apostrophierten Maximalziele der Vergleichung sollte keineswegs zur Grundlage für den Abbruch der hierauf gerichteten Bemühungen werden. Im Gegenteil ist es gerade die systematische Verfolgung dieser Ziele und idealiter beider zusammen -, welche den Gewinn jedenfalls der recht- und verwaltungssoziologischen Verständnisschärfung ermöglicht.

Wenn das Projekt demnach fortgeführt werden sollte, so gilt es zum Abschluss noch zu erörtern, wie es weitergehen könnte. An Anknüpfungspunkten - vor allem dies sollte zuvor illustriert werden - besteht kein Mangel, und eine Auswahl daraus zu treffen, überstiege die Bestimmung des vorliegenden Beitrags. Deswegen sollen hier nur zwei Prinzipien angedeutet werden, die bei den Überlegungen über den Zuschnitt der nächsten Projektschritte vielleicht Berücksichtigung finden könnten. 


\subsubsection{Ausgleich bestehender Asymmetrien}

Der bisherige Projektverlauf ist von einer doppelten Asymmetrie gekennzeichnet: einer disziplinären und einer regionalen. Es hätte anders auch kaum sein können, muss doch die Annäherung an ein solches Groß-Projekt irgendwo ihren Ausgangspunkt nehmen, um von dort aus dann allmählich das gesamte Feld zu erschließen.

Beim vorliegenden Projekt war dieser Ausgangspunkt im diszisplinären Sinne die Rechts- und Verwaltungswissenschaft. Dies wurde zuvor im Einzelnen dargelegt, einschließlich der Schritte, die bereits unternommen worden sind, um diese anfängliche Beschränkung zu transzendieren.

In regionaler Hinsicht lag der Schwerpunkt bisher auf der Betrachtung Europas. Dabei handelt es sich um eine Implikation weniger des ursprünglichen Projektdesigns, das von vornherein in zumindest gleicher Weise auf asiatische Länder fokussiert war. Vielmehr dürfte diese regionale Asymmetrie bislang schlicht der Lokalität der bisherigen Tagungen geschuldet sein, die allesamt in Deutschland stattfanden. Im Ergebnis freilich ist dadurch die Detailschärfe der auf Europa und insbesondere Deutschland bezogenen Betrachtungen insgesamt deutlich höher ausgefallen als bei ihren asiatischen Pendants.

Für die nächsten Schritte erscheint es angezeigt, diese Asymmetrien auszubalancieren. In regionaler Hinsicht wird man diesem - übrigens allseits konsentierten Petitum bereits durch die Wahl des nächsten Tagungsorts Genüge tun können. In disziplinärer Hinsicht dagegen handelt es sich um eine anspruchsvollere Aufgabe. Denn um jene „soziale Ambiance“, deren systematische Erkundung auf dem hier resümierten Workshop erst begonnen hat, weiter zu erforschen, wird man die verschiedenen einschlägigen Zweige der Sozial- und Kulturwissenschaften noch deutlich weiter verfolgen müssen.

Dass dies der Mühe wert ist, hat der Workshop demonstriert. Zugleich hat er hierfür Anknüpfungspunkte geliefert und auch die Richtungen angezeigt, in welche die künftigen Forschungsfragen weisen sollten. Die größte Herausforderung könnte demnach darin bestehen, Experten aus den jeweiligen Disziplinen und Regionen zu finden, die diese speziellen Fragen behandeln. Bekanntlich kommt es nicht selten vor, dass jene Klärungsbedarfe, die Rechts- oder Verwaltungswissenschaft mit Blick auf die übrigen Sozialwissenschaften formulieren, nicht beantwortet werden (können). Es ist insofern absehbar, dass man sich bei der Bestimmung künftiger Arbeitsschwerpunkte auch an der Verfügbarkeit entsprechender sozialwissenschaftlicher Expertise wird orientieren müssen.

\subsubsection{Exemplarische Fokussierung}

Mit der Erwähnung künftiger Arbeitsschwerpunkte ist auch der zweite Orientierungspunkt für die künftige Projektplanung bereits angesprochen. Eines der größten Verdienste der bisherigen Arbeitsschritte innerhalb des Projektes war es, dass die Breite und Komplexität des Themas erfasst und konturiert worden sind - zunächst, 
wie gesehen, mit Blick vor allem auf die Rechts- und Verwaltungswissenschaft, im dritten Schritt nun auch zunehmend in einer im weiten Sinne soziologischen oder kulturwissenschaftlichen Perspektive. Wenn dabei aber zugleich wiederholt auch das Bedürfnis nach weiterer Vertiefung augenfällig geworden ist, so versteht es sich nahezu von selbst, dass man derartige Vertiefungen aus praktischen Gründen nicht auf der gesamten Breite des über drei Tagungen hinweg immer weiter entfalteten Themas wird leisten können.

Bei den nun anstehenden Vertiefungen wird man daher nach einzelnen, enger definierten Betrachtungsgegenständen Ausschau halten müssen. Das bedeutet nicht, dass damit die bisher angestrebte Gesamtperspektive und die damit verbundenen Erklärungsambitionen aufgegeben werden müssten. Das Ziel sollte vielmehr sein, die Gegenstände möglicher Vertiefungen so zu wählen, dass die dabei gewonnenen Erkenntnisse einer Verallgemeinerung zugänglich sein könnten.

Welche Fragestellungen sich für solche exemplarischen Vertiefungen besonders eigenen, kann man und haben auch die Tagungsteilnehmer im Rahmen der Schlussdiskussion unterschiedlich beurteilt. In Frage käme beispielsweise, anknüpfend an unterschiedliche Behinderungsursachen vertiefende Betrachtungen anzustellen, also etwa von Geburt an bestehende Behinderungen einerseits und alterungsbedingte andererseits näher in den Blick zu nehmen. Dafür spräche nicht nur, dass sich die vermuteten kulturellen Unterschiede im Umgang mit Behinderten am Gegenstand „angeborener“ Behinderungen wahrscheinlich besonders deutlich manifestieren würden. Auch und vor allem könnte man bei dieser Einteilung mit dem Schwerpunkt auf alterungsbedingte Behinderungen ein Thema weiter verfolgen, dessen regionenübergreifende Brisanz der hier resümierte Workshop besonders unterstrichen hat.

Einer anderen Logik folgend, könnten bei den anstehenden Vertiefungen unterschiedliche Lebensphasen in den Blick genommen werden, also etwa Ausbildung, Erwerbsleben und die Phase danach. Diese Einteilung hätte zum einen für sich, dass die bestehenden Maßnahmen der Behindertenpolitik zum Teil ähnlich gegliedert sind, was die Aufbereitung erleichtern würde. Zum anderen wäre es auch bei diesem Zugriff wieder möglich, dem Konnex von demographischem Wandel und Behinderung weiter nachzugehen, und zwar beiden Facetten, die zuvor im Zusammenhang mit dem Beitrag von Kruse dargestellten wurden.

Eine Festlegung hinsichtlich der Gegenstände vertiefter Betrachtungen liegt, wie gesagt, jenseits der Zielsetzungen dieses Beitrags. Zumal bei einer solchen Festlegung neben systematischen ferner auch pragmatische Erwägungen eine wichtige Rolle spielen werden, dürften allzu enge Festlegungen im gegenwärtigen Stadium auch noch nicht tunlich sein. Stattdessen liegt es nahe, bis auf Weiteres auf Grundlage einer Sammlung möglicher Schwerpunktthemen zu operieren, zu der die hier genannten zählen, aber durchaus auch noch weitere hinzu treten könnten. 
MPI für ausländisches und internationales Sozialrecht, München

Deutsche Hochschule für Verwaltungswissenschaften, Speyer

Japan Zentrum der LMU, München

Referenten- und Teilnehmerliste des Workshops

„Kulturwissenschaftliche Grundlegung und Erklärungshypothesen divergenter Politiken, sowie Rechtsetzung für Menschen mit Behinderung in Europa und Asien unter den Bedingungen des demographischen Wandels“

gefördert von der Robert Bosch Stiftung

vom 20. - 24. Oktober 2007 im Kardinal Wendel Haus, München 
Prof. Dr. Makoto Arai, LL.M.

The University of Tsukuba

Soto Kanda 1-18-13

Tokyo 101-0021 / Japan

Tel.: +81-50-5518-2663

Fax: +81-3-3252-6741

e-mail: makotoar@guitar.ocn.ne.jp

Dr. Wolfgang Brenn; Leiter Projektmanagement

Japanisch-Deutsches Zentrum Berlin (JDZB)

Saargemünder Str. 2

D-14195 Berlin

Tel.: +49-30-83907-154

Fax: +49-30-83907-220

e-mail:wbrenn@jdzb.de

Prof. Dr. Kwang-Seok Cheon

College of Law

Yonsei University

134 Shinchon -dong, Sudaemun-ku

Seoul 120-749 / Korea

Tel.: +822-361-2987, 2988

Fax: +822-313-0822

e-mail: cheon@yonsei.ac.kr

Dr. Ding Na

Pfeuferstr. 33

D-81373 München

Tel.: +49-89-7553939

e-mail: xishui@aol.com

Mag. jur. Dipl.-Psych. Alexander Drewes, LL.M.

Am Sandkopf 44

D-34127 Kassel

Tel.: +49-561-2861020-0

Fax: +49-561-2861020-1

e-mail: Drewes.alexander@web.de 
Dr. William Gnanasekaran, Ph. D.

Selection Grade Lecturer

Madras Christian College, Chennai/Indien

Department of Public Administration

Tambaram, Chennai-600059

Tamil Nadu / India

Tel.: +44-22390-675

e-mail:dwgnanasekaran@hotmail.com

Prof. Dr. Alexander Graser

Professor of Comparative Public Law and Social Policy

Hertie School of Governance

Schlossplatz 1

D-10178 Berlin

Tel: $+49-30-212312-314$

Fax: +49-30-212312-888

e-mail: graser@hertie-school.org

Dr. Maksat Kachkeev, LL.M.

Institut für Ostrecht

Klosterstr. 79d

D-50931 Köln

Tel: +49-221-470-5591 oder -5575

Fax: +49-221-470-5582

e-mail: xmaksat@hotmail.com

Dr. Peter A. Köhler; Wissenschaftlicher Referent

Max-Planck-Institut für ausländisches und internationales Sozialrecht

Amalienstraße 33

D-80799 München

Tel.: +49-89-38602-424

Fax: + 49-89-38602-490

e-mail: koehler@mpisoc.mpg.de

Dr. Yasemin Körtek; Wissenschaftliche Referentin

Max-Planck-Institut für ausländisches und internationales Sozialrecht

Amalienstraße 33

D-80799 München

Tel.: +49-89-38602-408

Fax: +49-89-38602-490

e-mail: koertek@mpisoc.mpg.de 
Prof. Dr. Andreas Kruse

Ruprecht-Karls Universität Heidelberg

Institut für Gerontologie

Bergheimer Straße 20

D-69115 Heidelberg

Tel.: +49-6221-548180

Fax: +49-6221-545961

e-mail: andreas.kruse@gero.uni-heidelberg.de

Prof. Dr. Ming Cheng Kuo

Department of Law National Chengchi University

116 Mucha

Taipei/Taiwan ROC

Tel.: +886-2-2662-9518 oder 7518

Fax: +886-2-2939-0212

e-mail:mjguo@nccu.edu.tw

Klaus Lachwitz, Justitiar

Bundesvereinigung Lebenshilfe

Raiffeisenstraße 18

D-35043 Marburg

Tel.: +49-6421-491-159 oder 144 (Vorzimmer)

e-mail: klaus.lachwitz@lebenshilfe.de

Claudia Laes, Wissenschaftliche Mitarbeiterin

Max-Planck-Institut für ausländisches und internationales Sozialrecht

Amalienstraße 33

D-80799 München

Tel.: +49-89-38602-402

Fax: +49-89-38602-490

e-mail: laes@mpisoc.mpg.de

Prof. Dr. Jef van Langendonck

Katholieke Universiteit Leuven

Tiensestraat 41

3000 Leuven / Belgien

Tel.: +32-16-325-410

Fax: +32-16-325-424

e-mail: Jozef.vanlangendonck@law.kuleuven.ac.be 
Prof. Dr. Bernd Baron v. Maydell; Wissenschaftliches Mitglied und ehem. Direktor Max-Planck-Institut für ausländisches und internationales Sozialrecht

Amalienstraße 33

D-80799 München

Tel.: +49-89-38602-407

(priv.) Siebengebirgsstraße 58a

D-53757 Sankt Augustin

Tel.: +49-2241-338818

Fax: + 49-2241-342706

e-mail: c.v.maydell@t-online.de

Frau Prof. Miyoko Motozawa

Institut of Social Science

University of Tsukuba

1-1-1 Tennodai Tsukuba-shi

Tsukuba 305-8571 / Japan

Tel.: +81-298-53-4061

Fax: +81-298-53-4061

e-mail: motozawa@social.tsukuba.ac.jp

Prof. Dr. Frans Pennings

Universiteit van Tilburg

P.O. Box 90153 Room Y 141

5000 LE Tilburg

Niederlande

Tel.: +31-13-4662157

Fax: +31-13- 4663139

e-mail: f.j.l.pennings@uvt.nl

Univ. Prof. Dr. Dr. h.c. Rainer Pitschas, Master of Public Administration

Senatsbeauftragter für das Ausländer-(Aufbau-)Studium

Lehrstuhl für Verwaltungswissenschaft, Entwicklungspolitik und Öffentliches Recht

Deutsche Hochschule für Verwaltungswissenschaften

Freiherr vom Stein Straße 2

D-67346 Speyer

Tel.: +49-6232-654-345 oder 338 (Sekretariat)

Fax: +49-6232-654-305

e-mail: rpitschas@dhv-speyer.de 
Prof. Dr. Peter Pörtner; Leitung

Japan Zentrum der LMU München

Oettingenstraße 67

D-80538 München

Tel.: +49-89-2180-9830

Fax: +49-89-2180-9827

e-mail: p.poertner@Irz.uni-muenchen.de

Burkard Rappl; Ministerialdirigent

Abteilung Rehabilitation im Bayerischen Staatsministerium für Arbeit und Sozialordnung, Familie und Frauen

Winzererstraße 9

80797 München

Tel.: +4989-1261-1187 (Vorzimmer)

e-mail: burkard.rappl@stmas.bayern.de

Dr. Hans-Günther Ritz

Behörde für Soziales, Familie, Gesundheit und Verbraucherschutz

Ref. Soziale Entschädigung/ Rehabilitation (SGB IX)

Adolph-Schönfelder- Str. 5

D-22083 Hamburg

Tel. +49-40-42863-2845

Fax +49-40-427961-130

e-mail: hans-guenter.ritz@bsg.hamburg.de

Sarah Scholl; Studentische Mitarbeiterin

Max-Planck-Institut für ausländisches und internationales Sozialrecht

Amalienstraße 33

D-80799 München

Tel.: +49-89-38602-402

Fax: +49-89-38602-490

Dr. Bernd Schulte; Wissenschaftlicher Referent

Max-Planck-Institut für ausländisches und internationales Sozialrecht

Amalienstraße 33

D-80799 München

Tel.: +49-89-38602-426

Fax: $+49-89-38602-490$

Priv.: +49-89-7605791

e-mail: schulte@mpisoc.mpg.de 
Prof. Hitohiro Takizawa

Faculty of Law, Momoyamagakuin University

1-1 Manabino Izumi-Shi

Osaka 594-1198 / Japan

Tel.: +81-725-54-3131

Fax: +81-725-54-3202

e-mail: takizawa@andrew.ac.jp

Dr. Werner Tegtmeier; Staatssekretär a.D.

Am Park 85

D-53757 St. Augustin

Tel.: +49-2241-331394

Fax: +49-2241-342417

Prof. Dr. Peter Trenk-Hinterberger

Lehrstuhl für Arbeits- und Sozialrecht

Universität Bamberg

Feldkirchenstraße 21

D-96045 Bamberg

Tel.: +49-951-863-2636

Fax.: +49-951-863-2640

E-Mail: peter.trenk-hinterberger@sowi.uni-bamberg.de

Prof. Dr. Petr Tröster

Univerzity Karlovy V Praze

Právnická fakulta

Nám. Curieových 7

11640 Praha 1 / Tschechien

Tel: +42-2-21005-362

Fax: +42-2-21005-296

e-mail: troster@prf.cuni.cz

Soh-Yeon Won, Master of Public Administration

Gerhart-Hauptmann Straße 3

67346 Speyer

e-mail: sohyeonwon@googlemail.com

Ass. Prof. Dr. Urs Matthias Zachmann

Japan Zentrum der LMU München

Oettingenstraße 67

D-80538 München

Tel.: +49-89-2180-9823

Fax: +49-89-2180-9801

e-mail: U.Zachmann@Irz.uni-muenchen.de 